\title{
Analysis of historical solar observations and long-term changes in solar irradiance
}

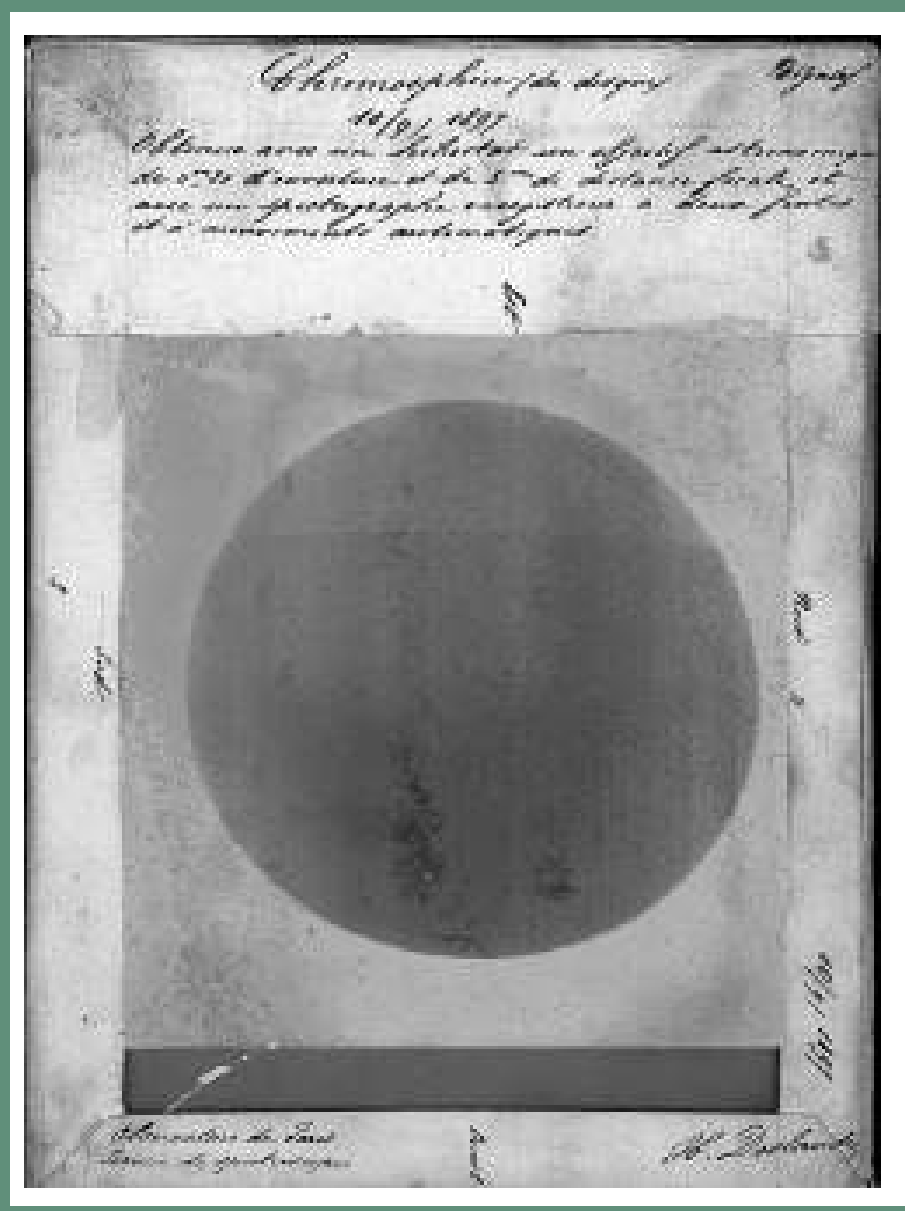

\section{Theodosios Chatzistergos}

International Max Planck Research School for Solar System Science at the University of Göttingen 



\title{
Analysis of historical solar observations and long-term changes in solar irradiance
}

\author{
Dissertation \\ zur Erlangung des mathematisch-naturwissenschaftlichen Doktorgrades \\ "Doctor rerum naturalium" \\ der Georg-August-Universität Göttingen \\ im Promotionsprogramm PROPHYS \\ der Georg-August University School of Science (GAUSS)
}

vorgelegt von

\section{Theodosios Chatzistergos}

aus Kos, Griechenland

Göttingen, 2017 
Betreuungsausschuss

Prof. Dr. Ansgar Reiners

Institut für Astrophysik, Georg-August-Universität, Göttingen

Prof. Dr. Sami K. Solanki

Max-Planck-Institut für Sonnensystemforschung, Göttingen

Dr. Natalie A. Krivova

Max-Planck-Institut für Sonnensystemforschung, Göttingen

Mitglieder der Prüfungskommision

Referent: Prof. Dr. Sami K. Solanki

Max-Planck-Institut für Sonnensystemforschung, Göttingen

Korreferent: Prof. Dr. Wolfram Kollatschny

Institut für Astrophysik, Georg-August-Universität, Göttingen

2. Korreferent: Dr. Yvonne Unruh

Imperial College London, Astrophysics, Blackett Laboratory, London

Weitere Mitglieder der Prüfungskommission:

Prof. Dr. Ariane Frey

Institut für Astrophysik, Georg-August-Universität, Göttingen

Dr. Natalie A. Krivova

Max-Planck-Institut für Sonnensystemforschung, Göttingen

Prof. Dr. Ansgar Reiners

Institut für Astrophysik, Georg-August-Universität, Göttingen

PD Dr. Olga Shishkina

Max-Planck-Institut für Dynamik und Selbstorganisation, Göttingen

Tag der mündlichen Prüfung: 02.06.2017 


\section{Bibliografische Information der Deutschen Nationalbibliothek}

Die Deutsche Nationalbibliothek verzeichnet diese Publikation in der Deutschen Nationalbibliografie; detaillierte bibliografische Daten sind im Internet über http: //dnb . d-nb . de abrufbar.

ISBN 978-3-944072-55-5

uni-edition GmbH 2017

http://www.uni-edition.de

(C) Theodosios Chatzistergos

(C) (i) This work is distributed under a

Creative Commons Attribution 3.0 License

Printed in Germany 



\section{Contents}

\begin{tabular}{ll}
\hline Summary & 7
\end{tabular}

\begin{tabular}{ll}
\hline Abbreviations & 9
\end{tabular}

\begin{tabular}{lll}
\hline & Introduction & 11
\end{tabular}

1.1 Sun-climate connection . . . . . . . . . . . . . . . . . 11

1.2 The Sun . . . . . . . . . . . . . . . . . . . . . . . . 13

1.2 .1 Solar interior . . . . . . . . . . . . . . . . . . . . . . . . . 14

1.2 .2 Solar atmosphere and limb darkening . . . . . . . . . . . . 14

1.3 Magnetic activity $\ldots \ldots \ldots \ldots \ldots \ldots$

1.3 .1 Magnetic field generation . . . . . . . . . . . . . . . . 16

1.3 .2 Active features . . . . . . . . . . . . . . . . . . . . . . . . . . . . . . .

1.3 .3 Solar cycle . . . . . . . . . . . . . . . . . 21

1.4 Solar irradiance . . . . . . . . . . . . . . . . . . . . . . 24

1.4.1 Measurements of irradiance . . . . . . . . . . . . . . . . . . 24

1.4 .2 Irradiance reconstructions . . . . . . . . . . . . . . . . . . . . . 25

1.4.2.1 Proxies of solar activity . . . . . . . . . . . 25

1.4.2.2 Irradiance models . . . . . . . . . . . . . . . . . . . . . 29

1.5 Motivation for this thesis . . . . . . . . . . . . . . . 31

\begin{tabular}{|ll|}
\hline Ca II K observations & 33 \\
\hline
\end{tabular}

$2.1 \quad$ Ca II K line . . . . . . . . . . . . . . . . . . . . . . . . . . . . 33

2.2 Observing instruments . . . . . . . . . . . . . . . . . . 34

2.2 .1 Spectroheliograph $\ldots \ldots \ldots \ldots$. . . . . . . . . . . . . . . . . . . . . . . . . . 34

2.2 .2 Filtergraph $\ldots \ldots \ldots \ldots \ldots \ldots \ldots$

2.3 Observational aspects $\ldots \ldots \ldots \ldots \ldots \ldots$

2.4 Photographic processes . . . . . . . . . . . . . . . . . . . . . . . . . . . . . .

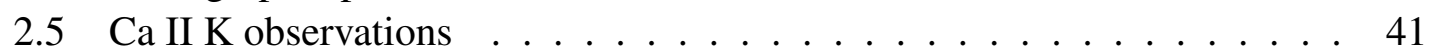

2.6 Processing techniques . . . . . . . . . . . . . . . . . . . . . . 44

2.6 .1 Photometric calibration methods . . . . . . . . . . . . . . . . . . . . . . . 44

2.6.1.1 Calibration wedges . . . . . . . . . . . . 44

2.6.1.2 Alternative calibration methods . . . . . . . . . . . 46

2.6 .2 Methods of the background computation . . . . . . . . . 47

2.7 Plage areas . . . . . . . . . . . . . . . . . . . . . . . 49

2.8 Conclusions $\ldots \ldots \ldots \ldots \ldots$ 
3 Photometric calibration and artefact correction of Ca II K observations 53

3.1 Introduction . . . . . . . . . . . . . . . 53

3.2 Method description . . . . . . . . . . . . . . . . 54

$3.2 .1 \quad$ Background computation . . . . . . . . . . . . . . . . . . . . . . . . . . . . 54

3.2 .2 CLV extraction . . . . . . . . . . . . . . . . . . . . . . . . . . . . . 58

3.2 .3 Photometric calibration . . . . . . . . . . . . . . . . 59

3.3 Performance of the method . . . . . . . . . . . . . . . . . 59

3.3 .1 Synthetic data . . . . . . . . . . . . . . . . 59

3.3.2 Analysis of the synthetic data . . . . . . . . . . . . . . 69

3.3.3 $\quad$ Performance on individual steps of the processing . . . . . . . . . 83

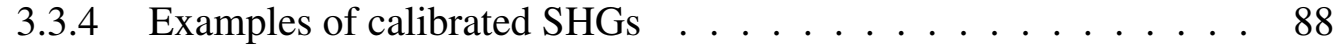

3.4 Comparison with other methods . . . . . . . . . . . . . . . . . 91

$3.4 .1 \quad$ Background calculation methods . . . . . . . . . . . . . . . . 91

3.4 .2 Calibration methods . . . . . . . . . . . . . . . . . . 92

3.5 Conclusions . . . . . . . . . . . . . . . . . 95

4 Plage areas time-series from Ca II K observations 103

4.1 Introduction . . . . . . . . . . . . . . . . . . 103

4.2 Segmentation schemes $\ldots \ldots \ldots$. . . . . . . . . . . . . . 103

4.3 Test of different segmentation schemes on modern data . . . . . . . . . . 105

4.3 .1 AR coverage areas . . . . . . . . . . . . 106

4.3.2 Average contrast of ARs . . . . . . . . . . . . . . . 107

4.3 .3 AR size-contrast relation . . . . . . . . . . . . . . . . . . 110

4.3 .4 Discussion . . . . . . . . . . . . . . . . . 112

4.4 Effect of photometric calibration on derived disc fractions . . . . . . . . 117

4.5 Segmentation of historical data . . . . . . . . . . . . . . . 121

4.5 .1 Processing . . . . . . . . . . . . . . . 121

4.5 .2 Plage areas . . . . . . . . . . . . . . . . . . . . . . . . . . . . 126

4.5 .3 Contrast of plage and network . . . . . . . . . . . . . 128

4.6 Conclusions . . . . . . . . . . . . . . . . . . . . . 130

5 Relationship between the Ca II K intensity and the photospheric magnetic field 135

$5.1 \quad$ Introduction . . . . . . . . . . . . . . . . . . . . . 135

5.2 Data and methods . . . . . . . . . . . . . . . . . 137

5.2 .1 Data . . . . . . . . . . . . . . . . . . 137

5.2 .2 Data reduction . . . . . . . . . . . . . . . . . 139

5.2 .3 Stray-light removal . . . . . . . . . . . . . . . . . . . . . 140

5.2 .4 Segmentation . . . . . . . . . . . . . . . . . . . . . 141

5.3 Results . . . . . . . . . . . . . . . . . . . . . . . . . . . 142

5.3 .1 Physical relationship . . . . . . . . . . . . . . . . 142

5.3.2 Exponents over time and different $\mu$ positions . . . . . . . . . 145

5.3 .3 Exponents for individual activity clusters . . . . . . . . . . . . 145

5.3 .4 Reconstructing unsigned magnetograms from Ca II K images . . 155

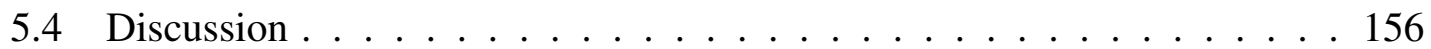

5.5 Summary and conclusions $\ldots \ldots \ldots \ldots 16 \ldots$ 
\begin{tabular}{llr}
\hline 6 & Group sunspot number series & 169
\end{tabular}

6.1 Introduction . . . . . . . . . . . . . . . . . . . . 169

$6.1 .1 \quad$ Sunspot observations . . . . . . . . . . . . . . . . . . . 169

6.1 .2 Sunspot classification schemes . . . . . . . . . . . . . 171

$6.1 .3 \quad$ Sunspot number series $\ldots \ldots \ldots$. . . . . . . . . . . . . . 172

6.2 Dependence of the Sunspot-Group Size on the Level of Solar Activity and

its Influence on the Calibration of Solar Observers . . . . . . . . . . . 179

6.2 .1 Data . . . . . . . . . . . . . . . . . . 180

6.2.2 Distribution of sunspot-group sizes5]The contents of this subsection are identical to the printed version of Usoskin, I. G., Kovaltsov, G. A., Chatzistergos, T., Solar Physics, 291, 3793-3805, 2016, with the permission of Springer. . . . . . . . . . . . 180

6.2 .3 Relation between records of observers with different acuities . . . 181

6.2 .4 A test: Wolf vs. Wolfer . . . . . . . . . . . . . . . . . . . 184

6.3 Summary and conclusions . . . . . . . . . . . . . . . . . 190

7 New reconstruction of the sunspot group numbers since 1739 using direct calibration and "backbone" methods 191

7.1 Introduction . . . . . . . . . . . . . . . . . . . . . . 192

7.2 Data . . . . . . . . . . . . . . . . . . . . . . . . . . . . . . . . . . 194

7.3 Calibration process $\ldots \ldots \ldots \ldots$. . . . . . . . . . . . . . . . . . . . . . . 195

7.3.1 Algorithm and primary observers . . . . . . . . . . . . . . . 195

7.3 .2 Secondary observers . . . . . . . . . . . . . . . . . . . 197

7.3 .3 Construction of the backbone series . . . . . . . . . . . . . 197

7.3.3.1 Sparse data: Schwabe and earlier backbones . . . . . . 201

7.3.3.2 Correcting for low quality observations: Flaugergues, Schubert, Zucconi, and Hagen backbones . . . . . . . . 201

7.3 .4 Inter-calibration of backbone series . . . . . . . . . . . . . . . . . . 201

7.3 .5 Construction of the final series . . . . . . . . . . . 203

7.4 Validation of the results . . . . . . . . . . . . . . . . . 204

7.4 .1 Comparison with other series . . . . . . . . . . . . . . . . . 204

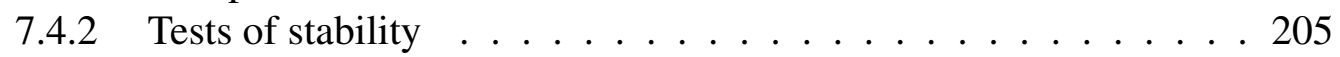

7.4.2.1 Choice of backbone observers . . . . . . . . . 205

7.4.2.2 Shape of the matrix . . . . . . . . . . . . 208

7.4.2.3 Quality of the RGO dataset . . . . . . . . . . . . . . 208

7.4.2.4 Other issues . . . . . . . . . . . . . . . . . 209

7.5 Summary and conclusions $\ldots . . \ldots 209$

\begin{tabular}{|ll}
\hline 8 & Summary and outlook \\
\hline
\end{tabular}

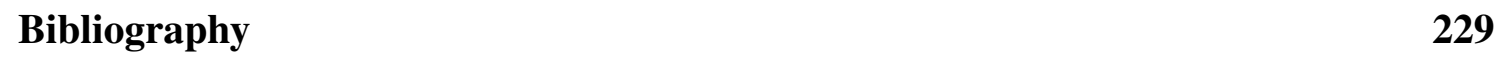

\begin{tabular}{ll}
\hline Scientific contributions & 261
\end{tabular}

\begin{tabular}{ll}
\hline Acknowledgements & 263
\end{tabular}

\begin{tabular}{ll}
\hline Curriculum vitae & 265
\end{tabular} 



\section{Summary}

The Sun is the main external driver of Earth's climate. Various mechanisms of the solar influence on climate have been proposed. The debate is ongoing, but variation in the radiative flux of the Sun is among the main candidates. Direct measurements of the solar irradiance exist for merely 40 years, which is a rather short period to derive conclusions about any possible long term changes in solar irradiance and their possible influence on climate.

The main driver of the irradiance variations on time-scales of days to decades, and possibly longer, is believed to be the solar surface magnetism. Models have been developed that reconstruct the irradiance by using appropriate proxies of the magnetic activity of the Sun. Irradiance models require input data representing both dark and bright magnetic features emerging at the solar surface. The most widely ever used proxies are the sunspot areas (available since 1874), the sunspot number (available since 1700), and the sunspot group number (available since 1610). However, these records do not provide direct information on bright features. Their evolution has to be inferred from the sunspot data via certain assumptions whose justification is still very unclear. Therefore, there is a strong need for a more direct facular proxy.

Ca II K full-disc spectroheliograms are uniquely suited for that purpose. Observations in the Ca II K line started as early as in 1892 at various sites, providing a good temporal coverage of the whole 20th century. However, these data suffer from a variety of problems hindering their immediate applicability. The historical Ca II K observations are stored in photographic plates or films, which have a non-linear response to the incident radiation. Information on this relation has not been recorded for the majority of the historical observations. Furthermore, a plethora of artefacts have been introduced on these images during their various life stages. These artefacts need to be accurately accounted for in order to provide meaningful results from such data.

We have developed a method to recover the relation for the response of the plates to the incident radiation by using information that is stored on the solar disc of the image. This method is based on the assumption that the darker parts of the quiet Sun regions remain unchanged in time. We have also developed a method of calculating the quiet Sun background of the images, which includes the centre-to-limb variation and takes into account various large-scale artefacts. We have shown that the accuracy of this method is greater than that of previously proposed techniques.

We have also reassessed the relation between the magnetic field strength and the $\mathrm{Ca}$ II K contrast, by using a larger number of $\mathrm{Ca}$ II images than was done in earlier such studies. We find that this relation can be well described with a power law function, and the best fit parameters are unaffected by the activity level or the position on the disc. Hence this relation potentially allows a reconstruction of pseudo-magnetograms from the 
available Ca II K observations covering almost the whole 20th century, that can be used by irradiance models.

The sunspot data (i.e. records of sunspot number), despite their extensive use, are not free of problems either. The process of cross-calibrating different records by individual observers has recently become a matter of debate. This debate brought to light that the majority of the methods used so far fail to take into consideration the non-linearity that arises due to different observing capabilities of the observers. We addressed the issue of the shape of the relation for the inter-calibration between different group sunspot number series. We have shown, with the aid of synthetic observations derived from the royal Greenwich observatory sunspot area records, that it is strongly non-linear, contrary to what is commonly assumed. We have developed a method to recalibrate the sunspot group number series with a non-linear non-parametric method based on daily statistics between the observers. Using Monte Carlo simulations we have accounted for the error propagation, which has not been done by any previous reconstruction. Our reconstruction of the group sunspot number favours moderate activity levels for the 18th and 19th century and supports the existence of the modern grand maximum of solar activity.

This thesis is divided into 8 chapters. Chapter 1 contains some background physics about the Sun and the motivation for this work. Chapter 2 gives an introduction to $\mathrm{Ca}$ II K observations and data processing, including information about the instruments, the photographic processes and the storage of the photographic plates. Various available observations are described along with the different methods that have been used to process them and to produce plage area time-series. Chapter 3 describes our method to process the historical Ca II K observations. All the tests on the synthetic data and results of processing and calibrating the historical data are described in detail, and the results are compared with those from other published works. In Chapter 4 we test various segmentation methods and present preliminary results for the plage areas derived from the calibrated historical Ca II K observations. In Chapter 5, we reassess the relation between the Ca II K contrast and the magnetic field strength. Chapter 6 first reviews available sunspot observations and their composites, and then our study on the dependence of the sunspot-group size on the level of solar activity and its effects on the calibration of records of different observers. Chapter 7 presents our recalibration of the group sunspot number series. Our results are also compared to the previously published series. Finally, Chapter 8 gives a summary and the outlook of the thesis. 


\section{Abbreviations}

$\begin{array}{ll}\text { AAVSO } & \text { American Association of Variable Star Observers } \\ \text { ACRIM } & \text { Active Cavity Radiometer Irradiance Monitor } \\ \text { ADF } & \text { Active Day Fraction } \\ \text { Ar } & \text { Arcetri Observatory } \\ \text { AR } & \text { Active Region } \\ \text { AR5 } & \text { Assessment Report 5 (of the IPCC) } \\ \text { BB } & \text { Backbone } \\ \text { BBSO } & \text { Big Bear Solar Observatory } \\ \text { CC } & \text { Characteristic Curve } \\ \text { CCD } & \text { Charge-Coupled Device } \\ \text { CDF } & \text { Cumulative Distribution Function } \\ \text { CEA16 } & \text { Clette et al. } \text { (2016) International Sunspot Number Series } \\ \text { CFDT } & \text { Cartesian Full Disk Telescope } \\ \text { CILi16 } & \text { Cliver and Ling }(2016) \text { Sunspot Number Series } \\ \text { CLV } & \text { Centre-to-Limb Variation } \\ \text { CMF } & \text { Constant Multiplicative Factor } \\ \text { CNO } & \text { Carbon-Nitrogen-Oxygen } \\ \text { Co } & \text { Coimbra Observatory } \\ \text { COROT } & \text { Convection, Rotation and planetary Transits } \\ \text { CT } & \text { Constant Threshold } \\ \text { DoF } & \text { Degree of Freedom } \\ \text { FITS } & \text { Flexible Image Transport System } \\ \text { GCR } & \text { Galactic Cosmic Ray } \\ \text { GSN } & \text { Group Sunspot Number } \\ \text { HMI } & \text { Helioseismic and Magnetic Imager } \\ \text { HoSc98 } & \text { Hoyt and Schatten }(1998) \text { Group Sunspot Number Series } \\ \text { INAF } & \text { Istituto Nazionale di Astrofisica } \\ \text { IPCC } & \text { Intergovernmental Panel on Climate Change } \\ \text { ISN } & \text { International Sunspot Number } \\ \text { JPG } & \text { Joint Photographic Experts Group } \\ \text { Ko } & \text { Kodaikanal Observatory } \\ \text { LFL } & \text { Linear Function of a Logarithm } \\ \text { LOS } & \text { Line-Of-Sight } \\ \text { MC } & \text { Monte Carlo } \\ \text { MDI } & \text { Michelson Doppler Imager } \\ \text { HO } & \end{array}$




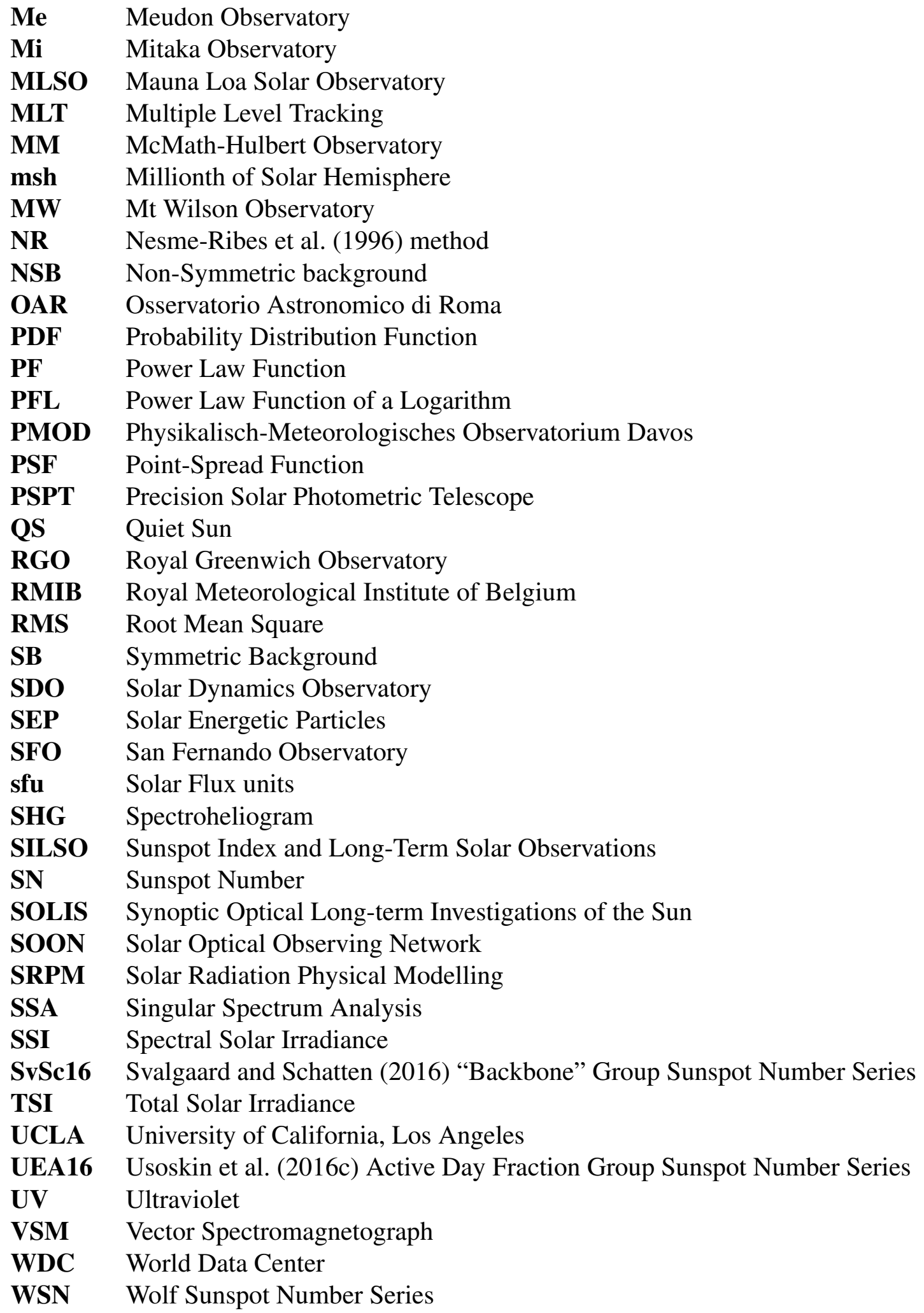




\section{Introduction}

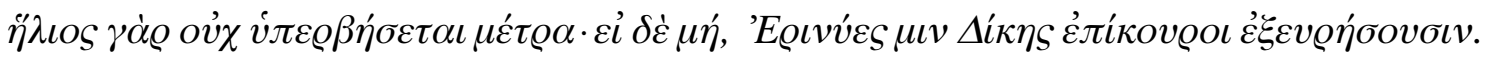

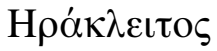

\subsection{Sun-climate connection}

The Sun has been worshipped since antiquity, being one of the first conceived deities. One of the reasons is its crucial influence on the conditions on Earth. Giving light and warmth, the Sun enables life to exist. Historically, the influence of the Sun on Earth had been considered unaltered, creating the idea of an unchanging Sun. However, sightings of "blemishes" or spots on the solar surface, with a roughly 11-year periodicity in their amount, were hinting that this was not the case. Galileo, Scheiner, and Riccioli were first to postulate a connection between the sunspots and the temperature on Earth (Benestad 2006). Herschel (1801) found a negative correlation between sunspot appearance and wheat price in London. His explanation was that the more spots are observed on the Sun the less rain will occur on Earth, thus modulating the wheat price. Since then, numerous studies searched for the evidence of solar influence on climate and its mechanisms (e.g. Haigh 1994, 1996, 1999, 2003, Cubasch et al. 1997, 2006, Hartley et al. 1998, Shindell et al.1999, Larkin et al. 2000, Kodera and Kuroda 2002, Tourpali et al.|2003, Krivova and Solanki|2004b, Matthes et al.|2006, Haigh|2007, Meehl et al.|2008, Frame and Gray 2009, Roy and Haigh 2012, Solanki et al.|2013, Xiang et al.2014, Cnossen et al. 2016, Dima and Voiculescu 2016, Foster et al. 2017, Sukhodolov et al. 2017). The exact mechanism (or possibly an interplay of multiple mechanisms) has not yet been identified. However, two of the most widely considered options are changes in the amount and spectral distribution of the solar energy entering the Earth system and changes in the galactic cosmic ray (GCR) flux and solar energetic particles (SEP).

Changes in the solar radiative energy flux entering the climate system have two main sources: changes in the Earth's orbital parameters and intrinsic solar variability. Changes in Earth's orbital elements, such as eccentricity, obliquity, and precession affect the amount of energy that reaches Earth and its distribution on the surface. Variations in Earth's orbital parameters introduce periodicities between about $20 \mathrm{Kyr}$ and $2 \mathrm{Myr}$ (Adhémar 1842, Croll 1875, Paillard 2001, Crucifix et al. 2006, Eldrett et al. 2015, Maher 2016). These are called Milankovitsch cycles and have a more or less known effect on climate, being considered the reason for the glacial and interglacial periods on Earth.

Intrinsic variability of the solar radiative flux includes changes in the total solar irradiance (TSI) and in the spectral solar irradiance (SSI). The TSI and SSI are defined as the spectrally-integrated and wavelength-resolved solar radiative flux at the top of the 
Earth's atmosphere at the mean distance of one astronomical unit. Figure 1.1 shows the spectrum of the Sun between 100 and 10,000 nm, its relative variation over the 11-year solar-cycle as well as the altitude in Earth's atmosphere to which radiation can penetrate. Two different mechanisms have been identified through which changes in solar irradiance can affect Earth's climate, the bottom-up and top-down mechanisms (Gray et al. 2010). The bottom-up mechanism involves irradiance reaching the surface, i.e. mainly in the visible or infra-red, where it causes heating. The globally averaged temperature variations over the solar cycle have been estimated to be roughly $0.07 \mathrm{~K}$ (White et al. 1997 , Gray et al. 2010). Heating of the surface introduces feedback mechanisms that increase the solar forcing. The most prominent such mechanism involves solar radiation that can reach sub-tropical sea surfaces. There it causes increased evaporation which eventually strengthens the hydrological cycle. This way the cloud coverage in the tropics and subtropics gets reduced thus allowing for more solar radiation to reach sea surfaces in these regions (e.g. Cubasch et al. 1997, Meehl et al. 2003, Cubasch et al. 2006, Meehl et al. 2008, Meehl and Arblaster 2009). The top-down mechanism involves the dynamical coupling between the stratosphere and troposphere (e.g. Haigh 1994, 1996, Hartley et al. 1998, Haigh 1999, Shindell et al. 1999, Kodera and Kuroda 2002, Shindell et al. 2006). The SSI in the UV varies by up to $100 \%$ (e.g. Floyd et al. 2003, Krivova et al.2006, Gray et al. 2010), which is much higher than variations of the TSI, and gets absorbed mainly in the stratosphere. The UV radiation causes ozone production as well as destruction, such that the net effect is an increase in the ozone production during high activity periods (Haigh 2007). The increased ozone concentrations during activity maxima reduce the irradiance in wavelengths $<330 \mathrm{~nm}$ and $>500 \mathrm{~nm}$ that can reach the troposphere. This affects the Earth-system non-linearly, and with strong spatial fluctuations.

There are also two suggested processes involving energetic particles. The first one involves the SEP events in the Earth's atmosphere. The SEP are accelerated into the heliosphere by solar flares or coronal mass ejections (Reames 2013) and hence their flux depends on the overall solar activity level (Hathaway 2015). SEPs, having rather high energies, are able to reach the Earth's atmosphere where they cause ionisation, dissociation and can eventually destroy ozone molecules (Solomon et al. 1982, Jackman et al. 2008, Sukhodolov et al. 2017). The second process involves the modulation of the GCR flux by solar activity. The interplanetary magnetic field and Earth's magnetic field act as a shield protecting Earth from charged particles. The solar wind carries the solar magnetic field, which varies with the activity of the Sun (e.g. Zank and Müller 2003). Therefore, the Sun modulates the flux of GCRs (e.g. Potgieter 2013). The higher the activity, the fewer energetic particles of GCRs are able to reach Earth. Cosmic rays within Earth's atmosphere have been suggested to destroy ozone, mostly at polar latitudes, where these particles are able to enter Earth's atmosphere. It has also been suggested that clouds can form due to cosmic rays (e.g. Ney 1959, Dickinson 1975, Svensmark and Friis-Christensen 1997, Marsh and Svensmark 2000, Svensmark et al. 2016). Nonetheless, the physical connection between GCRs and cloud formation has not been established yet and this effect is debated (e.g. Todd and Kniveton 2001, Laken et al. 2009, Pierce and Adams 2009, Čalogović et al. 2010, Kulmala et al. 2010, Dorman 2012, Laken et al. 2012, Laken and Calogović 2013). Possible energetic particle effects on the Earth's atmosphere are investigated by the CLOUD experiment at CERN. Although it has given some evidence for GCRs' influence on cloud formation, the significance level is low (Kirkby et al. 2011, 


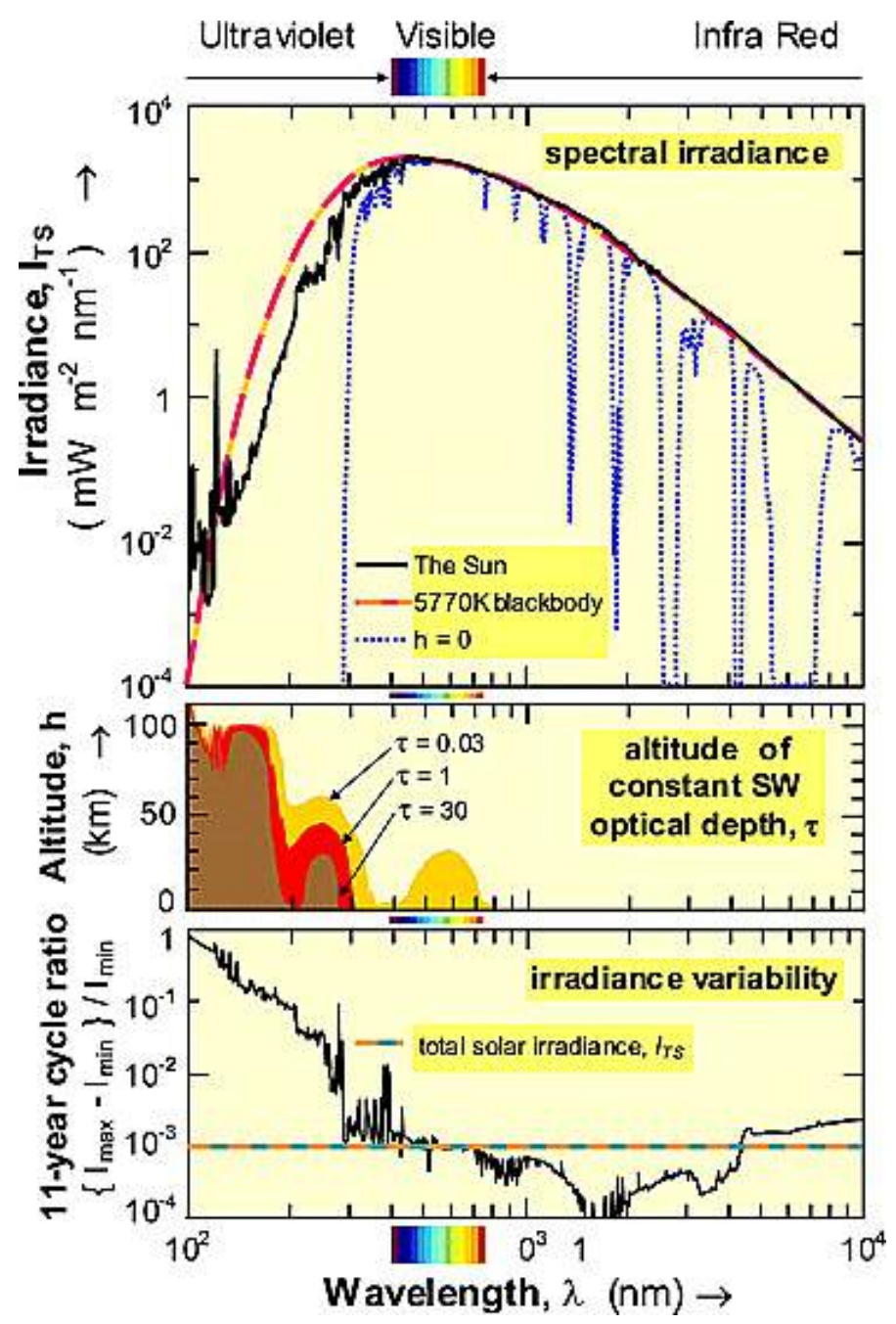

Figure 1.1: Top: Solar irradiance spectrum (black), and the spectrum of the radiation reaching the surface of the Earth (dotted blue). Also shown is the spectrum of a 5770 $\mathrm{K}$ blackbody (dashed red). Middle: Altitudes of radiation penetration at different wavelengths. Bottom: Relative variability of solar irradiance over the solar cycle. The variability level of TSI is marked with the horizontal dashed line. Taken from Gray et al. (2010).

Mironova et al. 2015, Dunne et al. 2016). Clouds and ozone concentrations increase Earth's reflectance. According to this conjecture, energetic particles affect the amount of energy reaching the surface.

The latest assessment report (AR5) by the Intergovernmental Panel on Climate Change (IPCC) estimates the radiative forcing due to solar irradiance changes to be $0.05 \pm 0.05$ $\mathrm{W} \mathrm{m}{ }^{-2}$ for the period $1750-2011$. With the anthropogenic forcing considered to be $2.3 \pm 1.0 \mathrm{~W} \mathrm{~m}^{-2}$, the Sun contributed up to $8 \%$ to the total radiative forcing over this period (Myhre et al. 2013). Importantly, however, the IPCC contends that the uncertainties in solar forcing are particularly high.

Accurate assessment of the solar influence on climate requires reliable records of solar irradiance over long periods, which, as we will discuss in Sect. 1.4, are not available. 
Without sufficiently long direct observations the secular trend of solar irradiance is rather speculative. Improving our knowledge of the Sun and understanding of the underlying physical mechanisms of the irradiance variations is the only feasible approach to infer the needed information in the past and reduce uncertainties in climate models.

\subsection{The Sun}

The Sun, being on average $1.496 \times 10^{8} \mathrm{~km}$ (Brumfiel 2012) from Earth (varying in the range $1.471-1.521 \times 10^{8} \mathrm{~km}$ within a year), is our nearest star that can be observed in great detail. The Sun is classified as an average G2V (Vaquero and Vázquez 2009) spectral type star lying on the main sequence of the Hertzsprung-Russell diagram. It is thought to be 4.6 Gyr old (e.g. Patterson 1956, Bonanno et al. 2002, Connelly et al. 2012) with mass $M=1.99 \times 10^{30} \mathrm{~kg}$ and radius $R=696,000 \mathrm{~km}$ (Schou et al. 1997, Antia 1998, Brown and Christensen-Dalsgaard 1998). It is considered to consist mostly of hydrogen and helium ( $\sim 75 \%$ and $\sim 24 \%$ by mass, respectively) with few heavier elements providing the remaining $\sim 1 \%$ in mass (Anders and Grevesse 1989, Grevesse and Sauval 2002, Lodders 2003, Basu and Antia 2008).

\subsubsection{Solar interior}

The solar interior is highly stratified and comprises a core (up to $\sim 0.25 R$ ), a radiative zone (up to $\sim 0.71 R$ Christensen-Dalsgaard et al. 1991, Basu and Antia 1997), and a convection zone reaching up to the solar surface. The temperature in the core is estimated to be $T_{c}=1.6 \times 10^{7} \mathrm{~K}$, while the density is $\rho_{c} \sim 1.5 \times 10^{5} \mathrm{~kg} \mathrm{~m}^{-3}$ (Carroll and Ostlie 2006), which are high enough values for hydrogen fusion to take place. The hydrogen fusion in the core is achieved mainly through the proton-proton chain reaction, and with a lower probability via the Carbon-Nitrogen-Oxygen (CNO) cycle (Adelberger et al. 2011). Both cycles have the same net effect of converting four hydrogen atoms into one helium atom, 2 positrons, and releasing electron neutrinos and gamma ray photons with total energy of 26.7 MeV (Caughlan and Fowler 1988, Stix 2004). Neutrinos barely interact with the matter of the Sun and mainly escape directly, while the photons take a more complex path. The core and radiation zones are very dense and hot, so that energy transfer occurs through direct interaction of particles and photons. Transfer of energy via conduction also occurs, but it is negligible due to the extremely small mean free path of particles compared to that of the photons (Stix 2004). Hence the energy is transported mainly by radiation. The photons get absorbed and re-emitted multiple times and it takes roughly 170,000 years for the energy from the core to reach the bottom of the convection zone (Mitalas and Sills 1992). The temperature decreases outward from the core, reaching $T \sim 5 \times 10^{5} \mathrm{~K}$ at the top of the radiative zone (Priest 2003).

In the convection zone the magnitude of the temperature gradient becomes greater than the adiabatic gradient, leading to the gas becoming unstable to convection (Stix 2004). The energy in this layer is transported by turbulent plasma motions. Energy transfer in the convection zone occurs much faster than in the radiation zone, and it takes approximately 35 days to transfer a parcel from the bottom to the top of the convection zone (Eggleton 2011). Convective layers extend until the opacity of matter becomes low 


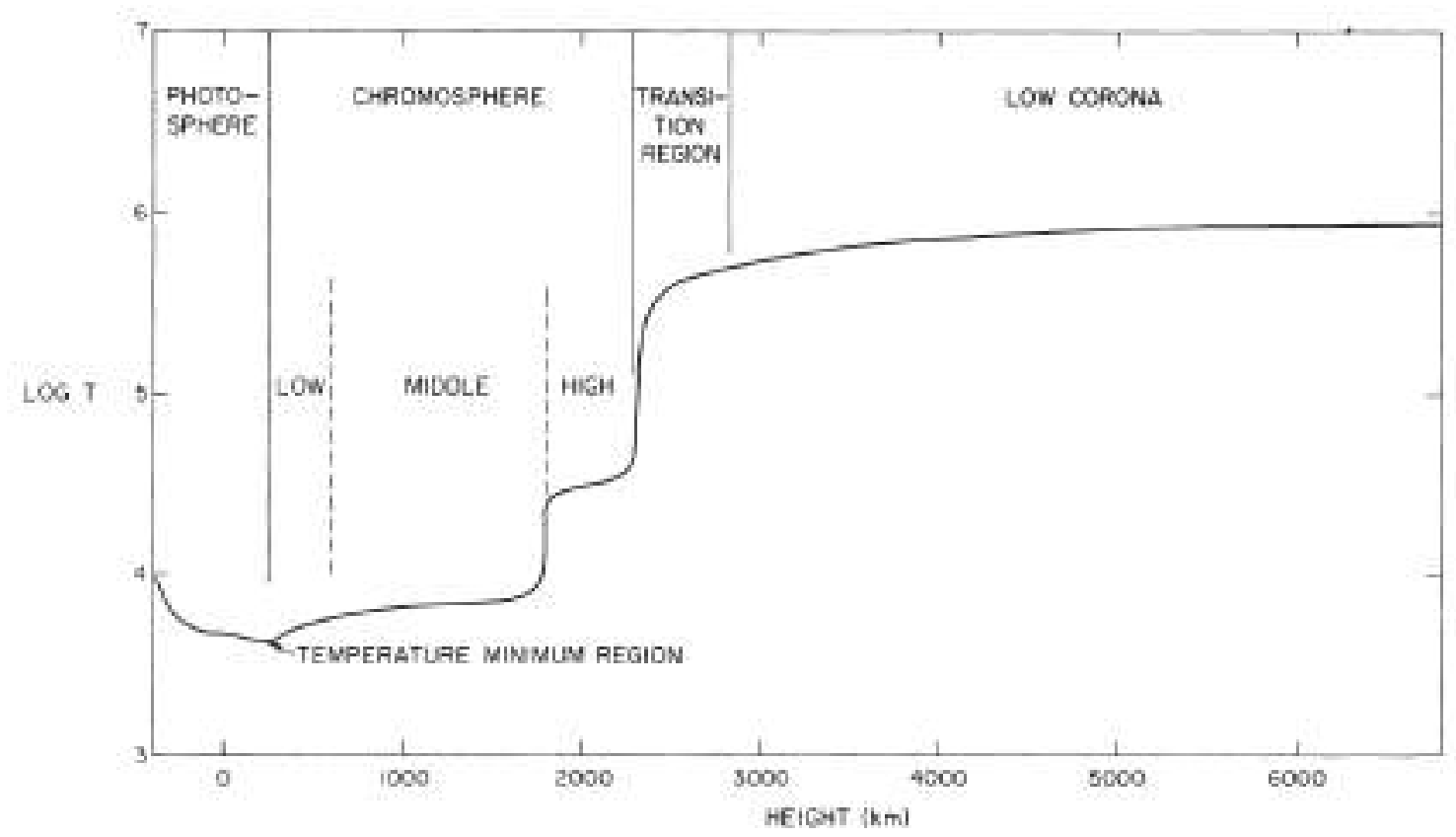

Figure 1.2: Temperature profile of lower solar atmosphere. Adapted from Athay (1976).

enough for the photons to escape by radiation. We define this layer as the solar surface and this point marks the bottom of the solar atmosphere.

The rotation of the radiative zone is that of a rigid body (Howe 2009). The rotation in the convection zone is differential and depends on the latitude and radius. At the surface it is roughly 25 days at the equator and 38 days at the poles as seen from a fixed point in space, i.e. sidereal rotation period (Schou et al. 1998, García et al. 2007). The synoptic period of 27.3 days at about $26^{\circ}$ latitude (Cox 2000) is usually taken to be the solar rotation period unless otherwise specified (called Carrington rotation period).

\subsubsection{Solar atmosphere and limb darkening}

The solar atmosphere is usually divided into the following regions: photosphere, chromosphere, transition region and corona. The division of these layers is rather loosely defined based on the temperature profile (Fig. 1.2).

In the photosphere the Sun radiates almost like a perfect black body with an effective temperature $\sim 5770 \mathrm{~K}$ (see Fig. 1.1). The continuous spectrum of the Sun along with some absorption lines originates predominantly in the photosphere. The temperature in the photosphere steadily decreases with height (see Fig. 1.2). The convection cells (granules) are visible here as bright cellular patterns. The centres of the granules appear brighter than the surroundings, being comprised of hot plasma that rises and flows outwards horizontally. Granules can be observed in a range of sizes, with typical diameters of 1-2 Mm and an average lifetime of $8 \mathrm{~min}$ (Roudier and Muller 1987, Title et al. 1989, Schrijver et al. 1997). Larger cells are also observed, which are thought to originate at greater depths in the convection zone. These are called super-granules and have typical diameters of roughly $30 \pm 20 \mathrm{Mm}$ and lifetimes of a few days (Hagenaar et al. 1997, Meunier et al. 2008, McIntosh et al. 2011, Williams and Pesnell 2011, Tlatov 2012). Existence of giant 
cells with diameters of $200 \mathrm{Mm}$ and lifetimes of about a month has also been suggested (Simon and Weiss 1968, Miesch et al. 2008, Hathaway et al. 2013).

In the chromosphere, the temperature starts to increase again, with acoustic or MHD waves (Liu 1974, Carlsson and Stein 1992, 1997) and magnetic reconnection (Morita et al. 2010) being sources of the heating required to achieve this. Figure 1.3 shows the approximate formation heights of some of the most important lines observed in the photosphere and chromosphere. The strong lines of Ca II K (393 nm) and H (397 nm), Mg II $\mathrm{K}(280 \mathrm{~nm})$ or $\mathrm{H} \alpha(656 \mathrm{~nm})$ are formed in the chromosphere.

Above the chromosphere lies the transition region. This is a rather narrow layer, where the temperature increases rapidly from $\sim 3 \times 10^{4} \mathrm{~K}$ in the chromosphere to $\sim 10^{6} \mathrm{~K}$ in the corona (Parenti et al. 2000, Lemaire and Stegen 2016, Warren et al. 2017). The corona is characterised by high temperatures and low density. The various atoms in this layer are highly ionized and bright emission lines are formed in this layer. A continuous stream of charged particles, called the solar wind, starts from the corona (Parker 1958, Owens and Forsyth 2013). During its outward expansion solar wind fills an area which is called heliosphere and is the last layer of the solar atmosphere. The heliosphere extends much further than the planets of our solar system to approximately a distance of 18.5 billion $\mathrm{km}$ or $123 \mathrm{AU}$ from the Sun (Krimigis et al. 2013, Webber and McDonald 2013, Stone et al. 2013, Gloeckler and Fisk 2015).

When we observe the photosphere of the Sun in the visible, near UV and IR, the centre of the disc appears brighter than the limb. This effect is called limb darkening and is illustrated in Fig. 1.4. The reason this effect takes place is that light coming from the centre of the disc originates from lower and hence hotter regions in the solar atmosphere than those closer to the limb. At the disc centre the line of sight crosses higher layers of the solar atmosphere, while closer to the limb, it only crosses the highest levels. This effect depends on the wavelength, with limb darkening being stronger at short wavelengths because of the wavelength dependence of the sensitivity of the Planck function to temperature. This is illustrated in Fig. 1.5 which shows the centre-to-limb variation (CLV) profiles as measured by Pierce and Slaughter (1977), Pierce et al. (1977), for the wavelength range $303-2402 \mathrm{~nm}$, normalised to the central intensity, $I_{0}$. The limb darkening at $730 \mathrm{~nm}$ is merely $0.25 I_{0}$, i.e. the intensity drops from $I_{0}$ at disc centre to $0.75 I_{0}$ at the limb, while at $303 \mathrm{~nm}$ it is $0.55 I_{0}$.

\subsection{Magnetic activity}

\subsubsection{Magnetic field generation}

The magnetic field plays an extremely important role on the Sun, making its observation always new and interesting thanks to their evolution with time and its interaction with the turbulent convection and with waves. The fields also make processes occurring on the Sun very complex. Magnetic field is thought to be generated below or in the convection zone through the dynamo mechanism (e.g. Parker 1955, Dikpati and Gilman 2009, Charbonneau 2010). This process requires a rotating and electrically conducting fluid. The time evolution of the magnetic field, $\boldsymbol{B}$, can be described by the induction equation: 


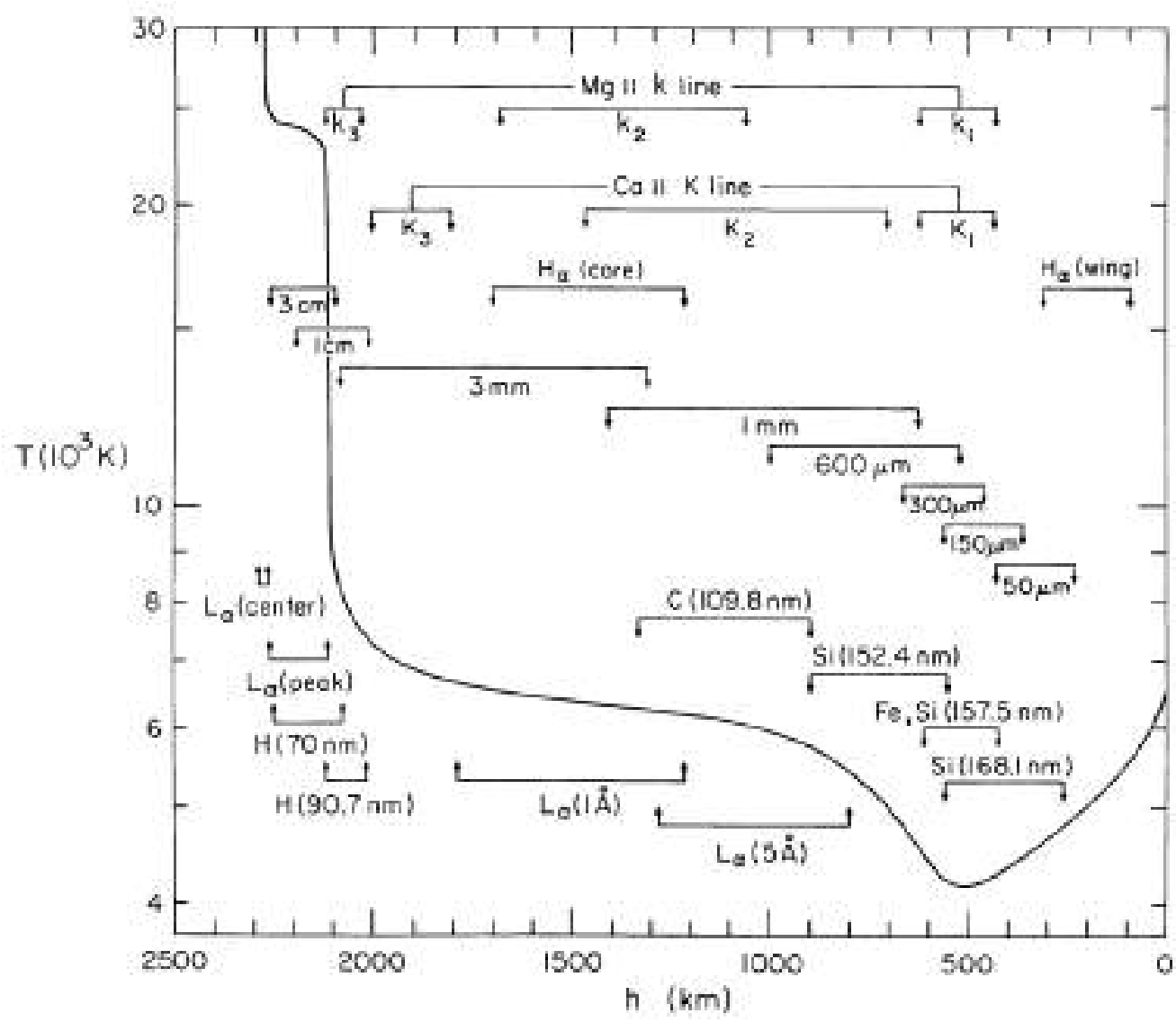

Figure 1.3: Approximate formation heights for some prominent lines in the lower solar atmosphere. The solid line is the temperature profile. Adapted from Vernazza et al. (1981).

$$
\frac{\partial \boldsymbol{B}}{\partial t}=\boldsymbol{\nabla} \times(\boldsymbol{v} \times \boldsymbol{B})-\boldsymbol{\nabla} \times\left(\frac{1}{\mu_{m} \sigma} \nabla \times \boldsymbol{B}\right),
$$

where $v$ is the fluid velocity, $\sigma$ the conductivity, and $\mu_{m}$ the magnetic permeability or magnetic constant. The first term on the right hand side of the equation describes the induction of the field by the plasma flow, while the second term the magnetic diffusion. The ratio of these two terms is defined as the magnetic Reynolds number,

$$
R_{m}=\mu_{m} \sigma V L,
$$

where $V$ and $L$ represent the typical velocity and length scales. $R_{m} \gg 1$ values mean that there is a coupling between the magnetic field and the plasma, usually termed frozenin magnetic field. In this case the magnetic field is advected with the plasma flow, the internal fields are maintained and no new field lines are able to penetrate them. $R_{m} \ll 1$ values mean that the field will tend to diffuse. In the Sun we get $R_{m} \gg 1$ and the magnetic field is frozen in the plasma (Priest 2003).

The ratio of the gas pressure to the magnetic pressure defines the plasma- $\beta$. $\beta \ll$ 1 means that the magnetic field dominates energetically (e.g., magnetic fields will be unaffected by motion of the plasma, but will drag the plasma with them if they move), 


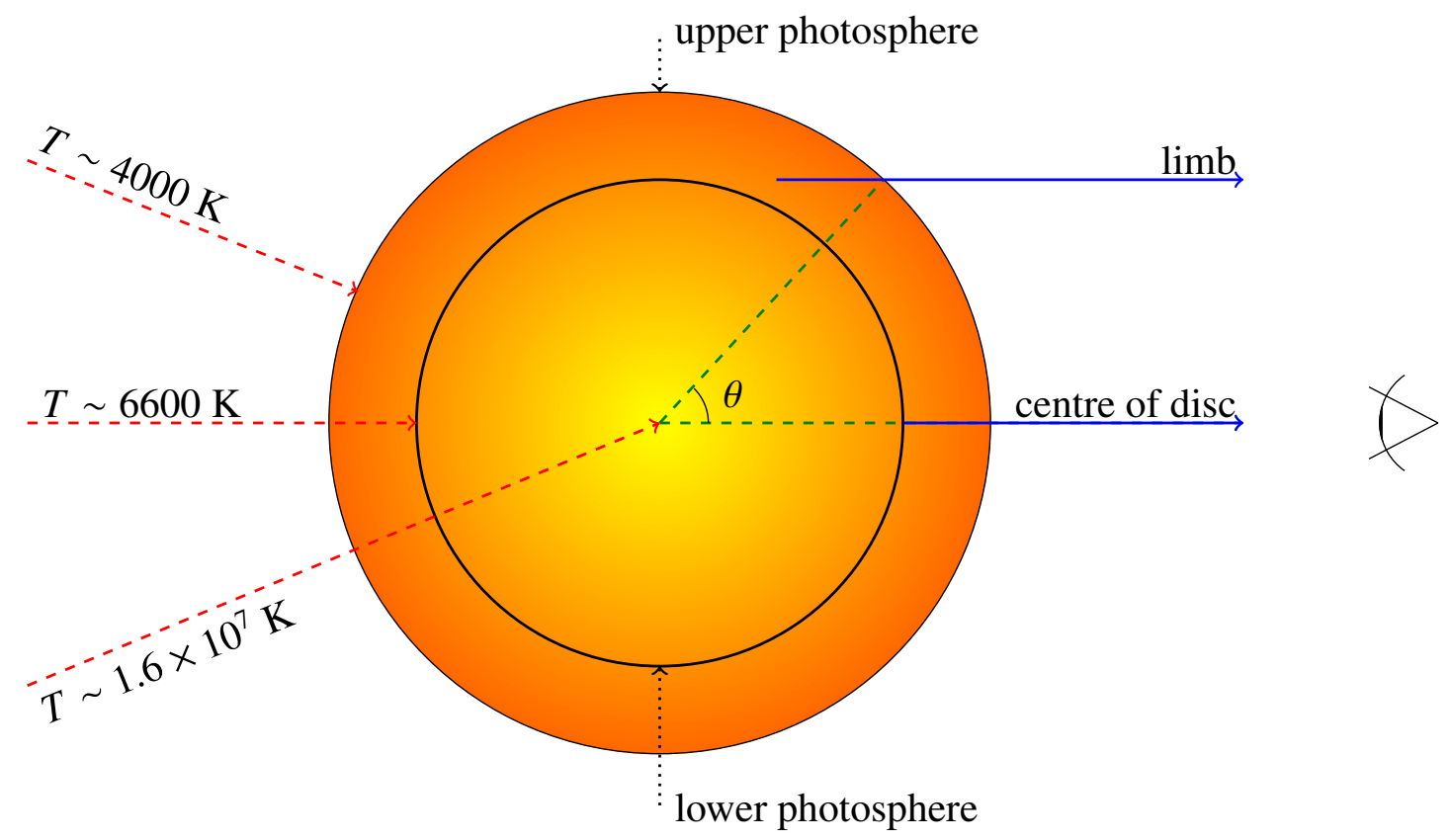

Figure 1.4: Schematic drawing of limb darkening. The blue rays show the different paths of photons originating at the disc centre and at the limb. $\theta$ is the heliocentric angle. Not to scale.

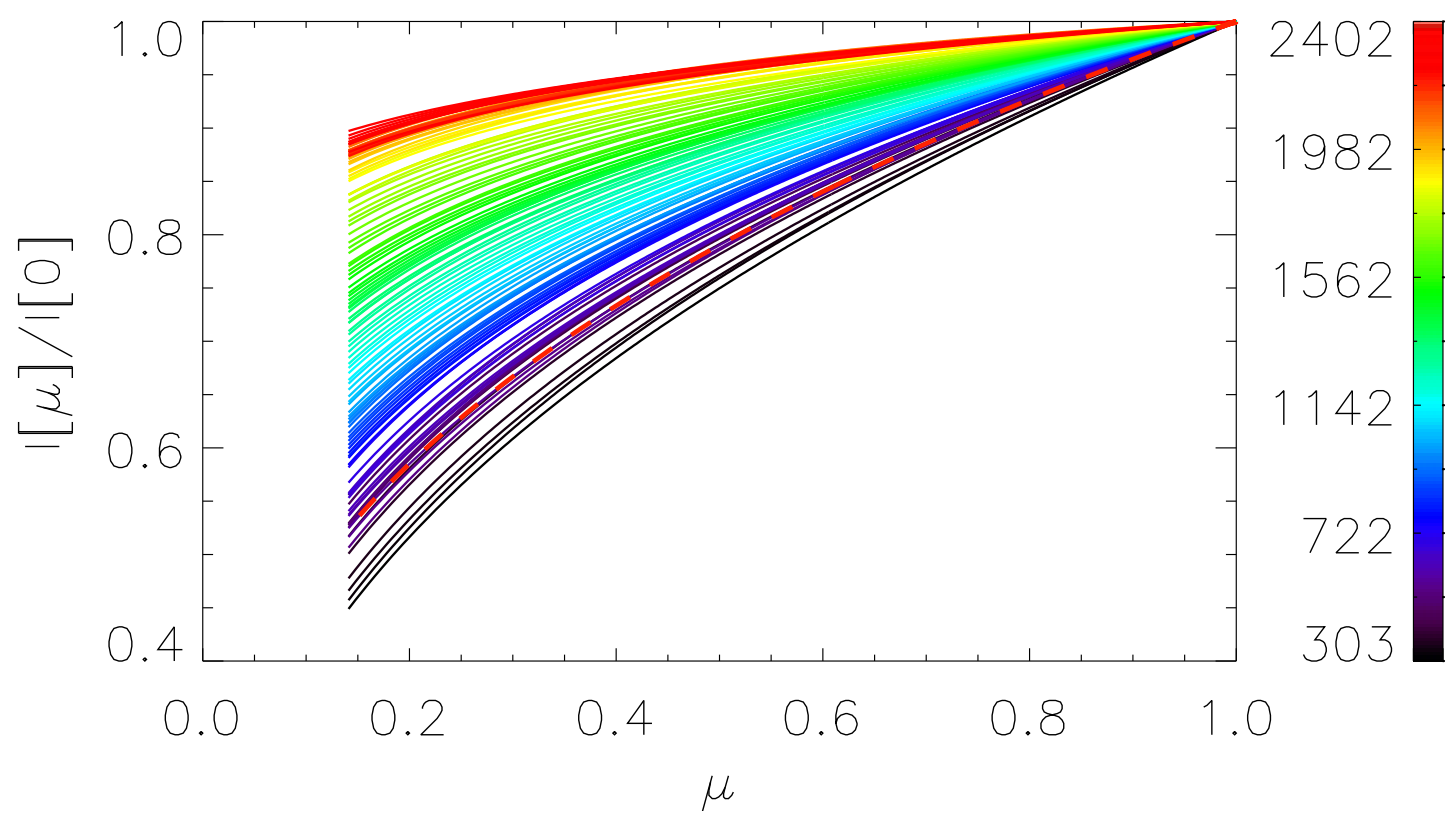

Figure 1.5: CLV profiles for wavelengths $303-2402 \mathrm{~nm}$ (black to red according to the colour bar) as a function of $\mu=\cos \theta$, where $\theta$ is the heliocentric angle, as tabulated by Pierce and Slaughter (1977), Pierce et al. (1977). The intensities are normalised to that disc centre $(I[0])$. The dashed red line corresponds to $395 \mathrm{~nm}$, the wavelength closest to the position of the Ca II K line considered in this thesis, among the wavelengths listed in Pierce and Slaughter (1977). 
while $\beta \gg 1$ means that the gas pressure dominates, i.e. the field will follow the motions of the plasma. In the convection zone and the photosphere of the Sun (outside sunspots and other localized magnetic flux concentrations) $\beta$ is $\gg 1$, but it decreases to values below unity in the chromosphere and increases again in the upper parts of the corona and the solar wind (Gary 2001).

In classical dynamo models solar differential rotation strengthens the magnetic field until it becomes unstable to buoyancy and rises (e.g. Parker 1955, Caligari et al. 1995). It typically reaches the surface as an $\Omega$-shaped loop, whose two foot-points form the two opposite polarity parts of active regions (or of smaller bipolar features, such as ephemeral active regions; Harvey and Harvey 1973). Field that is not sufficiently buoyant does not reach the surface, getting shredded by the turbulent convection (Rempel et al. 2000). It can then be transported further by the turbulent plasma. In addition, there is evidence for a small-scale turbulent dynamo acting closer to the solar surface, which produces a smallscale, turbulent magnetic field (Vögler and Schüssler 2007, Danilovic et al. 2010, Bühler et al. 2013, Lites et al. 2014). This has the consequence that the whole solar atmosphere is permeated by magnetic field in the form of small- and large-scale concentrations. The gas pressure drops with height and the magnetic pressure balances the decrease with an expansion of the flux tubes (Solanki and Steiner 1990). The various phenomena observed on the solar surface are categorised into active and quiet, depending on the amount of magnetic flux present.

\subsubsection{Active features}

The most prominent active or magnetic features in the photosphere are sunspots and pores. Figure 1.6 shows a close-up of a sunspot region. Pores are small isolated dark features with unipolar magnetic field $|B| \sim 2500 \mathrm{G}$ and diameters of a few hundred $\mathrm{km}$ to $6 \mathrm{Mm}$ (Brants and Zwaan 1982, Martinez Pillet 1997, Sütterlin 1998, Keil et al. 1999, Ermolli et al. 2017, Solanki et al. 2017). They are created when the magnetic flux exceeds a few times $10^{18} \mathrm{Mx}$. For even larger fluxes, a penumbra forms around the central umbra, so that a sunspot is formed. Pores are relatively short-lived with lifetimes of an hour to $\sim 1$ day (Stix 2004).

Sunspots typically appear as groups in large bipolar regions, where each spot forms a foot-point of a loop or set of loops of magnetic field lines. Within a pair, spots have opposite polarities. The leading one, typically the one closer to the equator, has the same polarity as the average polarity of the hemisphere (Hale et al. 1919). Sunspots (with pores) cover only a small fraction $(<1 \%$, e.g. Balmaceda et al. 2009, Hathaway 2015) of the solar disc and this amount varies with the activity level. The latitude of sunspot emergence also varies with time. At the beginning of the cycle they appear on average around $25^{\circ}$ latitude and gradually move to lower latitudes. This is called Spörer's law (Carrington 1863).

Sunspot latitudes as a function of time (shown in Fig. 1.7), define the so-called butterfly diagram (Arlt et al. 2013, Hathaway 2015, Leussu et al. 2017). The sunspot groups are tilted with respect to the equator and the tilt angle increases almost linearly with their latitude, which is referred to as Joy's law (Hale et al. 1919, Wang and Sheeley 1989). Sunspots can typically be divided into umbra and penumbra. The umbra is the dark central portion of the spot, while the penumbra is the brighter region surrounding the umbra 
(although it is still slightly less bright than the normal or quiet photosphere). The penumbra covers roughly 80\% of the total spot area (McIntosh 1981, Steinegger et al. 1990, Brandt et al. 1990, Beck and Chapman 1993, Solanki 2003). The effective temperature in the umbra is around $4200 \mathrm{~K}$, while it is $5400-5500 \mathrm{~K}$ in the penumbra (Solanki and Krivova 2009). These temperatures are lower than outside the spot ( $\sim 5770 \mathrm{~K})$, hence sunspots appear dark. The diameter of a sunspot usually lies in the range 3-40 Mm (Bray and Loughhead 1964, Martinez Pillet 1997, Sobotka 2003). Their lifetime is linearly related to their maximal area with the Gnevyshev-Waldmeier rule (Gnevyshev 1938, Waldmeier 1955, Petrovay and van Driel-Gesztelyi 1997, Solanki 2003) and varies between hours and months. The decay of their area with time has been described with linear and parabolic decay laws (Moreno-Insertis and Vazquez 1988, Howard 1992, Martinez Pillet et al. 1993, Petrovay and van Driel-Gesztelyi 1997, Baumann and Solanki 2005, Hathaway and Choudhary 2008, Litvinenko and Wheatland 2015). Sunspots have a magnetic field strength of $B \sim 2500-3500 \mathrm{G}$ near their centre which drops to $B \sim 700-1000$ $\mathrm{G}$ at the edges (Solanki and Marsch 2006). Their total magnetic flux is $\Phi>5 \times 10^{20}$ Mx (Zwaan 1985), Dacie et al. 2016, Sheeley et al. 2017). The central parts of the umbra have lower temperatures and densities hence are less opaque to radiation. Also, because of horizontal pressure balance, the interiors of sunspots are strongly evacuated. Together these effects cause the observed light from the umbra to originate from lower heights (by 400-800 km Loughhead and Bray 1958, Gokhale and Zwaan 1972, Martinez Pillet et al. 1993, Solanki et al. 1993, Mathew et al.|2004) than its surrounding, with the darkest parts having the highest magnetic field (Kopp and Rabin 1992) and lying lowest. This is called Wilson depression (Suzuki 1967), named after the observer who first noticed it in 1769. Observationally this effect manifests itself as a progressive diminishing of the disc-side penumbra and the umbra as the sunspot moves from the centre of the disc towards the limb. After a certain point the umbra is no longer visible.

Sunspots are usually surrounded by bright regions called faculae. These in turn are formed of magnetic concentrations or small flux tubes having a magnetic field strength that decreases from $\sim 1500 \mathrm{G}$ in the lower-middle photosphere to $\sim 200 \mathrm{G}$ at the temperature minimum (Stenflo and Harvey 1985, Zirin and Popp 1989, Rabin 1992, Rüedi et al. 1992, Bruls and Solanki 1995, Martínez Pillet et al. 1997). Near the centre of the solar disc they appear dark (in the infrared part of the spectrum, e.g. Foukal and Duvall 1985, Moran et al.|1992, Sütterlin et al.|1999, Sobotka et al.2000) or similar to their surroundings in the visible continuum, while they become bright towards the limb. They are observed as ensembles of flux tubes, hence forming large bright areas. Faculae as a whole have lifetimes of about 40 days and sizes of up to about $50 \mathrm{Mm}$ although the individual flux tubes forming them have diameters typically smaller than $300 \mathrm{~km}$ and lifetimes of minutes to hours. Note that when observed in chromospheric radiation, faculae are called plage and are bright all over the solar disc.

Small short-lived bipolar features are called ephemeral regions. Their life-times are on the order of a day and each carries a total flux of $\Phi<10^{20} \mathrm{Mx}$ (Harvey and Martin 1973, Zwaan 1985, Hagenaar et al. 2008, Wang et al. 2012, Zhao and Li 2012, Yang and Zhang 2014).

The magnetic features emerge in the form of loops that rise up to the corona. Magnetic field lines that close at distances more than $\sim 1.8-2.5 R_{\odot}$ from the Sun are termed open (Lee et al. 2011) as such fields are dragged away from the Sun by the solar wind. The 


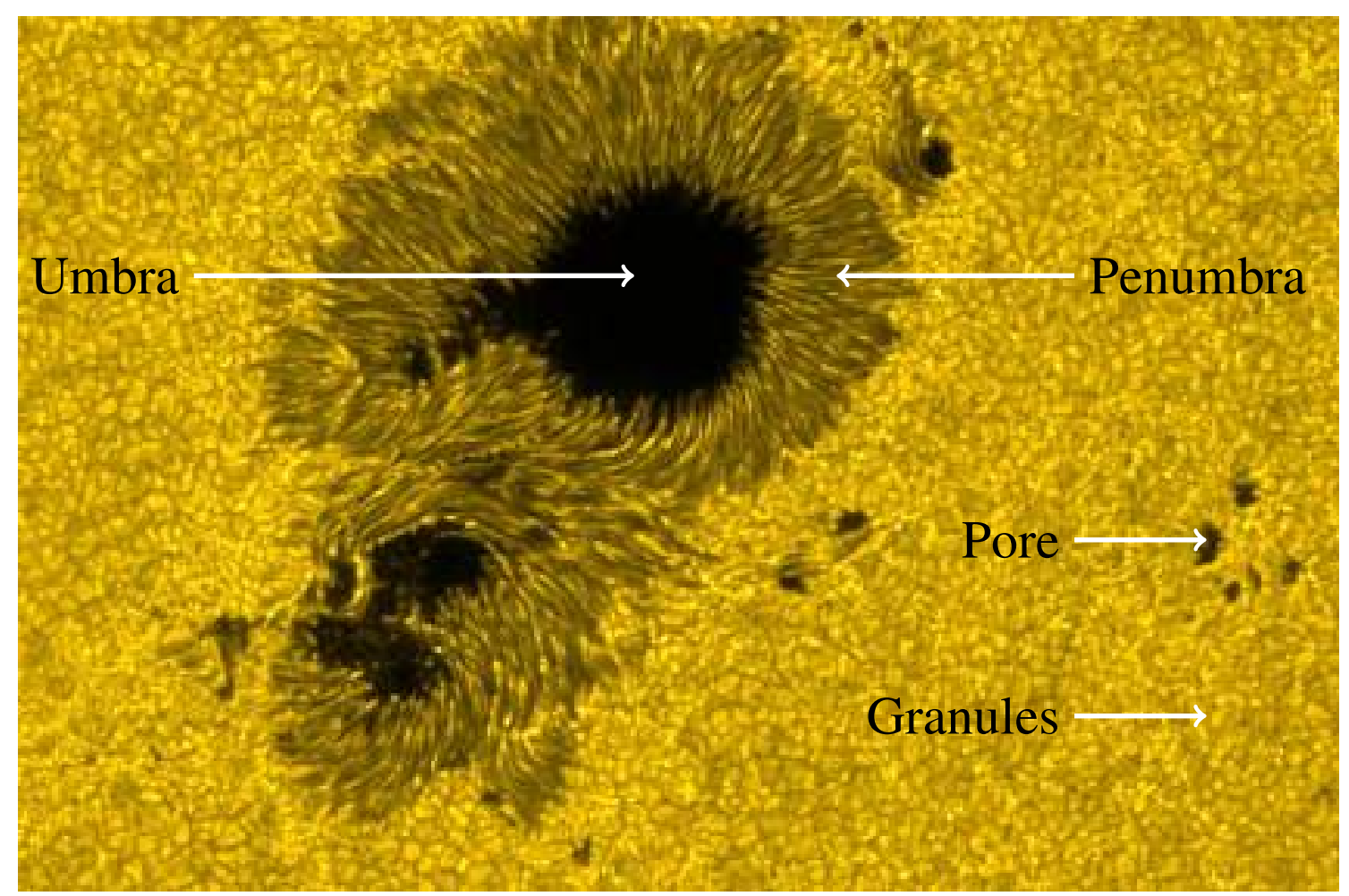

Figure 1.6: Observation of sunspots with solar optical telescope on board Hinode spacecraft. Adapted from www.nasa.gov.
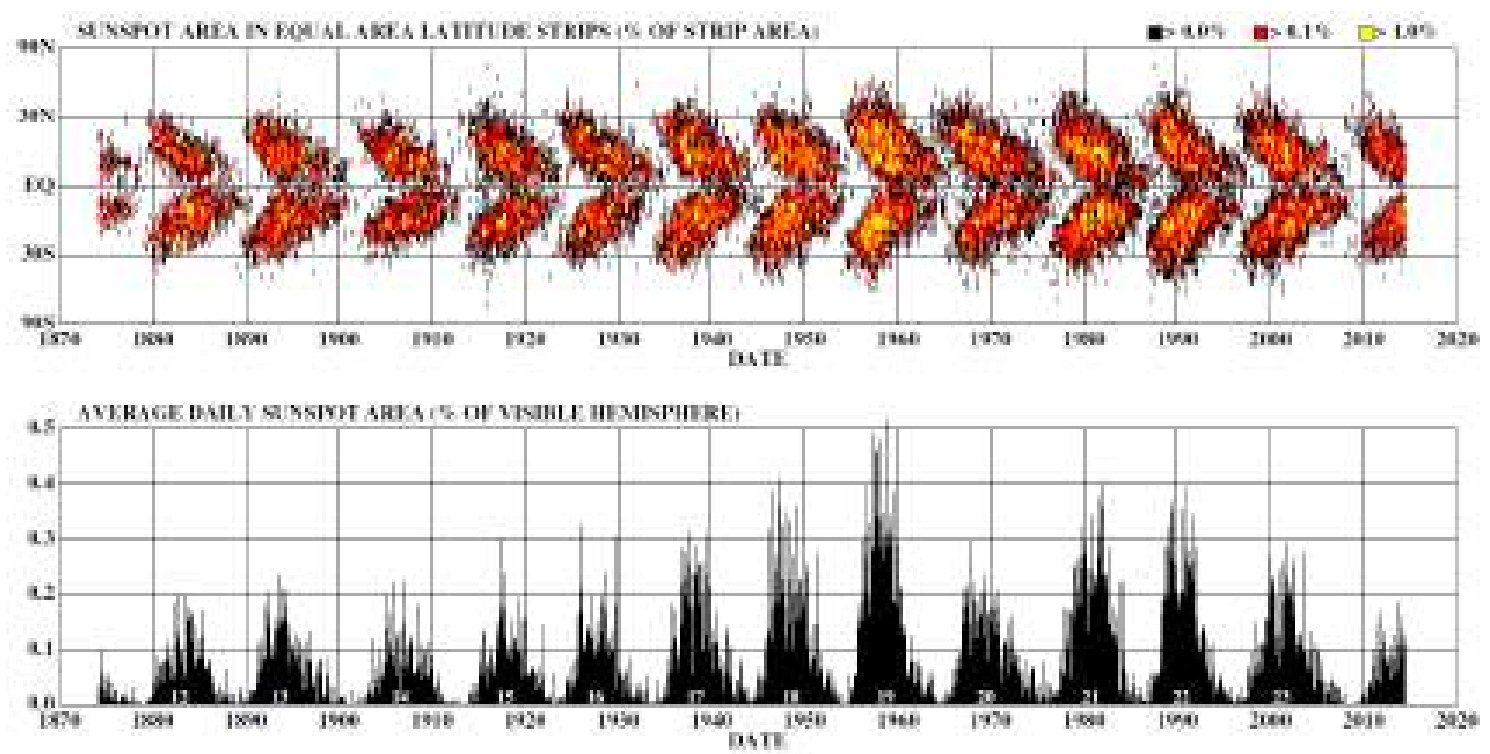

Figure 1.7: Top: Butterfly diagram, showing the spot emergence latitudes as a function of time. The colours indicate the percentage of the solar disc area covered by sunspots. Bottom: Daily sunspot areas in percentage of visible hemisphere as a function of time. The numbers near the bottom of the plot denote the conventional solar cycle numbering. Taken from Hathaway (2015). 


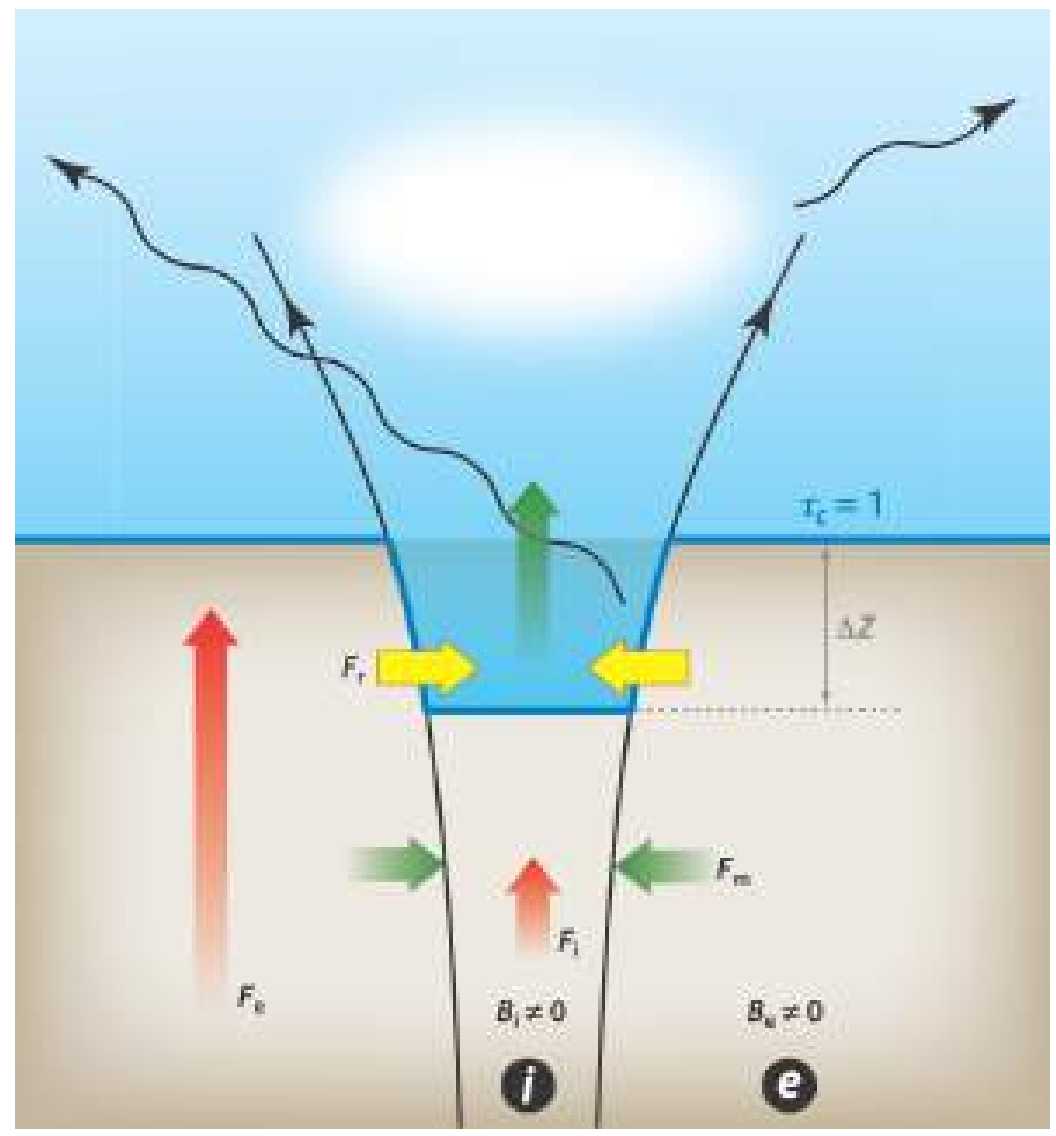

Figure 1.8: Schematic drawing of a magnetic flux tube. See text for details. Taken from Solanki et al. (2013) which is an adaptation from Zwaan (1978).

magnetic flux from the loops forming active regions constitutes the closed flux, while the one from the open field lines is the open solar magnetic flux.

Faculae, spots, and pores are all manifestations of the emerging magnetic field on the surface of the Sun. However, spots and pores appear as dark features, while faculae are bright. Figure 1.8 is a schematic drawing of the structure of a flux tube. The magnetic field within the flux tube suppresses the convective flows making the "bottom" of the feature (i.e. the optical depth unity surface inside the feature) to appear darker. Concurrently, there is also radiative heating of the walls of the flux tube from the surroundings. The flux tubes forming faculae are sufficiently narrow for the inflowing energy from the walls to render them bright especially near the limb where the walls can be seen best. Pores and sunspots are big enough with high magnetic field strengths inhibiting the convection and hence they appear dark. Simulations by Knölker and Schüssler (1988), GrossmannDoerth et al. (1994) showed a transition between bright and dark features to take place approximately for widths of 400-700 km.

In the chromosphere the magnetic pressure starts to dominate over the gas pressure and the flux tubes expand. Sunspots are still visible as dark structures, while plage (the extension of faculae) can be seen on the disc even at disc centre locations. The supergranulation pattern is seen in the chromosphere as dark cells, surrounded by bright weblike structures called network, populated by small magnetic flux concentrations. 


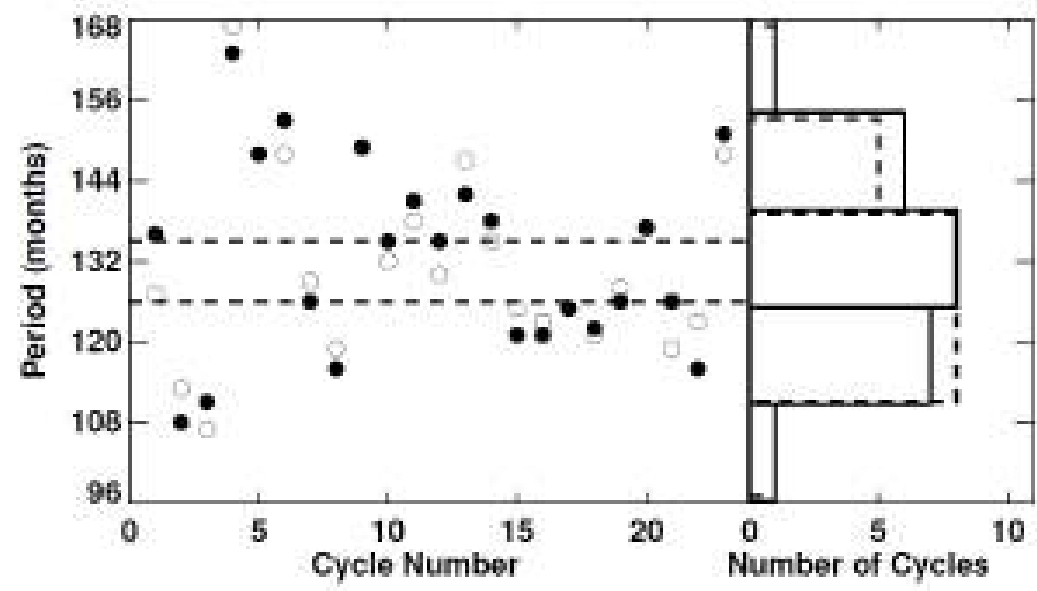

Figure 1.9: Left: The length of the solar cycle as a function of cycle number. The filled (open) circles show the periods derived with a 13-month mean (24-month Gaussian smoothing). Right: Distributions of periods (solid line for 13-month mean, dashed line for Gaussian smoothing). Taken from Hathaway (2015).

\subsubsection{Solar cycle}

The number of sunspots on the solar disc was the first quantity that was observed to exhibit a periodicity of roughly 11 years (Schwabe 1844), now known as the 11-year solar cycle (or Schwabe cycle). The period of the cycle is not constant and has been observed to vary between 9 and 14 years (see Fig. 1.9, e.g. Fligge et al.|1999, Hathaway 2015).

Solar activity varies significantly between activity minima and maxima. Also, the strength of individual cycles changes with time. It has been suggested that the strength of a cycle is related to the period of the preceding cycle (Hathaway et al. 1994, Solanki et al. 2002).

Shorter periodicities of approximately 1.3 years and 156 days have been suggested (Lean and Brueckner 1989, Oliver et al. 1998, Lockwood 2001, Krivova and Solanki 2002). There is also a 22-yr periodicity of the polarity of the average polar magnetic field, called Hale's magnetic polarity cycle (Babcock 1959). This occurs due to crossequator cancellation of flux in magnetic elements. This results in an excess of magnetic elements with polarities opposite to the average of the given hemisphere (following polarities). Magnetic field elements are transported towards the poles by the meridional circulation, which at the surface is directed from the equator towards the poles. The magnetic elements of the following polarity mostly having the polarity opposite to the polar field, so that their flux cancels with that already present at the poles. This process eventually leads to a reversal of the polar field every $\sim 11$ years and is illustrated in Fig. 1.10 . Longer periodicities have also been found, e.g. with an average period of $\sim 100 \mathrm{yr}$, called the Gleissberg cycle (e.g. Gleissberg 1939, Garcia and Mouradian 1998, Ogurtsov et al. 2002, Feynman and Ruzmaikin 2014, Vázquez et al. 2016, Le Mouël et al. 2017). A longer $\sim 210$ year periodicity has also been noticed in the cosmogenic radioisotope data, called Suess or de Vries cycle (e.g. Suess 1980, Wagner et al. 2001, Usoskin et al. 2004, Vonmoos et al.2006, Steinhilber et al. 2012).

There are also periods of extended high or low activity, called grand maxima and min- 


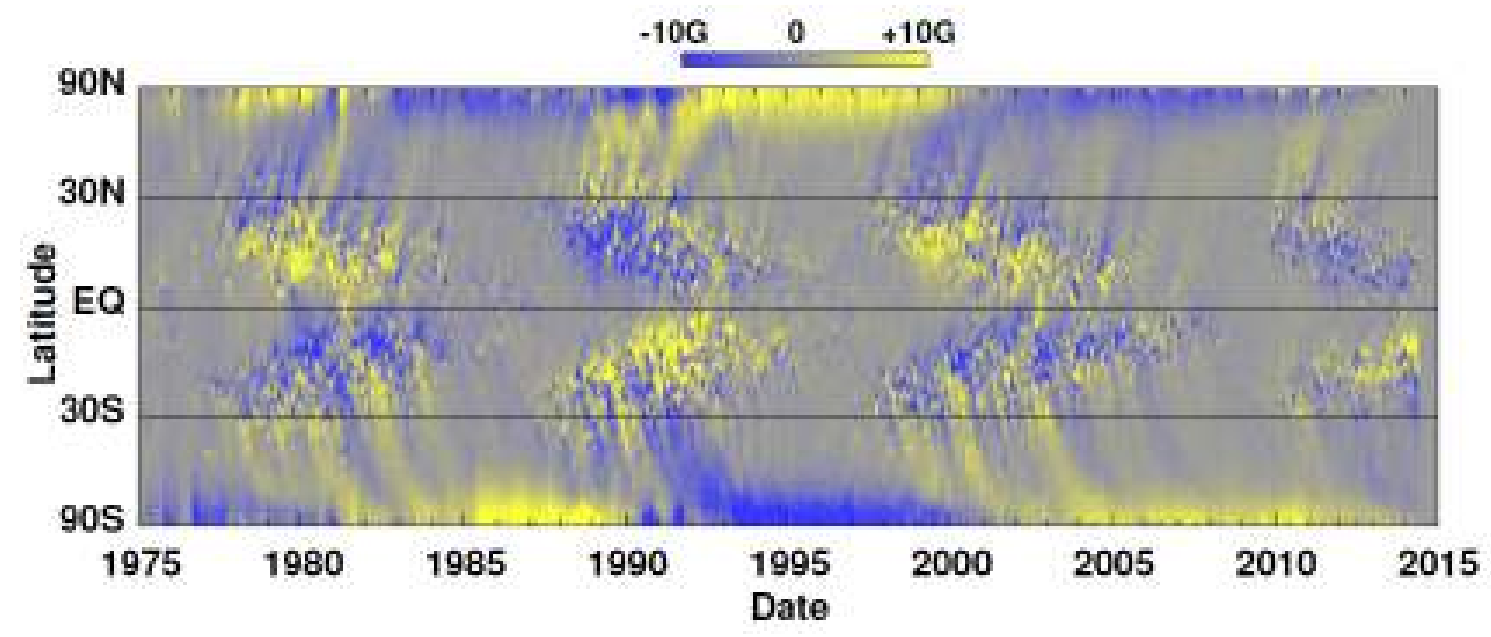

Figure 1.10: Magnetic butterfly diagram derived from longitudinally averaged radial magnetic field measurements from Kitt Peak and SOHO. Taken from Hathaway (2015).

ima. Figure 1.11 shows the sunspot number series (Usoskin et al. 2016a) reconstructed from the concentration of the cosmogenic radioisotope ${ }^{14} \mathrm{C}$ for the last 9000 years (see Sect. 1.4.2.1. Red stars and blue circles highlight the periods of grand maxima and grand minima, respectively. The period between 1930 and 2010 is considered as the modern grand maximum (Solanki et al. 2004, Usoskin et al. 2007). This has recently been debated by Svalgaard and Schatten (2016), and will be discussed in more detail in Chapter7. The period between 1640 and 1720 is called the Maunder minimum and is the only grand minimum covered by direct observations (Eddy 1976, Sokoloff 2004). The existence or the strength of the Maunder minimum has also been a matter of debate recently (Usoskin et al. 2015, Zolotova and Ponyavin 2015, 2016, Carrasco and Vaquero 2016, Svalgaard and Schatten 2016, Usoskin et al. 2017). The period of low sunspot numbers between 1790 and 1830 is called Dalton minimum, although the activity was not low enough to be considered a grand minimum. The sunspot series reconstructed by Usoskin et al. (2016a) suggests that the Sun is in a grand maximum (minimum) state for $\sim 10 \%$ $(\sim 20 \%)$ of the time, although these percentages depend on how exactly grand maxima and minima are defined.

\subsection{Solar irradiance}

The solar irradiance, like other changes wrought by the magnetic field, also follows the solar activity cycle. Evidence exists that irradiance might vary on longer time-scales, too, which is of particular importance for Earth's climate. In this section we give an overview of the available irradiance measurements and models.

\subsubsection{Measurements of irradiance}

Early ground-based measurements performed before the late 1970s were unable to detect variations in TSI so it was considered to be invariant and given the name "solar constant". Pouillet (1838) was the first to measure the "solar constant" and determined its value 


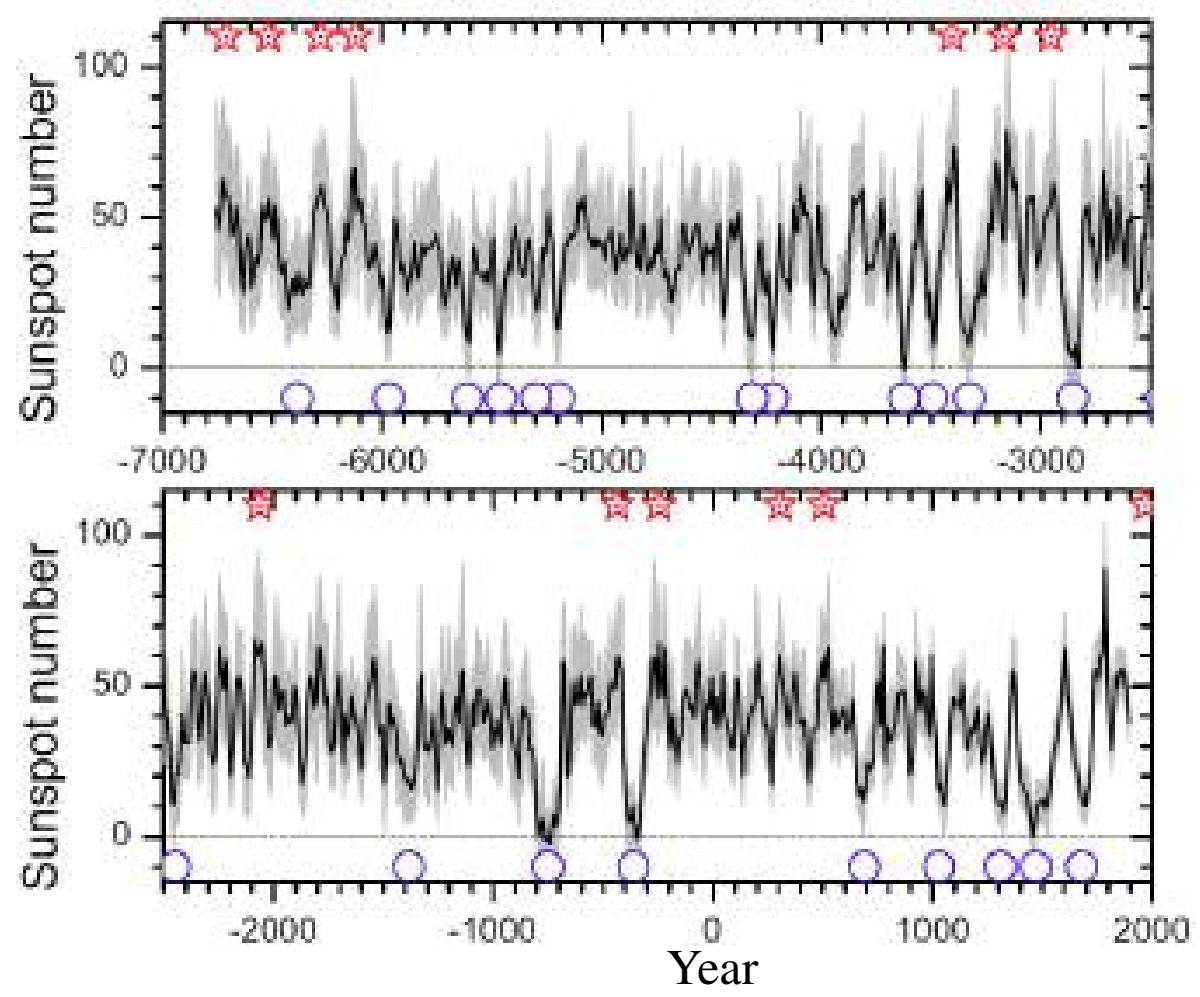

Figure 1.11: Decadal sunspot numbers over the last 9000 years reconstructed from the cosmogenic radioisotope ${ }^{14} \mathrm{C}$. Red stars (blue circles) denote the grand maxima (minima). From Usoskin (2017).

to be roughly $1227 \mathrm{~W} \mathrm{~m}^{-2}$. Several ground-based programs to measure the "solar constant" were initiated in the beginning of the 20th century. Two such programs took place at the Smithsonian astrophysical observatory (1902 - 1957, Langley and Abbot 1900, Abbot 1933, 1952, 1963) and Mt Wilson observatory (Abbot 1919). Abbot (1919) derived the value of $1360 \mathrm{~W} \mathrm{~m}^{-2}$ for the "solar constant", while Abbot (1933, 1952, 1963) inferred a $0.1 \%$ variability. However, these measurements cannot be considered very reliable because they suffered from high ambiguities connected to processes in the Earth's atmosphere. For example, most of the UV part of the spectrum does not reach the surface at all, and thus cannot be measured (see middle panel in Fig. 1.1). More precise space-based measurements started in 1978 (e.g. Hickey et al. 1980, Willson and Hudson 1988, Kopp et al. 2005, Fröhlich 2006, Schmutz et al. 2013). Figure 1.12 shows the space-based measurements of TSI. These measurements show, in general, a very similar relative variability, but different absolute levels of TSI. The currently adopted value of TSI is approximately $1360.8 \mathrm{Wm}^{-2}$ for the period of solar minimum in 2008 (Kopp and Lean 2011). Measurements of SSI exist since 1978 as well, however they cover mostly the UV part of the spectrum (Deland and Cebula 2012, Ermolli et al.2013, Yeo et al. 2015a). The wavelength coverage is not complete and for various wavelengths there are gaps in time.

Measurements show that TSI and SSI vary on all time-scales (Fröhlich 2013, Kopp 2016). $p$-mode oscillations are responsible for variations over minutes, while convection, flares or planetary transits for minutes to hours (e.g. Fröhlich and Lean 2004, Kopp et al. 2005). Longer-period variations are attributed to the evolution of the magnetic features on the solar surface (e.g. Seleznyov et al. 2011). The effect of the magnetic features is 
illustrated in Fig. 1.13, which shows the variation of TSI due to the passage of a sunspot group. There is an appreciable dip in TSI when the sunspot group is visible, and the dip becomes deeper when the sunspot reaches the disc centre. A small increase in TSI is often observed prior to and after the sunspot passage due to the brightening produced by the faculae surrounding the sunspot, which is strongest near the limb.

Since the Sun is evolving on the main sequence, its radiative output is expected to increase approximately by up to $1 \%$ every 100 million years (Gough 1981). For the current observing capabilities, this is, however, a negligible amount.

Very few TSI radiometers covered periods longer than one solar cycle (see Fig. 1.12). The instruments also suffer from degradations affecting their stability in time. Various methods have been adopted to account for these degradations (Kopp 2014), however residual uncertainties remain. With problems besetting inter-calibration and potential residual errors due to instrument degradations, these measurements do not allow deriving reliable conclusion about potential long term solar variability, e.g. on the change in TSI since the end of the Maunder minimum. In the past three different composites were created to homogenise these observations. The composites are ACRIM (Active Cavity Radiometer Irradiance Monitor, which is the instrument taken as the reference by Willson 1997, Willson and Mordvinov 2003), PMOD (named after Physikalisch-Meteorologisches Observatorium Davos; Fröhlich 2006), RMIB (named after Royal Meteorological Institute of Belgium, in french called IRMB; Dewitte et al. 2004). The calibration of these timeseries is achieved with daisy-chaining (i.e. using a series of data, where each one gets calibrated to the level of the reference data-set and then acts as the reference for the next data, see also Sect. 6.1.3 keeping values from only one instrument per day.

Figure 1.14 shows daily values from the PMOD composite marking with different colours the various instruments whose data entered it. Issues with the calibration of these composites result in them having significantly different trends (Zacharias 2014, Kopp 2016). Comparing the periods of minima in 1986 and 2009 one notices almost no trend in RMIB composite, while ACRIM and PMOD show weak opposite trends of $\sim 0.001 \%$ annual increase and decrease, respectively. PMOD shows the same decreasing trend over all three minima that measurements exist, though this is not the case for ACRIM. ACRIM exhibits a larger increase of $\sim 0.005 \%$ per year between 1986 and 1996 (Willson and Mordvinov 2003), but then decreases by $\sim 0.004 \%$ per year between 1996 and 2009.

More recently, Dudok de Wit et al. (2017) presented a new purely statistical methodology for the creation of a composite. It considers all instruments for a given day with weights which are based on their estimated uncertainties. Somewhat problematically, extrapolations of datasets from other days also enter the composite. The uncertainty of this composite is rather large and encompasses all previously existing composites. Due to the high uncertainties of the early data, the long-term TSI trend cannot be recovered. Positively, however, it is the first time that such an uncertainty was provided at all. The 4 existing TSI composites are plotted in Fig. 1.15 .

\subsubsection{Irradiance reconstructions}

In the absence of direct space-based measurements of solar irradiance before 1978, there is a need to develop models to reconstruct it (e.g. for Earth's climate studies) using other available data. The models reconstruct the solar irradiance by accounting for the com- 


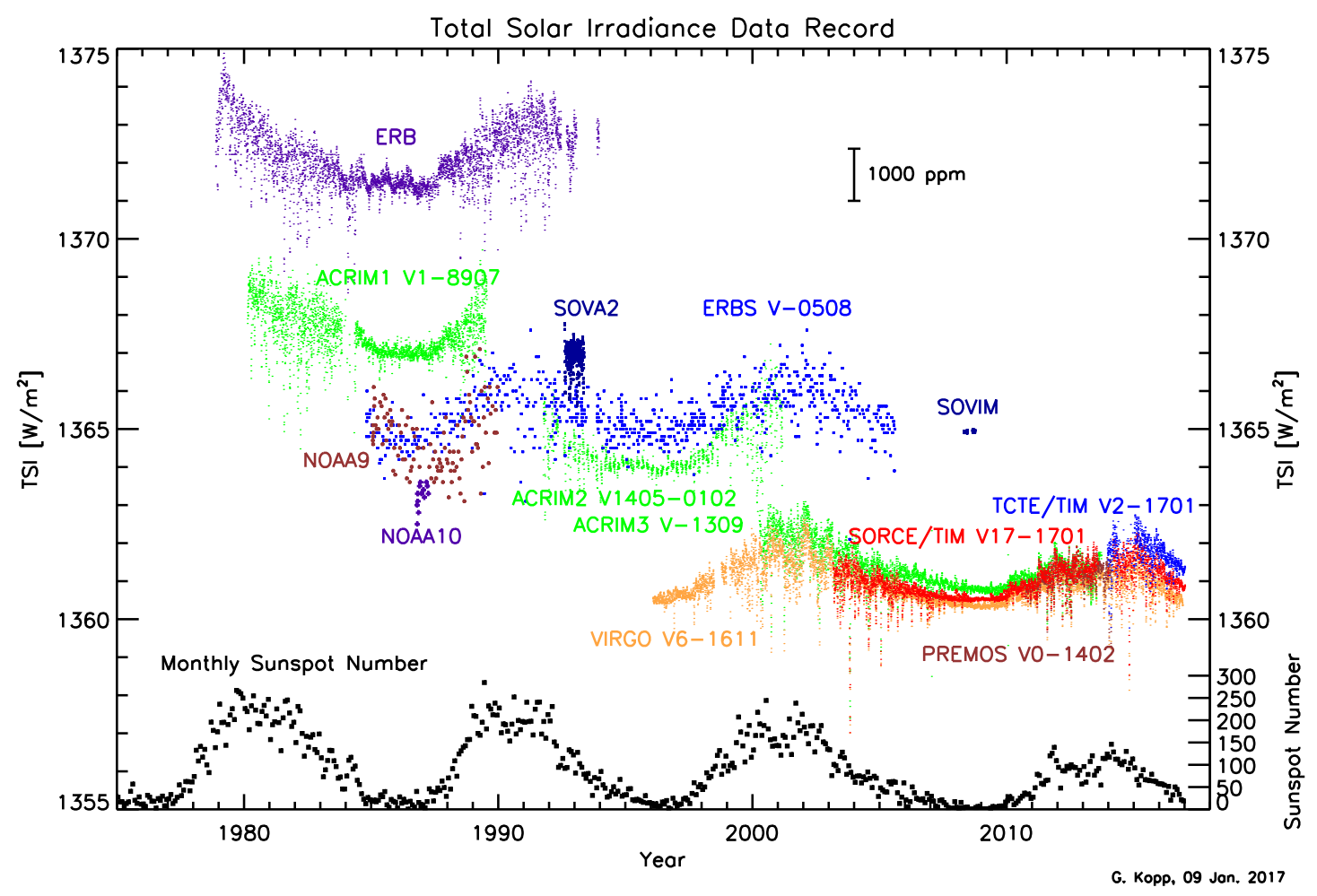

Figure 1.12: Space-based TSI measurements. The monthly sunspot number is shown in the lower part of the plot (black dots). From Kopp (2016).

peting dark and bright contributions from magnetic features on the solar surface. Data that have been used to describe the evolution of the magnetic features are magnetograms, which are are direct measurements of the solar magnetic field and are available since 1974 (e.g. Livingston et al. 1976, Howard et al.|1983, Scherrer et al. 1995, Schou et al.|2012a), and various proxies of the solar magnetic activity.

\subsubsection{Proxies of solar activity}

The various proxy data that have been used to reconstruct solar irradiance can be divided into direct observations of the Sun, and indirect proxies.

The direct observations of the Sun can be further split into disc-integrated, full-disc spatially resolved, and sunspot observations. Disc-integrated direct observations of the Sun are Sun-as-a-star observations taken at different wavelengths. Proxies that have been used include the Mg II core-to-wing ratio (available since 1978, e.g. Heath and Schlesinger 1986, Viereck and Puga 1999, Viereck et al.|2004, Snow et al. 2014), Ca II K 1 Å index (available since 1974, e.g. White and Livingston 1981, Donnelly et al. 1994, Keil et al. 1998), Ly- $\alpha$ flux (available since 1969, e.g. Vidal-Madjar 1975, Woods et al. 2000), He I equivalent widths (available since 1974, e.g. Harvey and Recely 1984), and the F10.7 index (available since 1947, e.g. Covington 1969, Tanaka et al. 1973, Tapping 1987, Tapping et al. 2007). The F10.7 index is the radio flux at $10.7 \mathrm{~cm}(2800 \mathrm{MHz})$ and is expressed in solar flux units ( $\mathrm{sfu}$, where $1 \mathrm{sfu}=10^{-22} \mathrm{Wm}^{-2} \mathrm{~Hz}^{-1}$ ).

Full-disc resolved observations have been used to derive information on the areas and locations of active regions. Full-disc observations that have been used have been taken in 


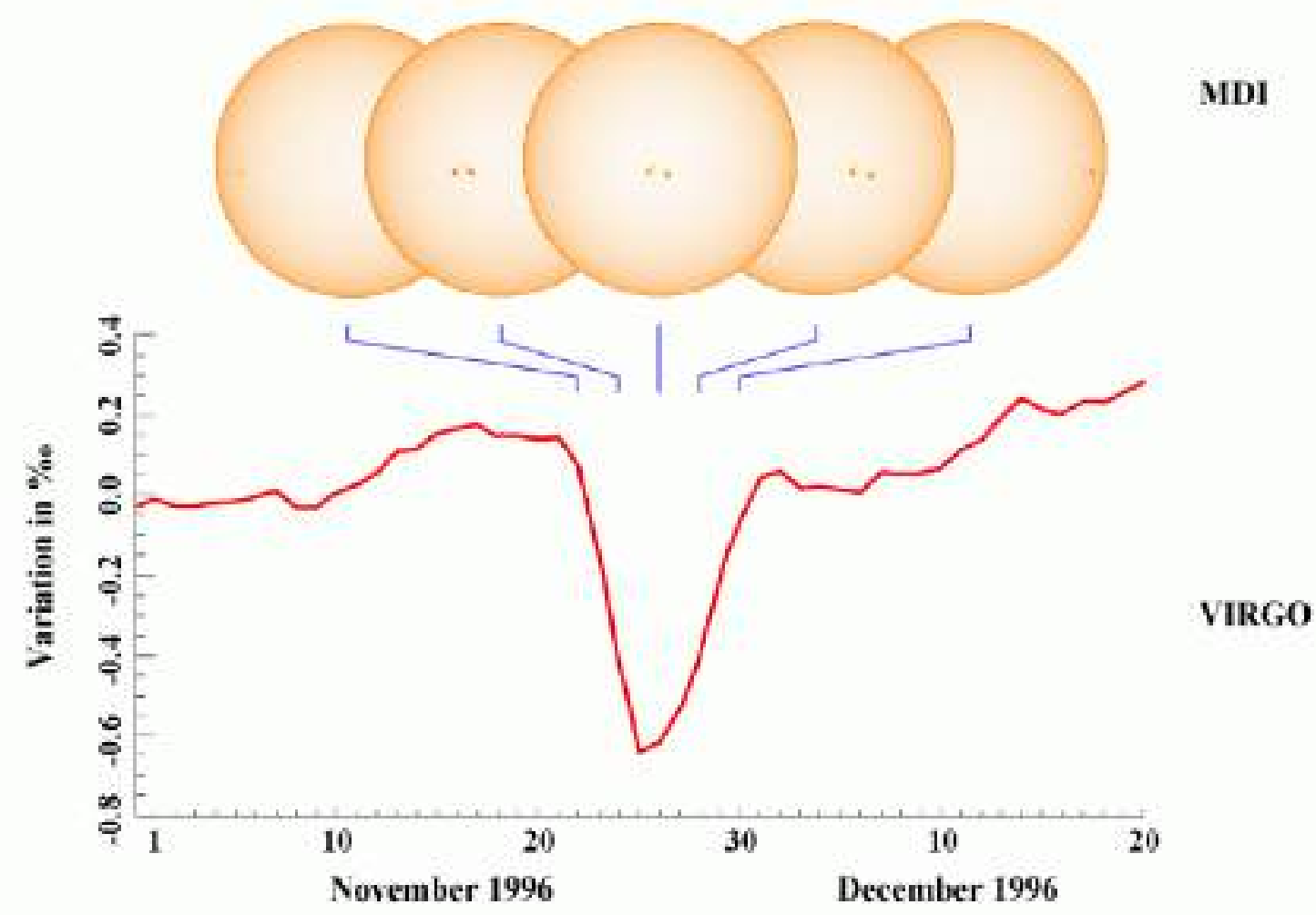

Figure 1.13: Relative change in TSI measured by SOHO/VIRGO in November - December 1996. On top are shown Michelson Doppler Imager (MDI) continuum images for the periods marked in the plot. Taken from Solanki and Fligge (2002).

the Ca II K line (photographic spectroheliograms since 1892, and CCD filtergrams since 1986 e.g. Hale 1893, Chapman et al. 1997, Ermolli et al. 2007b), and in continuum wavelengths (available since 1874, e.g. Ermolli et al. 1998, Scherrer et al. 2012, Willis et al. 2016a). Observations in the continuum are used to derive information about sunspots and faculae in the photosphere. Ca II K observations sample the chromosphere (see Chapter 2) and are used to derive information about plage areas. Ca II K images can act as a substitute for magnetograms (although without information on the magnetic polarity) and can be used to trace the evolution of the magnetic field on the Sun. The reason for this is the reported relation between Ca II K brightness and magnetic field strength averaged over a pixel (e.g. Babcock and Babcock 1955, Skumanich et al. 1975, Schrijver et al. 1989, Harvey and White 1999, Loukitcheva et al. 2009, Kahil et al. 2017). However, the exact characteristics of this relation are not well defined (see Chapter 5). Use of Ca II K data has been limited so far mostly to the CCD filtergram observations, available since about 1986, while earlier photographic filtergrams and spectroheliograms suffer from the lack of proper photometric calibration, besides suffering also from artefacts and other defects (see Chapters 2 and 3). Analysis of the uncorrected and uncalibrated data carries high uncertainties.

Three types of sunspot records have been used, sunspot areas, sunspot number, and group sunspot number. The photometric sunspot index (PSI) is derived from the sunspot areas by taking the average spot contrast into account and describes the deficit in irradi- 


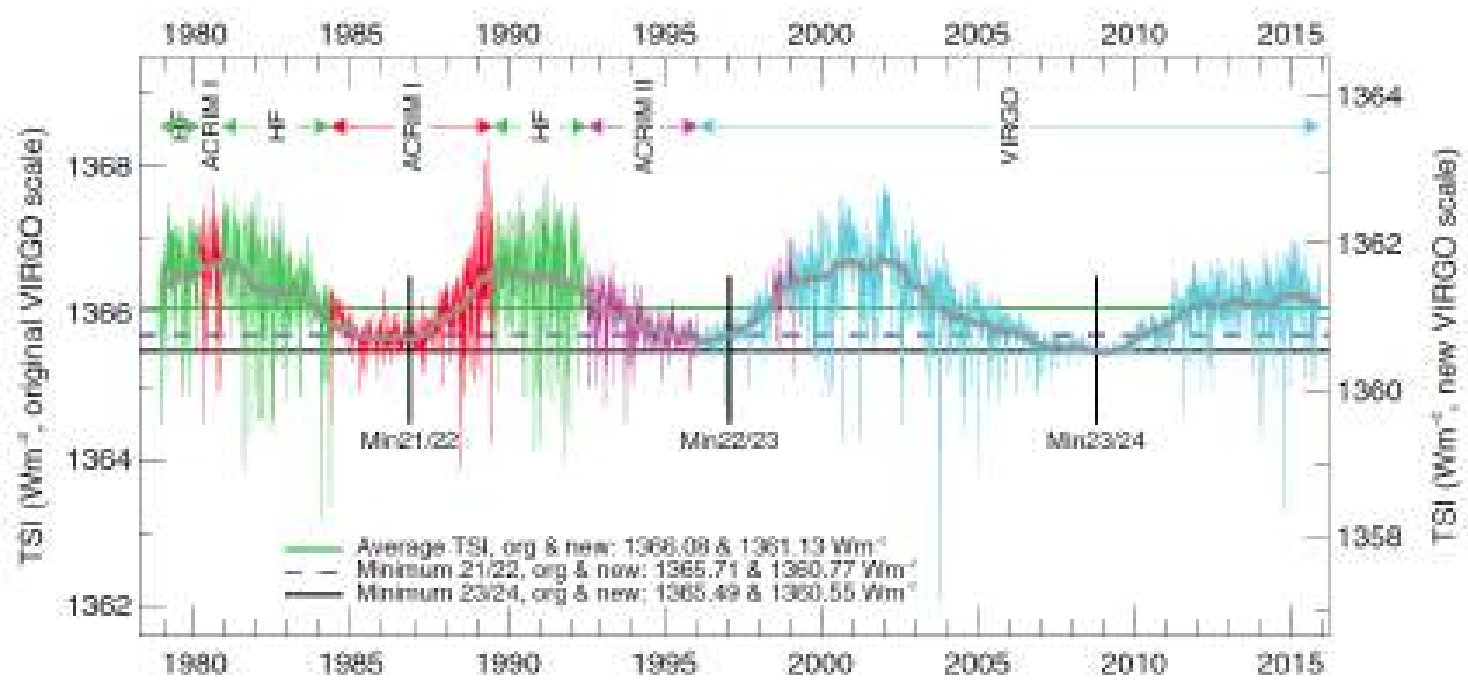

Figure 1.14: The PMOD TSI composite. The values on the $y$ axis on the left correspond to the original Virgo scale, while on the right after the corrections. The different colours correspond to values from different instruments according to the labels at the upper part of the plot. The horizontal lines mark the average value of TSI over the whole interval (green) and the average values during the minima between cycles 21/22 (blue dashed) and cycles 23/24 (black). The TSI values of the horizontal lines are noted at the lower part of the plot. Taken from http://pmodwrc.ch/pmod.php?topic=tsi/ composite/SolarConstant

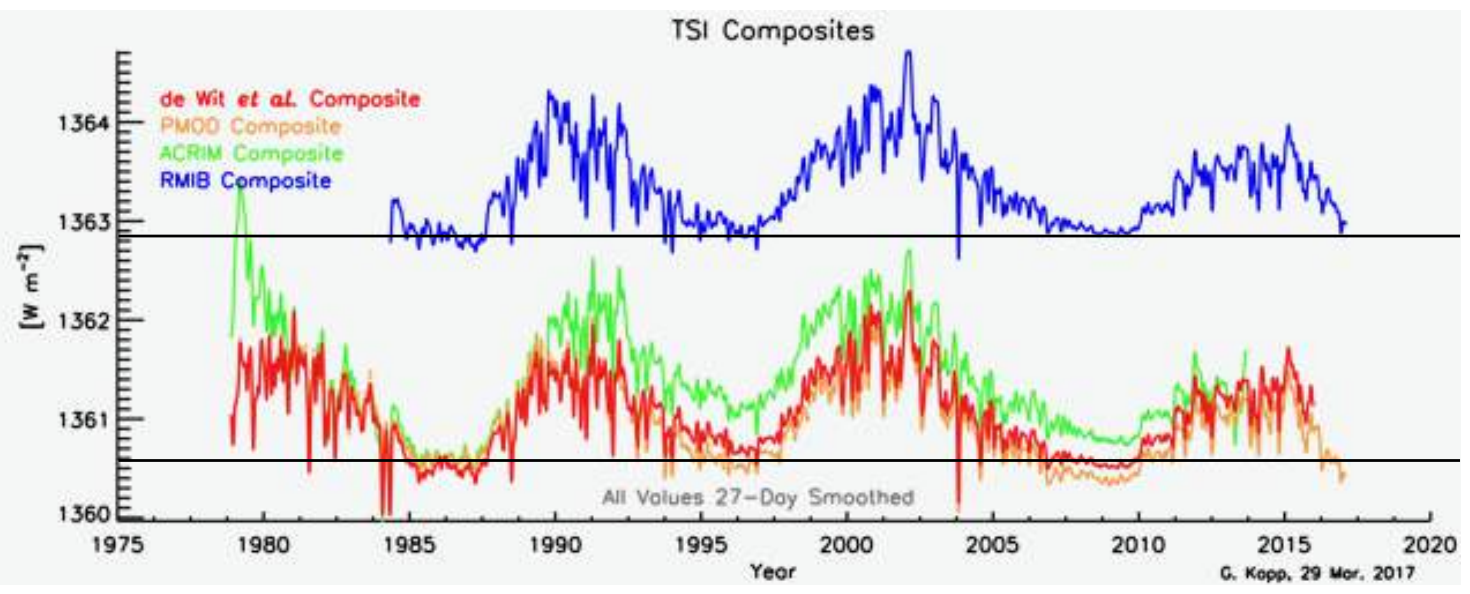

Figure 1.15: Composites of TSI: PMOD (yellow), ACRIM (green), RMIB (blue), and the one by Dudok de Wit et al. (2017). The composites are plotted in 27-day smoothed values. The horizontal lines denote the approximate level of TSI around 1986 in PMOD and RMIB composites. Courtesy of Greg Kopp.

ance due to the sunspots (e.g. Willson et al. 1981). Measurements of sunspot areas exist since 1874, sunspot number records are available since 1700 (see Chapter 6 for more details), and the group sunspot number series extend back to 1610. These data can provide information on the bright features only using some assumptions. A more detailed discussion of the available sunspot number series and issues concerning their calibration is 
given in Chapters 6 and 7 .

Indirect proxies of solar activity are, e.g., the aa-index, cosmic ray flux, and concentrations of cosmogenic isotopes ${ }^{10} \mathrm{Be}$ and ${ }^{14} \mathrm{C}$. The aa-index (Mayaud 1973) is a geomagneticactivity index measured from two almost antipodal observatories in the UK and Australia. It is correlated with solar activity (Pulkkinen et al. 2001, Beer et al. 2006) through the induction of currents when the Sun's open magnetic field lines are passing the Earth change. That in turn leads to fluctuations of the geomagnetic field. Measurements of the aa-index go back to 1868. The cosmic ray flux has been measured since 1951 from various neutron monitors around the globe (Moraal and Stoker 2010, Mavromichalaki et al. 2016, Jung et al. 2016, Usoskin 2017). The cosmic ray influx in the heliosphere is considered constant for the time-scales considered here (periodicities of $~ 143 \mathrm{Myr}$ in the cosmic ray flux have been identified due to the passage of the Solar system through the spiral arms of our galaxy Shaviv 2002). Within the heliosphere, the cosmic ray flux is affected by solar activity via the Sun's open magnetic flux. Hence, the cosmic ray flux measured on Earth is higher during low activity periods and vice versa (Forbush 1954, 1958).

Cosmogenic isotopes ${ }^{10} \mathrm{Be}$ and ${ }^{14} \mathrm{C}$ are formed due to interactions of cosmic rays mostly with nitrogen and oxygen in Earth's atmosphere and eventually get stored in plant material and ice, respectively (Lal and Peters 1967, Beer et al. 2012). Just as the cosmic ray fluxes are affected by solar activity, so are the production rates of ${ }^{10} \mathrm{Be}$ and ${ }^{14} \mathrm{C}$. Therefore, by measuring the abundances of ${ }^{14} \mathrm{C}$ along different rings of tree trunks or the concentrations of ${ }^{10} \mathrm{Be}$ at different depths in ice cores enables us to infer the amount of the isotopes that was deposited and hence the amount of incoming cosmic rays. This enables deriving the heliospheric modulation potential which describes how the Sun modulates the flux of cosmic rays and is hence related to solar activity. The ${ }^{10} \mathrm{Be}$ data that have been recovered in ice cores go back to $\sim 1,000,000 \mathrm{BP}$ (e.g. Beer et al. 1990, Berggren et al. 2009, Beer et al. 2012), while ${ }^{14} \mathrm{C}$ has been measured from various sources and go back to $\sim 100,000,000$ BP (e.g. Goslar et al. 2000, Kitagawa and Plicht|2006, Beer et al. 2012). In particular, measurements of ${ }^{14} \mathrm{C}$ that have been done in tree rings go back to $\sim 14,000$ BP (e.g. Stuiver and Polach 1977, Pilcher et al. 1984, Spurk et al. 1998, Hua et al. 2009, Beer et al. 2012, though disconnected measurements have been recovered in sub-fossil kauri trees covering 3500 years within the period 23,000-43,000 BP Turney et al. 2010), in corals 50,000 BP (e.g. Bard 1998, Druffel et al. 2008, Beer et al. 2012, Reimer et al. 2013), in lake or sea sediments $\sim 100,000,000$ BP (e.g. Goslar et al. 2000, Kitagawa and Plicht 2006, Beer et al. 2012), and in speleothems 50,000 BP (e.g. Beck et al. 2001, Hoffmann et al. 2010, Reimer et al. 2013).

The radioisotopes undergo radioactive decay and hence their concentrations get reduced with time. Based on their known half-lives one can estimate how many years it will take for their concentrations to become unmeasurable, which is found to be $\sim 15 \mathrm{Myr}$ for ${ }^{10} \mathrm{Be}$ (10 times its half-life) and $\sim 40,000 \mathrm{yr}$ for ${ }^{14} \mathrm{C}$ (Beer et al. 2012). The measurements further back in time have significantly reduced resolution and considerably increased uncertainties (Beer et al. 2012). Use of ${ }^{14} \mathrm{C}$ after $\sim 1900$ is hindered, because of the burning of $\mathrm{C}$ from coal and oil, which are so old that all ${ }^{14} \mathrm{C}$ in them has decayed, during the industrial era. This introduces great uncertainties in the dating of ${ }^{14} \mathrm{C}$. This is called the Suess effect (Suess 1955).

Among other products of interactions of cosmic rays in the atmosphere, ${ }^{14} \mathrm{C},{ }^{36} \mathrm{Cl}$, ${ }^{39} \mathrm{Ar}$, and ${ }^{81} \mathrm{Kr}$ get deposited in ice cores too, but in lower abundance than ${ }^{10} \mathrm{Be}$. ${ }^{44} \mathrm{Ti}$ is 
produced after interactions of GCRs with $\mathrm{Fe}$ and $\mathrm{Ni}$ (Bonino et al. 1995). It has been measured in stony meteorites that have fallen on Earth, but as a time-series it has, at the moment, only 20 values covering the period 1766 - 2001 (Usoskin et al. 2006, Asvestari et al. 2017). However, it is a very important proxy since, in contrast to ${ }^{14} \mathrm{C}$ and ${ }^{10} \mathrm{Be}$, it is not affected by Earth's atmosphere (e.g. climate, which can influence the terrestrial archives storing ${ }^{10} \mathrm{~B}$ and ${ }^{14} \mathrm{C}$ ). Auroral sightings extending back to $567 \mathrm{BC}$ (e.g. Legrand 1987, Schröder 1992, Stephenson et al.|2004) provide indirect information on solar activity (Siscoe 1980, Pulkkinen et al. 2001, Stephenson et al. 2004, Beer et al. 2006, Vázquez and Vaquero 2010). Various other data have been suggested to potentially carry information about solar activity, but their use has been rather limited so far due to poor cadence, poor understanding of the connection to solar activity, or high uncertainties. Varved lake sediment data can give information on the yearly rate of snow melting based on the thickness of detrital lamina. The snow melting rate is suggested to be anti-correlated with the TSI (Saarni et al. 2016). These data extend back 3600 years (Saarni et al. 2016). However, they suffer from potential layer mixing and high uncertainties in their dating, quite aside of the very indirect and controversial connection with solar activity. Variations in tree-ring formation in fossil forests going back for almost $300 \mathrm{Myr}$ have been suggested to carry information on solar activity too (Luthardt and Rößler 2017).

\subsubsection{Irradiance models}

Models have been developed that use magnetograms and the proxies described in Sect. 1.4.2.1 to account for the contributions of the magnetic features on the solar surface to solar irradiance. These features include sunspots (usually separated into umbra and penumbra), faculae, and network. In some models faculae and network are subdivided into further subcategories (e.g. Ermolli et al. 2011, Fontenla et al. 2011). Everything not belonging to these classes is tagged as the quiet Sun (QS, hereafter). Several models have been successful in reproducing the measured TSI (Fröhlich and Lean 1997, Fligge et al. 2000b, Preminger et al.|2002, Krivova et al. 2003, Wenzler et al.|2006, Ermolli et al.|2011, Ball et al.2012, Yeo et al. 2014b). Two distinct categories of such models exist:

- proxy (or empirical) models (e.g. Hudson et al. 1982, Oster et al. 1982, Heath and Schlesinger 1986, Tapping 1987, Tapping and Morton 2013, Foukal and Lean 1988, 1990, Lean et al. 1995, Chapman et al. 1996, 2012, 2013, Keil et al. 1998, Lean 2000, Worden et al. 2001, Preminger et al. 2002, Wang et al. 2005, Steinhilber et al. 2009. Delaygue and Bard 2011, Coddington et al. 2015, Foukal 2015, Georgieva et al. 2015, Tebabal et al. 2015, Yeo et al. 2017).

- semi-empirical models (e.g. Fligge et al. 1998, Fligge and Solanki 2000, Fligge et al. 2000a, Solanki and Fligge 1998, 1999, Ermolli 2001, Ermolli et al. 2003b, 2011, Krivova et al. 2003, 2007, 2009, 2010, 2011, Penza et al. 2003, Fontenla et al. 2006, 2011, Wenzler et al. 2006, Crouch et al. 2008, Haberreiter et al. 2008, 2014, Shapiro et al. 2011, Vieira et al. 2011, Ball et al. 2012, Bolduc et al. 2014, Dasi-Espuig et al.2014, 2016, Yeo et al. 2014b).

The empirical models make use of proxies of solar magnetic activity and apply regressions to them to match the direct measurements of TSI and SSI. Proxy-based models 
have used the Mg II index (e.g. Heath and Schlesinger 1986, Donnelly et al. 1994, Coddington et al. 2015, Tebabal et al. 2015, Yeo et al. 2017), Ca II K 1 $\AA$ index (e.g. White and Livingston 1981, Donnelly et al. 1994, Keil et al. 1998), Ly $-\alpha$ flux (e.g. Foukal and Lean 1990), He I equivalent widths (e.g. Foukal and Lean 1988), the F10.7 index (e.g. Lean and Foukal 1988, Foukal and Lean 1990, Yeo et al. 2017), white-light observations (e.g. Foukal 2015), Ca II K full-disc observations (Oster et al. 1982, Foukal and Lean 1986, Chapman et al. 1996, Worden et al. 2001, Foukal and Milano 2001, Foukal et al. 2009. Chapman et al. 2012, 2013), sunspot number series (e.g. Hudson et al. 1982, Lean et al. 1995), and the modulation potential derived from cosmogenic radioisotopes (e.g. Steinhilber et al. 2009, Delaygue and Bard 2011). One of the main problems of the empirical models is that they rely heavily on actual measurements of solar irradiance to derive information about the long-term trend. However, the limited availability of direct measurements and problems with instrument degradations prohibit any robust conclusion about such trends at this moment. This becomes yet more problematic with the spectral irradiance where the available data are even scarcer. The empirical models also make an intrinsic assumption that linear combinations of proxy series can reproduce the irradiance variations at different time-scales and wavelengths (Yeo et al. 2015b).

The semi-empirical models use solar observations to derive information on the locations and areas covered by magnetic features. The brightness of these features is computed with spectral synthesis codes from semi-empirical model atmospheres. The sunspot contribution to irradiance has in all models been derived from continuum observations or the sunspot number series. A number of different observations have been used to describe the facular contributions. Magnetograms, being direct measurements of the magnetic field, provide the most accurate reconstruction of the solar irradiance (Fligge et al. 2000a, Krivova et al. 2003, Wenzler et al. 2006, Ball et al. 2012, Yeo et al. 2014b), however magnetograms exist for almost the same period of time as the direct measurements of irradiance. Full-disc CCD-based Ca II K filtergrams have been used in a similar manner to magnetograms (e.g. Ermolli 2001, Ermolli et al. 2003b, 2011, Fontenla et al. 2006, 2011). The sunspot areas, or a reconstructed version of them from the sunspot number series, have been used to infer the filling factors of faculae via some model (e.g. Solanki and Fligge 1999, Fligge and Solanki 2000, Krivova et al.,2007, 2010), or used to compute magnetograms with the help of surface flux transport simulations (e.g. Dasi-Espuig et al. 2014, 2016).

The cosmogenic radioisotope concentrations have also been used to reconstruct the open solar magnetic flux and the sunspot numbers from which the irradiance is finally computed (e.g. Vieira et al. 2011). In these reconstructions, the geomagnetic field needs to be known as well. Besides the direct measurements of the geomagnetic field (available since 1834 Courtillot and Le Mouel 1988), it has been reconstructed back to $\sim 10,000 \mathrm{BC}$ based on information found in lava flows, lake or marine sediments, and archaeological artifacts (Donadini et al. 2009, 2010). This is currently limiting the use of the cosmogenic radioisotope data for irradiance reconstructions back to $\sim 10,000 \mathrm{BC}$. However, both the sunspot observations and the cosmogenic radioisotope concentrations provide information about the bright regions only in a rather indirect way under some assumptions.

Relying on solar observations to describe the distribution of the magnetic features on the solar surface, the semi-empirical models are also susceptible to uncertainties from these data. However, semi-empirical models are able to take into account the CLV of the 
magnetic features for all wavelengths. Furthermore, the use of intensity spectra derived from radiative transfer and model atmospheres allows for more accurate SSI reconstructions. The semi-empirical models also have the potential of improving our understanding about the physics of the Sun. This can be achieved because the models use approximations and assumptions based on our current knowledge of the Sun, which can be validated by directly comparing the output of the irradiance models with actual irradiance measurements.

Figure 1.16 shows six TSI reconstructions based on sunspot records, or ${ }^{10} \mathrm{Be}$ and ${ }^{14} \mathrm{C}$ data back to 1600. It is obvious that the reconstructions differ in the magnitude of the changes in TSI between the present time and the Maunder minimum. Shapiro et al.(2011, not shown in Fig. 1.16) derived the largest difference of $6 \mathrm{~W} \mathrm{~m}^{-2}$, while Schrijver et al. (2011, this was just an estimate of the irradiance changes not a complete irradiance reconstruction) found a difference of only $0.7 \mathrm{~W} \mathrm{~m}^{-2}$. Other reconstructions favour a more moderate difference of 1.0-1.5 W m${ }^{-2}$ (Wang et al. 2005, Krivova et al. 2007, 2010, Vieira et al. 2011, Dasi-Espuig et al. 2014). This illustrates that there is a significant disagreement among the various reconstructions on the secular trend of the solar irradiance. These discrepancies arise due the differences in the models used, but also due to the ambiguities of the data used for the reconstructions, in particular the lack of information on the bright component, which alone determines the secular trend since the Maunder minimum. It is imperative to improve the quality of the available data in order to reduce the uncertainties in the irradiance reconstructions as well.

\subsection{Motivation for this thesis}

As explained in Sect. 1.4.1 and 1.4.2.2 the existence or the magnitude of any secular trend in irradiance variations is uncertain both from measurements and reconstructions. This is due to the long-term uncertainties in the direct irradiance measurements, in the proxy data used by models, and due to the missing information on the evolution of bright features. In this context, Ca II K spectroheliogram (SHG) observations are a really critical dataset. They can provide information on plage regions and their locations on the disc, allowing for more accurate irradiance reconstructions back to the beginning of 1900's. Furthermore, this can be used as a test of existing models to provide constraints on the magnitude of variations over the 20th century. Thus, Ca II K data can set the grounds for an improvement in the irradiance reconstructions also further back in time than the 20th century. However, these old SHG data were stored in photographic plates or films, which are not linear detectors (as will be discussed in Chapter 2). A plethora of largescale artefacts have been introduced during the storage of the plates, that also need to be accounted for.

A lot of existing irradiance reconstructions are currently based on the sunspot number record. However, even this dataset is far from being consistent. Being a composite of observations by different observers made with different instruments and capabilities, it is predominantly made by stitching the data together through simple linear scaling. However, as we will demonstrate in Chapter 6, the relation for the inter-calibration of individual observers is not linear. Hence the available series carry significant errors that increase vastly further back in time.

The work presented here aims at enhancing our understanding of solar variability on 


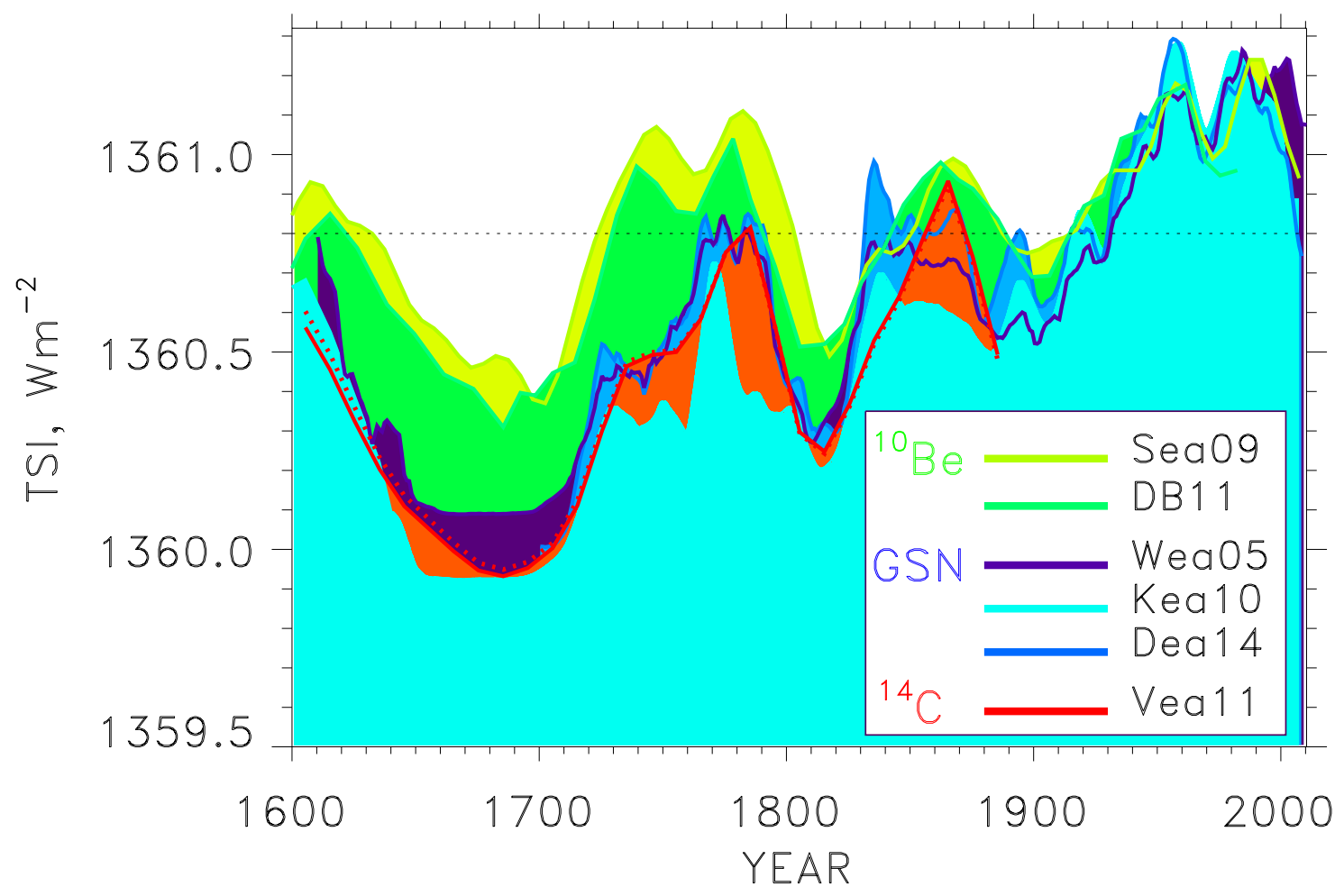

Figure 1.16: TSI reconstructions since 1600 based on sunspot areas (blue colours) and cosmogenic radioisotopes ${ }^{10} \mathrm{Be}$ (green colours), and ${ }^{14} \mathrm{C}$ (red). Sea09 is for Steinhilber et al. (2009), DB11 for Delaygue and Bard (2011), Wea05 for Wang et al. (2005), Kea10 for Krivova et al. (2010), Dea14 for Dasi-Espuig et al. (2014), and Vea11 for Vieira et al. (2011). From Usoskin et al. (2015).

time-scales of decades to centuries by improving or producing new long-term proxies of solar magnetic activity. In particular, we develop a method to photometrically calibrate the available digitised Ca II K spectroheliograms and to correct them for various artefacts. The data are described in detail in Chapter 2 and the method in Chapter 3 . In Chapter 4 we test various segmentation methods and present preliminary results for the plage areas derived from historical $\mathrm{Ca}$ II K observations. The relation between the bright features and the magnetic field strength, studied in Chapter 5, allows a conversion of these data to magnetograms. In Chapter 6 we discuss the dependence of the sunspot group areas on the level of solar activity. This has important implications on the relation between the sunspot group measurements by two observers, and hence is important for evaluating the aptness of existing calibration methods. In Chapter 7 we propose a new method for a more accurate reconstruction of the group sunspot number series. This reconstruction takes into account the non-linearity of the relation between observers, enabling also to account accurately for error transport. Finally, in Chapter 8 a brief outlook is given and some future steps described. 


\section{Ca II K observations}

This chapter provides some general information about observations of the Sun in wavelength ranges centred in the $\mathrm{Ca}$ II K line. We first discuss characteristics of the solar spectrum around this line. We continue with a brief description of the instruments used for solar observations in this wavelength. This is followed by some basic aspects of photographic processes with focus on the response of the plates to the incident radiation. We give an overview of the numerous observational programs performed in the $\mathrm{Ca}$ II $\mathrm{K}$ line for the Sun since the late 19th century. Finally we briefly review previous studies aimed at processing such data.

\subsection{Ca II K line}

The Ca II K line is centred at $393.367 \mathrm{~nm}$, and is a rather broad and deep line having a core intensity that is roughly $5 \%$ of that in the continuum. It is characterised by 2 peaks $\left(\mathrm{K}_{2 \mathrm{~V}}\right.$, $\left.\mathrm{K}_{2 \mathrm{R}}\right)$, a reversal at the centre $\left(\mathrm{K}_{3}\right)$, and 2 secondary minima $\left(\mathrm{K}_{1 \mathrm{~V}}, \mathrm{~K}_{1 \mathrm{R}}\right)$. The labels $\mathrm{V}$ and $\mathrm{R}$ denote whether the peak/minimum is towards the violet $(\mathrm{V})$ or red $(\mathrm{R})$ part of the spectrum relative to $\mathrm{K}_{3}$. Figure 2.1 shows two intensity profiles of the $\mathrm{Ca}$ II $\mathrm{K}$ line corresponding to a QS and a plage region superimposed on each other. All individual features introduced above, occur within the $1 \AA$ interval marked in Fig. 2.1. The formation height in the solar atmosphere of the Ca II K line can be seen in Fig. 1.3. From the wings of the $\mathrm{Ca}$ II $\mathrm{K}$ line towards the $\mathrm{K}_{1}$ minima it samples the photosphere with increasing height until the $\mathrm{K}_{1}$ minimum, which is formed at the temperature minimum, at the boundary between the photosphere and chromosphere. The decreasing temperature with height in the photosphere results in a normal absorption line. Closer to the line core than the $\mathrm{K}_{1}$ minima the line gets brighter with increasing height. In the lower chromosphere the source function follows the Planck function. This is the case nearly up to the heights where the $\mathrm{K}_{2}$ peaks are formed. Above these heights the source function becomes smaller than the Planck function. As a result absorption dominates again and causes the $\mathrm{K}_{3}$ feature to be the darkest in the line (Stix 2004).

A very important aspect of Ca II K line that can be seen in Fig. 2.1 is the ability of this line to provide information about the magnetic features. Thus the change in intensity of the line core is significantly stronger when sampling a plage region (upper curve in Fig. 2.1) compared to a QS region (lower curve in Fig. 2.1). 


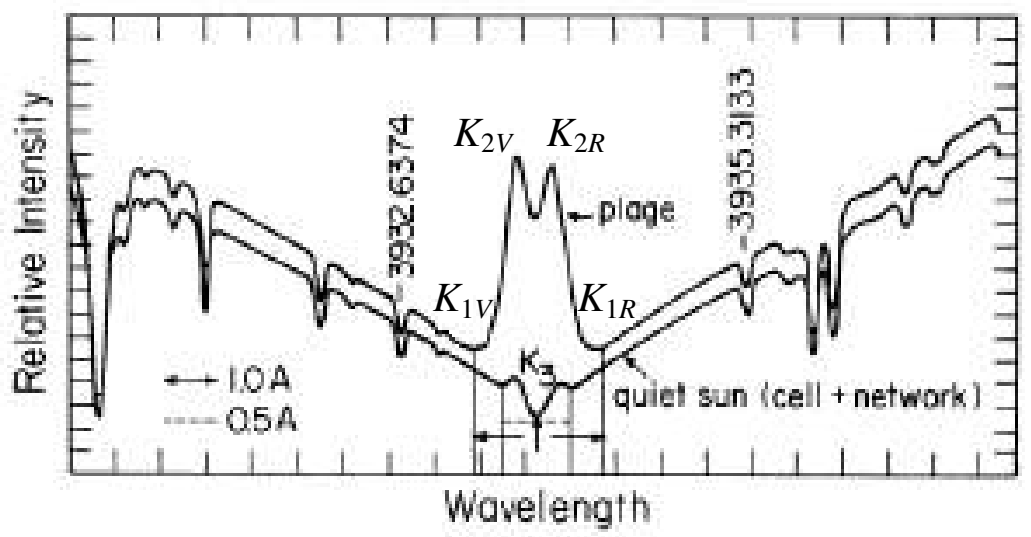

Figure 2.1: Ca II K line profile for quiet Sun (lower curve) and plage (upper curve). The horizontal arrows and dashed line below $\mathrm{K}_{3}$ denote $1 \AA$ and $0.5 \AA$ intervals, respectively. Taken from Skumanich et al. (1984).

\subsection{Observing instruments}

Two general groups of optomechanical instruments have been used to image the Sun in the core of the Ca II K line. The first one is a spectroheliograph, which was first used in 1889 and is still employed in various observatories (see Table 2.1). More modern observatories, however, usually use a filtergraph (see Table 2.2). The observations then are stored on photographic plates or films and more recently as digital files recorded with CCD cameras.

\subsubsection{Spectroheliograph}

A spectroheliograph is a device used to produce images of the Sun in a specific (narrow) band of wavelengths. It was invented by George E. Hale in 1889 (Hale 1893). The principle of this instrument is the use of a prism or a diffraction grating to separate the incoming light into different wavelengths followed by a narrow slit to allow the passage of the desired range of wavelengths based on its width. At the entrance of the instrument there is a narrow slit allowing light from only a narrow strip of the Sun to enter, and this strip is then photographed. The entrance slit and the photographic material are then synchronously moved to photograph the next strip on the Sun. Figure 2.2 shows the schematic of the spectroheliograph employing 2 prisms used at the Kodaikanal observatory. The siderostat (Fig. 2.2 a)) that was used to follow the movement of the Sun was an 18-inch diameter plane mirror (marked as M1 in Fig. 2.2b)). A 12" in diameter triplet lens (L1) focused the rays on the first slit (S1). A set of two prisms (P1, P2) were used. Then an achromatic lens (L3) focused the rays on the second slit (S2), while also passing through a violet filter $(\mathrm{F} 1)$. Finally the rays are sent to the photographic plate $(\mathrm{P})$.

Scanning the full solar disc with a spectroheliograph requires several minutes. The movement of the instrument has to be extremely accurate and constant, otherwise various image distortions are introduced. Archived observations that were stored on photographic material show that a very common problem they suffered from was that the subsequent strips overlapped. Being exposed twice, these overlapping regions appear brighter. Other 
a)

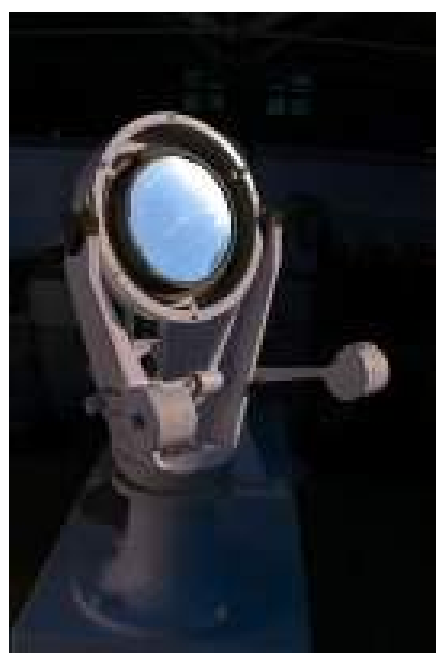

b)

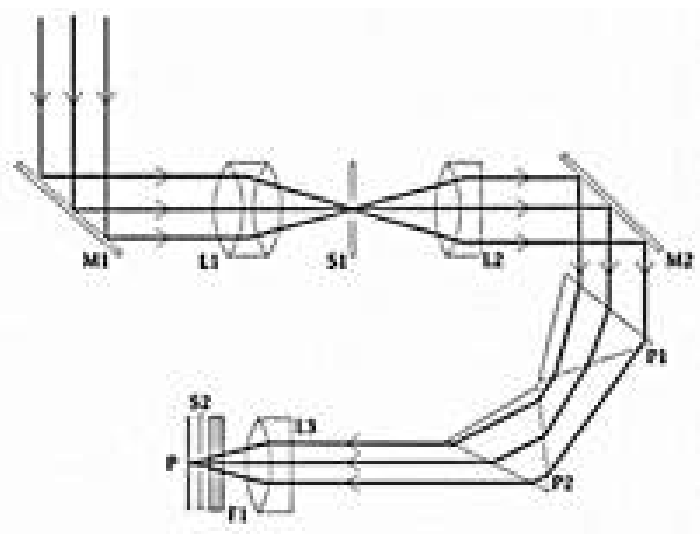

Figure 2.2: Siderostat (a)) and schematic of spectroheliograph (b)) used at the Kodaikanal observatory. The telescope-spectroheliograph combination includes a siderostat (M1); a triplet lens (L1) focusing the rays to the first slit (S1); a doublet lens (L2); a plane mirror (M2); two prisms (P1, P2); a doublet lens (L3), a violet filter $(\mathrm{F} 1)$, a second slit (S2) and the photographic material (P). Images taken from https://kso.iiap.res.in//observatory/instruments

issues include the varying spectral passband, because the slit width was not maintained at a constant value. Small unwanted movements of the slit can also change the sampled wavelength band.

\subsubsection{Filtergraph}

Filtergraphs are much simpler configurations than the spectroheliographs. They simply consist of a telescope with appropriate filters that allow light only within the desired wavelength band to pass and focus it on to the detector (photographic or electronic) located at the end. The great advantage of filtergrams is that the exposure can be very short, of the order of a few seconds. Also it is possible to record the whole solar disc simultaneously without the need of complex and sensitive mechanisms, thus minimizing geometrical distortions in the images.

\subsection{Observational aspects}

All ground-based observations taken with spectroheliographs and filtergraphs suffer to some extent from certain problems. The most common among those are seeing, straylight, and vignetting.

Seeing is produced by variations in the refractive index in the Earth's atmosphere. Seeing can manifest itself as distortions in the image due to variable displacement of different parts of the image. Displacements over shorter time than the exposure will show up as motion blurring, while if they last longer than the exposure they will appear as distortions in the image. Changes in temperature and/or wind speed in the Earth's atmosphere are the common sources of this phenomenon. Consequently, the time of day at which 


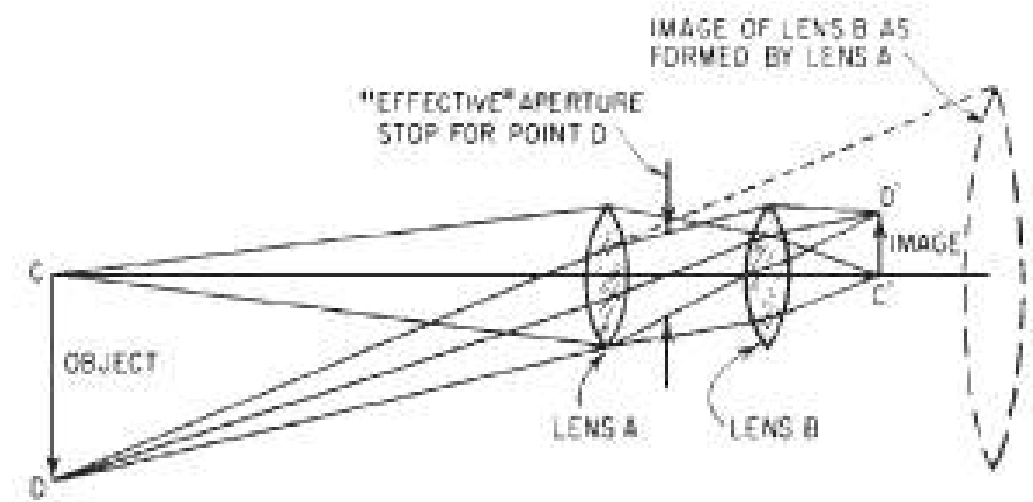

Figure 2.3: Schematic of optical vignetting, demonstrating how a finite distance between two lenses can introduce vignetting in the recorded image from an off axis point (D). Taken from Smith (2007).

the observation is carried out plays a significant role, and usually early morning is the optimum period for solar observations to minimise seeing. The location of the telescope affects the seeing conditions as well, thus mountaintop locations near flat surfaces are preferred.

Stray-light includes any light rays that originate from a source different from the target or taking a path different than the intended one. Stray-light reduces the signal-to-noise ratio and lowers the dynamic range. This can occur due to another bright light source near the telescope, due to scattering in Earth's atmosphere or even within the telescope. Since the Sun is an extended, relatively homogeneously bright source, most stray-light in solar images comes from another part of the solar disc.

Vignetting is the effect of reducing the intensity at the edges of the recorded image. It is divided into mechanical, optical, and natural vignetting. Mechanical vignetting is caused by abstraction of incident light due to external mechanical components (Smith 2007). Figure 2.3 shows a simplified schematic for optical vignetting (Smith 2007). There is a finite distance between lens $\mathrm{A}$ and $\mathrm{B}$ which acts to limit the light coming from off axis points. For example light arriving from point $\mathrm{D}$ is bounded by the lower end of lens $\mathrm{A}$ and the upper end of lens B. Hence, less light comes from point D than from C. Natural vignetting is caused due to the geometry of the optics (Gardner 1947). It manifests itself as a $\cos ^{4} \phi$ decrease in brightness from the centre towards the edges of the image, where $\phi$ is the angle between the object and the normal to the lens. The $\cos ^{4} \phi$ law is valid provided that the lens has no distortions, and that the diaphragm is between the lens and the object which is at an infinite distance (Gardner 1947).

\subsection{Photographic processes}

In this section we follow Mees (1942), James and Higgins (1960), and Dainty and Shaw (1974) to give a brief overview of photographic processes. The photographic material consists of a base, usually a glass plate or a paper or plastic sheet, coated with some photosensitive material (termed emulsion). The emulsion usually consists of silver ions $\left(\mathrm{Ag}^{+}\right)$and halides forming a cubic lattice, commonly referred to as grains (Dainty and 
Shaw 1974), that are embedded in gelatine. The halide used in photographic material is predominantly bromide $\left(\mathrm{Br}^{-}\right)$, sometimes along with small amounts of iodide ( $\left.\mathrm{I}^{-}\right)$(James and Higgins 1960).

Upon incident radiation (or thermal fluctuations), photoelectrons can be released into the conduction band, where they can be trapped due to imperfections of the crystal. Thus a "latent" image is formed, when the electrons attach to the silver ions producing metallic silver. This image is not visible, unless the exposure is excessive. The conversion to metallic silver occurs at a very low rate, and ends when every exposed silver halide has been eliminated (Mees 1942). In order to speed up this phase and reveal the image that has been created, developing agents that react preferentially with the exposed grains are applied. This process is called development. Some of the unexposed grains also interact with the developing agents and convert into metallic silver, thus introducing what is called photographic fog.

The relation between the plate darkening and the incident radiation is called characteristic curve (CC, hereafter). Abney (1889) was the first to investigate quantitatively the changes in the plate darkening with a series of increasing exposures. He suggested that the CC is described as a square function. This form can now be considered appropriate only for the lower part of the CC. Hurter and Driffield (1890) expanded this analysis by increasing the accuracy of the instruments involved. They introduced the nomenclature used even today for the photographic processes and the $\mathrm{CC}$ is also called Hurter-Driffield curve.

A simple response relation between the incident radiation and the darkening it causes on the photographic material can be derived by making the following considerations. The silver halides are assumed to be identical and separate photoreceptors, independent of their surroundings, uniformly spaced in the emulsion with only one layer of grains. These conditions do not represent the real case. However, they enable us to derive a simple model to describe the underlying relation. Departures from these assumptions will only slightly distort the resulting curve. We also make the assumption that counts below $(L)$ and above $(H)$ a certain threshold do not get recorded. By assuming that the illumination over the emulsion is uniform, the statistics of the incident quanta can be described with a Poisson distribution.

With $q$ being the average number of incident quanta, the probability to count $r$ quanta becomes

$$
P_{r}=q^{r} \frac{e^{-q}}{r !}
$$

The average count of each photoreceptor, $l$, would be

$$
\begin{aligned}
l & =\sum_{r=1}^{\infty} r P_{r} \\
& =\sum_{r=1}^{\infty} r q^{r} \frac{e^{-q}}{r !} \\
& =\sum_{r=0}^{L-1} 0 q^{r} \frac{e^{-q}}{r !}+\sum_{r=L}^{H-1}(r-L+1) q^{r} \frac{e^{-q}}{r !}+\sum_{r=H}^{\infty}(H-L+1) q^{r} \frac{e^{-q}}{r !}
\end{aligned}
$$




$$
\begin{aligned}
l & =q^{L} \frac{e^{-q}}{L !}+2 q^{L+1} \frac{e^{-q}}{(L+1) !}+\cdots+(H-L+1) q^{H} \frac{e^{-q}}{H !}+(H-L+1) q^{H+1} \frac{e^{-q}}{(H+1) !}+\cdots \\
& =\left(q^{L} \frac{e^{-q}}{L !}+\cdots+q^{H-1} \frac{e^{-q}}{(H-1) !}+\cdots\right)+\left(q^{L+1} \frac{e^{-q}}{(L+1) !}+\cdots+q^{H-1} \frac{e^{-q}}{(H-1) !}+\cdots\right)+\cdots \\
& =\sum_{r=L}^{\infty} q^{r} \frac{e^{-q}}{r !}+\sum_{r=L+1}^{\infty} q^{r} \frac{e^{-q}}{r !}+\cdots+\sum_{r=H}^{\infty} q^{r} \frac{e^{-q}}{r !} \\
& =\sum_{r=0}^{\infty} q^{r} \frac{e^{-q}}{r !}-\sum_{r=0}^{L-1} q^{r} \frac{e^{-q}}{r !}+\sum_{r=0}^{\infty} q^{r} \frac{e^{-q}}{r !}-\sum_{r=0}^{L} q^{r} \frac{e^{-q}}{r !}+\cdots+\sum_{r=0}^{\infty} q^{r} \frac{e^{-q}}{r !}-\sum_{r=0}^{H-1} q^{r} \frac{e^{-q}}{r !} \\
& =e^{-q}(H-L+1) \sum_{r=0}^{\infty} \frac{q^{r}}{r !}-\sum_{r=0}^{L-1} q^{r} \frac{e^{-q}}{r !}-\sum_{r=0}^{L} q^{r} \frac{e^{-q}}{r !}-\cdots-\sum_{r=0}^{H-1} q^{r} \frac{e^{-q}}{r !} \\
& =(H-L+1)-\sum_{r=0}^{L-1} q^{r} \frac{e^{-q}}{r !}-\sum_{r=0}^{L} q^{r} \frac{e^{-q}}{r !}-\cdots-\sum_{r=0}^{H-1} q^{r} \frac{e^{-q}}{r !} .
\end{aligned}
$$

This can be expressed in the form:

$$
l=(H-L+1)\left(1-f_{1} e^{-q}\right),
$$

where

$$
f_{1}=\frac{1}{H-L+1}\left(\sum_{r=0}^{L-1} \frac{q^{r}}{r !}+\sum_{r=0}^{L} \frac{q^{r}}{r !} \cdots+\sum_{r=0}^{H-1} \frac{q^{r}}{r !}\right) .
$$

When this image is digitised, it is scanned by different photoreceptors that we consider to be ideal. These photoreceptors have an aperture, $A$, that is different to the grain size. The image that gets recorded depends on the opacity of the plate, $O$. The transmitted (recorded) intensity $\left(I_{t}\right)$ is the incident radiation $\left(I_{i}\right)$ with a fraction removed due to the opacity of the plate. The amount that is subtracted can be considered proportional to the average count of each grain, so that $I_{t}=I_{i}-l b I_{i}$, where $b$ is a proportionality constant. Therefore, the values measured on the photographs (termed as transparency $T$ ) can be described as:

$$
T=\frac{I_{t}}{I_{i}}=\frac{I_{i}-l b I_{i}}{I_{i}}=(1-l b)=1-b(H-L+1)\left(1-f_{1} e^{-\frac{A E}{N_{A}}}\right) .
$$

Here $E$ is the exposure per unit image area and is related to $q$ with the equation:

$$
E=\frac{N_{A} q}{A}
$$

where $N_{A}$ is the number of photoreceptors.

The density, $d$, of the photographic material is defined as

$$
\begin{gathered}
d=\log _{10} O=\log _{10} \frac{1}{T} \\
d=-\log _{10}\left(1-b(H-L+1)\left(1-f_{1} e^{-\frac{A E}{N_{A}}}\right)\right) .
\end{gathered}
$$


In reality we expect the grain size to vary, also the emulsion will have multiple grain layers instead of just one. However, these simple considerations bring out the qualitative behaviour of the photograph to different exposures.

This relation between $d$ and $E$ is the $\mathrm{CC}$ and has a sigmoid shape if plotted against $E$ in logarithmic scale. The CC in general is defined as the relation $d=f(\log E)$. Here, $E=I \times t, I$ is the incident energy per unit area and $t$ the exposure time. Assuming that the plate was exposed evenly, the $\mathrm{CC}$ becomes $d=f(\log I)$. Application of the specific appropriate relation between $d$ and $I$ converts the density values of the photographs to intensity units. This process is called photometric calibration.

Figure 2.4 shows a typical example of a CC, where one can see four distinct regions. The middle section (denoted with label c in Fig. 2.4 is to a good approximation linear, and the response is directly proportional to the incident radiation. This is the region of proper exposure and the main object of the photograph should be aimed to be within this region. The slope of the line along this region is called the $\gamma$ of the plate. It is the slope of the dotted line in Fig. 2.4 .

The region on the lower left part of the curve (label a in Fig. 2.4) represents the fog level, where silver halides turn to metallic silver mostly due to thermal fluctuations. This is essentially the sensitivity of the film/plate to light. The more sensitive it is, the lower the value of the fog level should be.

Immediately after the fog level there is the underexposure region (label b in Fig. 2.4), where the incident photons affect the plate increasingly more with increasing incident radiation.

Right after the linear section (label c in Fig. 2.4), there is the overexposure region (label d in Fig. 2.4), also called the shoulder. In this region the incident photons have a constantly decreasing impact on the plate, eventually leading to a saturation level where no more increase in density occurs.

There is a 5th region, called solarisation (label e in Fig. 2.4), which did not arise from this simple consideration described here. It occurs if the exposure is even greater than the one that results in saturation. In this region the resulting density decreases further when yet more photons arrive at the plate. Solarisation causes the reflectance of these regions to change and introduces bronzed shadows. It has been shown that solarisation can be compensated with extended development (Wilsey and Pritchard 1926, Mees 1942), however this does not correct for the saturation. Figure 2.5 shows the effect of increasing development time on the $\mathrm{CC}$ and the appearance of solarisation.

The photographic material has a range of exposure times within which the ratio of brightness to exposure time is constant. With exposures outside that range this is no longer the case and we have the effect called reciprocity failure. At low light levels, during the exposure several atoms of silver are formed within an unexposed crystal. If that happens, then there is the possibility for the whole crystal to develop into silver. In order to reduce such effects various techniques have been used, such as the use of sensitizers on the film prior to exposure (Babcock 1976).

The characteristics of the $\mathrm{CC}$ depend on a variety of factors, e.g. the exposure time, the gelatine of the photographic plate, the composition of the developers (reducing and fixing baths), the wavelength of observation, the duration of plate development, the temperature, and the degree of stirring during this step.

The emulsion has different sensitivity to different wavelengths. This is illustrated in 


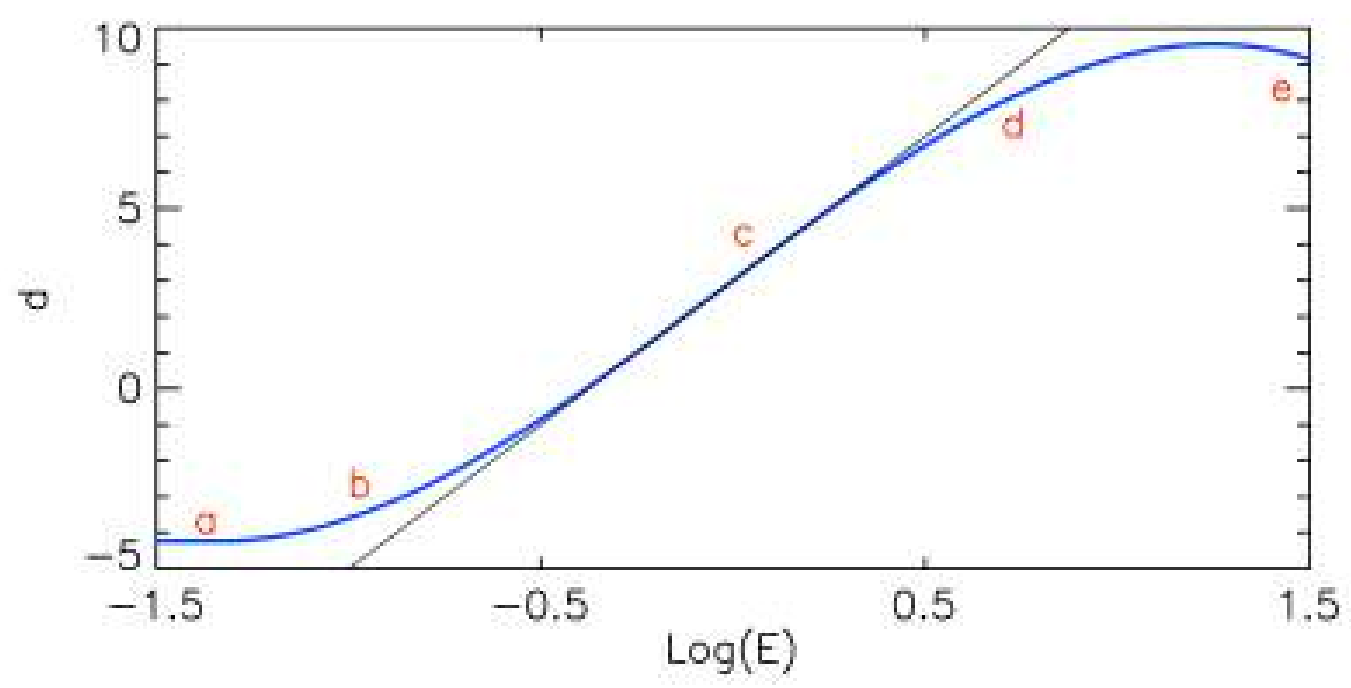

Figure 2.4: A typical form of a characteristic curve. Details are given in Sect. 2.4 .

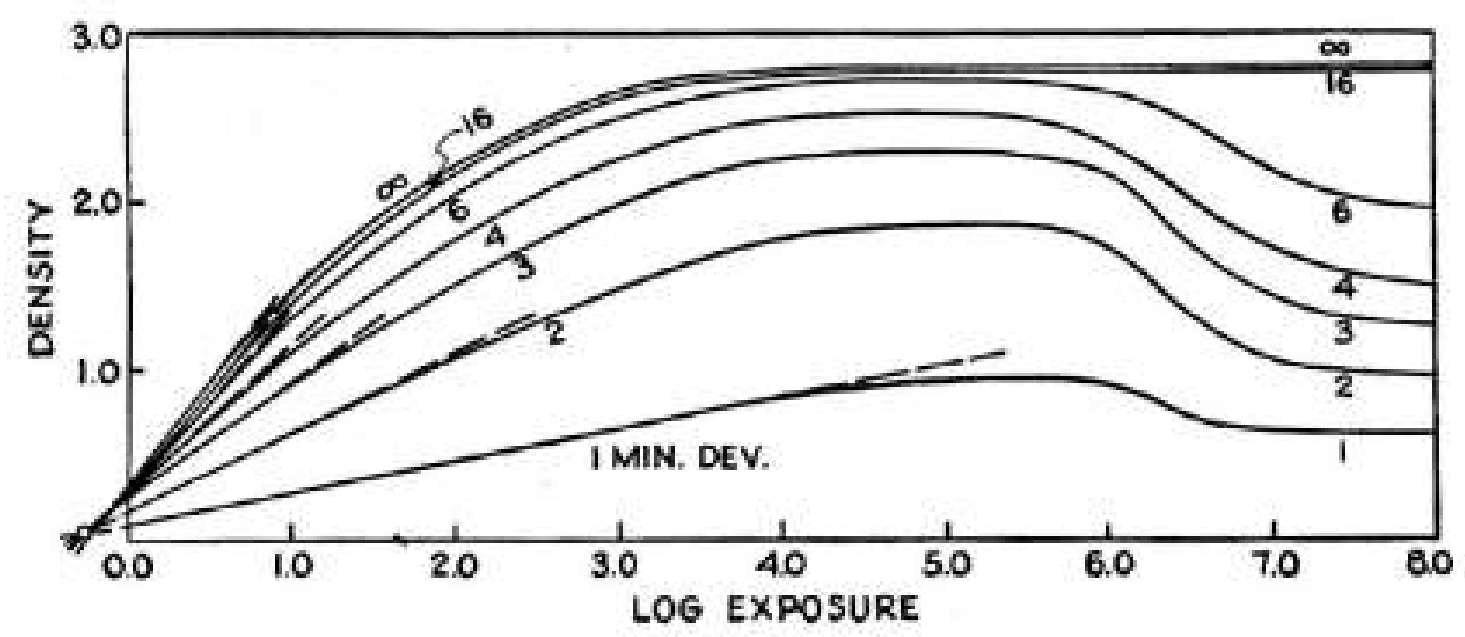

Figure 2.5: $\mathrm{CC}$ derived for different development times (given in minutes on the right end of the curves) illustrating the effect of development on the $\mathrm{CC}$ and solarisation. Taken from Wilsey and Pritchard (1926).

Fig. 2.6 that shows the resulting $\gamma$ of a medium-speed emulsion as a function of the wavelength of observation for different sensitizers. One can notice an almost consistent increase in $\gamma$ with increasing wavelength for all sensitizers up to $\sim 460 \mathrm{~nm}$. The behaviour for higher wavelengths depends strongly on the sensitizer. The sensitivity of a high-speed emulsion to wavelength of observation shows a similar behaviour, but with a smaller change in $\gamma$ (Mees 1942). 


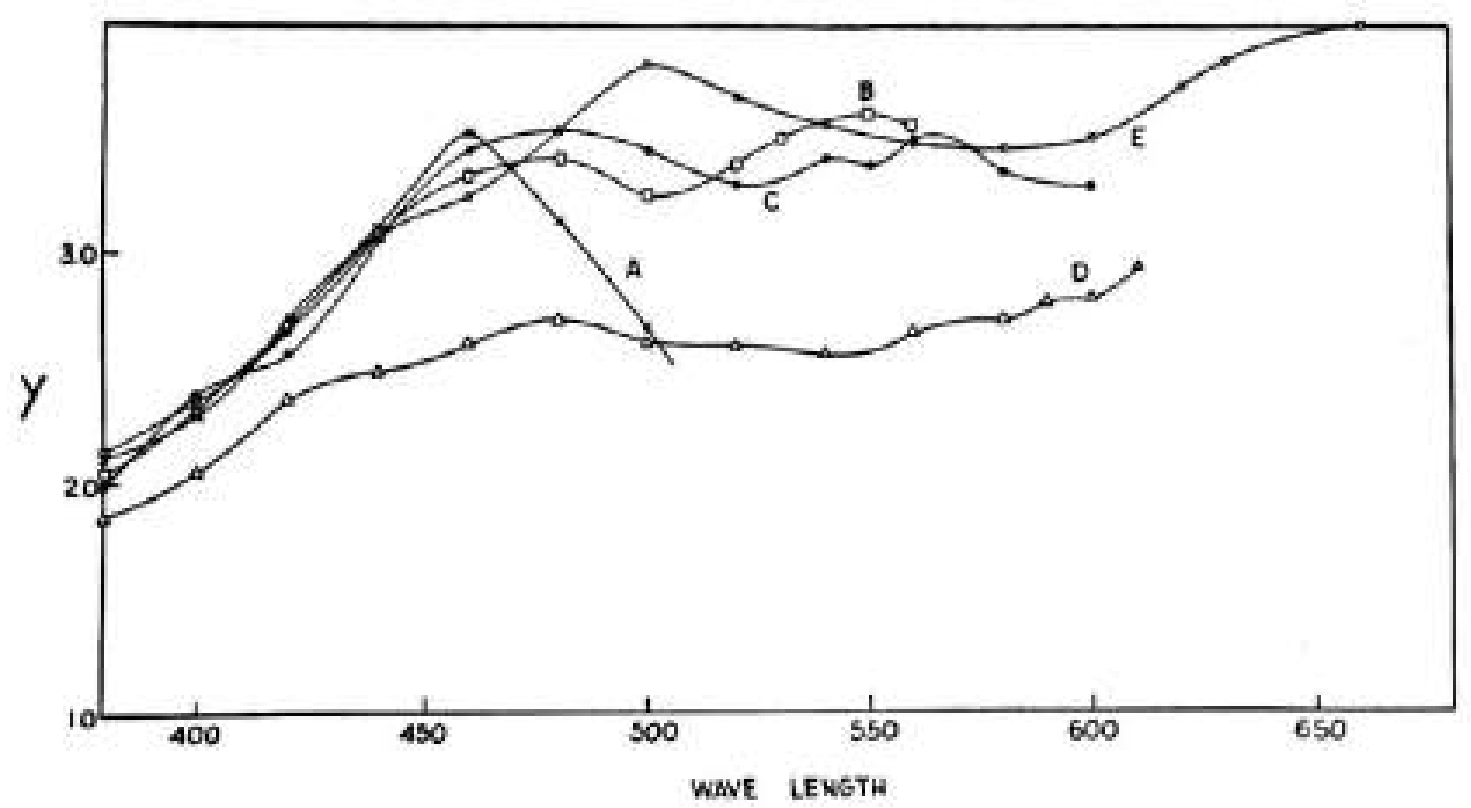

Figure 2.6: Dependence of $\gamma$ to wavelength (in $\mathrm{nm}$ ) of observation for a medium-speed emulsion. The curves show results for different sensitizers: unsensitized (A), pseudocyanine (B), meroocyanine (C), thiacarbocyanine (D), dibenzthiacarbocyanine (E). Taken from Mees (1942).

\subsection{Ca II K observations}

Regular observations in the Ca II K line have been performed throughout the 20th century. Table 2.1 lists some of the observatories that took photographs in the Ca II K line with a spectroheliograph and the most relevant reference(s) for each site. The table is not intended to be a comprehensive list, but rather to illustrate the wealth of available data. Information about such datasets in some cases is difficult to be found, since not all observational programs have been sufficiently described in publications, or the documentation of the observatories might not be publicly available. Another issue concerns the period and number of observations that is reported. The reported values are sometimes the operational period of the observatory, but sometimes these are only the periods covered by the discovered plates. For instance, the plates reported here to stem from the Hamburg observatory are only those found in the archive of the Kiepenheuer-Institut für Sonnenphysik, the rest of the plates are probably lost.

The plates from the Arcetri, Catania, Coimbra, Kodaikanal, Kyoto, McMath-Hulbert, Mitaka, Mt Wilson, Rome, and Sacramento Peak observatories have been digitised and made publicly available. However, the digitizing devices and methods varied not only among datasets but also with time. This results in solar images with different sizes and characteristics depending on the archive. The status of the plates from other observatories is unclear. Samples of digitised observations from Meudon, Wendelstein, and Schauinsland observatories have been made available on-line. For certain datasets there have been multiple digitisations. For instance, Mt Wilson and Kodaikanal data have been digitised twice. The observatories of Coimbra, Meudon, and Kislovodsk continue performing ob- 
servations with the spectroheliograph, though the data are now taken with CCD cameras.

Table 2.2 lists various observatories that performed observations with a filter centred in the $\mathrm{Ca}$ II K line. There are still a number of observatories continuing surveys in Ca II $\mathrm{K}$ with a spectroheliograph or a filtergraph. All observatories functioning now employ a CCD camera and do not use photographic plates any more.

Figure 2.7 shows some examples of digitised spectroheliograms (SHG) from the archives of Arcetri, Catania, Kodaikanal, Meudon, Mitaka, McMath-Hulbert, Mt Wilson, Wendelstein, and Yerkes observatories. The images from Arcetri, Catania, Kodaikanal, Mitaka, and Mt Wilson are shown as negatives containing values of transparency. The images by McMath-Hulbert, Meudon, Wendelstein, and Yerkes are converted to positives, containing values of density. Visual inspection of historical data shows QS density patterns (2D QS background, hereafter) that are in general strongly non-symmetric and inhomogeneous. This is due to a plethora of problems affecting the data, which are introduced either during the observation (e.g. partial coverage by clouds, vignetting, uneven movement of the slit, seeing, stray-light), the development (e.g. non-homogeneous bathing, touching the plate before the process was finished), the storage period (e.g. dust accumulation, scratches, humidity, ageing burns), or the digitization (e.g. dust, hair, loss of dynamic range) of the plate. The solar disc is also often distorted (see e.g. Fig. 2.7 g)).

The analysis presented in this work, was restricted to the digitised SHGs from the Arcetri (Ar), Coimbra (Co), Kodaikanal (Ko, first digitisation), Meudon (Me), Mitaka (Mi), McMath-Hulbert (MM), and Mt Wilson (MW, second digitisation) observatories. These images were taken in the Ca II K line at $\lambda=393.367 \mathrm{~nm}$, with nominal bandwidths ranging from $0.01 \mathrm{~nm}$ to $0.05 \mathrm{~nm}$ and scanning time of several minutes to cover the solar disc. The bandwidths presented for the different archives are the nominal ones, while the real values can vary even within a single photograph. The varying bandwidths change the height of the atmosphere that is sampled. Broader bandwidths increase the photospheric contributions and plage regions appear less bright while sunspots are better visible. Figure 2.8 shows an example of a MW observation where the right part of the image was clearly taken with a broader bandwidth than the left part, possibly due to instrumental problems.

In this work we also use modern observations. They were taken at the Istituto Nazionale di Astrofisica (INAF) Osservatorio Astronomico di Roma (OAR) with the Precision Solar Photometric Telescope (PSPT, Ermolli et al.|1998, 2007a). The Rome/PSPT has been acquiring daily full-disk observations since July 1996, but with the final instrument only from mid 1997 onwards. A second PSPT telescope operated at the Mauna Loa solar observatory (MLSO Rast et al. 2008) between 1998 and 2015. The PSPT telescopes acquire full-disk solar images on $2048 \times 2048$ CCD arrays with various narrow-band interference filters within a few minutes of each other. The observations were carried out with filters centred on the Ca II K line and were processed to apply the standard instrumental calibration (Ermolli et al. 2003a, Rast et al. 2008). The exposure time is much shorter than the historical observations, usually about $0.06 \mathrm{~s}$.

Modern observations suffer significantly fewer problems than the historical ones, however they are still not free of artefacts. Large-scale inhomogeneities are present in modern observations too, mostly due to passage of clouds. Stray-light, seeing, and vignetting can also affect modern data.

It is therefore clear that processing of both historical and modern observations requires recovering the centre-to-limb variation (CLV) profile and accounting for all possible arte- 

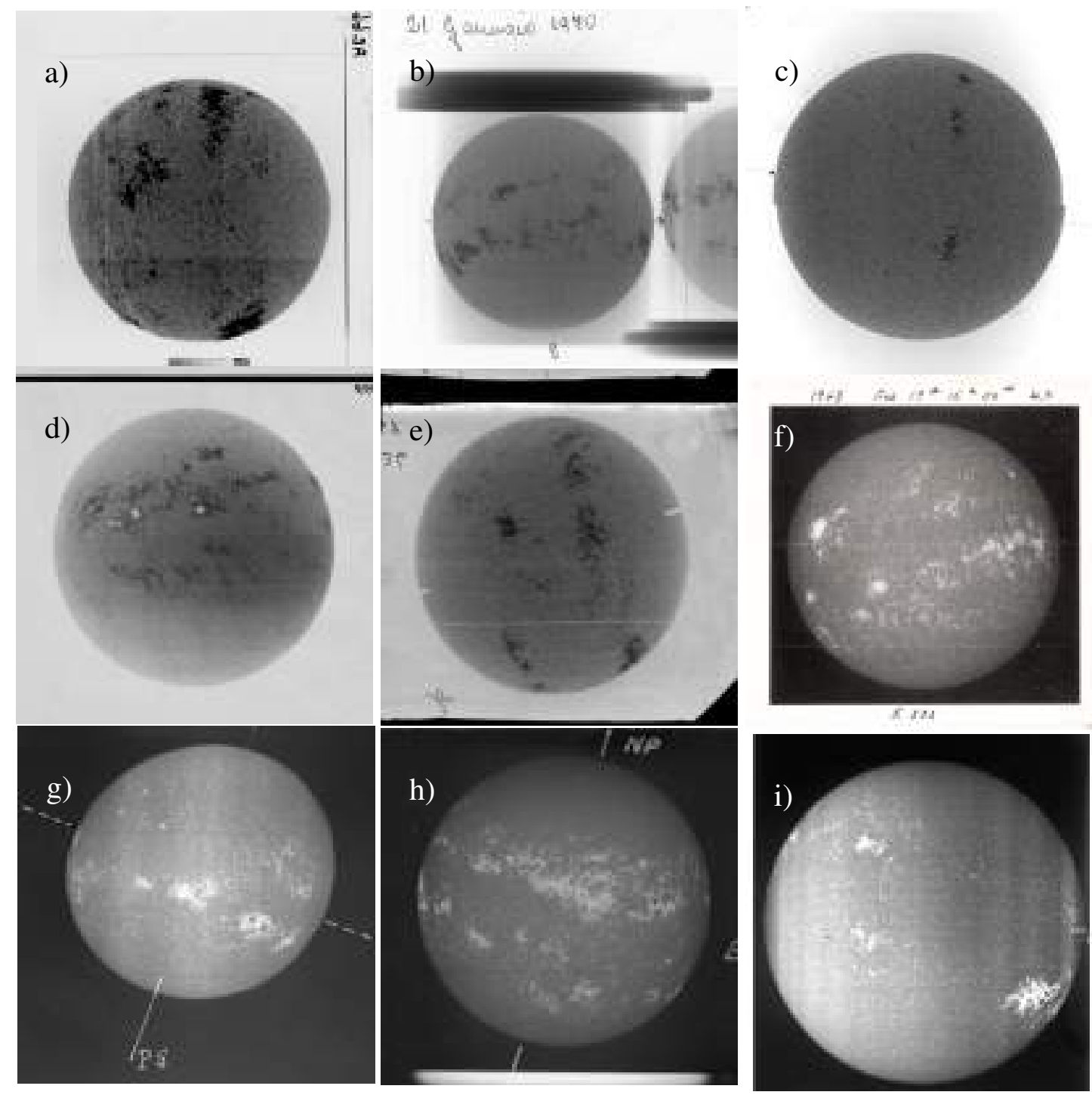

Figure 2.7: Examples of digitised SHGs taken at the: (a)) Arcetri (06/09/1957), (b)) Catania (21/01/1970), (c)) Kodaikanal (14/09/1912), (d)) Mitaka (25/01/1959), (e)) Mt Wilson (01/01/1969), (f)) McMath-Hulbert (19/02/1948), (g)) Meudon (18/05/1894), (h)) Wendelstein (13/08/1947), and (i)) Yerkes (16/02/1907) observatories. (a)-e)) are shown in transmittance values (negative), (f)-i)) are shown in density values (positive).

facts and large-scale inhomogeneities affecting the images. Processing of the historical observations requires one additional step, the photometric calibration of the images.

\subsection{Processing techniques}

Ca II K photographic observations have been used to derive the area, position, and contrast of plage regions, as well as their relation to sunspots. In this section we describe all earlier methods developed to analyse digitized photographic SHG, in particular, the methods used to photometrically calibrate the photographic plates, to calculate the limb-darkening with various inhomogeneities of the solar disc, and to segment them to identify the plage 
Table 2.1: List of historical full-disc SHG Ca II K archives including the period of observations, rough estimate of the number of images, whether they have been digitised, and the bibliography entry.

\begin{tabular}{|c|c|c|c|c|}
\hline Observatory & Years & Plates & Digitised & Reference \\
\hline Arcetri & 1931-1974 & 6000 & yes & 1 \\
\hline Cambridge & $1913-1941$ & 4000 & no & 2,3 \\
\hline Catania & $1963-1977$ & 5000 & yes & 4 \\
\hline Coimbra $^{1}$ & $1926-2007$ & 16000 & yes & 5 \\
\hline Crimea $^{2}$ & 1955-1979 & & no & 6 \\
\hline Ebro & $1905-1937$ & & no & 7 \\
\hline Hamburg ${ }^{3}$ & $1943-1958$ & 200 & partially & 8 \\
\hline Kenwood & $1892-1895$ & & no & 9 \\
\hline Kislovodsk $^{1}$ & $1957-2002$ & & no & 10 \\
\hline Kodaikanal & 1904-2007 & 30000 & yes & 11 \\
\hline Kyoto & 1926-1969 & 7500 & yes & 12 \\
\hline Madrid & $1912-1917$ & & no & 13 \\
\hline Manila & 1963-1976 & & no & 14,15 \\
\hline McMath-Hulbert & 1942-1979 & 10000 & yes & 16 \\
\hline Meudon ${ }^{1}$ & 1894-2002 & & partially & 17 \\
\hline Mitaka & 1917-1974 & 8500 & yes & 18 \\
\hline Mt Wilson ${ }^{4}$ & $1915-1985$ & 40000 & yes & 19 \\
\hline Sacramento Peak & 1963-2002 & 6000 & yes & 20 \\
\hline Schauinsland $^{3}$ & 1944-1964 & 3000 & partially & 8 \\
\hline South Kensington ${ }^{5}$ & 1902-1912 & & no & 21 \\
\hline Wendelstein ${ }^{3}$ & 1943-1977 & 5000 & partially & 8 \\
\hline Yerkes ${ }^{4}$ & 1895-1907 & & partially & 22 \\
\hline
\end{tabular}

References. (1) Ermolli et al. (2009a); (2) Hubrecht (1912); (3) Moss (1942); (4) Zuccarello et al.(2011); (5) Garcia et al. (2011); (6) http://craocrimea.ru/ru/; (7) Curto et al. (2016); (8) Wöhl (2005); (9) Hale (1893); (10) Tlatov et al. (2015); (11) Makarov et al. (2004); (12) Kitai et al. (2013); (13) Vaquero et al. (2007); (14) Hennessey (1969); (15) Mattig and Schroeter (1977); (16) Mohler and Dodson (1968); (17) Mein and Ribes (1990); (18) Hanaoka (2013); (19) Lefebvre et al. (2005); (20) Tlatov et al. (2009); (21) Lockyer (1909); (22) Hale and Ellerman (1903);

\section{Notes.}

${ }^{1}$ Have implemented a CCD camera after that period and continue to make observations.

${ }^{2}$ These are the earliest and latest dates we could find that mention Ca II K observations, but they do not necessarily coincide with the start and end of the program.

3 These data were found in the Fraunhofer-Institut (now Kiepenheuer-Institut für Sonnenphysik) and the numbers correspond only to the recovered plates, not the total amount of observations.

${ }^{4}$ The dates are estimated, Hale created Kenwood observatory in 1889 , but managed to take the first full-disc photograph of the Sun with a spectroheliograph in 1892. He moved along with the telescope and spectroheliograph to Yerkes observatory in 1895. Moved again in 1905 to Mt Wilson along with the Snow telescope constructed in Yerkes observatory, in order to improve the seeing conditions.

${ }^{5}$ This was the Solar physics observatory, which was moved to Cambridge in 1912. 
Table 2.2: List of full-disc filtergram Ca II $\mathrm{K}$ archives including the period of observations, and bibliography entry.

\begin{tabular}{lccc}
\hline Observatory & \multicolumn{2}{c}{ Years } & Reference \\
& Plates & CCD & \\
\hline Anacapri & $1968-1973$ & - & $1,2,3$ \\
Baikal $^{6}$ & $1995-2003$ & $2003-$ & 4 \\
Big Bear & $1971-1995$ & $1996-2005$ & 5,6 \\
Chrotel & - & $2009-$ & 7 \\
Kandilli ${ }^{7}$ & $1968-1994$ & - & 8 \\
Kanzelhöhe & - & $2010-$ & 9 \\
Kodaikanal twin & - & $2008-2013$ & 10 \\
Mees & - & $1988-$ & 11 \\
MLSO/PSPT & - & $1998-2015$ & 12 \\
Rome/PSPT & - & $1996-$ & 13 \\
Rome Monte Mario & $1964-1979$ & - & 14 \\
Brussels & - & $2012-$ & 15 \\
Pic du Midi & - & $2007-$ & 16 \\
San Fernando & - & $1986-$ & 17 \\
Schauinsland & $1968-1984$ & - & 1,18 \\
\hline
\end{tabular}

References. (1) Kiepenheuer (1969); (2) Kiepenheuer (1974); (3) Antonucci et al. (1977); (4) Golovko et al. (2002); (5) Naqvi et al. (2010); (6) Zirin (1974); (7) Bethge et al. (2011); (8) Dizer (1968); (9) Hirtenfellner-Polanec et al. (2011); (10) Singh and Ravindra (2012); (11) http://www.solar.ifa.hawaii.edu/; (12) Rast et al. (1999); (13) Ermolli et al. (2007a); (14) Cimino (1967); (15) http://sidc . be/uset/; (16) Koechlin (2015); (17) Chapman et al. (1997); (18) Münzer et al. (1989);

Notes.

${ }^{6}$ Observations before 2003 were stored on $80-\mathrm{mm}$ film, therefore still need photometric calibration.

${ }^{7}$ Observation were stored in $35-\mathrm{mm}$ film.

regions.

\subsubsection{Photometric calibration methods}

\subsubsection{Calibration wedges}

The importance of recovering the $\mathrm{CC}$ of the images used for scientific purposes was not always realised. This led to no attempt to photometrically calibrate such images for almost half a century. When its importance was realised, calibration wedges started to be recorded as well. These are multiple exposures (usually from 7 to 21) that were imprinted by using entrance slits on the same optical components employed for the solar observations or by exposing the plate to a controlled light-box after the solar image was obtained. Therefore, they provide information needed to recover the CC. One example of a calibration wedge imprint can be seen in Fig. 2.9.

Despite their extended use (e.g. Fredga 1971, Kariyappa and Sivaraman 1994, 


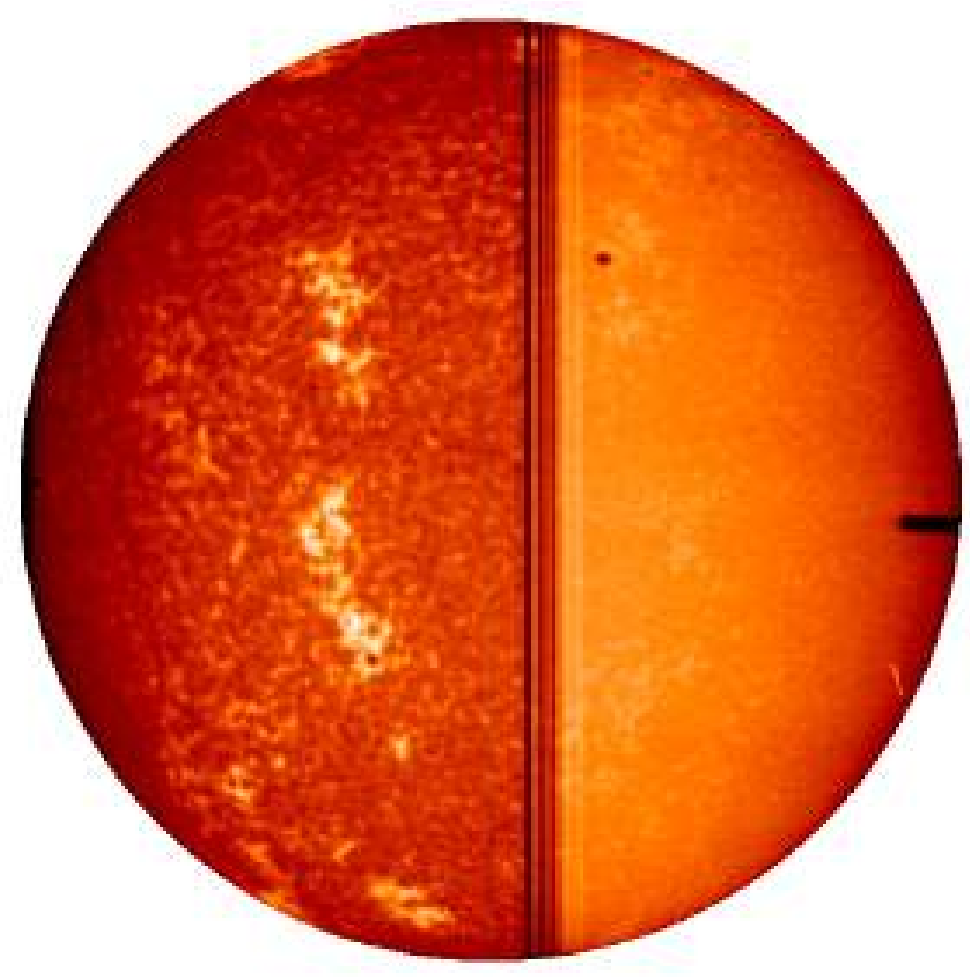

Figure 2.8: MW observation taken on 28/06/1979. The right part of the image was taken with a broader bandwidth than the left part, possibly due to problems of the instruments.

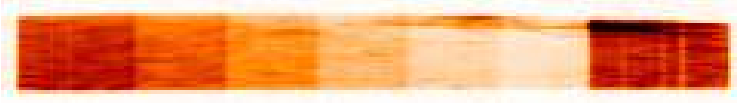

Figure 2.9: Example of a calibration wedge.

Kariyappa and Pap 1996, Worden et al. 1998a, Giorgi et al. 2005, Ermolli et al. 2009a), calibration wedges have serious shortcomings. First of all, these wedges were not stored in the majority of the historical data. For example, SHGs were taken regularly since 1915 at the MW Observatory, but calibration exposures were imprinted on the plates only from 1961 onwards; at the Ko site observations started in late 1904 while step-wedges were first exposed on the plates in 1958. Recovery of the CC from the step-wedge exposures was problematic until numerical procedures allowed to overcome computational difficulties associated with its sigmoidal shape. The photographic calibration was frequently carried out by considering only those portions of the plate exposure where the relation between $d$ and $\log E$ was linear. Another issue is that the intensity range covered by the wedges does not necessarily coincide with that of the solar disc. This means that 7 points can be insufficient to reconstruct the $\mathrm{CC}$ if the range of intensities they cover is small compared to the range for which the $\mathrm{CC}$ changes. Figure 2.10a) shows one such example, where the points from the wedge lie only on the linear part of the CC. A more important issue is that very often the multiple exposures for the same intensities yielded significantly different values of density (Fig. 2.10b)). This makes the resulting CC highly uncertain. 

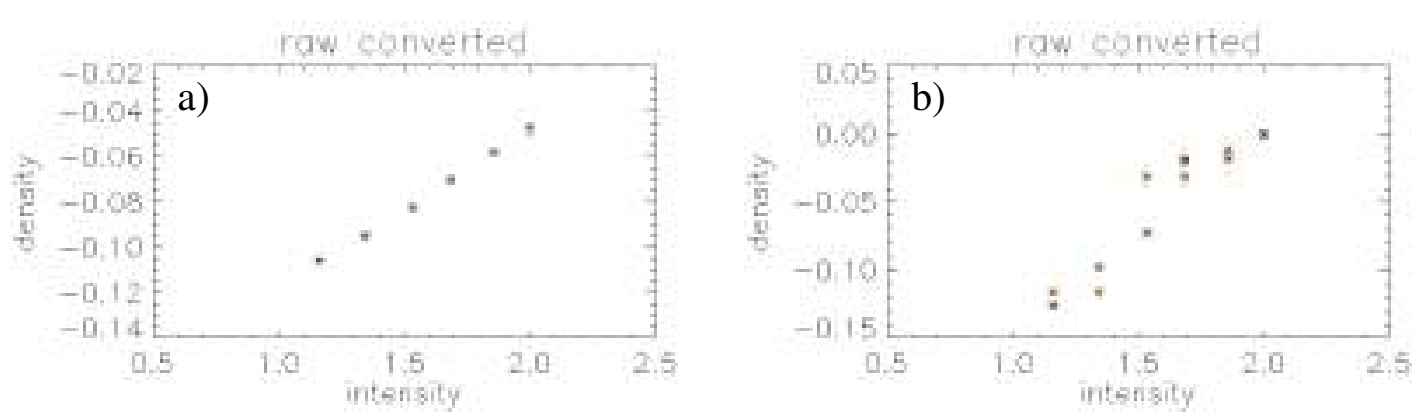

Figure 2.10: Values determined from the calibration wedge of Arcetri observation of (a)) 16/05/1938, (b)) 28/06/1938.

\subsubsection{Alternative calibration methods}

Three alternative ways to calibrate historical SHGs have been presented in the literature.

Priyal et al. (2013) and Steinegger et al.(1996) calibrated Kodaikanal and Sacramento peak data, respectively by applying to them a CC averaged over all available step-wedge exposures. As discussed in Sect. 2.4, the $\mathrm{CC}$ is expected to vary significantly among different observations, thus making this approach questionable (see also Sect. 3.4.2).

Ermolli et al. (2009b) have used the method by Mickaelian et al. (2007) originally derived to calibrate photographic plates of star surveys. This method uses information stored in the unexposed and darker parts of the plate. The CC is derived as $I_{i}=(V-$ $B) /\left(T_{i}-B\right)$, where $V$ is the average value of the unexposed part of the plate, $B$ is the average of the darkest pixels and $T_{i}$ is the transparency value of the $i$ th pixel.

Tlatov et al. (2009) suggested to calibrate SHGs by using a linear relation between a standardized CLV profile from Pierce and Slaughter (1977) and the average image values at two radial distances. The standardized CLV they used was derived with a 2 nd degree polynomial fit at $390.928 \mathrm{~nm}$. The image values were scaled linearly to the values of the standardized profile, without conversion to density values as required by the photographic theory. Essentially this method is simply a linear scaling of the image to match a desired range of intensity values (see also Sect. 3.4.2).

\subsubsection{Methods of the background computation}

Various methods have been used to process Ca II K images and to compensate for the CLV. These methods can be divided into 5 categories.

1. Sampling values radially:

- Brandt and Steinegger (1998) divided the disc in 50 concentric annuli of equal area. Within each annulus they calculated the cumulative histogram of the pixel values. The threshold value of 5\% for the cumulative histogram defined the QS level of each annulus. The 1D QS CLV was calculated as a 2nd degree fit to the average values of the annuli.

- Walton et al. (1998) computed the median value within concentric annuli of equal area. The final CLV was a cubic Hermite fit to the median values.

- Johannesson et al. (1998) applied an azimuthal median filter, but calculated the values at disc centre with a polynomial fit. They then smoothed the resulting map 
with a low-pass filter with a variable window width that decreases in size towards the limb.

- Denker et al. (1999) calculated the median intensity within a 200" $\times 200$ " window at disc centre. They then transformed the images to polar coordinates through a bilinear interpolation. A median filter is applied to different radial positions. A second degree polynomial fit to the average profile is used to replace the values close to the centre. They further smooth this map with a low-pass filter with a varying window, which gets smaller towards the limb.

- Zharkova et al. (2003) applied the same method as Denker et al. (1999) but additionally performed a fast Fourier transform and applied a Butterworth low-pass filter. In this way they were able to remove radial artefacts.

- Tlatov et al. (2009) divided the disc in four radial and azimuthal segments. Within each segment they calculated the mode pixel values and iteratively repeated the calculation up to two times by halving the segments each time. In each step they consider only the pixels with $I<I_{\text {mode }}+2 \sigma$. The final background is derived by interpolating the mode values to the rest of the disc.

2. Running window median filter methods: (e.g. Lefebvre et al. 2005, Bertello et al. 2010, Chatterjee et al. 2016). In these methods the background is computed by assigning to each image pixel the median value of the pixels that are within \pm half the window width in pixels.

3. Methods applying 2D polynomial fits to the whole disc:

- Worden et al. (1998b) fitted a 2D polynomial to the whole disc. The authors did not specify the order of the polynomial.

- Caccin et al. (1998) fitted a 2D 4th order polynomial to the whole disc. They ignored regions lying outside the $2 \sigma$ interval.

4. Method applying 1D polynomial fits to rows and columns of the image:

- Worden et al. (1998a) fitted a 1D 5th degree polynomial along image rows and columns to density values of the original observation and on the image resulting from its $45^{\circ}$ rotation. Regions with values outside the $\pm 2 \sigma$ interval of the image were excluded from the analysis and the background was estimated by applying a median, low-pass filter to the average surface derived from the fits.

5. Methods applying combinations of the other methods:

- Fuller et al. (2005) used the method of Zharkova et al. (2003) to remove the limb darkening. To identify and account for any residual large scale inhomogeneities, they applied a running-window median filter twice, first with a large window and then with half its dimensions.

- Ermolli et al. (2009b) used the method by Walton et al. (1998) with 100 annuli and then divided each row and column of the image by the result of a 2 nd degree polynomial fit to their intensity values.

- Singh et al. (2012) used the method of Denker et al. (1999) and a variant of the 
approach suggested by Worden et al. (1998a) applying a 3rd degree polynomial fit to the image values instead of a 5 th degree polynomial.

- Priyal et al. (2013) used the method of Denker et al. (1999) and a variant of the approach suggested by Worden et al. (1998a), applying a 3rd degree polynomial fit instead of a 5th degree one. Priyal et al. (2013) fitted only the rows of the solar observation and kept about a quarter to half of the darker pixels in each row.

The majority of these methods are not suitable for historical observations. The methods from the first category (except the one by Tlatov et al. 2009) result in radially symmetric backgrounds, failing to account for all large scale inhomogeneities. The methods from the second and third category fail to account for density variations between adjacent lines, which is one of the most common artefacts found in the historical observations. The method by Worden et al. (1998a) (and effectively all others as well) are affected by inaccurate exclusion of active regions (AR, hereafter), or in a few cases a complete lack of such an exclusion (see Sect. 3.4.1). This may introduce artificial trends in the results that depend on the activity level.

\subsection{Plage areas}

Modern and historical $\mathrm{Ca}$ II $\mathrm{K}$ data have been used to derive plage area coverage of the solar disc. Various works used different sets of images from different observatories, and more importantly employed different processing and segmentation methods. Another important aspect is that even though all these studies aimed at deriving the plage areas, the definition of plage was not always the same.

Foukal (1996) used uncalibrated MW data from the first digitisation and produced a time series by manually selecting plage regions in every image. As an extension of this work, Foukal (1998, 2002) used again uncalibrated MW data, but segmented them with a constant multiplicative factor to the standard deviation of the contrast images obtained after the removal of the CLV. In this work they identify plage including parts of the network component. This index is called Apn. With the same processing, Foukal and Milano (2001) derived network areas from uncalibrated MW data. Foukal and Milano (2001) argued that the network area coverage did not change over the 20th century, which would imply a weak secular change in the irradiance or even no change at all.

A series of plage areas was also derived from MW data at the university of California, Los Angeles (UCLA). The Ca II K images were calibrated with CCs derived from the available step-wedge measurements and processed as described in Lefebvre et al. (2005) (see Sect. 2.6.2). The histogram of contrast image values was calculated and a Gaussian function was fit to it. This process was repeated iteratively by keeping only the regions on the disc that correspond to the central part of the histogram. The additive parameter of the function is defined as a Ca II K plage index, called UCLA plage index (Foukal et al. 2009). Bertello et al. (2010) processed uncalibrated data in a similar way (see Sect. 2.6.2) and produced another plage index. The index is defined in the same way as in the UCLA series, with differences in the fitting process of the Gaussian function. These two indices do not carry information on the absolute disc fraction, and need to be calibrated again. 
Tlatov et al. (2009) processed the data from the Ko, MW and Sacramento Peak observatories. The method they used to identify the QS was described in Sect. 2.6.2, and the calibration was performed as described in Sect. 2.6.1.2. The segmentation was performed on the calibrated image without compensating for the CLV. The thresholds were defined as a constant multiplicative factor to the full width at half maximum of the intensity distribution of pixels within each sector (see Sect. 2.6.2). They found good agreement with the Apn index (Foukal 1998) and plage areas from UCLA (Lefebvre et al. 2005) (Spearman correlation 0.94 and 0.95 respectively).

Ermolli et al. (2009b) processed all Ar, Ko, and MW as described in Sect. 2.6.1.2 and then segmented them with constant thresholds. They found a good agreement (Pearson coefficient 0.95) with the results by Foukal (1998), even though the reported values by Ermolli et al. (2009b) are systematically lower than those by Foukal (1998). They found good agreement between their series and the UCLA index too, with Pearson coefficient of 0.97 and differences usually within 3\%, which reach $40 \%$ for cycle 19 though.

Ermolli et al. (2009a) processed all Ar data that were calibrated with the available CCs. An average $\mathrm{CC}$ from those derived from the available wedges was applied on the data lacking wedge measurements. The segmentation was done in two steps, first they applied a low-pass filter on the image and identified regions with lax constant thresholds in contrast and size. Then they applied another constant threshold derived with the method by Nesme-Ribes et al. (1996) to the original image this time and only within the regions identified with the first threshold.

Priyal et al. (2013) processed Ko data as described in Sect. 2.6.1.2 and 2.6.2 and then segmented them with constant thresholds. They found a good agreement between their time-series and that by Foukal (1996) for MW data. However, the results by Priyal et al. (2013) for cycle 19 are lower by $20 \%$ than those by Foukal (1996).

Chatterjee et al. (2016) applied the same methodology as Bertello et al. (2010) on uncalibrated Ko data and identified the plage regions as those with $C>C_{\text {median }}+\sigma$, where $C$ is the contrast of each pixel, $C_{\text {median }}$ the median contrast of the disc and $\sigma$ the standard deviation of contrasts. They found a Pearson coefficient of 0.96 between their series and the one by Bertello et al. (2010). They also reported that the plage areas over cycle 19 from Ko data are lower than those obtained from MW data as derived by Bertello et al. (2010).

Four other plage area records have been derived from Ca II K filtergrams taken at the Big Bear solar observatory (BBSO Johannesson et al. 1998), the San Fernando observatory (SFO Chapman et al. 2011), Rome/PSPT (Ermolli et al. 2010b), and MLSO/PSPT (Rast et al. 2008).

The BBSO series ${ }^{8}$ is a composite, using observations from the MM observatory over the period 1942-1979, MW over 1979-1981, and BBSO over 1981-1987 (Foukal 1993). Gaps were filled with data from Sacramento Peak observatory. The data were used uncalibrated and the segmentation was performed with a constant threshold relative to the disc-centre intensity. However, the analysis of data of poor quality, as well as corrections to all data, were done with manual inspection.

The San Fernando observatory (SFO) index is derived from data obtained with two telescopes, CFDT1 and CFDT2 (Cartesian Full Disk Telescope). The SFO photometric

\footnotetext{
${ }^{8}$ Available at http://www.ngdc .noaa.gov/nndc/struts/form?t=102827\&s=34\&d=8, 350, 9
} 
indices provide values of the total area, in millionths of hemisphere, covered by sunspots and faculae. The facular regions are identified with a constant threshold of $4.8 \%$ in contrast (Chapman et al.2011).

The Solar Radiation Physical Modelling (SRPM) values provide the disc fractions covered by the various classes of features as described in Fontenla et al. (1999). Red continuum images are used to identify sunspots, while all other (bright) features are identified on Ca II K images. There are two SRPM series, the first one employs the Rome/PSPT observations described in Sect. 2.5. while the second one uses MLSO/PSPT observations. The AR areas are derived with a constant contrast threshold.

\subsection{Conclusions}

There is a wealth of solar observations taken in the core of the Ca II K line. With the earliest observation taken in 1892, the available data cover the entire 20th century. The recognition of the potential of such data for solar activity studies has led to a process of digitizing the available data, which is an ongoing process. The instruments used to record the observations vary among dataset and sometimes even changed with time. The historical observations were predominantly stored in photographic plates or films. All these result in the digitised archives to be highly inhomogeneous and each image suffering from a different set of problems. We gave an overview of the various methods that have been used to process the historical data. However, the efficiency of these methods has not been studied quantitatively. In the next two chapters we describe our work on the historical Ca II K images. Where we introduce a new method to correct the images for various problems, perform the photometric calibration, and identify the plage areas on the solar disc. 



\section{Photometric calibration and artefact correction of Ca II K observations}

*This chapter is based on a journal article in preparation (Chatzistergos et al.2017a)

\subsection{Introduction}

This chapter describes the processing of full-disc Ca II K images. Such data have been sometimes used to derive plage areas as an index of solar activity (see Sect. 1.4.2.1 and 2.7). However, these data suffer from a plethora of problems, that need to be accounted for before employment of these data. The historical observations were stored on photographic plates that are non-linear detectors and the information to recover the relation between the incident and recorded radiation is missing for the majority of the available data. Moreover, the images exhibit a variety of artefacts and large scale inhomogeneities (see Sect. 2.2 - 2.5). Many methods have been proposed to perform the photometric calibration and to identify the centre-to-limb variation (CLV) with the inhomogeneities and artefacts (outlined in Sect. 2.6). However, all available methods have shortcomings and fail to some extent to account for all known problems affecting such data. Furthermore, there has been no quantitative study of the errors introduced by these methods.

Here we present a new method to calibrate the historical photographic Ca II K spectroheliograms (SHG) as well as a novel method to compensate for various artefacts in the data. This includes correction of the CLV of the solar intensity and of patterns of non-solar origin introduced by observational and archival processes. This method, which works without knowledge of the specific characteristic curve (CC) of the analysed plate and in the absence of step-wedge imprints, is based on the computation of the CLV of density values on quiet Sun (QS) regions. Importantly, we rigorously test our technique on modern CCD-based data artificially degraded to correspond to photographically obtained SHGs.

In this study we used the historical archives from Arcetri (Ar), Coimbra (Co), Kodaikanal (Ko), Meudon (Me), Mitaka (Mi), McMath-Hulbert (MM), and Mt Wilson (MW) observatories and modern observations from Rome/PSPT, which were described in Sect. 2.5. Key information about the datasets used in this study is summarised in Table 3.1.

The method is presented in Sect. 3.2. In Section 3.3 we assess the accuracy of the method with synthetic data, that have known characteristics, and artefacts and test the proposed method on a large sample of SHGs. In Section 3.4 we compare it with other approaches presented in the literature. 


\subsection{Method description}

Our method of calibration and processing of the SHG is based on the assumption that, in these observations, the CLV of intensity in the internetwork regions, i.e. the quietest part of the QS, does not vary with time, in agreement with White and Livingston (1978, 1981), Livingston and Wallace (2003), and Livingston et al. (2007). This assumption is also supported by the results of Bühler et al. (2013) and Lites et al. (2014) that the internetwork magnetic flux, which is the main component of magnetic field populating these quietest parts of the solar surface, remains unchanged over the solar cycle. Before applying the proposed method to the historical images, we convert their values to densities according to Eq. 2.4. Figure 3.1 a) shows the density image corresponding to the Ko observation displayed in Fig. 2.7c). The main steps of our method can be summarised as:

- Calculation of the 2D map of QS regions in each image (including CLV and inhomogeneities);

- Extraction of the 1D QS CLV profile in each image;

- Construction of CC by relating the $1 \mathrm{D}$ QS CLV to a reference $1 \mathrm{D}$ radial intensity QS CLV from CCD-based Rome/PSPT observations;

- Calibration of the image using the $\mathrm{CC}$;

- Compensation for the intensity CLV.

We assume that all departures of the 2D internetwork map in SHG from a radially symmetric map are image artefacts of non-solar origin. This is supported by results from modern observations that indicate the radial uniformity of radiative emission of the internetwork (1D radial QS, Livingston and Sheeley 2008, Peck and Rast 2015). We also assume that the bandwidth of the observations has remained constant over the disc and for all data within an archive. This might not always be the case, but this assumption is unavoidable since information on the real bandwidth is not available.

\subsubsection{Background computation}




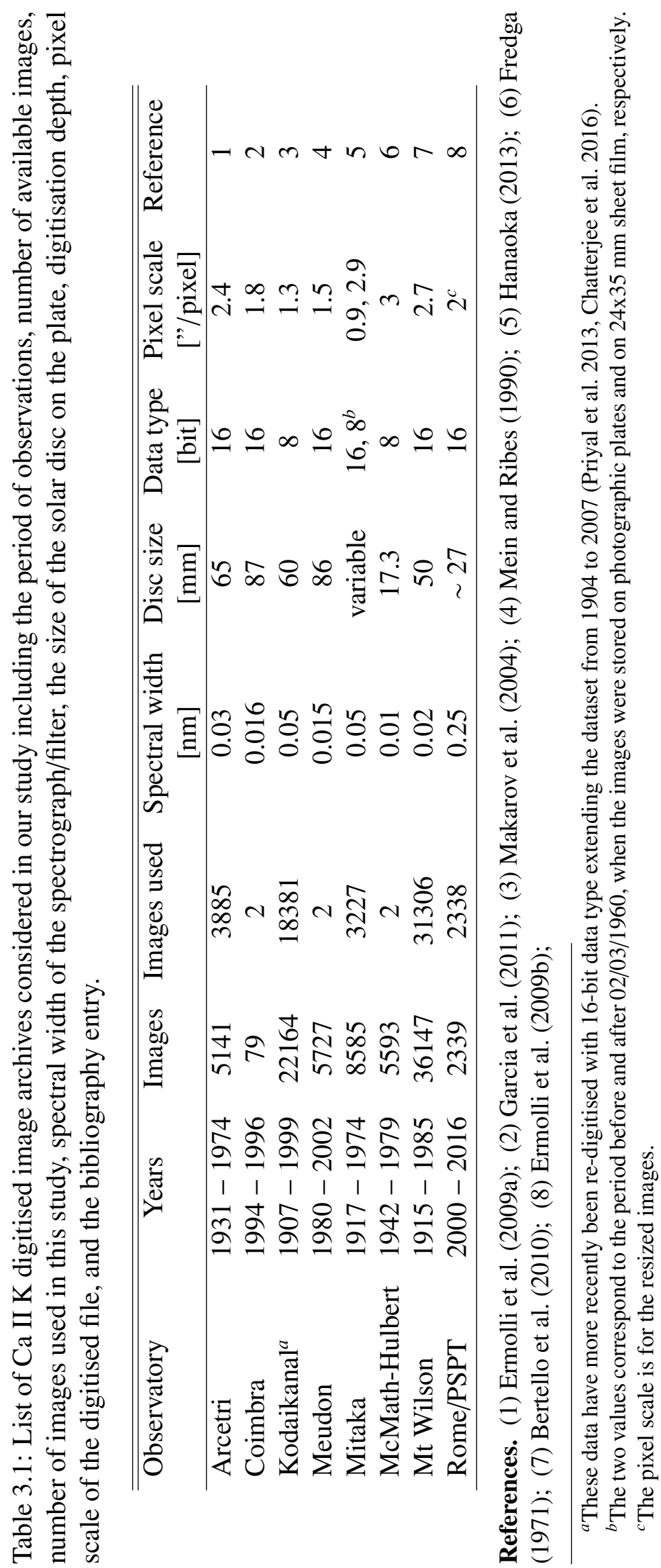


a)

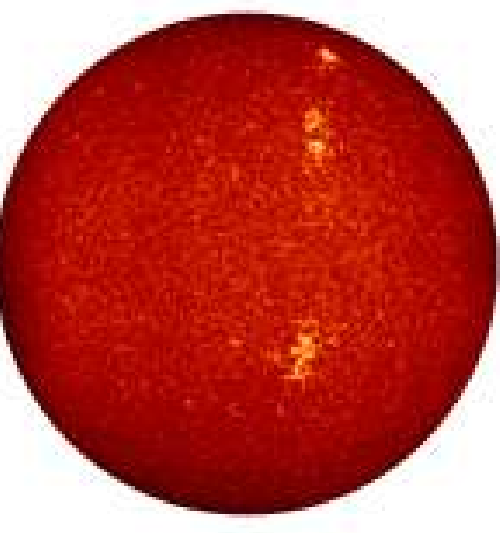

e)

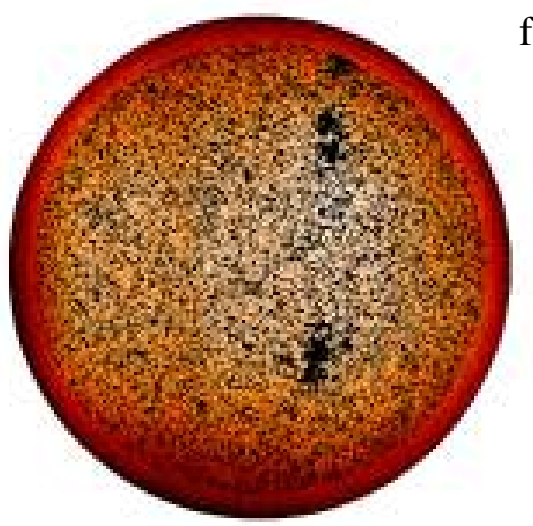

b)

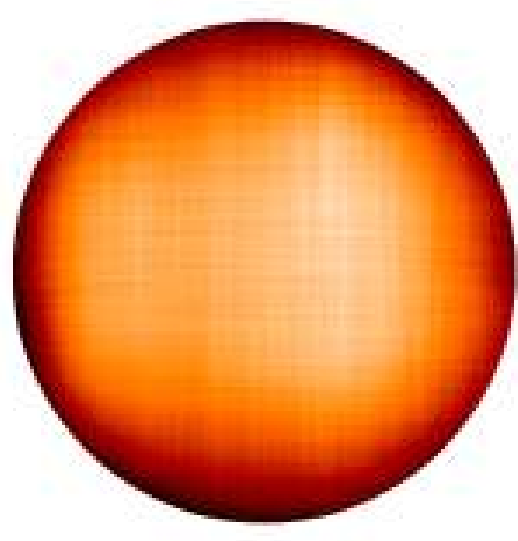

f)

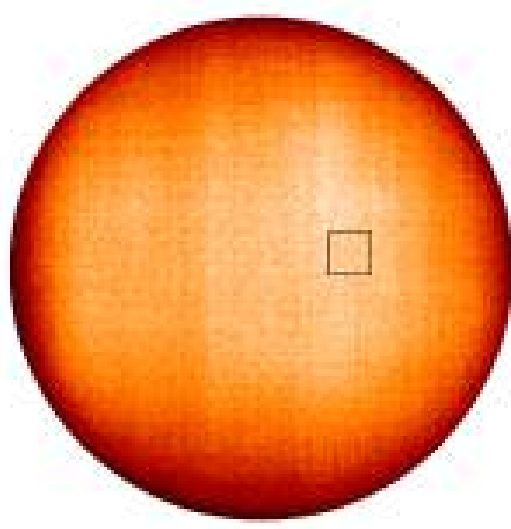

c)

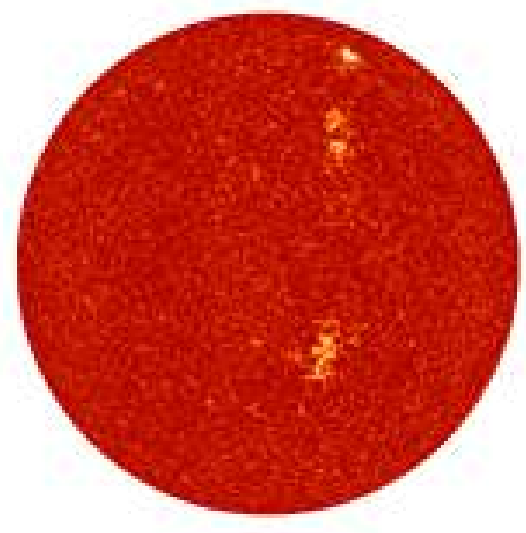

g)

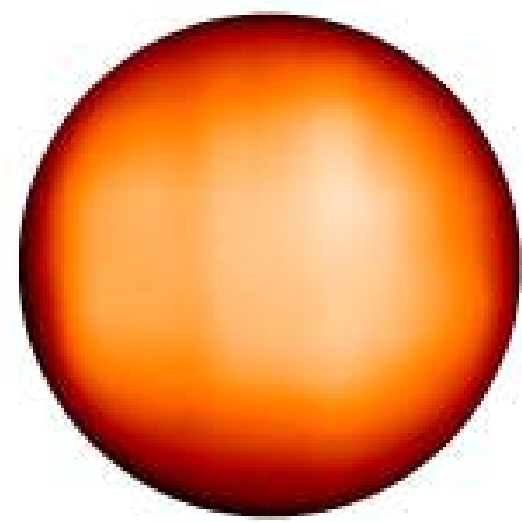

d)

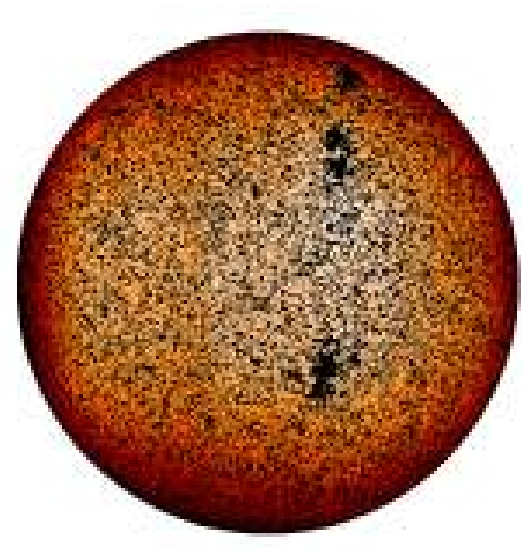

h)

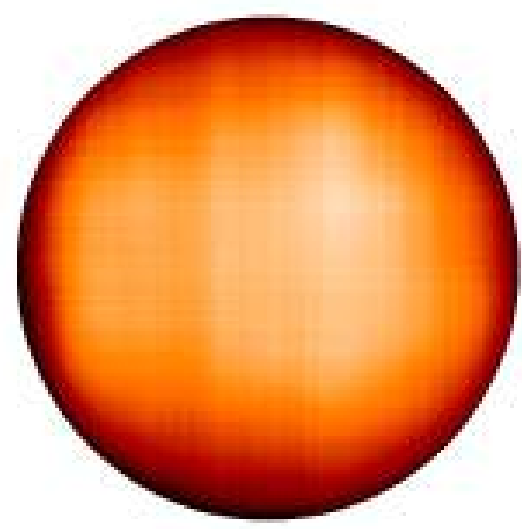

Figure 3.1: Selected processing steps of Ko observation taken on 14/09/1912: (a)) original density image, (b)) first estimate of background map, (c)-h)) results of each step of the iterative process (2.1-2.5), (e) and f)) correspond to Step 2.3. The black square in panel f) shows the dimensions of the window used for the median filter in Step 2.4 (see Sect. 3.2.1 for details). 
We first derive the 2D QS background. For this it is essential to accurately exclude active regions (AR), otherwise there is a risk of overestimating the background due to the contribution of remaining ARs. The process of calculating the 2D QS background can be outlined as follows:

- Get a rough estimate of the background;

- Use the last estimate of the background to identify and exclude ARs;

- Iteratively repeat the process of calculating the background and identifying the ARs until sufficient accuracy is achieved;

The first rough estimate of the background is obtained in 3 steps.

- Step 1.1. The solar disc is divided into azimuthal slices of $30^{\circ}$ that cover the disc in steps of $5^{\circ}$. Within each slice we apply a 5th degree polynomial fit of the form $d=f(\mu)$, where $\mu=\cos \theta$ and $\theta$ is the heliocentric angle. The best fit values of $d$ are assigned to all pixels within a given slice. Results obtained from contiguous slices are gradually averaged and stitched together (this method will be referred to as rotating slices).

- Step 1.2. We identify the regions in the density image lying outside $1 \sigma$ of the result of Step 1.1. These are tentatively identified as AR and are excluded from the further analysis (note that the removal of non-AR pixels in this process does not influence the results; it is more important to discard as many AR pixels as possible).

- Step 1.3. We apply a 5th degree polynomial fit to the density image, excluding ARs defined in Step 1.2, along each column and row of the image separately (similarly to Worden et al. 1998a, but without the $45^{\circ}$ rotation of the disc). To all pixels of each analysed column/row we assign the density values resulting from the best fit. We get a background map by stitching together the results obtained from the best fit; multiple values derived for the same location on the solar disc are averaged (we will refer to this method as column/row fittings).

The calculations described in Steps 1.1-1.3 provide a rough, first estimate of the background. However, the identification of ARs at this stage is rather inaccurate. During this preliminary calculation ARs lead to overestimates of the values of the background, while the varying contrast for different $\mu$ positions renders the identification near the limb less accurate.

Therefore in order to improve the AR identification and the calculation of the background, we iteratively repeat the following steps:

- Step 2.1. We compute the density contrast image $C_{i}^{d}=\left(d_{i}-d_{i}^{\mathrm{QS}}\right) / d_{i}^{\mathrm{QS}}$, where $d_{i}$ is the original density image and $d_{i}^{\mathrm{QS}}$ the density background resulting from the previous calculation at the $i$ th pixel. During the first iteration $d^{\mathrm{QS}}$ is the rough background estimate (from Step 1.3), afterwards we use the result of the previous iteration.

- Step 2.2. We remove the AR in the original density image, retaining only the pixels that fulfil the threshold criteria $\left.C_{i}^{d}\right\rangle\left\langle C^{d}\right\rangle+l_{1} \sigma$ or $C_{i}^{d}\left\langle\left\langle C^{d}\right\rangle-l_{2} \sigma\right.$, where $l_{1}, l_{2}$ are the applied thresholds. For the first 3 iterations $l_{1}=1$ and $l_{2}=4$, afterwards $l_{1}=0.5$ and $l_{2}=1$. These threshold values were chosen following a series of tests which showed that they effectively removed plage, network and sunspots, leaving just internetwork regions. See discussion in Sect. 3.3.3. 
- Step 2.3. To fill in the gaps left by the AR over the whole disc in the original density image we apply the column/row fittings on the image obtained in Step 2.2, where the fitting is repeated three times to improve the accuracy of the computation (see discussion in Sect. 3.3.3). To avoid artefacts of the fit at the limb due to missing values, the gaps by the ARs in the outer $0.1 R$ are first filled in with the rotating slices method.

- Step 2.4. We apply a running window median filter on the image resulting from Step 2.3. The filter window width used is $R / 6$ (shown with a rectangle in Fig. 3.1f; see discussion in Sect. 3.3.3). To avoid inconsistencies, the outer $R / 12$ part of the disc is re-sampled outside the disc to fill the space between $R$ and $R+R / 12$ and the pixel values of the re-sampled section are adjusted so that the median filter is not affected by the pixel values outside the disc.

- Step 2.5. We identify dark and bright linear artefacts affecting many of the SHGs (for instance see Fig. 2.7 (e)) by separately fitting a polynomial to every row and column of the residual image between the original density image and the result of Step 2.4, excluding the AR.

The sum of the maps derived in Step 2.4 and Step 2.5 is the final background map of each iteration. The five-step processing described above is repeated until the difference between two subsequent background maps does not improve the accuracy of the QS background further. Usually 4 iterations allow lowering the relative unsigned mean difference between maps from two consecutive iterations to $<0.1 \%$.

The result of the processing described so far is usually an asymmetric map (nonsymmetric background, NSB hereafter) that describes the 2D QS background of the original image. The asymmetry is caused by a residual pattern that includes small- and largescale inhomogeneities due to image problems, e.g. dark and bright bands and linear artefacts, stray light features, image gradients. Figure 3.1 shows different steps of the processing on a randomly selected Ko SHG. In particular, the original density image is shown in panel a), the result of the first column/row fittings process in panel b), while panels c)-h) show the results of each step (2.1-2.5) of the iterative process.

Finally, we apply the method of Nesme-Ribes et al. (1996) on the residual image between the original and the NSB to identify and compensate any offset in the average level of the computed NSB and the analysed image. The method by Nesme-Ribes et al. (1996) identifies the level of the QS as the minimum of the average densities of disc regions with density values within $\pm k \sigma$, where $\sigma$ is the standard deviation and $k$ takes values between 0.1 and 3.0.

\subsubsection{CLV extraction}

We compute the radially symmetric background (SB hereafter), which in turn gives the 1D QS CLV, by applying a 5th degree polynomial fit to the density values $d=f(\mu)$ of the deduced NSB. All image pixels are considered with their respective $\mu$ position and the fit is weighted with the local $\sigma$ defined within 100 concentric and equal area annuli. This is the most important step for the calibration process, because the way the 1D QS CLV is defined, it determines the CC.

Our calculation of NSB includes possible vignetting affecting the original SHGs, however this leads to miscalculation of the 1D QS CLV. Under-exposure depends on the intensity level, while vignetting is a purely geometric effect. Unfortunately, the geometry 
is such that their effects are similar, and vignetting is known to darken solar observations towards the limb, resembling the effects of under-exposure. The effects of vignetting are expected to be restricted mostly at the edges of the image, in which case it might not affect the solar disc at all or it is almost indistinguishable from under-exposure. Notwithstanding the similarities of their effects on the solar observation, some differences in the signatures of vignetting and under-exposure on the QS CLV and CC exist. In fact, vignetting can potentially affect larger parts of the solar observation than under-exposure, by modifying the CLV even near the image centre in contrast to under-exposure that mainly affects observations near the solar limb (see Fig. 3.21). This is valid as long as the under-exposure is not extreme. However, in extreme cases the image quality is anyway very poor and such images cannot be processed meaningfully.

We attempt to estimate the vignetting on the analysed image and account for it in the calculation of the 1D QS CLV, by comparing the SB with a rescaled version of the logarithm of the CLV retrieved from the CCD-based Rome/PSPT observations. If there is no vignetting, the SB from the polynomial fit is kept. Otherwise, the SB is computed by rescaling the CLV from the modern observations. Its lower values are adjusted so as to find the minimum density for which the difference between SB and the CLV derived with the fit continuously increases towards the limb.

\subsubsection{Photometric calibration}

In our approach, we perform the photometric calibration by seeking for information stored on the solar disc that can be used in a similar manner as the often missing calibration wedges.

For each analysed image, we deduce the $\mathrm{CC}$ by relating the density values obtained from its SB at a given $\mu$ position to the logarithm of intensity values derived from modern Rome/PSPT observations at the same disc location. The amount of equal area annuli we use to acquire this relation and apply a linear fit to it is equal to the nearest integer of $2 R$. This is consistent with the assumption that the QS density values in good-quality observations lie on the linear part of the CC. From the fit we only exclude the last value that corresponds to the regions very close to the limb, due to the higher uncertainties that characterise these regions. We linearly extrapolate the computed relation to the whole range of density values on the image and make use of the fit parameters to photometrically calibrate the original density image. The result of this procedure is illustrated in Fig. 3.2 a). We also photometrically calibrate the estimated NSB. Removal of the QS CLV from the calibrated image and normalization to it then provides the contrast image of the analysed SHG (Fig. 3.2b)).

Figure 3.3 shows the $\mathrm{CC}$ derived from the processing of the Ko observation displayed in Fig. 3.1 a).

\subsection{Performance of the method}

\subsubsection{Synthetic data}

We tested the method proposed in Sect. 3.2 on a large number of synthetic images that were obtained from contrast Rome/PSPT images by imposing on them known radially 
a)

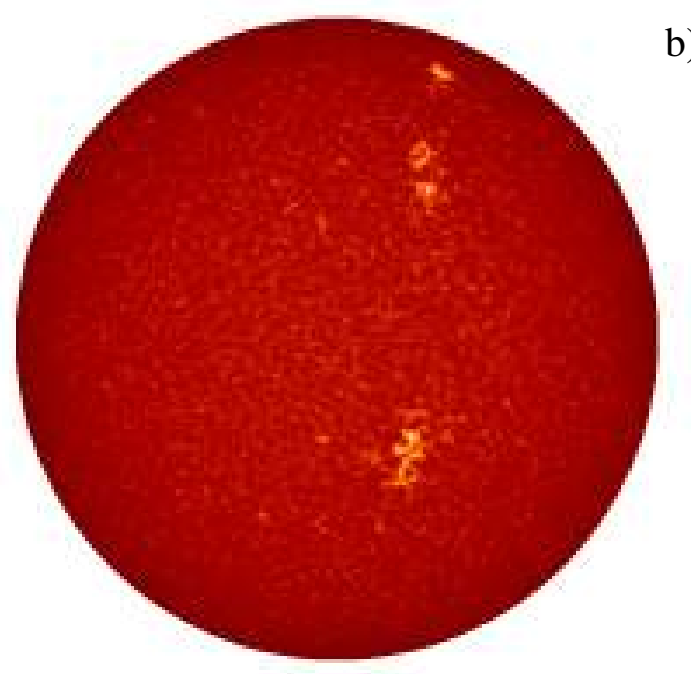

b)

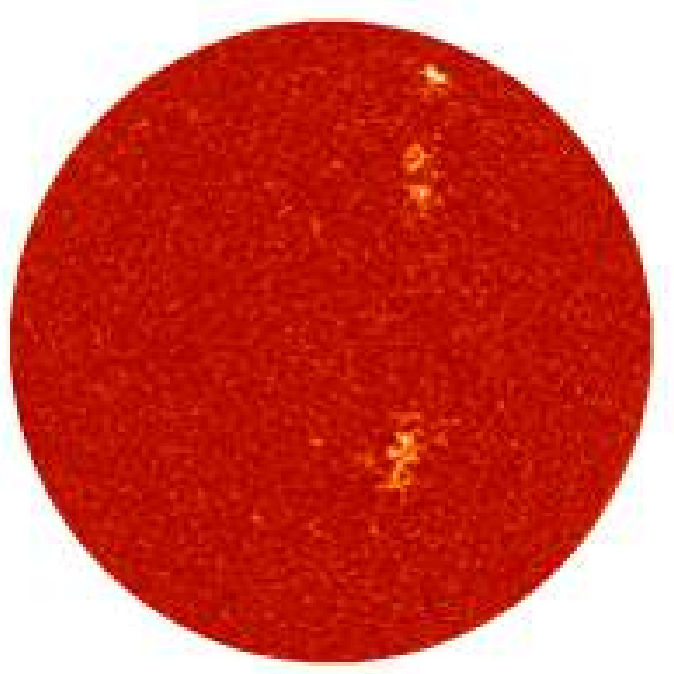

Figure 3.2: (a) Photometrically calibrated, and (b) contrast image corrected for the CLV for the Ko observation shown in Fig. 3.1 .

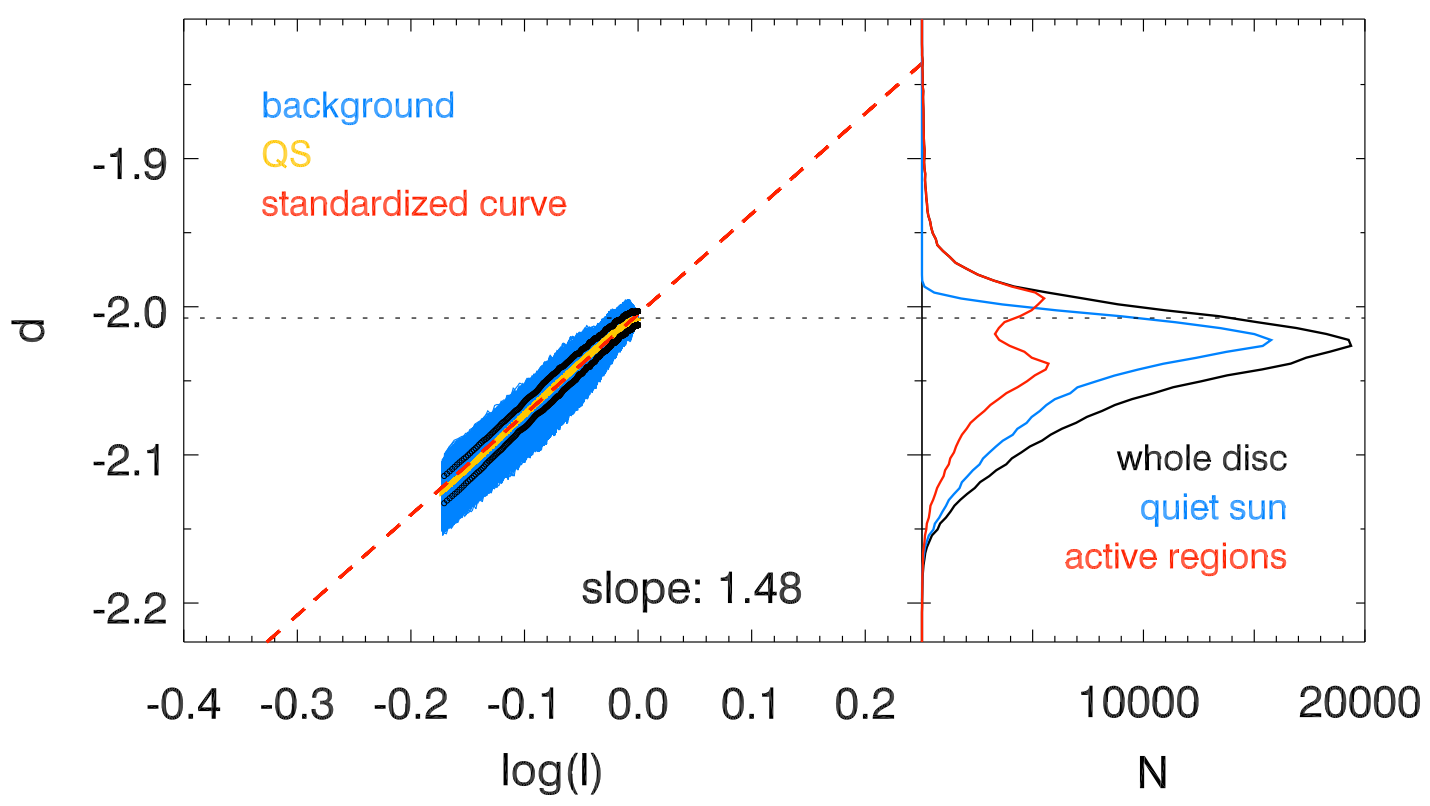

Figure 3.3: Left: CC derived from the processing of the Ko observation shown in Fig. 3.1 (red), measured CC for the QS (orange) with $1 \sigma$ uncertainty (black) and the whole background (blue). The slope of the derived CC is also shown. Right: Distribution of density values for the QS (blue), AR (red) and whole disc (black). The horizontal dashed line in both panels denotes the highest value of the QS CLV.

symmetric CLVs and a variety of inhomogeneities identified in historical observations (and converted to intensity). We then converted the degraded images from intensities to densities by applying various CCs, thus emulating historical observations. We used 2000 Rome/PSPT images taken between 21/08/2000 and 07/10/2014 after the usual reduction steps (dark current removal, flat fielding etc.) that greatly reduce instrumental effects. 
They were also resized to half their original dimension ( 2 arcsec per pixel after resizing), to roughly match the size of the historical data. The Rome/PSPT images were processed as presented in Section 3.2.1 to derive contrast images. These contrast images are defined as $C_{i}^{I}=\left(I_{i}-I_{i}^{Q S}\right) / I_{i}^{Q S}$, where $I_{i}$ is the intensity, and $I_{i}^{Q S}$ the intensity of the QS of the $i$ th pixel. The imposed CLVs are in the form of a 5 th degree polynomial function of $\ln \mu$, as presented by Pierce and Slaughter (1977), with parameters determined from the Rome/PSPT observations. The range of values of the imposed intensity CLV is $[0.6,1.0]$, while the contrast range of the facular pattern is $[0.1, \sim 0.6]$, and of the network pattern is $[0.026,0.1]$. These ranges match the ones found on images of the Ko, Ar and Mi archives.

We produced 8 subsets of synthetic images with the following features:

- Subset 1: Ideal density images with symmetric backgrounds, no linear artefacts or bands, to estimate the precision of the proposed method and the sensitivity of results to the level of solar activity.

- Subset 2: Images with imposed CCs that are linear functions with varying slopes, to test the sensitivity of the method to different slopes of CC within the range $0.1-4.0$.

- Subset 3: Images with imposed CCs that are non-linear functions with various levels of over- and under-exposure (shown in Fig. 3.4), to estimate errors in the retrieved calibration due to exposure problems.

- Subset 4: Images with different sizes, to test the accuracy of the method on the various datasets. The disc diameter was varied between 200 and 1100 pixels.

- Subset 5: A vignetting function with different strength levels was added to images, to test the ability of the method to identify possible vignetting effect and to distinguish it from other artefacts.

- Subset 6: Various large scale density patterns of non-solar origin were imposed (shown in Fig. 3.5), to evaluate the efficiency of the method to distinguish between the solar and non-solar patterns and the accuracy of accounting for the latter.

- Subset 7: Images with a different CLV (shown in Fig. 3.6) than the one used during the standardization of the $\mathrm{CC}$, to test the errors of calibrating data obtained with different bandwidths.

- Subset 8: A combination of all the artefacts mentioned above was added randomly on each image, to produce an inhomogeneous dataset resembling the historical ones. The $\mathrm{CC}$ is defined as a 3rd degree polynomial function with randomly selected parameters within the following ranges: $[-4.6,-1.3]$ for the constant term, $[0.5,4.0]$ for the linear term, $[-0.03,0.00]$ for the quadratic term, and $[-0.03,0.00]$ for the cubic term. The range of intensities within the undegraded images varies and the logarithm of it lies between -1.2 and 0.5 (similar to those shown with the light grey area in Fig. 3.4).

Figures 3.7 - 3.10 show some examples of the synthetic images derived from the same contrast Rome/PSPT observation (taken on 21/08/2000), illustrating the variety of problems we aim at addressing with the application of the proposed method on these data. In the same figures we show the results obtained for these synthetic data and the pixelby-pixel errors in the NSB calculation and the calibrated contrast images. The contrast images used to derive the errors are offset so that the mean QS contrast value is 1 . 


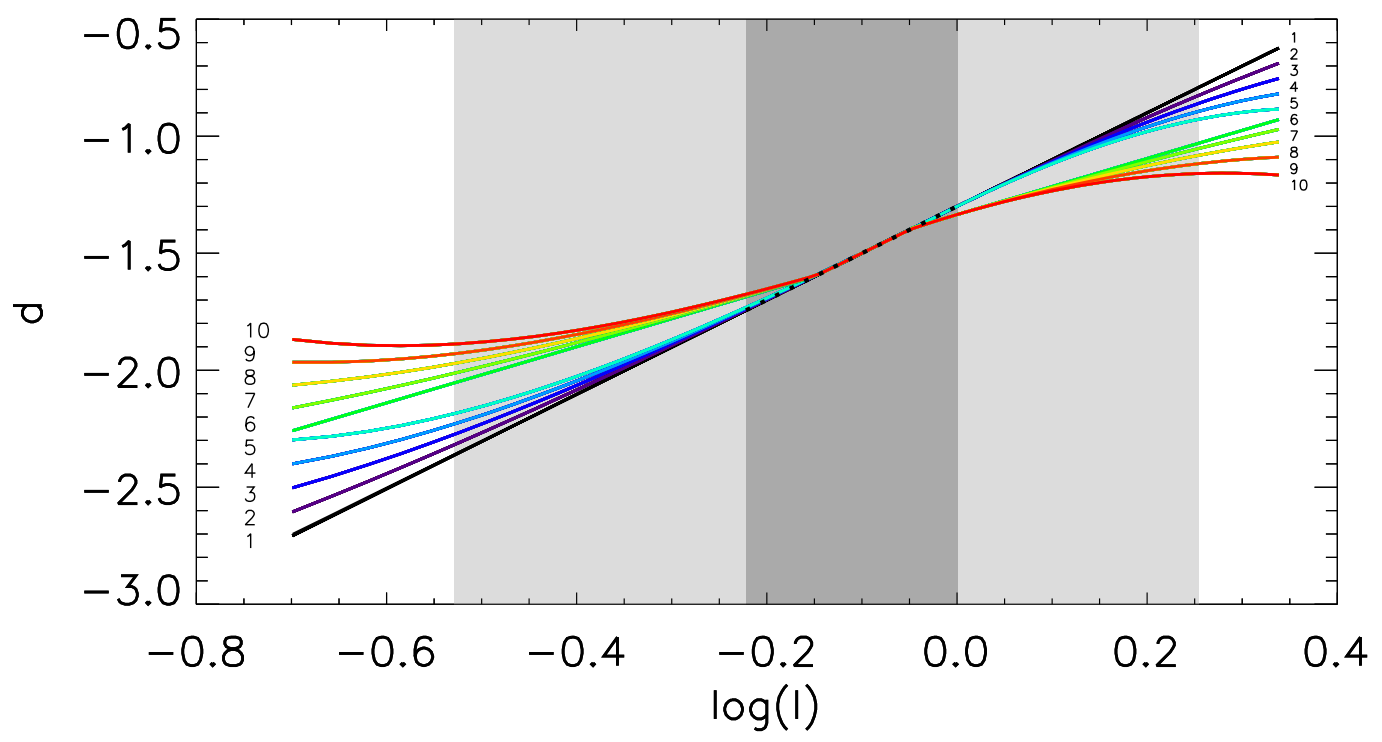

Figure 3.4: CC relations imposed on the data of subset 3. Note that the parts of each curve for $\log I<-0.1$ (corresponding to underexposure) can be combined with the part of the same or any other curve for $\log I \geq-0.1$ (overexposure). E.g. 10,1 implies very strong underexposure, no overexposure, while 10,10 corresponds to very strong underand overexposure. The dark (light) grey shaded areas denote the range of values of the QS (entire disc) for Rome/PSPT image taken on 21/08/2000 which was used to create the synthetic images shown in Fig. 3.7-3.10). The dotted black line denotes the CC imposed on data of subset 1. Find details in Section 3.3.1 
1)

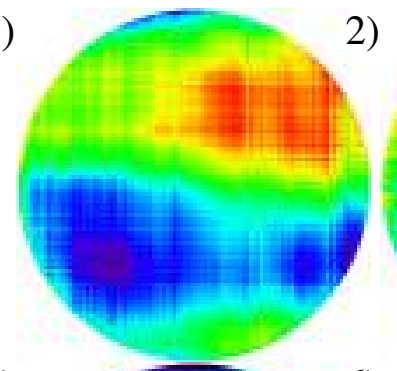

5)

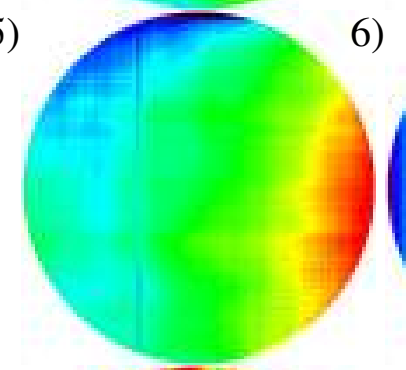

9)

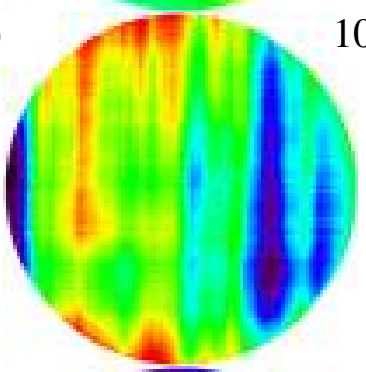

13)

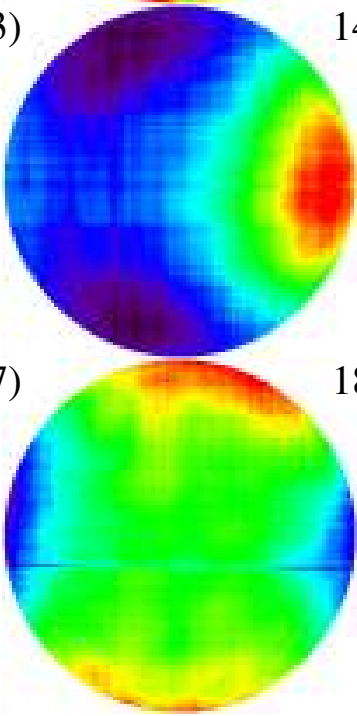

2)

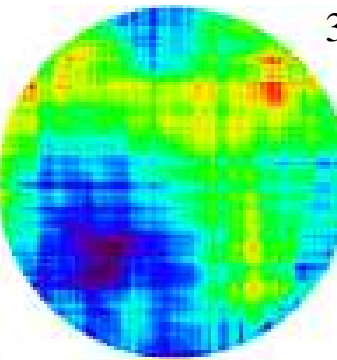

6)

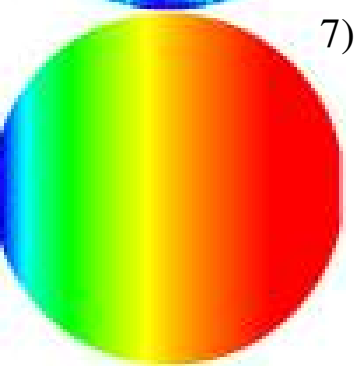

10)

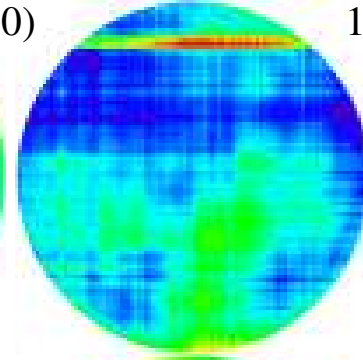

14)

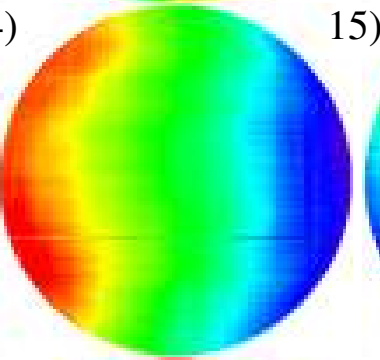

18)

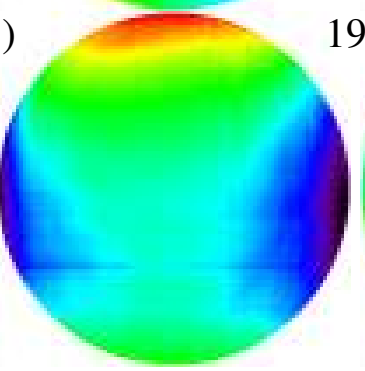

3)

11)

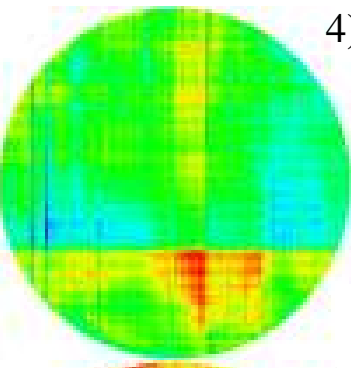

8)
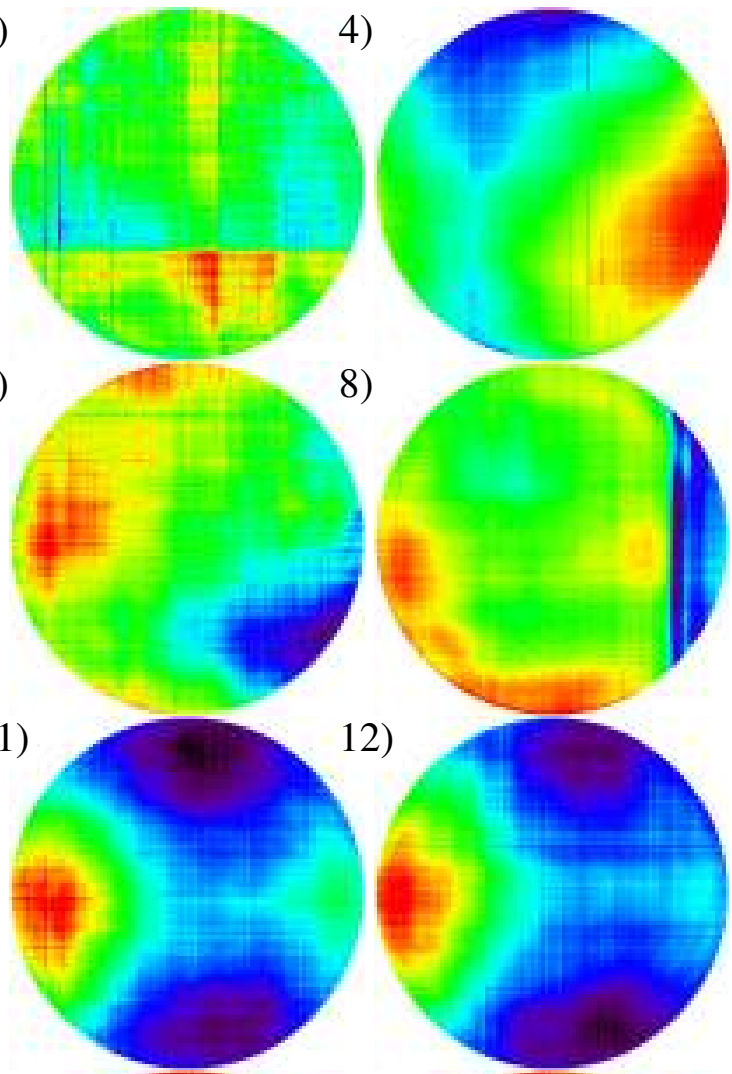

12)

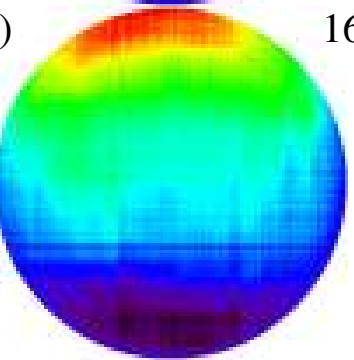

16)
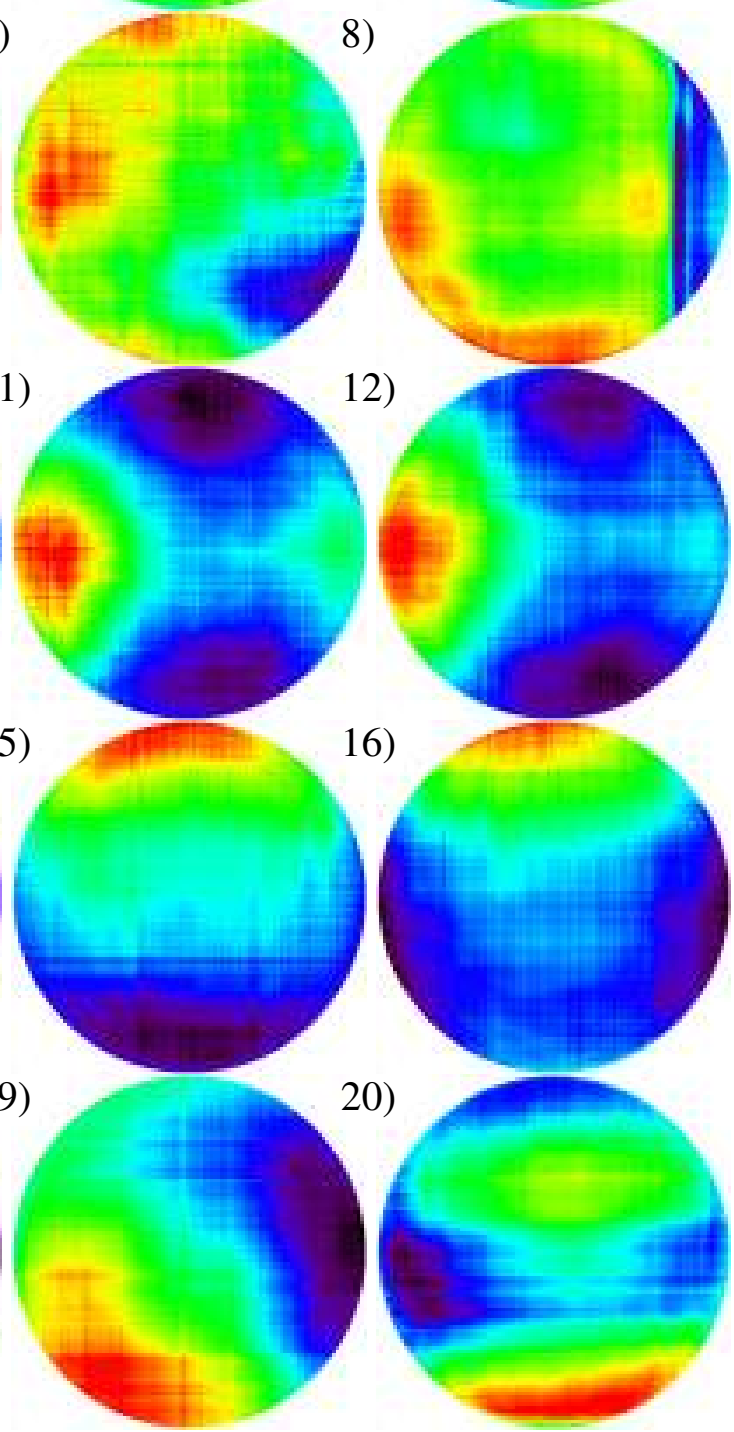

20)

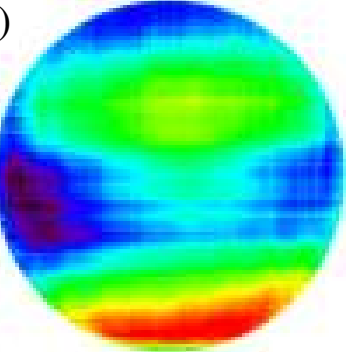

Figure 3.5: Inhomogeneities used to create images of subsets 6 and 8. The first 5 backgrounds were derived from Ar observations, the sixth one is artificial and introduces a gradient of a fourth root function over the disc, 7-10 from Ko, 11-18 from MW, and 19-20 from Mi images. 


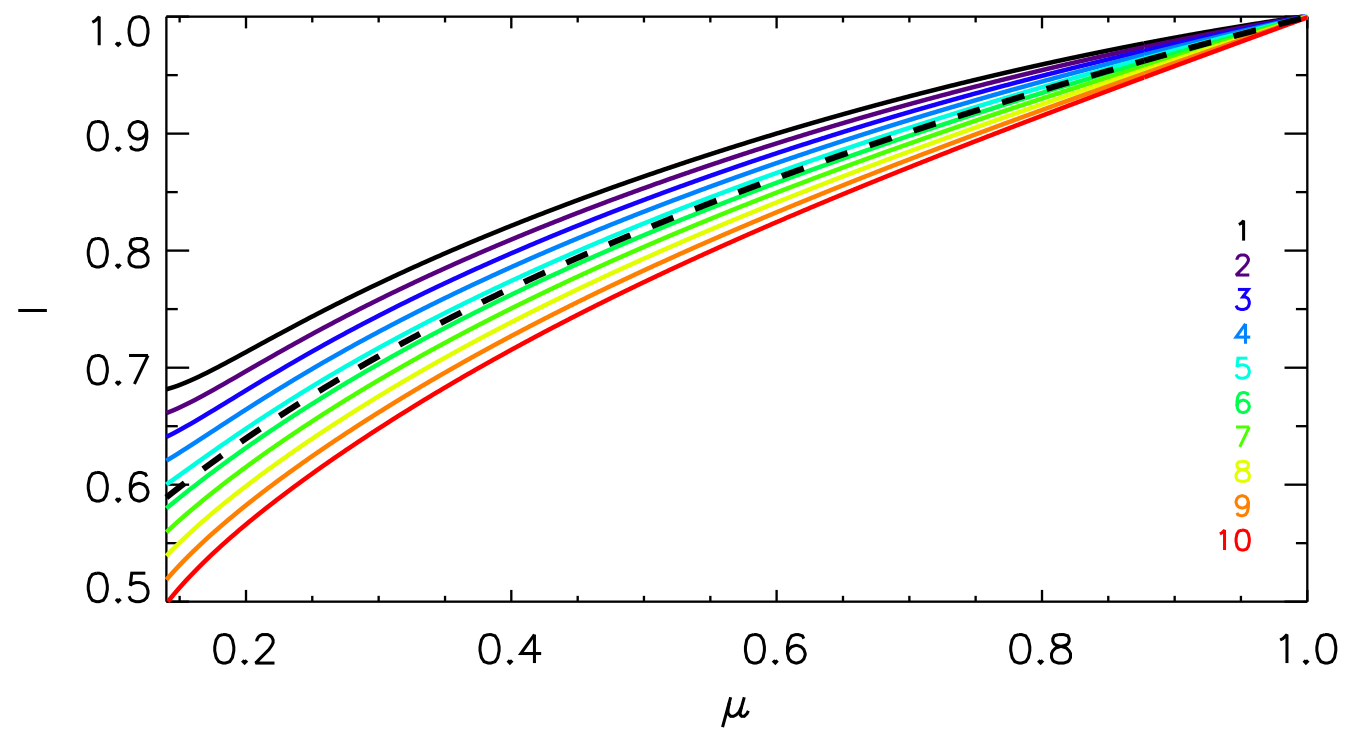

Figure 3.6: Intensity CLV imposed on the images of subset 7. The dashed black line denotes the average CLV measured on Rome/PSPT observations. The colours from black to red correspond to the cases 1-10 shown in Fig. 3.23 . 


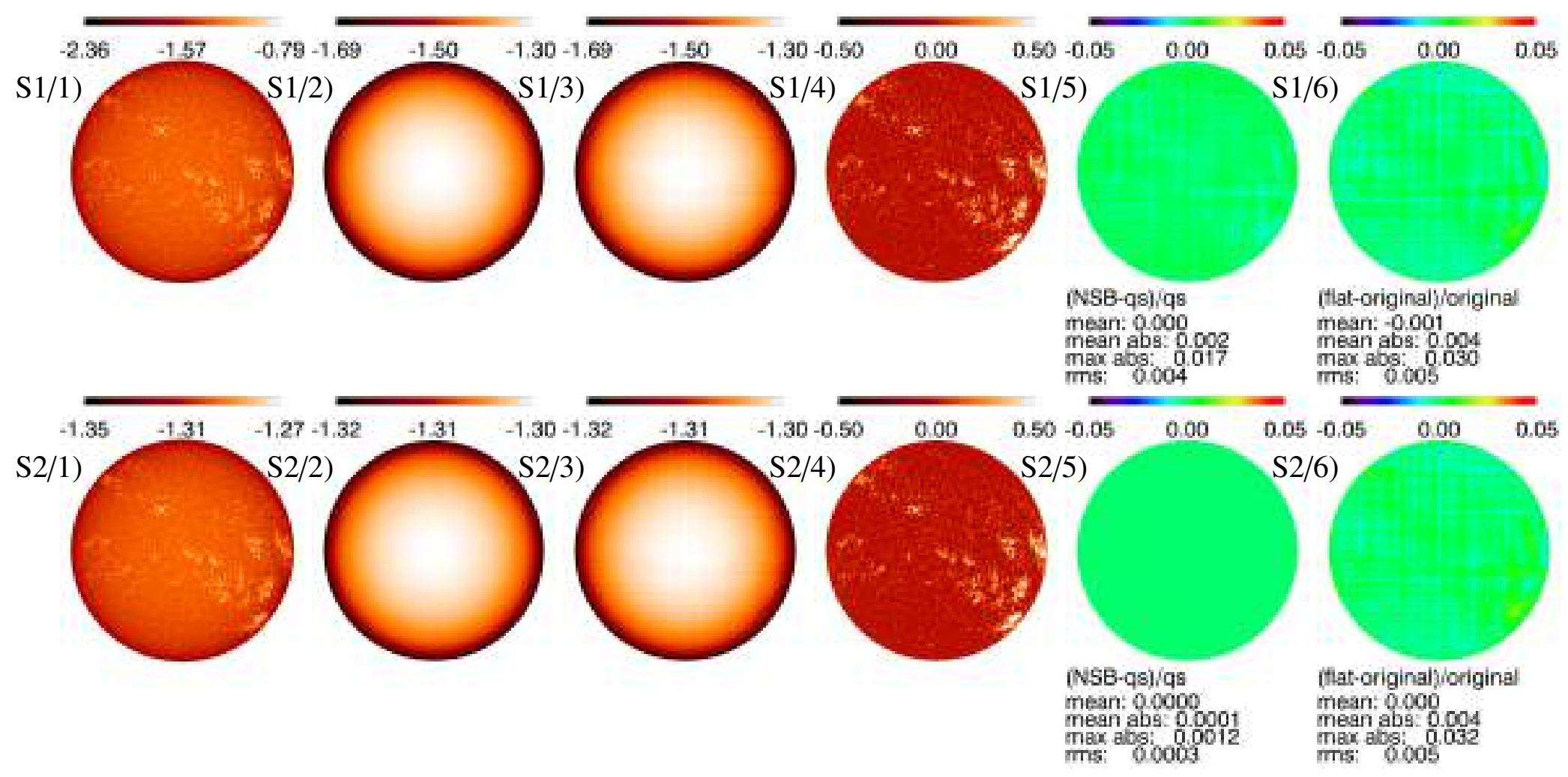

Figure 3.7: Examples of the calibration procedure on synthetic images from subsets 1 and 2 (from top to bottom) produced by Rome/PSPT image taken on 21/08/2000. From left to right: (1)) density images, (2)) imposed backgrounds, (3)) calculated backgrounds, (4)) calibrated contrast images, (5)) pixel-by-pixel relative errors in NSB, and (6)) relative errors in calibrated contrast images. Here we show the following cases: subset $2-\mathrm{CC}$ with slope of 0.1 . Also given (below the images in columns 5 and 6 are the values of the RMS, mean, mean absolute and maximum relative differences within the disc up to $0.98 R$. 
Figure 3.8: Same as Fig. 3.7 but for subsets 3 and 4. Here we show the following cases: subset 3 - combination of the strongest under- and over- exposure (case 10 underexposure and 10 overexposure in Fig. 3.4; subset 4 - disc diameter of 1100 pixels. 


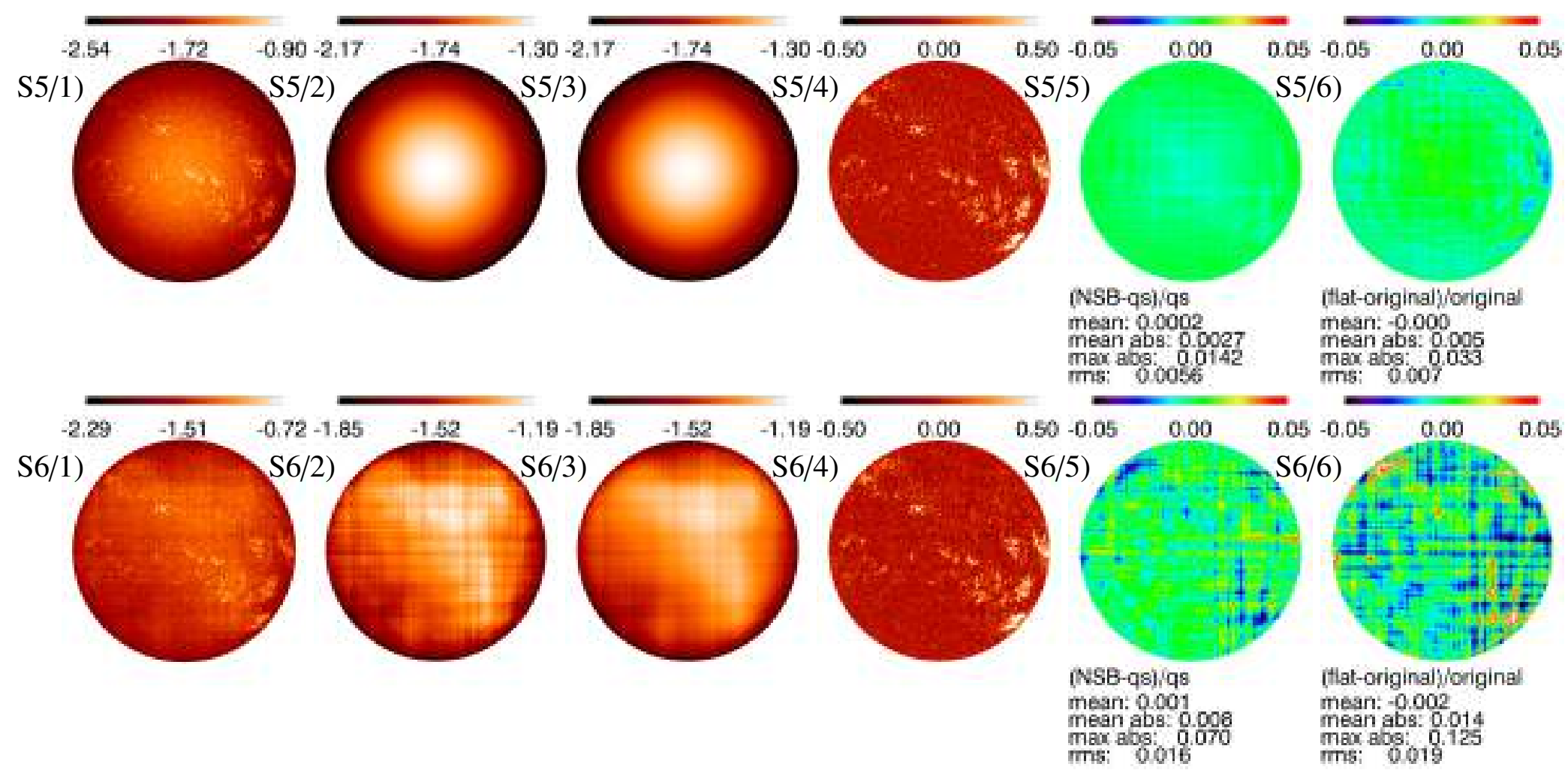

Figure 3.9: Same as Fig. 3.7 but for subsets 5 and 6. Here we show the following cases: subset 5 - strongest vignetting (red curve in Fig. 3.21; subset 6 - level 5 of inhomogeneity No. 2 (shown in Fig. 3.5. 
Figure 3.10: Same as Fig. 3.7 but for subsets 7 and 8. Here we show the following cases: subset 7 - CLV No. 4 (shown in Fig. 3.6); subset 8 - vignetting No. 2, level 5 of inhomogeneity No. 4, CC $d=-3.6+3.6 \log I-0.01(\log I)^{2}-0.004(\log I)^{3}$, and CLV used $d=1.0+0.33 \log (\mu)+0.06(\log (\mu))^{2}-0.04(\log (\mu))^{3}-0.05(\log (\mu))^{4}-0.01(\log (\mu))^{5}$ that lies roughly between cases 6 and 7 in Fig. 3.6 . 


\subsubsection{Analysis of the synthetic data}

Table 3.2 summarises key aspects of the various synthetic datasets, and quantitative results obtained by testing the proposed method on them. The results are briefly presented in the following and are later described in greater detail by introducing each subset of synthetic data individually. Table 3.2 summarizes the results derived from the analysis of all data within the various subsets. An exception is made for subsets 3 and 6 , for which the presented values correspond to results restricted to images representative of historical data of reasonable quality, i.e. excluding synthetic images made to suffer from extreme exposure problems affecting the QS regions or images with superposed large-scale patterns whose amplitudes were larger than 0.6 of the CLV.

Briefly, the results obtained for subsets 1-6 show that our method recovers the QS density CLV with average relative error $<3 \%$ in the absence of strong non-solar patterns affecting the image, and error $<6.5 \%$ if the extreme cases of inhomogeneities encompassed in subset 6 are also included. The results derived from subset 7 prove that the above accuracy is maintained as long as the CLV differs by roughly $<10 \%$ from the one we imposed on the data. The results on subset 8 show that we can produce consistent results from a rather inhomogeneous dataset.

\section{Subset 1: Linear relation without inhomogeneities}

We selected 500 Rome/PSPT images that sample the period 2000-2014 regularly, we computed the logarithm of each image and applied a linear $\mathrm{CC}$ to them to emulate the response of the plate. This set thus represents almost ideal density images without any large scale inhomogeneities or exposure problems, allowing to estimate the precision of the proposed method in the presence of AR.

Figure 3.11 shows an example of results obtained on one image from the subset 1, where a) is the density image derived from the Rome/PSPT observation, b) is the imposed CLV, $\mathbf{c}$ ) is the calculated NSB and $\mathbf{d}$ ) is the calculated SB.

Figure 3.12 shows results derived by comparing the NSB retrieved from the proposed method and the imposed background for all the data of subset 1 (disc positions within $0.98 R)$. Throughout this analysis the relative differences are given in absolute values (unsigned), but we also provide the signed average difference. The red curve displays the maximum relative difference divided by 10 to improve the clarity of the plot. The mean values of the maximum and average relative difference between the retrieved NSB (SB) and the CLV intensity imposed on all images of subset 1 are $0.025(0.006)$ and 0.002 (0.0007), respectively; the RMS difference is 0.005 (0.002). On average only $0.2 \%$ of the pixels show differences exceeding the average value $\pm 3 \sigma$ for the NSB. All above values show that the proposed method is able to retrieve radially symmetric backgrounds with great accuracy, even though this assumption is not made during processing.

Figure 3.13 shows results obtained by comparing the contrast image derived with the proposed method and the original data; the mean values of the maximum and average relative difference are 0.024 and 0.003 , respectively, and the RMS difference is 0.004 .

The calculated slopes for the $\mathrm{CC}$ derived from the method are in good agreement with the imposed one. The results we get have a slope of on average 0.505 with standard deviation 0.001 , compared to the imposed one of 0.5 .

To estimate the accuracy of the method when applied on data unaffected by intensity 


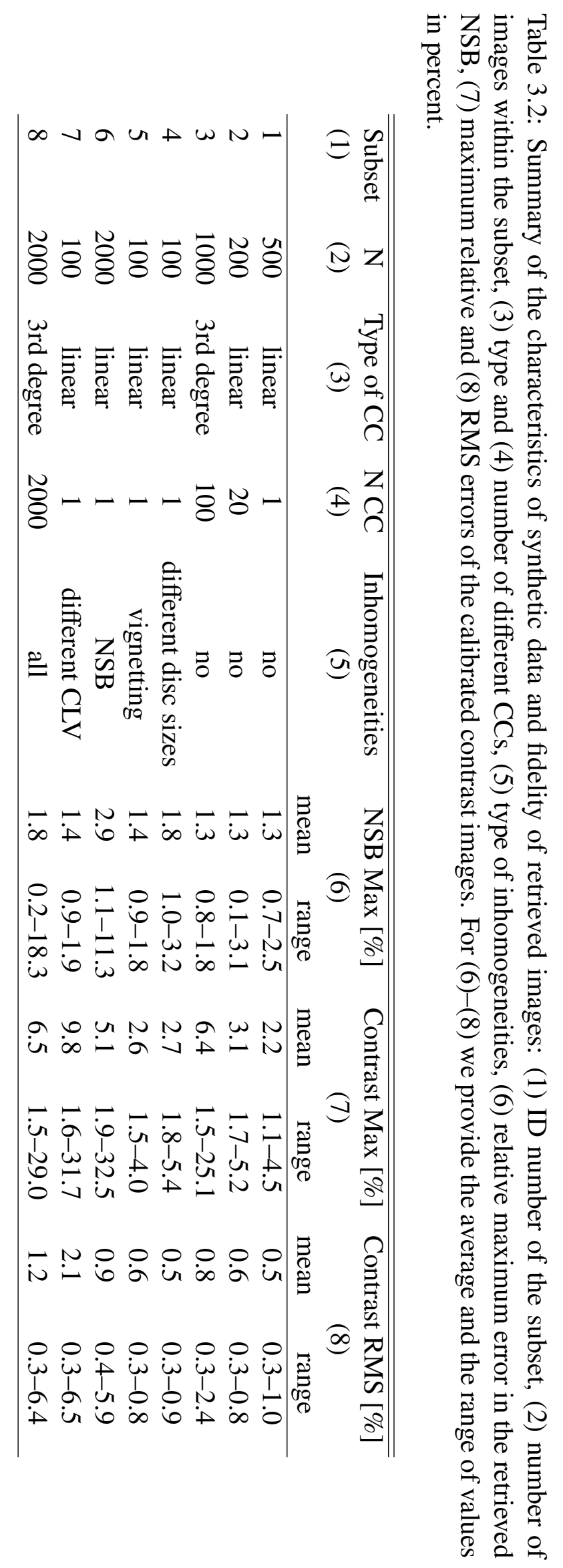


variations between adjacent lines, we analysed subset 1 one more time but now bypassing step 2.5 of the background calculation (Sect. 3.2.1). We found that the method's accuracy is on average a factor of 2 higher than obtained by applying the line fittings. In particular, the mean values of the maximum and average relative difference between the retrieved NSB (contrast) and the CLV intensity (background) imposed on all images of subset 1 are lower than $0.02(0.013)$ and $0.006(0.001)$, respectively; the RMS difference is 0.0027 (0.002). The errors of NSB can be seen in Fig. 3.14, while those for the calibrated contrast images in Fig. 3.15
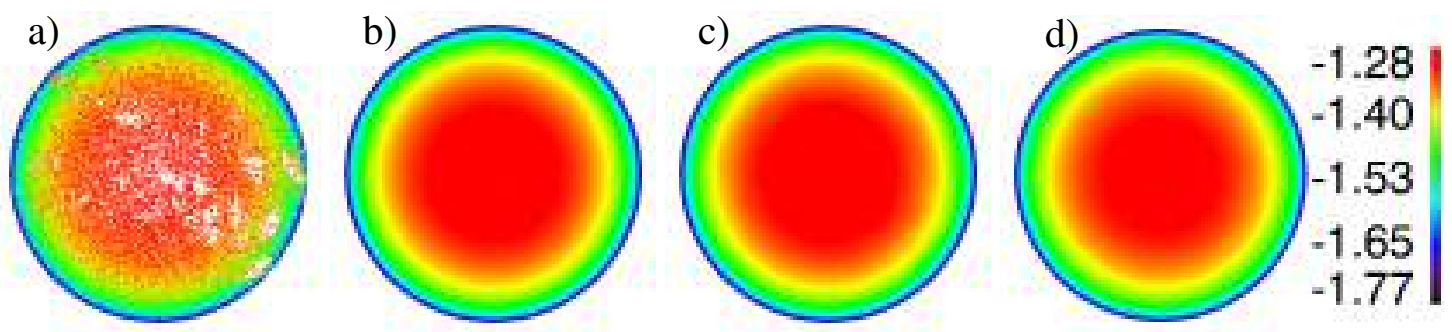

Figure 3.11: Example of application of our method to one image from subset 1. a) is the density image derived from the Rome/PSPT observation (saturated to the range of the QS for illustration purposes), $\mathbf{b}$ ) is the imposed CLV, $\mathbf{c}$ ) is the calculated NSB and $\mathbf{d}$ ) is the calculated SB. The colour bar is the same for all 4 images.

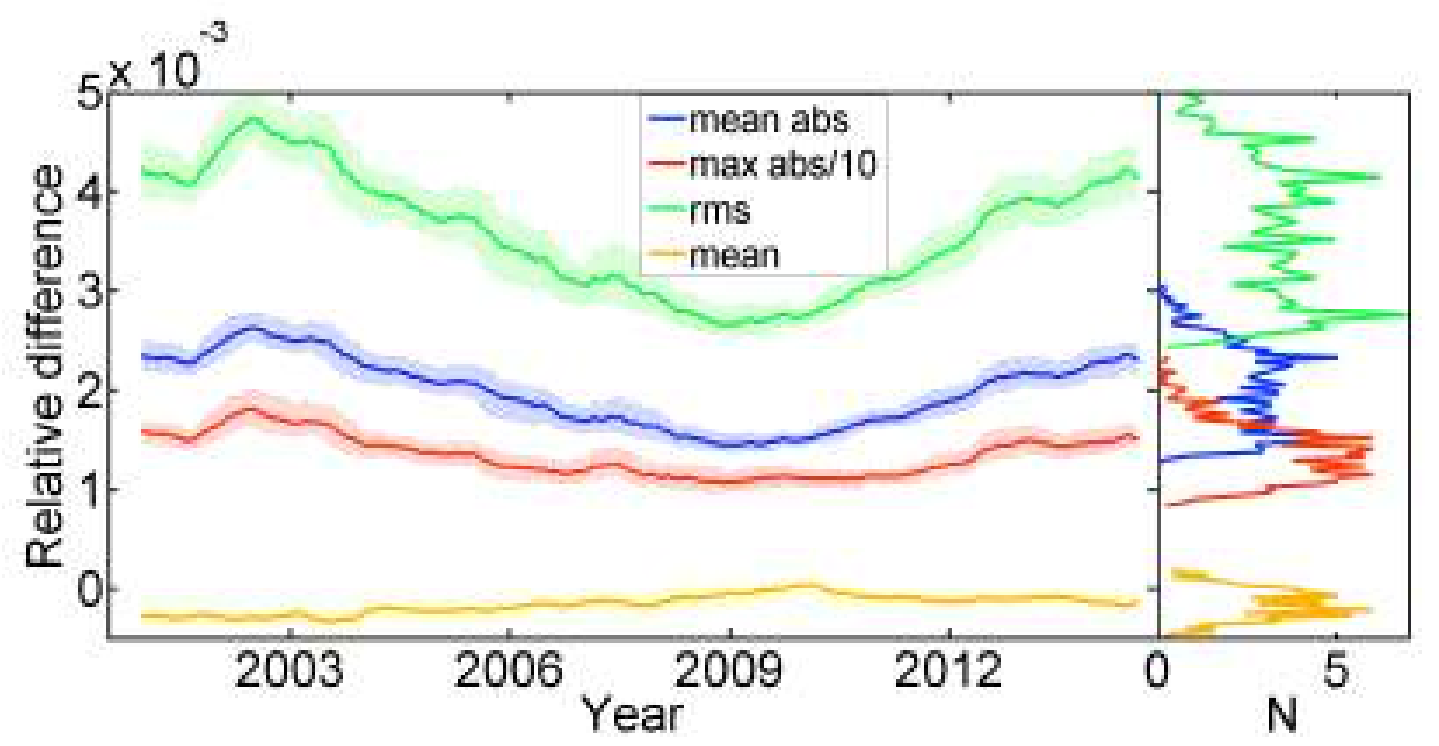

Figure 3.12: Left: Relative difference between the NSB and the imposed background for all images of subset 1 (disc positions within $0.98 R$ ). For clarity, the maximum difference is divided by 10 . The solid lines are 30 point averages and the shaded surfaces denote the asymmetric $1 \sigma$ intervals. Right: Distribution functions of the difference values. 


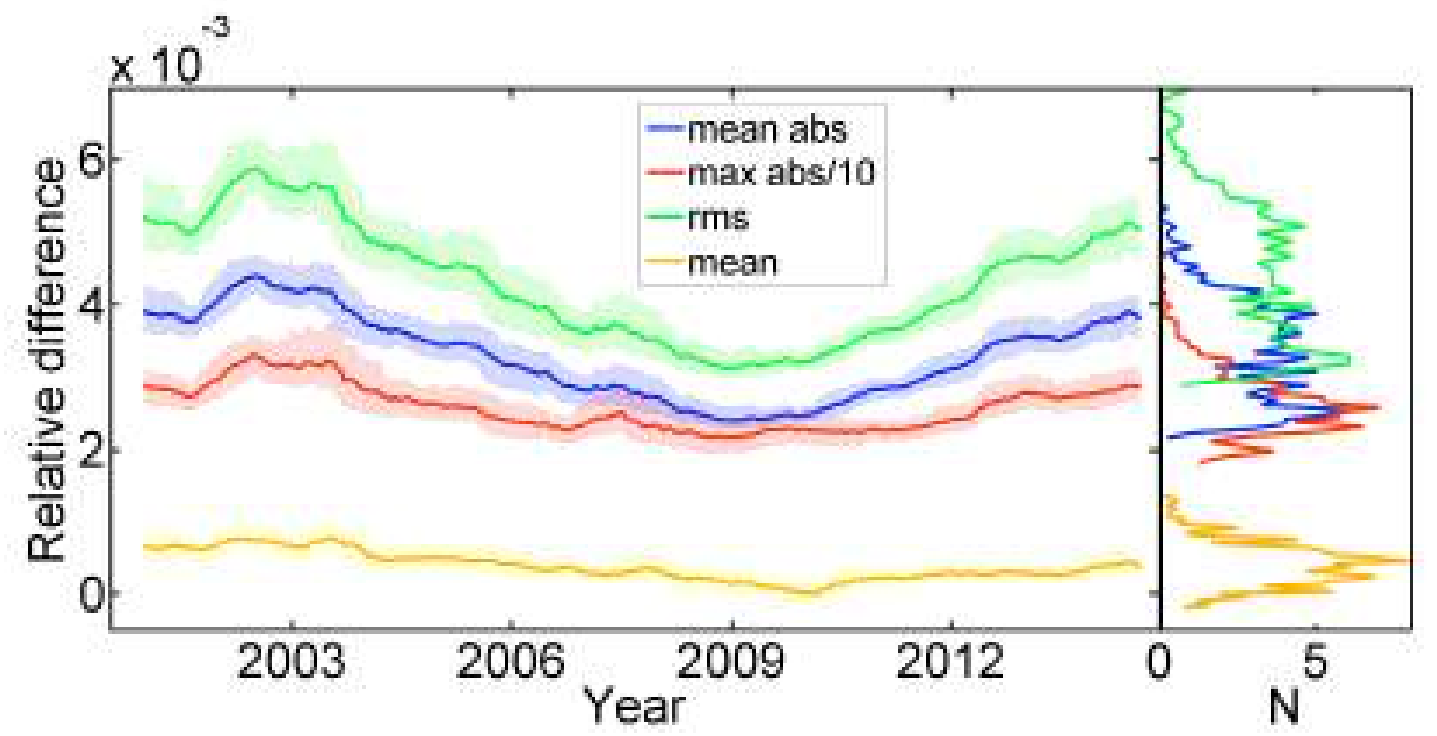

Figure 3.13: Left: Relative difference between the contrast image retrieved using our method and original undegraded data for all images of subset 1 (disc positions within 0.98R). Labels are as in Fig. 3.12. Right: Distribution functions of the difference values.

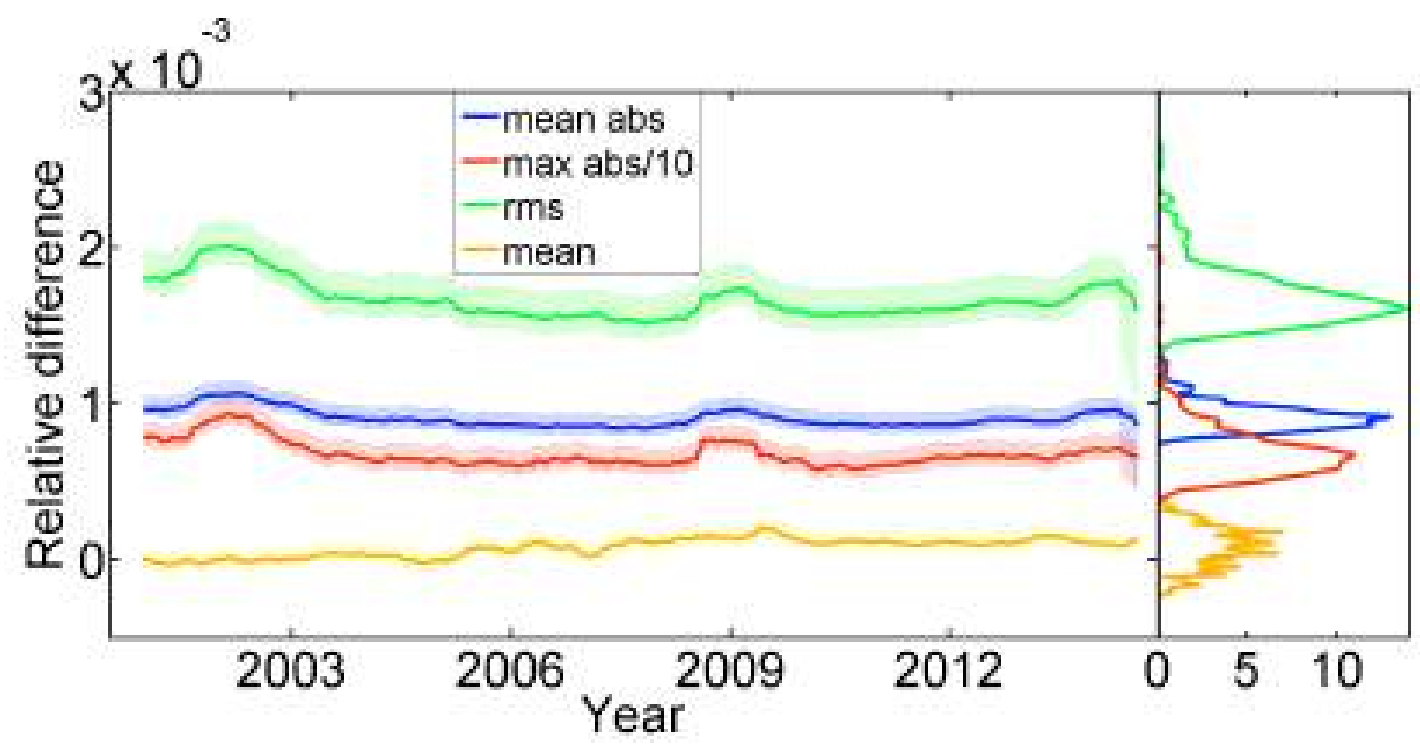

Figure 3.14: Same as Fig. 3.12 but without performing the line fittings (Step 2.5). 


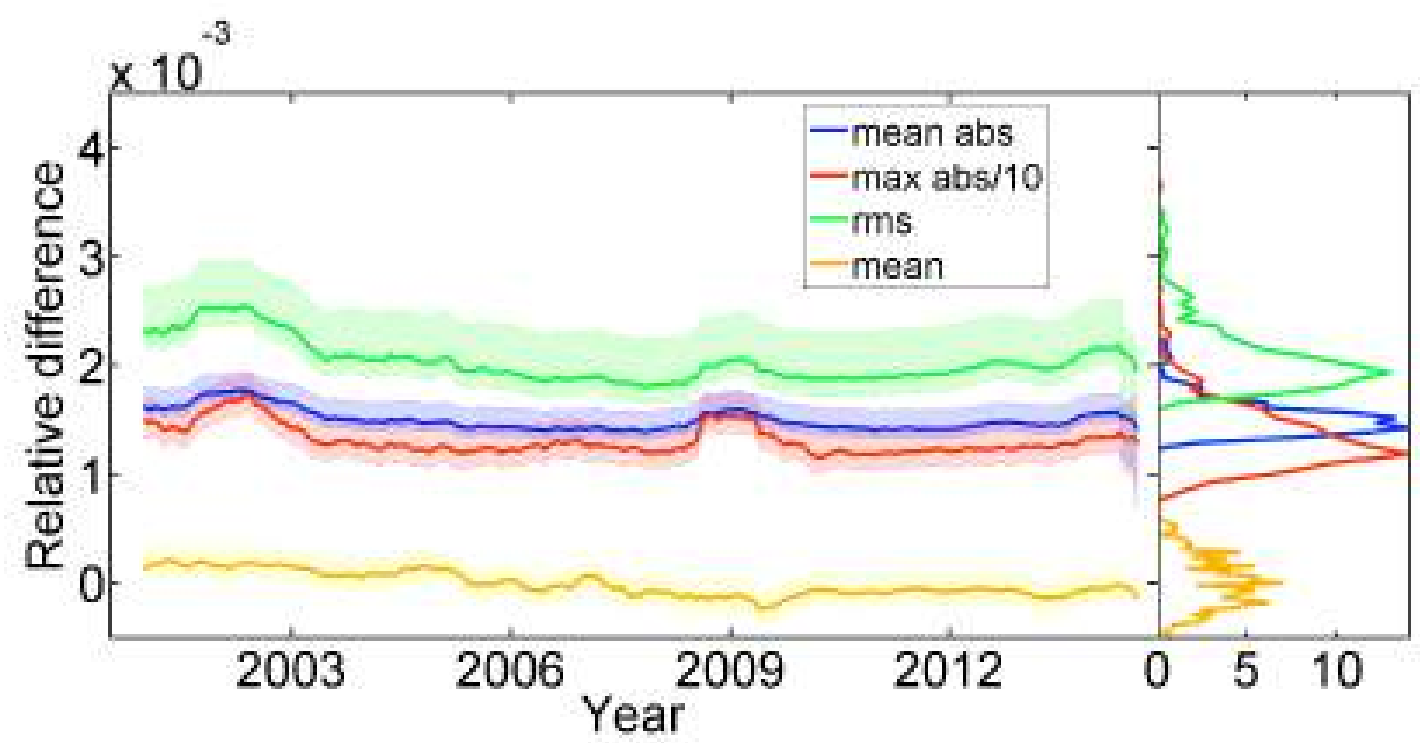

Figure 3.15: Same as Fig. 3.13 but without performing the line fittings (Step 2.5).

\section{Subset 2: Different linear relations without inhomogeneities}

This subset was created to test the efficiency of the proposed method to account for different linear relations of the CC. Due to the changes in the photographic plates or films that were used, the different conditions of observations, development or digitization, the historical data have different $\mathrm{CC}$. Therefore this subset tests the accuracy of our method to restore good quality images with different $\mathrm{CCs}$, but with no large-scale inhomogeneities or exposure problems. We selected 10 Rome/PSPT images covering the period 20002014 and imposed on them 20 different linear relations with slopes in the range 0.1-4.0. Subset 2 therefore consists of 200 images.

Figure 3.16 shows the relative differences obtained by comparing images retrieved using our method and original undegraded data; panels a) and b) (c) and d)) refer to values of NSB (contrast) images, respectively. Each box represents a different image of subset 2 , the rows (columns) show values derived from synthetic images created with different Rome/PSPT contrast observations (different imposed CC relations). The values on the $y$-axis represent the year when the used contrast Rome/PSPT observations were taken. The different colours give the errors according to the colour bar next to the plot. The relative differences between the retrieved NSB (SB) and imposed background increase with increasing slope of the relation applied, remaining however relatively small. The maximum difference measured is 0.03 (0.01) for the extreme case, but on average remains $<0.0009$ and maximum of RMS difference is 0.009 (0.004).

The relative difference between the contrast image derived from our method and the original data reaches up to 0.06 for individual pixels in the worst case (on average < 0.0009); the RMS difference is less than 0.006 .

\section{Subset 3: Non-linear relations without inhomogeneities}

This subset was created to test the effects of applying a linear calibration on data that were constructed by imposing non-linear $\mathrm{CC}$ relations. We used the same 10 images as 


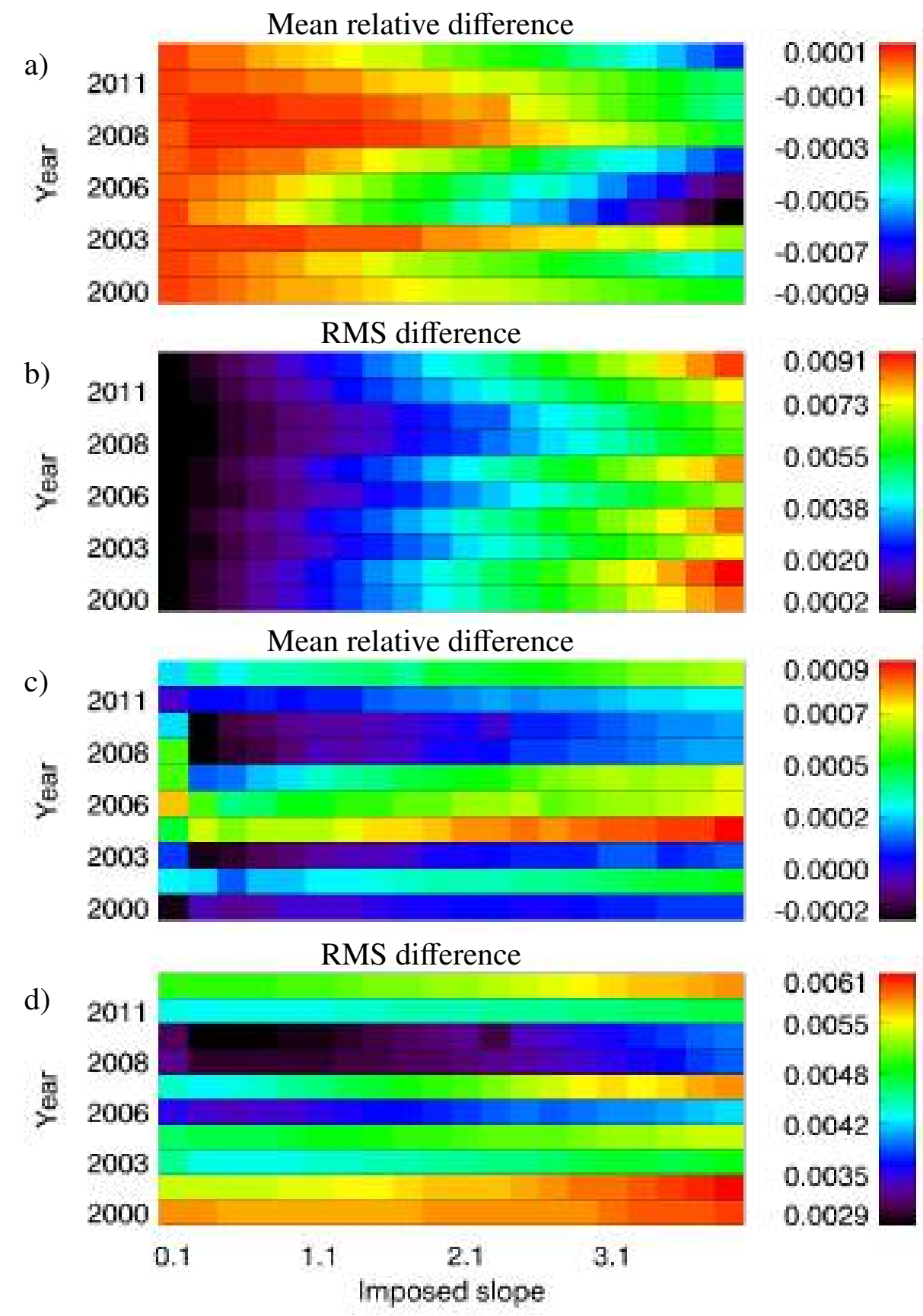

Figure 3.16: Colour-coded errors resulting from application of our method to images of subset 2. Each box of a given colour corresponds to a different observation (there are 200 boxes in all). Each row (column) of boxes shows results derived from a given observation (imposed CC relation). The colour bar applies to all boxes in a given subfigure. a) mean relative and b) RMS difference between the NSB and the imposed background for all images, c) mean relative and d) RMS difference between the retrieved contrast image and the original data. The values are given for disc positions within $0.98 R$. 
employed for subset 2, computed the logarithm of each image and applied a series of nonlinear relations on them. This way we can evaluate the accuracy of the proposed method for data suffering over- and under-exposure and to quantify the expected deviations introduced when applying a linear calibration on them. Figure 3.4 shows the imposed relations. We used 2 separate functions to describe under-exposure and over-exposure. For each exposure problem we used 10 different strength levels and considered all combinations of these functions (in total 100 cases per image). The first 5 cases of over- and under-exposure (labelled 1-5 in Fig. 3.4) produce images with intensity values belonging to the QS lying on the linear part of the $\mathrm{CC}$, while for all the rest of the cases only a small part of the QS lies on the linear part of the CC. Subset 3 consists of 1000 images.

Figure 3.17 shows the relative differences between the images derived with our method and the original undegraded data of subset 3; panels a) and b) (c) and d)) refer to values of NSB (contrast) images, respectively. Each $10 \times 10$ box is for synthetic images derived from different Rome/PSPT contrast images. In each panel, rows (columns) show values derived from different levels of over-exposure (under-exposure).

Overall the accuracy introduced by the method decreases with increasing exposure problems. Nonetheless the errors remain relatively small (maximum relative difference is 0.02 for the extreme case and 0.006 maximum RMS difference) and are always comparable with the results from Subset 1. The inaccuracy increases in the cases when over-exposure affects the QS. The differences in SB remain extremely low for no underexposure problems affecting the QS, maximum relative difference is 0.007 , while maximum RMS difference is 0.005 .

The maximum relative difference obtained by comparing the retrieved and original undegraded contrast images is usually $>0.1$ and reaches up to 0.95 for the extreme exposure problems (case 10 in Fig. 3.4); RMS is $<0.62$. These errors affect mostly the bright features. The maximum differences remain $<0.2$ for the maximum over-exposure and under-exposure considered which does not affect the QS (cases 1-5 in Fig. 3.4) and decrease with decreasing exposure problems. The errors are usually reasonable for the cases when there is no over-exposure on the QS.

Figure 3.18 shows the CC constructed for the extreme exposure problems considered. The green, blue, and red curves display the imposed relation, the measured QS CLV, and the standardized linear relation, respectively. For all the data, our method allows calculating the NSB accurately enough so that the obtained CLV reproduces the imposed relation for almost the whole range of QS values. In particular, the maximum relative difference between the imposed $\mathrm{CC}$ and the one recovered by the method is less than 0.005 . The accuracy drops slightly for the last point at the limb and only for the cases of severe under-exposure, rising to 0.017 .

\section{Subset 4: Different disc sizes with linear $\mathrm{CC}$ without inhomogeneities in the image}

The photographic plates from different observatories have different sizes. Even among the images of the same archive there are variations due to changes of the spectroheliograph and of the photographic plates. The digitization affects the number of pixels covered by the disc as well. The disc size is also affected by the seasonal variation of the Sun-Earth distance. Therefore, this subset was created to test the efficiency of the proposed method when applied to datasets that have a different disc size. We selected 10 Rome/PSPT 


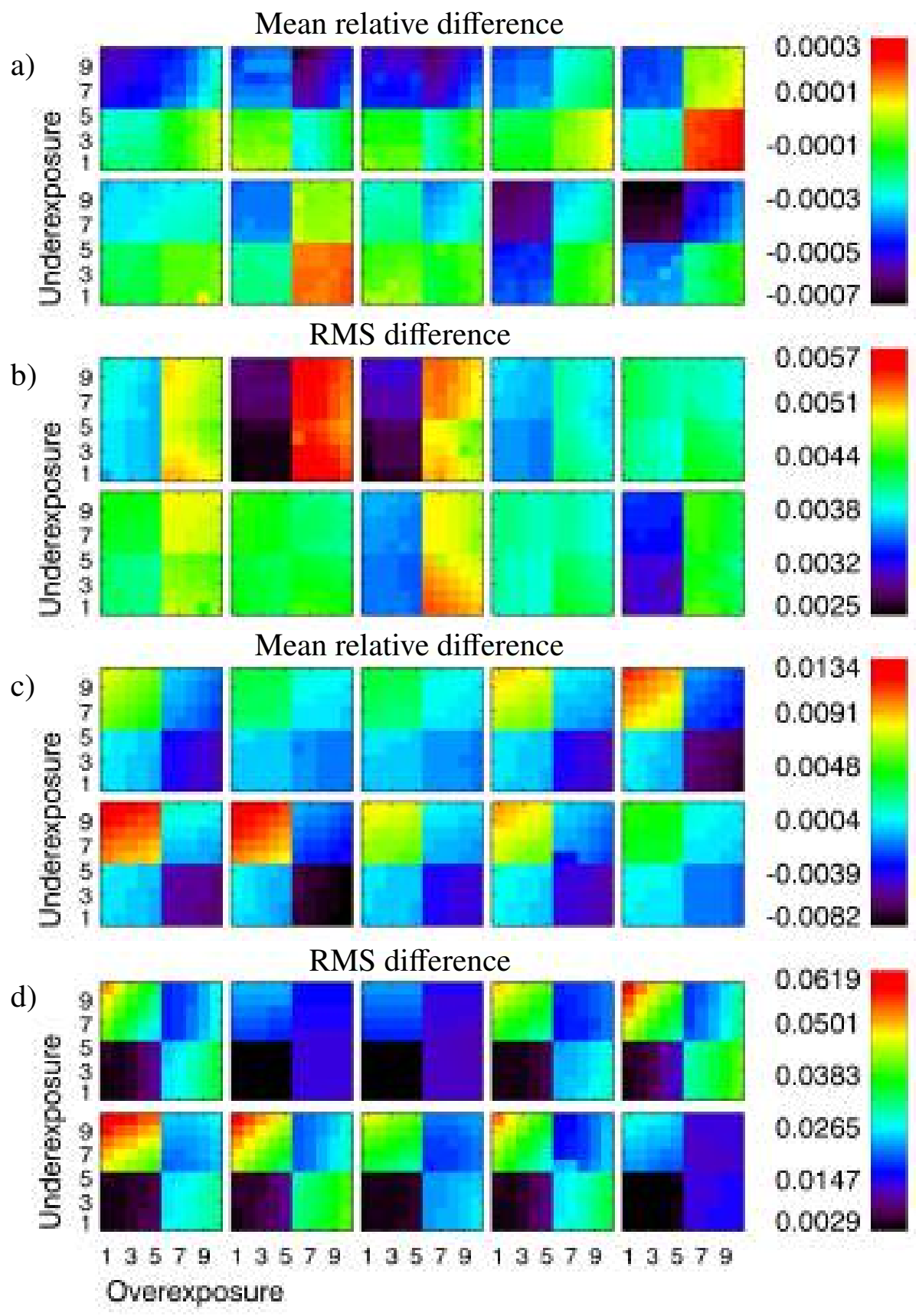

Figure 3.17: Colour-coded errors from application of our method to images of subset 3. The colour of each box (so-to-say each pixel of the plotted pattern) represents the error introduced when processing one particular Rome/PSPT image with different introduced exposure problems. Each square composed of $10 \times 10$ boxes in a given panel corresponds to synthetic images created from a given Rome/PSPT image, with the rows (columns) within the square showing the errors for different combinations of under- (over-) exposures considered (the employed CC curves are shown in Fig. 3.4). The colour bar applies to all boxes in a given panel. a) mean relative and b) RMS difference between the NSB and the imposed background for all images, c) mean relative and d) RMS difference between the retrieved contrast image and the original data. The values are given for disc positions within $0.98 R$. 


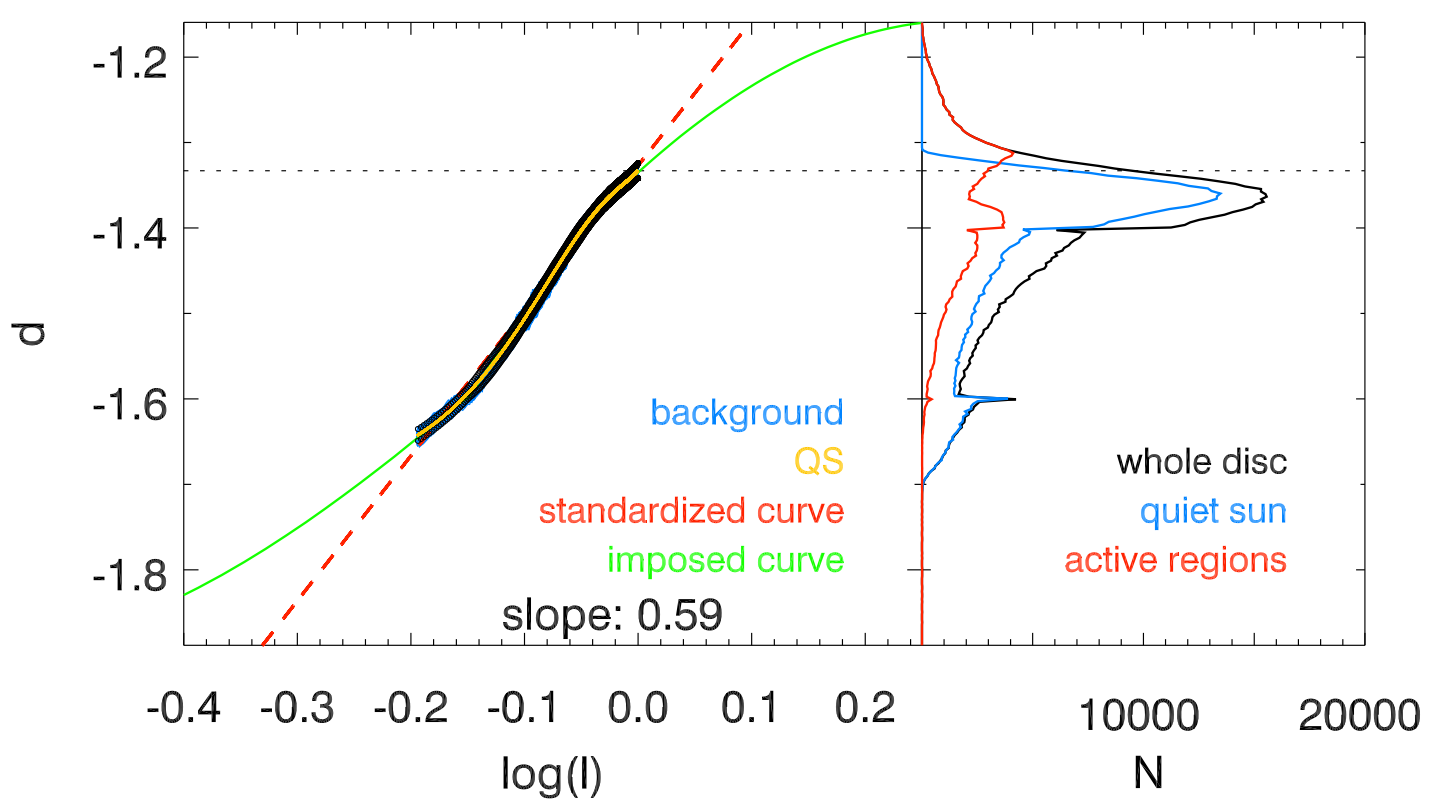

Figure 3.18: Left: Imposed CC (green), standardized CC derived with our method (red), CC estimated from the QS (orange) with its $1 \sigma$ level (black circles), and the whole background (blue, lying almost perfectly behind the black circles) of the synthetic image of subset 3 derived from Rome/PSPT observation taken on 21/08/2000 (shown in Fig. 3.7 S3/1)), which corresponds to an extreme case of over- and under-exposure on the QS considered in our study (level 10 for both under- and over- exposure). Shown also is the slope of the derived CC. Right: Distribution of density values for the QS (blue), the AR (red) and the whole disc (black). The dashed line in both panels denotes the highest value of the QS CLV.

images covering the period 2000-2014 and re-sampled them, so that each was represented in the form of solar images with 10 different radii between 100 and 550 pixels. The range of radii was defined to include the sampling present in most available datasets, however the upper limit was dictated by the number of pixels covered by the disc on Rome/PSPT observations. Subset 4 consists of 100 images.

Figure 3.19 shows the relative differences between the images derived with our method and the original Rome/PSPT data; panels a) and b) (c) and d)) refer to relative differences between NSB (contrast) images, respectively, each (coloured) box for a different image of subset 4. In each panel, rows (columns) show values derived from different observations (imposed radii). The relative difference between the retrieved NSB (SB) and imposed background decreases with increasing radius of the disc; the maximum difference measured is $0.03(0.007)$ for the images with the smallest radius, average difference is $<0.0008$, and maximum of RMS difference is $0.006(0.002)$.

The relative difference between the contrast image derived with our method and the original data reaches up to 0.053 ; the average differences are $<0.0013$; the RMS differences are $<0.007$. 


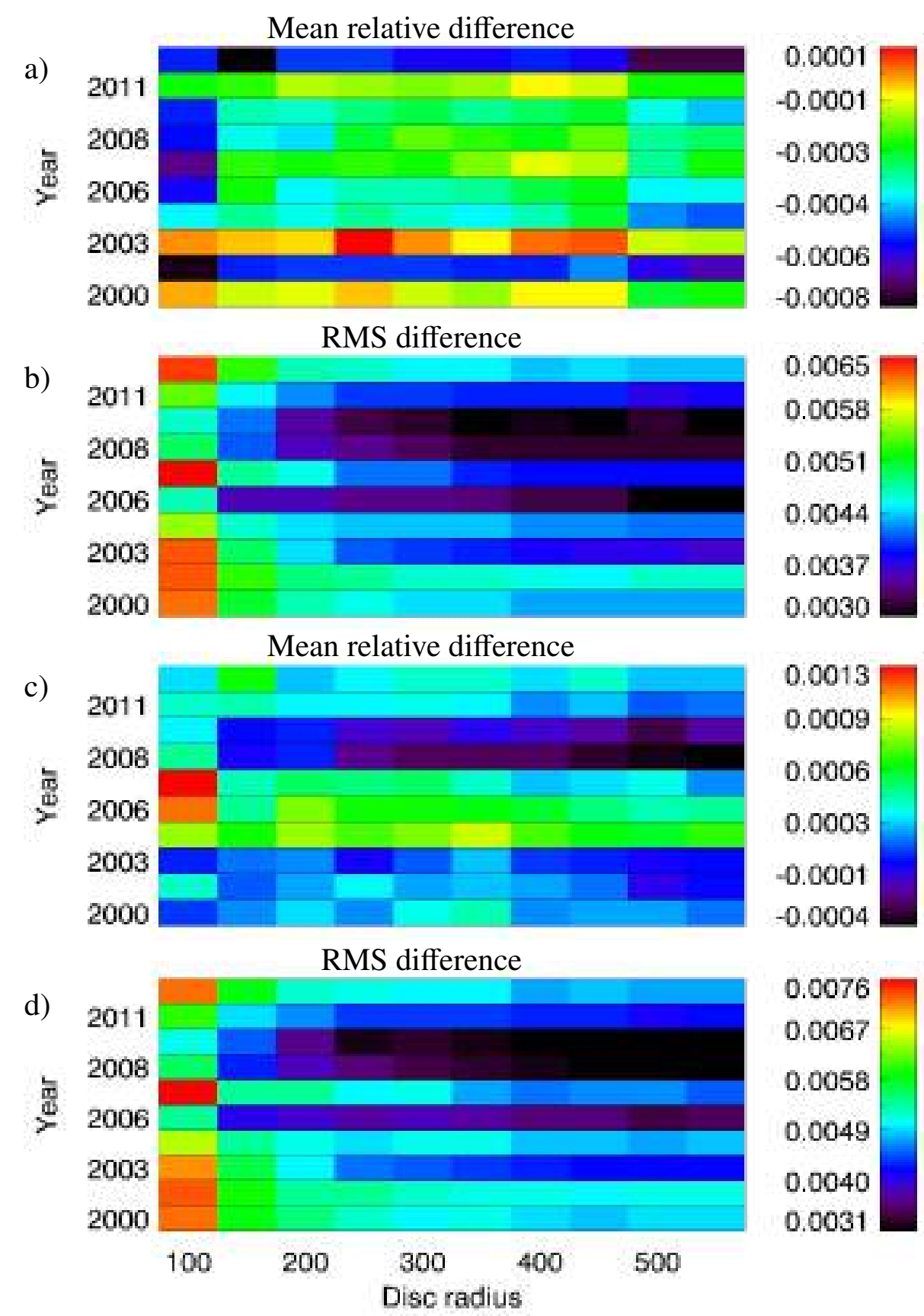

Figure 3.19: Colour-coded relative differences between the images retrieved by applying our method to images of subset 4 and the underlying original, undegraded Rome/PSPT images. Each box (with a given colour) corresponds to a different synthetic image; row (column) shows results derived from a given Rome/PSPT observation (imposed radius). The colour bar corresponds to all boxes. a) mean relative and b) RMS difference between the NSB and the imposed background within a given subfigure, c) mean relative and d) RMS difference between the retrieved contrast image and the original data. The values are given for disc positions within $0.98 R$. 


\section{Subset 5: Vignetting with linear CC without inhomogeneities}

This subset was created to evaluate the effects of vignetting on the performance of the proposed method. We took the same 10 Rome/PSPT observations as employed above and imposed on them the same radially symmetric CLV as in subset 1 , along with the vignetting function $c \times \cos ^{4} s$ (Gardner|1947, Goldman and Chen 2005) in intensity, where $s$ is the distance of each pixel to the centre of the disc and $c$ is a constant that takes 10 discrete values between 0 and 0.5 . This vignetting function causes maximum decrease in normalised intensities between 0.0 near the disc centre and 0.3 (which corresponds to $50 \%$ ) near the limb in the density images. Subset 5 consists of 100 images.

Figure 3.20 shows the relative differences between the images derived with our method and the original data. Panels a) and b) (c) and d)) refer to values of NSB (contrast) images, respectively, each box corresponds to a different image of subset 5 . In each panel, rows (columns) show values derived from different observations (imposed vignetting). The relative difference between the retrieved NSB (SB) and imposed background increases with increasing strength of vignetting (value of $c$ ); the maximum difference measured is $0.018(0.014)$ for the extreme case, the average differences are $<0.0004$, and maximum of RMS difference is 0.006 (0.016).

The relative difference between the contrast image derived with our method and the original data reaches up to 0.047 ; the average differences are $<0.0003$; the RMS differences are $<0.007$.

Figure 3.21 shows the effect of the vignetting on the $\mathrm{CC}$. As intended, vignetting decreases non-linearly the density towards the limb. This results in significantly wrong slopes for the computed $\mathrm{CC}$ if the vignetting is not accounted for. Calibrating the data with the wrong slope of the $\mathrm{CC}$ results in lower contrasts than the ones from the undegraded data and the contrast tends to decrease with increasing magnitude of vignetting.

\section{Subset 6: Varying magnitude of inhomogeneities with linear CC}

This subset was created to evaluate the effects of the varying levels of inhomogeneities on the results derived with the proposed method. The magnitude of the inhomogeneities is compared to the range of values of the CLV and we construct for each background 10 cases with inhomogeneities whose amplitude lies in the range [0.1-1.0] of the CLV. The backgrounds used here are shown in Fig. 3.5. The facular patterns overlain on these backgrounds were derived from 10 Rome/PSPT observations in the period 2000-2014. Subset 6 consists of 2000 images, resulting from 20 backgrounds, 10 amplitudes for each background, each applied to the 10 chosen Rome/PSPT images.

The relative differences of the NSB derived with our method and the original data of subset 6 can be seen in Fig. 3.22. We show the results for only two Rome/PSPT images, one taken at high and one at low solar activity periods, in order to make it easier to discern the differences between the results obtained from the various inhomogeneities and levels. In particular, in Fig. 3.22 the $\mathrm{x}$ axis represents the different backgrounds, the circles show results for the high activity image, while the squares for the low activity image and the colours signify the varying levels of inhomogeneities (black implying low amplitudes, while red is for large amplitudes).

Unsurprisingly, the errors introduced when retrieving the intensity image using the proposed method increase with magnitude of the inhomogeneities on the analysed im- 


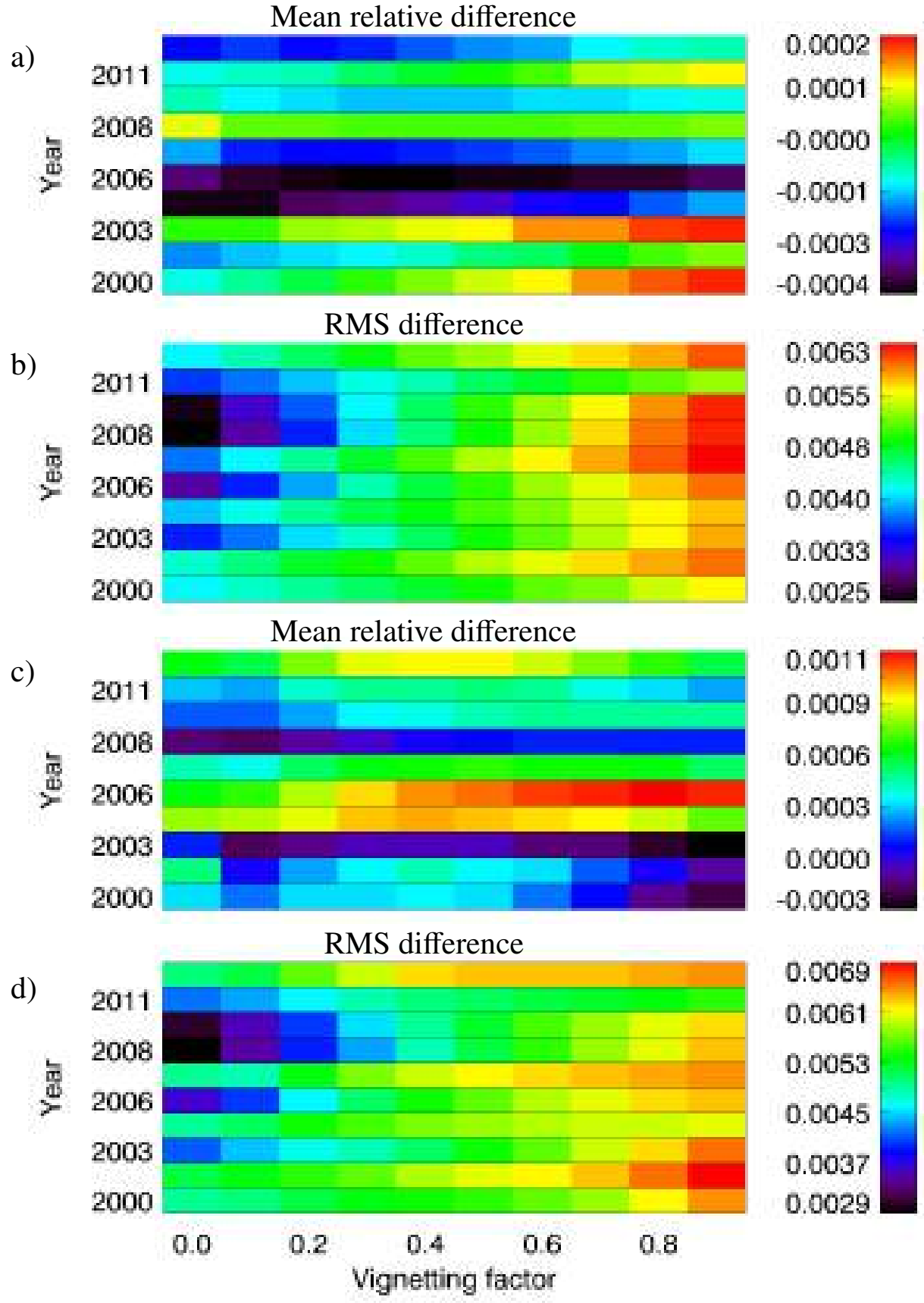

Figure 3.20: Colour-coded relative differences between the images retrieved by applying our method to images of subset 5 and the underlying original, undegraded Rome/PSPT images. Each box corresponds to a different synthetic image. Each row (column) shows results derived from a given Rome/PSPT observation (imposed vignetting). The colour bar corresponds to all boxes within a subfigure. a) mean relative and b) RMS difference between the NSB and the imposed background for all images, c) mean relative and d) RMS difference between the retrieved contrast image and the original Rome/PSPT data. The values are given for disc positions within $0.98 R$. 


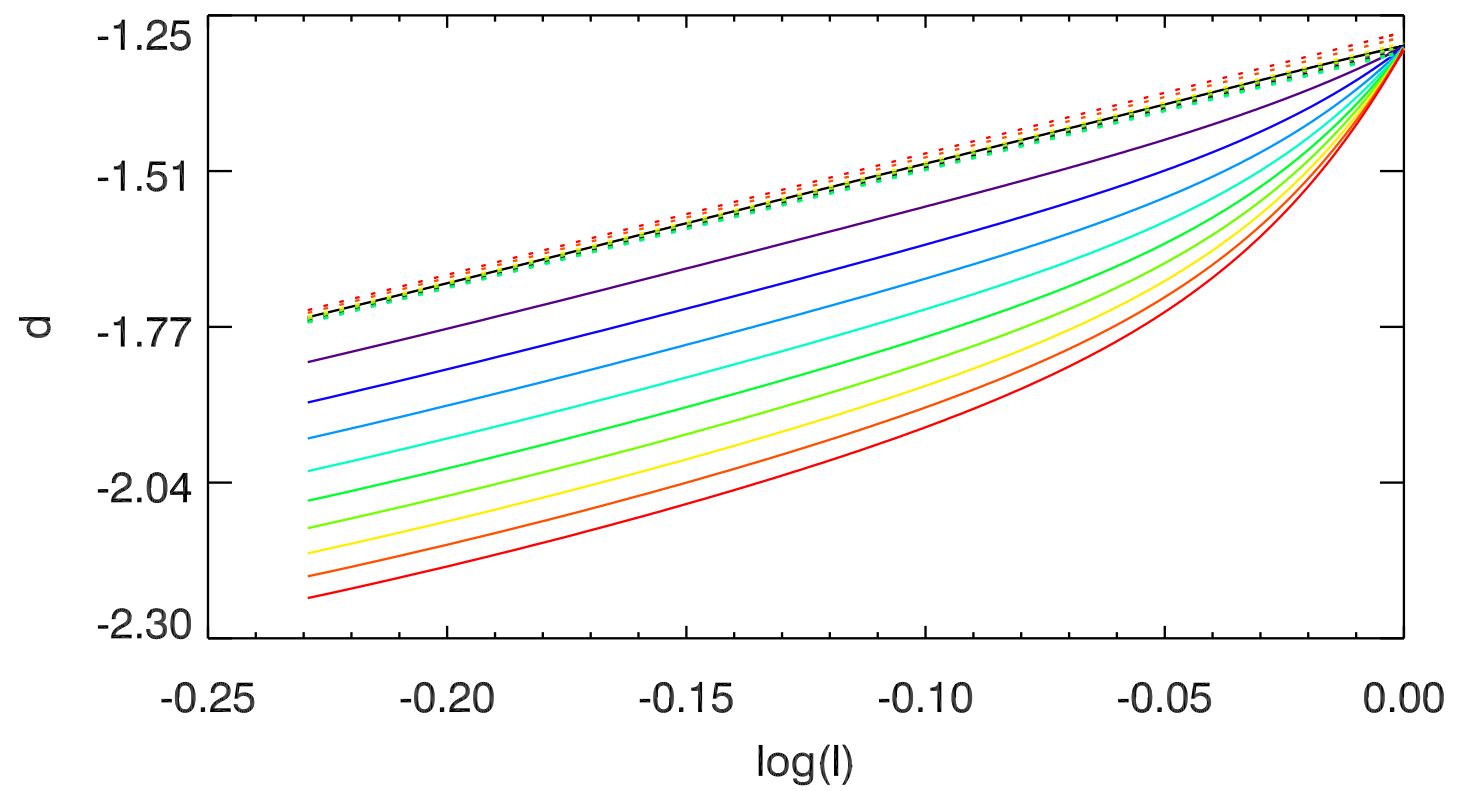

Figure 3.21: CC for synthetic images with vignetting (subset 5) created from Rome/PSPT observation taken on 21/08/2000. The colours denote the magnitude of the vignetting, with red for the strongest case and black for no vignetting. The solid curves are derived with the values measured on the density image, while the dotted curves are after the correction for the vignetting by our method.

age. In many images the errors remain relatively low, e.g. background No. 6 in Fig. 3.5. where the maximum difference is 0.007 . The maximum difference is obtained for the images with very bright/dark small artefacts, while larger-scale inhomogeneities and small artefacts with mild brightness are reliably processed by our method. The errors are highest when density variations in adjacent image lines are significantly brighter than their surroundings, with a maximum relative difference of 0.5 . The maximum differences for NSB remain $<0.1$ for most of the backgrounds even in the extreme cases that were considered.

The results for the rest of the data in subset 6 are very similar to the ones presented here. We did not find any significant dependence of the results on the level of solar activity.

\section{Subset 7: Different CLVs with linear CC}

Subset 7 was created to study the effects of applying our method on data that have a different CLV than the one we use to standardize them. The data could have a different CLV because the observation was centred at a different wavelength than the core of the Ca II $\mathrm{K}$ line, or possibly due to a different bandwidth (e.g. due to a different spectral resolution or slit width). We used again 10 Rome/PSPT images to derive the facular pattern and on each one of those imposed 10 different functions for the quiet Sun (seen in Fig. 3.6), describing deviations from the one we measure in modern Rome/PSPT observations. Subset 7 consists of 100 images.

Figure 3.23 shows the relative differences between the corrected and calibrated images 


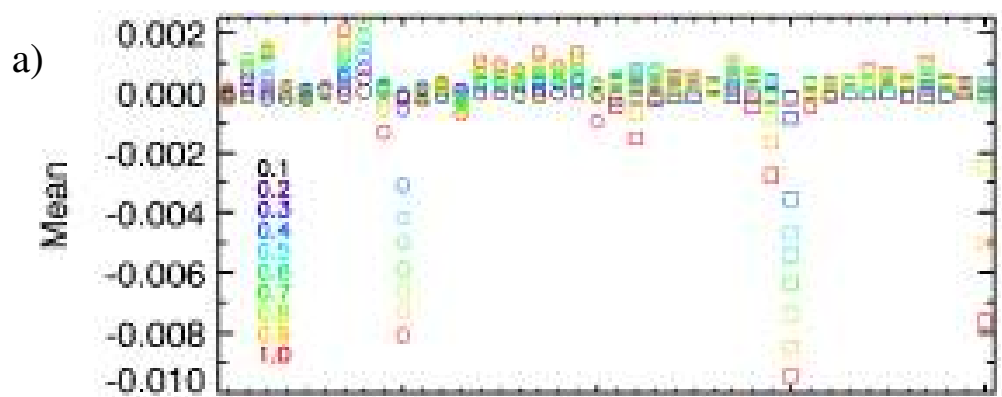

b)
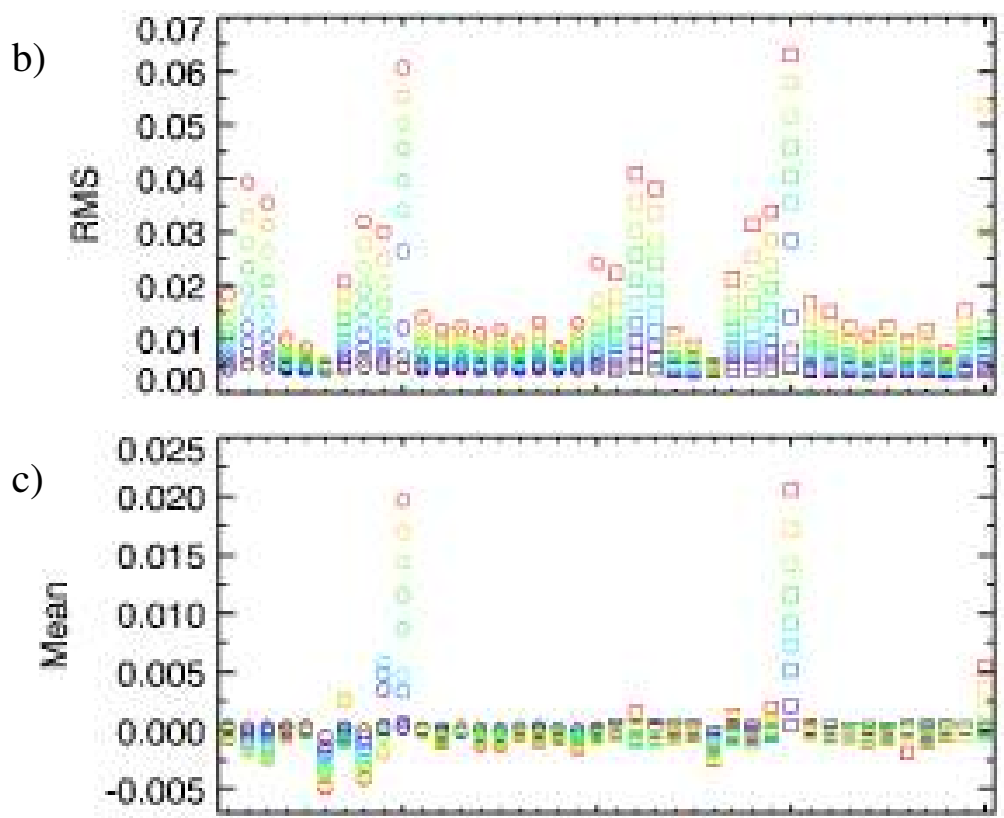

d)

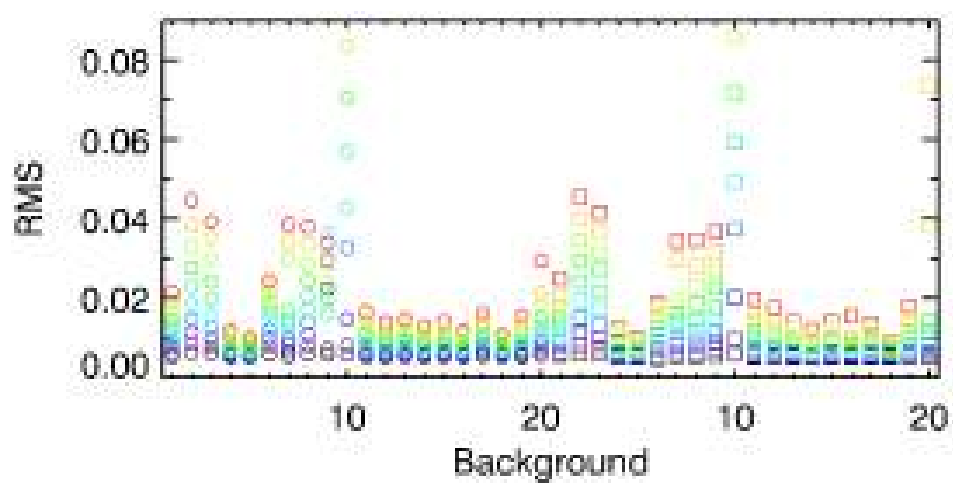

Figure 3.22: Errors in the images retrieved with our method applied to subset 6 . The circles (squares) correspond to errors in images based on Rome/PSPT data taken at a high (low) activity period. The colours denote the different magnitudes of the inhomogeneities. The scale (relative to the background intensity variation from the limb to disc centre) is given in the topmost frame. The various backgrounds are numbered following Fig. 3.5 , a) mean relative and b) RMS difference between the NSB and the imposed background for all images, c) mean relative and d) RMS difference between the retrieved contrast image and the original data. The values are given for disc positions within $0.98 R$. 
derived with our method and the original data; panels a) and b) (c) and d)) refer to values of NSB (contrast) images, respectively, each box for a different image of subset 7 . In each panel, rows (columns) show values derived from different observations (imposed CLV). The relative difference between the retrieved NSB (SB) and imposed background has a maximum value of $0.019(0.004)$, the average differences are $<0.0004$, and the maximum RMS difference is $0.004(0.0015)$.

We found that the different CLVs affect more the calculation of the slope of the CC and the errors for the contrast images increase, where the changes in the RMS differences reach up to 0.038 , the maximum differences remain $<10 \%$ for 3 CLVs surrounding the average curve we use.

\section{Subset 8: Random problems}

Subset 8 was created to study the performance of our method on data where every image suffers from a different set of problems. We used 2000 Rome/PSPT images to derive the facular pattern and on each one of those imposed a random function for the quiet Sun (shown in Fig. 3.6), describing deviations from the one we measure in modern Rome/PSPT observations. We also added a random pattern of inhomogeneities that were used for subset 6 (Fig. 3.5), with a random level of strength for each image. We convert the images to density by applying a $\mathrm{CC}$ in the form of a 3rd degree polynomial with randomly selected parameters within the following ranges: $[-4.6,-1.3]$ for the constant term, $[0.5,4.0]$ for the linear term, $[-0.03,0.00]$ for the quadratic term, and $[-0.03,0.00]$ for the cubic term. A random level of vignetting was also added. Subset 8 consists of 2000 images.

Figure 3.24 shows the relative errors for the background calculation on data of subset 8. The results demonstrate that the proposed method allows retrieving the NSB affecting the observation with maximum relative error $<2 \%$ averaged over all the analysed images. We found that, similarly to artefacts on the historical images, the presence of gradients on the synthetic images affects the range of values in the calibrated data, due to errors in the calculated SB.

Figure 3.25 illustrates the accuracy of the proposed method on the most challenging subset 8 . Shown are the maximum and average values of the relative difference as well as the RMS difference between the calibrated and processed contrast image and the original undegraded synthetic contrast image. These values quantify the final errors of our image processing which is comprised of the photometric calibration and the removal of the CLV and other image patterns from the analysed image and finally provides the corrected contrast image. We found that the maximum differences are on average $<6.5 \%$, while the average differences are $<1 \%$. There is, however, a tail consisting of images with higher maximum or average differences. For images representative of low activity periods, maximum errors are approximately $2 \%$ lower than those found for images at high activity periods, which illustrates the need for a very careful removal of ARs prior to carrying out any image processing.

\subsubsection{Performance on individual steps of the processing}

Our method includes original ideas applied to the purpose of processing SHG (e.g. rotating slices), but it also partly uses ideas from the previously published methods described 


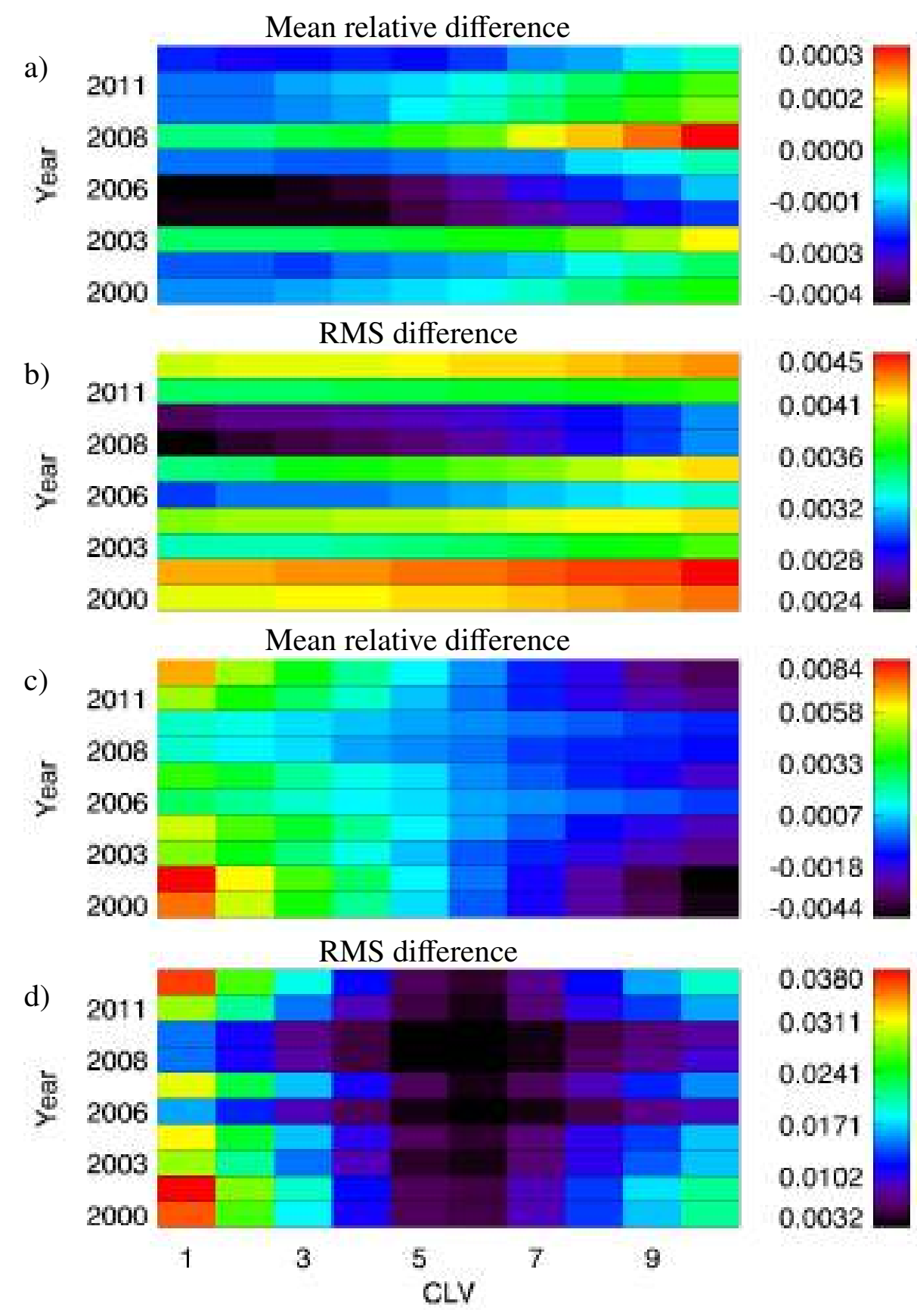

Figure 3.23: Colour-coded relative differences between the images retrieved by applying our method to images of subset 7 and the underlying original, undegraded Rome/PSPT images. Each box corresponds to a different observation. Each row (column) of boxes shows results derived from a given observation identified by the year in which it was recorded (imposed CLV, numbered as in Fig. 3.6. The colour bar is applicable to all boxes in the corresponding sub-figure. a) mean relative and b) RMS difference between the NSB and the imposed background for all images, c) mean relative and d) RMS difference between the retrieved contrast image and the original data. The values are given for disc positions within $0.98 R$. 


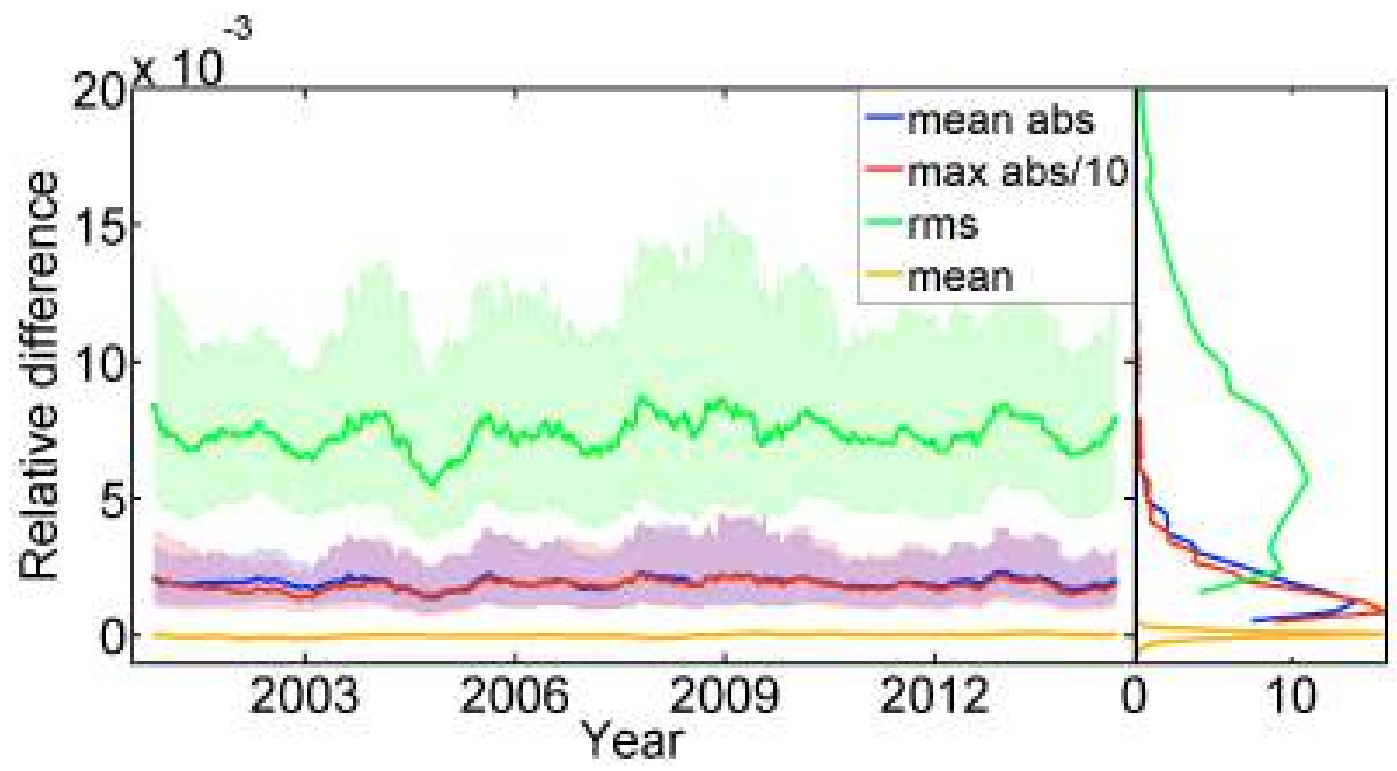

Figure 3.24: Left: Relative difference between the NSB and the imposed background (within $0.98 R$ ) for all the synthetic data of subset 8. RMS difference (green), mean absolute difference (blue), mean difference (orange) and maximum difference (red). These differences are plotted vs. the date on which the original Rome/PSPT images (that were randomly distorted) were recorded. Note that the maximum difference values have been divided by 10 to plot them on the same scale as the other quantities. The solid lines are 100 point averages and the shaded surfaces denote the asymmetric $1 \sigma$ interval. Right: Distribution of the relative difference values.

in Sect. 2.6. By testing all these methods on synthetic data we identified their drawbacks, which helped us to optimize the steps of our proposed method.

For example, in our calculation of the background, we do not rotate the image by $45^{\circ}$ as proposed by Worden et al. (1998a). We found that the rotation does not improve the accuracy of the image processing further, if the outcome of other processing steps has been optimized. In contrast, our iterative fitting improves the accuracy of the AR identification and, in turn, of the QS estimation by allowing more accurate results than obtained from both non-iterative computations with a 5th degree polynomial function as suggested by Worden et al. (1998a) and iterative computations with higher degree functions. However, we also noticed that on average more than three computations of the fit per iteration step merely results in an increase of the noise of the final NSB map derived from the processing without improving the accuracy of the result.

In Step 2.2 to identify and exclude ARs we apply a thresholding scheme with asymmetric limits, by using the values ${ }_{-1}^{+0.5} \sigma$ instead of the more widely employed $\pm 2 \sigma$. The asymmetric range allows us to account for both, the potentially inaccurate identification of AR near the solar limb at earlier processing steps and the small disc fraction of dark features in Ca II K observations. For example, Fig. 3.26 shows the relative errors in the NSB calculation on the synthetic image shown in Fig. 3.7 S1/1) if symmetric limits of $\pm 1 \sigma$ and $\pm 2 \sigma$ are used. The errors derived with our method and the asymmetric limits are shown in Fig. 3.7 S1/5) and are 1.8\%. The use of the symmetric limits tends to give results overestimating the QS background around AR. The errors increase and reach $3.5 \%$ 


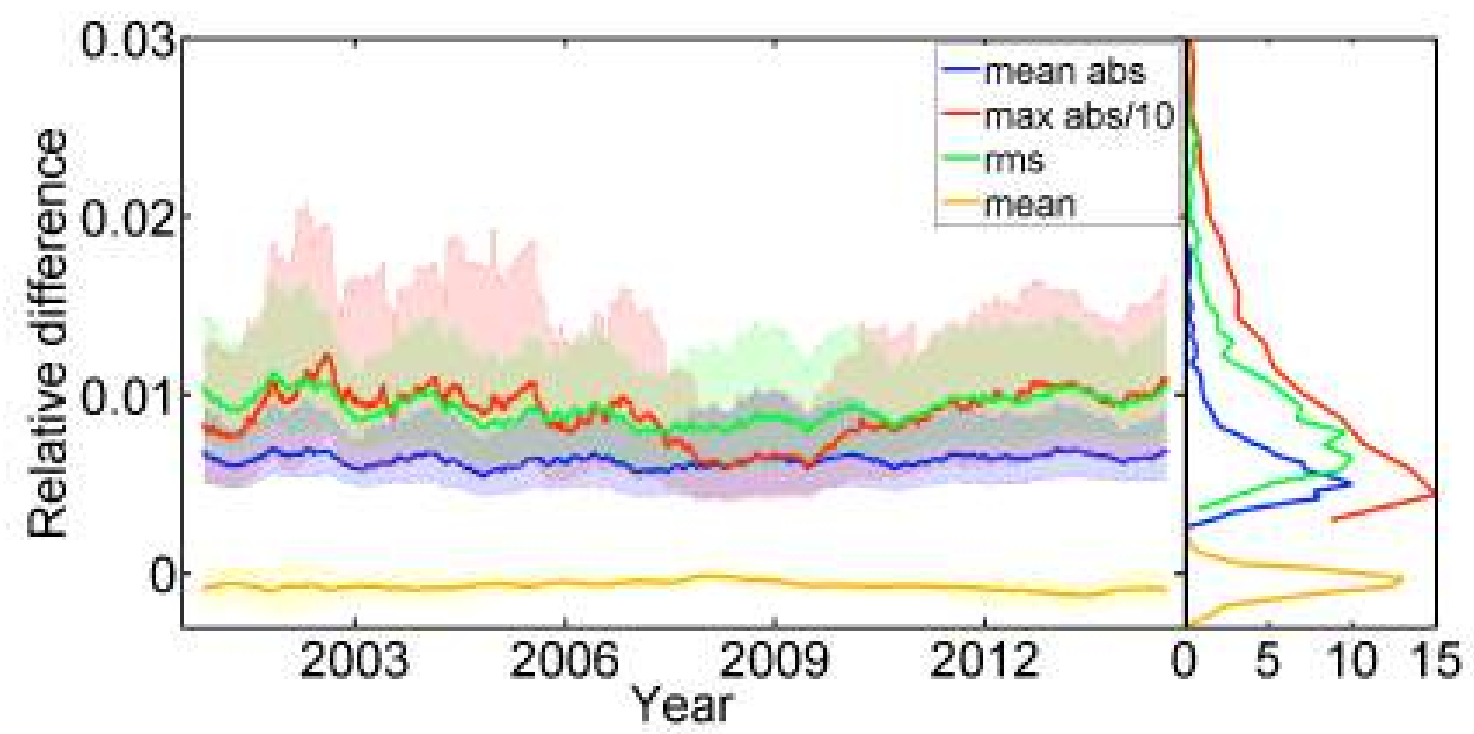

Figure 3.25: Left: Relative difference between the calibrated and CLV corrected image with our method and the original image (within $0.98 R$ ) for all the synthetic data of subset 8. RMS difference (green), mean absolute difference (blue), mean difference (orange) and maximum difference (red). Each of these values refers to a single image at a time (e.g. the difference averaged over all pixels, or the maximum value found in one pixel of the image). These differences are plotted vs. the date on which the original Rome/PSPT images (that were randomly distorted) were recorded. Note that the maximum difference values have been divided by 10 to plot them on the same scale as the other quantities. The solid lines are 100 point averages (i.e. averages over the values obtained for 100 images) and the shaded surfaces denote the asymmetric $1 \sigma$ interval. Right: Distribution of the relative difference values.

and $6.3 \%$ for $\pm 1 \sigma$ and $\pm 2 \sigma$ limits, respectively. The employed upper limit of $+0.5 \%$ was found to be a good compromise. Larger values led to the inclusion of significant portions of ARs in the QS background, while lower values for the lower limit risk to wrongly exclude large regions of the solar observations near the limb from the 2D QS background calculation.

The window width for the median filter used in Step 2.4 was chosen to scale with $R$, to achieve consistent results from different data with varying disc size. The adopted width is larger than the typical scale of the network on the analysed observations, in order to avoid effects of small-scale density patterns of solar origin on image processing results, but is small enough to account for rapid changes of the background near the solar limb. We found that window widths in the range $R / 6-R / 8$ perform best on all available data and we adopted the more conservative value of $R / 6$. This finding is in contrast to that of Chatterjee et al. (2016) who used a window width of $\sim R / 13$. Figure 3.27 shows one example of testing different window widths on an image from subset 1 (shown in Fig. $3.7 \mathrm{~S} 1 / 1)$ ). We show widths of $R / 2, R / 4, R / 13$, and $R / 20$ pixels. Large window widths fail very close to the limb, while smaller widths progressively fail with ARs and network. Window width of $R / 4$ gives comparable results with those of our adopted value. 

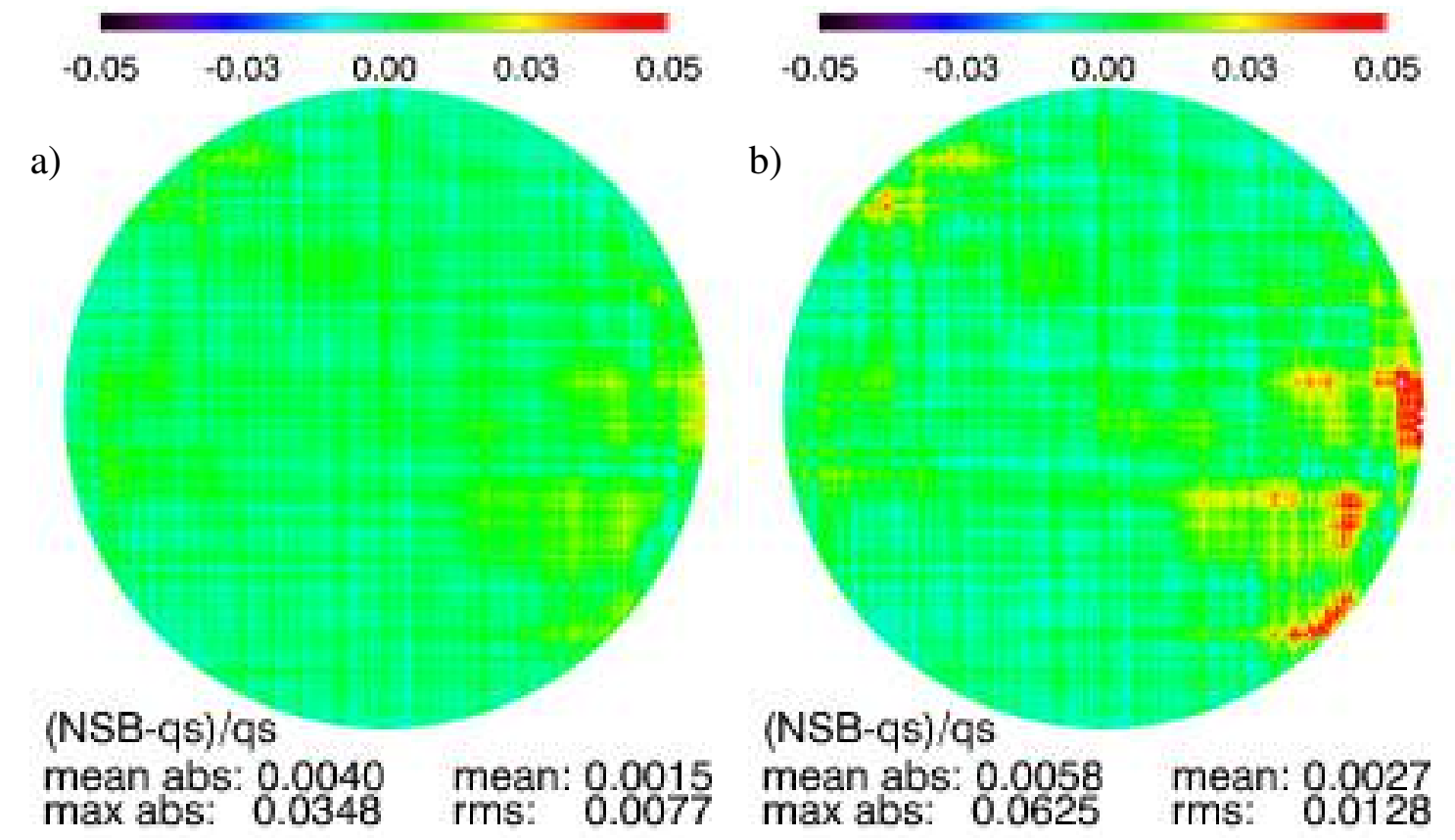

Figure 3.26: Relative error in NSB calculation for one image of subset 1 derived from Rome/PSPT observation taken on 21/08/2000 excluding ARs with symmetric limits. The limits used are (a)) $\pm 1 \sigma$ and (b) $\pm 2 \sigma$. Also shown are the values of the RMS, mean, mean absolute, and maximum relative differences by comparing image regions within $0.98 R$. The colour bars apply to the images below them. The errors from processing with our method are shown in Fig. 3.7 S1/5).

The accuracy of our processing also decreases if we do not identify the density variations of adjacent image lines $\mathrm{2}^{2}$ and simply derive a smooth background map. Furthermore, if these variations are not properly accounted for in the analysed image its subsequent analysis aimed at the estimation of e.g. the photometric properties of AR returns inaccurate results.

Tests on subset 3 that includes non-linear CCs, showed that our method is very accurate in recovering the shape of the $\mathrm{CC}$ even on observations with strong exposure problems, with relative errors in the computed $\mathrm{CC}$ being usually $<0.5 \%$ under typical conditions as well as for the other synthetic subsets. Even in the extreme cases of overor under-exposure the relative error in the computed CC lies below 1.7\%.

The analysis of the images of the subset 2 suggests that the CC slope only mildly affects the results. Our method also accurately disentangles the vignetting contribution on the CC (see Fig. 3.21). Figure 3.28 shows an example of processing an image from subset 5 without attempting to recover the vignetting (see Fig. 3.9 S5/6) for the results with the vignetting recovery). The vignetting contribution increases the slope of the $\mathrm{CC}$ which in turn reduces the contrast values of the plage regions. The errors over these regions become 35 times greater than if we account for the vignetting with our method.

\footnotetext{
${ }^{2}$ Such lines may have been introduced during the observation due to problems of the spectroheliograph employed, e.g. irregular drive.
} 


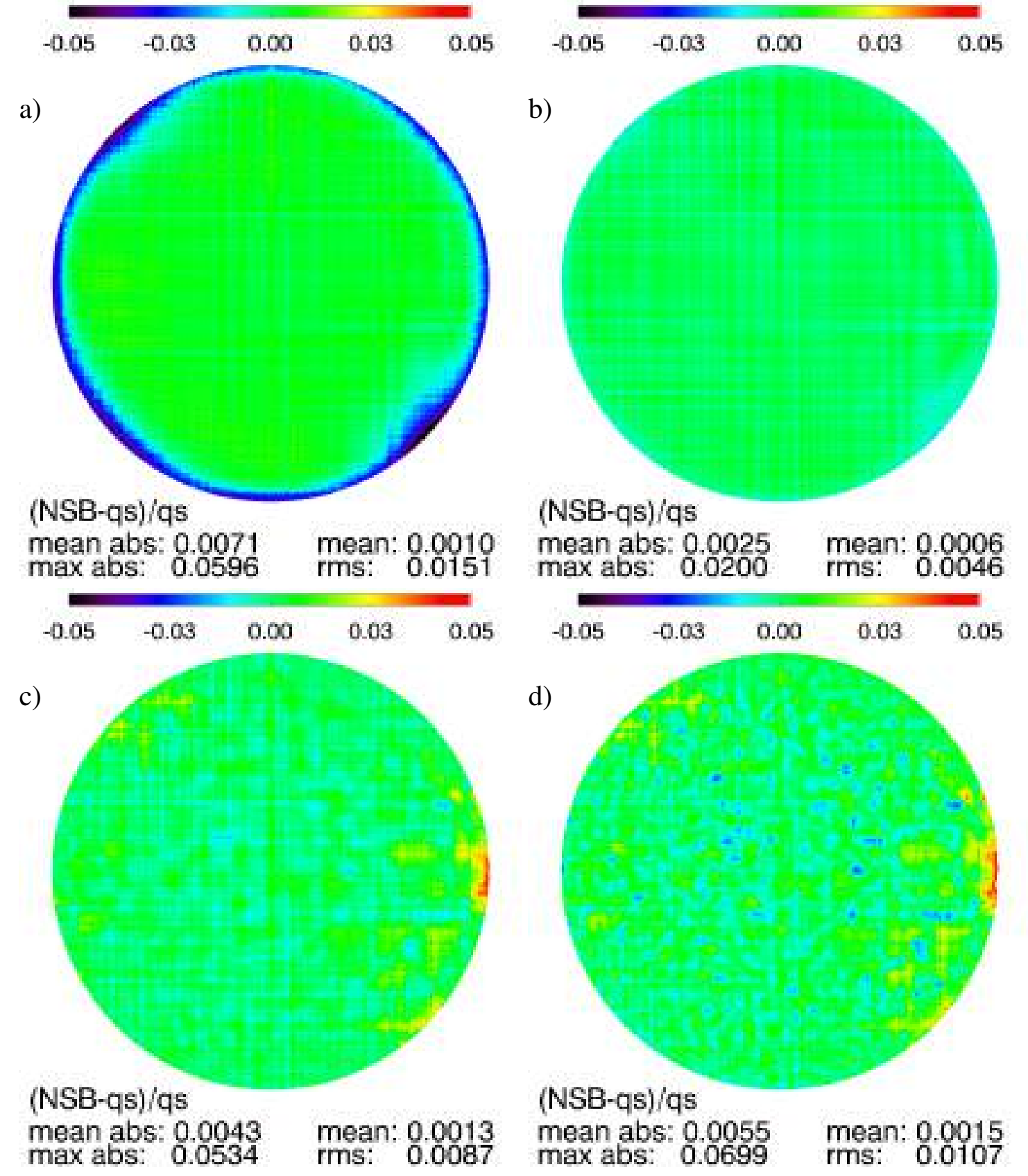

Figure 3.27: Relative error in NSB calculation for one image of subset 1 derived from Rome/PSPT observation taken on 21/08/2000 shown in Fig. 3.7 S1/1). The NSB was derived with our method and running window median filter width of (a)) $R / 2$, (b)) $R / 4$, (c)) $R / 13$, and (d)) $R / 20$ pixels. Also shown are the values of the RMS, mean, mean absolute, and maximum relative differences by comparing image regions within $0.98 R$. The colour bars apply to the images below them.

\subsubsection{Examples of calibrated SHGs}

We have applied the proposed method to many SHG randomly selected from the seven available historical (photographic) archives. Figures 3.29 and 3.30 show examples of the results obtained from observations taken at periods of high and low solar activity, re- 

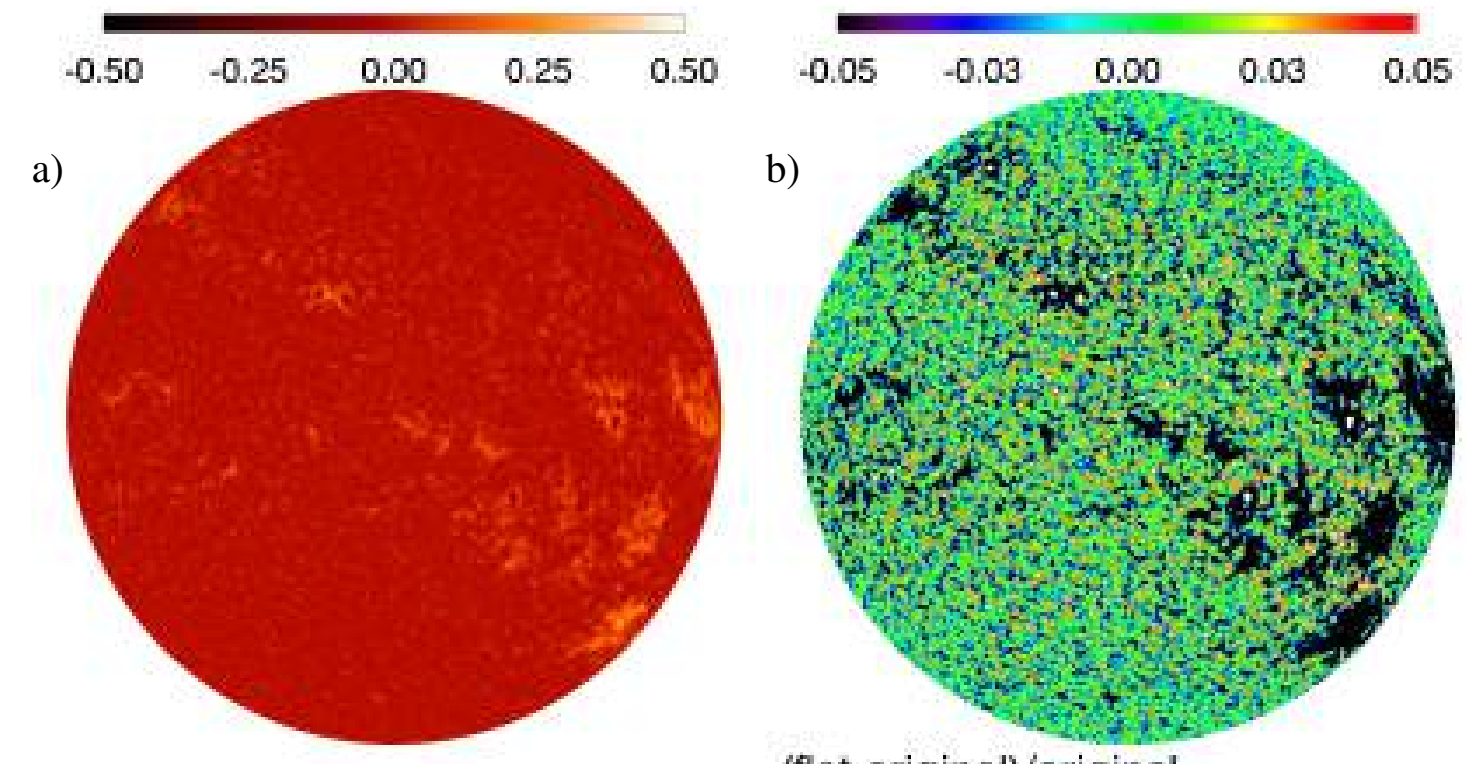

(flat-original)/original

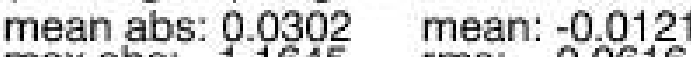

max abs: 1.1645 rms: 0.0616

Figure 3.28: Examples of applying our method on a synthetic image of subset 5 derived from Rome/PSPT observation taken on 21/08/2000: (a)) contrast image derived after processing of the synthetic image without compensation for the vignetting; (b)) relative error of the calibrated contrast images. Also shown are the values of the RMS, mean, mean absolute, and maximum relative differences by comparing image regions within $0.98 R$. The colour bars apply to the images below them.

spectively. From left to right, each panel shows the original observation (density image), the density image saturated to show the background, the background (CLV plus inhomogeneities) deduced from the proposed processing, the calibrated image, the identified inhomogeneities of the analysed image, and the final contrast image after the QS CLV removal. From top to bottom each such set of images is shown for SHG extracted from the Ar, Co, Ko, Me, Mi, MM, and MW archives, respectively. All the calibrated images are shown within the intensity range $[0.0,2.0]$, while the contrast images (i.e. images compensated for the CLV) are plotted within [-0.5, 0.5]. For the rest there is a colour bar denoting the range of values. Further examples can be found in Chatzistergos et al. (2016).

The deduced backgrounds describe the different patterns in the analysed images quite accurately. Figures 3.29 and 3.30 clearly show that the method works consistently with data extracted from various photographic archives, taken at different activity levels. All calibrated images lie within the same range of values and show a similar CLV pattern. The same is true for the contrast images that return plage regions within the same intensity ranges and no obvious residual large scale artefacts. The method is able to account even for strong inhomogeneities and rather peculiar patterns (e.g. Fig. 3.29 Ar, or Fig. 3.30 Ko) without affecting the plage regions. The inhomogeneities identified here show a CLV that is usually off-centred, in many cases having its highest value towards the limb. Furthermore, images show many dark/bright bands that could occur due to something 


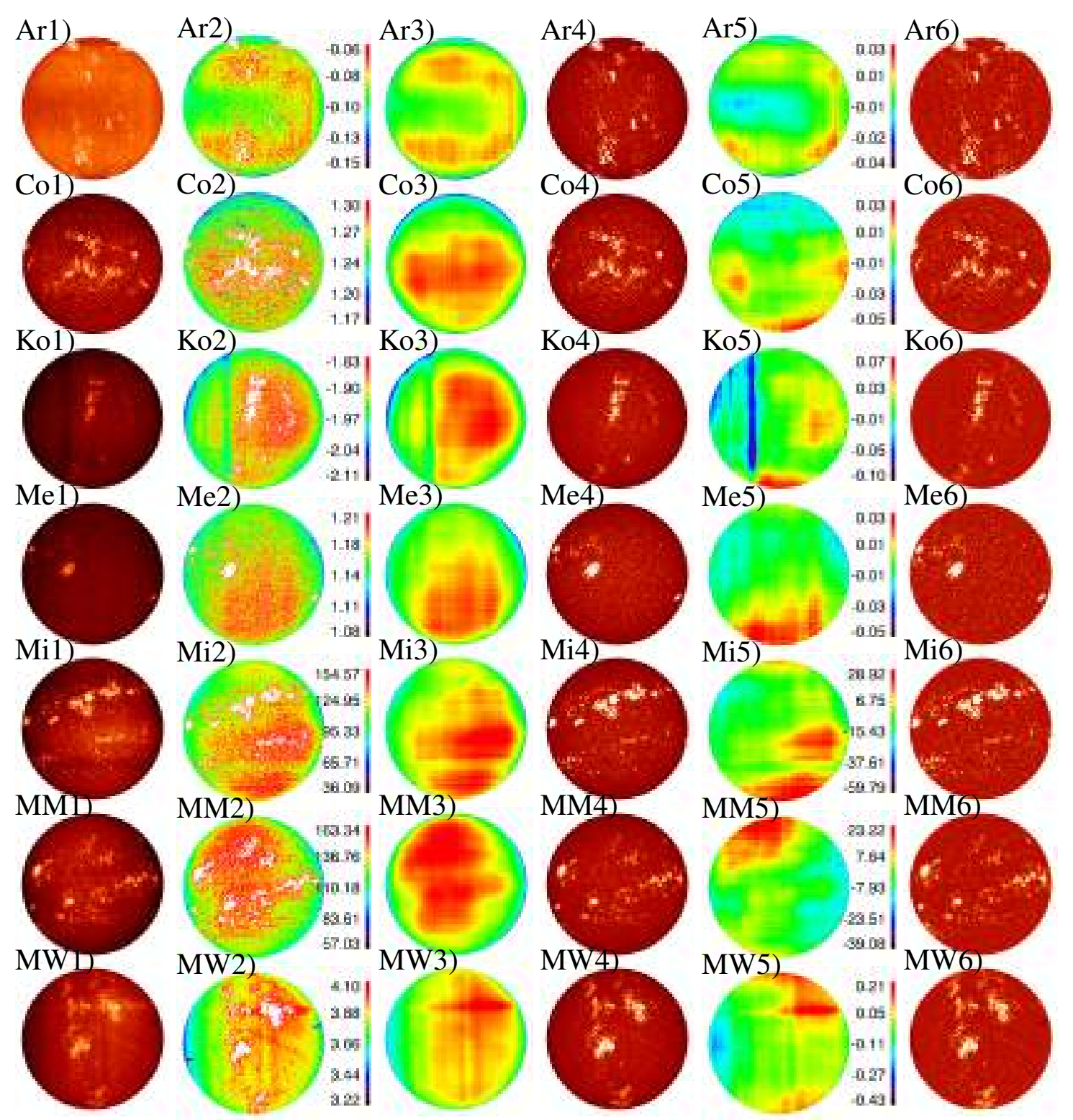

Figure 3.29: Examples of the calibration procedure of historical images from the Ar, $\mathrm{Co}, \mathrm{Ko}, \mathrm{Me}, \mathrm{Mi}, \mathrm{MM}, \mathrm{MW}$ archives (from top to bottom) taken at high activity periods. From left to right: (1)) density images, (2)) density images saturated such as to clearly show the backgrounds, (3)) calculated backgrounds, (4)) calibrated images, (5)) identified inhomogeneities, and (6)) images corrected for QS CLV. The colour bar between images in columns 2) and 3) applies to both images.

occluding the Sun for a short period, or not constant exposure over the different rasters.

The tests on historical observations with good quality, resulted in 1D QS CLVs very similar to the one from the Rome/PSPT data. This strengthens our argument that the CCs of the good quality historical data can be described by a linear relation. 


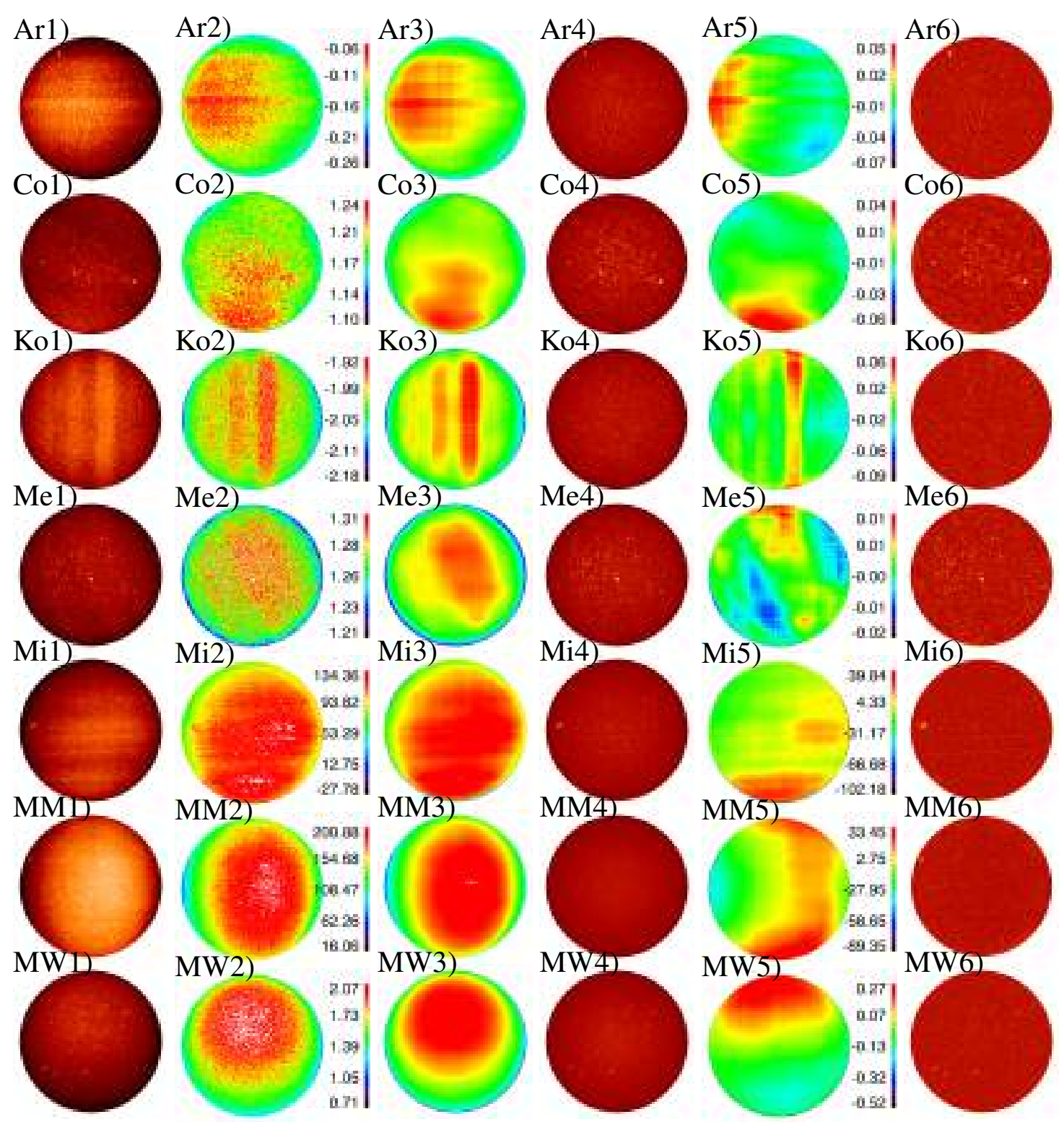

Figure 3.30: As in Fig. 3.29 for images taken at low activity periods.

\subsection{Comparison with other methods}

\subsubsection{Background calculation methods}

We found that the methods presented in the literature that apply radially symmetric computations suffer from their inability to account for the asymmetric patterns affecting the images and do not account for small-scale image artefacts, e.g. the density variations in adjacent lines. Therefore they cannot be used with the historical observations. However, they can potentially be used in modern observations in the absence of large-scale inhomogeneities. For that purpose we tested these methods on the data of subset 1, which have no such problems. Figure 3.31 shows the errors we get in recovering the SB of one image from subset 1 (shown in Fig. 3.7 S1/1)) with our method and those by Brandt and Steinegger (1998), Walton and Preminger (1999), and Caccin et al. (1998). These methods are 
applied as they were described in Sect. 2.6.2. However, for the method of Caccin et al. (1998) we stretched the disc to the regions outside the disc in the same way as is used in our method. We notice that our method succeeds in getting the level of the QS more accurately than the other methods. The method by Brandt and Steinegger (1998) shows an offset in the level of the QS along with significant errors. The method by Walton and Preminger (1999) gets the level of the QS more closely, but with higher errors than from application of our method. By applying the methods of Caccin et al. (1998) and Worden et al. (1998b) on the degraded synthetic SHGs we found that these techniques provide inaccurate QS CLV values towards the limb, with errors that reach $15 \%$. These methods also fail at the disc centre.

Application of median filtering suffers under presence of AR and its inability to account for density variations along adjacent image lines. The method by Tlatov et al. (2009) is also unable to account for these lines.

Figure 3.32 shows the pixel by pixel relative differences between the NSB and the imposed background derived with our method and that by Worden et al. (1998a) , on two synthetic images from subset 6 . Part of the AR remained undetected by the latter method and so enters the computation of the background. Thus the method by Worden et al. (1998a) overestimates the actual background in some plage areas and introduces processing errors. The maximum relative errors in NSB are lower for observations taken at low solar activity (3.7\%), than at periods of high solar activity (26\%), but on average the method by Worden et al. (1998a) introduces one order of magnitude higher errors over the disc than obtained from our proposed method.

We applied the method by Priyal et al. (2013) on all data of subset 6 (shown in Fig. 3.33) and we found that the method by Priyal et al. (2013) works reasonably well on images with weak anisotropies, but consistently fails to account for the large inhomogeneities affecting the data, by introducing up to 16 (25) times larger maximum (RMS) errors than those from our method. Besides, the method by Priyal et al. (2013) does not allow recovering any image patterns that occur in a direction different from the one considered for the fit.

\subsubsection{Calibration methods}

We tested the accuracy of the method by Priyal et al. (2013) by applying an average CC to calibrate a whole dataset with our synthetic data. We derived the average $\mathrm{CC}$ from the curves we imposed to all the data of subset 8 , and studied the differences between contrast images obtained from the original data and the ones resulting from the calibration with the average CC. We used the imposed background of each image to compensate for the limb darkening, in order to avoid any other uncertainties of our procedure. This error estimate can be considered only as a lower limit, since the CCs used to derive the average were the imposed ones, therefore without taking into consideration any errors in the calculation of the individual CCs. Figure 3.34 shows that the errors introduced by the calibration with the average $\mathrm{CC}$ as proposed by the method by Priyal et al. (2013) are on average

\footnotetext{
${ }^{3}$ When we applied the method by Worden et al. (1998a) we did not perform the last step of the low-pass filtering, because the information of the window-width or the way they applied it on regions very close to the limb is not described. Applying this step could potentially reduce the errors of isolated pixels, but it would not make a difference in the misidentification of the AR.
} 

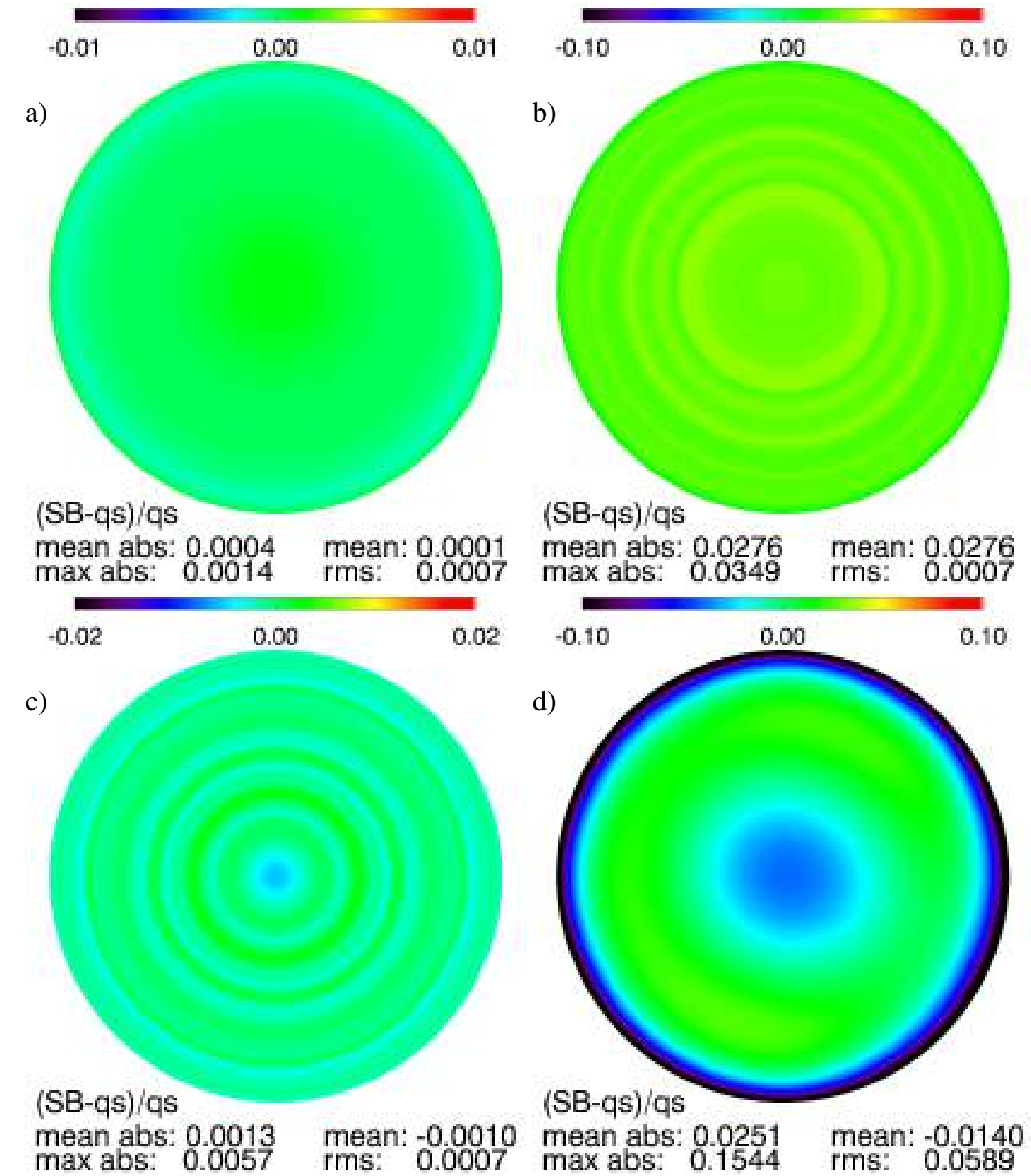

mean abs: 0.0013 max abs: 0.0057

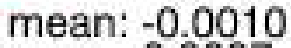

rms: 0.0007

max abs: 0.1544

Figure 3.31: Relative error in SB calculation for one image of subset 1 derived from Rome/PSPT observation taken on 21/08/2000 shown in Fig. 3.7 S1/1). The SB was derived (a)) with our method, and the methods by (b)) Brandt and Steinegger (1998), (c)) Walton and Preminger (1999), and (d)) Caccin et al. (1998). Also shown are the values of the RMS, mean, mean absolute, and maximum relative differences by comparing image regions within $0.98 R$. The colour bars apply to the images below them and are different for each image.

$\sim 50 \%$. These errors reach values as high as $300 \%$ for few cases. We stress, however, that we cannot rule out that in actual historical data sets the CCs display a smaller variation than in our subset 8 . Therefore, the above test mainly shows the greater versatility of the 
present technique for handling a range of $\mathrm{CC}$ values.

Figure 3.35 shows two examples of Ko images processed with our method (left panels) and by Priyal et al. (2013) The input data employed for this comparison come from different digitizations of the same observation. This limits our analysis of the results to qualitative aspects only. Our images were saturated at the same level to illustrate all ARs clearly, however the data by Priyal et al. (2013) were provided in JPG file format and hence we are unable to saturate the images to the same level. Still, Fig. 3.35 clearly shows that images processed by Priyal et al.(2013) are affected by uncorrected inhomogeneities, to a significantly larger extent than images processed by our method.

Figure 3.36 shows the relative difference between results derived from the application of the method by Tlatov et al. (2009) and our method to one synthetic image from subset 1 produced from the contrast Rome/PSPT observation shown in Fig. 3.7 S1. The image calibrated with the method of Tlatov et al. (2009) displays a significant offset $\sim 0.7$ and fainter plage and dark regions than in the original image (contrast of $\sim 0.1$ and $\sim 0.5$ obtained by the method of Tlatov et al.2009, and ours, respectively).

The method by Ermolli et al. (2009b) cannot be tested on the synthetic data, since it relies on information of the unexposed regions of the plate that we cannot replicate in a meaningful way in the synthetic data.

Finally, we compared the CC computed with our technique and from the calibration wedges stored on Ar data.

Since our method sets the QS near the solar disc centre to be around 1, whereas the wedges describe the response of the whole plate and contain no information as to which range the QS corresponds, there is a scaling factor between the images calibrated with our method and with the wedges. This factor depends mostly on the digitization (i.e. the range of values of the QS in the digital files), but also on other factors (e.g. slightly different exposure time should change the location of the QS in the CC). Thus, a direct comparison of the CCs derived from the two methods is not straightforward.

To account for the difference in values range, we derived the $\mathrm{CC}$ from the wedges by applying a polynomial fit to the wedge measurements (Ermolli et al.2009a). We used this CC to calibrate the density CLV that was calculated with our method. We then rescaled it to match the range of values in the CLV that was calibrated with the CC derived with our method. Since information is lost with the rescaling, this approach does not allow any conclusion on the slope of the CC. Nonetheless, in this way we can test the assumption of using a linear curve to calibrate these data, provided the fitting of the wedge measurements is done accurately enough. However, this may not happen for all Ar data, because of insufficient or inaccurate information stored on the wedges. The wedges of the Ar data usually consist of 3 scans for 7 known exposures, giving 7 points in intensities to fit the sigmoid CC. These values do not necessarily cover the whole range of values on the disc, or even if they do their number may be too low to describe a sigmoid function (as was discussed in Sect. 2.6.1.1).

Figure 3.37 shows an example of a derived $\mathrm{CC}$ for one Ar observation including the rescaled wedge measurements and fit. This is one of the good cases where the CC derived with our method matches almost perfectly the one derived from the wedges, missing only a small part of underexposed regions. It is important to note that the scatter of the

\footnotetext{
${ }^{4}$ Available at http://kso.iiap.res.in/data
} 
values derived from the wedges is almost the same as the scatter in the background of the image. We achieve pixel by pixel relative differences between the image calibrated with our method and that with the wedge that are $<5 \%$, which is consistent with the results presented with the synthetic data from subset 6 (the example tested here was found to have inhomogeneities with a range within 0.6 that of the CLV).

\subsection{Conclusions}

We have developed a new method to photometrically calibrate the historical full-disc Ca II K SHGs and to correct them for various artefacts of solar and non-solar origin. The method is based on the standardization of the QS CLV intensity pattern to the one resulting from modern observations, under the assumption that it does not vary with time. Modern observations suggest that this holds within the accuracy of the proposed method. We showed that the errors introduced by the above assumptions are relatively small and have minor impact on the CLV estimation unless the analysed observation is of very poor quality. We assume that QS regions store all the information required to construct the $\mathrm{CC}$ for the range of brightnesses covered by the QS anywhere on the solar disc. This is not fulfilled for observations with strong over-exposure effects, as these introduce errors into the bright plage regions near the centre of the solar disc, that cannot be calibrated away by our method. Therefore very poor quality observations are rejected. However, they constitute a very small fraction of the available data. In addition, it can be that only part of the characteristic curve is accurately represented by the QS density values and the assumed linear relation. This would affect mostly plage regions near disc centre or fainter regions towards the limb and can limit the accuracy of our processing. However, we showed that our method results in much lower errors than different approaches presented in the literature

To test the accuracy of the proposed method, we created a large number of synthetic images emulating various problems encountered in historical observations. The maximum error of our method is $<6.5 \%$ averaged over all the degradations studied here, while the average error is $<1 \%$. These errors were derived on synthetic data including extreme cases of imposed artefacts. The maximum errors reduce to $<2 \%$ if we exclude images with the most extreme artefacts. Application of other methods for the processing of SHGs presented in the literature, returns errors that are between 3 and 300 times larger than those derived from our method.

We estimated the accuracy of processing modern Ca II K data by applying the proposed method to synthetic images unaffected by linear artefacts. The error estimates decreased by almost a factor of 2 with respect to those reported earlier, with maximum relative errors being on average $<0.6 \%$.

We have also applied the proposed method to images from seven historical SHG archives. We showed that the method allows us to process images from different archives consistently, without adjustments for observations taken with different instruments at various observatories and at times of different levels of solar activity.

It is worth noting that our method to derive the CLV can be applied with minor adjustments to full-disc solar observations taken in other spectral ranges than Ca II K. Examples are archival white-light photographic images used for identifying and measuring sunspot properties (e.g. Ravindra et al. 2013, Willis et al. 2013, Hanaoka 2013), or SHG in the 
H $\alpha$ line (e.g. Mein and Ribes 1990, Pötzi 2008, Garcia et al. 2011, Hanaoka 2013, Kontogiannis et al.2016). 
a)

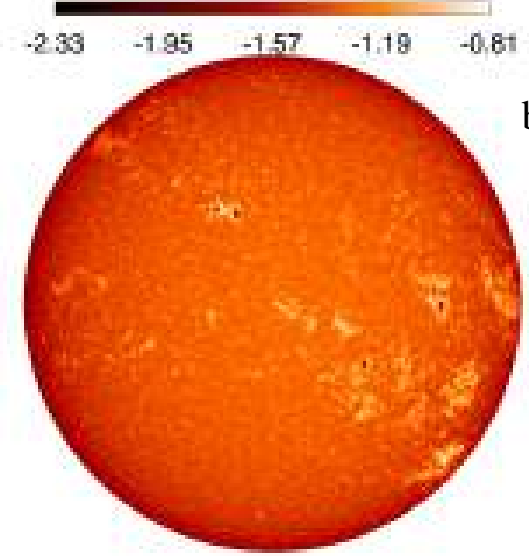

c)
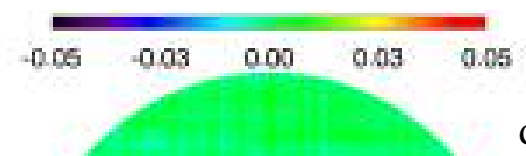

e)
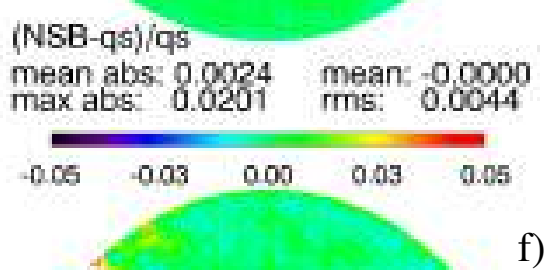

(NSB-qs)/qs

mean abs: 0.0066

max abs 0.2612 b)

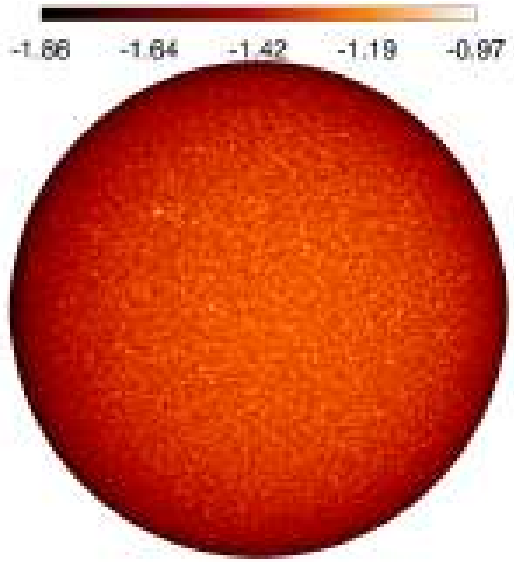

d)
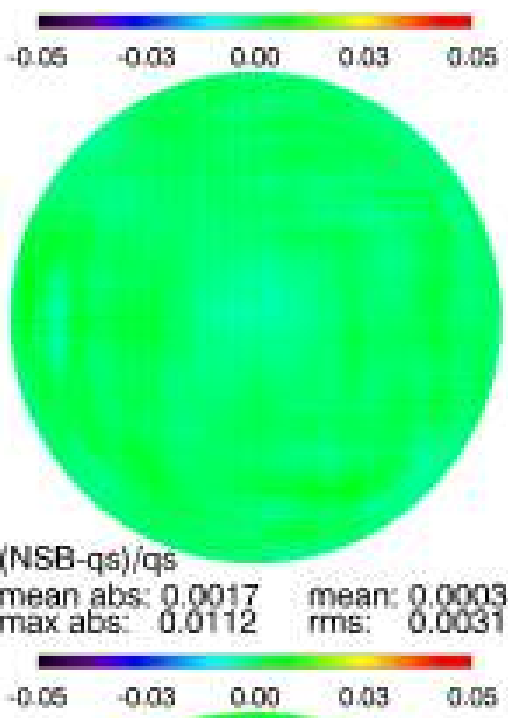

f)

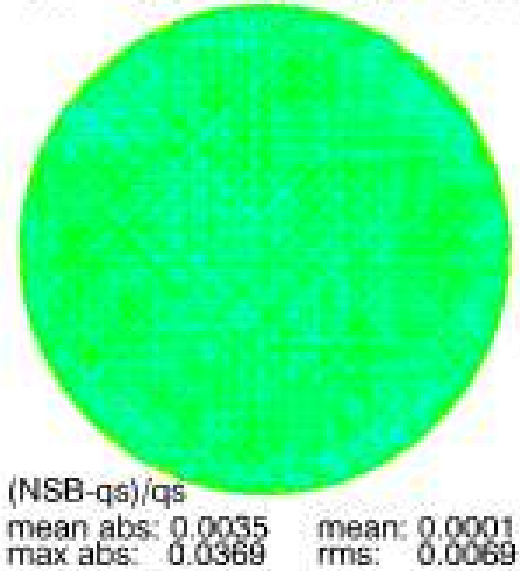

Figure 3.32: Relative error in NSB calculation with our method (c)-d)) and the method by Worden et al. (1998a, e)-f)) for two images of subset 6 with the lowest level of inhomogeneities (density images shown on $\mathbf{a})-\mathbf{b})$ ). The facular pattern was derived from the Rome/PSPT observations taken on 21/08/2000 (left) and 09/08/2008 (right). Also given (below the images) are the values of the RMS, mean, mean absolute and maximum relative differences within the disc up to $0.98 R$. 


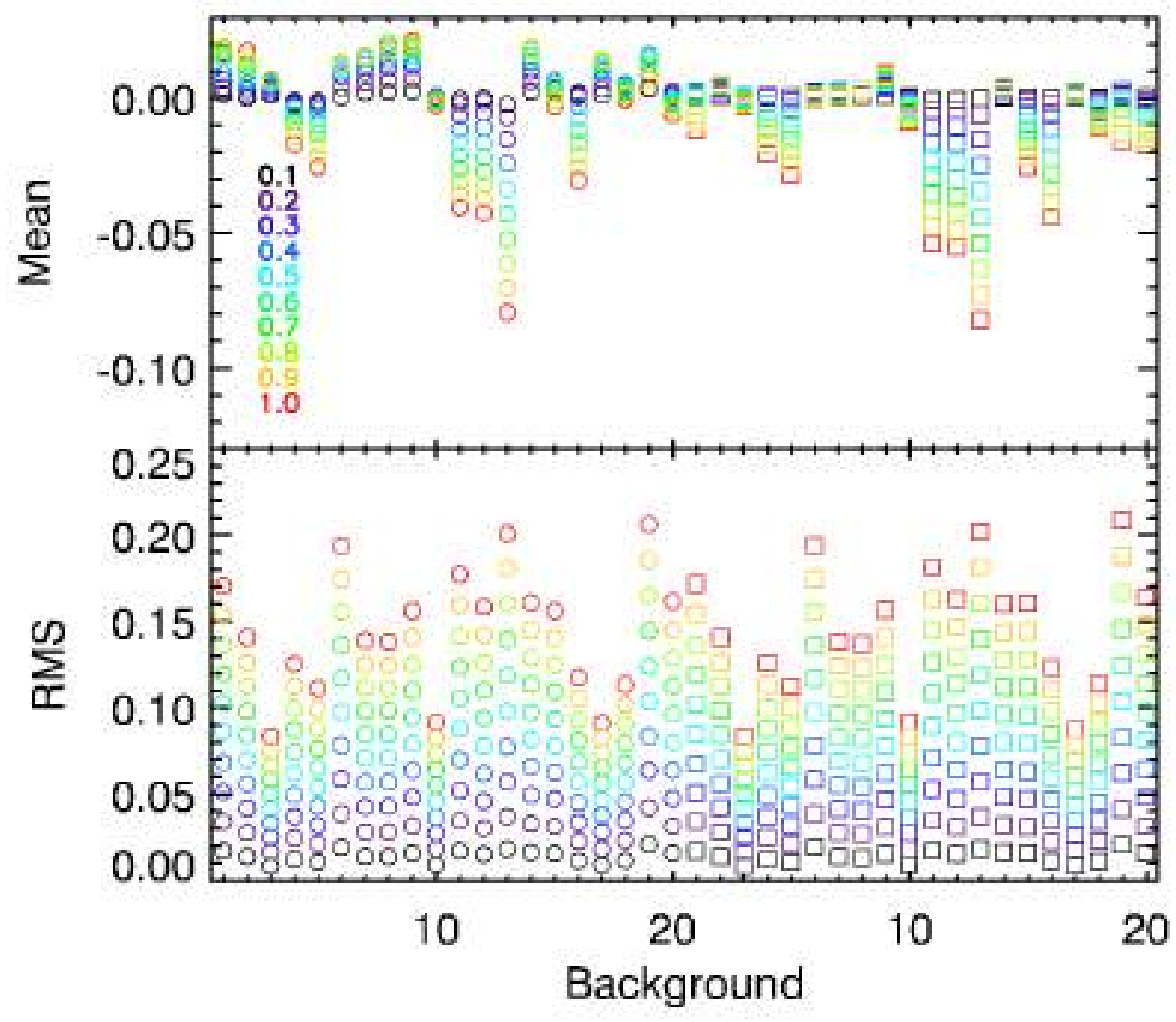

Figure 3.33: Relative difference between the NSB and the imposed background up to $0.98 R$ for the synthetic images of subset 6 as obtained by applying the method of Priyal et al. (2013). See Fig. 3.22 for results with our method. The circles (squares) correspond to results obtained from images taken at a high (low) activity period. The colours denote the different levels of the magnitude of the inhomogeneities. Top: mean relative difference, Bottom: RMS difference. 


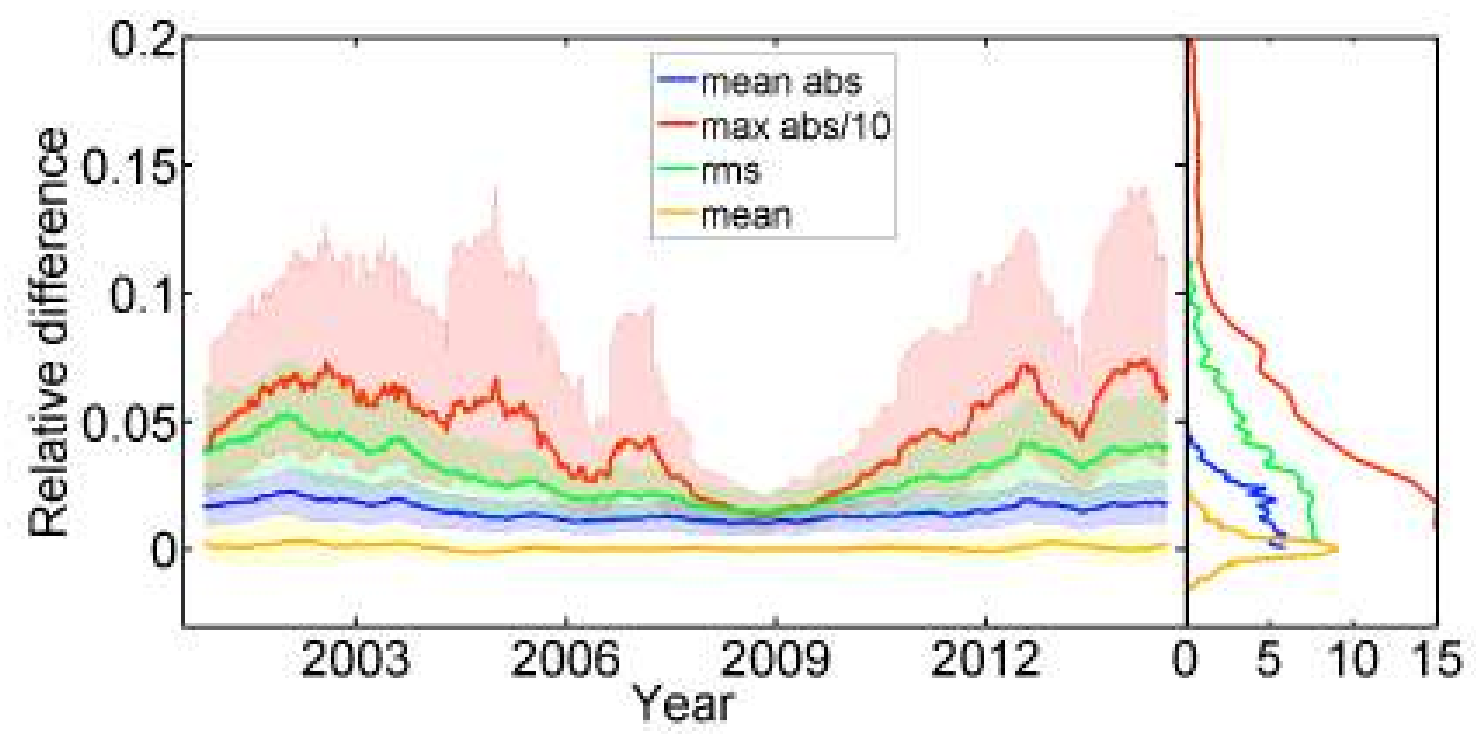

Figure 3.34: Left: Relative error for the contrast images derived from the calibration with the average CC as suggested by Priyal et al. (2013) of all the synthetic images of subset 8. Note that the red line (maximum value of the unsigned relative difference) has been divided by 10 to allow it to be plotted together with the other curves. The solid lines are 100 point averages and the shaded surfaces denote the asymmetric $1 \sigma$ interval. Right: Distribution of the relative difference values. 
a)

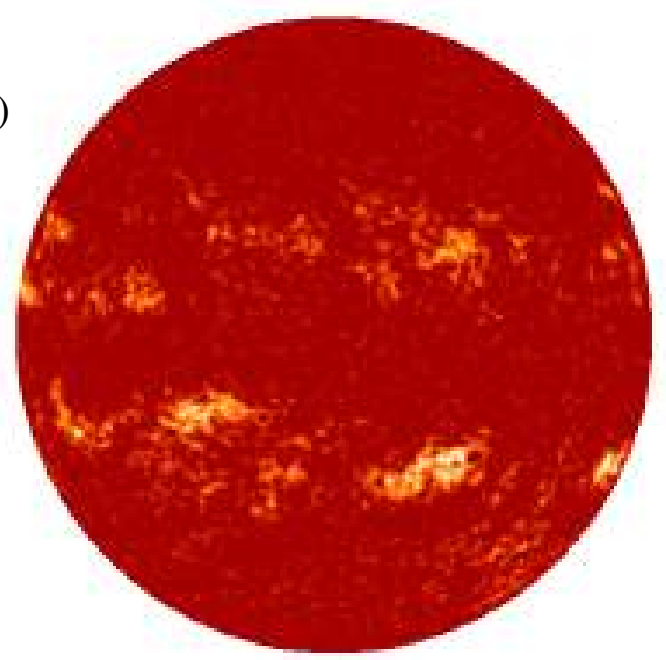

c)

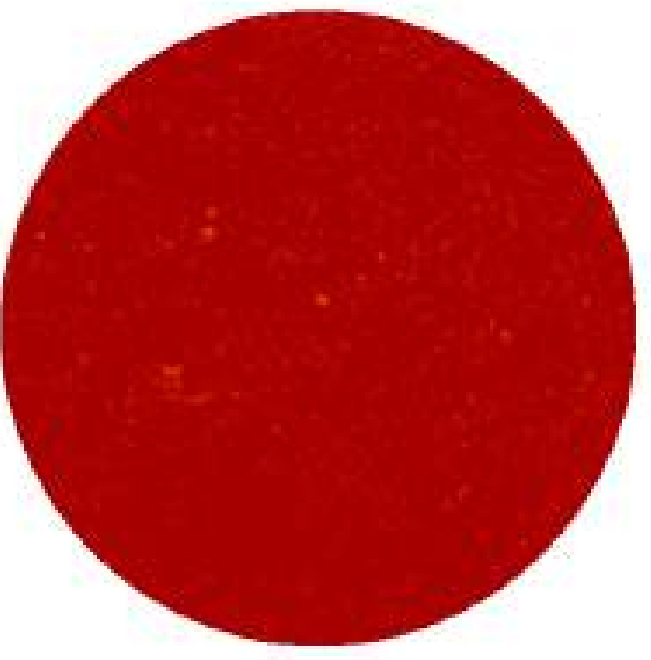

b)

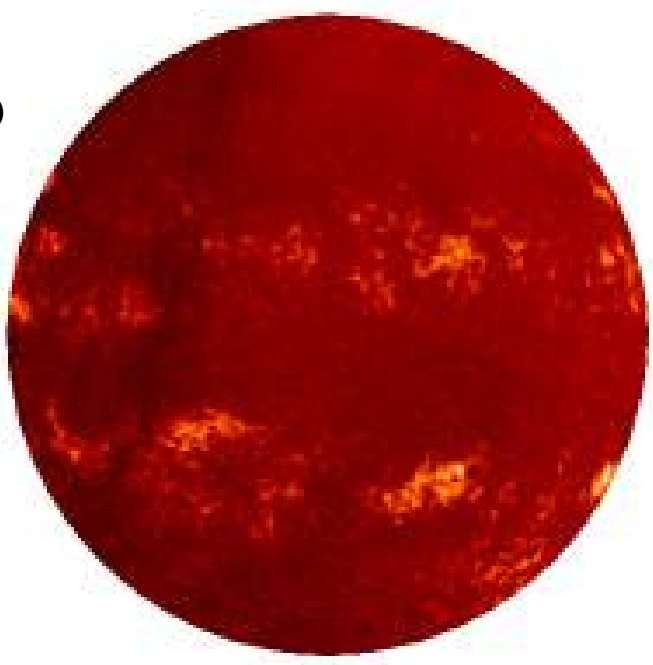

d)

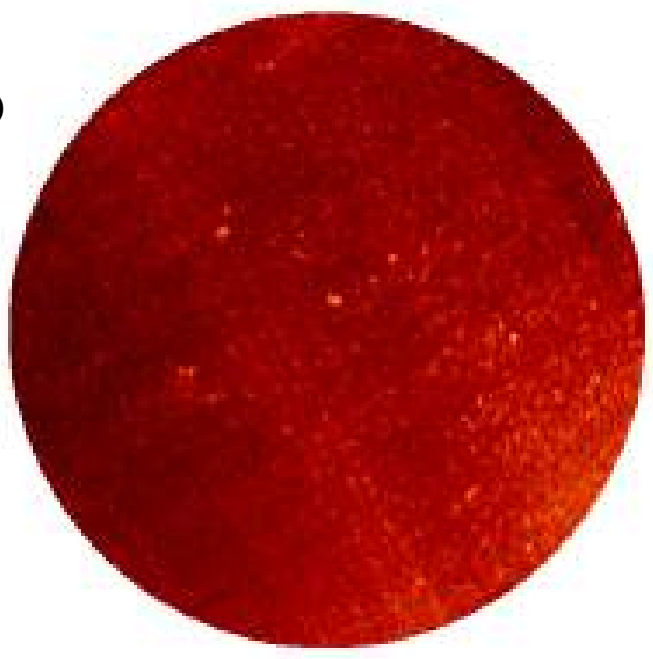

Figure 3.35: Examples of calibrated and CLV compensated images from the Ko archive derived with our method (left) and with the method of Priyal et al. (2013, right); the latter data were taken from the Kodaikanal website (http://kso.iiap.res.in/data). The results from Priyal et al. (2013, right) are given in JPG files and shown here unsaturated, while the results from our processing are saturated in the range $[-0.5,0.6]$. The observations were taken on 05/08/1947 (top) and 01/01/1964 (bottom). 


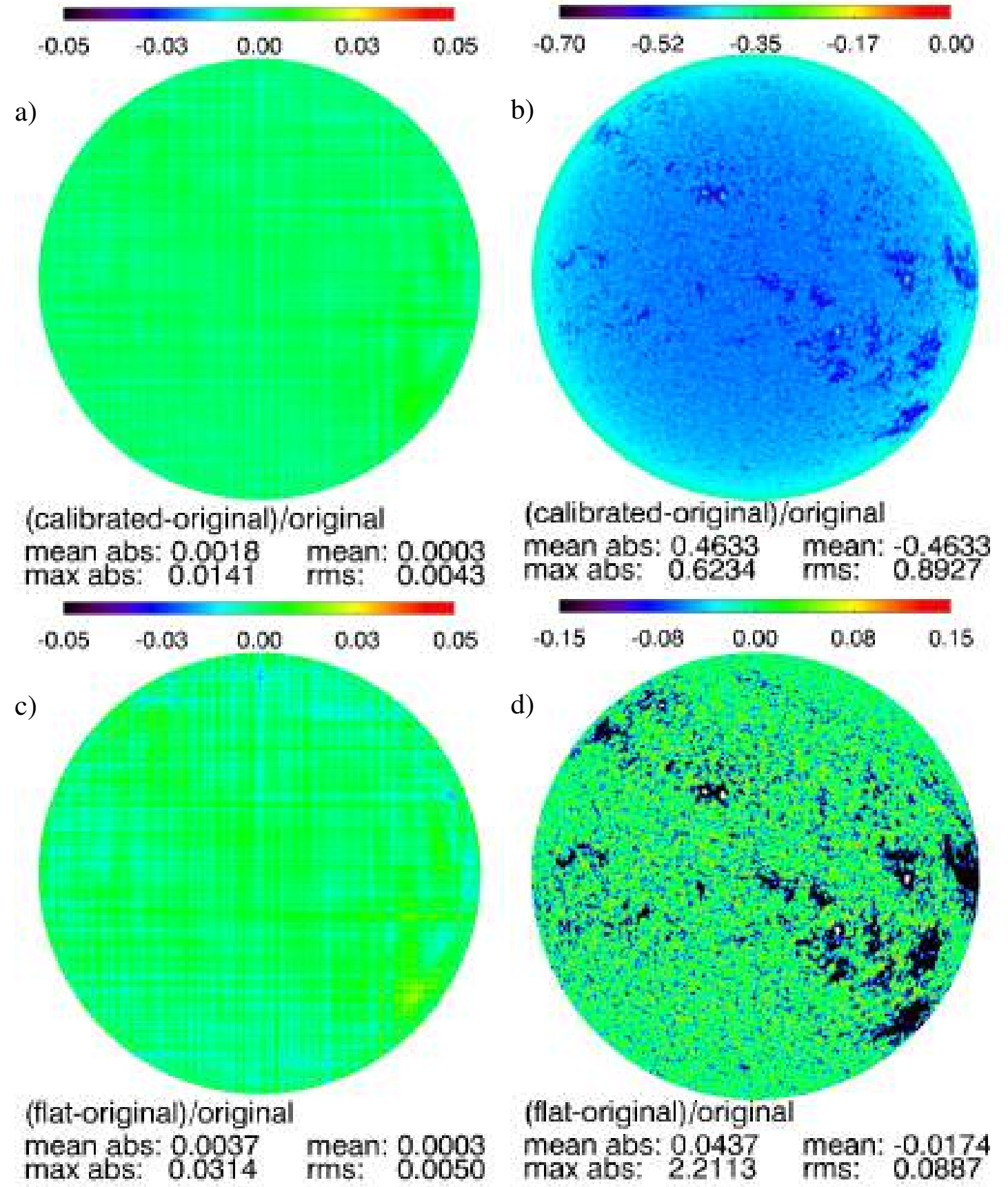

Figure 3.36: Relative error of the calibrated (top) and the contrast images (bottom) produced after linear calibration with our method (left) and the method of Tlatov et al. (2009, right) for an image of subset 1 . The facular pattern was derived from a Rome/PSPT observation taken on 21/08/2000 (shown in Fig. 3.7 S1). Also listed are the values of the RMS, mean, mean absolute and maximum relative differences by comparing image regions within $0.98 R$. The colour bars apply to the images below them and are different for each image. 


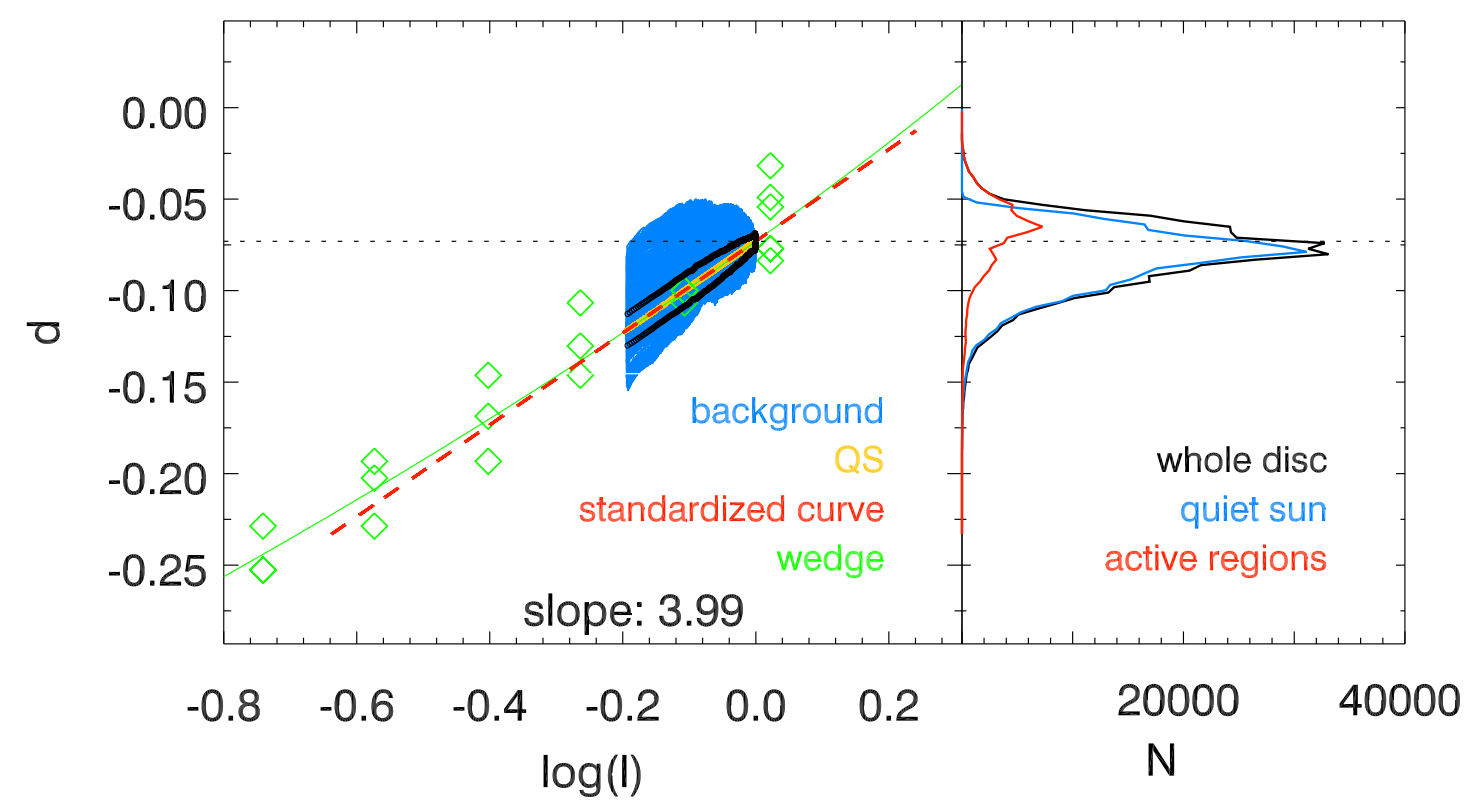

Figure 3.37: Left: Standardized CC derived from our method (red, extrapolated to the range of values of the whole disc), measured CC for the QS (orange) with $1 \sigma$ uncertainty (black), the whole background (blue), calibration wedge measurements and fit (green rhombuses and curve, respectively) of Ar observation taken on 20/07/1948. Shown also is the slope of the derived CC. Right: Distribution of densities for the QS (blue), AR (red) and whole disc (black). The horizontal dashed line in both panels denotes the highest value of the QS CLV. 


\section{Plage areas time-series from Ca II K observations}

*This chapter will be part of a journal article in preparation (Chatzistergos et al. 2017b)

\subsection{Introduction}

This chapter describes our work on deriving plage area time-series from the historical $\mathrm{Ca}$ II K spectroheliograms. We first test different segmentation methods (described in Sect. 4.2) on modern CCD-based Ca II K observations to assess their efficiency and to allow us to estimate expected errors when analysing the historical observations (Sect. 4.3). We also study the relation between the average contrast and the size of active regions (AR). Then in Sect. 4.4 we use the synthetic data (introduced in Sect. 3.3.1) to evaluate effects of segmenting historical Ca II K observations that are either uncalibrated or photometrically calibrated with the method introduced in Chapter 3. Finally in Sect. 4.5 we present preliminary results for the plage and network areas from 4 of the available historical $\mathrm{Ca}$ II $\mathrm{K}$ archives.

\subsection{Segmentation schemes}

Here we have tested the following four segmentation methods, which are the most widely used ones for studies with Ca II K observations.

1. A threshold in contrast values, $K$, that is kept constant over the solar disc (this method is hereinafter called CT for Constant Threshold. e.g. Steinegger et al. 1996, Singh et al. 2012, Priyal et al. 2013, 2014).

2. A threshold of a constant multiplicative factor, $n$, to the standard deviation of the contrast values, $\sigma$, over the whole disc calculated for each image separately (CMF for Constant Multiplicative Factor, hereafter. e.g. Foukal 1998, Chatterjee et al. 2016):

$$
K=\operatorname{median}(C)+n \sigma,
$$

where $C$ are the contrast values of the image. This method has been used in the literature to derive areas for plage, but not for network. Here we use it for the network as well in order to compare with the other methods.

3. A variant of the method presented in Nesme-Ribes et al. (1996) (NR, hereafter) as used e.g. by Ermolli et al. (2009b). This method assumes a Gaussian background 
brightness distribution, while magnetic features add a non-Gaussian contribution in the wings of the distribution: at the low and high contrast for dark spots and bright plage, respectively. We first compute the mean contrast, $\bar{C}$, and the standard deviation of the contrast, $\sigma_{C}$, over the disc. For an array of values of $k$ (typically in the range 0.1 to 3.0), we identify pixels that have contrasts within $\bar{C} \pm k \sigma$. For these locations we calculate the mean contrast and the standard deviation. The minimum of the calculated mean contrasts, $\bar{C}_{\min }$, best represents the background quiet Sun (QS) regions. The idea is that network and plage skew the distribution and cause $\bar{C}$ to be shifted to positive values, while the darker spots and pores cover a sufficiently small part of the solar surface as not to dominate over the effect of plage and network. The value of $k$ that gives the lowest mean contrast is adopted as the best representation of the QS contrast. Nesme-Ribes et al. (1996) find that the optimum threshold $K$ is the contrast that corresponds to $k$ being right after $k_{\bar{C}_{\min }}$. They consider values of $k$ between $0.3+k_{\bar{C}_{\text {min }}}$ and $0.6+k_{\bar{C}_{\text {min }}}$. However NR aimed at identifying the QS, while we aim at segmenting the image, i.e. identifying various magnetic features. For this purpose we consider a multiplicative factor, $m$, to the standard deviation within the disc and the contrast threshold used to identify plage and network is then given by the following equation:

$$
K=\bar{C}_{\min }+m \sigma_{\bar{C}_{\min }} .
$$

4. A method based on the Multiple Level Tracking (MLT hereafter) code by Bovelet and Wiehr (2001, 2007). Note that we will refer to this method as MLT hereafter, even though it is not exactly the same. Plage and network are initially identified as in CT. Then we use a second constant threshold $\left(K_{2}\right)$ for network and tag pixels that exceed this second threshold if they are adjacent to those already identified to belong to network. These regions are maintained as network regions till the end of the process. We separately identify all pixels with contrast above the second network threshold and we merge all neighbouring pixels into separate individual regions. The values within each region are separately normalized such that the maximum value within each region is 1 . Finally we apply yet another threshold $\left(K_{3}\right)$, in the normalised contrast values this time. All pixels above this third threshold are appended to the previously defined network regions.

Preminger et al. (2001) suggested the three-trigger algorithm to segment Ca II K images, but since it is similar to MLT we do not consider it separately here.

In all these schemes we applied an additional weak limit on the feature sizes of 20 pixels for the plage and 4 pixels for the network, which in the latter case often corresponds to the spatial resolution element (and even below it on days with poor seeing).

To derive plage and network areas from the $\mathrm{Ca}$ II $\mathrm{K}$ observations we create masks for each image separately. These masks have the value of unity over the selected regions of interest and the value of 0 elsewhere. For each image we can determine the fraction of the disc covered by ARs by simply getting the ratio of the number of pixels identified as ARs to that of the whole disc. We also consider feature areas corrected for projection effects and express the results as the fraction of the hemisphere area.

The independent threshold parameters in each segmentation scheme were set to identify different contrast regimes as the plage and network. The values that provide a rea- 


\begin{tabular}{lcc}
\hline & Plage & Network \\
\hline CT & $K=0.21$ & $K=0.029$ \\
CMF & $n=2.70$ & $n=0.5$ \\
NR & $m=6.73$ & $m=1.05$ \\
MLT & $K=0.21$ & $K_{1}=0.034, K_{2}=0.014, K_{3}=0.35$ \\
\hline
\end{tabular}

Table 4.1: Segmentation parameters used on the various schemes for plage and network. The parameters used in MLT for the network correspond to upper $\left(K_{1}\right)$, lower threshold $\left(K_{2}\right)$, and the one for the normalised values $\left(K_{3}\right)$.

sonably good match with the results from the Rome Solar Radiation Physical Modelling (SRPM, see Sect. 2.7) were adopted and are listed in Table 4.1. We do not identify the sunspot regions to derive their areas. The masks derived with CT, NR, MLT, and CMF count the sunspots to the QS regions. Due to some ambiguities very close to the limb, we consider only the disc within $0.99 R$, or equivalently for $\mu>0.14$.
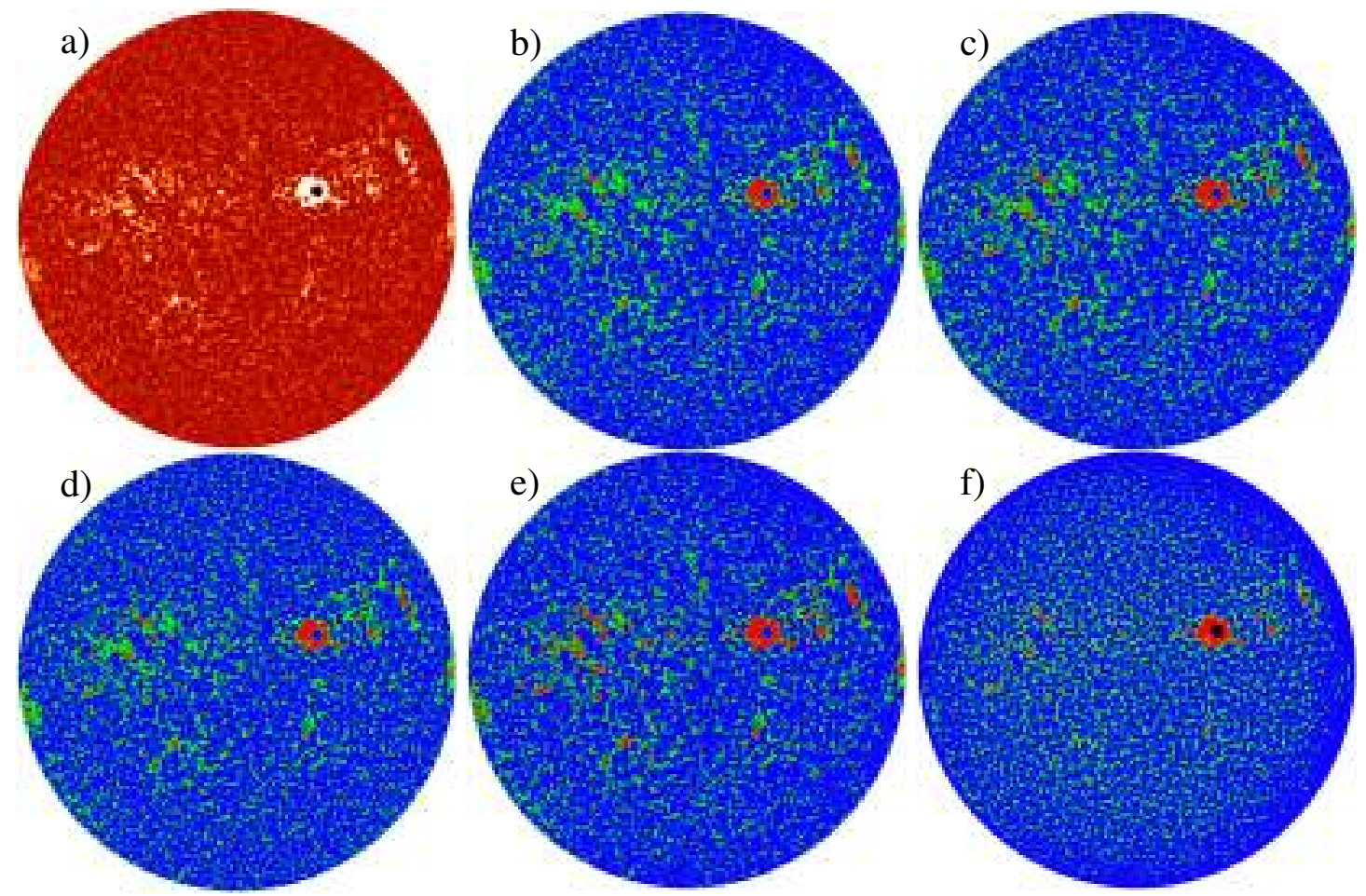

Figure 4.1: Examples of the masks for the observation taken on 18/02/2005 with Rome/PSPT (a)), produced with (b)) CT, (c)) NR, (d)) MLT, (e)) CMF, and (f)) SRPM. Red marks plage, green the network, blue the QS, and black the sunspots.

\subsection{Test of different segmentation schemes on modern data}

To assess the efficiency of different segmentation approaches and estimate expected errors when analysing the historical observations, we first apply the four segmentation ap- 

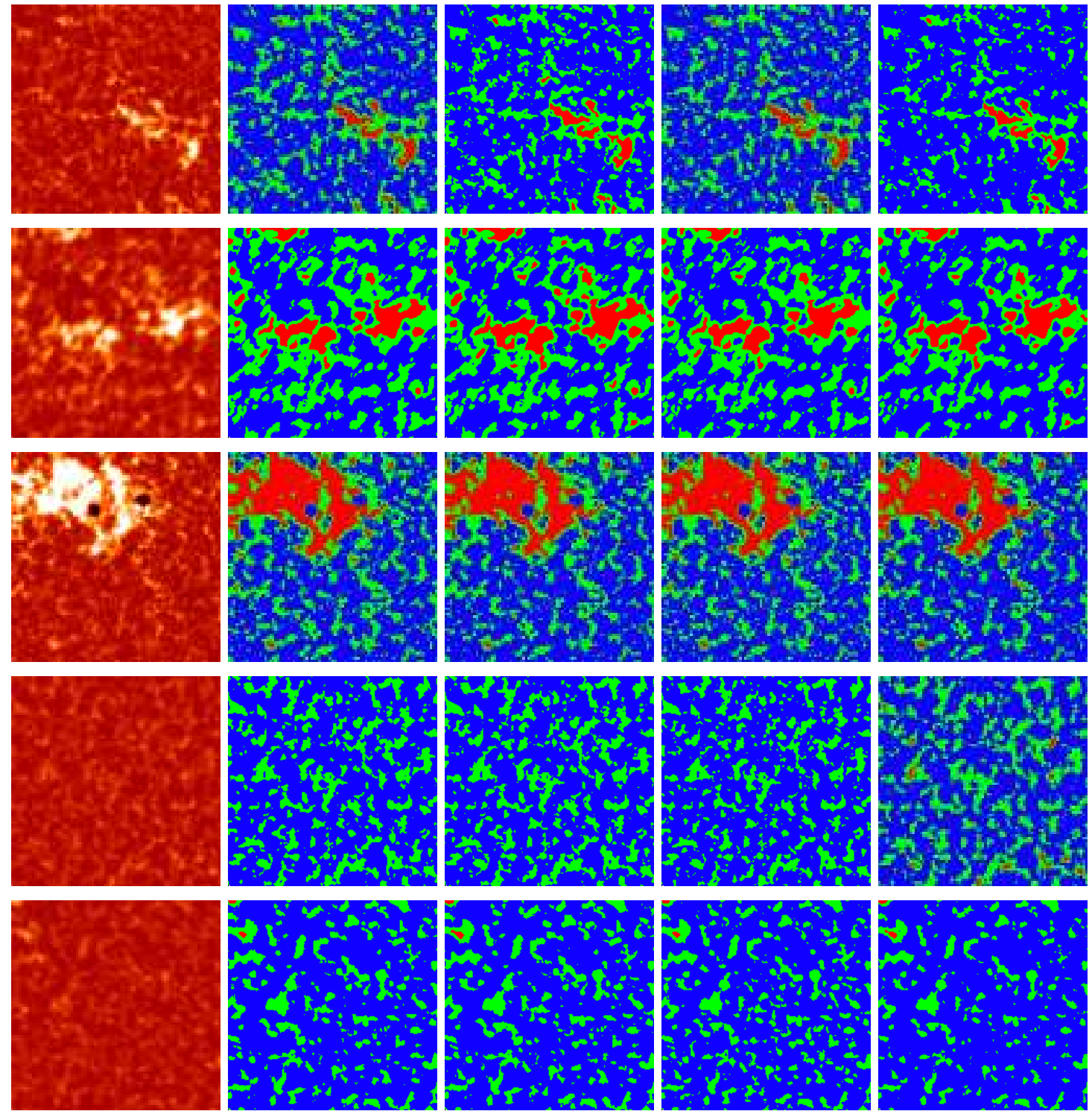

Figure 4.2: Magnified $400 \times 400 \operatorname{arcsec}^{2}$ sub-arrays at disc centre of Rome/PSPT observations (column 1) and corresponding masks derived with CT (column 2), NR (column 3), MLT (column 4), and CMF (column 5). The observations were taken on (from top to bottom) 21/08/2000, 10/02/2005, 07/06/2007, 12/11/2009, and 09/04/2011.

proaches described in Sect. 4.2 on modern CCD-based Ca II K observations taken with the Rome Precision Solar Photometric Telescope (PSPT, see Sect. 2.5). We used 2338 observations taken over the period 08/2000-04/2016 (see Table 3.1). The centre-to-limb variation (CLV) has been compensated in the same way as was described in Sect. 3.2.1, bypassing Step 2.5 since the Rome/PSPT images do not suffer from any linear artefacts (i.e. lines of a different exposure produced by problems in the movement of the instrument). 


\subsubsection{AR coverage areas}

Figure 4.1 shows examples of masks derived from a Rome/PSPT observation taken on $18 / 02 / 2005$. We show masks derived with each of the four segmentation methods tested here, as well as the SRPM mask for comparison. Different features are shown by different colour. The differences between the masks are small, and not easily discerned by eye. However, we notice that MLT identifies features seemingly covering the whole disc, while with all other methods there are small regions with lower concentration of network.

Figure 4.2 shows magnified $400 \times 400 \mathrm{arcsec}^{2}$ sub-arrays of five Rome/PSPT observations and the corresponding masks with all four segmentation methods. These sub-arrays were taken at disc centre and cover active and quiet periods. The seeing conditions are also different, for instance the image in the second row was taken under worse seeing than the rest. The masks derived with CT, NR, and MLT are very similar, with only subtle differences. The masks with CMF, however, show greater variations. The features with CMF in the fourth row are more expanded, while in the fifth row they are smaller compared to those in the masks derived with the other methods.

Time-series of plage and network areas in disc fractions derived with all aforementioned segmentation methods are plotted in Figs. 4.3 and 4.4 respectively. MLT and CT results match well those by SRPM. For the series derived from all images for the network and plage areas together, we find correlation coefficients (RMS differences) with the SRPM series of 0.97 (0.012), 0.93 (0.012), 0.68 (0.023), and -0.58 (0.06) for CT, MLT, $\mathrm{NR}$, and CMF, respectively.

We notice that CT and MLT show a very similar evolution of the disc fractions with time for both plage and network regions. Both methods give results that are in good agreement with the SRPM values. NR and CMF show similar behaviour with each other for the network component. Both show an anti-cyclic behaviour, even though the effect on the results of NR is much less pronounced and the disc fractions are essentially constant in time within the $1 \sigma$ uncertainty. For the plage component NR returns results that are in good agreement with those of SRPM, CT, and MLT, with RMS differences of the order of 0.001 . The plage component derived with CMF is roughly $1 \%$ higher during activity minima than found with any other method. Overall, CMF results appear to be strongly affected by the solar activity.

Figures 4.5 and 4.6 show the disc fractions as functions of $\mu$. The results with all segmentation methods show the same behaviour for the plage disc fractions, which are higher near disc centre and reach an almost constant value towards the limb. This is consistent with the fact that ARs preferentially emerge in middle to low latitudes. The disc fractions of network identified with all segmentation schemes decrease almost consistently with $\mu$, but seem to reach a constant value with MLT for $\mu<0.6$.

\subsubsection{Average contrast of ARs}

Figures 4.7 and 4.8 show the change of the average contrast of the plage and network features with time. The contrasts of plage and network increase with activity. However, the variation of the average contrast values with time depends on the segmentation method as well. CT and MLT show the smallest variation in contrast with time, which is on average 30\% (20\%) for network (plage). The temporal variation of contrast for either plage or network identified with CMF is $\sim 60 \%$. 


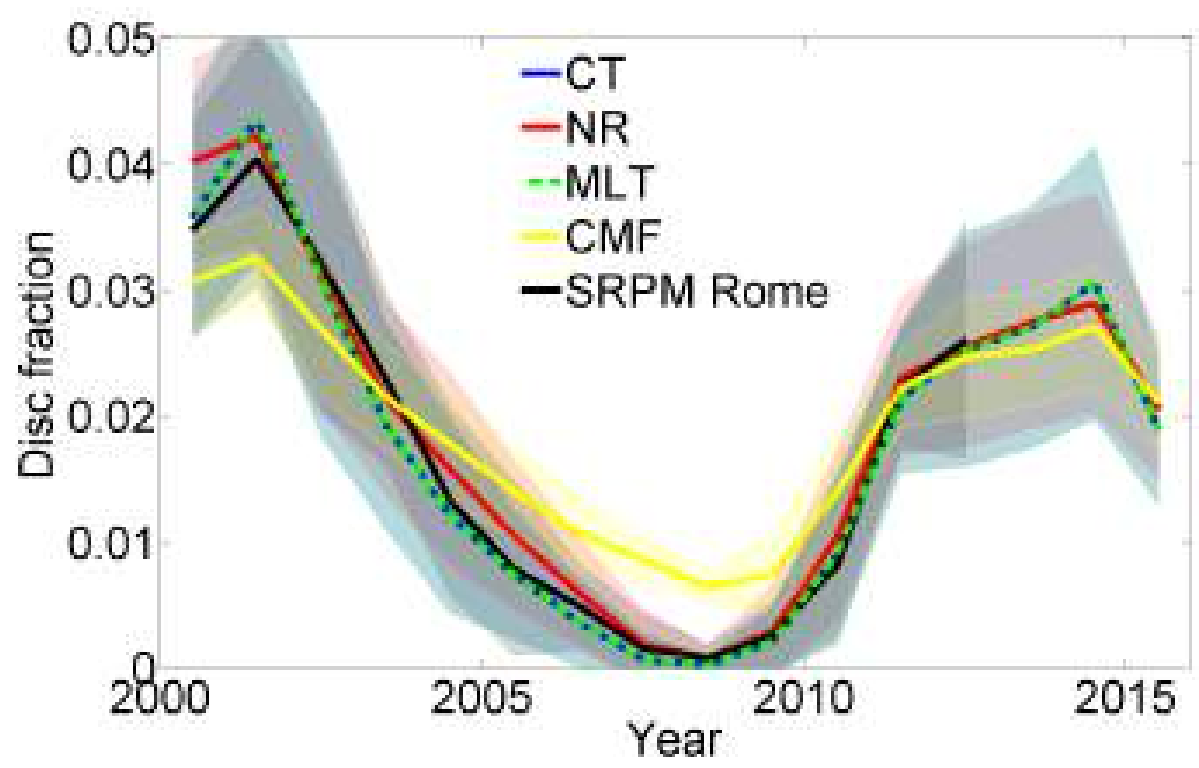

Figure 4.3: Change of the plage disc fraction over time derived from Rome/PSPT observations by applying different segmentation techniques. The curves are yearly median values: blue for the CT, red for NR, green for MLT, yellow for CMF, and black for Rome SRPM. The shaded areas are the standard deviations among all derived disc fractions within each year.

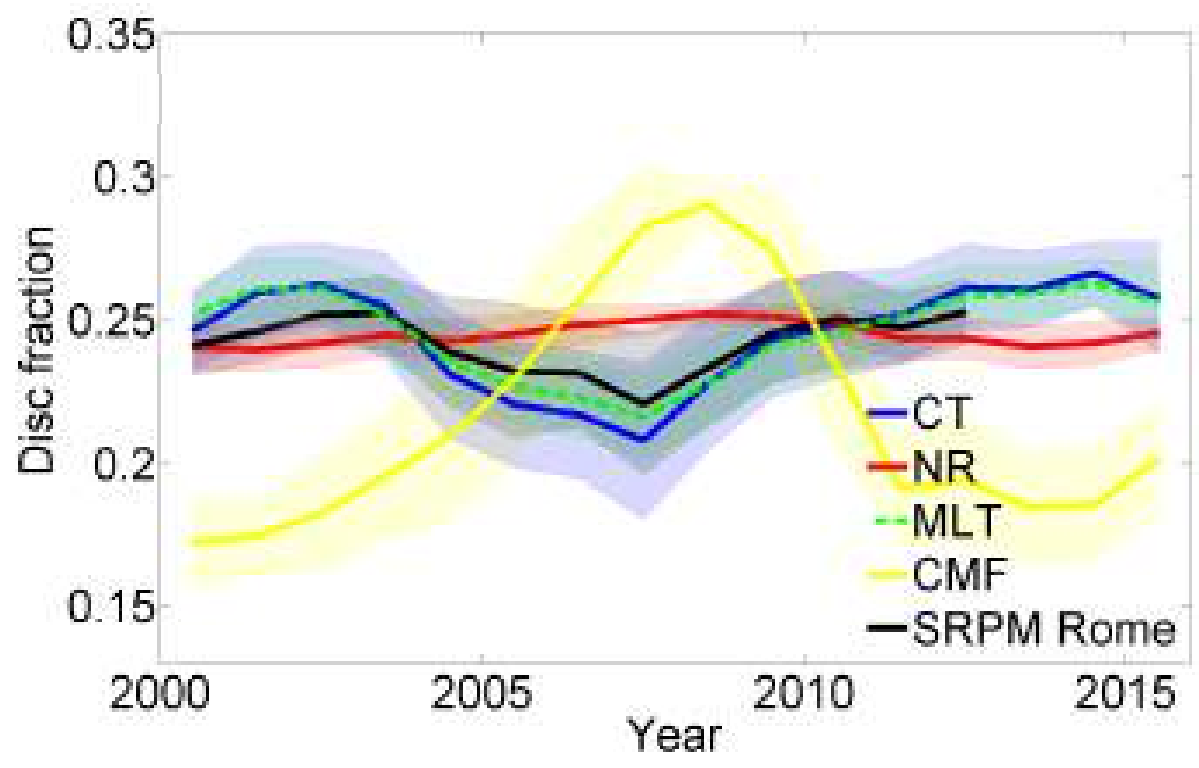

Figure 4.4: Same as Fig. 4.3 but for the network. 


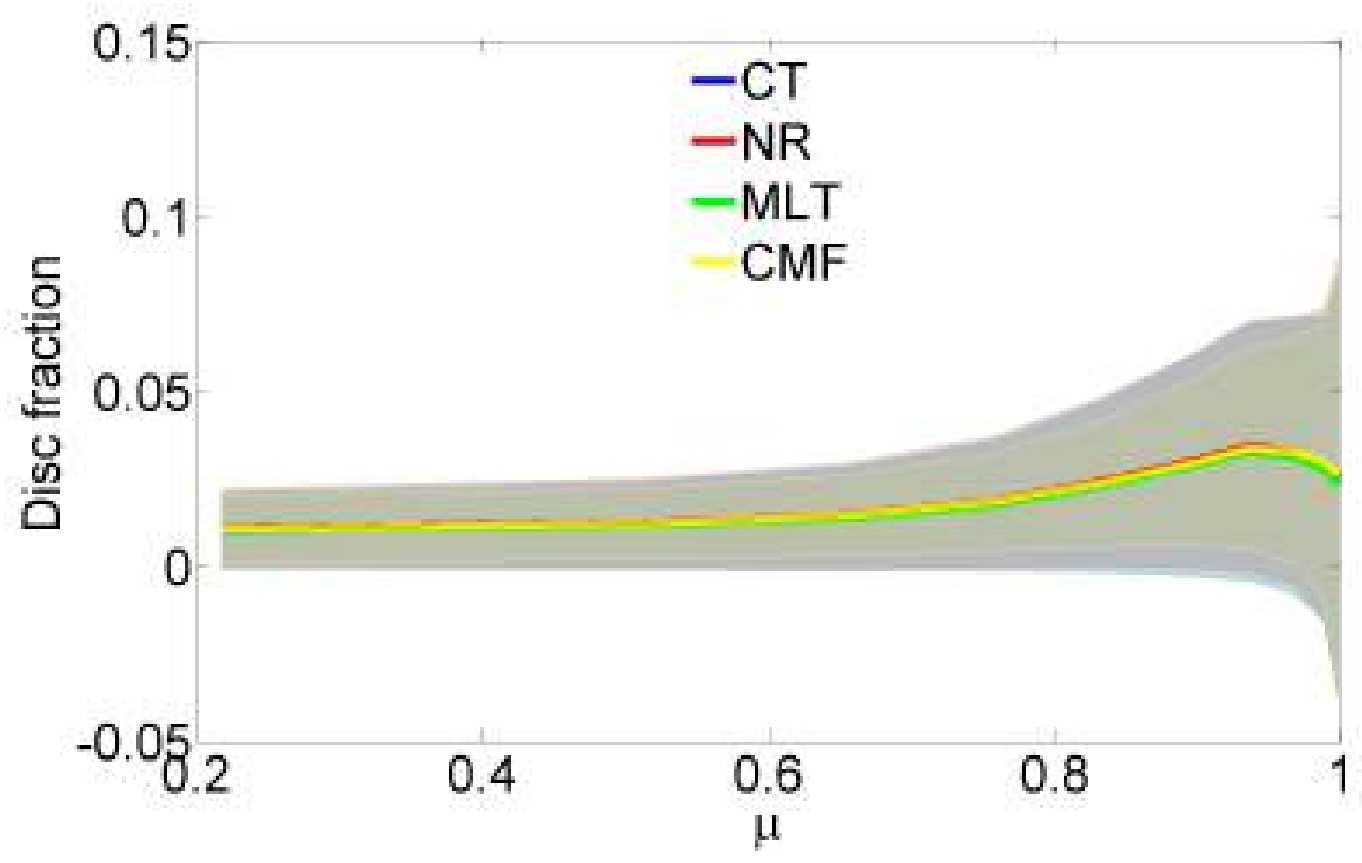

Figure 4.5: Disc fraction of plage as a function of $\mu$ as derived with CT (blue), NR (red), MLT (green), and CMF (yellow). The disc fractions were calculated within 10 equal area annuli and the solid lines are median values within each annulus, while the shaded areas are the standard deviations of the disc fractions.

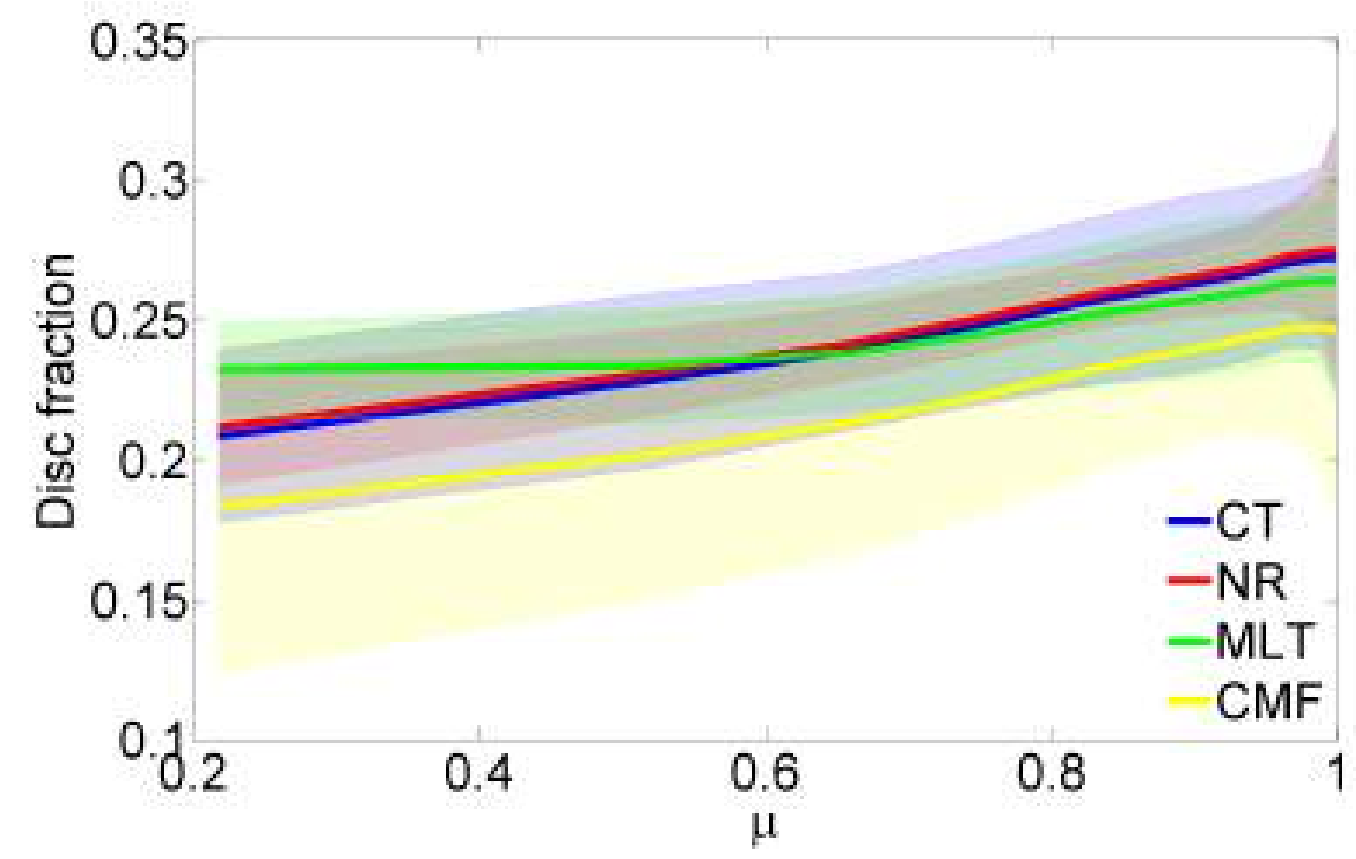

Figure 4.6: Same as Fig. 4.5 but for the network. 


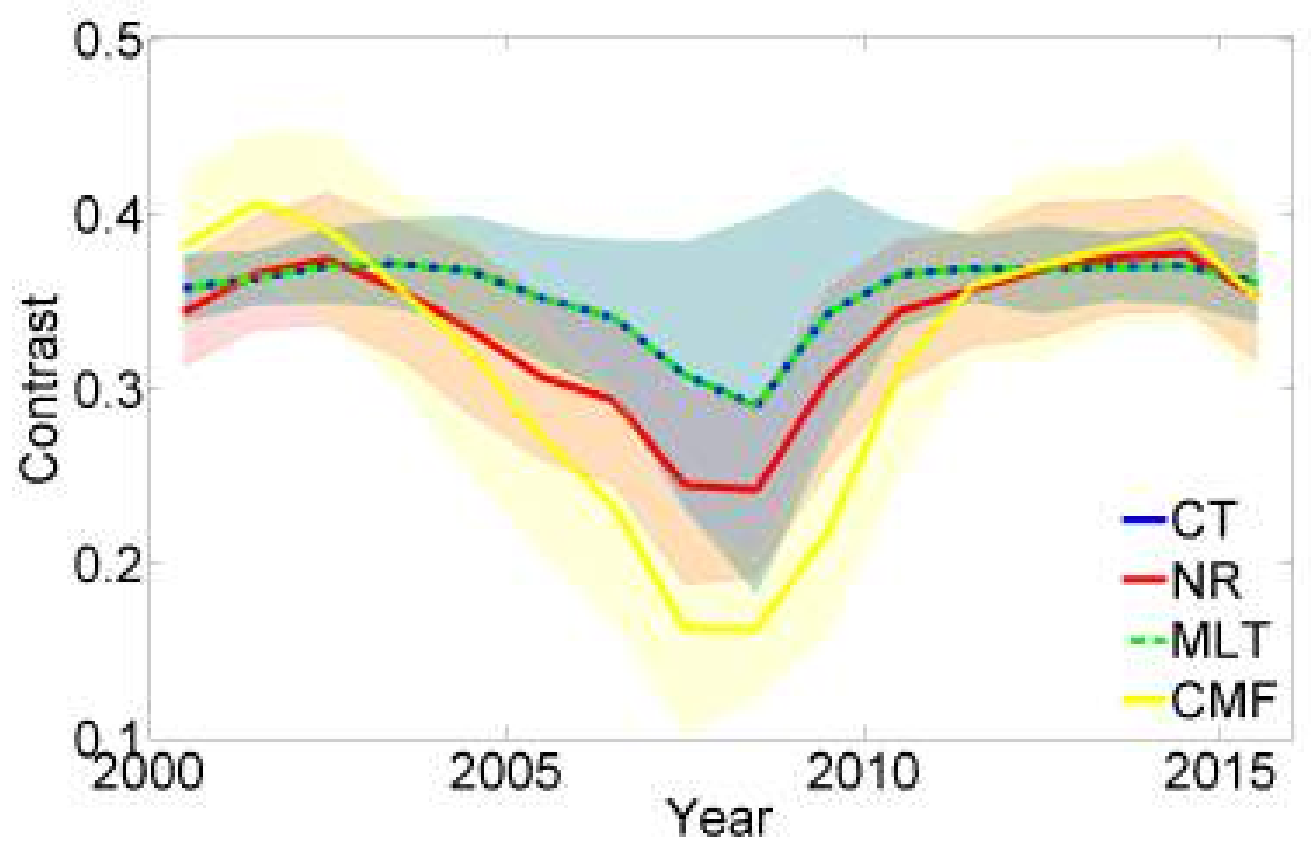

Figure 4.7: Change of the average contrast of plage over time derived from Rome/PSPT observations by applying different segmentation techniques. The curves are yearly median values: blue for the CT, red for NR, green for MLT, and yellow for CMF. The shaded areas are the standard deviations among all derived contrasts within each year.

Figures 4.9 and 4.10 show the dependence of the average contrast of plage and network on the heliocentric angle. The average contrasts of plage exhibit the same variation over $\mu$ with all tested segmentation methods, which is to slightly increase towards the limb. There are almost constant offsets in the average contrasts of plage among the different segmentation methods for the used parameters. The contrasts of network show the opposite trend and decrease with $\mu$. The average contrasts derived with MLT, CT, and NR are almost identical near disc centre, but the contrasts derived with MLT diverge towards the limb to lower values. This shows that the difference between MLT, CT, and NR are likely caused by the fact that MLT is identifying more faint network features close to the limb.

\subsubsection{AR size-contrast relation}

We now look at the average contrast of the identified features as a function of their size. All contiguous pixels identified as plage or network are categorised as separate features and we describe their size in terms of disc fraction. These dependencies are shown in Figs. 4.11 and 4.12 for $\mathrm{CT}$ and NR, respectively. The plots show a well defined relation, with small differences for the two segmentation schemes. The contrasts increase with increasing feature size, but saturate for disc fractions greater than $\sim 0.02$. The results presented here are similar to those found by Ermolli et al.(2007a). We found a generalised logistic function to best fit the data:

$$
C=p_{0}+p_{1}\left(1-e^{-p_{2} A}\right)^{1 / p_{3}},
$$




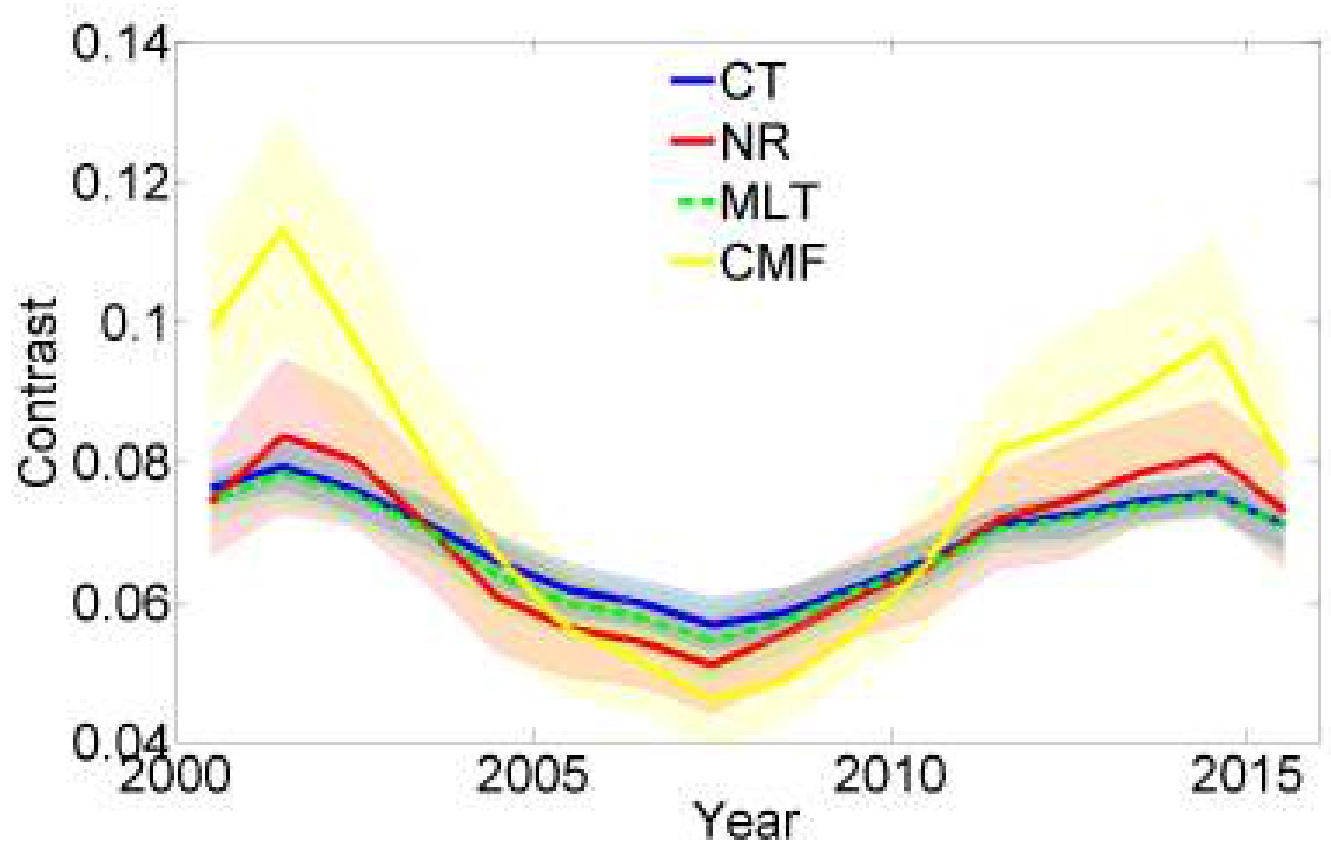

Figure 4.8: Same as Fig. 4.7 but for network.

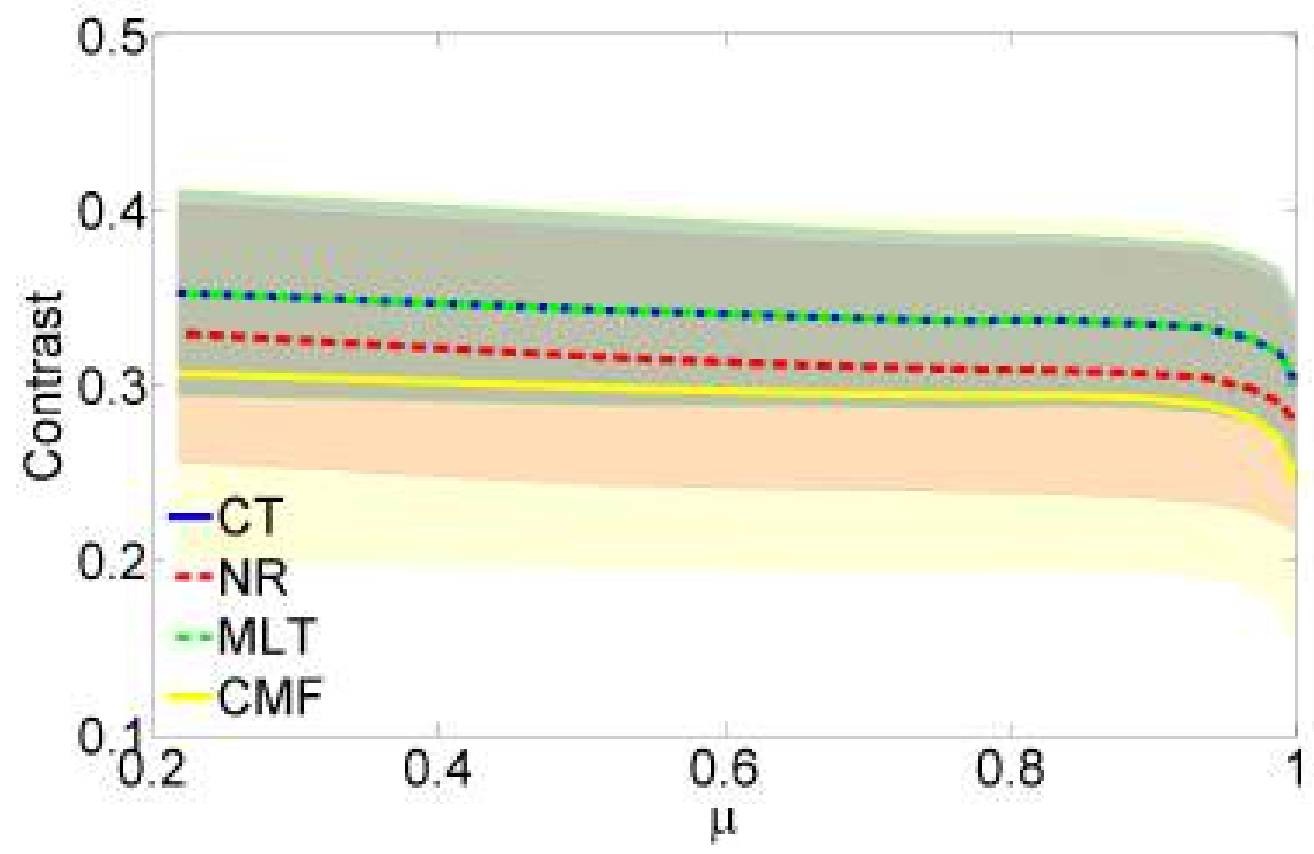

Figure 4.9: Average contrast of plage as a function of $\mu$ as derived with CT (blue), NR (red), MLT (green), and CMF (yellow). The contrasts were calculated within 10 equal area annuli and the solid lines are median values within each annulus, while the shaded areas are the standard deviations of the contrasts. 


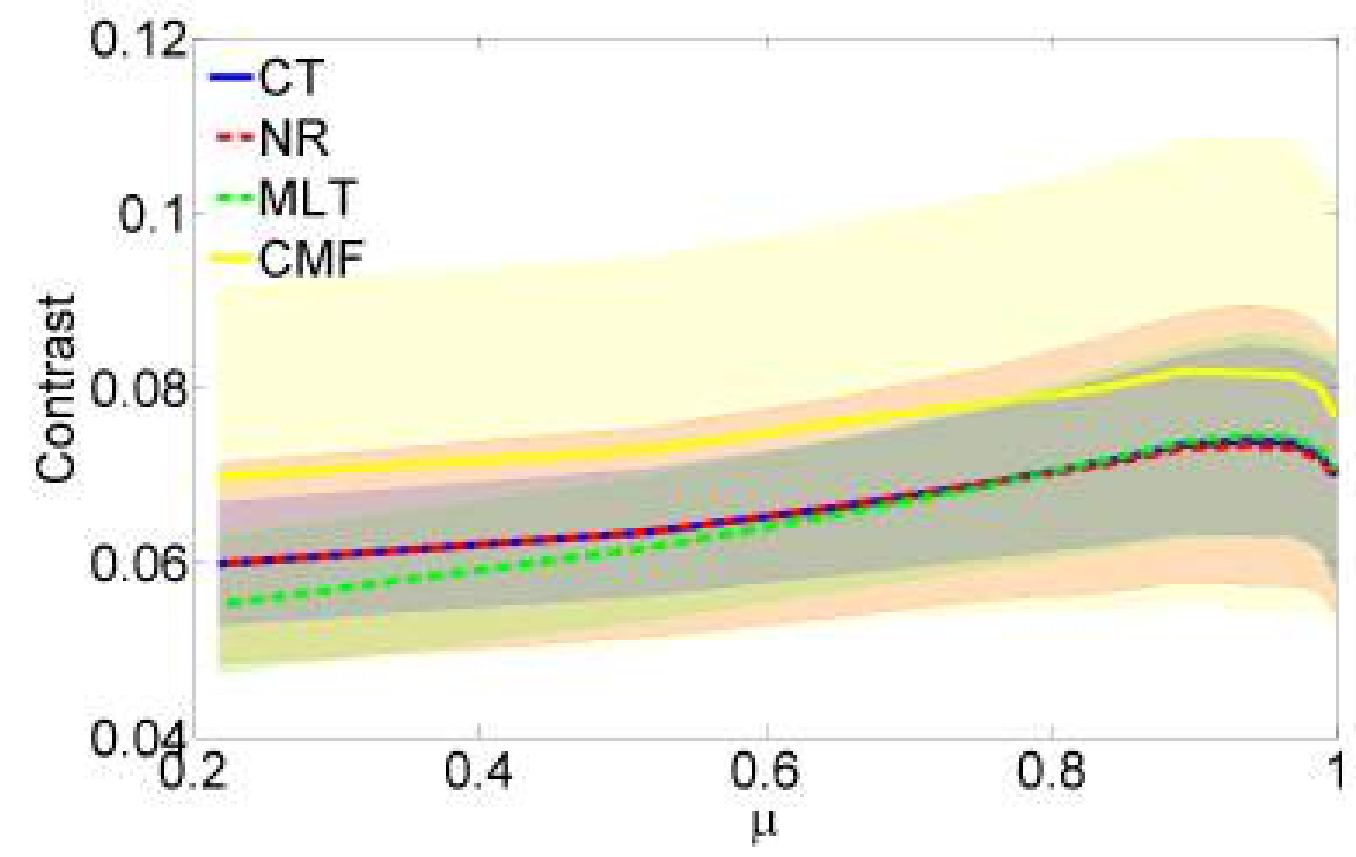

Figure 4.10: Same as Fig. 4.9 but for network.

where $A$ is the area of the features, $C$ their average contrast and $p_{0}$ to $p_{3}$ the free parameters of the fit. The parameters of the fit for the values over the whole disc are shown in Figs. 4.11 and 4.12 as well. The relation of contrast as a function of size was then derived again for features within 10 equal area annuli. The results are also shown in Figs. 4.11 and 4.12 for different $\mu$ locations. We do not find any significant variation over the disc.

Figure 4.13 compares the dependence of the average contrast of the identified features on their size as derived with CT, NR, CMF, and MLT. The curves are the averages of the results obtained from each observation over the whole disc. Compared to CT, all other methods result in saturation at higher contrasts. The curve by NR follows the one by CT very closely, while CMF is systematically higher.

Figures 4.14 and 4.15 show the dependence of the average contrast of the identified features on their size as a function of time for every Rome/PSPT image, segmented with $\mathrm{CT}$, and CMF respectively. The evolution of the size-contrast relation for NR, and MLT is similar to that for CT. We notice that fewer features with size greater than $10^{-3}$ of the disc are observed during activity minimum periods with all segmentation methods. There are also brighter features during activity maxima and the relation shows a more rapid increase in contrasts with size during activity maxima. The variations between activity minima and maxima are more pronounced in the results with CMF. There is no obvious physical reason why the brightness of magnetic features of a certain size should change over the solar cycle. Therefore Figs. 4.14 and 4.15 show the limitations of the methods. In particular, Fig. 4.15 speaks against the use of the CMF segmentation technique.

\subsubsection{Discussion}

Results shown in Figs. 4.3, 4.4, 4.7, and 4.8 indicate that segmentation methods applying a simple multiplicative factor to $\sigma$, such as our tested CMF, produce feature area val- 


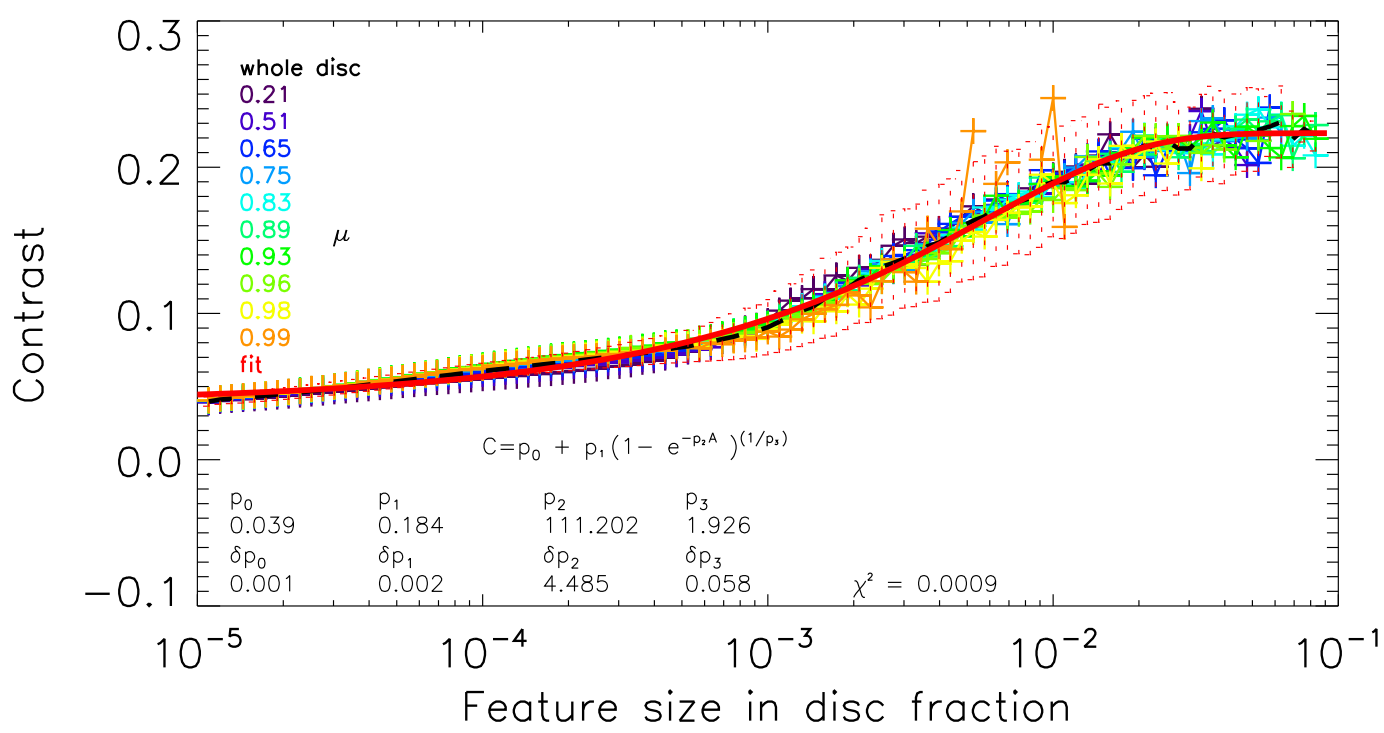

Figure 4.11: The dependence of the average contrast values of the features on their size for Rome/PSPT observations. The segmentation was done with CT. The black dashed line shows the disc averaged values, the red line is the result of the fit of a function of the form of Eq. (4.3). The error bars are the standard deviations in the measured values derived over the whole disc among all images. The coloured lines show the same relation within equal area annuli with $\mu$ shown in the legend. The function of Eq. (4.3), the parameters of fitting Eq. (4.3) to the size-contrast relation, along with their $1 \sigma$ uncertainties and the $\chi^{2}$ of the fit are provided in the lower part of the plot.

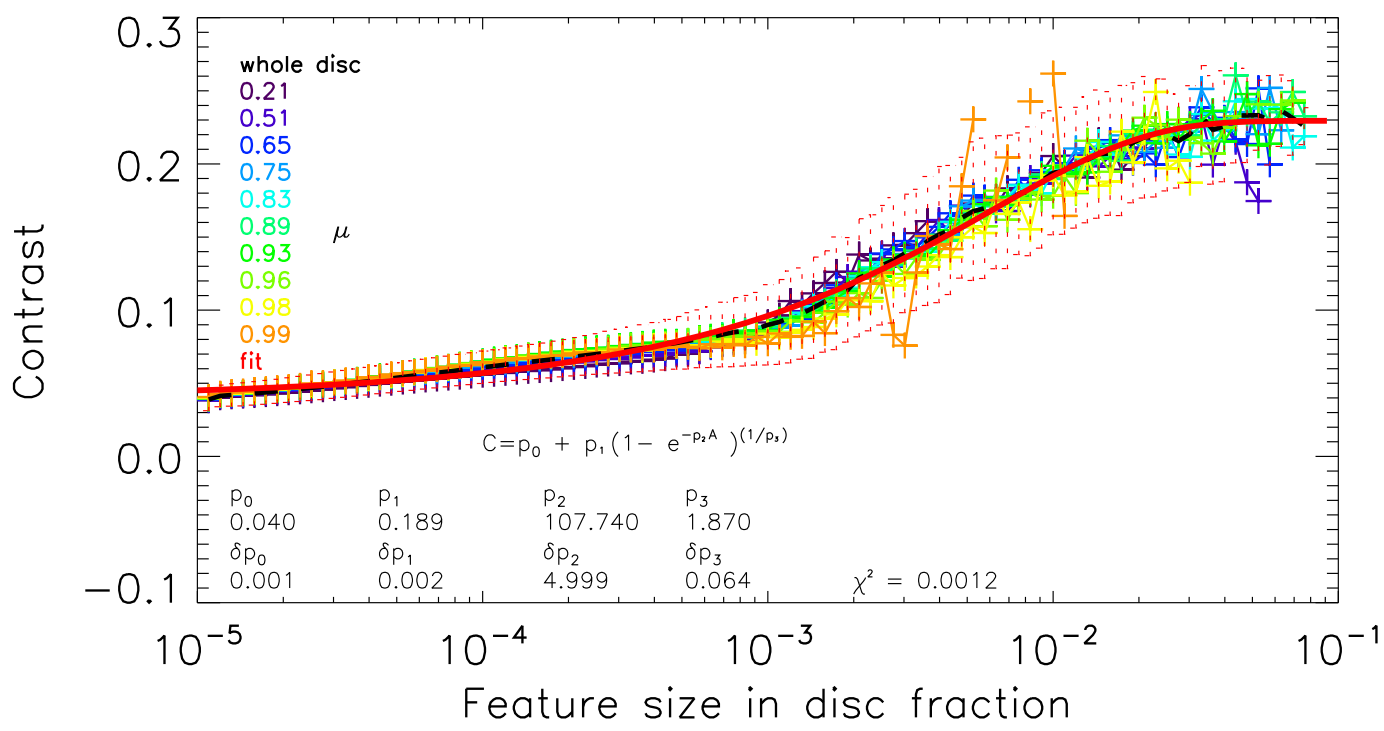

Figure 4.12: Same as Fig. 4.11 but derived with the NR method. 


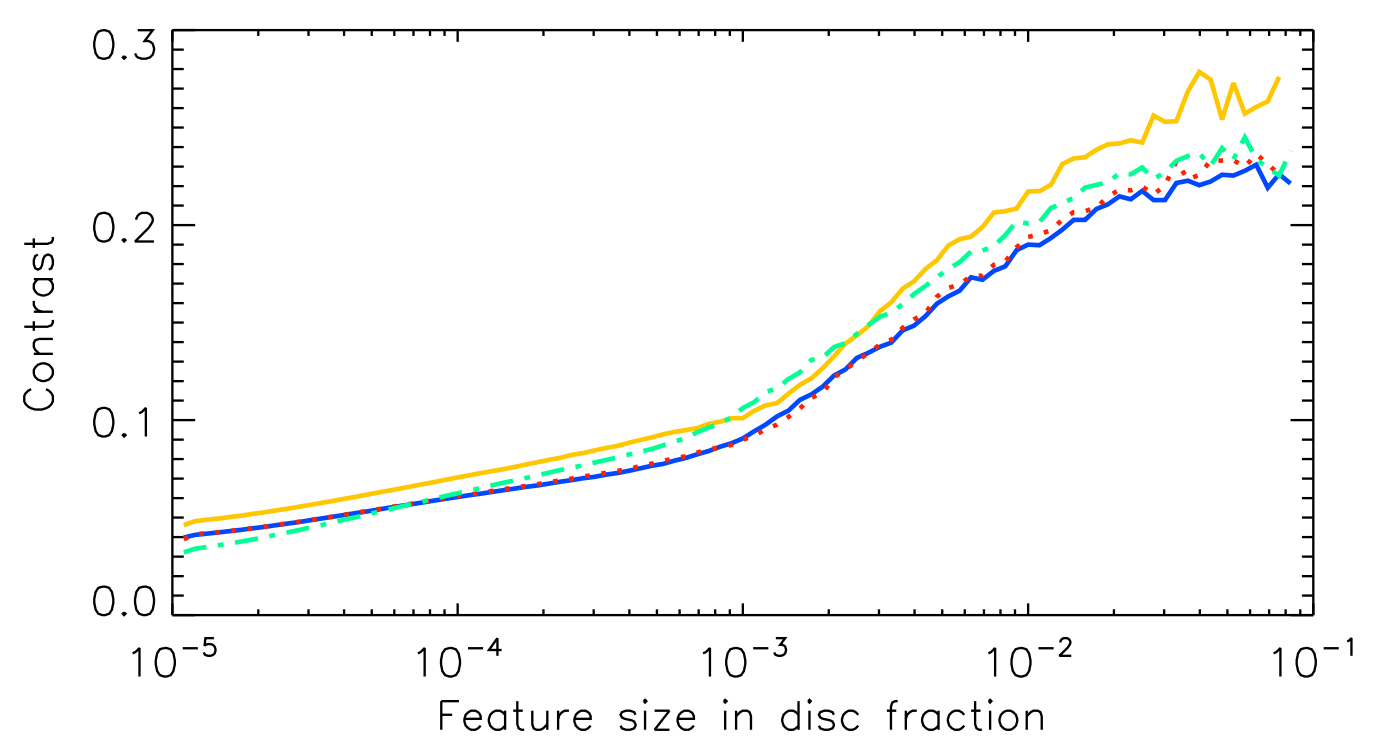

Figure 4.13: The dependence of the average contrast values of the features on their size for Rome/PSPT observations as derived with CT (blue), NR (red), MLT (green), and CMF (yellow). The curves are the averages over the whole disc among all available images.

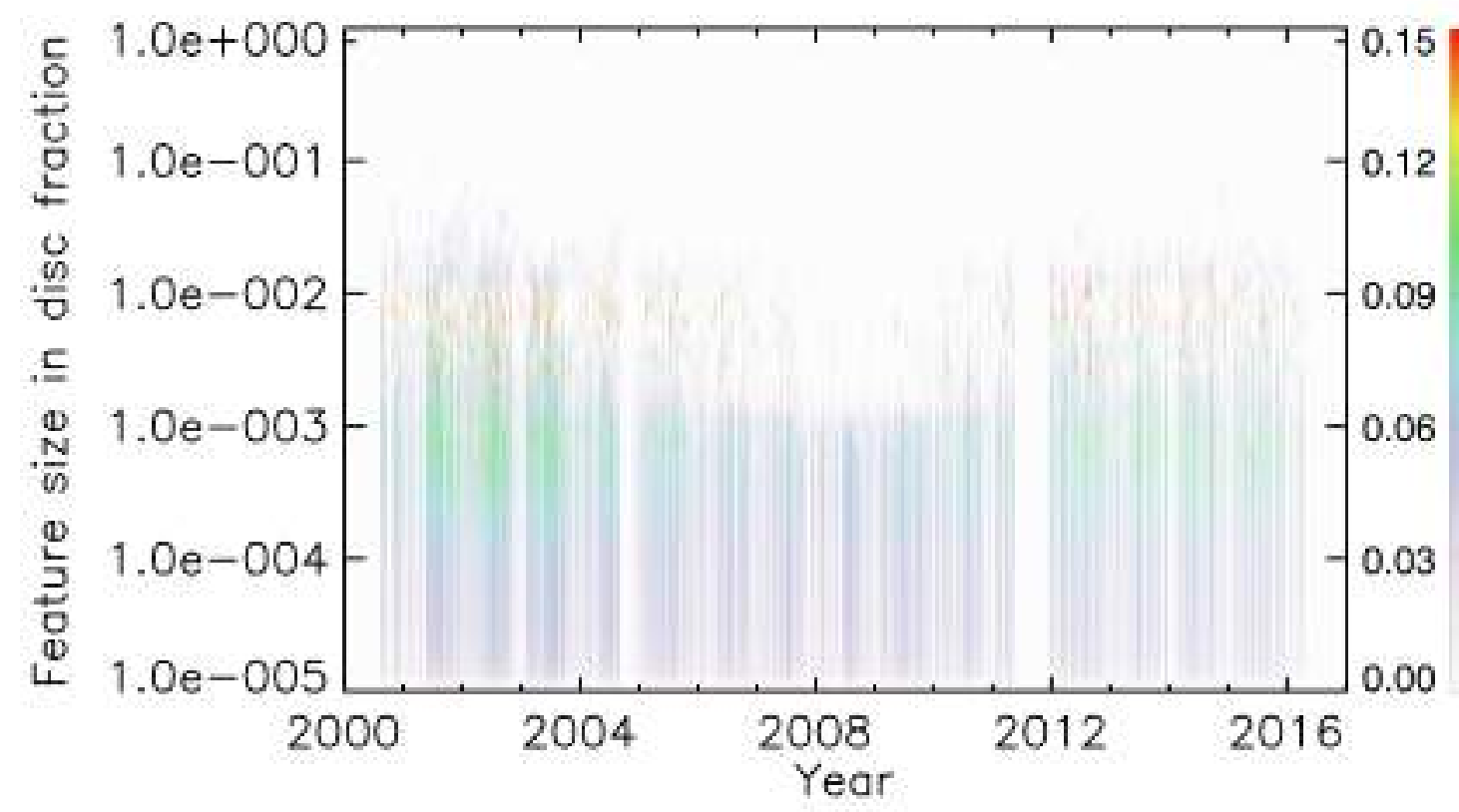

Figure 4.14: Colour-coded average contrast values of features identified in Rome/PSPT images as a function of their size (in disc fraction, $y$ axis) and time ( $x$ axis). The segmentation was done with CT. The colour bar is in units of contrast. 


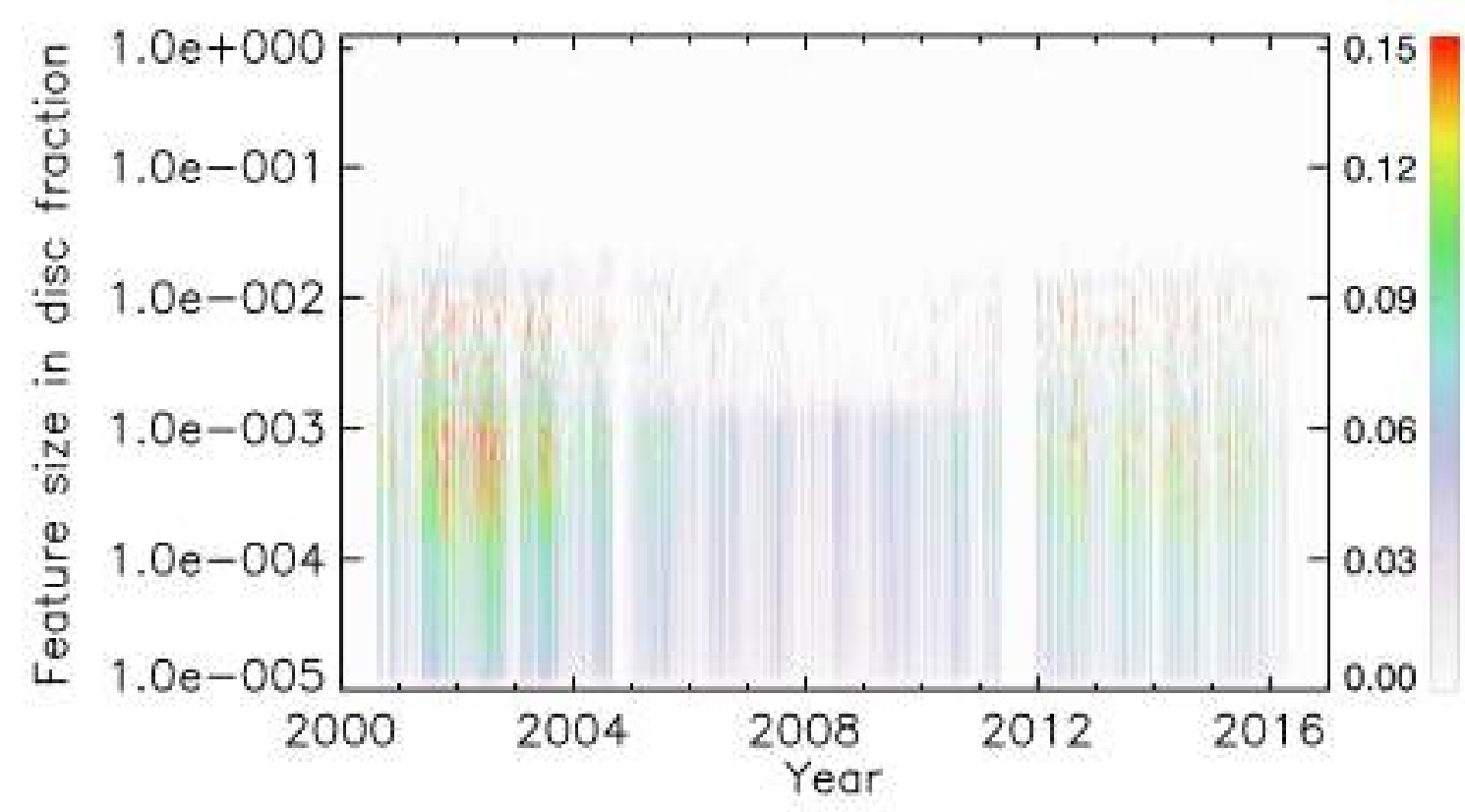

Figure 4.15: Same as Fig. 4.14 but the segmentation was done with CMF.

ues that are strongly affected by the solar activity level. In particular, CMF results in a contrast-size relationship that is strongly dependent on the activity, which casts doubt on the reliability of this method. Therefore application of these methods should be avoided.

Methods such as the tested CT, NR, and MLT produce similar results for the plage areas. For the network, MLT turns out to produce more homogeneous masks, identifying network features in regions that are too dark to be identified by all other techniques mentioned here. MLT gives disc fractions closer to those of the SRPM series.

Results with CT are found to be consistent with the SRPM values as well, however CT works only under the assumption that the image preprocessing was accurate enough and images are free of inhomogeneities and effects of solar activity.

The average contrast of the QS, $\bar{C}_{\min }$ as derived with NR, for all Rome/PSPT images analysed here is plotted in Fig. 4.16. $\bar{C}_{\text {min }}$ exhibits a negligible variation $(\sim 0.001)$ among the different images and even less so for low activity periods. This demonstrates that the processing of the images to compensate the CLV was done quite accurately. Figure 4.17 shows the standard deviation, $\sigma$, of the contrast values over the disc, as well as $\sigma_{\bar{C}_{\text {min }}}$ derived with NR. The value of $\sigma$ varies by 0.1 with the solar cycle. The value of $\sigma_{\bar{C}_{\text {min }}}$ shows a similar pattern, however the variation is one order of magnitude smaller, being merely 0.01 .

The variation of $\sigma_{\bar{C}_{\min }}$ over the solar cycle is greater than that of $\bar{C}_{\min }$ and the thresholds derived with NR are dominated by the behaviour of $\sigma_{\bar{C}_{\min }}$. Hence, this scheme is still affected by the level of activity, but the effects are one order of magnitude smaller than in CMF.

In Fig. 4.16 and 4.17 one can notice a discontinuity in $\bar{C}_{\text {min }}, \sigma_{\bar{C}_{\text {min }}}$, and $\sigma$ in September 2001. The Rome/PSPT was initially located in Monte Mario in Rome, but it was relocated to Monte Porzio Catone in September 2001. Also visible in the plots are the seasonal variations due to varying average seeing conditions. Therefore, application of NR and 


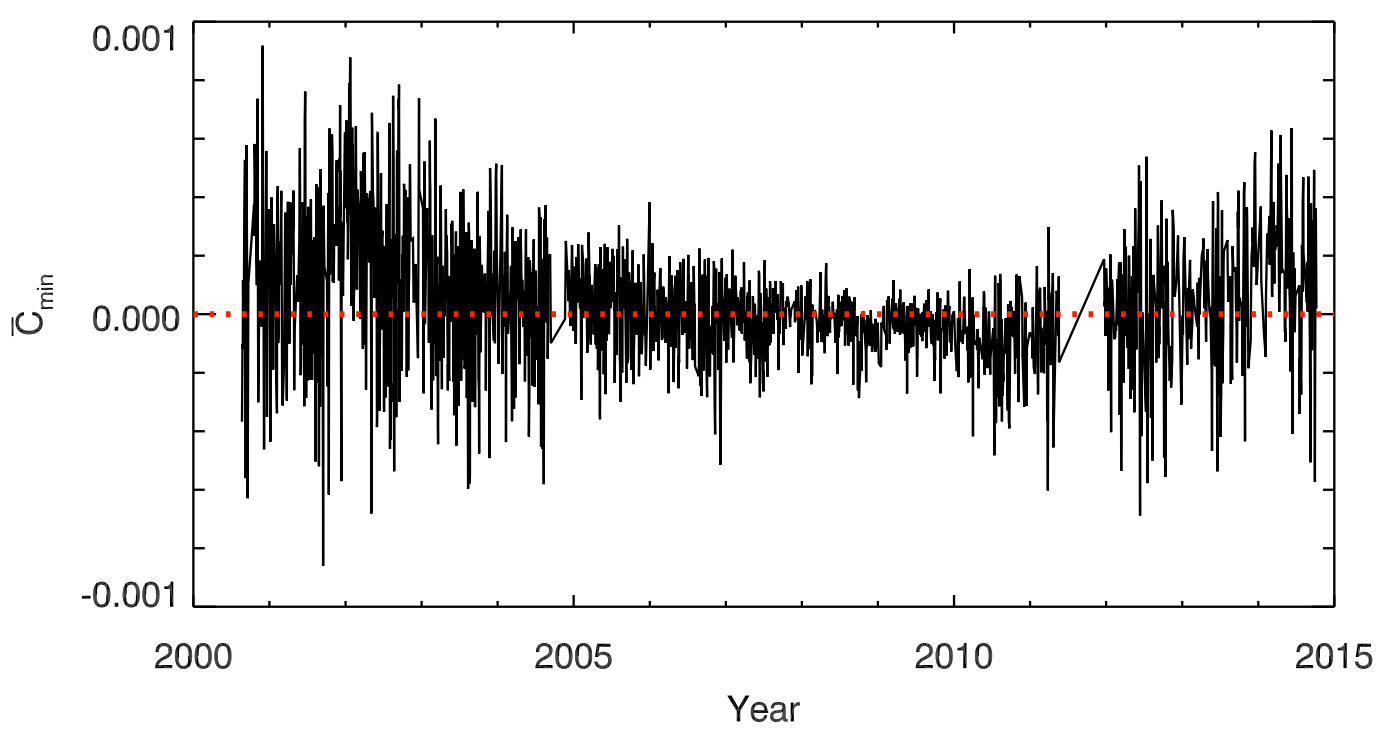

Figure 4.16: Contrast corresponding to the average QS, $\bar{C}_{\min }$, as a function of time, as derived by applying NR on Rome/PSPT observations. The red dotted line denotes $\bar{C}_{\text {min }}=$ 0 .

CMF accounts for the discontinuity in 2001 and greatly reduces the seasonal variations of image quality from the derived time-series.

For the rest of our analysis here we will use the NR method and CT for the tests in Sect. 4.4. NR is the only method, among those tested here, that is not significantly affected by the solar activity level and which can at the same time also work on data with various inhomogeneities and varying dynamic ranges. However, at this point we cannot rule out residual effects of the activity level on results derived with the NR method.

\subsection{Effect of photometric calibration on derived disc fractions}

Before applying our processing and segmentation method to historical data, we test its accuracy on synthetic data. We use subset 8 of the synthetic data, described in Sect. 3.3.1, which includes 2000 images obtained from the Rome/PSPT observations taken during the period 2000-2014 and degraded to simulate historical observations. The degrading was done by adding random amount and strength of inhomogeneities and converting them to density images with random non-linear characteristic curves (CC). Thus this sample of synthetic image represents a rather inhomogeneous dataset, emulating the historical datasets. Figure 4.18 shows the relative difference between the disc fraction of plage and network identified on the processed images of subset 8 compared to those computed on the original undegraded synthetic data. The segmentation was done with CT. The thresholds on contrast values used here were the same as those we defined on the Rome/PSPT data (see Sect. 4.2), specifically $t h_{p}=0.21$ and $t h_{n}=0.029$ for the plage and network, 


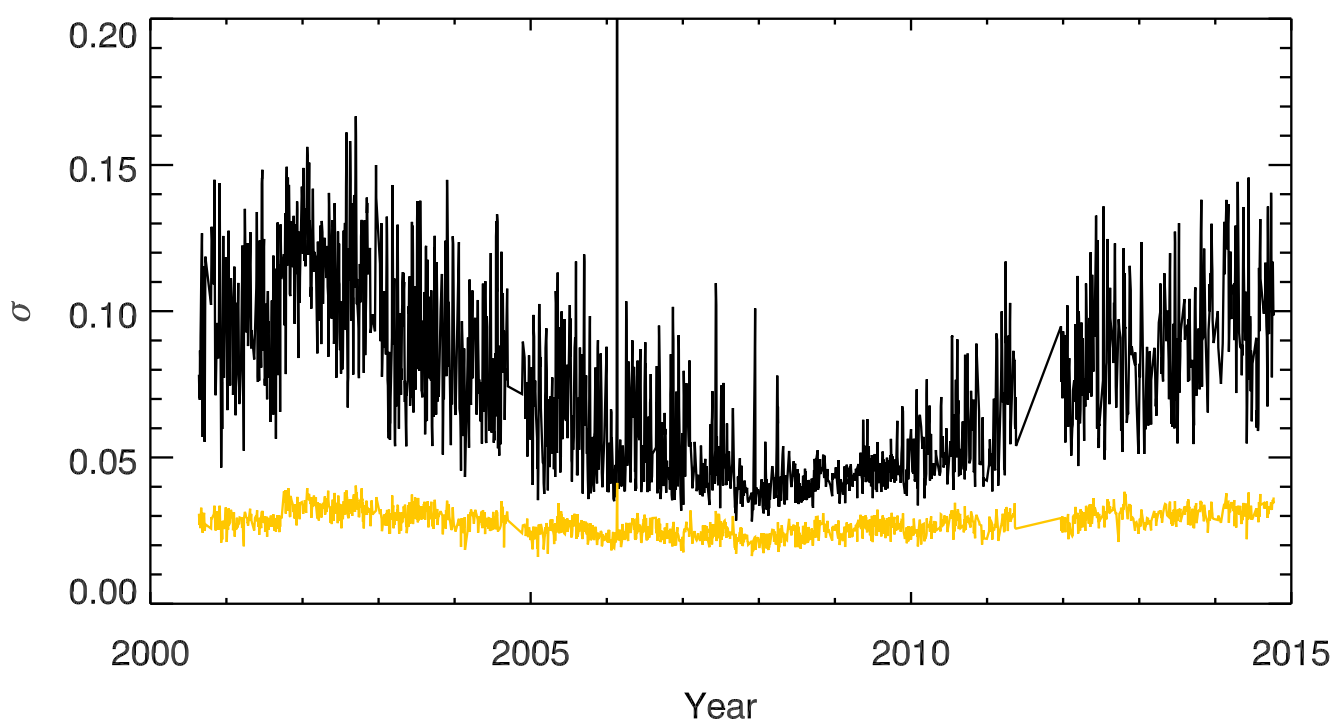

Figure 4.17: Changes of the standard deviation $\sigma$ of the contrast values of the disc with time (black), and $\sigma_{\bar{C}_{\min }}$ derived by applying NR (yellow) on Rome/PSPT observations.

respectively. Figure 4.18 demonstrates that the estimated disc fractions for the synthetic data after our processing typically lie within $2 \%$ of the values derived from the original Rome/PSPT observations that were used to create the synthetic data.

Foukal (1996, 1998), and Foukal and Milano (2001) derived plage areas from uncalibrated spectroheliograms (SHG). Since these papers do not provide adequate information about the method applied to remove the CLV, we cannot directly compare the accuracy of the processing applied to the data to our method (see Sect. 3.2.1). Therefore, we resorted to making use of the synthetic data that were created to test the accuracy of the calibration process and are described in Sect. 3.3.1. With these data we can study how accurately we can recover the AR areas if no photometric calibration was performed on the data. We use subsets 1 and 6 (described in Sect. 3.3.1), which were degraded with a linear CC. Subset 6 included various strength levels of inhomogeneities which were extracted from historical data.

Figure 4.19 and Table 4.2 illustrate the effects of calibration and CLV removal on the AR disc fractions derived for subset 1 . The segmentation was done with CT, but the parameters in each case were adjusted to match the disc fractions of the undegraded Rome/PSPT observations used to create these data. We show the difference between the disc fractions obtained for the original Rome/PSPT observations and for subset 1, which we refer to as residual disc fractions. We find the smallest variation with time of the residual disc fractions to those derived from the undegraded Rome/PSPT images for the uncalibrated data flattened, i.e. CLV compensated, with the imposed background. This might sound peculiar, but recall that the CC is a logarithmic function and has to be applied on the images that include the CLV in order to mimic the historical data. Using the imposed background is, though, an idealised situation where there are no errors in the background calculation. These results are very close to those we derive with our 


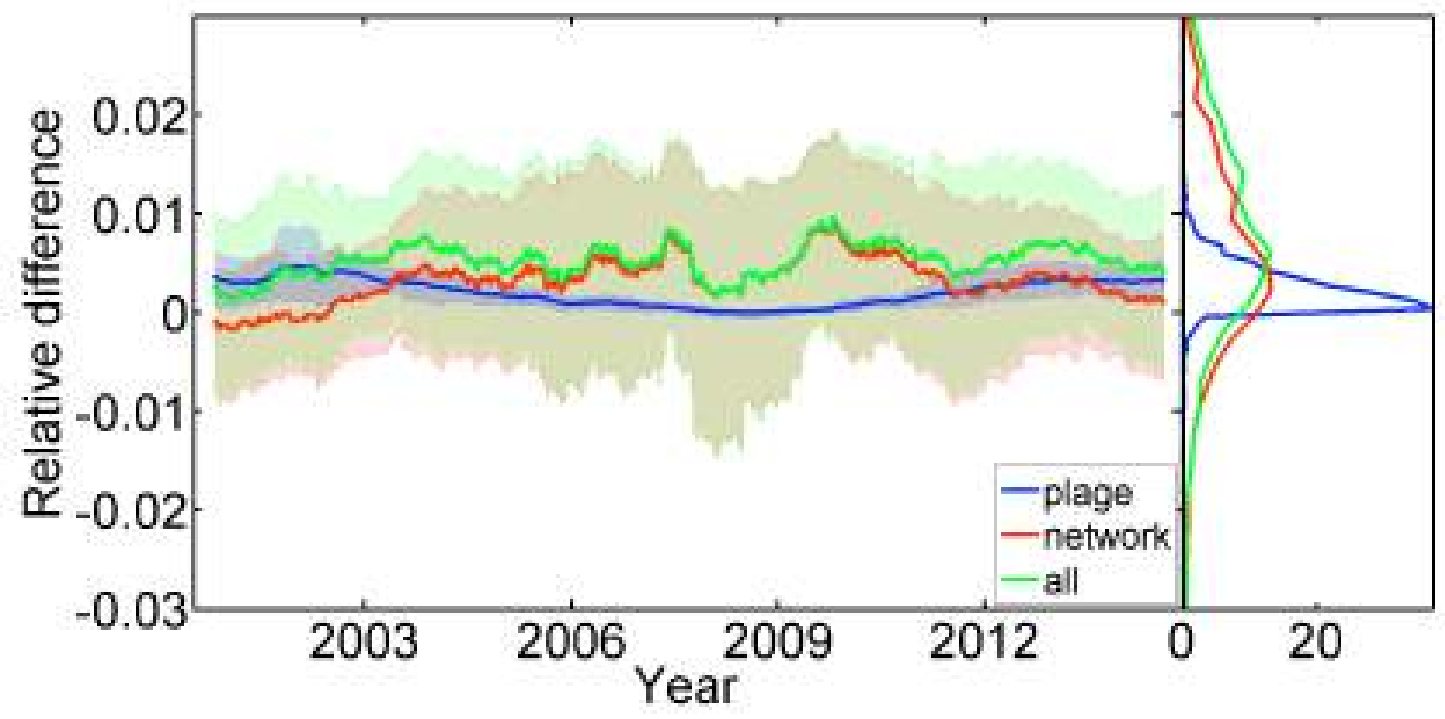

Figure 4.18: Left: Relative difference between the disc fractions of ARs obtained on the images processed with our method and on the original undegraded images of subset 8 . Disc fractions are shown separately for plage (blue), and network (red), as well as for their sum (green). The features were identified with constant thresholds. The solid lines are 100 point averages and the shaded areas show the asymmetric $1 \sigma$ intervals. Right: Distribution of the relative difference values.

calibration method (keep in mind that a linear relation was initially imposed on these data, these results might be different if the imposed relation was non-linear). The variations in time increase if we use uncalibrated data where the CLV was removed with our method. The differences become significant if we use uncalibrated data that were compensated for the CLV with other methods, e.g. the one by Worden et al. (1998a). This illustrates the importance of an accurate computation of the CLV. For instance, if the CLV was removed with the method of Worden et al. (1998a), we notice variations in the residual disc fractions that are in phase with the solar cycle, something we do not observe in the other cases. This confirms that the method of CLV removal by Worden et al. (1998a) is affected by the activity level and returns erroneous results for the AR areas.

In order to test effects of various image inhomogeneities on the segmentation results, we repeated this analysis for the data of subset 6 . The segmentation now is done with NR (described in Sect. 4.2). In fact, the presence of inhomogeneities affect the dynamic range of the images rendering the datasets rather inhomogeneous. A segmentation with a CT cannot produce consistent results on these data. We compare the results obtained from the reconstructed data, with the disc fractions derived on the original Rome/PSPT images with the same segmentation scheme. Figure 4.20 shows the relative errors in the calculation of the disc fractions from the data of subset 6 for all features if they are derived from data that were linearly calibrated with our method (red squares and dashed line for the lowest level of inhomogeneities), uncalibrated data that were flattened with our method (light blue circles) and uncalibrated data that were flattened with the imposed background (green circles and dashed line for the lowest level of inhomogeneities). For low to medium 
Table 4.2: Differences in disc fractions derived for subset 1. The first column denotes if the data were calibrated with our method or were uncalibrated. The second column gives information about the method used to compensate for the CLV. Then are given the maximum absolute, mean absolute, and RMS differences between the disc fractions calculated in each case and those from the original Rome/PSPT images.

\begin{tabular}{lcccc}
\hline \hline Calibration & CLV compensation & Max abs. & Mean abs. & RMS \\
\hline Linear & Our method & 0.0036 & 0.0006 & 0.0008 \\
Uncalibrated & Our method & 0.0115 & 0.0021 & 0.0026 \\
Uncalibrated & Imposed CLV & 0.0019 & 0.0005 & 0.0006 \\
Uncalibrated & Worden et al.(1998a) & 0.0142 & 0.0036 & 0.0044 \\
\hline
\end{tabular}

levels of inhomogeneities affecting the analysed image, we achieve the lowest errors if we perform a linear calibration on the data. The disc fractions derived on uncalibrated data flattened with the imposed background exhibit smaller deviations to those from the undegraded data, and the errors are comparable to the ones we get with the linear calibration. However this is an idealised situation since we do not know the background for historical observations. The errors and the scatter of the results increase dramatically (reaching $100 \%$ in some cases) if we use uncalibrated data that were flattened with the background calculated with our method. It is worth noting that potentially the results can be different if one applies a different segmentation scheme. On average the error is $2 \%$ for the disc fractions derived from the linearly calibrated data or the uncalibrated data flattened with the imposed background.

These results indicate that although the photometric calibration of the image is important, this step is, however, not the most critical one if one wants to simply derive plage areas. The results from the uncalibrated data, are significantly affected by the errors in the calculation of the CLV.

Bertello et al. (2010) presented a method to derive a plage coverage index from uncalibrated data. Density contrast images were produced with a running window median filter. Then a Gaussian function was fit to the histogram of the whole image. A second histogram was calculated, this time keeping only the regions within $x_{-2 \sigma}^{+7 \sigma}$, where $x$ is the centre of the Gaussian. The histogram was split into 30 bins and divided by the total number of pixels. Another four-parameter Gaussian was fitted to these normalised bins, and the $\mathrm{Ca} \mathrm{K}$ index was defined as the additive parameter from the fit. Here we study the efficiency of this index with the synthetic data and compare it with the results derived with our processing. Bertello et al. (2010) do not provide information on how the images were compensated for QS CLV patterns. Therefore, we used our method to remove the CLV that we have tested for its accuracy (in Sect. 3.3).

Figure 4.21 shows plage regions derived from all synthetic data of subsets 1 and 6 with our method and that by Bertello et al. (2010). The values derived with the method by Bertello et al. (2010) were linearly scaled using parameters of the linear fit to the original Rome/PSPT values. In this way we achieve on average the best match with the original Rome/PSPT values, however we obtain negative values of disc fractions which is unrealistic. Requiring that all values be positive reduces significantly the agreement between the two series. The $\mathrm{Ca} \mathrm{K}$ index by Bertello et al. (2010) exhibits higher scatter 


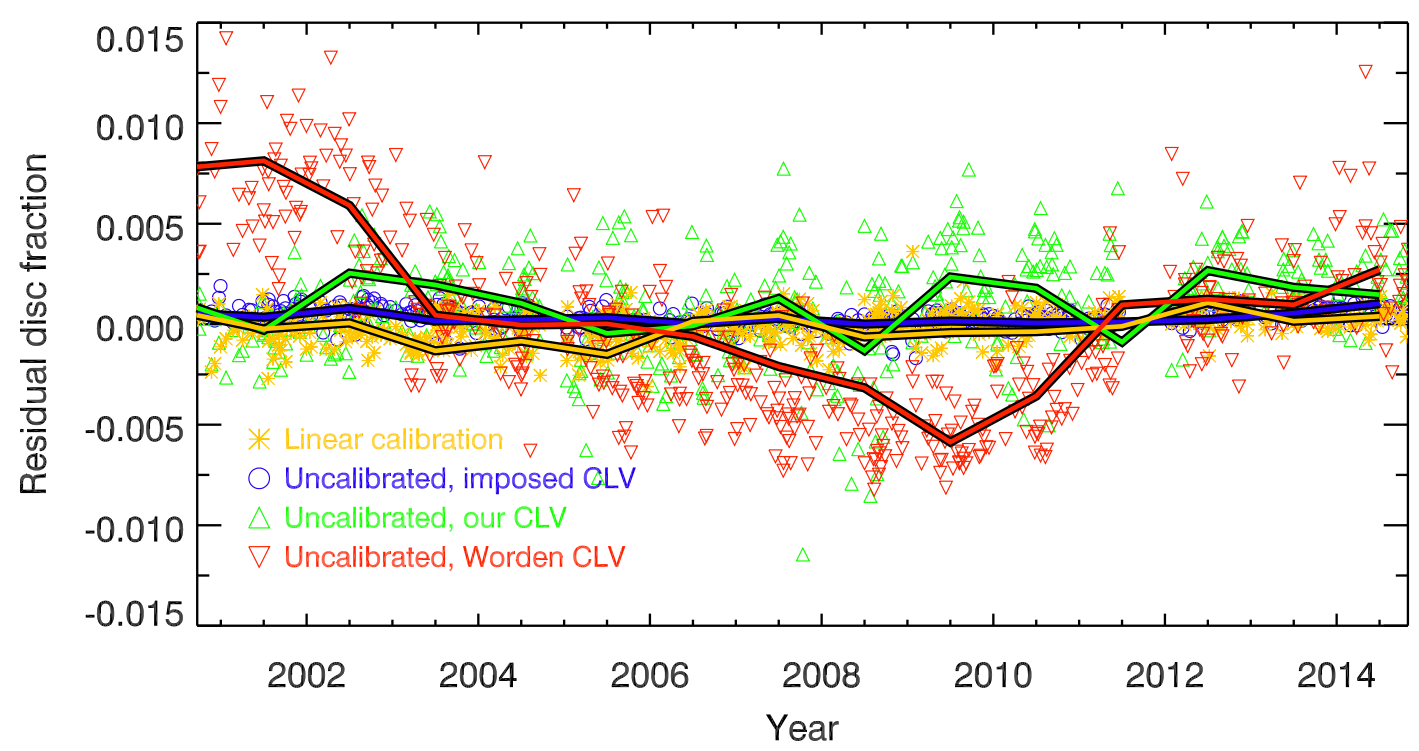

Figure 4.19: Difference between the disc fractions for all ARs obtained for the original flat Rome/PSPT data and for subset 1 . The data from the subset 1 were processed as follows: linearly calibrated with our method (Sect. 3.2, yellow), uncalibrated but the imposed CLV was used to remove the CLV (blue), uncalibrated but with the CLV removed with our method (green), uncalibrated but with the CLV removed following Worden et al. (1998a) (red). The segmentation was done with a constant threshold, the parameters in each case were adjusted to match the average disc fractions over all original Rome/PSPT data.

over the whole period, but follows the same trend. The maximum absolute difference we get between the result obtained with our method and the original data is $0.1 \%$, showing that calibrating the data leads to more accurate results. For subset 6 , when including all backgrounds and levels of inhomogeneities, we get a higher scatter for the $\mathrm{Ca} \mathrm{K}$ index by Bertello et al. (2010). However part of the differences might be due to different definitions of the contrast threshold of the plage regions. Considering that the $\mathrm{Ca} \mathrm{K}$ index by Bertello et al. (2010) does not reach a plateau during activity minimum it might partly include the network component.

\subsection{Segmentation of historical data}

In this section we present preliminary results of the segmentation of the historical Ca II K observations. The images were first calibrated and processed with the methods described in Sect. 3.2. Analysis of multiple archives, specifically of those from the Ar, Ko, Mi, and MW observatories, helps us to better assess systematic changes and sources of artefacts in individual data sets. We calculate the disc fraction and areas of active features in millionths of a hemisphere (msh, hereafter) corrected for projection effects. We complement the results from historical data with areas from modern observations as well. In particular plage areas from Rome/PSPT data that were processed as described in Sect. 4.3 and from the San Fernando observatory (SFO, these data were provided by Angela M. Cookson). The computed series are then compared with other results found in the literature. The Mi 


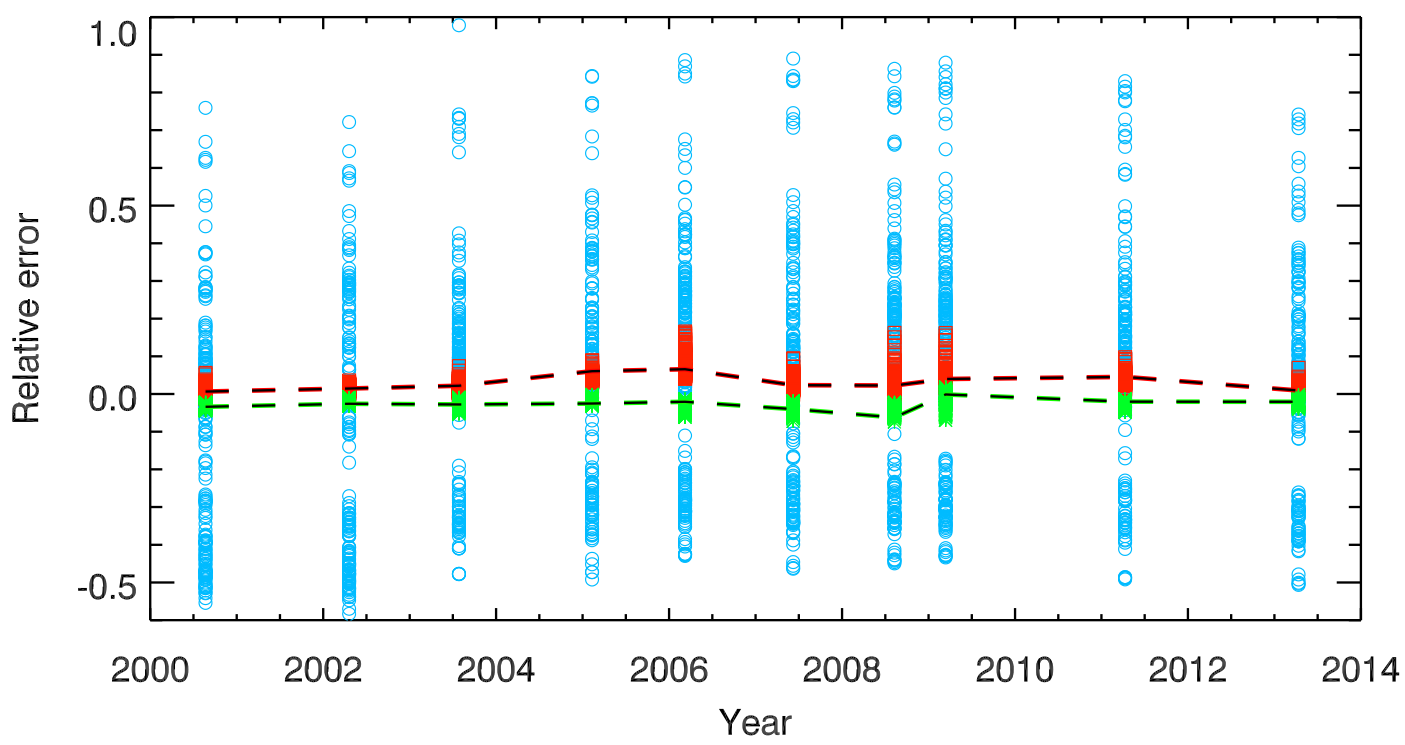

Figure 4.20: Relative errors in disc fractions of ARs between the original flat Rome/PSPT data and the images from subset 6 . The green asterisks are for uncalibrated data flattened with the imposed background, the blue circles are uncalibrated data flattened with the background calculated with our method (Sect. 3.2.1), the red squares are for data that were linearly calibrated with our method (Sect. 3.2.3). The dashed lines connect the errors for the images of subset 6 created with the lowest strength level of inhomogeneities for our method (red) and the uncalibrated data (green).

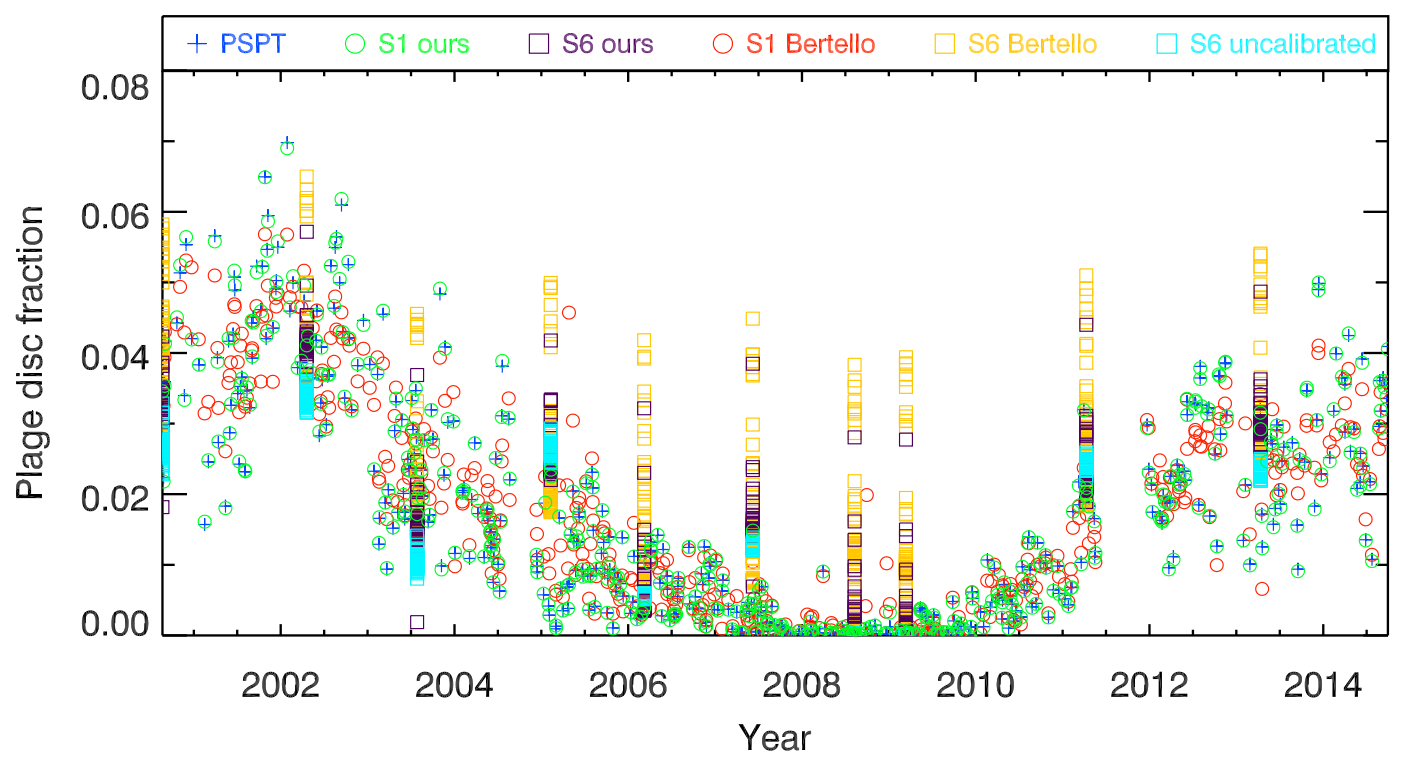

Figure 4.21: Comparison between disc fractions derived from the original Rome/PSPT data (blue plus signs), calibrated data of subset 1 (green circles, notice that they lie almost perfectly on top of the blue plus signs) and subset 6 (dark blue squares) with our method, and the $\mathrm{Ca} \mathrm{K}$ index reproducing Bertello et al. (2010) for subset 1 (red circles) and subset 6 (orange squares). Note, subset 6 is available on for 10 days during the considered period. 
archive has not been studied earlier.
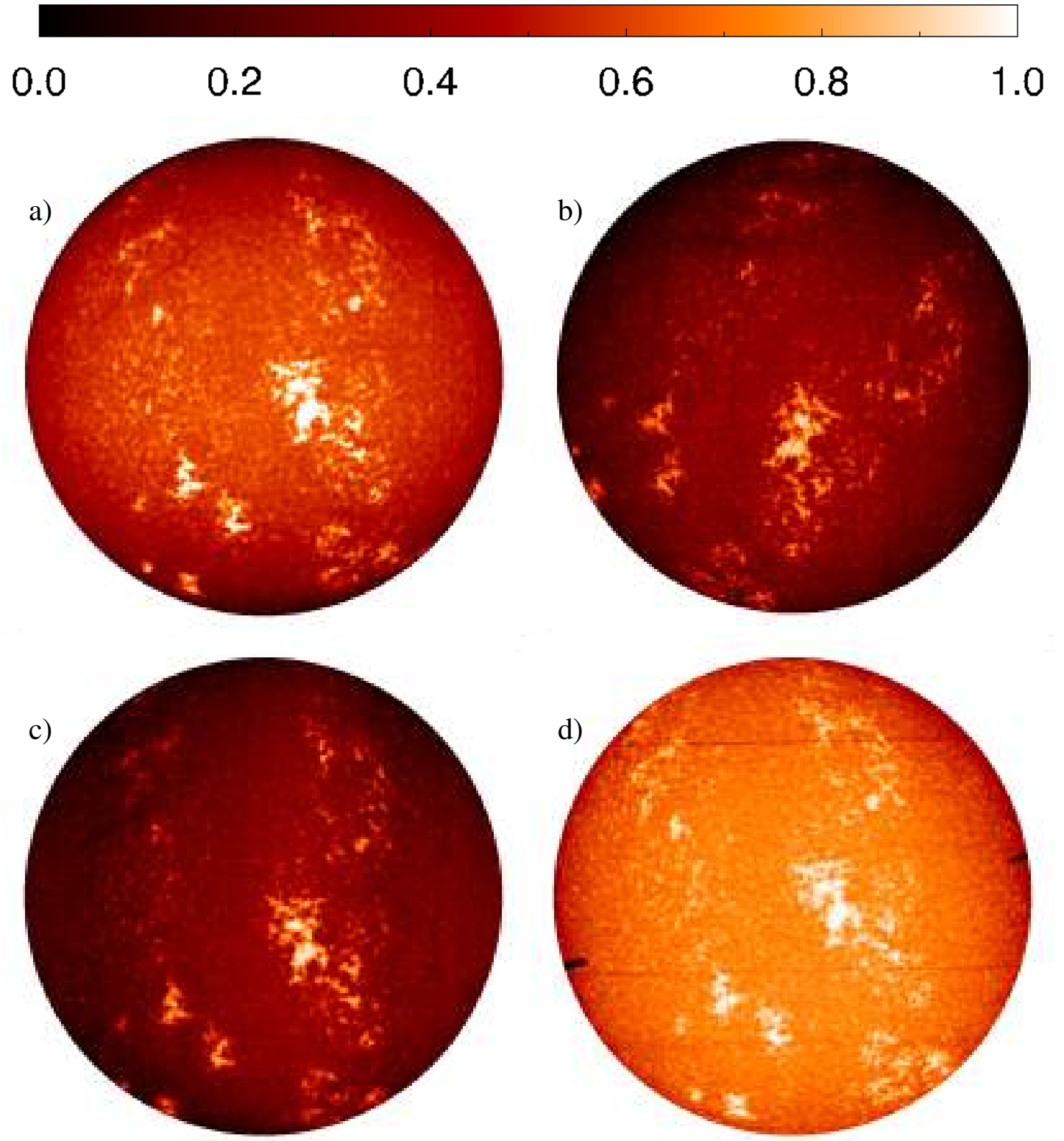

Figure 4.22: Examples of the historical observations analysed in this study. Shown are the raw density images taken on 23/01/1968 at the: (a)) Arcetri, (b)) Kodaikanal, (c)) Mitaka, and (d)) Mt Wilson observatories. The colour scale of each image covers the full range of brightness (density) on the disc, from maximum (white) to minimum (black). The colour bar at the top is in units of normalised densities and corresponds to all images. The images are not compensated for solar ephemeris.

Figure 4.22 shows an example of a typical density image from each historical data set taken on the same day. The brightness scale is individually normalized to cover the values from the minimum (black) to the maximum (white) within the disc in each figure. This shows for instance, saturated regions in the Ar observation. 


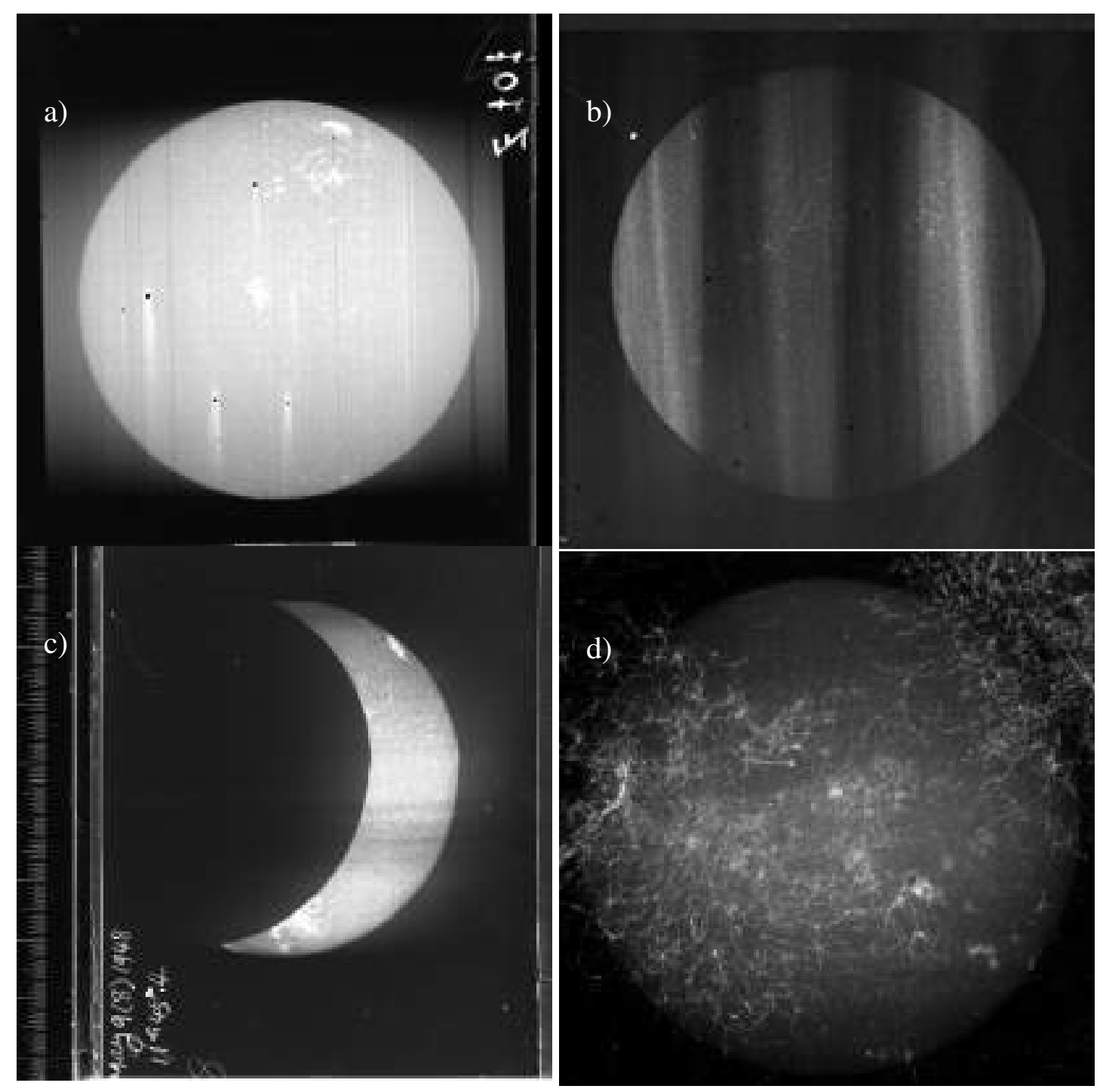

Figure 4.23: Examples of historical observations that were excluded from this study. Shown are the raw density images taken at the: (a)) Arcetri (13/02/1942), (b)) Kodaikanal (12/12/1909), (c)) Mitaka (09/05/1948), and (d)) Mitaka (05/11/1971) observatories. The images are not compensated for solar ephemeris.

\subsubsection{Processing}

For the analysed historical data, estimates of the centre of the solar disc and radius are listed in their FITS file headers. These estimates, with a few exceptions, have adequate accuracy. They are, however, less accurate for Mi data and had to be recalculated. This task is complicated because historical SHG very rarely show a regular solar disc. This is due to distortions or misalignments of subsequent slit observations. The main complication of getting the coordinates of the disc centre and radius automatically are the artefacts affecting the image near the limb and outside the disc, e.g. stray light, markings, dust, scratches, missing portions of the disc. To identify the edge of the disc, we first apply a Sobel filter on the image. We create a mask containing only regions within appropriate thresholds in the image that resulted from the Sobel filter set to 1. However, at this stage 

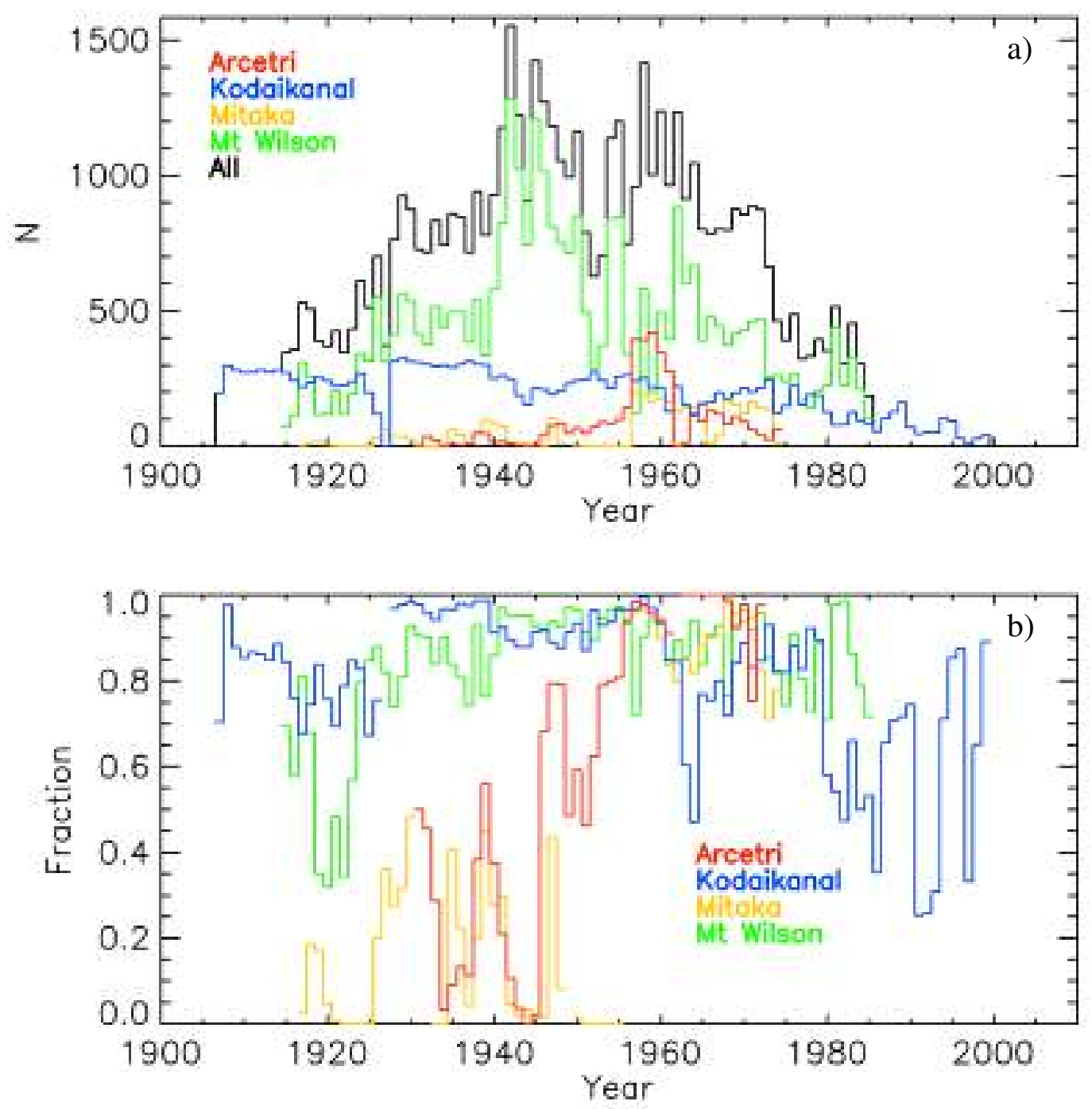

Figure 4.24: The number of images per year used in this study (a)) and the fraction of available images per year that was used (b)) from the Ar, Ko, Mi, and MW archives.

the result of the Sobel filter is strongly affected by artefacts within the images and is not sufficient to define the edge of the disc. Therefore, we do the following steps to improve the determination of the edge of the disc. We dilate and erode the mask to make its edges smooth and then remove all small-size and isolated regions in the mask that are most likely due to image artefacts. We apply another Sobel filter to the smoothed mask to identify the edge of the disc creating yet another mask. We perform a bootstrap Monte Carlo simulation where we randomly choose half of the points from the mask and fit a circle. We repeat this process 1000 times and then adopt the average parameters for the radius and centre coordinates. We then exclude from the previous mask the regions with the distance to the centre of the disc greater than $1.03 R$ and perform one last fit. This method, however, does not work on Mi data. The reason is that regions outside the disc in Mi data suffer from significant problems. Therefore we ended up defining the centre coordinates and the radius for Mi by fitting a circle on three manually selected points. This allows an 



Figure 4.25: Examples of produced masks with the NR segmentation method. Column 1 shows the contrast images saturated within the range [-0.3,0.3], column 2 the masks showing all features and column 3 the masks of the network component for Ar (top), Ko (2nd row), Mi (3rd row), MW (bottom) observations respectively. Note that the images have not been turned to align solar north.

estimate of the disc centre and the radius with accuracy sufficient for further processing. 
This method was also used to correct problematic data from the other datasets. For our analysis, we only consider pixels within $0.99 R(0.98 R$ for Ko) to avoid uncertainties in the radius estimates. This corresponds to $\mu=\cos \theta=0.14$ ( $\mu=0.2$ for Ko), where $\theta$ is the heliocentric angle.

To remove remaining small artefacts and outliers, we smoothed the image with a running window median filter with a very small width (4 pixels), produced a contrast image and replaced the pixels of the original image with $C>1.2$ or $C<0.5$ with the corresponding values from the smoothed image. These limits were chosen to be very conservative, in order to ascertain that the bright plage or the dark parts of the sunspots are not affected by this procedure.

We found a lot of duplicate files in the MW dataset by applying a code that checked for the pixel-by-pixel similarity of the images. Of these, more than 80 pairs of files contained the same image but listed different dates. The date of these recordings was identified by comparing with alternative datasets. The duplicate images with the wrong date were excluded from our analysis. Other datasets sometimes have incorrect dates too, usually due to mistakes in naming files during the digitisation. These kind of errors in the data are unsurprising considering that the digitisation process (which was not limited to $\mathrm{Ca}$ II $\mathrm{K}$ images) had to deal with $\sim 10^{4}-10^{5}$ files and usually lasted several years. This highlights the need to analyse multiple SHG series to reduce effects of these errors.

Many historical images suffer from various problems that do not allow any meaningful analysis. Images with high noise levels, or extremely low contrast regions (hinting at exposure problems), or with missing parts of the disc were excluded from this work. Examples of such images are shown in Fig. 4.23. Some ARs in the Ar data are saturated. This could have resulted from the digitization, or from overexposure and saturation of the photographic plates. The results we can derive from such data is limited, however we tag the saturated regions as plage regions and use such images to gain information on fainter features.

Figure 4.24 shows the annual distribution of the number and the fraction of the images from the four archives we used for our analysis. In particular, we used: 3885, 18381, 3227, and 31306 images from Ar, Ko, Mi and MW respectively.

The data have been photometrically calibrated and compensated for the limb darkening as described in Sect. 3.2. The segmentation was done with the NR method (see Sect. 4.2). Examples of the derived masks of plage and network features can be seen in Fig. 4.25. The parameters for the segmentation are listed in Table 4.1 and were the same for all datasets. These masks are used to calculate the disc fractions covered by various magnetic structures. We also produce time-series of the plage areas in millionths of solar hemisphere. To do that we first correct the pixel areas by dividing with the corresponding $\mu$. The areas are derived by summing up all the pixel areas corresponding to a feature and normalising them with the area of the hemisphere $2 \pi R^{2}$.

\subsubsection{Plage areas}

Figure 4.26 shows the daily and the annual median values of the combined plage and network disc fractions for all the processed SHG data along with the Rome/PSPT data. The Rome/PSPT data were processed in the same way as the historical data. The sunspot areas compiled by Balmaceda et al. (2009) are plotted in the lower panel for comparison. 


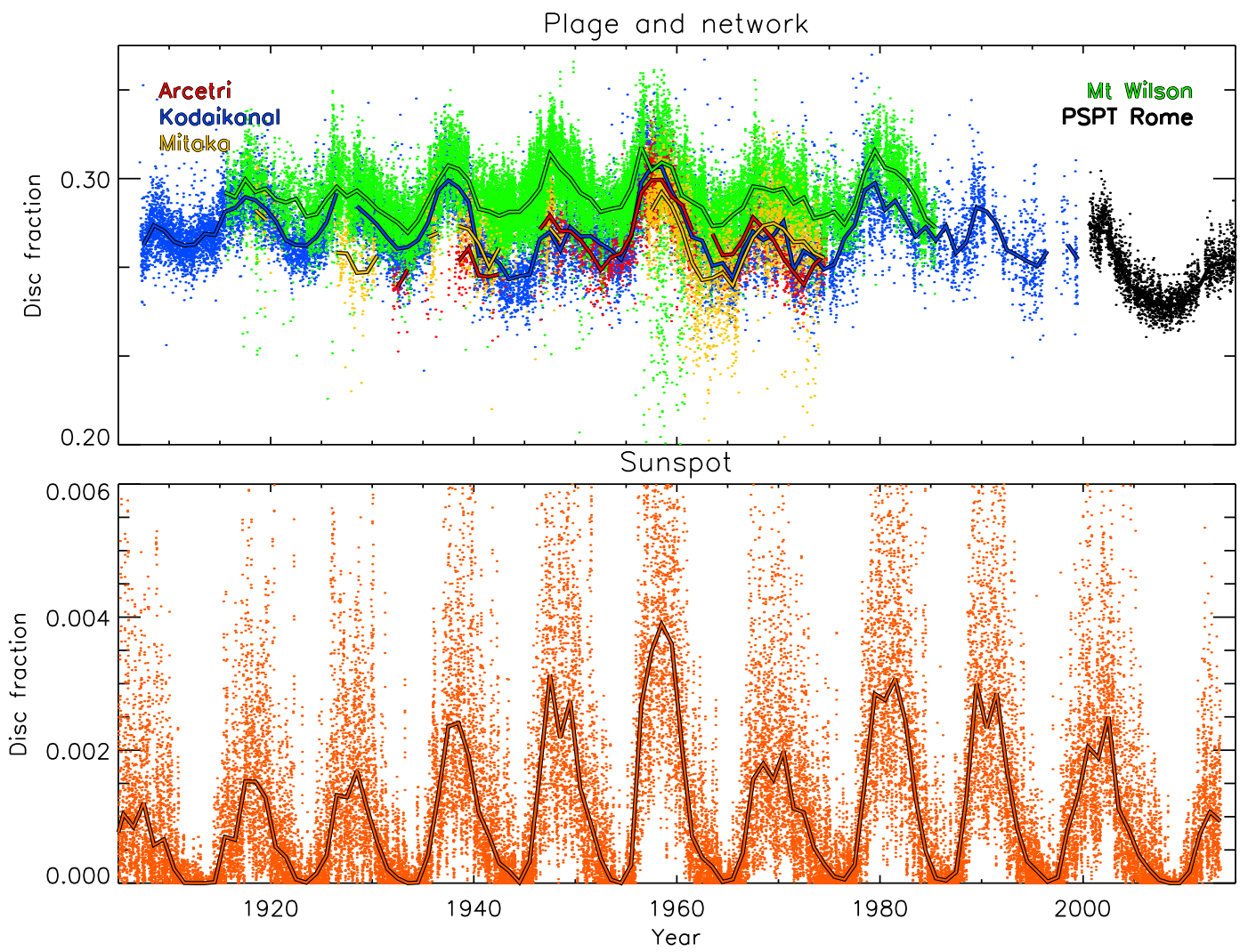

Figure 4.26: Disc fraction of plage and network (top) over time, derived with the NR thresholding scheme with the same parameters for the datasets of Arcetri (red); Kodaikanal (blue); Mt Wilson (green); Rome/PSPT (black). Also shown are the sunspot areas from Balmaceda et al. (2009) (bottom). Individual small dots represent daily values, while the thick lines indicate annual median values.

The derived plage disc fractions generally agree well between the different datasets. The results for MW are slightly higher than those from the other datasets. The differences could be because of the narrower slit width used in MW than in the other observatories.

Figure 4.27a) shows the derived plage disc fraction for the historical archives as well as Rome/PSPT and SFO data. The disc fractions agree well between all datasets, except for the MW dataset before 1970's which gives disc fractions that are higher by up to 5\% (cycle 18) than values derived from all other datasets. The values from SFO are overplotted to bridge the Rome/PSPT and Ko series. These records appear to be consistent. In the same figure panels b) - d) compare annual means of our results for individual archives with those available in the literature. Panel e) shows the areas derived in this study for Ar and Ko data together with the published Big Bear solar observatory (BBSO) series (see Sect. 2.7). The various published series are given in $\mathrm{msh}$ for data from all observatories but Ko and SFO, which are given in disc fractions. Therefore to enable to compare our results with the published series we show values of disc fractions in panels a) and c), and areas in msh in panels b), d), and e). Figure 4.28 compares our results from Ko data to those by Ermolli et al. (2009b). The areas derived by Ermolli et al. (2009b) were corrected for projection effects and had to be presented separately to those by Tlatov et al. (2009), 
Priyal et al. (2013), and Chatterjee et al. (2016) that provide areas uncorrected for the projections effects.

Ar data were used by Ermolli et al. (2009b) to derive plage areas. Our series and that by Ermolli et al. (2009b) agree rather well after 1950, but our series is consistently higher for earlier periods. The differences reach 10,000 msh during cycle 17. According to Fig. 7 of Ermolli et al. (2009b) the plage areas from Ar data during that cycle they derived are too low compared to the results they obtained from MW and Ko data. This supports the area values obtained here. However, the amount of data used in our study before 1950 is significantly low, which could also be the reason for the differences.

The Ko data were analysed by Ermolli et al. (2009b), Tlatov et al. (2009), Priyal et al. (2013), and Chatterjee et al. (2016). Our time-series lies close to those by Priyal et al. (2013) and Tlatov et al. (2009). Compared to ours, the series by Ermolli et al. (2009b) and Chatterjee et al. (2016) are systematically lower and higher, respectively.

Our MW series shows a good agreement with that of Bertello et al.(2010), however it should be noted that the series by Bertello et al. (2010) was rescaled by applying a linear relation derived with our series. Our plage areas and those from Bertello et al. (2010) are systematically higher or equal (cycle 20) than those derived by Ermolli et al. (2009b) and Foukal (1996), except for cycle 19. The areas by Foukal (1996) are slightly lower than those by Ermolli et al. (2009b), except for cycle 19 that are considerably higher.

The published time-series are provided in annual values, and the data included in each year may vary among the different series. This might partly explain the differences between the time-series obtained here and in earlier studies. However, the main differences come from the calibration, CLV compensation and segmentation schemes. For instance, Foukal (1996) segmented the images by manually selecting the plage regions potentially introducing bias of selection. Another critical issue is the definition of plage, which differs from study to study and is rather arbitrary.

Our Ar and Ko disc fractions agree rather well with the BBSO series before the 1980's. For this period The BBSO series consists of data from the MM observatory, but when the Big Bear and MW data are used the BBSO series becomes significantly lower than ours. This suggests that there might be unaccounted systematic differences between the 3 observatories contributing to the BBSO composite.

Figure 4.29 shows scatter plots between our series and those of Ermolli et al. (2009b), Foukal (1996), Priyal et al. (2013), and Bertello et al. (2010). The plots show that there is an almost linear relation between our series and those by Ermolli et al. (2009b), Bertello et al. (2010), Foukal (1996), and Priyal et al. (2013). The scatter is slightly higher for Foukal (1996) and Priyal et al. (2013).

\subsubsection{Contrast of plage and network}

Figure 4.30 shows the variation of the average contrast of plage (top) and the network (bottom) with time for the historical SHGs analysed here. The mean contrast in the Ko data declines. MW contrasts do not show any systematic trend, but features appear on average significantly brighter than in other datasets or at other periods around 1960. This is important and should be discussed, as further analysis is needed to understand whether this is a problem of the data or whether our method fails during this period. Bertello et al. (2010) encountered the same problem with the MW data around 1960 and they explained 

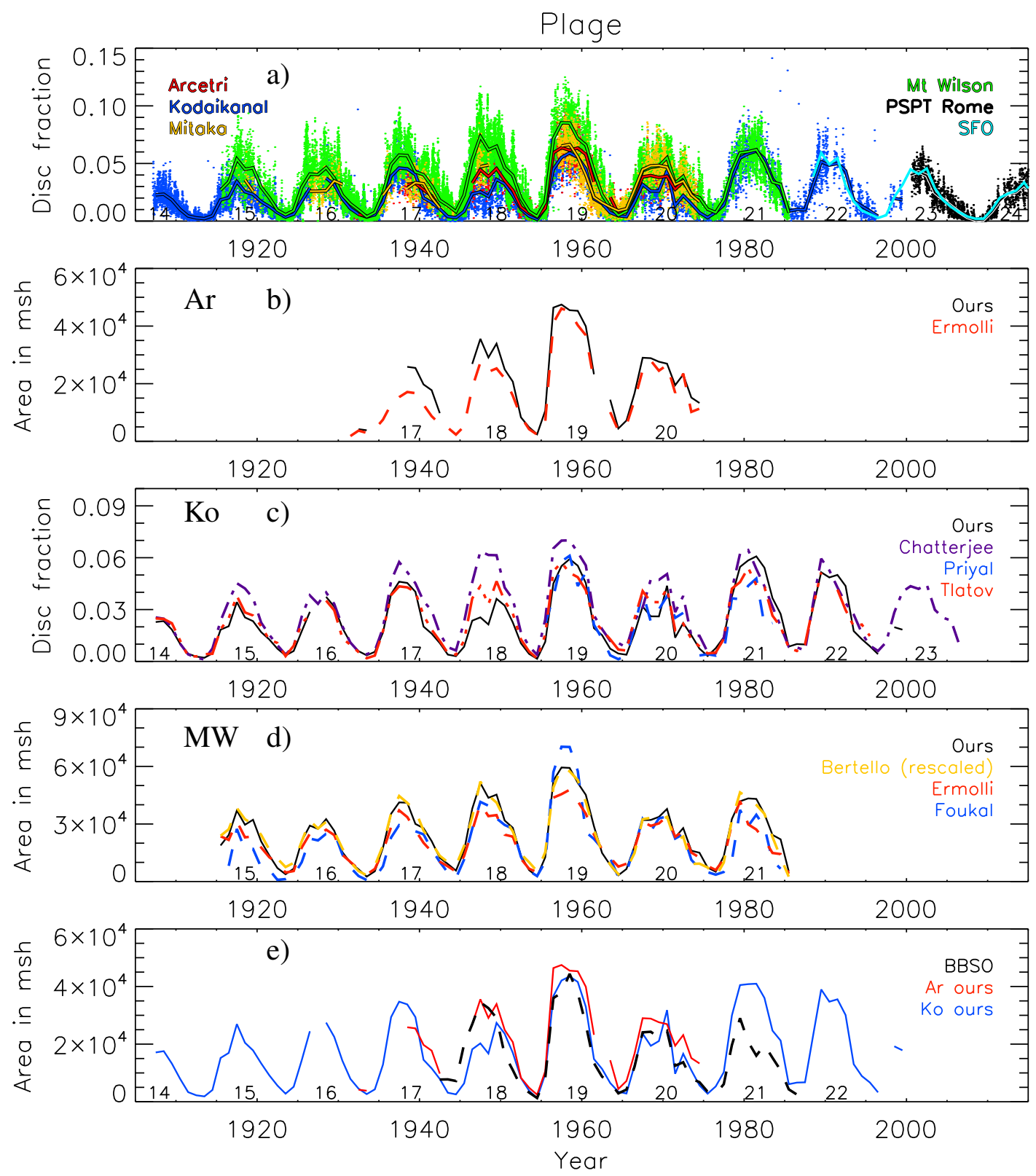

Figure 4.27: Plage area variation with time: (a)) our results for different datasets derived with NR thresholding scheme (see Table 4.1 for the values of segmentation parameters) and the same parameters. Plotted are the areas for the datasets of Arcetri (red); Kodaikanal (blue); Mitaka (orange), Mt Wilson (green); Rome/PSPT (black). Also shown are the SFO values (light blue). Panels (b) - d)) compare our results to those by other authors: (b)) Ermolli et al. (2009b, red) for Ar data; (c)) Chatterjee et al. (2016, purple), Priyal et al. (2013, blue), Tlatov et al. (2009, green) for Ko data; (d)) Bertello et al. (2010, red), Ermolli et al. (2009b, red), Foukal (1996, red) for MW data. Panel (e)) compares our results for $\mathrm{Ar}$ (red) and Ko (blue) data with the published BBSO series (black). The curves are annual median values. Panels b), d), and e) give area values in millionths of hemisphere corrected for projection effects, while panels a) and c) are in disc fraction. The numbers near the $x$ axis denote the conventional solar cycle numbering. 


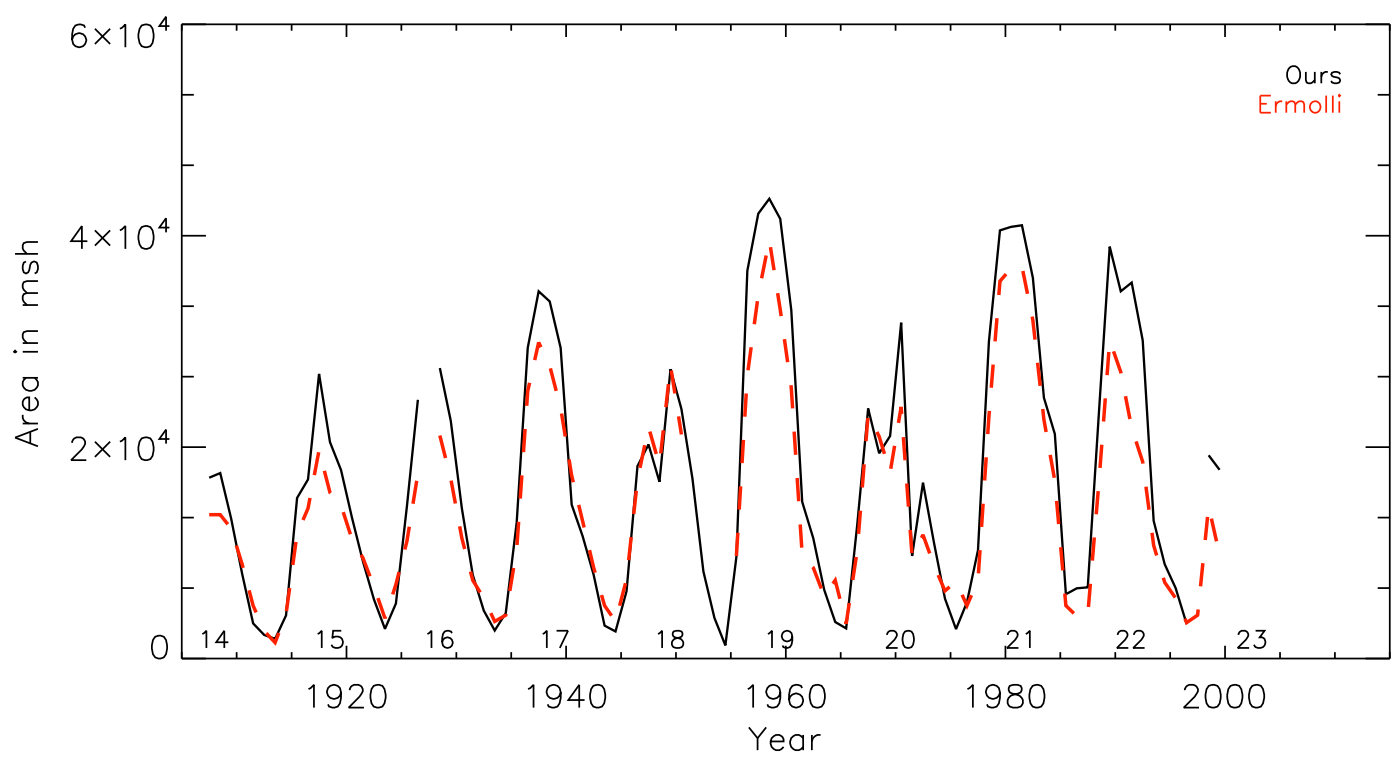

Figure 4.28: Plage area variation with time as derived from Ko observations with our method (black) and that of Ermolli et al. (2009b, red). The curves are annual median values and are given in millionths of hemisphere corrected for projection effects. The numbers near the $x$ axis denote the conventional solar cycle numbering.

this with a narrower band pass. Contrast values from Ar, Ko, Mi, and MW archives are comparable with those from the Rome/PSPT observations.

Figure 4.31 shows the average contrast of the network among all analysed images from the Ar, Ko, Mi, MW, and Rome/PSPT data for different heliocentric angles. The average contrast of the network components remains almost constant over the disc for all datasets. For Ko data we find a difference between inner and outer bin on average of 0.007 .

We have also analysed the dependence of the contrast on the size of the features. Figure 4.32 shows the results for all the processed Ko data. The dependence is similar to that obtained with the modern Rome/PSPT observations (Fig. 4.12), however with a higher scatter, reaching 0.15 (compared to 0.05 that we found for Rome/PSPT). Figure 4.33 shows the average size-contrast relation over all images of each historical archive and Rome/PSPT. We notice that the relation saturates at higher contrast values in the historical archives, except for Ko. The curve from Ko is the one closest to that from Rome/PSPT. The curve derived from Ar is not reliable due to the saturated regions. The much higher contrast from MW may have to do with the narrower slit, i.e. narrower wavelength range covered by MW SHGs, as the contrast tends to increase the core of Ca II K. Remaining artefacts and processing errors can explain the increase in contrast. These require further investigation. 
a)

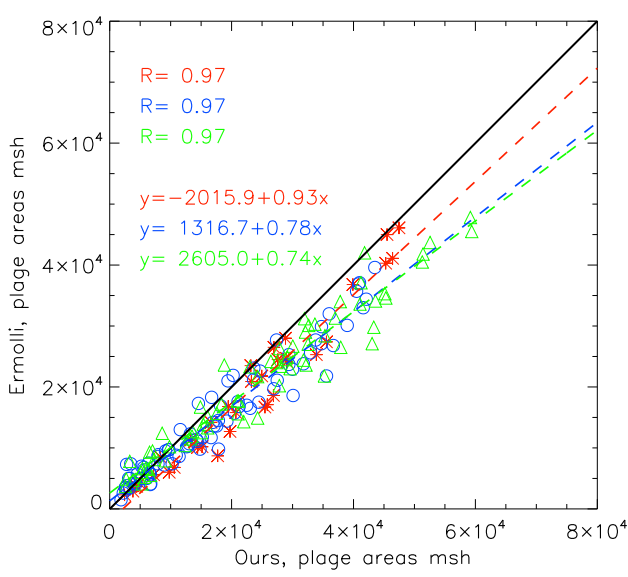

c)

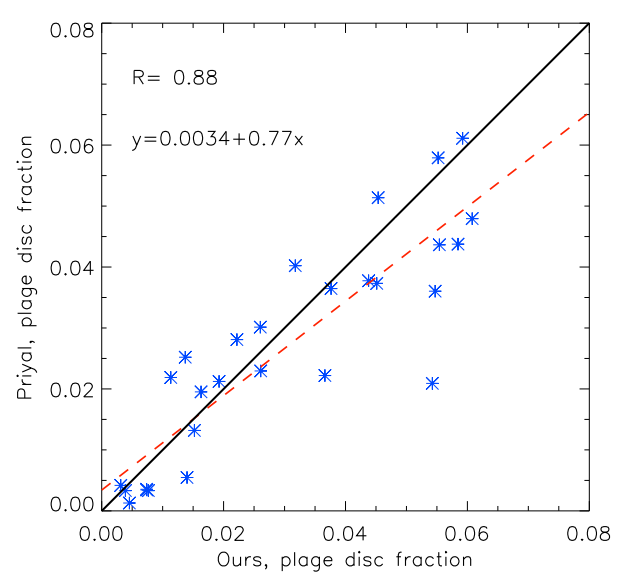

b)

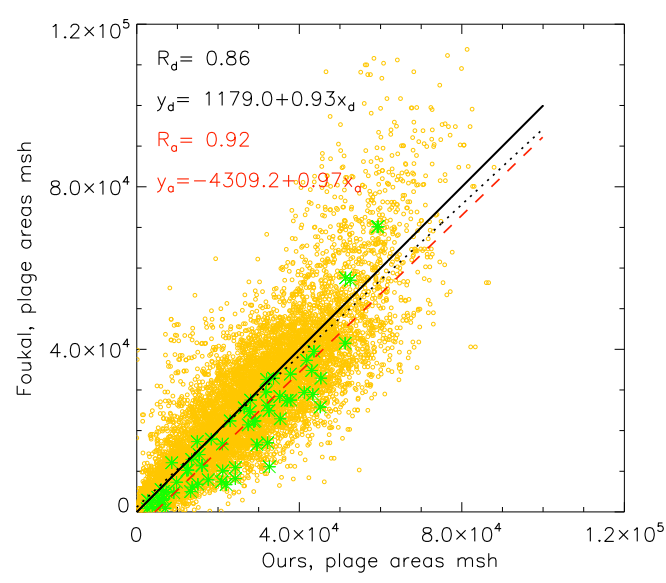

d)

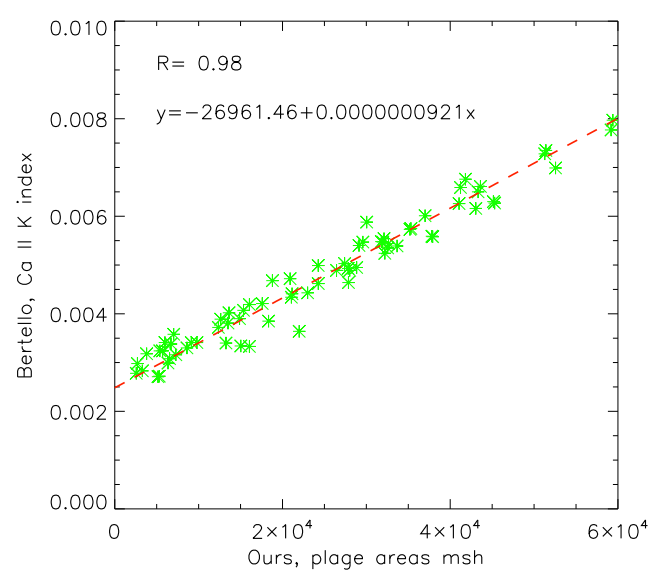

Figure 4.29: Scatter plots between our plage area series and those presented in (a)) Ermolli et al. (2009b), (b)) Foukal (1996), (c)) Priyal et al. (2013), and (d)) Bertello et al. (2010) for data recorded at Ar (red), Ko (blue), and MW (green). Annual values are compared in all panels, but in panel (b)) we also compare the daily values (orange circles). The solid black lines have a slope of unity. The dashed (dotted) lines are linear fits to the annual (daily) data. Also shown is the linear correlation factor between the different series and the parameters of the linear fits.

\subsection{Conclusions}

We tested different segmentation schemes on modern CCD-based Ca II K observations. We showed that the method of applying a multiplicative factor to the standard deviation of the intensity values on the disc as a threshold (CMF) is strongly affected by the activity and should be avoided. All other published methods we tested return consistent results with each other. However, CT and MLT require accurate processing to remove the CLV prior to the segmentation. With NR we identify residual effects due to active features, though to a lesser extend than for CMF.

Finally, we segmented SHG data and calculated preliminary plage areas over the whole period they are available. We compared our results to those presented in the literature. We showed the feasibility of accurate processing of different historical SHG time-series and to derive consistent results in terms of plage and network areas and con- 

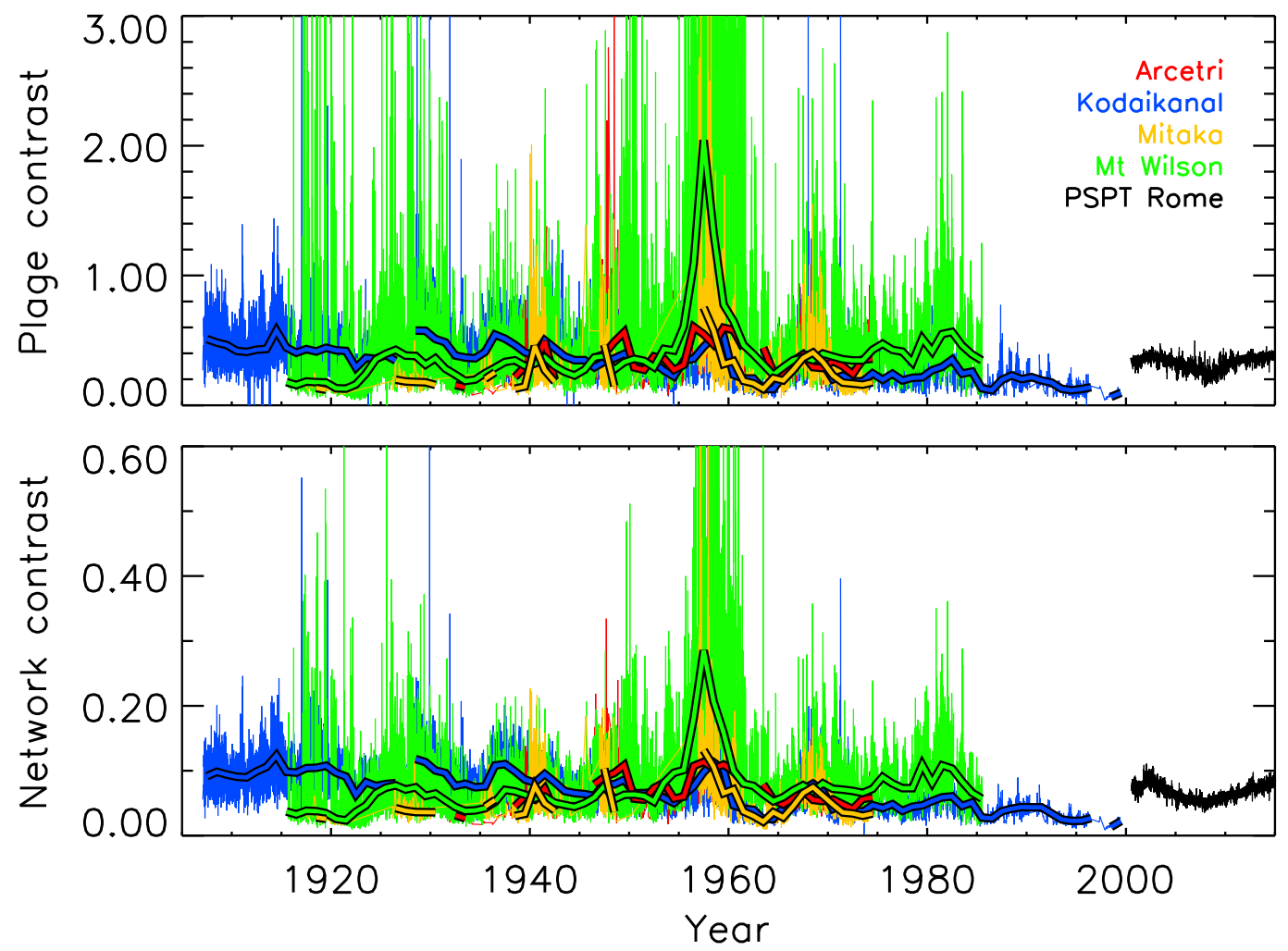

Figure 4.30: Average contrast of plage (top) and network (bottom) as a function of time derived with the NR thresholding scheme with the same parameters for the datasets of Ar (red); Ko (blue); Mi (yellow); MW (green); Rome/PSPT (black). Thin lines show daily values, while thick show lines annual median values.

trasts. We showed that the photometric calibration is not the most critical step if the aim is to only segment the data and get plage areas. The most important step is an accurate estimate of the background of the image (i.e. the quiet Sun CLV) and accounting for all possible artefacts. This is only true under the assumption that the data had been properly exposed. By testing other suggested methods of processing uncalibrated data, we showed that processing with the method we developed and presented here returns much lower uncertainties. Further work is required to improve the segmentation method. The differences between the various archives need to be studied, too. 


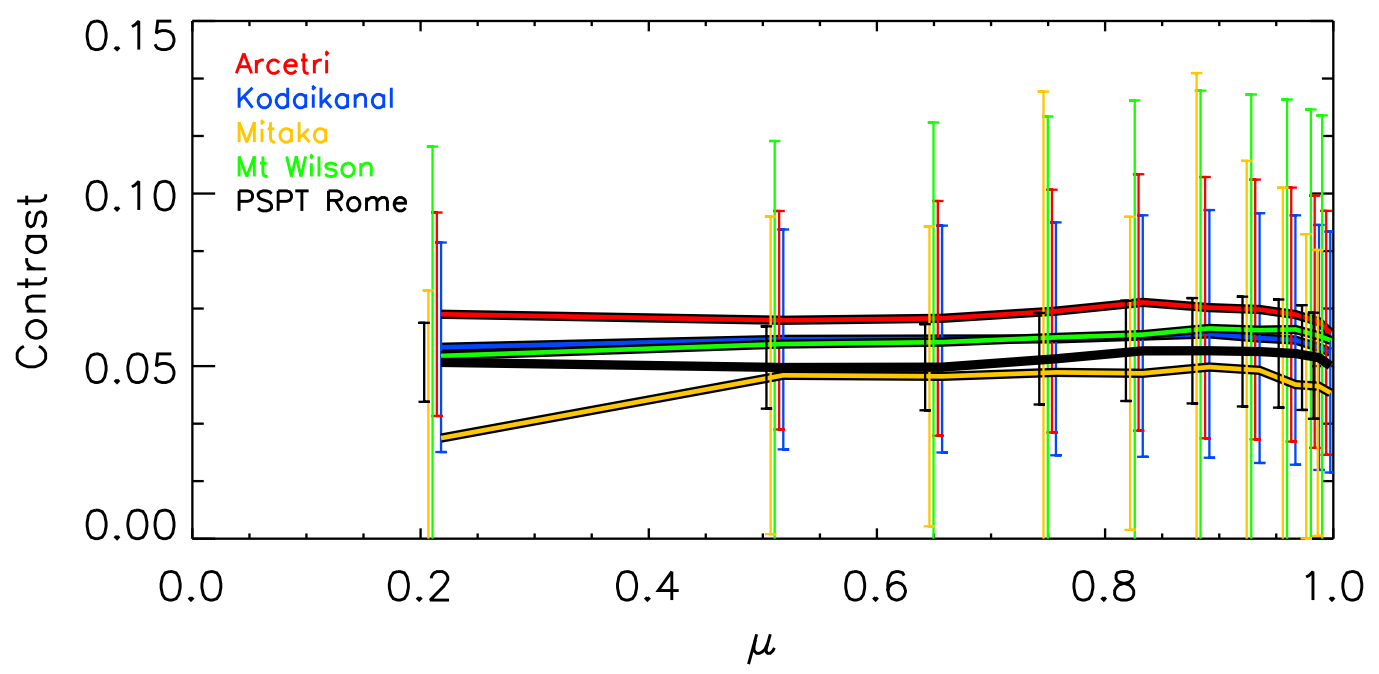

Figure 4.31: Average contrast of the network over all images of each dataset as a function of the heliocentric angle. The segmentation was done with the NR thresholding scheme with the same parameters for all datasets: Ar (red); Ko (blue); Mi (yellow); MW (green); Rome/PSPT (black). The error bars denote the $1 \sigma$ level and have been slightly offset in $\mu$ to improve visibility. 


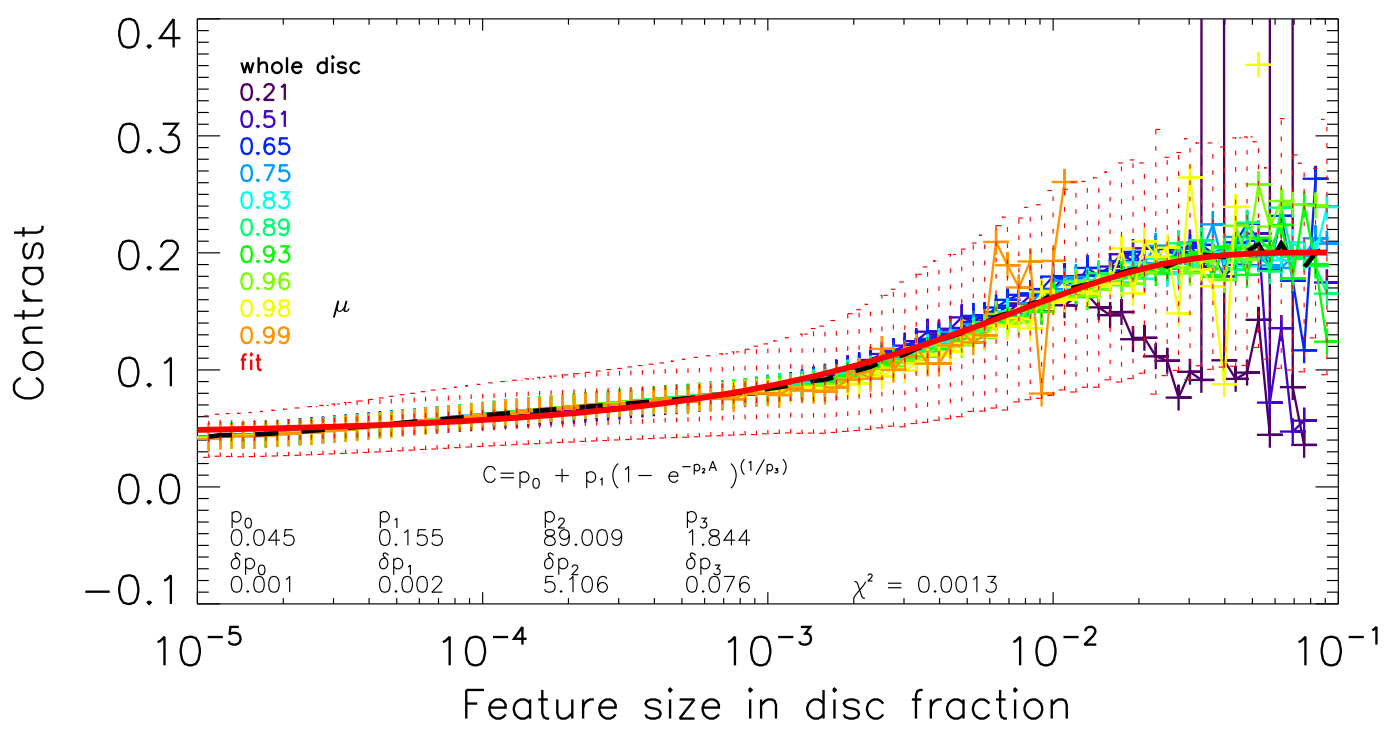

Figure 4.32: Dependence of the contrasts of Ca II K bright features on their size for Ko data. The segmentation was done with NR. The black dashed line shows the disc averaged values, the red line is the result of the fit of a function of the form of Eq. (4.3). The error bars are the standard deviations in the measured values derived over the whole disc among all images. The coloured lines show the same relation within equal area annuli with $\mu$ shown in the legend. The function of Eq. (4.3), the parameters of fitting Eq. (4.3) to the size-contrast relation, along with their $1 \sigma$ uncertainties and the $\chi^{2}$ of the fit are provided in the lower part of the plot.

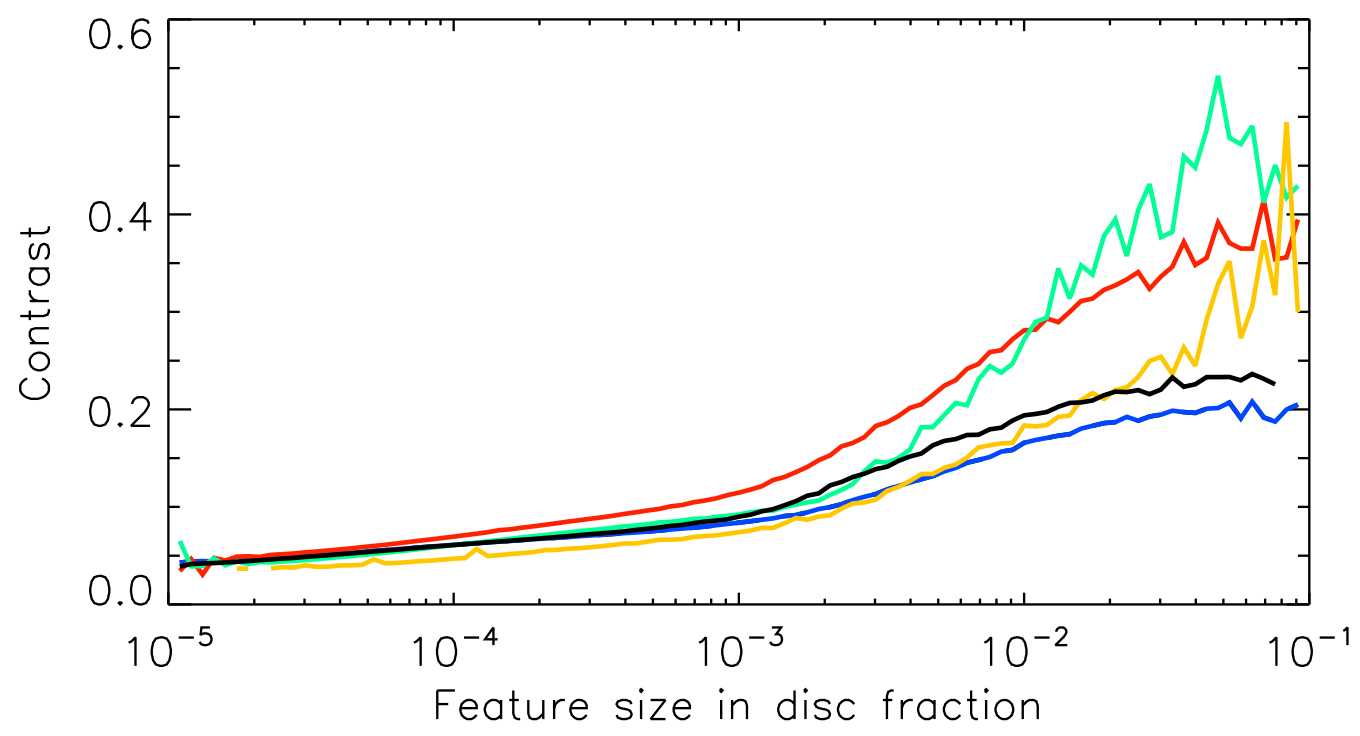

Figure 4.33: The dependence of the average contrasts of the features on their size for Ar (red), Ko (blue), Mi (yellow), MW (green), and Rome/PSPT (black) observations. The curves are the averages over the whole disc among all available images. 


\section{Relationship between the Ca II K intensity and the photospheric magnetic field}

*This chapter is based on a journal article in preparation (Chatzistergos et al. 2017c)

\subsection{Introduction}

Since the first comparative analysis of full-disk solar magnetograms and Ca II K spectroheliograms by Babcock and Babcock (1955) there has been consistent evidence of a link between the magnetic field strength and the excess Ca II K emission. Babcock and Babcock (1955) mentioned a "one-to-one correspondence" between bright regions in Mt Wilson Ca II K spectroheliograms and magnetic regions in magnetograms. This reported association, which was promptly confirmed by Howard (1959) and Leighton (1959), has led to numerous studies of solar and stellar Ca II data aiming to determine its exact characteristics.

Considerable efforts have been devoted to understand the relation between magnetic field strength and Ca II K intensity for different solar magnetic regions of the Sun. In particular, by following active regions (AR) over the disc, Frazier (1971) found a polynomial function, while Skumanich et al. (1975) obtained a linear relationship for a quiet Sun (QS, hereafter) region at the disc centre. Schrijver et al. (1989) reported that, after the subtraction of the basal flux from the observed Ca II $\mathrm{K}$ data, the relation between magnetic field strength and Ca II K intensity was best described by a power law with an exponent of about 0.6. Nindos and Zirin (1998) found that the linear relationship applies when magnetic field strengths below a particular threshold $B$ are excluded. Looking separately at ARs, decaying ARs, enhanced network and quiet network, Harvey and White (1999) obtained a power law relationship between Ca II K intensity and line-of-sight magnetic flux density. The study by Harvey and White (1999) was the first one to consider the whole solar disc. The exponents they derived varied between 0.47 and 0.78 for both the active and QS and were constant over the disc. The different results for the exponents they presented could be due to fitting of features at different positions on the solar disc. More recently, Rast (2003a) and Ortiz and Rast (2005) found a power law exponent value of 0.66 for the QS in full-disc data, while Rezaei et al. (2007) estimated the exponent to be 0.2 in a QS region and $0.4-0.5$ in network regions. They also noticed that the exponent depends on the magnetic field threshold below which the data are excluded from the fitting. Vogler et al. (2005) studied 60 pairs of full-disc Ca II K observations and mag- 
netograms found a power law exponent with exponents that were consistent with those derived by Harvey and White (1999). By analysing a QS region observed in three wavelength ranges including the Ca II K-line, Loukitcheva et al. (2009) found a power-law relation between the magnetic field strength and the Ca II K intensity, with an exponent of 0.3. Kahil et al. (2017) analysed high resolution Ca II H observations of QS regions taken with Sunrise balloon-borne telescope (Solanki et al. 2010, Barthol et al. 2011) and reported a logarithmic function to fit the data best. This was the first study with data that have resolved strong magnetic field elements in the internetwork. From an analysis of Kitt Peak magnetograms, uncalibrated Mt Wilson Ca II K spectroheliograms, and SOLIS/VSM observations of Ca II taken at $854.2 \mathrm{~nm}$ and magnetograms, Pevtsov et al. (2016) reported a linear relation between the magnetic flux and the area covered by plage in two Ca II lines ( $\mathrm{K}$ and infra-red). They concluded that $\mathrm{Ca}$ II observations are unreliable proxies for the magnetic field strength. However, they did not differentiate between plage and sunspots or consider the fact that the Ca II data they used were not photometrically calibrated (see Chapters 2 and 3). Table 5.1 summarises the main features of the studies discussed here.

Major efforts have been invested in measuring the disk integrated $\mathrm{Ca}$ II $\mathrm{H}$ and $\mathrm{K}$ emission of many stars. In fact, such emission has been regularly analysed e.g. by the synoptic programs carried out with the ground-based Mt Wilson Observatory $\mathrm{H}$ and $\mathrm{K}$ photometers (1966-2003, Wilson 1978, Duncan et al. 1991, Baliunas et al. 1995) and Lowell Observatory Solar-Stellar Spectrograph (1994-present, Hall et al.2007), and by the space-born photometer on-board the CoRoT mission (Michel et al. 2008, Auvergne et al. 2009, Gondoin et al. 2012). Indeed, based on the fact that such emission is an indicator of the strength of, and area covered by, magnetic fields on the Sun (Leighton 1959), and that in many active stars the $\mathrm{Ca}$ II $\mathrm{H}$ and $\mathrm{K}$ variations due to magnetic regions can be easily detected ${ }^{1}$, the $\mathrm{Ca}$ II H and $\mathrm{K}$ measurements have been used to trace long-term changes in surface activity of stars caused by e.g. the activity cycle, rotation, and convection (e.g. Sheeley 1967, White and Livingston 1978, Keil and Worden 1984, Baliunas et al. 1985, etc.).

The Ca II H and K measurements of stars have led to an improved knowledge of stellar rotation and activity, and of the degree to which the Sun and other stars share similar dynamical properties (for reviews, see e.g. Lockwood et al. 2007, Hall 2008, Reiners 2012, Lockwood et al. 2013). For example, the variation of the disc-integrated Ca II K line over the activity cycle (Sheeley 1967, Skumanich et al. 1984) was found to be quite common among main-sequence stars, albeit not universal, often with quite a different amplitude than the $40 \%$ change reported for the Sun (Schrijver et al. 1989). Stellar brightness is affected by the level of the stellar magnetic activity. Indeed, the Sun and stars with low magnetic activity levels become photometrically brighter when their activity increases, while magnetically more active stars display the opposite behaviour and become fainter when their activity increases (Radick et al. 1990, Lockwood et al. 2007, Shapiro et al. 2014). Stellar Ca II observations are per force integrated over the whole stellar disk. However, except for the study of Harvey and White (1999), restricted to a few images, no investigation has determined the relation between Ca II brightness and magnetic field strength covering the full solar disc.

\footnotetext{
${ }^{1}$ Being of the order of a few tens of percent.
} 
Many studies require long data series of the solar surface magnetic field, e.g. to derive information on the structure, activity, and variability of the Sun, or for related applications such as e.g. Earth's climate response to solar irradiance variability. Regular magnetograms are, however, available only for the last four solar cycles. In recent years, following the availability of a few digitized series of historical Ca II K observations some attempts have been made to reconstruct magnetograms from Ca II K observations, based on the relation between the Ca II K intensity and magnetic field strength. In particular, Pevtsov et al. (2016) reconstructed magnetograms from Ca II K synoptic charts made from Mt Wilson observatory images. For their reconstruction they used sunspot records to get information about the polarity and assigned to each plage area a single magnetic field strength value based on the sunspot measurements. The areas and locations of plage regions were derived from photometrically uncalibrated Ca II K images, which were processed in a similar way as done by Bertello et al. (2010). There were two more studies (Sheeley 2008, Muñoz-Jaramillo et al. 2012) that inferred information about the polar magnetic field with a linear scaling to the amount of polar faculae found on Mt Wilson white light and Ca II K observations, respectively. Sheeley et al. (2011) and Chatterjee et al. (2016) constructed carrington maps with Ca II K images from the Mt Wilson and Kodaikanal observatories, respectively. These maps can be used to trace the evolution of the plage regions, however they provide $\mathrm{Ca}$ II $\mathrm{K}$ contrast and need to be converted into magnetic field strength.

In this chapter we present a new observational study of the relation between magnetic field strength and $\mathrm{Ca}$ II K intensity from full-disc observations derived from two archives of high quality solar observations. We used new observations from the space-borne Helioseismic and Magnetic Imager (HMI, Scherrer et al. 2012, Schou et al. 2012b) aboard the Solar Dynamics Observatory (SDO, Pesnell et al.2012) and the ground-based Precision Solar Photometric Telescope at the Rome Observatory (Rome/PSPT, Ermolli et al. 1998, 2007a), whose quality is superior to most previously available data. The amount of available data is also far greater than that used in previous studies, thus allowing us to extend the previous studies by analysing the relation between magnetic field strength and Ca II K intensity more accurately, over the whole disc and at different levels of solar activity during the current cycle. Here we test the accuracy of using the results derived from our study to model the variability of the solar surface magnetic field by its signature on available Ca II K data. This allows us to produce more accurate reconstructions of magnetograms. We provide additional constraints for modelling the stellar variability by studying the relation between the magnetic field strength and the $\mathrm{Ca}$ II $\mathrm{K}$ intensity for almost the entire solar disc and as a function of the size of the magnetic region. In this way we can make use of the wealth of available historical Ca II K spectroheliograms to reconstruct magnetograms throughout the whole 20th century.

This chapter is organised as follows. Section 5.2 describes the data and methods employed for our analysis. In Section 5.3 we present our results and discuss them in Section 5.4. In Section 5.5 we summarize the results of this study. 


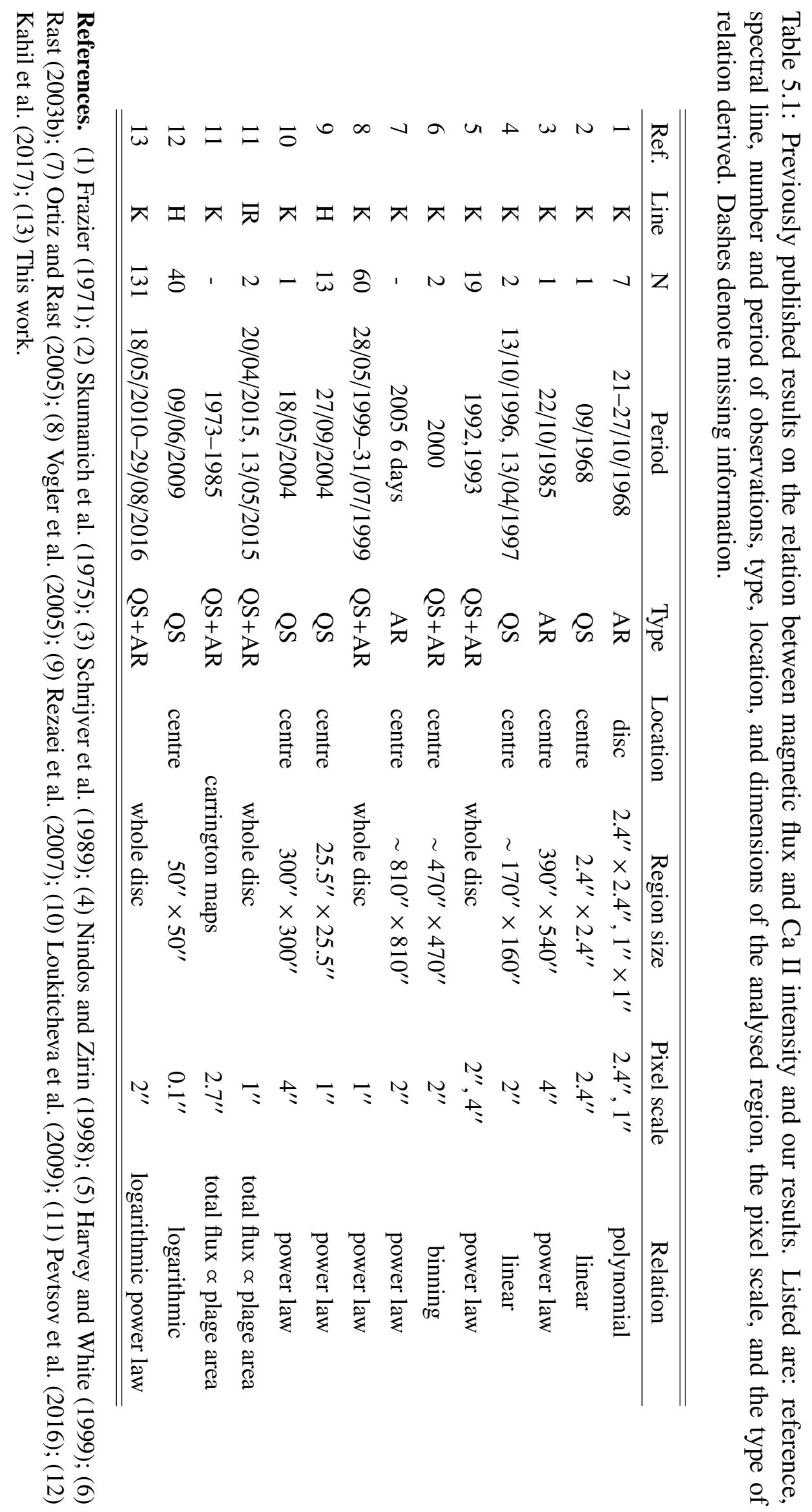




\subsection{Data and methods}

\subsubsection{Data}

We analysed full-disc photospheric longitudinal magnetograms and continuum intensity images from the SDO/HMI, and full-disc filtergrams taken at the $\mathrm{Ca}$ II $\mathrm{K}$ line and red continuum from the Rome/PSPT.

Rome/PSPT, in operation since 1996, is a $15 \mathrm{~cm}$ telescope designed for photometric solar observations characterized by $0.1 \%$ pixel-to-pixel relative photometric precision (Coulter and Kuhn 1994). The images ${ }^{2}$ analysed in this study were acquired with narrow-band interference filters within 3 minutes from each other, by single exposure of a $2048 \times 2048 \mathrm{CCD}$ array. The filter employed for these observations is centred at the $\mathrm{Ca}$ II $\mathrm{K}$ line core $(393.3 \mathrm{~nm})$ with bandwidth of $0.25 \mathrm{~nm}$, and in the red continuum at 607.2 $\mathrm{nm}$ with bandwidth of $0.5 \mathrm{~nm}$. At the acquisition, the data were reduced to a pixel scale of $2^{\prime \prime}$ to account for typical conditions of local seeing. Standard instrumental calibration has been applied to the data (Ermolli et al. 1998, 2010a).

SDO/HMI, in operation since April 2010, takes full-disc 4096×4096 pixel filtergrams at six wavelength positions across the Fe I $617.3 \mathrm{~nm}$ line at $1.875 \mathrm{~s}$ intervals. The filtergrams are combined to form simultaneous continuum intensity images and longitudinal magnetograms with a pixel scale of 0.505 arcsec and $45 \mathrm{~s}$ cadence. For each Rome/PSPT image pair, we took the 360s average of the SDO/HMI images and magnetograms taken close in time (on average less than 2 minutes apart and no more than 8 minutes). The averaging was done to suppress intensity and magnetogram signal fluctuations from noise and $p$-mode oscillations.

For our analysis, we have selected data with the highest spatial resolution (for Rome/PSPT), closest time between SDO/HMI and Rome/PSPT observations and highest signal-to-noise ratio. In our selection we avoided winter periods and kept observations mostly during summer months, when the seeing conditions are best. Our data sample consists of 131 sets of near-simultaneous observations covering the period 18/05/2010 to 29/08/2016.

\subsubsection{Data reduction}

We have ignored the pixels with flux density below $20 \mathrm{G}$. The value of $20 \mathrm{G}$ corresponds roughly to three times of the noise level as evaluated by Yeo et al. (2013, 2014b). Since the magnetic flux tubes making up network and faculae tend towards an orientation normal to the surface, while magnetograms measure the line-of-sight component of it ( $\left.B_{\mathrm{LOS}}\right)$, we have corrected the magnetograms for foreshortening by dividing the pixel signal by the corresponding $\mu$ (cosine of the heliocentric azimuthal angle). We also removed the polarity information from the SDO/HMI data, and only consider the absolute value of the magnetic flux density, $\left|B_{\mathrm{LOS}}\right| / \mu$ (i.e. the magnetic field strength averaged over the effective pixel). The Rome/PSPT images were initially rescaled to match the size of SDO/HMI so that we can align both observations with highest accuracy. The Rome/PSPT images were then rotated and aligned to the SDO/HMI observations, by applying compensations for ephemeris. Both observations were then re-scaled to the original dimensions of

\footnotetext{
${ }^{2}$ Available at http://www.oa-roma.inaf.it/solare
} 
a)

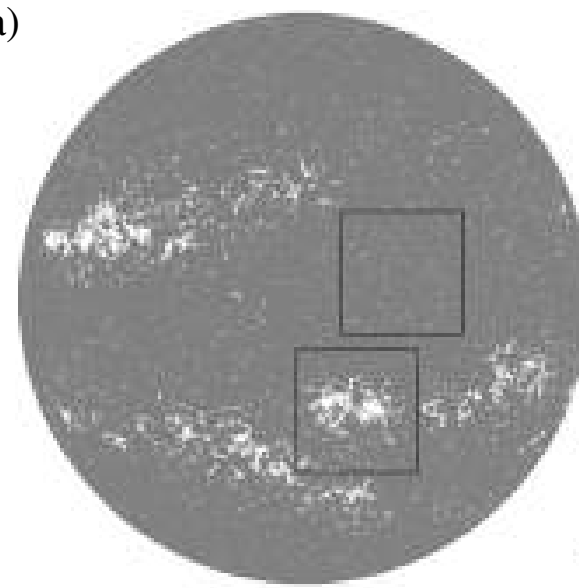

c)

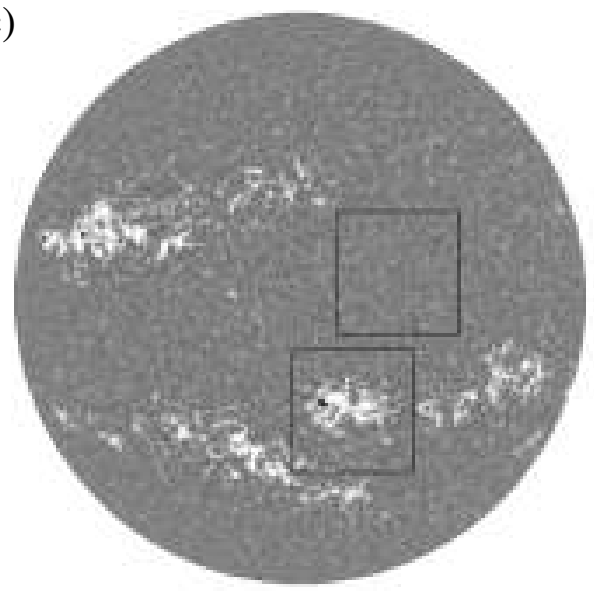

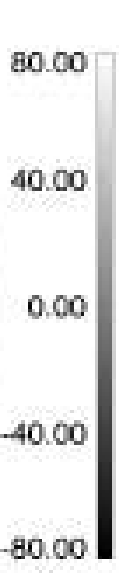

b)
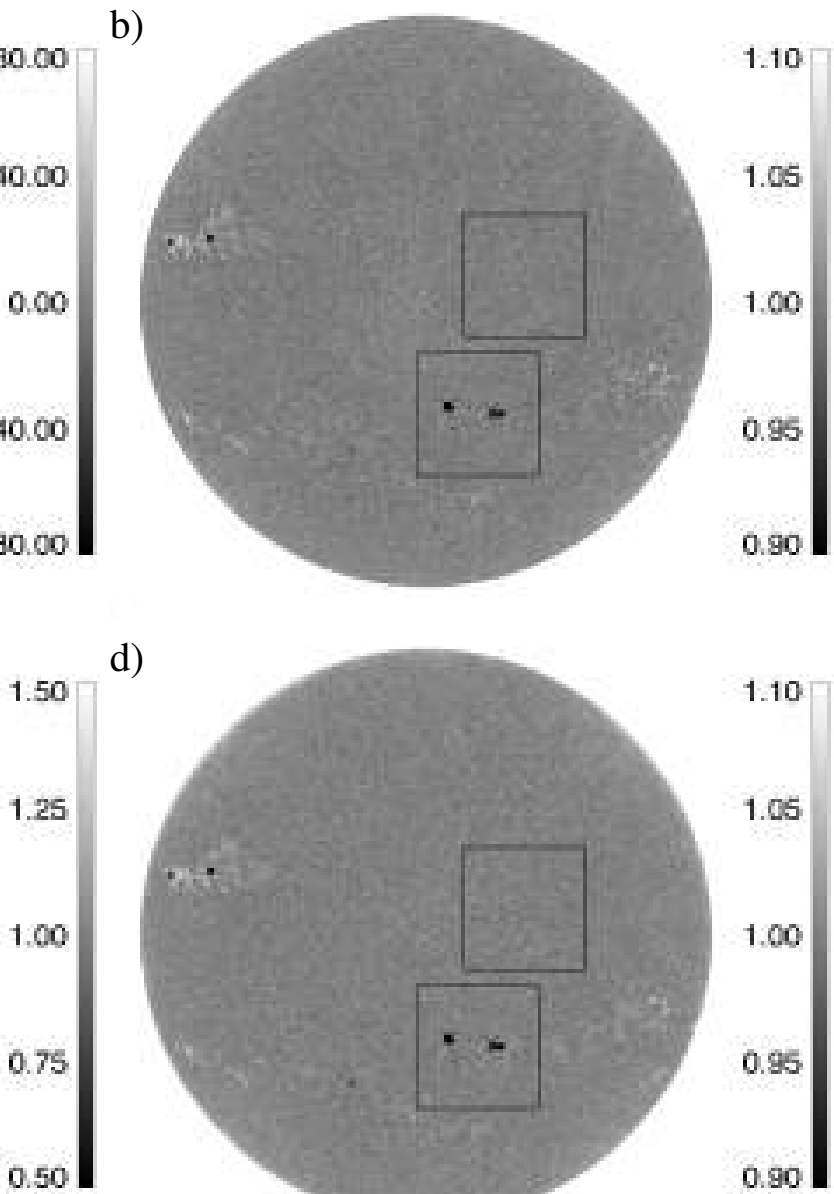

d)

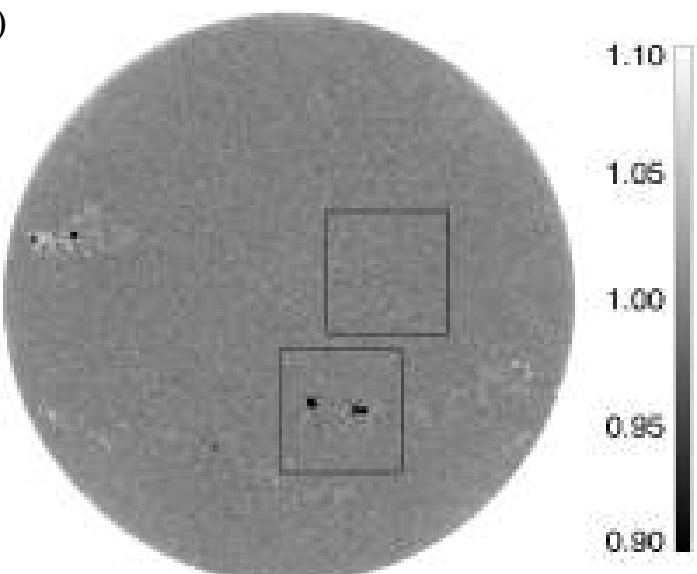

Figure 5.1: Examples of the observations analysed in this study taken on 01/04/2011 at 09:06:00 UT: (a)) SDO/HMI unsigned $B_{\text {Los }}$ magnetogram, (b)) SDO/HMI continuum contrast image (i.e. compensated for intensity CLV), (c)) Rome/PSPT Ca II K and (d)) red continuum contrast images. The grey-scale bars on the right-hand side of each panel show values in $\mathrm{G}$ and contrast, respectively. The squares denote the insets shown in Fig. 5.4 .

Rome/PSPT. To further reduce effects due to seeing, we also reduced the resolution of the SDO/HMI data to that of the Rome/PSPT by smoothing them with a low-pass filter with a 2 pixel running window width. In the following, we refer to the SDO/HMI data so obtained as SDO/HMI degraded magnetograms.

For each Ca II K image we removed the limb darkening and obtained a contrast map. In particular, for each image pixel $i$, we define its contrast $C_{i}$ as $C_{i}=I_{i} / I_{i}^{\mathrm{QS}}$ where $I_{i}$ is the measured intensity of pixel $i$, and $I_{i}^{\mathrm{QS}}$ is the intensity of the QS at the same position. The latter was derived from the iterative procedure described in Sect. 3.2.1. In short, we mapped $I^{Q S}$ by centring a window on each disc position and calculating the median. Bright features were excluded by replacing them with the results of fifth order polynomial fitting in the horizontal, vertical, and radial directions. Then the whole procedure was repeated until the result did not change any more. 
a)

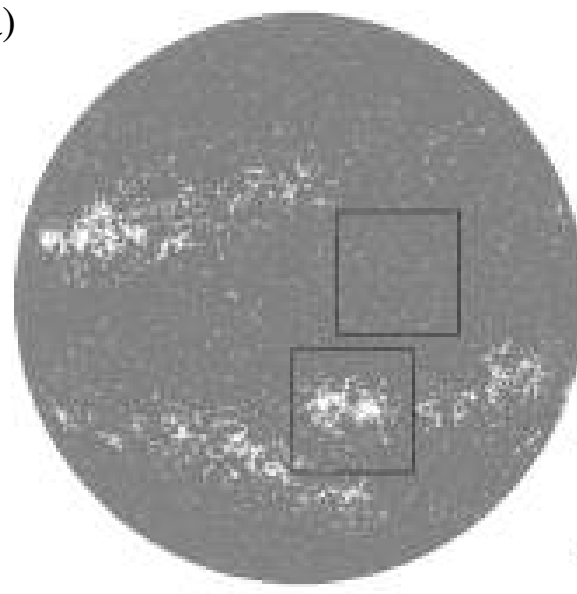

c)

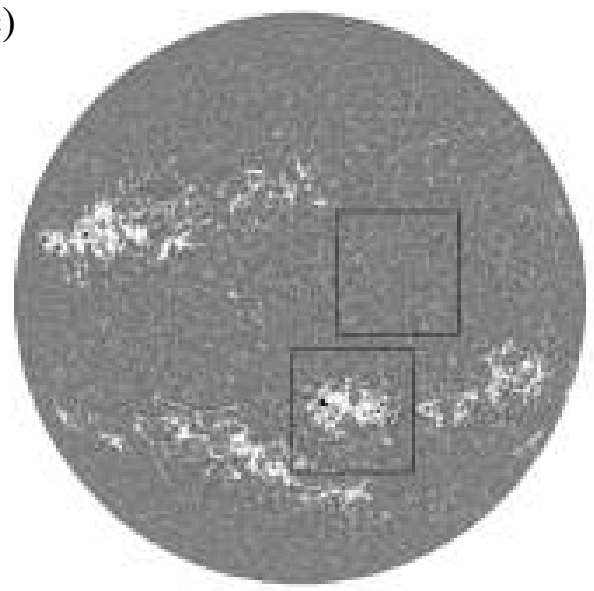

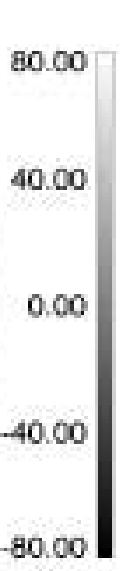

b)
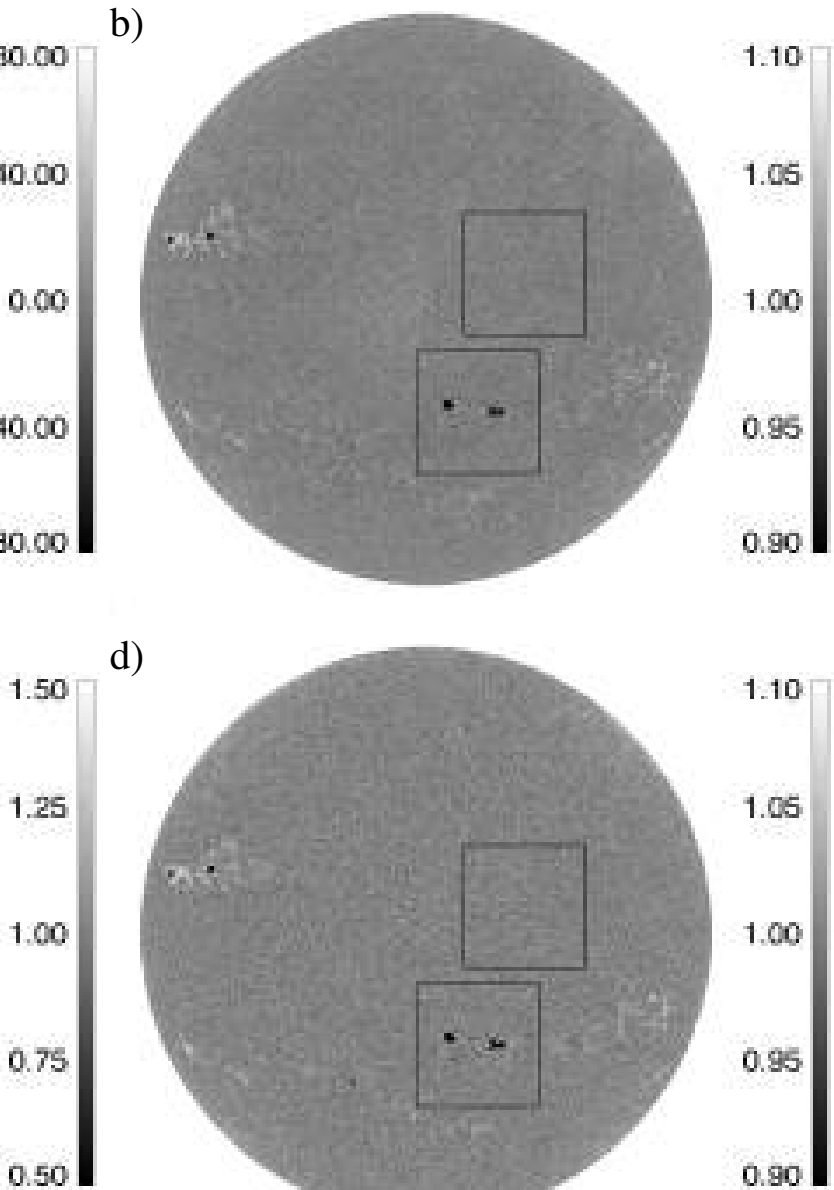

d)

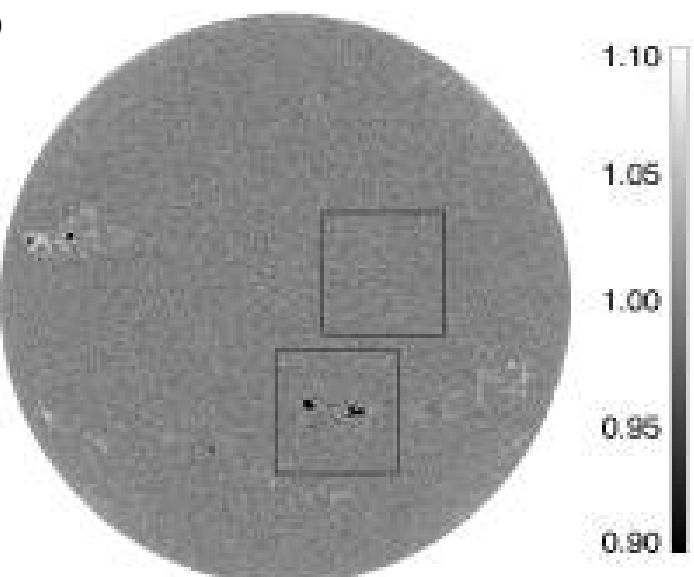

Figure 5.2: Same as Fig. 5.1 but for stray-light corrected data.

In this study we are interested in bright magnetic regions. Therefore, we masked out sunspots in the magnetograms and in Ca II K observations. Sunspots were identified in SDO/HMI continuum intensity as regions having intensity contrast lower than 0.89 (following Yeo et al. 2013) and in Rome/PSPT red continuum images lower than 0.95. The threshold for the red continuum images is derived as the average value of $\bar{I}-3 \sigma$ from all Rome/PSPT red continuum and SDO/HMI continuum images separately. The plage regions immediately surrounding sunspots were excluded as well, as they may be affected by stray-light and by extended low-lying sunspot canopies (e.g. Giovanelli and Jones 1982, Solanki et al. 1994, 1999), as was shown by Yeo et al. (2013). This was done by expanding the sunspot regions with a varying size kernel, corresponding to $10 \times 10$ and $30 \times 30$ pixel $^{2}$ at disc centre and limb, respectively.

Figure 5.1 shows examples of the analysed SDO/HMI and Rome/PSPT images.

\subsubsection{Stray-light removal}

We also investigate whether removal of stray-light on the analysed data affects our results. To this end, we restored 51 of the SDO/HMI and Rome/PSPT data as proposed by Yeo et al. (2014a) and Criscuoli and Ermolli (2008), respectively. In particular, for 
the SDO/HMI observations, the point-spread function (PSF) of the instrument was deconvolved from Stokes I and V observables that were then used to produce the stray-light corrected magnetograms; for the Rome/PSPT, the data were deconvolved by using analytical functions defined by modelling the centre-to-limb variation of intensity in the data and instrumental PSF. Figure 5.2 shows the same observations as in Fig. 5.1 after the stray-light removal.

\subsubsection{Segmentation}

For our analysis we also selected pixels that correspond to magnetic regions in magnetograms and bright regions in $\mathrm{Ca}$ II $\mathrm{K}$ images. Identifying individual bright magnetic features helps to investigate potential differences in the relation between magnetic field strength and $\mathrm{Ca}$ II $\mathrm{K}$ intensity in regions with different magnetic field strength. We identified features of interest with two different methods.

In the first method, we consider two different classes of magnetic features and separate them with simple cut-off thresholds depending on their contrast ranges in Ca II K images and $\left|B_{\mathrm{LOS}}\right| / \mu$ ranges in SDO/HMI magnetograms. Pixels within the same intensity range from different locations on the disc are grouped together. These features are labelled network, and plage, respectively. The ranges are $20 \mathrm{G} \leq\left|B_{\mathrm{LOS}}\right| / \mu<75 \mathrm{G}$ and $1.1 \leq$ $C<1.21$ for network and $\left|B_{\mathrm{LOS}}\right| / \mu \geq 75 \mathrm{G}$ and $C \geq 1.21$ for plage. The threshold for the plage in Ca II K images is the same as the one in Sect. 4.2 for CT. The threshold for the network in the magnetograms of $20 \mathrm{G}$ is roughly 3 times the noise level in HMI magnetograms. The threshold of plage for the magnetograms and the one for the network for Ca II K images were adapted to minimise the differences between the disc fractions calculated in the magnetograms and Ca II K images. Figure 5.3a) (b)) shows the derived mask of Rome/PSPT Ca II K (SDO/HMI magnetogram) with this segmentation method on the image shown in 5.1c) (a)). Plage regions are shown in red, network in green and QS in blue.

Figure 5.4 displays examples of insets of one active and one quiet region from the observations shown in Fig. 5.1. Also plotted are the corresponding masks of plage and network combined, illustrating that the bright features in the $\mathrm{Ca}$ II $\mathrm{K}$ images belong to magnetic regions and network in the magnetograms. The ARs appear slightly smaller and show smaller-scale features in the magnetograms than in the Ca II K data. This can occur for a variety of reasons. The flux tubes comprising the ARs expand with height in the solar atmosphere, therefore the ARs are expected to be more extended. Other possible reasons include lower spatial resolution and seeing effects due to Earth's atmosphere that are smearing the features in the Ca II K observations. However, these effects should be minimised after the degrading we applied to the magnetograms. The choice of the segmentation thresholds has an effect as well, if they are not consistent between the magnetograms and $\mathrm{Ca}$ II $\mathrm{K}$ images. We evaluated a variety of threshold combinations, but we were unable to match better the AR areas in the two observations without introducing even smaller scale features in the magnetograms. Therefore, we consider that the differences are mostly due to the expansion of the flux tubes.

The second method is similar to that used by Harvey and White (1999). With this method we aim at isolating individual activity clusters (which may be composed of multiple close or overlapping ARs). In this way we can study how the relation between the 

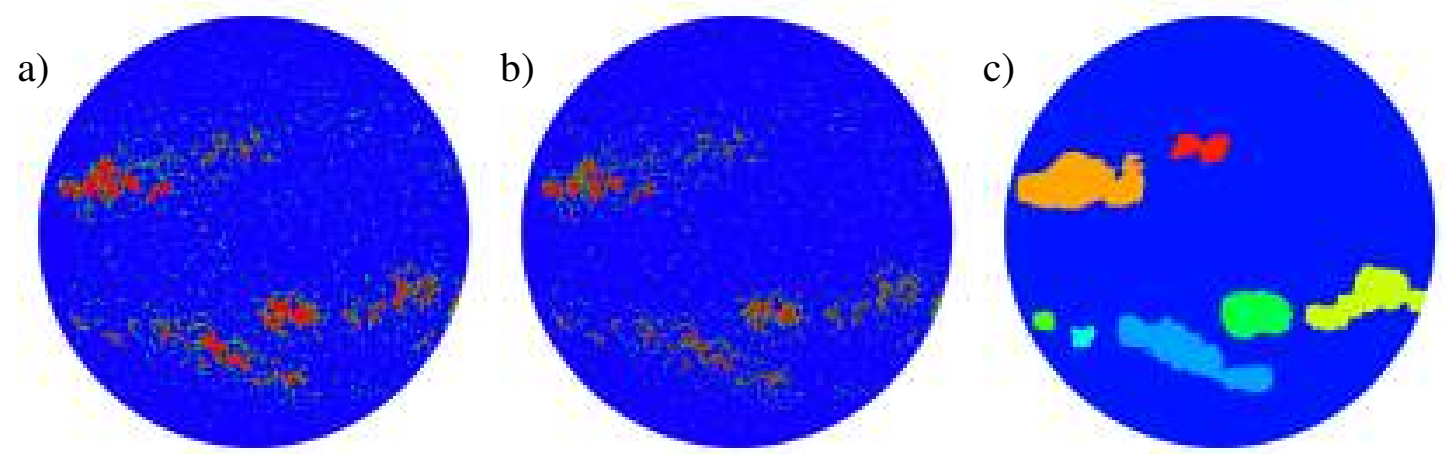

Figure 5.3: Segmentation masks of bright magnetic features derived with the two methods applied in our study for observations shown in Fig. 5.1. (a)) Mask of Ca II K image and (b)) magnetogram showing plage (red), network (green), and QS (blue). (c)) Mask of magnetogram aiming at isolating individual activity clusters, shown with different colours and the QS in blue. The masks are shown prior to the exclusion of the sunspot regions.

magnetic field strength and the Ca II K contrast varies among features of different sizes and locations on the disc. We apply a low pass-filter with a 50 pixel window width to the degraded magnetogram and a constant threshold of $\left|B_{\mathrm{LOS}}\right| / \mu=15 \mathrm{G}$ to isolate individual magnetic regions. Contiguous pixels are grouped together, and all isolated regions are considered as separate clusters. We also apply a 50 pixel threshold in the size of the clusters. Pixels not being assigned to any cluster are categorised as QS, though they include the network as well. Figure 5.3c) shows the mask derived with this segmentation method on the image shown in Fig. 5.1 a). The different features are shown with different colours, while the QS is shown in blue.

In our analysis we exclude all pixels with $\mu<0.14$ (outermost 0.01 of the solar radius) to restrict errors due to projection effects. Finally, the sunspot regions were excluded from all masks as described in Sect. 5.2.2.

\subsection{Results}

\subsubsection{Physical relationship}

We start by considering the data without the corrections for stray-light and without performing any segmentation other than excluding the sunspot regions. We take pairs of pixels from all the available degraded magnetograms and the corresponding $\mathrm{Ca}$ II K observations for all disc locations with $\mu>0.14$. Figure 5.5 shows the relation between the $\mathrm{Ca}$ II K brightness and $\left|B_{\mathrm{LOS}}\right| / \mu$ for all pairs of the degraded magnetograms and corresponding Ca II K images considered in our study. Each dot represents one pair of pixels.

The Spearman correlation coefficient between $\left|B_{\mathrm{LOS}}\right| / \mu$ and Ca II K contrast supports a monotonous relationship. The coefficient obtained for individual images is on average $\rho=0.60$, while it is $\rho=0.98$ for all pixels from all data. The significance level is zero with double-precision accuracy, implying a highly significant correlation.

Figure 5.5 shows that the Ca II K contrast increases with increasing magnetic field strength, but tends to saturate at high $\left|B_{\mathrm{LOS}}\right| / \mu$. The purple curve in Fig. 5.5 is a run- 
ning mean over $\left|B_{\mathrm{LOS}}\right| / \mu$ values. It suggests that the relation saturates at around $400 \mathrm{G}$. However, binning the data over the Ca II K contrast values (light blue curve), suggests a somewhat different relation. Attenuation bias due to errors in the independent variable in each case can cause these relations to skew compared to the true relationship. The choice of the quantity over which the binning is performed affects the exact form of the relation between the magnetic field strength and the $\mathrm{Ca}$ II $\mathrm{K}$ intensity. Therefore, we also perform the fit on the bisector between the binned curves assuming each of the two different quantities as independent variables (yellow curve).

In order to find the best relation describing the data, we considered three different functions: a) a power law with an offset (PF) as commonly used in the literature (e.g. Schrijver et al. 1989, Harvey and White 1999, Ortiz and Rast 2005, Rezaei et al. 2007, Loukitcheva et al. 2009); b) a logarithm (LFL) as proposed by Kahil et al. (2017); and c) a power law function of the logarithm of $\left|B_{\mathrm{LOS}}\right| / \mu$ (PFL). These three functions can be described by the following equation:

$$
C=a_{1}+a_{2} x^{a_{3}},
$$

where $x=\left|B_{\mathrm{LOS}}\right| / \mu$ for PF, and $x=\log \left(\left|B_{\mathrm{LOS}}\right| / \mu\right)$ for PFL and LFL (with $a_{3}$ being 1 for LFL). We perform these fits on the bisector of the two running means based on all selected pixel pairs from all images, where $\left|B_{\mathrm{LOS}}\right| / \mu>20 \mathrm{G}$. However, just for comparisons we also perform the fits on the curve derived by binning the data in $\left|B_{\mathrm{LOS}}\right| / \mu$ intervals, we refer to these fit as $\mathrm{PF}^{*}, \mathrm{PFL}^{*}$, and LFL*. Table 5.2 lists the derived parameters. Both $\mathrm{PF}$ and PFL give low values for $\chi^{2}$, being $\simeq 0.03$ and $\simeq 0.01$, respectively. The fit is not satisfactory for the LFL which gives $\chi^{2} \simeq 3.31$.

The fits with the three tested functions are also shown in Fig. 5.5. PF and PFL closely follow each other up to about $400 \mathrm{G}$, but slightly diverge at higher magnetic field strengths, with PFL following the binned curve more closely. They also differ in the extrapolated region for $\left|B_{\mathrm{LOS}}\right| / \mu<20 \mathrm{G}$, with PFL giving higher contrasts. However, the differences between the two curves are minute. LFL fails to reproduce the binned curve for low magnetic fields, but follows the curve binned over $\left|B_{\mathrm{LOS}}\right| / \mu$ at high magnetic field strengths.

The exponent derived for PF is consistent with those obtained by Schrijver et al. (1989), Harvey and White (1999), Ortiz and Rast (2005) favouring an exponent of 0.6, $0.69,0.65$, respectively for all bright features considered. This value is higher than those derived by Rezaei et al. (2007), Loukitcheva et al. (2009) and Vogler et al. (2005). The difference to the results by Loukitcheva et al. (2009) (exponent of 0.31) can potentially be explained by the different threshold in the magnetic field strength used by the two studies. Loukitcheva et al. (2009) used a threshold of $1.5 \mathrm{G}$, and showed that the exponent increases to roughly 0.6 if a threshold of $20 \mathrm{G}$ is used. The same stands for Rezaei et al. (2007) who found the exponent to increase to 0.51 when the threshold was $20 \mathrm{G}$. Our results for LFL differ from those presented by Kahil et al. (2017): $a_{1}=0.29 \pm 0.003$ and $a_{2}=0.51 \pm 0.004$ for $\left|B_{\mathrm{LOS}}\right| / \mu>50 \mathrm{G}$ in $\mathrm{Ca}$ II H data. The differences can be due to different atmospheric heights sampled in the analysed data, as well as due to the lower spatial resolution of the observations used here compared to those used by Kahil et al. (2017). Thus, LFL might be not an appropriate function for the data we use here.

We also studied how these results depend on the $\left|B_{\mathrm{LOS}}\right| / \mu$ threshold applied. Figure 5.6 shows the parameters derived by applying all three functions to the data shown in Fig. 
Table 5.2: Parameters of the three fit functions (Eq. 5.1) to all pixel pairs of all degraded magnetograms and Ca II K observations (as described in Sect. 5.3.1). Also listed are the $\chi^{2}$ of the fits. Also listed are the results of the fits of the three functions to the curve derived after binning in $\left|B_{\mathrm{LOS}}\right| / \mu$.

\begin{tabular}{lccccc}
\hline \hline Function & $x$ & $a_{1}$ & $a_{2}$ & $a_{3}$ & $\chi^{2}$ \\
\hline PF & $\left|B_{\mathrm{LOS}}\right| / \mu$ & $1.004 \pm 0.004$ & $0.014 \pm 0.001$ & $0.67 \pm 0.01$ & 0.03 \\
PFL & $\log \left(\left|B_{\mathrm{LOS}}\right| / \mu\right)$ & $1.064 \pm 0.002$ & $0.015 \pm 0.001$ & $3.93 \pm 0.06$ & 0.01 \\
$\mathrm{LFL}$ & $\log \left(\left|B_{\mathrm{LOS}}\right| / \mu\right)$ & $0.622 \pm 0.003$ & $0.347 \pm 0.002$ & 1.00 & 3.31 \\
$\mathrm{PF}^{*}$ & $\left|B_{\mathrm{LOS}}\right| / \mu$ & $0.995 \pm 0.001$ & $0.015 \pm 0.001$ & $0.62 \pm 0.01$ & 0.03 \\
$\mathrm{PFL}^{*}$ & $\log \left(\left|B_{\mathrm{LOS}}\right| / \mu\right)$ & $1.089 \pm 0.001$ & $0.035 \pm 0.001$ & $2.84 \pm 0.01$ & 0.14 \\
LFL $^{*}$ & $\log \left(\left|B_{\mathrm{LOS}}\right| / \mu\right)$ & $0.980 \pm 0.001$ & $0.105 \pm 0.001$ & 1.00 & 4.31 \\
\hline \hline
\end{tabular}

5.5 and varying the threshold in $\left|B_{\mathrm{LOS}}\right| / \mu$ between 1 and $50 \mathrm{G}$. For PF and PFL we show only the exponents, though the other parameters of the tested functions are affected as well. The exponents for PF and PFL reach a plateau after a threshold of $\sim 8$ and $\sim 18 \mathrm{G}$, respectively, and after that they tend to slightly decrease. The two parameters of LFL show opposite behaviours: $a_{1}$ is constantly decreasing, while $a_{2}$ is increasing over the range of thresholds considered here. The curves derived with PF and PFL show a stable behaviour for thresholds greater than $\sim 8$ and $\sim 18 \mathrm{G}$, respectively showing that the differences in the exponents are due to compensation in the other parameters of the fit. The PFL exponents are more stable against the choice of the $\left|B_{\mathrm{LOS}}\right| / \mu$ threshold.

\subsubsection{Exponents over time and different $\mu$ positions}

We also study if the exponents of the fits change with the activity level and the position on the disc. To understand the change with time we performed the fits to every image separately first for all pixels with $\mu>0.14$, and then for the plage and network regions separately, as defined with the first segmentation method. To study the variation of the exponent we fixed $a_{1}$ and $a_{2}$ for the PF and PFL fits to the values derived in Sect. 5.3.1 and are listed in Table 5.2. The parameters were let free for LFL though. Figure 5.7 shows the coefficients of the fits as a function of time. The resulting exponents for PF and PFL depend on the type of feature and are slightly higher for plage than the network. Performing the fit to all pixels on the disc with $\mu>0.14$ and each image separately, we find an average exponent of $0.66 \pm 0.01$ and $3.9 \pm 0.1$ for PF and PFL, respectively. The average parameters for LFL are $a_{1}=0.64 \pm 0.06$ and $a_{2}=0.33 \pm 0.04$. The errors are the $1 \sigma$ intervals among all the daily calculated values. These values agree within the $1 \sigma$ uncertainty level with those we derived in Sect. 5.3.1 for all three functions. As seen in Fig. 5.7 the scatter of the resulting exponents is such that within the limits of the current analysis, we see no evidence that the relationship between $\left|B_{\mathrm{LOS}}\right| / \mu$ and Ca II K intensity varies over the solar cycle. We notice exactly the same behaviour for the plage component for PF and PFL. There are some changes in the network component that result in higher exponents for the low activity period in 2010 for PF and PFL, but they are still constant in time within the derived uncertainties. Harvey and White (1999) analysed data from 
three observatories and segmented the features into 4 sub-categories. Their exponent for the feature class they term enhanced network is higher than the one we derived here for network or plage. For all other feature classes the results of Harvey and White (1999) for Kitt Peak data are consistent with ours, favouring exponents of 0.62-0.73. However, their analysis for data from Sacramento Peak or Big Bear Solar Observatory returns lower exponents (0.47-0.58).

We have also studied the dependence of the relationship on the heliocentric angle. Figure 5.8 shows the coefficients of the various features as a function of their position on the solar disc. We considered 10 concentric annuli of equal area covering the solar disc up to $\mu=0.14$. The mean values of the exponents computed over the various annuli slightly decrease towards the limb, but their standard deviation increases so that the exponents do not show any significant variation with the position on the disc (within the $1 \sigma$ uncertainty). In particular, the relative difference between the average value of the exponents within the innermost and outermost annuli for PF (PFL) is 6\% (10\%). The same behaviour is seen even when network and plage regions are considered separately. Our finding of such a small CLV of the computed exponents supports the assumption that these are mainly vertical magnetic fields that lead to the Ca II K excess brightness.

\subsubsection{Exponents for individual activity clusters}

To test if the results are the same for individual activity clusters, we segmented the images with the second method and repeated fitting the three tested functions to each individual cluster separately. We also considered the QS (including the network) separately. Figure 5.9 shows the Ca II K contrast plotted against $\left|B_{\mathrm{LOS}}\right| / \mu$ for the images shown in Fig. 5.1 . The individual pixels lying within the patches identified as an activity cluster with the second segmentation method are shown with coloured dots. Figures 5.10 and 5.11 show scatter plots for the same images, but now including only the pixels corresponding to activity clusters and QS, respectively. The binned curves from different activity clusters are in agreement with each other, with the exception of one cluster. However, this cluster is very small in size and the statistics are worse than for the other clusters. The relation shown by QS regions shows a smaller slope than the one obtained for active regions. However, this is probably due to a much lower number of QS and network pixels with strong magnetic fields resolved in the analysed SDO/HMI degraded data.

Results for different clusters agree well with each other within the accuracy of the fit. Table 5.3 lists the results for each cluster for observations taken in June 2014 (close to the maximum of cycle 24). Averaging all exponents derived for clusters (QS and network) from all images give on average the values of $0.67 \pm 0.03(0.64 \pm 0.01)$, and $3.9 \pm 0.2$ $(3.7 \pm 0.1)$, for PF and PFL respectively. The exponents derived here are in agreement with those derived in the previous subsections. We find no dependence on $\mu$ for the derived exponents with this segmentation method either.

Figure 5.12 shows the exponents derived with PF (red) and PFL (blue) as a function of the disc fraction of the clusters. We see no dependence of the exponent on the feature size, however the uncertainty of the derived parameters is obviously higher for smaller features. This happens because of poorer statistics. Also localised effects of potential misalignment become more significant in this case. 
a)

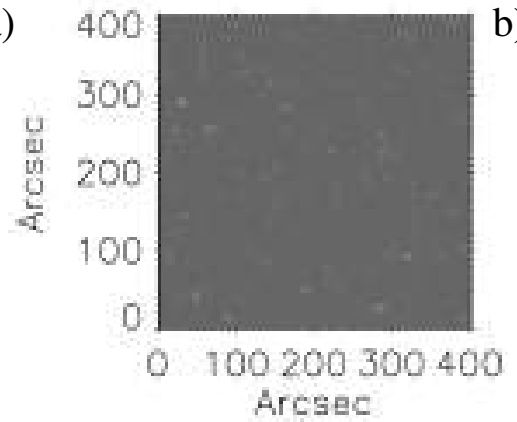

c)

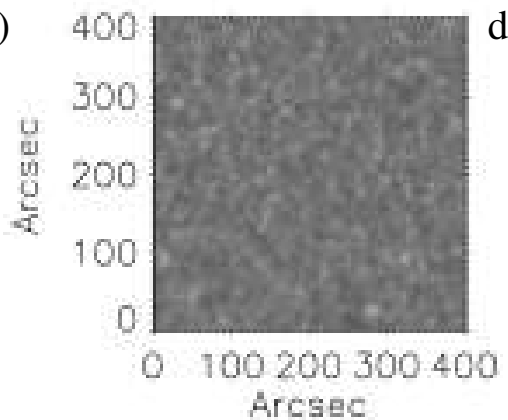

e)

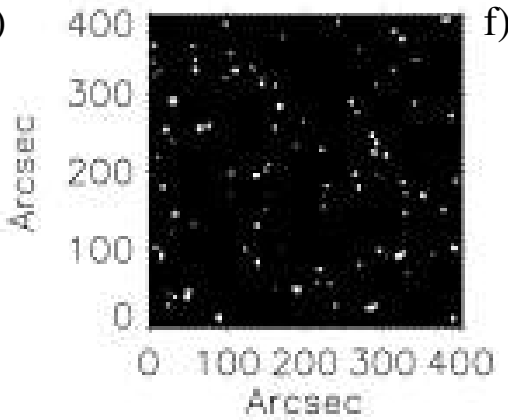

g)

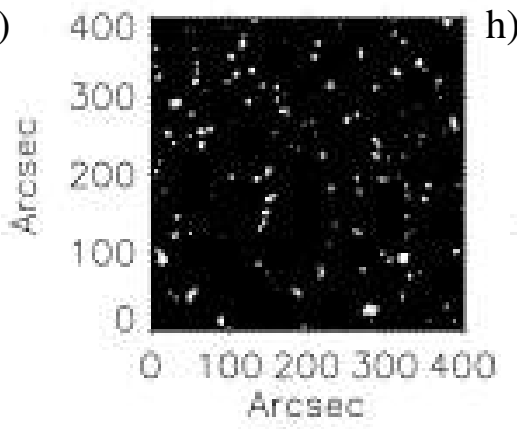

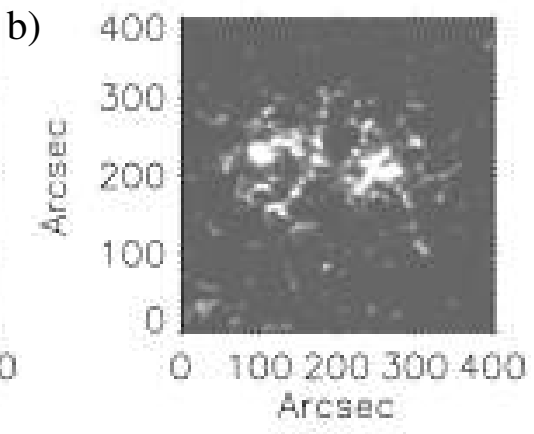

d)

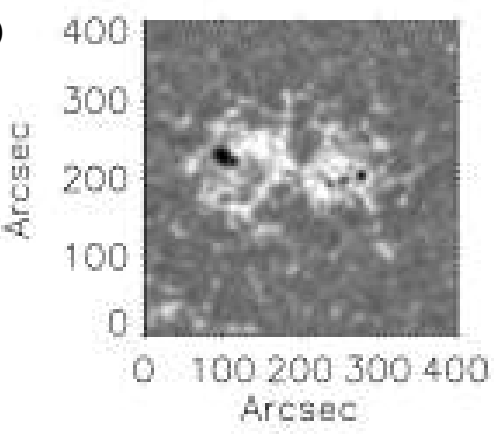

f)

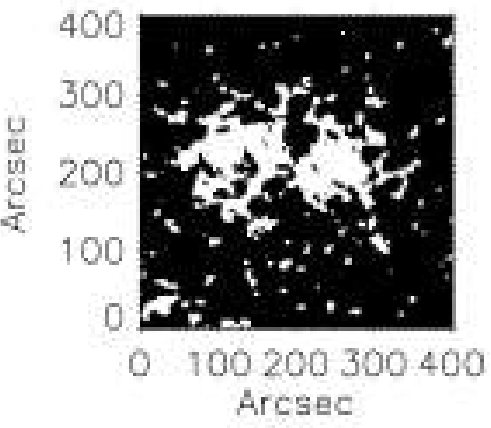

h)

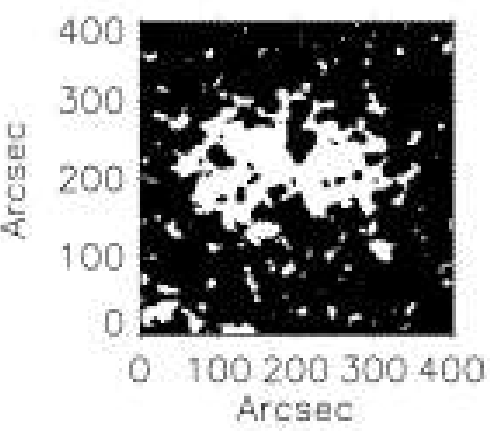

Figure 5.4: Magnified $400 \times 400 \operatorname{arcsec}^{2}$ parts of the images shown in Fig. 5.1 for a network (left) and a faculae region (right). SDO/HMI unsigned (and spatially degraded) magnetogram (a), b)); Rome/PSPT Ca II K (c), d)), and their segmentation masks derived with constant thresholds (e), f)) from the magnetograms and $(\mathbf{g}), \mathbf{h})$ ) from the Ca II K images. The magnetograms are saturated in the range [-300,300] $\mathrm{G}$ (the negative value was chosen merely to improve visibility of the pixels), while the Ca II K observations in the range $[0.5,1.6]$ (the QS has the average value of 1). 


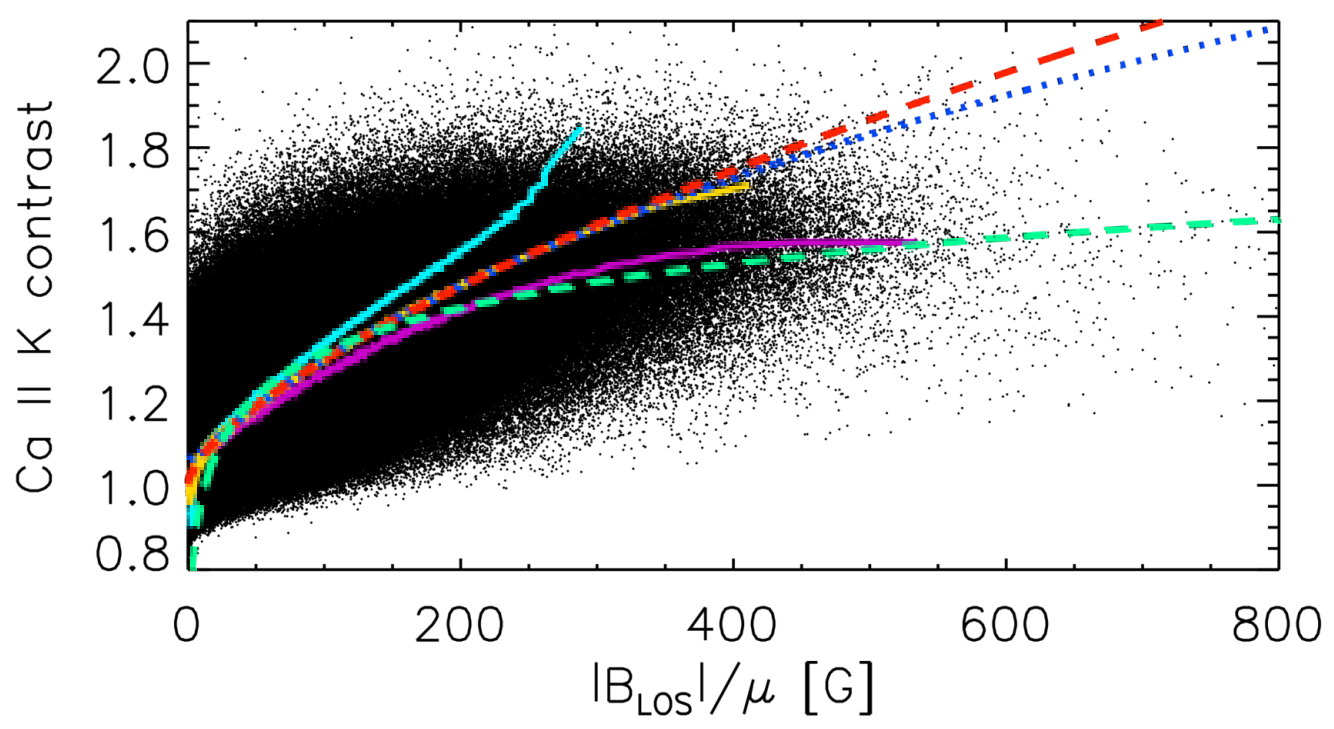

Figure 5.5: Ca II K contrast plotted against the unsigned line-of-sight magnetic flux density divided by $\mu\left(\left|B_{\mathrm{LOS}}\right| / \mu\right)$ for all pixel pairs (excluding sunspots) in all available images (black dots). Curves show 5000-point running means (over $\left|B_{\mathrm{LOS}}\right| / \mu$ in purple, over $C$ in light blue, and their average in yellow), as well as power law function (PF, red), power law function of $\log \left(\left|B_{\mathrm{LOS}}\right| / \mu\right)$ (PFL, blue) and logarithmic function (LFL, green) fits on the bisector between the binned curves assuming each of the two different quantities as independent variables (yellow curve). 

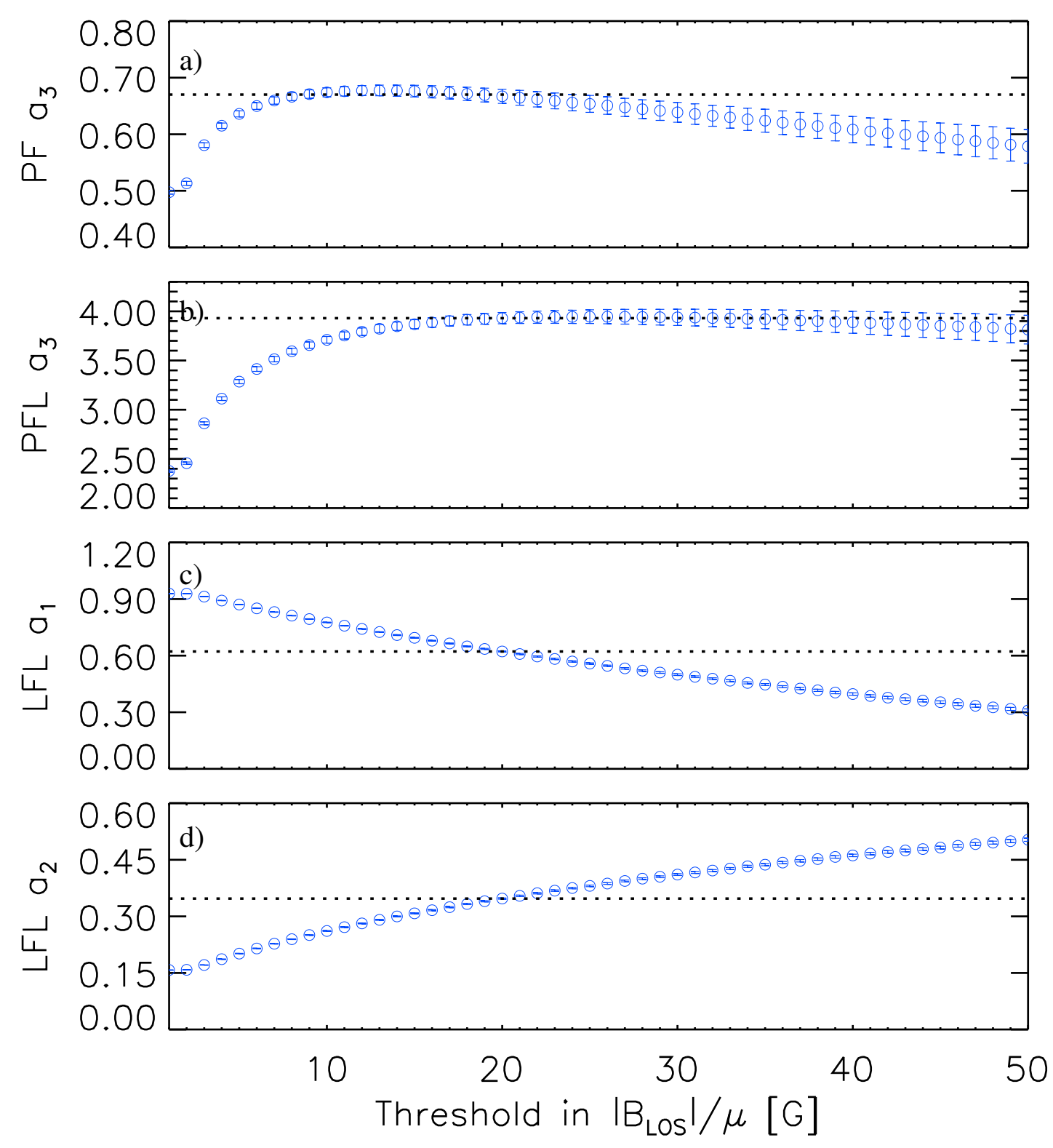

Figure 5.6: Parameters of the fits (Eq. 5.1) as a function of the threshold in $\left|B_{\mathrm{LOS}}\right| / \mu$, derived for the whole disc for PF (a)), PFL (b)), and LFL (c),d)) fits. The dotted line in each panel is the best fit parameter derived with the threshold of $\left(\left|B_{\mathrm{LOS}}\right| / \mu\right)=20 \mathrm{G}$. 

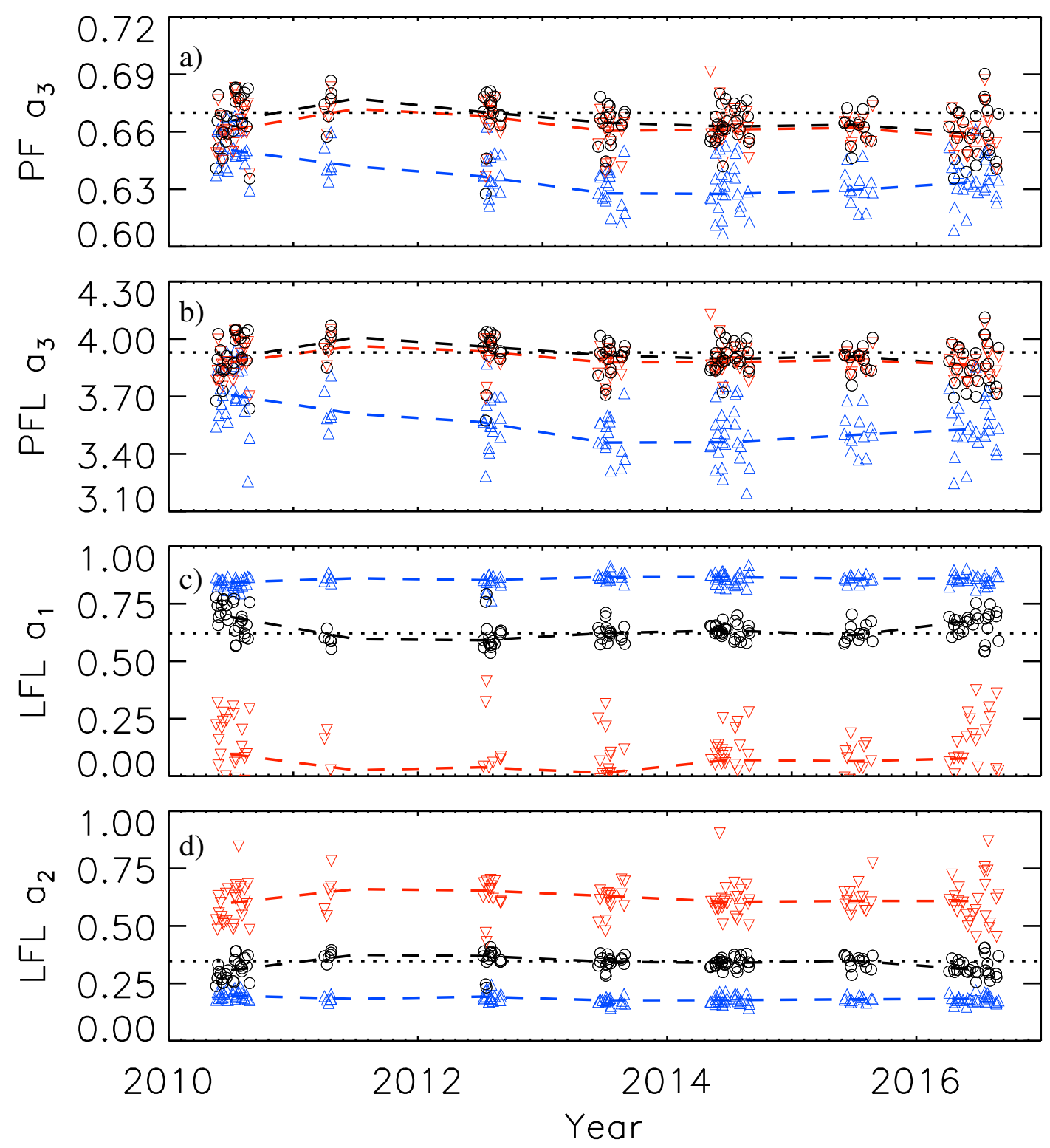

Figure 5.7: Parameters of the fits (Eq. 5.1) as a function of time, derived for the whole disc for all bright features (black), as well as for the network (blue), and plage (red) separately, for PF (a)), PFL (b)), and LFL (c),d)) fits. The dashed lines connect the median values obtained from all analysed images within a given year, while the dotted lines mark the values of the parameters of the best fit derived in Sect. 5.3.1. 

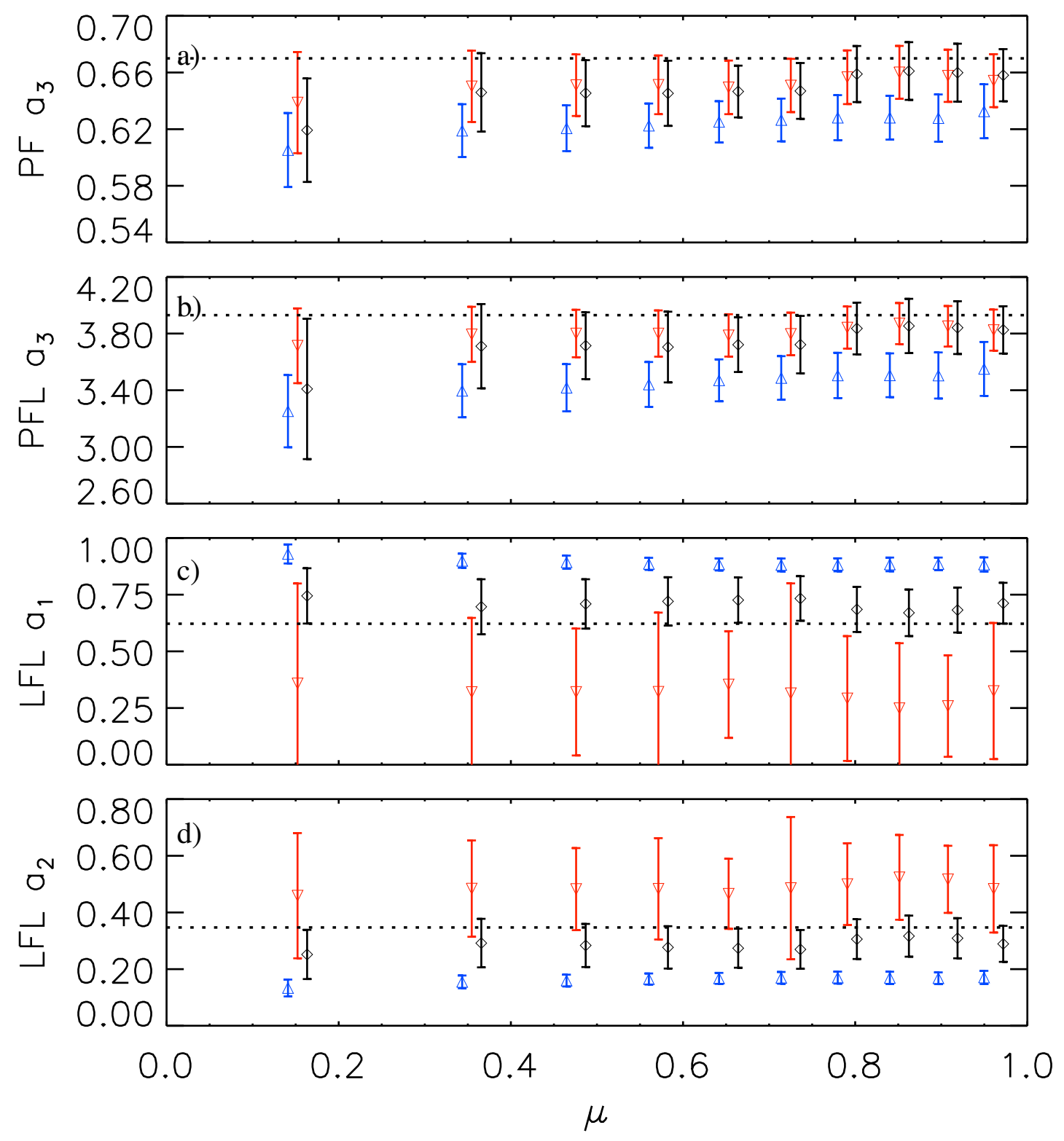

Figure 5.8: Parameters of the fits (Eq. 5.1) as a function of $\mu$, derived for 10 annuli of equal area for all bright features (black), as well as for the network (blue), and plage (red) separately, for PF (a)), PFL (b)), and LFL (c),d)). Results for the network are shown in the middle of the $\mu$ interval they represent, while the others are slightly shifted in $\mu$ to improve the clarity of the plot. The dotted lines mark the values of the best fit parameters derived in Sect. 5.3.1 


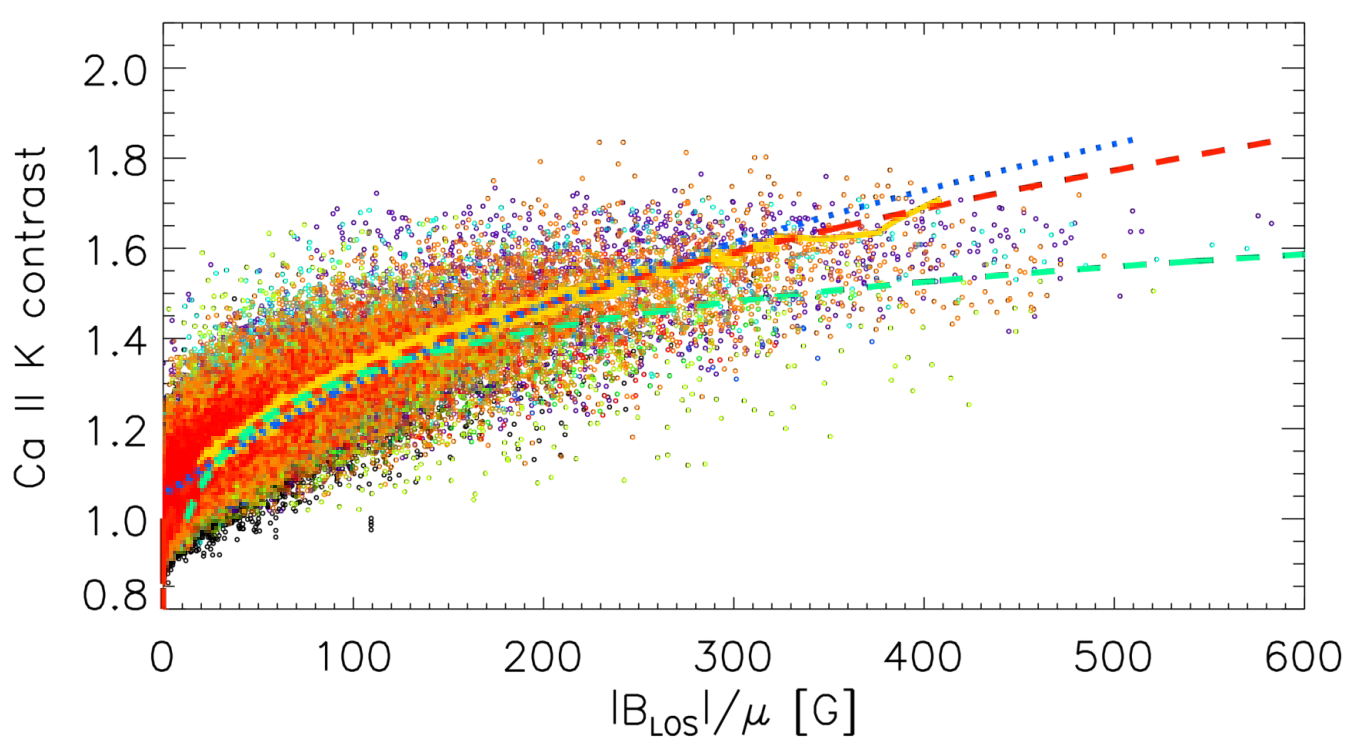

Figure 5.9: Ca II K contrast plotted against $\left|B_{\mathrm{LOS}}\right| / \mu$ for all individual activity clusters identified with the second method in observations shown in Fig. 5.1 (coloured dots). Each cluster is shown with the same colour as in Fig. 5.3 c). The yellow curve is the bisector of 50-point running mean curves assuming each of the two different quantities as independent variables independently of clusters. Also shown are the PF fit (red), PFL fit (blue), and LFL (green) fits.

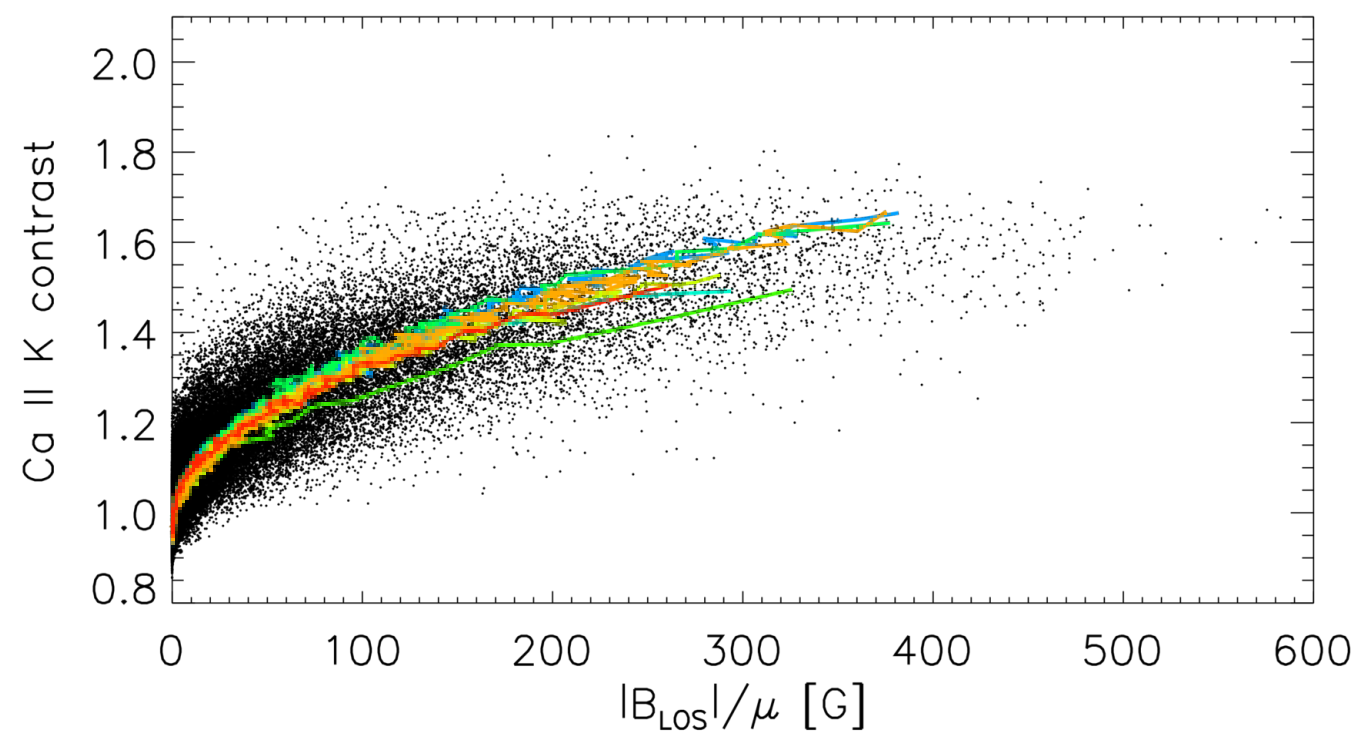

Figure 5.10: Ca II K contrast plotted against $\left|B_{\mathrm{LOS}}\right| / \mu$ for all activity clusters identified with the second method in observations shown in Fig. 5.1. The coloured curves are 50point running means for the individual clusters, shown with the same colours as in Fig. $5.3 \mathrm{c})$. 


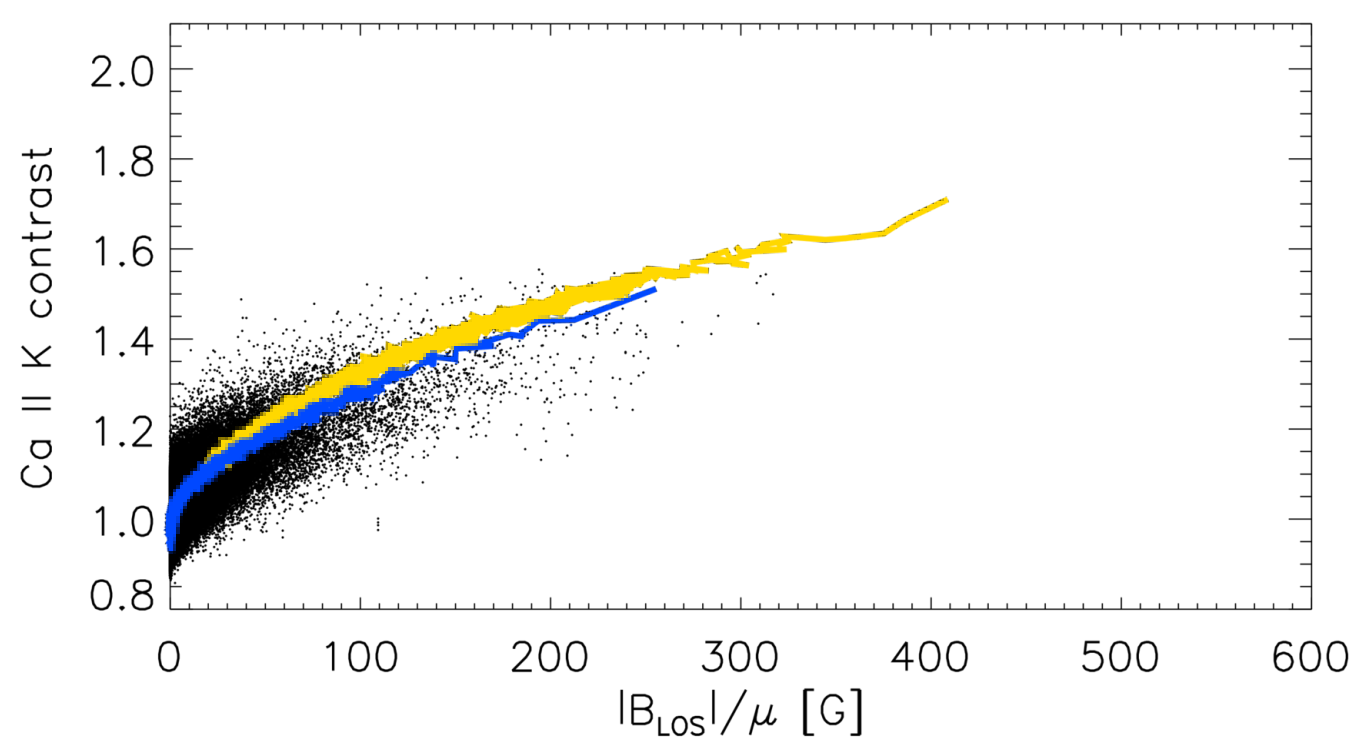

Figure 5.11: Ca II K contrast plotted against $\left|B_{\mathrm{LOS}}\right| / \mu$ for all QS and network regions identified with the second method in observations shown in Fig. 5.1. Shown also are 50-point running means for the QS (blue) and all (yellow) regions.

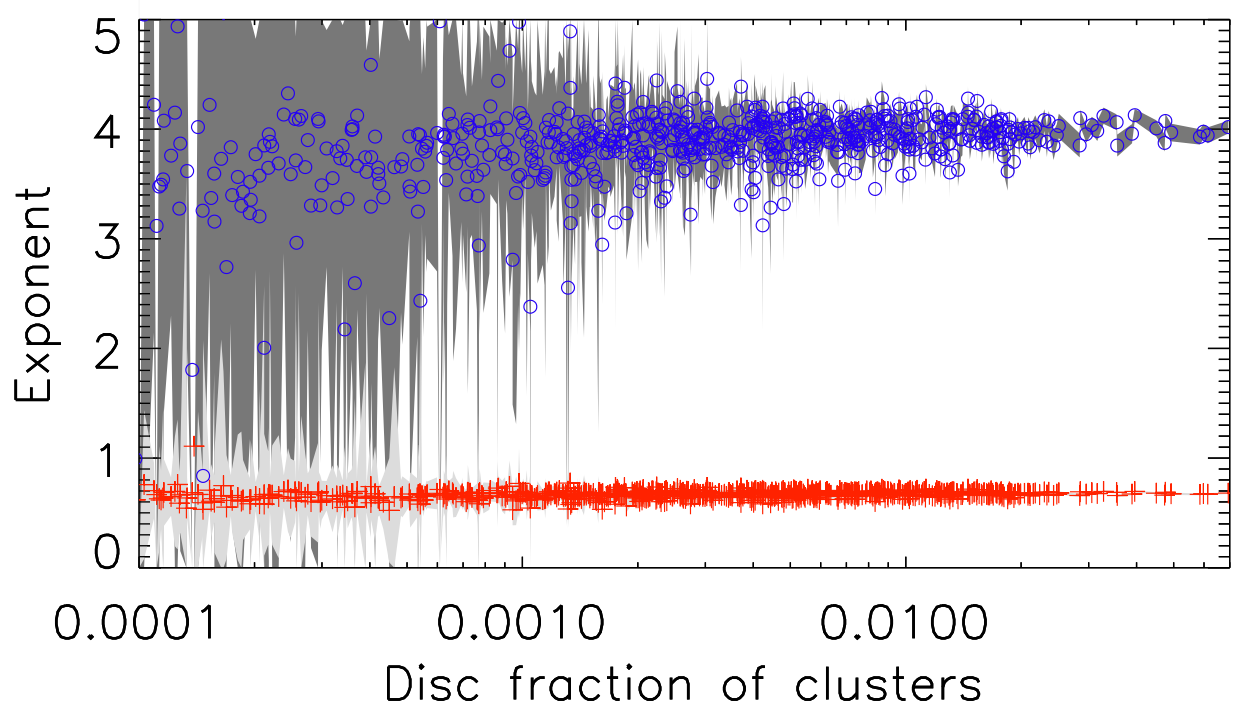

Figure 5.12: Exponents of the PF (red) and PFL (blue) fits as a function of size for individual activity clusters. The shaded areas denote the $1 \sigma$ error in the fit parameters. 


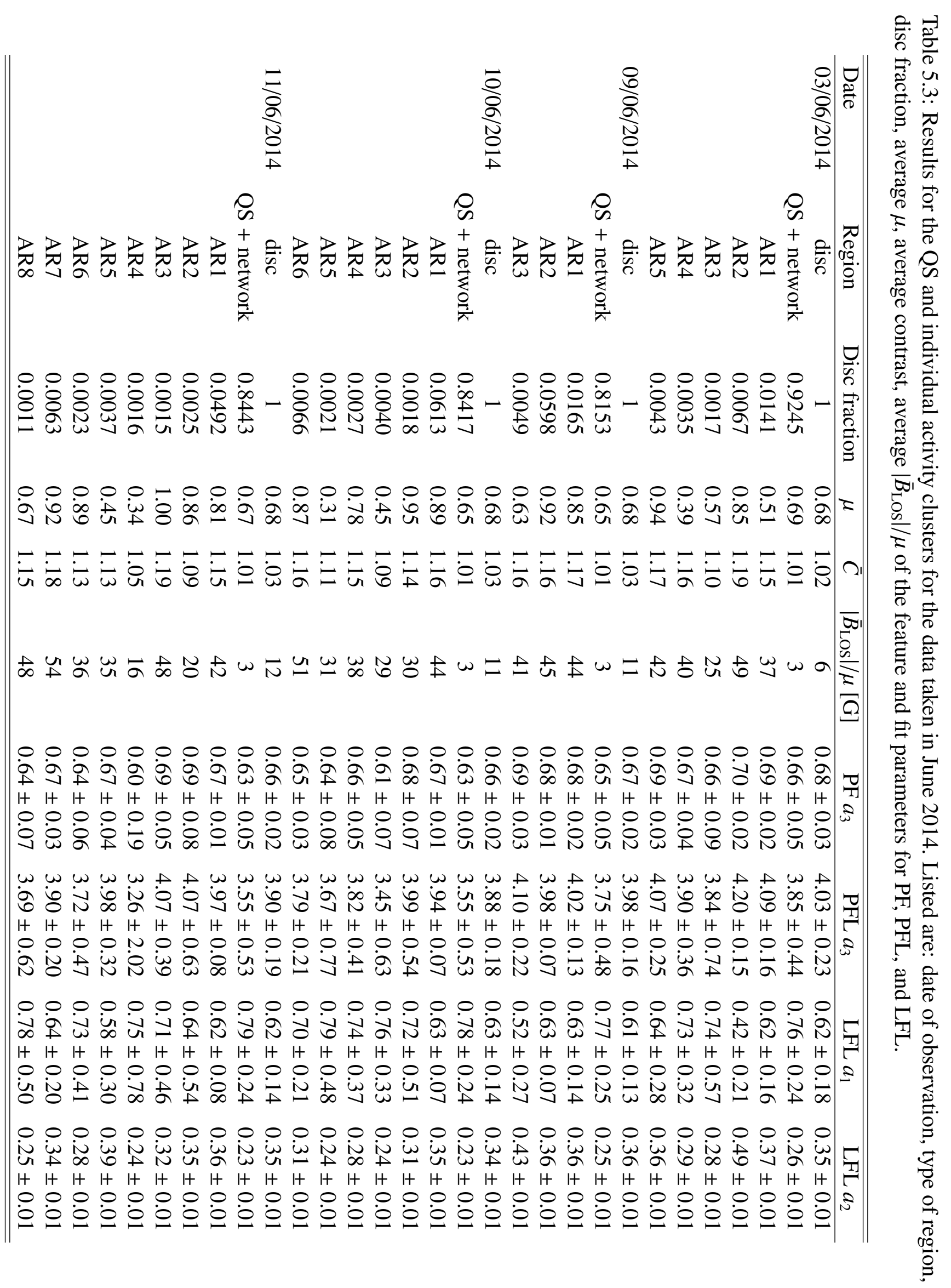




\subsubsection{Reconstructing unsigned magnetograms from Ca II K images}

In the previous sections we showed that the exponents of the functions tested in our study remained constant in time and did not depend on $\mu$. This allows us to reconstruct pseudomagnetograms from the full-disc Ca II K observations simply by using of the derived parameters. We investigate the accuracy of such process by comparing the reconstructed magnetograms to the original magnetograms. Applied to earlier Ca II K observations, this approach would allow a reconstruction of magnetograms back to the beginning of the 20th century.

We use the Ca II K observations and apply on them the 3 tested relations with their respective best fit parameters derived in Sect. 5.3.1 and listed in Table 5.2. Here we also use $\mathrm{PF}^{*}$ to reconstruct magnetograms in order to compare the results with the PF. The pixels with $C \leq 1$ are set to $0 \mathrm{G}$. We thus produce four synthetic magnetograms from each Ca II $\mathrm{K}$ observation. This approach suffers from the limitation that it does not allow the polarity of the magnetic field to be recovered. However, this is not a problem for a number of studies and applications, e.g. irradiance reconstructions, where the models do not require the polarity of the bright features. Figures 5.13 and 5.14 show examples of reconstructed magnetograms for an active and a quiet day using the PFL on the Rome/PSPT Ca II K (panel a)) and the corresponding SDO/HMI magnetogram (panel b)), as well as the pixel by pixel absolute differences between the reconstructed and the original magnetograms by using PF (panel c)), PFL (panel d)), LFL (panel e)), and PF* (panel f)). Prior to getting the differences, the original and reconstructed magnetograms were multiplied with $\mu$, so that the compared quantity is $|B|$ and not $|B| / \mu$. In this reconstruction we only made use of the information we get from the Ca II K image to identify the regions on which we apply the relation obtained in Sect. 5.3.1. This means that we are not identifying the sunspots accurately and their immediate surroundings are the regions with the highest errors, reaching differences of up to $\sim 1000 \mathrm{G}$. These regions have been masked out in Fig. 5.13 and the errors reported in the plots do not include sunspots. Furthermore, we consider only the regions with $\left|B_{\mathrm{LOS}}\right| / \mu \geq 20 \mathrm{G}$ in the original (and degraded) magnetogram to calculate the differences. Comparing the errors between the reconstructed and the original magnetograms after excluding the immediate surroundings of sunspots we get similar errors for both PF and PFL. In particular we find absolute differences with RMS $\simeq 30 \mathrm{G}$ and $\mathrm{RMS} \simeq 20 \mathrm{G}$ for the active and quiet day, respectively for both PF and PFL. We discern no significant difference between these two reconstructed magnetograms. The differences for the quiet day show that we slightly underestimate the weak fields. The differences for the LFL reach up to $2500 \mathrm{G}$ on plage regions. These high errors arise due to the fact that there are numerous very bright pixels in the Ca II K observations that would correspond to very strong fields in this case, as the fitted curve increases very slowly. This is a problem encompassed by reconstructions that use a relation derived from a fit with binning in $\left|B_{\mathrm{LOS}}\right| / \mu$. We also show the differences for $\mathrm{PF}^{*}$, that has been commonly used in the literature. In this case, the errors are slightly higher than for PF or PFL, but the errors with PF, $\mathrm{PFL}$, and $\mathrm{PF}^{*}$ are comparable with each other. Figure 5.15 shows scatter plots between the four reconstructed magnetograms and the original one for the active day. The reconstructed magnetograms with PF and PFL show the best correspondence. The one with LFL and that with the result of $\mathrm{PF}^{*}$ tend to overestimate the magnetic field. Figure 5.16 shows the pixel by pixel RMS differences between the original and the reconstructed mag- 
netograms obtained using the derived best fit parameters of the three functions we tested, without masking the surroundings of the sunspots this time. Figure 5.16 reveals that the RMS differences remain less than $100 \mathrm{G}$ for all unsigned magnetograms reconstructed with the PF and PFL, but reach $500 \mathrm{G}$ for LFL.

We now check how good the regions in the reconstructed magnetograms correspond to magnetic regions and network in the original magnetograms. For this, we derive the disc fractions covered by features with the first segmentation method applying constant thresholds on contrast in the Rome/PSPT and constant thresholds on $\left|B_{\mathrm{LOS}}\right| / \mu$ in the original SDO/HMI and reconstructed images, respectively. The disc fractions derived from the original (and degraded) magnetograms and the reconstructed ones from all three functions are shown in Fig. 5.17. We also show separately the disc fractions derived from the original-sized and smoothed SDO/HMI magnetograms. When doing so we use the same degraded parameters for both cases. Figure 5.18 shows the residual disc fractions with respect to the ones derived from the degraded magnetograms. There is a good agreement between the five series, with correlation coefficient of 0.98 for all $\left|B_{\mathrm{LOS}}\right| / \mu$ ranges. For all feature classes the difference of the disc fractions derived from degraded magnetograms and Rome/PSPT (original-sized magnetograms) are on average $0.3 \%(0.8 \%)$ and are always below $1.3 \%(1.8 \%)$. The differences between the degraded magnetograms and the reconstructed ones with PF (PFL) fits are on average $1.0 \%(0.8 \%)$ and are always below $2.3 \%(2.0 \%)$. We notice that the reconstructed magnetograms exhibit higher disc fractions for network by $\sim 1 \%$.

We have calculated the total flux from the reconstructed and the original magnetograms; results are plotted in Fig. 5.19 for the same $\left|B_{\mathrm{LOS}}\right| / \mu$ ranges as in Fig. 5.17 . The day by day correlation coefficients between the total flux in the original and the reconstructed magnetograms with both PF and PFL fits is 0.98 for all bright features and remains at similar levels for plage and network. We notice that the slightly higher network disc fractions result in an almost constant offset in the total flux of the network components. The total flux in the degraded magnetograms is reduced compared to that from the original-sized magnetograms, which is consistent with the results of Krivova and Solanki (2004a).

\subsection{Discussion}

In Sect. 5.2.2 we mentioned that we had to re-align Ca II K images to match the magnetograms. Here we present results obtained by testing the sensitivity of our results to potential misalignment of the images. We repeated our analysis by using Rome/PSPT images shifted by a random number of pixels, in both the $x$ and $y$ direction, with respect to the data considered in Sect. 5.3 and compared results with findings reported there. We used offsets up to 9 pixels and performed 100 computations for each value. Figure 5.20 shows the relative difference of the derived exponents on the images with the offsets to the original ones. We show the errors for the exponents derived for PF and PFL separately. With offsets up to 9 pixels, the errors for the derived parameters with PF (PFL) remain below $8 \%(12 \%)$, while they are less than 5\% (7\%) and 2\% (3\%) for 5 and 1 pixel offsets, respectively. We notice that errors due to the offsets are higher during low activity periods, since the small-scale fields dominating at such periods are more prone to misalignment errors. 

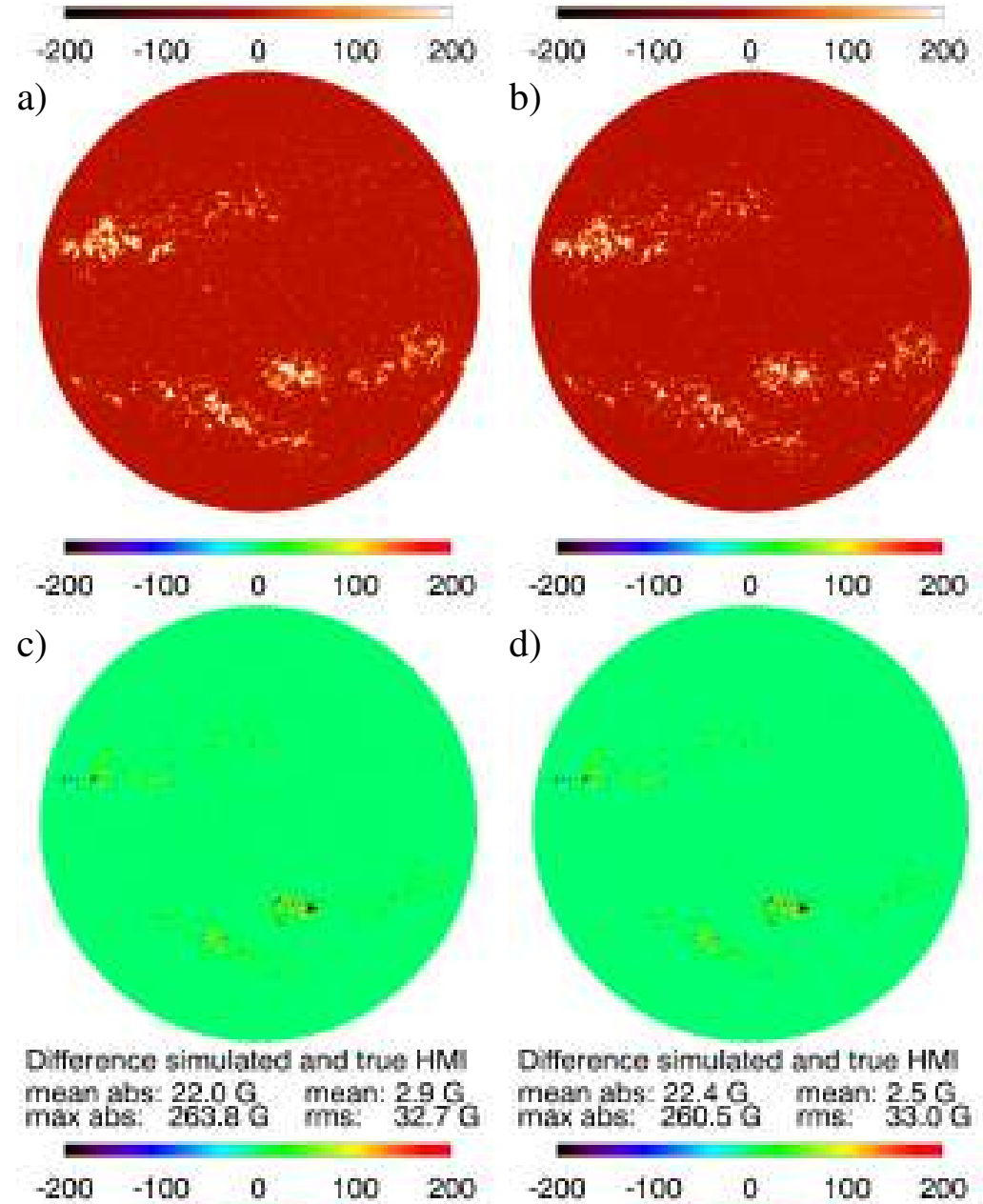

d)

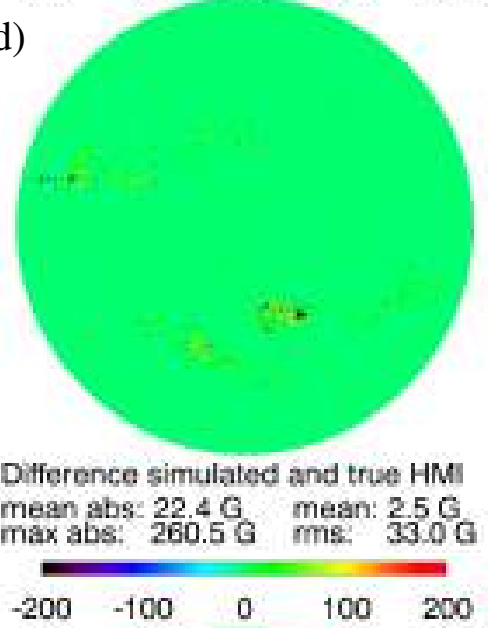

e)

$\begin{array}{lllll}200 & -100 & 0 & 100 & 200\end{array}$

f)

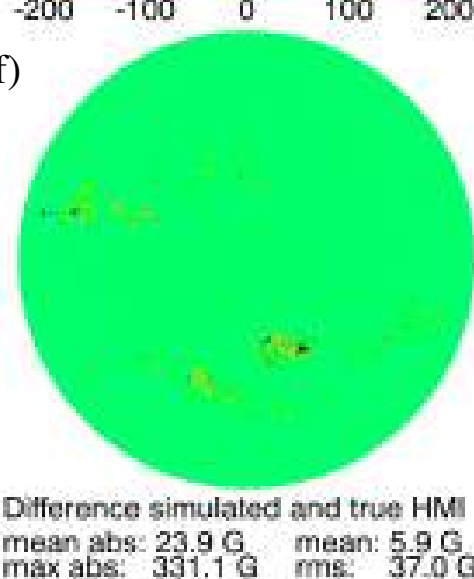

Difference simulated and true HM: mean abs: $34.2 \mathrm{G}$ mean: $18.3 \mathrm{G}$ mean abs: $23.9 \mathrm{G}$ mean: $5.9 \mathrm{G}$ max abs: $331.1 \mathrm{G}$ ms: $370 \mathrm{G}$

Figure 5.13: Magnetogram reconstructed from the Ca II K image taken on 01/04/2011 using the average parameters for PFL (a)), SDO/HMI magnetogram on the same day (b)) (both are saturated at $200 \mathrm{G}$ ), difference between the reconstructed magnetogram from Ca II K data and original SDO/HMI magnetogram with PF (c)), PFL (d)), LFL (e)), and $\mathrm{PF}$ if the fit was performed on the binned data over $\left.\left|B_{\mathrm{LOS}}\right| / \mu(\mathbf{f})\right)$. The RMS, mean, mean absolute, and maximum absolute relative differences are listed under each panel (c)-f)).

As described in Sect. 5.2.2, we removed the polarity information prior to resizing 

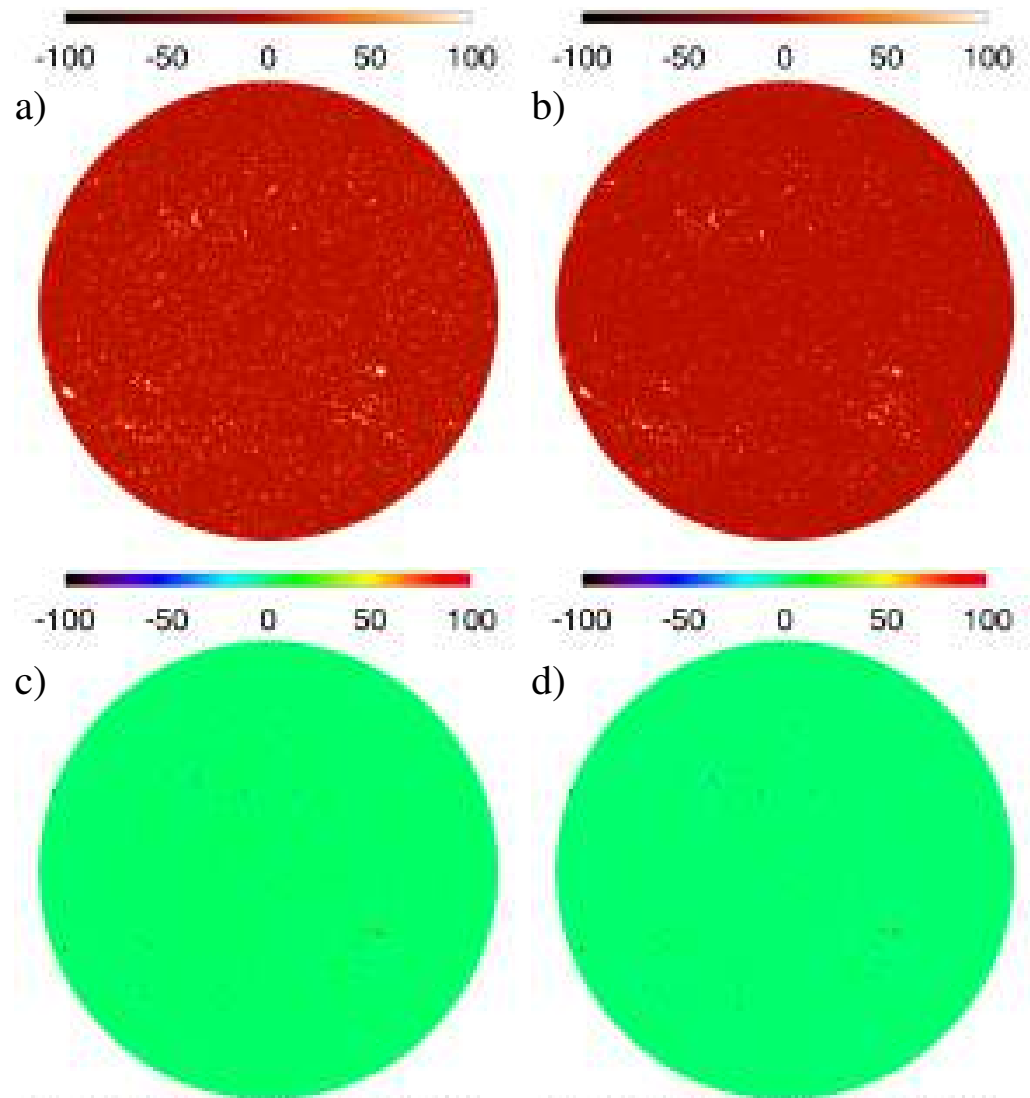

Difference simulated and true HMI

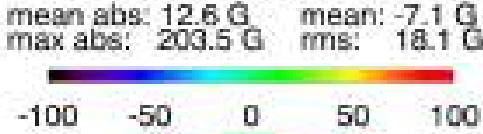

d)

e)
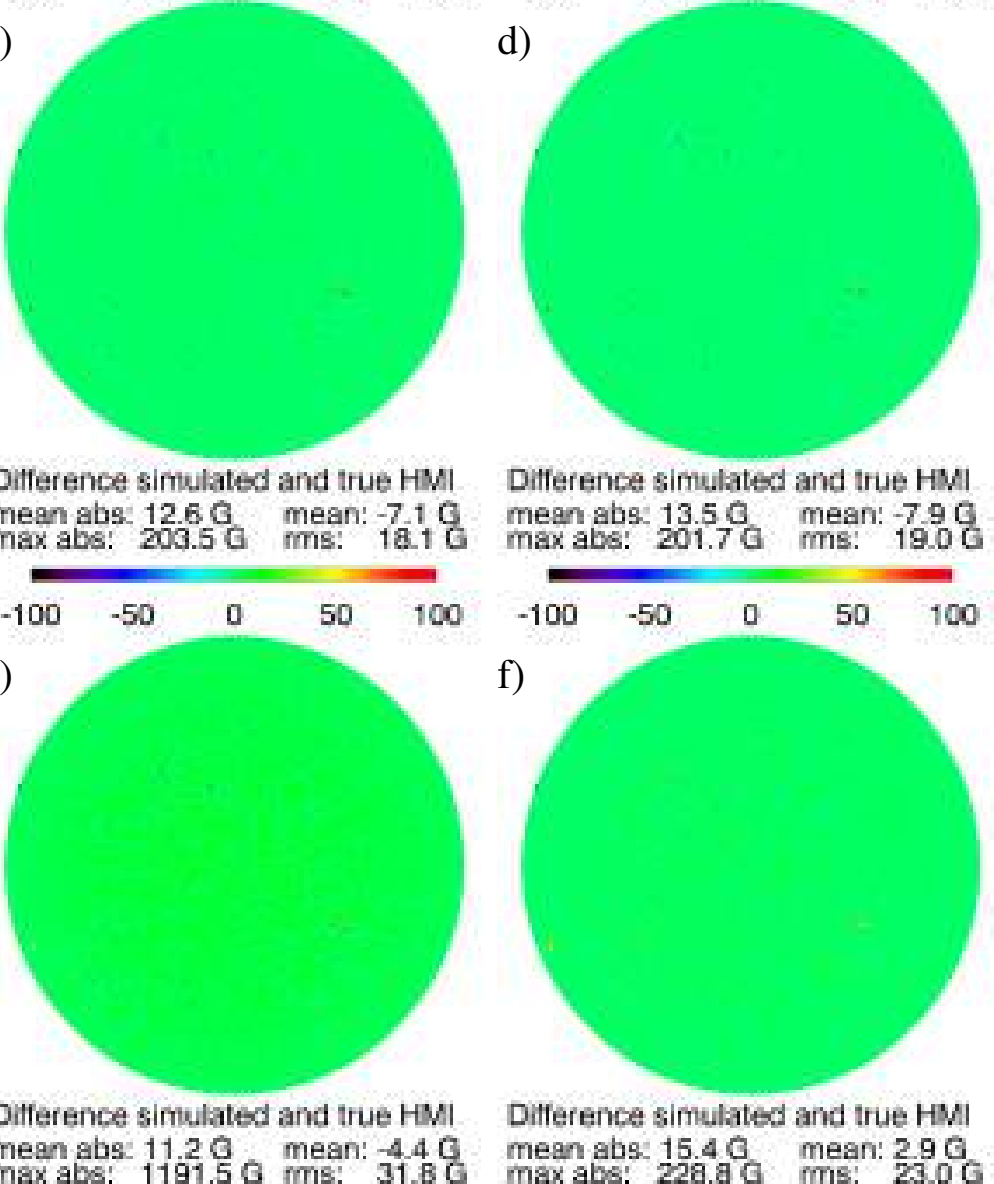

f)

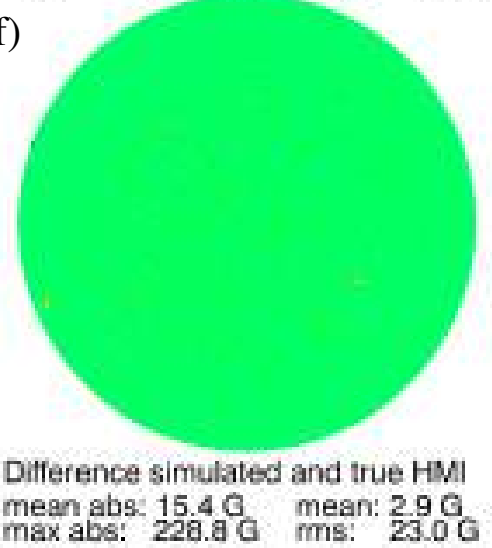

Figure 5.14: Magnetogram reconstructed from the Ca II K image taken on 07/06/2010 using the average parameters for PFL (a)), SDO/HMI magnetogram on the same day (b)) (both are saturated at $100 \mathrm{G}$ ), difference between the reconstructed magnetogram from Ca II K data and original SDO/HMI magnetogram with PF (c)), PFL (d)), LFL (e)), and $\mathrm{PF}$ if the fit was performed on the binned data over $\left.\left|B_{\mathrm{LOS}}\right| / \mu(\mathbf{f})\right)$. The RMS, mean, mean absolute, and maximum absolute relative differences are listed under each panel (c)-f)).

the magnetograms. The magnetic regions outside spots appear bright in Ca II K images 
a)

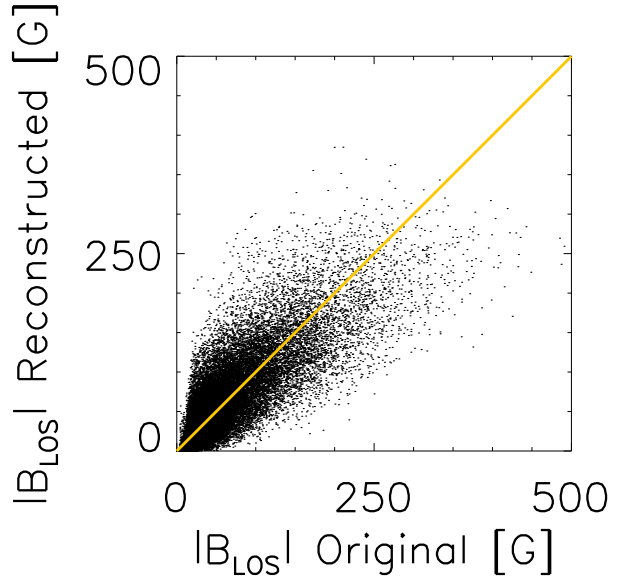

c)

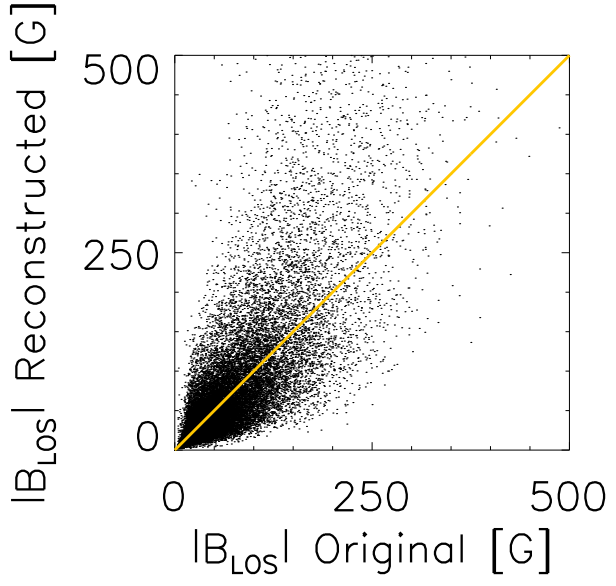

b)

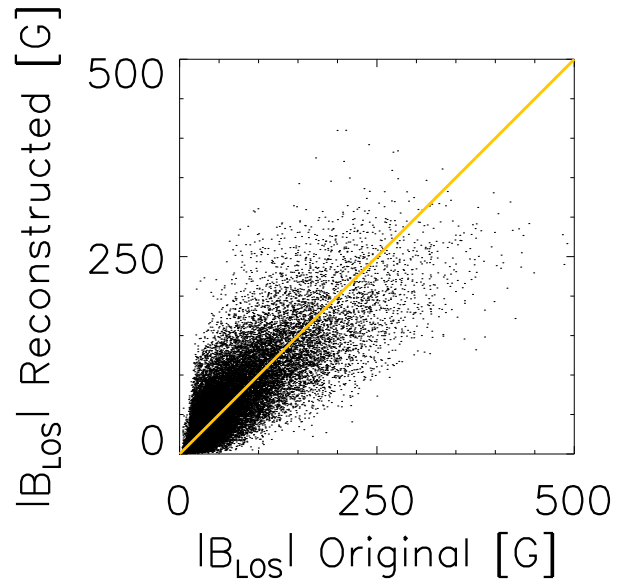

d)

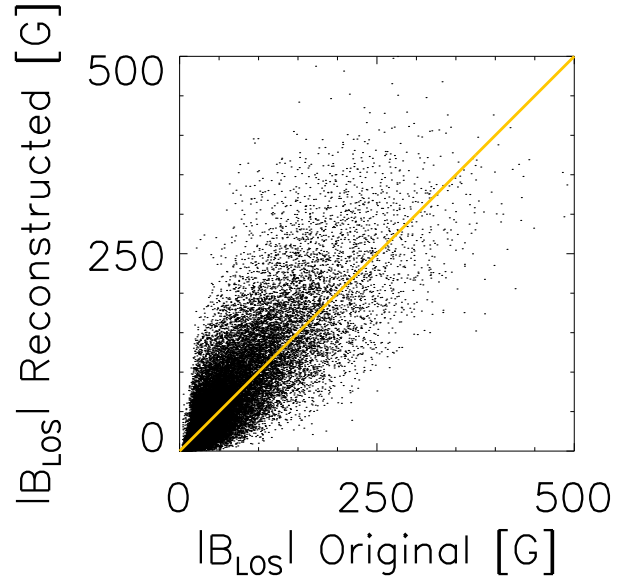

Figure 5.15: Scatter plots between original (degraded) magnetograms and those reconstructed from the Ca II K image taken on 01/04/2011 using the average parameters for PF (a)), PFL (b)) LFL (c)), and PF if the fit was performed on the binned curve over $\left|B_{\mathrm{LOS}}\right| / \mu$ (d)). The yellow line has a slope of unity. The axes are shown in the range from the original magnetogram.

independently of their polarity. If we resized signed magnetic maps, the magnetic flux of opposite polarities would have been cancelled out when falling into the same new bin, and bright $\mathrm{Ca}$ II pixels would be related to pixels with rather low magnetic field strengths. Here we investigate how the results change if the polarity removal is done after the resizing. Figure 5.21 shows the fraction of all pixels unaffected in each image, as well as the fraction of the affected pixels that are isolated or have $\left|B_{\mathrm{LOS}}\right| / \mu<20 \mathrm{G}$. The fraction of pixels affected within an image is between $2 \%$ and $7 \%$, while $84 \%$ of all these are isolated pixels. Furthermore, $71 \%$ of these pixels have values less than $20 \mathrm{G}$ in the original magnetogram, and are thus below the noise threshold. We find on average a difference of $15 \mathrm{G}$ (RMS of $36 \mathrm{G}$ ) between the values of the resized magnetograms if 


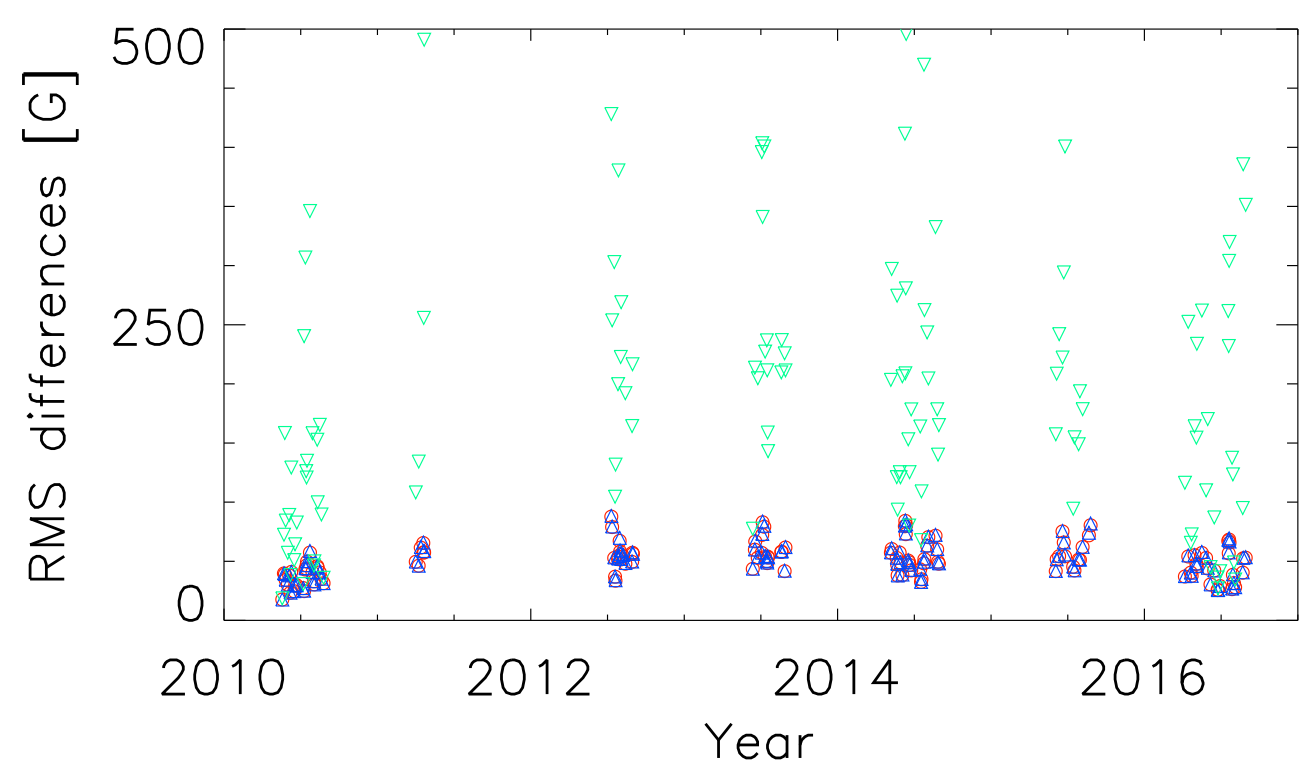

Figure 5.16: RMS pixel by pixel differences in $\mathrm{G}$ between the original magnetograms and the reconstructed ones using the parameters (listed in Table 5.2) derived from the PF (red), PFL (blue), and LFL (green) fits.

the polarity was removed prior or after the resizing for all pixels within $\mu>0.14$. Figure 5.22 shows the ratio of the total flux in the magnetograms with the polarity removed prior and after the resizing. We find on average $\Phi_{\text {abr }} / \Phi_{\text {aar }} \simeq 1.008$, but exceed 1.04 for two observations during low activity periods. Therefore, we find that removing the polarity before or after the resizing does not affect our results in a significant way.

It is worth noting that the accurate computation of the CLV for the Rome/PSPT Ca II $\mathrm{K}$ and red continuum data and SDO/HMI continuum images is critical for this kind of analyses. Any residual effects of limb darkening could introduce artificial trends into the derived fits. This would affect mostly the CLV of the relation, but can also introduce artefacts if bright regions were not accurately disentangled from the CLV. The accuracy of the processes has been extensively discussed in Sect. 3.3 and was found to be satisfactory, with an average error in the contrast images being less than $0.6 \%$.

We have also studied the effect of the stray-light on our results. For this, we have repeated the same analysis on images that have been corrected for stray light (see Sect. 5.2.3). Since these images have a higher contrast, the segmentation parameters for different features had to be adapted (increased by 0.02 in contrast and $10 \mathrm{G}$ ). Otherwise the process that we applied was exactly the same. Figure 5.23 is similar to Fig. 5.5, but now for the stray-light corrected data. The scatter in Ca II K contrast is higher. Qualitatively, our results remain unchanged, with almost constant exponents over the disc and time. Also, quantitatively, the values of the exponents are almost the same, with best fit parameters $0.65 \pm 0.01$ and $3.89 \pm 0.08$ for PF and PFL respectively. Figures 5.24 and 5.25 show the exponents as a function of time and $\mu$, respectively, derived from the analysis of stray-light corrected data. 


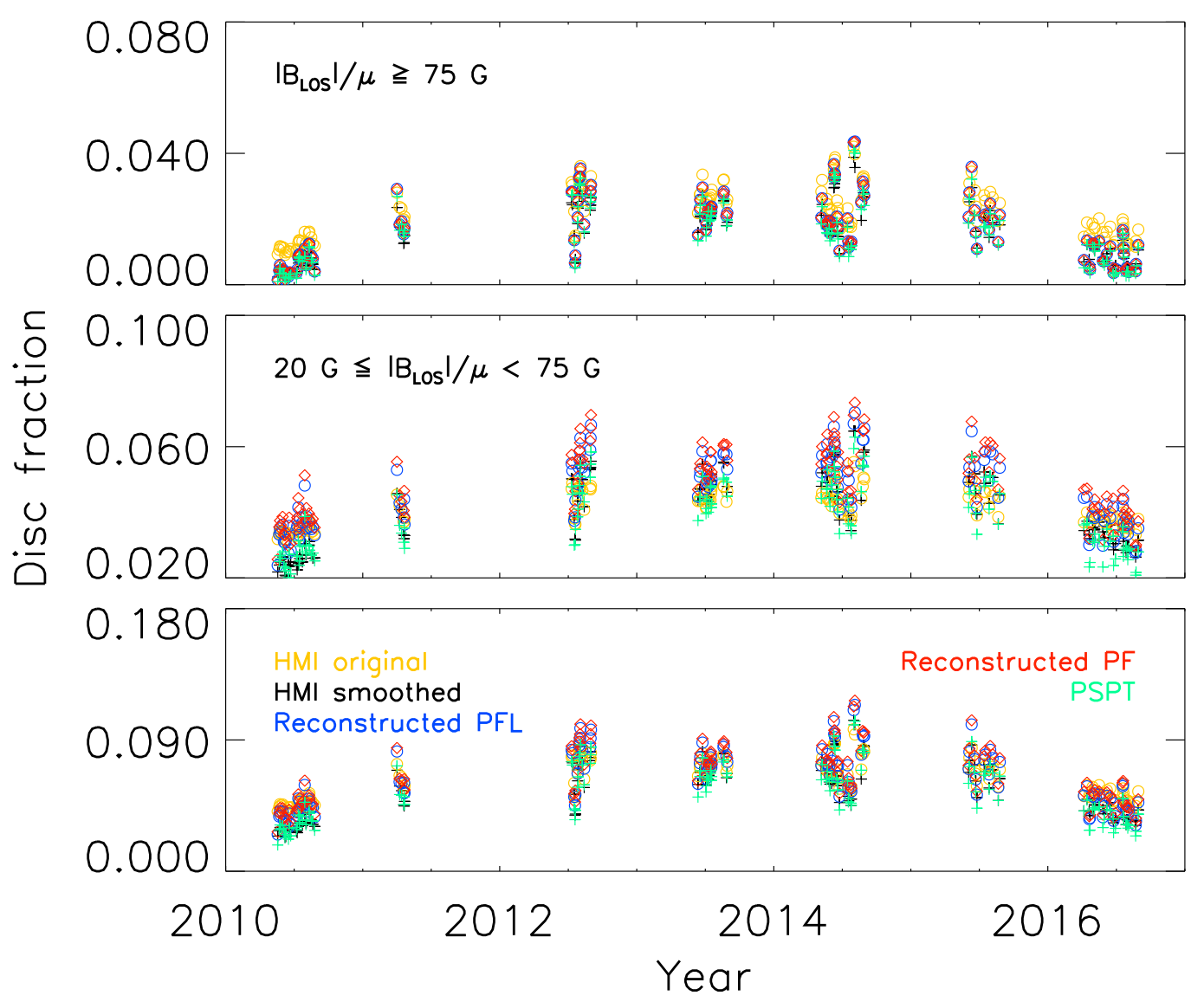

Figure 5.17: Disc fractions of magnetic features derived from SDO/HMI observations (original and reduced spatial resolution in yellow and black, respectively), Rome/PSPT Ca II K images (green), and reconstructed magnetograms with PF (red) and PFL (blue). Each panel corresponds to different features (as marked in each panel) identified with the first segmentation method, while the bottom panel is for all features together.

Pevtsov et al. (2016) claim that Ca II contrast images are unreliable proxies for the magnetic flux due to the large scatter between $\mathrm{Ca}$ II $\mathrm{K}$ brightness and the magnetic flux they found. Furthermore, the scatter plot they show implies a reversal of the relationship at higher magnetic fluxes. However, it should be noted that the data they analysed are of significantly lower quality than the ones we used. Moreover, their reported reversal of the relation at high magnetic fluxes is perfectly consistent with the inclusion of sunspots in their analysis. Inclusion of sunspots explains the lack of correlation reported by Pevtsov et al. (2016) also for Ca II $854.2 \mathrm{~nm}$ observations.

\subsection{Summary and conclusions}

We analysed the relation between the excess $\mathrm{Ca}$ II $\mathrm{K}$ emission and the magnetic field strength. For this, we used 131 sets of co-aligned near-co-temporal SDO/HMI magnetogram/continuum observations and Rome/PSPT observations taken in the core of the $\mathrm{Ca}$ II $\mathrm{K}$ line and in the red continuum. We confirm the existence of a close relation between 


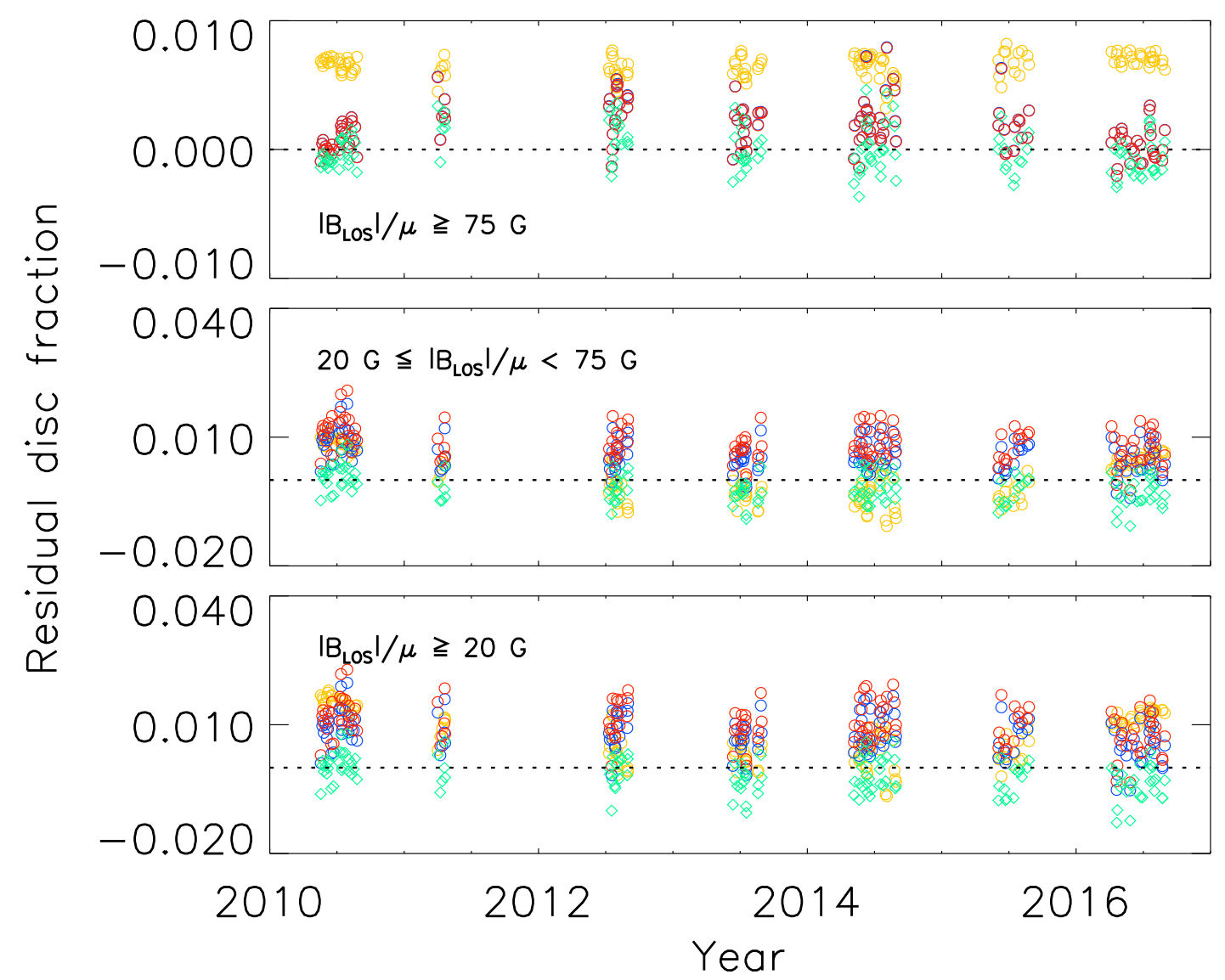

Figure 5.18: Difference between the disc fraction of magnetic features derived from SDO/HMI observations with reduced spatial resolution and Rome/PSPT (green), $\mathrm{SDO} / \mathrm{HMI}$ (original spatial resolution in yellow), reconstructed magnetograms with PF (red), and PFL (blue). Each panel corresponds to different features (as marked in each panel) identified with the first segmentation method, while the bottom panel is for all features together. The dashed horizontal lines are for a difference of 0 .

the excess $\mathrm{Ca}$ II $\mathrm{K}$ emission and the magnetic field strength. We fit the relation between the $\mathrm{Ca}$ II $\mathrm{K}$ intensity and the vertical component of the magnetic field $\left(\left|B_{\mathrm{LOS}}\right| / \mu\right)$ with a power law function of the logarithm of $\left|B_{\mathrm{LOS}}\right| / \mu$ with an offset, and test it against a power law function and a logarithmic function of $\left|B_{\mathrm{LOS}}\right| / \mu$ that have been presented in the literature. The parameters we derived for the power law function are consistent with those presented in the literature. The results for a power law function are also very similar to those derived with power law function of the logarithm of $\left|B_{\mathrm{LOS}}\right| / \mu$. A logarithmic function is found to be not representative of bright features in Ca II K images.

The observations analysed here greatly extend the sample of studied data with respect to other studies. In particular, we examined a greater amount and higher quality data than has been done before for such studies. The data are spanning half a solar cycle, and for this time-scale we report no significant variation of the exponents with time. We find no variation of the exponents over the disc positions up to $\mu=0.14$. The results for the exponents remain the same if stray-light is taken into account. The constancy of 


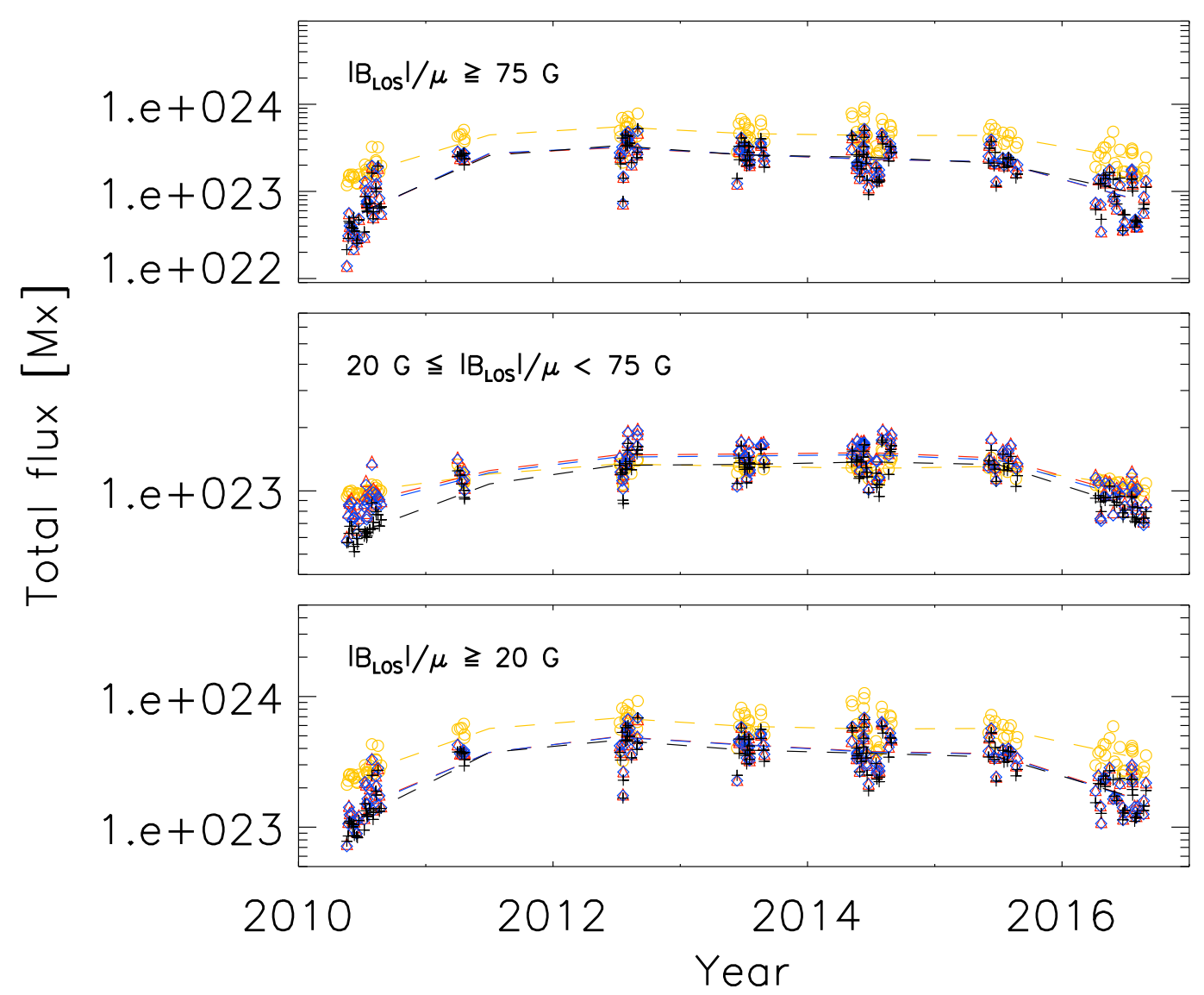

Figure 5.19: Total flux in Mx of active features derived from the magnetograms (yellow circles for the original and black plus signs for the reduced spatial resolution ones) and from the unsigned magnetograms reconstructed from Ca II K observations with PF (red triangles) and PFL (blue rhombuses). Each of the upper two panels corresponds to a different type of features (as listed in the panels) identified with the first segmentation method, while the bottom panel is for all features together. The dashed lines connect the annual median values.

the exponents in time and $\mu$ suggests that maps of the unsigned LOS magnetic field can be reconstructed from $\mathrm{Ca}$ II $\mathrm{K}$ observations with merely the knowledge of the exponent derived here. If other data are also used, for instance sunspot measurements, it might be possible to recover the polarity of the ARs as well. Having studied this relation for almost the entire disc, up to $\mu=0.14$ or $0.99 R$ makes this study more applicable to stellar work than most earlier investigations.

In conclusion, we show that it is possible to use Ca II K observations to reconstruct magnetograms of the Sun, however without information on the polarity of the field. The total magnetic flux in the original magnetograms and in those reconstructed from the corresponding $\mathrm{Ca}$ II $\mathrm{K}$ observations agree very well with the correlation factor of 0.98 for the daily values. This means that historical Ca II K spectroheliograms, when properly processed and calibrated, can be used to extend the series of magnetograms throughout the whole 20th century. 
a)
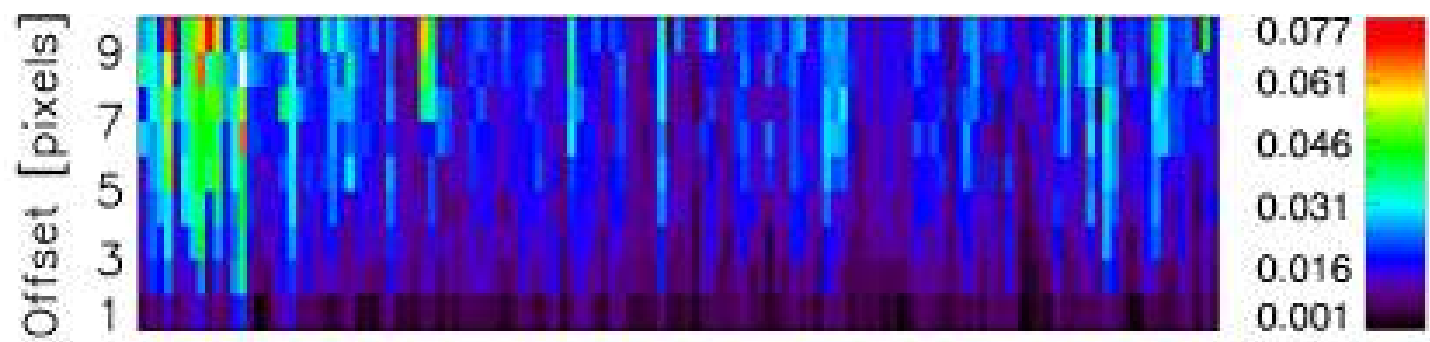

0
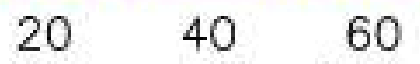

80

100

120

Number of image

b)
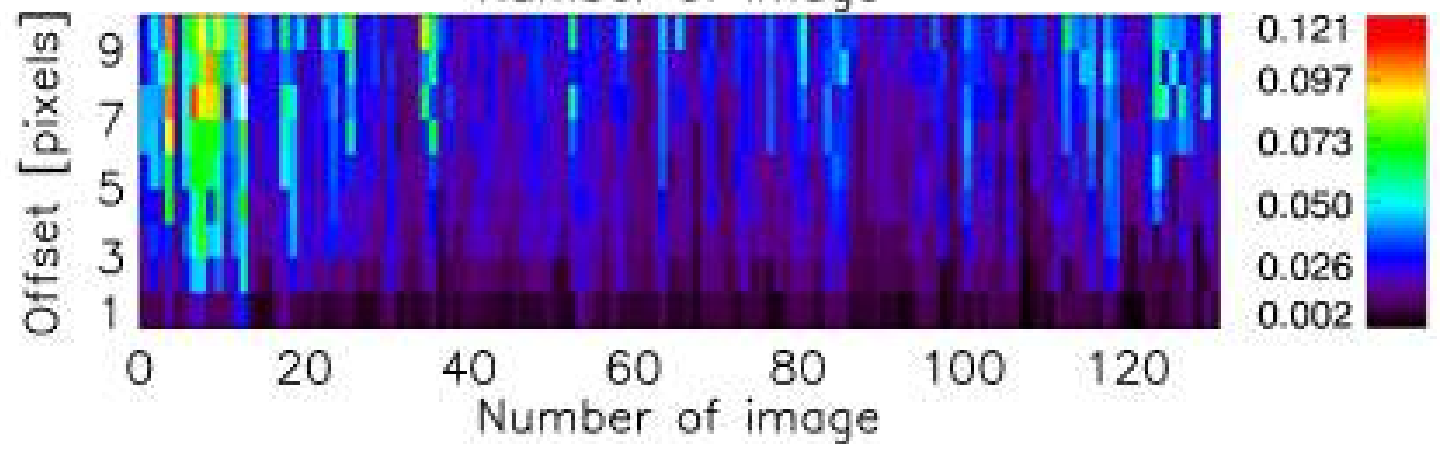

Figure 5.20: Colour-coded relative errors in the exponent derived with PF (a)) and PFL (b)) due to random offsets in position of the Ca II $\mathrm{K}$ image. Each $\mathrm{Ca}$ II $\mathrm{K}$ image was randomly shifted in both $x$ and $y$ directions up to 9 pixels. The boxes give the average error after 100 realisations. The $y$ axis gives the value of the maximum possible offset in any direction. The $x$ axis indicates the analysed images ordered by date covering the period 2010-2016.

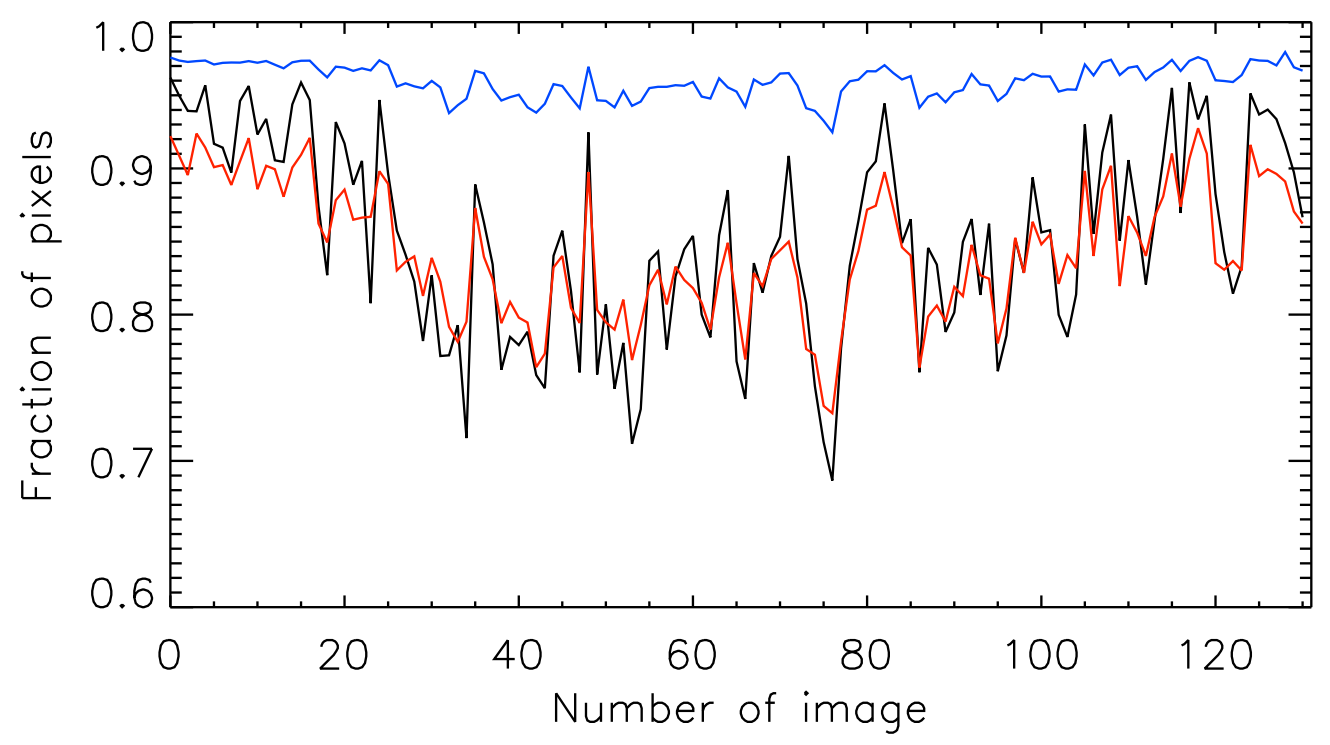

Figure 5.21: Fraction of pixels per image unaffected by flux cancellation due to resizing (blue). Also shown is the fraction of the affected pixels with $\left|B_{\mathrm{LOS}}\right| / \mu<20 \mathrm{G}$ (black) and the fraction of these pixels that are isolated pixels (red). 


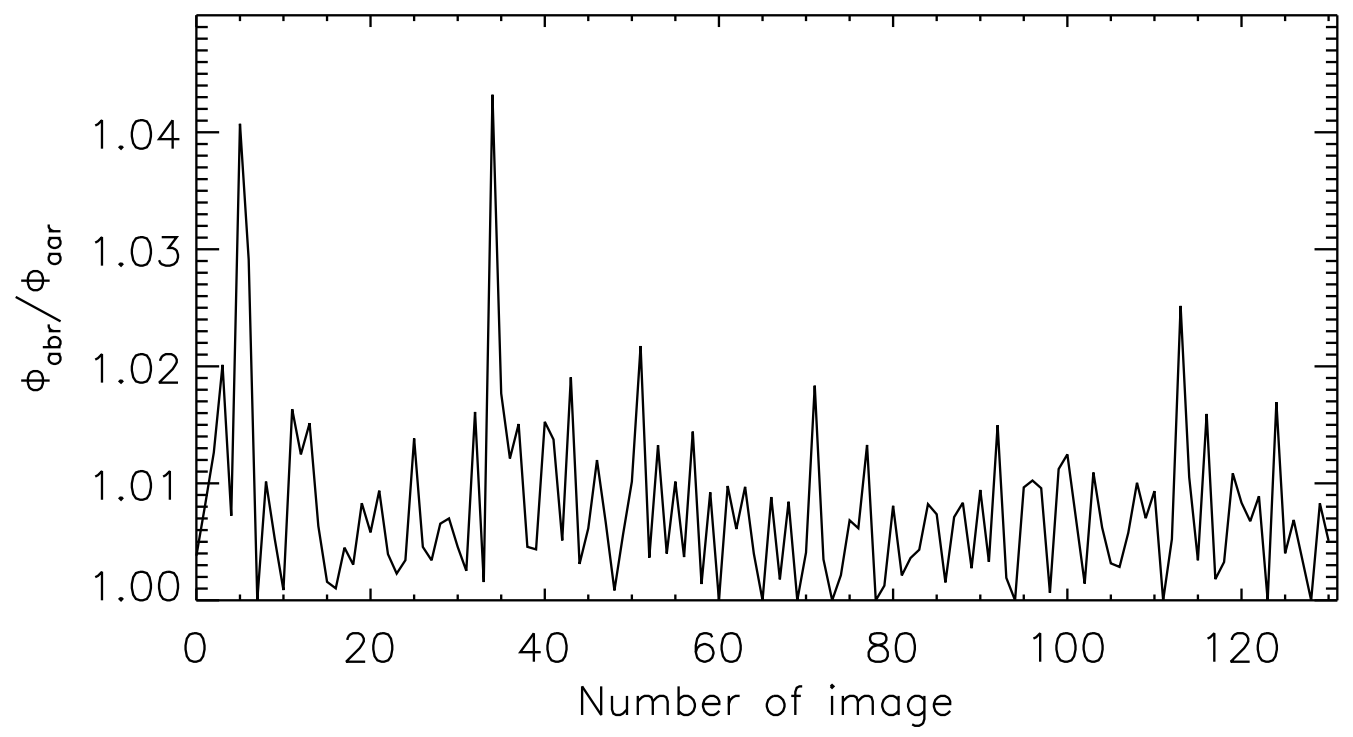

Figure 5.22: Ratio of total magnetic flux measured in the magnetograms where the polarity was removed prior and after the resizing.

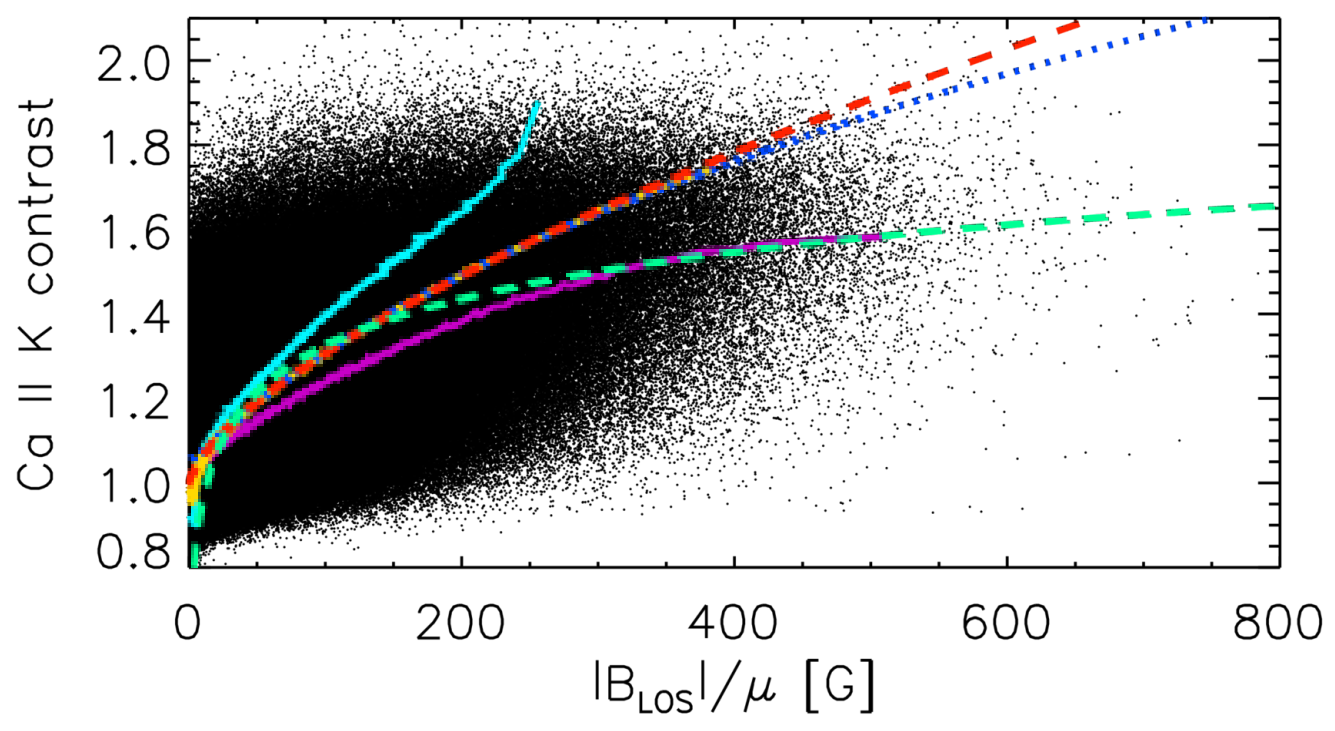

Figure 5.23: Same as Fig. 5.5 but for stray-light corrected data. 

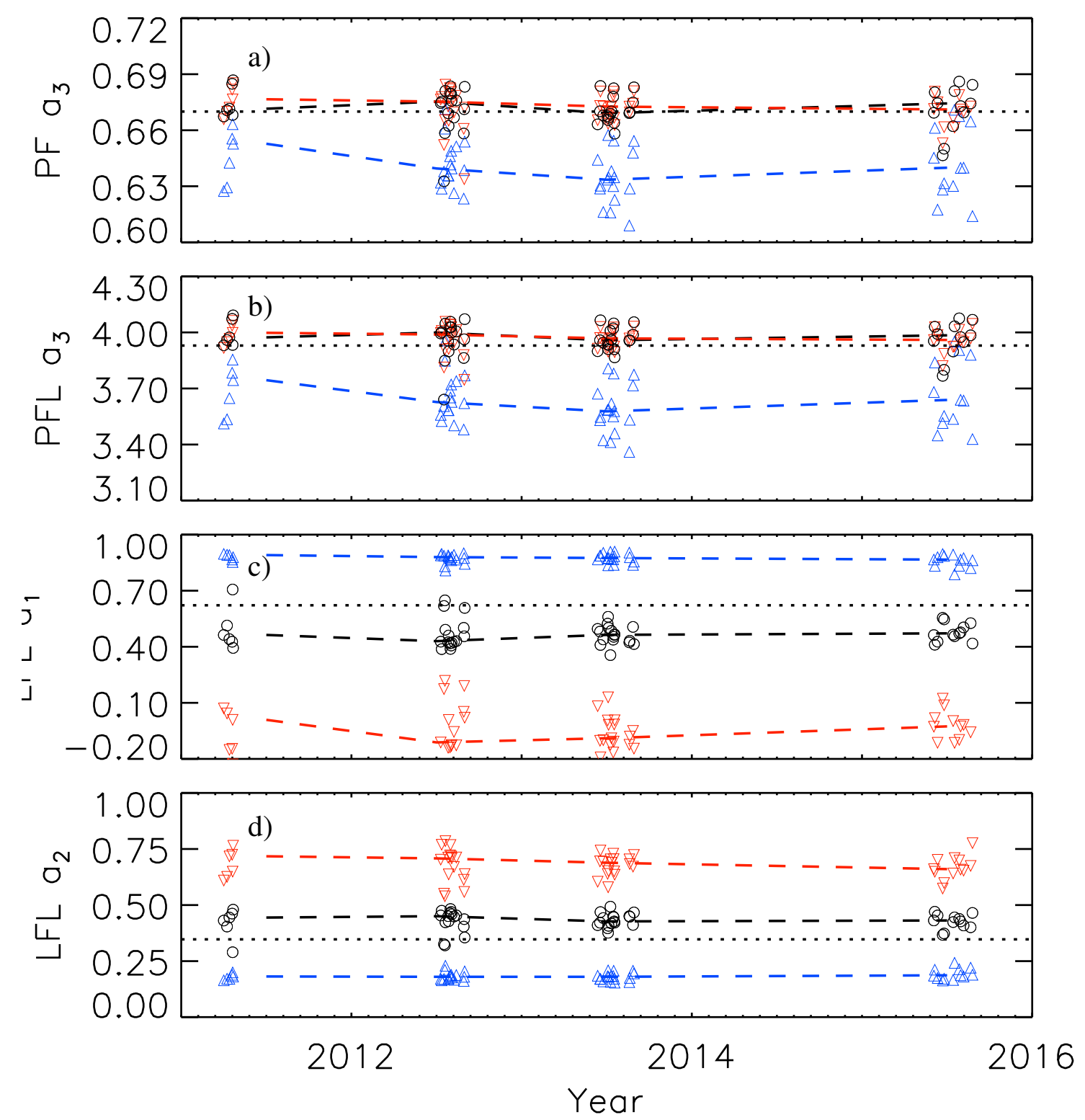

Figure 5.24: Same as Fig. 5.7 but for stray light corrected images. 

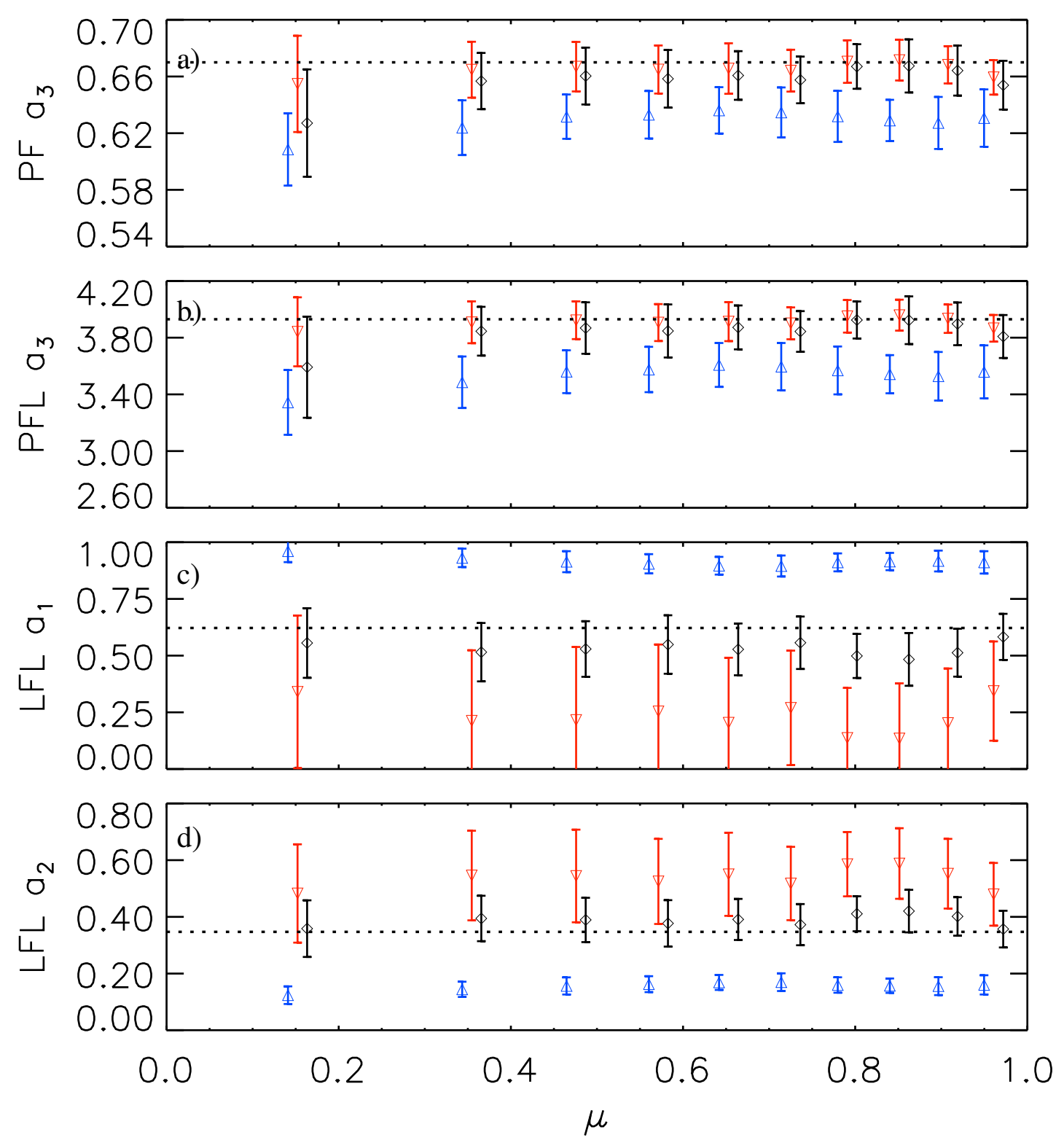

Figure 5.25: Same as Fig. 5.8 but for stray light corrected images. 



\section{Group sunspot number series}

*This chapter is based on a published journal article (Usoskin et al.|2016b).

\subsection{Introduction}

This chapter describes our analysis of the historical sunspot number observations. We start (Sect. 6.1.1) with an overview of the available sunspot observations and the earlier attempts to produce a homogeneous series from them. We also discuss problems affecting these previous reconstructions. In Sect. 6.2, we study the dependence of the sunspot size on the activity level and the relationship between the records by two individual observers with different acuities.

\subsubsection{Sunspot observations}

Sunspots are the most observed feature among all the various solar surface magnetic phenomena. Since 1610 the number of sunspots on the solar disc has been primarily recorded with telescopes, but observations have also been made with the naked eye or a camera obscura.

Observations of sunspots with the naked eye are possible if the solar brightness is diminished, by for example fog or smoke. There have been many reports of spots in ancient literature, with possibly the oldest one dating back to $350 \mathrm{BC}$ in ancient Greece by Theophrastus (Hardy 1991). However the majority of such records come from south-east Asia. The records of naked eye sightings mostly consist of vague statements mentioning the existence of spots on the sun rather than accurately quantifying their number or size. Furthermore, the very low frequency of the recovered observations make a proper calibration impossible. Wittmann and Xu (1987), Yau and Stephenson (1988), Stephenson (1990) compiled a dataset with such data covering the period $165 \mathrm{BC}-1918$ AD. Figure 6.1 shows the recovered naked-eye observations with decadal cadence and illustrates the small amount of available data and the large gaps without any observation.

The available observations with a camera obscura are rather limited too. Kepler reported the first known viewing of a sunspot in 1607 (Vaquero 2007) with a camera obscura. However, the oldest drawing of the sun using a camera obscura dates back to 1544 (Gemma Frisius et al.1545) showing no sunspots on the solar disc. Absence of sunspots in the reports before the 1600s could be explained by the lower activity level of the Sun (Spörer minimum). It is possible though that sunspots were there, but not reported due to the fact that observations were mainly made during eclipses or because of the limited observing capabilities of the camera obscuras. Observations with camera obscura continued until the middle of the 18th century. 


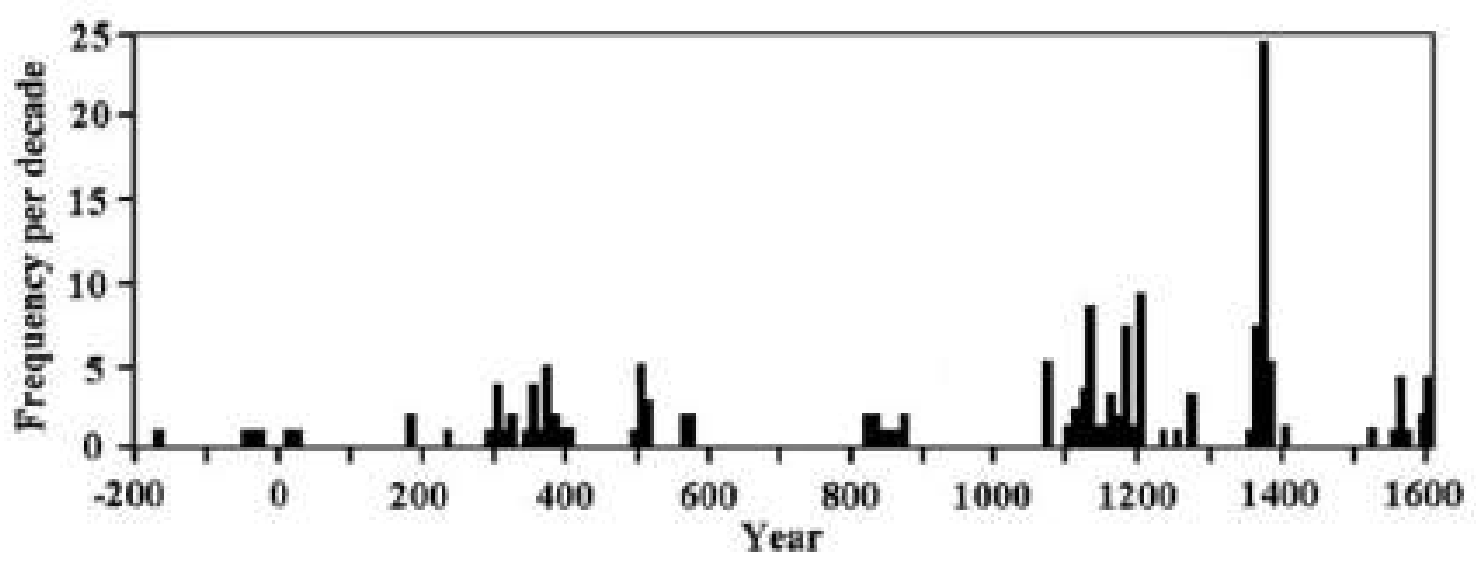

Figure 6.1: Naked eye observations of sunspots. Taken from Vaquero and Vázquez (2009) as adapted from Yau and Stephenson (1988).

With the invention of the telescope in 1610, there have been more regular observations of the Sun. The use of a telescope enabled smaller and finer structures to be seen than was possible before. The data that survived are either in the form of documentation of the observations, stating what was seen by observers on the disc, or drawings. More recently photographs in white light have been used as well. The drawings and photographs allow for a complete recalculation of the series carrying information on locations and areas of the spots as well. The documentations in some cases still include vague statements and mainly give information for the groups.

There have been three major attempts to compile a single homogeneous database with sunspot number data. The first one was done by Wolf in 1849 (Wolf 1850), who along with his own observations also started gathering up those from Schwabe, Staudacher, and Flaugergues. Wolf's database was maintained and updated at the Zurich observatory and extended with more recent observations. Hoyt and Schatten (1998) updated this database and extended it back to 1610 including only the data for the counts of sunspot groups. However various typos and questionable values were found in this database which led to the more recent major revision by Vaquero et al. (2016). In this database, all ambiguous values were removed, and various newly-found observers were added. This database includes 1,052,413 daily values from 738 different observers covering the period 1610 - 2010. The majority of these observers were located in Europe. Figure 6.2 shows the number of days with sunspot records per decade in the Hoyt and Schatten (1998, grey) and Vaquero et al. (2016, black) databases. One can see that there is almost full coverage after 1800s, but earlier data are more scarce. The scarcity of sunspot data around 1790 gave rise to the debate as to whether a solar cycle was lost and cycle 4 is actually 2 solar cycles (Usoskin et al.|2001, 2002, 2003a b, 2009, Krivova et al.|2002, Arlt 2008, Zolotova and Ponyavin 2011, Karoff et al. 2015, Owens et al. 2015).

All these observations have been carried out by various people, with different instruments, at various locations, and did not always aim at counting spots (e.g., meridian observations of the Sun or eclipse observations). There are large gaps within the series of individual observers, leaving the observations after 1739 disconnected to the earlier ones, and many series do not directly overlap with others. Even for the same observer, 
the quality of the record may vary with time as a result of gaining experience, the ageing of the observer (e.g. deterioration of eyesight) or the varying conditions at the observing location (e.g. seeing).

\subsubsection{Sunspot classification schemes}

Grouping of sunspots was performed rather arbitrarily for most of the available database. In an attempt to make the process more consistent, three different classification schemes were suggested.

The first one was devised by Waldmeier (1939). This scheme identified 9 different stages in the life of a sunspot group, and the groups were assigned a letter depending on which stage they were at. Figure 6.3 shows the different classes of sunspots, usually referred to as Zürich classes. Following the evolutionary track of a group affects the grouping. Newly emerged small spots near other spots would be considered as a separate group, that otherwise would have been counted as one group. Furthermore, Waldmeier introduced a group weighting scheme based on the size of the spots (Cortesi et al. 2016, Svalgaard et al.2017). Adopting this scheme would increase the counts of the observers. However, it is unclear when Waldmeier started using this scheme, and it is also ambiguous as to whether other observers adopted it too. Therefore the adoption or not of the Waldmeier scheme would introduce another discordance between the various series.

A different classification scheme was proposed by McIntosh (1981, 1990), shown in Fig. 6.4. In this scheme each group is assigned three letters, the first is a modified version of the Zürich classes (G, J classes were dropped), the second describes the type of the penumbra (size and symmetry), while the third depends on how compact the group is.

Yet another classification scheme was used at Mt Wilson observatory. It is based on the magnetic properties of the sunspot groups (Notes from Mt Wilson observatory 1947). The spot groups are divided into three classes, as unipolar $(\alpha)$, bipolar $(\beta)$, and complex $(\gamma)$. Subcategories of bipolar regions exist based on size comparison between the following and preceding spot. For the unipolar groups the subcategories define if the spot came from a decaying bipolar group and if it was the following or preceding one.

\subsubsection{Sunspot number series}

A number of composite series have been produced by cross-calibration of the available sunspot (group) number data. Here we give an overview of the different series that have been produced either by calibrating existing data or with extrapolation.

The first series was compiled by Rudolf Wolf from Zürich who introduced the Wolf sunspot number (WSN) in 1848 (Wolf 1850, continued and updated as the international sunspot number, ISN), given by the formula

$$
R_{s}=k(10 G+S)
$$

where $k$ is a constant factor to normalize the different observers with each other, $S$ the number of sunspots, and $G$ the number of sunspot groups. The observations of Wolf were chosen as the reference. Data from only one observer were kept for each day, giving priority to those from the chosen primary observer, ignoring all other available data. Data from the secondary, tertiary, etc., observer were used, if none were available from the primary 


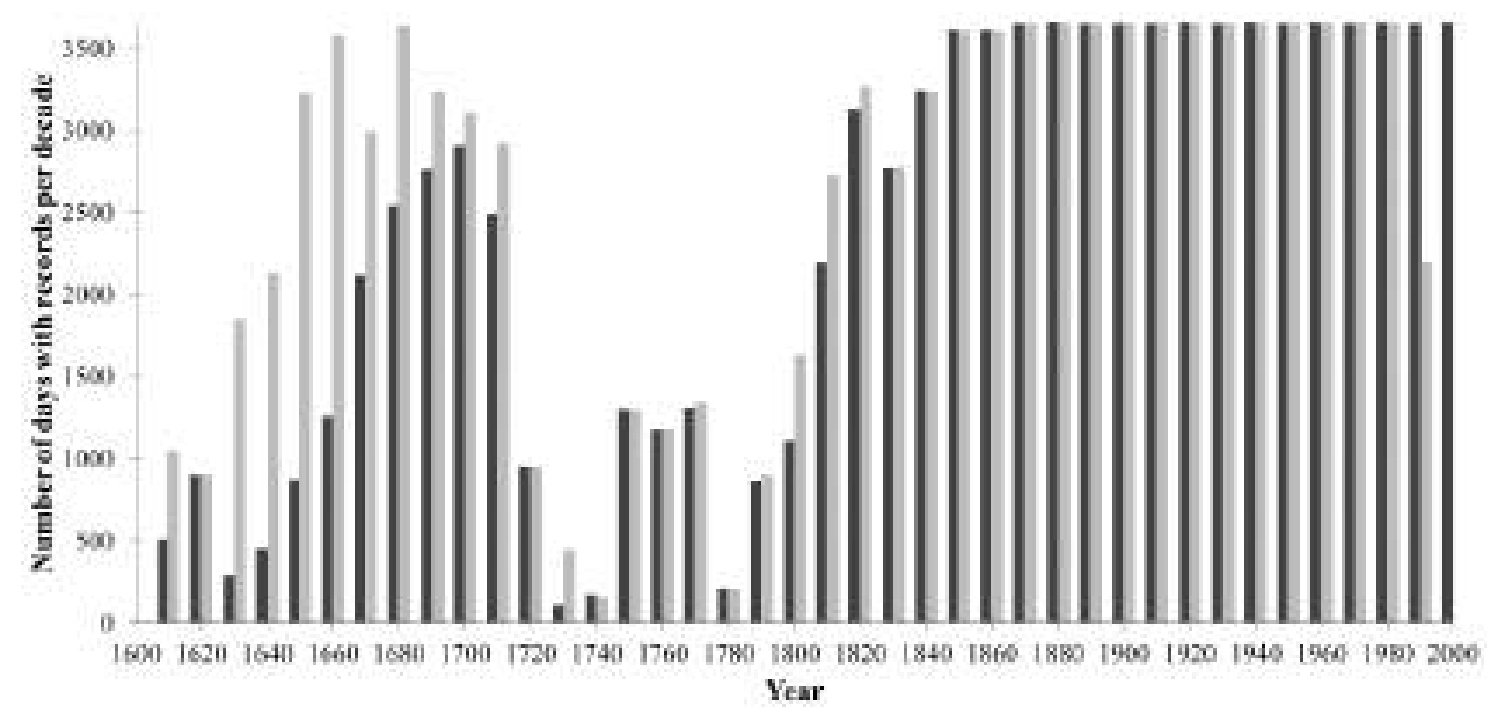

Figure 6.2: Number of days with sunspot records per decade in the Hoyt and Schatten (1998, grey) and Vaquero et al. (2016, black) databases. Taken from Vaquero et al. (2016).

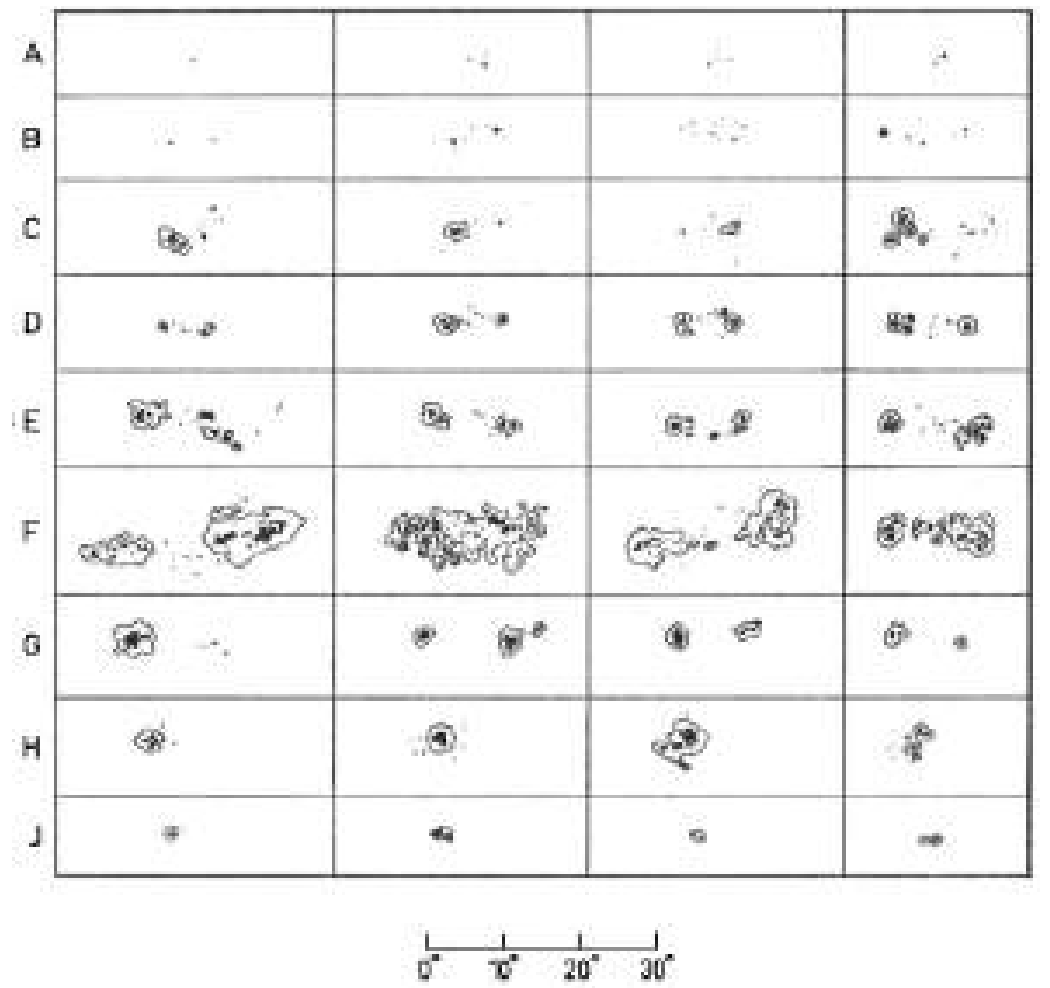

Figure 6.3: The different classes of sunspot groups as introduced by Waldmeier (1939). The scale at the lower part of the figure is in degrees of heliographic longitude. Taken from Svalgaard and Schatten (2016). 


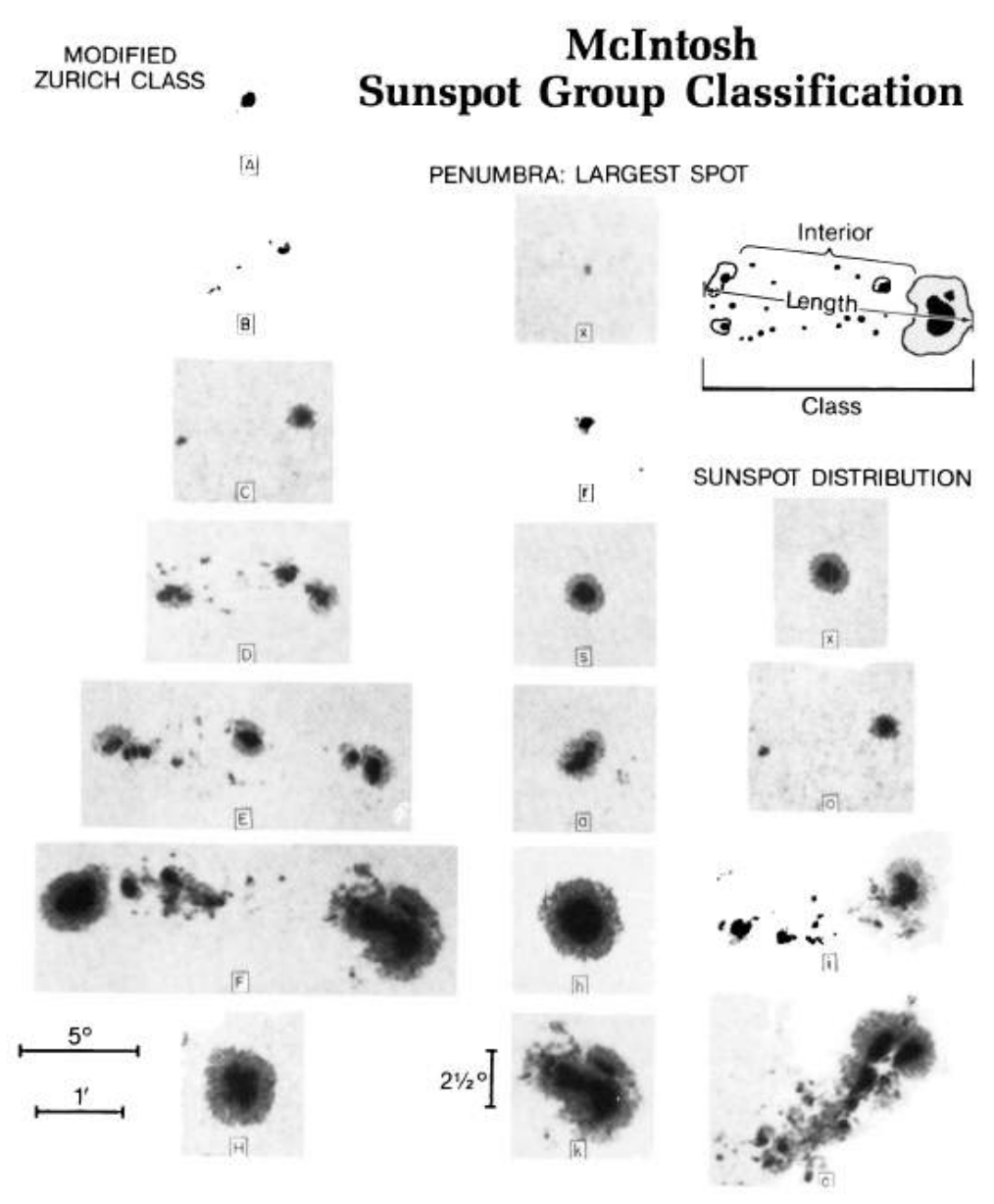

Figure 6.4: The different classes of sunspot groups as introduced by McIntosh (1990). Taken from McIntosh (1990).

observer. Wolf would not count small and short-lived sunspots that had no penumbra, or would not separate sunspots if they were surrounded by the same penumbra (Clette et al. 2014). These criteria were dropped when Wolfer became the primary observer in Zürich and a scaling factor of 0.6 was used for the data after 1882 to equalize them with the older observations. This series comprises annual values back to 1700 , monthly values back to 1749, and daily values back to 1818 .

Since 1981 the WSN/ISN series is synthesized by the Royal Observatory of Belgium (Clette et al. 2007), adapted to include all available observers for each day, not only the primary one. The WSN/ISN series was updated as version 2.0 in 2015 by changing the reference to that of Wolfer (dropping the 0.6 factor) and correcting for some proposed inhomogeneities (Clette and Lefèvre 2016).

Since 1944 another SN series has been maintained by the American association of variable star observers (AAVSO), commonly referred to as the American index (Shapley 1946, Taylor 1985). This series was making use of more than 40 observers situated in the US. The ISN series is taken as the reference, while a secondary observer is used as reference for all other observers. The correction factor for the secondary observer is taken to be the average $k$-factor with comparison to ISN and a tertiary observer for the last 4 
months. All other observers are then calibrated based on the corrected secondary observer.

These were the only available sunspot number series for more than 50 years, until Hoyt and Schatten (1998) introduced the group sunspot number (GSN, hereafter) series (HoSc98, hereafter). This series reports the number of sunspot groups alone, neglects individual spots and averages data from all observers on the same day after they have been normalised to the same level. The GSN series reduces uncertainties in the counts of individual sunspots and hence is more robust than WSN/ISN. In addition, there are more raw data about the group numbers than sunspot numbers and the GSN series extends back in time to 1610 . The daily GSN is defined as

$$
R_{g}=\frac{12.08}{N} \sum_{i} k_{i} G_{i}
$$

where $k_{i}$ is the individual correction factor of the $i$-th observer, $G_{i}$ is the GSN reported by the $i$-th observer, $N$ is the total number of observers on the given day, and the constant 12.08 was introduced to match the average level of $R_{g}$ to that of $R_{s}$ over the period 1874-1976. The $k$-factors were derived by dividing the total amount of sunspot groups reported by the secondary observer to that from the reference observer for their overlapping period and using only those days that both observers saw at least one sunspot. Hence these $k$-factors are simple linear scaling parameters. Royal Greenwich Observatory (RGO, hereafter) was set to be the reference (including all available observations), while Horrebow and Galileo were also considered as reference observers for their respective periods. The cross-calibration of the data recorded by earlier observers was done with a daisy-chaining sequence of $k$-factors. Daisy-chaining is the process of starting with a reference observer, and calculating the $k$-factors for other overlapping observers. The latter observers, after their scaling, are in turn used as the reference for other observers with direct overlap, and so on. Figure 6.5 shows a schematic of the daisy-chaining process. Multiple routes were used and in the end the average $k-$ factor was used. However, there is no detailed documentation for the routes that were used in each case, making it almost impossible to replicate this series exactly. An issue with the daisy-chaining scheme is that it leads to error accumulation in time when going further away from the reference observer. Galileo, as well as all other disconnected observers (before the gap in 1739), were arbitrarily calibrated with $k=1.255$, which is the average value found for the modern observers. Therefore the whole series before 1739 is highly ambiguous.

The realisation that these series suffered from various problems, led to the creation of various new series. Lockwood et al. (2014) applied corrections to the ISN and GSN series. The corrections to the ISN include an $11.6 \%$ decrease for the values after 1940 to account for the Waldmeier discontinuity. Another correction is the decrease of the values before 1848 by $20 \%$ due to inconsistencies between the observations of Schwabe and Wolf (Leussu et al. 2013). Lockwood et al. (2014) also extended the ISN back to 1610 by rescaling the GSN, $R_{S}=1.3 R_{G}$. For the GSN series they adopted some corrected database values from Vaquero et al. (2011). They made use of the averaging scheme presented by Usoskin et al. (2003c) to calculate monthly and annual means for periods with sparse observations. They also extended the series with data by the Solar Optical Observing Network (SOON).

Cliver and Ling (2016, ClLi16, hereafter) revised the GSN series by using the same methodology as Hoyt and Schatten (1998). They claim, however, that the early data from 
RGO (i.e. 41 years before 1915) suffer from unsteady quality due to the alleged learning curve process (Cliver et al. 2015). Therefore, they normalised the RGO data before 1915 to those by Wolfer with a second degree polynomial fit. However, it is not clear yet if the early RGO data suffer from inhomogeneities or how extensive the problem might be. Studies by Sarychev and Roshchina (2009), Clette et al. (2014), Lockwood et al. (2016c), Willamo et al. (2017) found no problem with RGO data after 1880, 1900, 1877, and 1890, respectively. The ClLi16 series shows the highest levels of activity for the 19th century among all available GSN series. The ClLi16 series covers the period 1841-1980.

Svalgaard and Schatten (2016, SvSc16, hereafter) produced their series as the weighted average of the series derived with three different methods. For the period 1749-2015 they used daisy-chaining of $k$-factors and Wolfer as the reference observer. They introduced five key observers (called "backbones", BB hereafter) to calibrate the records of each overlapping secondary observer to their (see Fig. 6.5). In particular the BB observers were Locarno (1958-2015), Koyama (1947-1980), Wolfer (1878-1928), Schwabe (1826-1867), and Staudacher (1749-1799). The BB approach was used in an attempt to reduce the number of daisy-chain steps. However, most of the BB observers they used did not directly overlap with each other and their inter-calibration was performed through series that were extended with lower quality secondary observers. Essentially this means that three steps are needed to calibrate each BB observer to another. With non-overlapping $\mathrm{BBs}$ there is also the problem that there can be observers with records that have no direct overlap to the BB and hence cannot be calibrated (e.g. Billwiller, Zurich and Sawyer, E.F. Cambridgeport that have no direct overlap with either Wolfer or Schrabe BBs). The $k$-factors for each calibration are determined with linear regression on annually averaged values, while the fit is forced to go through the origin. For the period 1749-1860 SvSc16 used an extra normalisation to account for differences between the various observers in this period. They split observers within 1790-1835 into 2 categories, the high and low observers, and the ratio of their annual averaged counts yielded an average $k=1.58$. This $k$-factor was then applied to the low observers to match their level to that of the high observers. They also scaled the Staudacher BB to match the high observers with $k=1.68$. Finally, the third method is applied for the period 1610-2015. This method is illustrated in Fig. 6.6 and is the only method used for this series to calibrate the observers prior to 1749 . They produced another series, where for each year they keep the highest count by any observer. They divide the whole period into 4 intervals based on the period of observation of 4 of the main BB observers they used. The interval of the Staudacher BB is extended back to 1610 . Within each interval they derived one $k$-factor as the average ratio between the series they got from the BB method and that of the annual maxima. This $k$-factor is used to normalise the series of the annual maxima. SvSc16 also applied a 7\% reduction to the number of sunspot groups after 1940. This was suggested to be able to compensate for possible effects of the introduction of the Waldmeier classification of sunspot groups by the observers used in the series (Waldmeier 1939). However, it is unclear if, when, and which observers adopted this scheme. Furthermore, Lockwood et al. (2016b a) have suggested that such a correction is not needed for the GSN. This is the only series other than HoSc98 that goes back to 1610 and suggests a somewhat higher level of solar activity compared to HoSc98, except for the 20th century.

Clette et al. (2016, CEA16, hereafter) produced a new GSN series for the period 19452015 by including mostly data from the Sunspot Index and Long-term Solar Observations 
Daisy-chaining

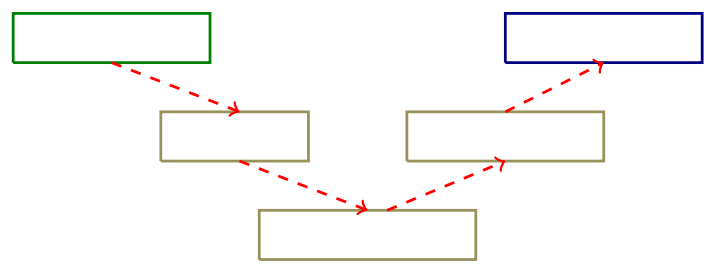

Backbones

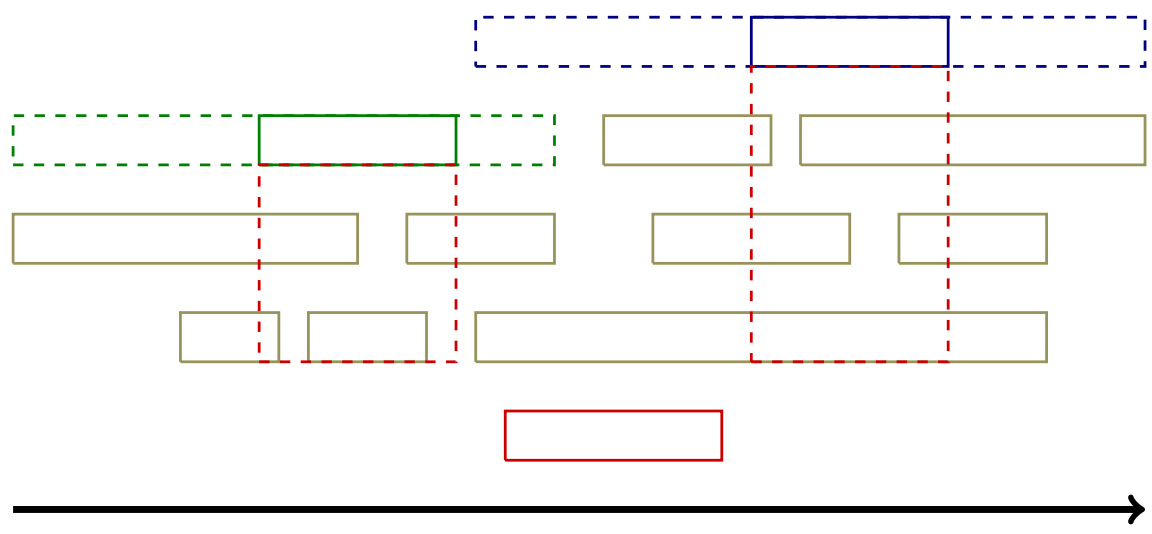

Time

Figure 6.5: Schematic of daisy-chaining and backbone methods used for the crosscalibration of sunspot records between observers. The rectangles signify time periods covered by different observers. Green colour is used for the reference observer, blue for the observer whose records are aimed at being calibrated, yellow for the secondary observers, and red for observers whose records have no direct overlap with the reference observer. The red arrows show the progression of calibration of secondary observers in the daisy-chaining. The dashed red rectangles show the overlapping periods of the secondary observers with the reference observers. The dashed green and blue rectangles show the extended backbone series after all secondary observers have been calibrated and integrated in each backbone.

(SILSO) World Data Center (WDC). Using Locarno as their reference, they calculated a series of daily $k$-factors as the ratio of the group number and the raw Wolf number. With these $k$-factors they constructed an average GSN series that then acted as the reference to derive the $k$-factors again. CEA16 assume that ISN and GSN should have constant ratios (cf. Georgieva et al. 2016). They fit a 2nd degree polynomial to the ratio of ISN to GSN to remove the activity dependent differences that are found. Any residual differences after the corrections are attributed to variations in the number of sunspots within groups.

Usoskin et al. (2016c, UEA16, hereafter) have presented the only non-linear calibration method so far. This method also works without the need for the observers to have directly overlapping records: it is free of daisy-chaining and the associated error accumulation. This method is based on the active day fraction (ADF, hereafter) statistics of an observer. ADF is the ratio of the days an observer reported at least one spot to the number of days they performed observations within a month. However, observers have different 


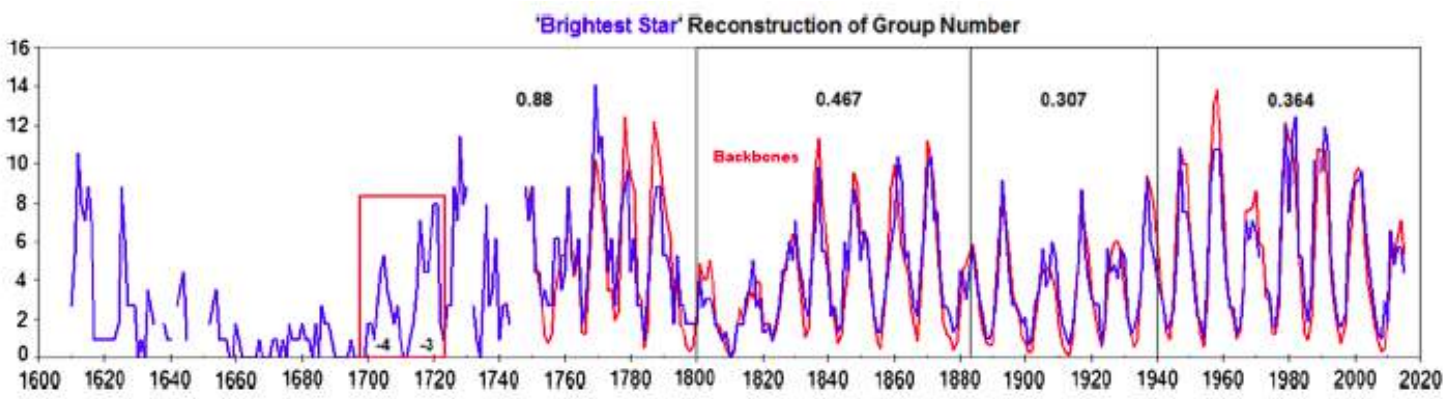

Figure 6.6: "Brightest star" method used for the SvSc16 series. The $x$ axis represents the year and the $y$ axis the group count. The red curve is the series after calibration of the backbones, while the blue curve has the maximum group count of any observer per year. The periods of the backbones are separated with vertical lines (the Koyama and Locarno backbones are combined here). Also shown is the average $k$-factor derived with this method for each backbone. Taken from Svalgaard and Schatten (2016).

observing capabilities which are expressed in having different acuity thresholds. This threshold corresponds to the smallest size of a spot an observer can detect, and anything smaller than that size will be missed. A set of artificial observers (created the same way as those we introduce in Sect. 6.2.1) based on the RGO sunspot area records were created in order to reproduce the ADF statistics for observers with different acuities. The acuity threshold of each observer was derived by a direct comparison of their ADF statistics to those of the artificial observers. The acuity threshold was used to construct the calibration matrix (in a similar way to the PDF matrices described in Sect. 7.3.3), which was then applied on the daily values of the observer. Staudacher has a peculiarity in his observations, which show very few spotless days and the ADF method would consider him an unrealistically good observer. Therefore, Staudacher was calibrated in a different way. A direct comparison matrix was built for all overlapping days ( \pm 1 days) with Horrebow (in a similar way to the PDF matrices described in Sect. 7.3.3). This matrix was used to bring Staudacher to the same level as Horrebow. Then the calibration curve that was derived for Horrebow was applied on Staudacher as well. UEA16 manually selected 20 observers that cover the period 1749-1995. This series yields a moderate level of sunspot activity in the 18th and 19th centuries, lying between the HoSc98 and SvSc16 series. This series was recently updated to include data from the revised database by Vaquero et al. (2016) and it now includes 29 observers (Willamo et al.2017).

Besides the previous series that attempt to calibrate existing observations, there have been attempts to extend or produce SN series for periods with poor coverage by extrapolation of existing series. Schove (1955, 1979) used naked eye observations and auroral sightings and extrapolated the sunspot number series to cover the period $649 \mathrm{BC}-2000$ AD. In a similar manner Nagovitsyn (1997) extrapolated the series back to 1100. Rigozo et al. (2001) extrapolated the ISN series back to 1000 with a method based on a wavelet transformation. Nagovitsyn et al. (2016) applied a similar method with a wavelet transformation to extrapolate the ISN v2 series back to 1610 using the SvSc16 GSN series as well. The reconstruction by Nagovitsyn et al. (2016) shows higher activity during the Maunder minimum than was previously considered. Travaglini (2017) used Bayesian method to reproduce the $\mathrm{SN}$ series for the period $1610-1715$. As basis he used the ISN 
v2 series, which was unchanged. The extrapolation further back in time was done using concentrations of cosmogenic radioisotopes ${ }^{14} \mathrm{C}$ and ${ }^{10} \mathrm{Be}$. These reconstructions differ significantly with each other, however all of them raise the Maunder minimum period to levels similar to that of the 20th century.

The main problem of such reconstructions is that they are not based on physics of the Sun, but rather assume that the SN series is to some extent stationary and the available measurements provide enough information to predict their evolution. Based on these assumptions the series of Schove (1955) had predicted the activity until the year 2000 and proved to be wrong. Another issue is the use of the ISN v2 and SvSc16 GSN series that have potentially inflated the SN prior to the 20th century, removing any secular trend.

There are also reconstructions of sunspot numbers from the available cosmogenic radioisotope ${ }^{14} \mathrm{C}$ and ${ }^{10} \mathrm{Be}$ data back to $7000 \mathrm{BC}$ (Usoskin et al. 2007, 2014, 2016a).

Additionally, there are two composite series of sunspot areas compiled by Hathaway and Harvey (1990) ${ }^{1}$ and Balmaceda et al. (2009) ${ }^{2}$. The series by Hathaway and Harvey (1990) used the data of RGO (1874-1976) and appended them with data from SOON for the period after 1976. The series by Balmaceda et al. (2009) is a composite of the RGO (1874-1976), Pulkovo (1977-1986), and SOON (1987-2008) records. Gaps were filled with additional data from Rome or Yannan observatories. RGO was taken as the reference and all other series were calibrated with linear regression forced through the origin.

Here we will focus on the sunspot group number series that are created by a direct calibration of existing sunspot number observations. One important implication of these series is that they imply different long-term trends for the activity of the Sun (Lockwood et al. 2016c, Kopp et al. 2016). HoSc98 and UEA16 show a trend of increasing solar activity, while ClLi16 and SvSc16 show an almost constant level of activity.

All of these series have been criticised and extensively evaluated, with none yet found to be consistent with all of the available indirect data of solar activity. For instance SvSc16 and ClLi16 disagree with cosmogenic radioisotope $\left({ }^{14} \mathrm{C},{ }^{10} \mathrm{Be},{ }^{44} \mathrm{Ti}\right)$ records while HoSc98 and UEA16 show a better agreement (Asvestari et al. 2016, Asvestari 2016, Asvestari et al.2017); SvSc16 disagree with auroral data (Lockwood et al.2016d); no series agrees with the solar wind magnetic field measurements, although $\mathrm{SvSc} 16$ agree better than other series during the maxima (Cliver 2016).

Most of the previously described series use an outdated methodology that is based on constant $k$-factors. The computation of the $k$-factors is done with ordinary linear least square regression that is forced to go through the origin. Application of this method is associated with various assumptions and their validity is not usually mentioned. These assumptions are listed here:

1. The relation between two variables $x$ and $y$ is linear for all $x$-values. However, the relationship between the sunspot (group) number series of two observers has been demonstrated to be non-linear by Lockwood et al. (2016e), Usoskin et al. (2016c), and Chatzistergos et al. (2017d). This is also discussed in Sect. 6.2.3.

2. The pairs of $x$ - and $y$-values are taken randomly from the same population and have sufficient lengths. This assumption is valid for the sunspot series.

\footnotetext{
${ }^{1}$ Available at https://solarscience.msfc.nasa.gov/greenwch.shtml

${ }^{2}$ Available at http://www2 .mps.mpg.de/projects/sun-climate/glchange.html
} 

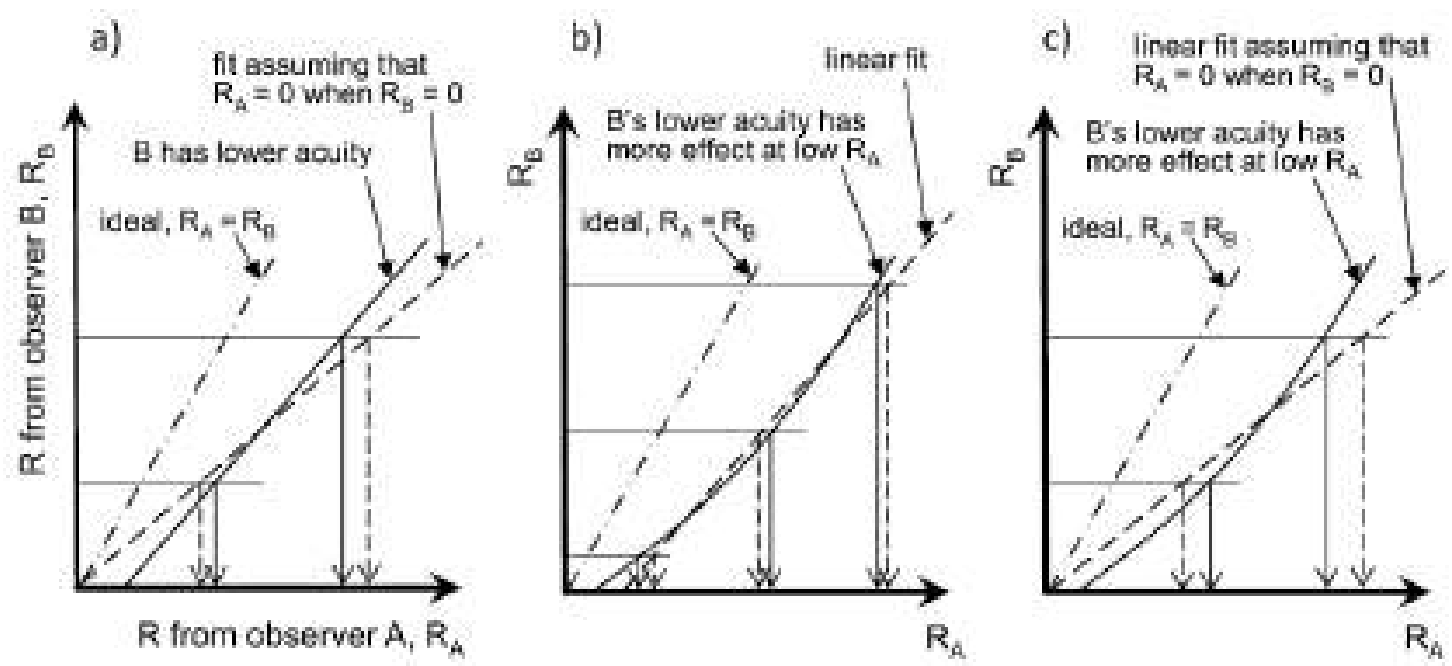

Figure 6.7: Schematic drawings showing the effects of applying a linear calibration on sunspot data with $(\mathbf{a}), \mathbf{c})$ ) and without $(\mathbf{b})$ ) forcing the relation to go through the origin for the cases that the assumed relation between the counts of the observers is linear (a)) and non-linear (b),c)). Illustrating that forcing the regression through the origin results in a worse fit. Taken from Lockwood et al. (2016a)

3. The errors follow a normal distribution around the true values and are independent of each other. This assumption is also invalid for the sunspot numbers, since they have been shown to be asymmetric and not normal (Usoskin et al.|2016c).

4. The sample has constant variance (homoscedasticity). This assumption is also not valid since the variance of the data is larger for periods of high activity than around solar minima.

5. $x$-values are known precisely and are error-free. This assumption is invalid, since both variables have uncertainties. Furthermore, in these studies the $x$-axis corresponds to the observer to be calibrated, and it is most likely to be the one having greater uncertainties.

6. The relation is assumed to go through the origin. This is also invalid (Lockwood et al.2016e) since, if no spots were reported by an observer, it does not necessarily mean that another observer with better acuity would not see any spots as well (see Fig. 6.7).

Among the six assumptions made by the $k$-factor methodology for the sunspot numbers, only one is valid and hence such practices are erroneous. It becomes obvious that the series needs to be revised and reconciled. 


\subsection{Dependence of the Sunspot-Group Size on the Level of Solar Activity and its Influence on the Calibration of Solar Observers}

The contents of this section are based on the journal article Usoskin, I. G., Kovaltsov, G. A., Chatzistergos, T., Solar Physics, 291, 3793-3805, 2016, with the permission of Springer.

In Sect. 6.1.1 we mentioned some of the reasons that can make the records from different observers disagree with each other. The effect of all these cases on the individual series is difficult to disentangle. However, we assume that the main difference arises because all observers are imperfect, each one having their own observational acuity threshold (Lockwood et al. 2016e, Usoskin et al.2016c). The different acuities can arise due to the telescope capabilities, the eyesight of the observer or the average seeing conditions at the location of the observations. This is nicely illustrated in Fig. 6.8 which shows drawings of the Sun made by Galileo and Jungius on the same day. Galileo was able to differentiate between small and large spots, but Jungius could not resolve the smaller spots and so only saw "blobs" over these regions. Therefore, both observers would only return the same number of sunspot groups in the absence of small isolated spots. The relation between the counts of two observers depends strongly on the number of isolated small spots and their proximity to the larger ones. The relation can only be linear if the fraction of missed to detected groups is constant over the solar cycle. However, various studies found that the fraction of small groups was higher around solar activity minima and decreased with the level of solar activity (Kilcik et al. 2011, Jiang et al. 2011, Nagovitsyn et al. 2012, Obridko and Badalyan 2014). In this section, we study the dependence of the sunspot size on the level of the solar activity. We create pseudo-observers with different acuities to study how the relation between their counts depends on the acuity.

\subsubsection{Data}

In this work, we used the sunspot group number records from the Vaquero et al. (2016) database as well as the sunspot group area records from $\mathrm{RGO}^{3}$. Catania ${ }^{4}$, and Rome ${ }^{4}$ observatories. In this section we only consider the RGO data within the period 1916-1976 (17943 days) to exclude data with uneven quality due to the previously suggested learning-curve process (see Sect. 6.1.3 and 7.4.2.3). We also do not use the SOON data because of a possible transition inhomogeneity (Balmaceda et al. 2009, Lockwood et al. 2014, Hathaway 2015). We have checked qualitatively that all of our results are unaffected if we use different periods for RGO. The data from the Catania observatory cover the period 1978-1999 with 4000 days, while the data from the Rome observatory cover the period 1958-2000 with 8311 days.

We created a number of artificial observers using the sunspot area records from RGO, Rome, and Catania observatories by imposing various acuity thresholds. These are thresh-

\footnotetext{
${ }^{3}$ Available at: http://solarscience.msfc.nasa.gov/greenwch.shtml

${ }^{4}$ Available at: https://www.ngdc.noaa.gov/stp/solar/sunspotregionsdata.html
} 
olds $(A)$ on the minimum size of a sunspot group that the observer would count. Therefore all sunspot groups with size greater than $A$ are counted and all smaller ones are missed. We used whole sunspot group areas in msd uncorrected for foreshortening, in order to make these synthetic data similar to what a real observer would see from Earth. We used thresholds between $0 \mathrm{msd}$ (defining the "ideal" observer) and $200 \mathrm{msd}$ ("poor" observer). This range of acuities corresponds to actual observers with proper telescopes (e.g., Vaquero and Vázquez 2009, Arlt et al. 2013, Neuhäuser et al. 2015, Usoskin et al. 2016c). Using 3 different archives to perform the analysis in this section ensures that our findings are not artefacts of one archive.

\subsubsection{Distribution of sunspot-group sizes ${ }^{5}$}

Here we investigate how the size of sunspot groups changes with solar activity. This is usually studied using the mean size of sunspot groups (Jiang et al. 2011), but this may be confusing because the size distribution of spots is highly asymmetric and the mean value is not a robust feature. Figure 6.9a) depicts the cumulative distribution function (CDF) of sunspot-group sizes for the $\mathrm{RGO}$ synthetic data. $\mathrm{CDF}(A)$ is defined as the fraction of the sunspot groups with area not smaller than the given value of the area threshold $[A]$. By definition $\operatorname{CDF}(0)$ is equal to unity, since each group has a non-zero size. The black curve shows the global CDF for all the sunspot groups (about 119300 groups) in the reference dataset. The blue dotted line represents the CDF for 920 groups (46 days) for high activity days with 20 groups $(G=20)$ reported. The red dashed curve depicts the $\mathrm{CDF}$ for 2781 days with low activity (only one group reported, $G=1$ ). One can see that there is a significant fraction of large groups even for low-activity days: $\approx 10 \%$ of groups have area greater than $500 \mathrm{msd}$. The group-size distribution changes with the level of solar activity: while the CDF for low-activity days is lower than the global CDF, the distribution for high-activity days is significantly higher. For example, as one can see from Figure 6.9 a), the relative contribution of large sunspot groups $(A \geq 500 \mathrm{msd})$ doubles for high-activity days $(G=20)$ with respect to low-activity days $(G=1)$. This implies that the rise of activity is mostly due to the emergence of large sunspot groups, indicating that the sunspot-group size distribution changes with the level of solar activity. To generalize the study of the CDF dependence on the level of solar activity, we plotted in Figure 6.9 $\mathrm{b}$ a contour plot of the CDF as a function of the activity level (quantified in $G$ ) and the group size (quantified in msd). All the CDF were normalized to that at the low-activity level $(G=1$, see red dashed line in Figure 6.9a) ) so that $\operatorname{CDF}(G=1)$ is unity for all $A$. One can see that the shape of CDF changes with the level of solar activity so that the fraction of large spots is growing, while the fraction of small spot decreases with activity. For example, the brown spot in the top-right corner implies that the relative fraction of groups with area $>500 \mathrm{msd}$ is nearly doubled for high-activity days $(G=20)$ compared to lowactivity days $(G=1)$, as discussed above for Figure 6.9a). In the subsequent section we study how the fact that the size distribution of sunspot groups changes with the level of solar activity affects solar observers of different quality and their mutual inter-calibration.

\footnotetext{
${ }^{5}$ The contents of this subsection are identical to the printed version of Usoskin, I. G., Kovaltsov, G. A., Chatzistergos, T., Solar Physics, 291, 3793-3805, 2016, with the permission of Springer.
} 


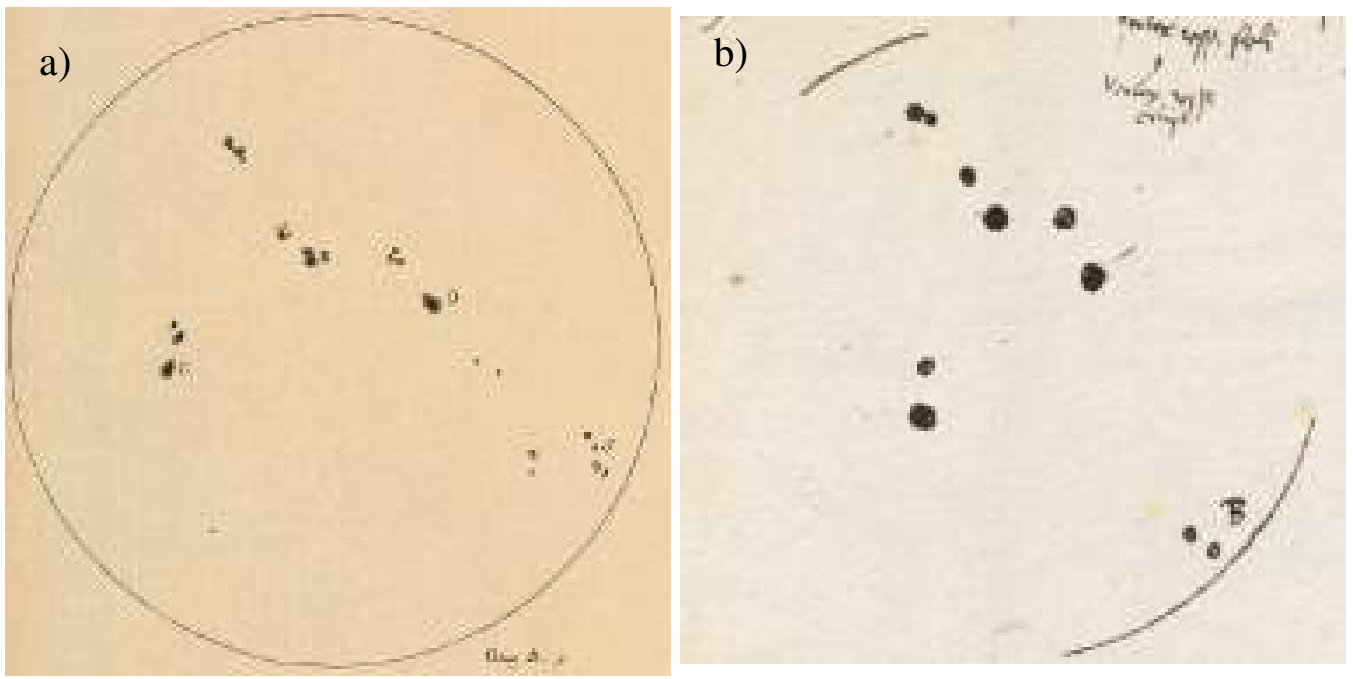

Figure 6.8: Observations of the Sun made on 09/06/1612 by Galileo (a)) and by Jungius (b)). Taken from Neuhäuser and Neuhäuser (2016).

\subsubsection{Relation between records of observers with different acuities}

We define the correction factor $c$ to be the ratio of the sunspot group counts between two observers with different acuity thresholds. This is a function of $G$.

$$
c\left(G_{A}\right) \equiv \frac{G_{\text {ref }}}{G_{A}},
$$

where $G_{\text {ref }}$ and $G_{A}$ are the group counts of the reference observer and the one with acuity threshold $A$.

Subtracting 1 from $c$ we get:

$$
c\left(G_{A}\right)-1=\frac{G_{\mathrm{ref}}-G_{A}}{G_{A}} .
$$

The numerator corresponds to the number of spots seen by the reference observer but missed by the secondary one. The denominator corresponds to the number of spots seen by the secondary observer. Therefore, $c\left(G_{A}\right)-1$ represents the ratio of missed to observed spots for an observer with acuity threshold $A$. Figure 6.10 shows $c\left(G_{A}\right)-1$ as a function of $G_{A}$ for 4 different values of $A$ using the synthetic data from RGO group areas. The figure illustrates that an observer with a certain acuity threshold would miss higher fraction of spots for low than for high activity periods. For instance an observer with acuity threshold of 100 msd would report $\sim 60 \%$ and $\sim 20 \%$ fewer groups for a low $(G=1)$ and a high $(G=15)$ activity period, respectively. Therefore, applying a linear scaling between the counts of observers with different acuities would return erroneous results.

To evaluate the relation between the records of two observers that have different acuities, we used the artificial observers described in Sect. 6.2.1. In this way we mimic an observer with finite acuities or telescope limitations.

We related the counts by each synthetic observer to the reference observer, using all daily values. Thus, we created a conversion matrix between the reference observer $(A=0)$ and the one with acuity threshold $A$. This matrix represents the probability for a count $G^{*}$ 

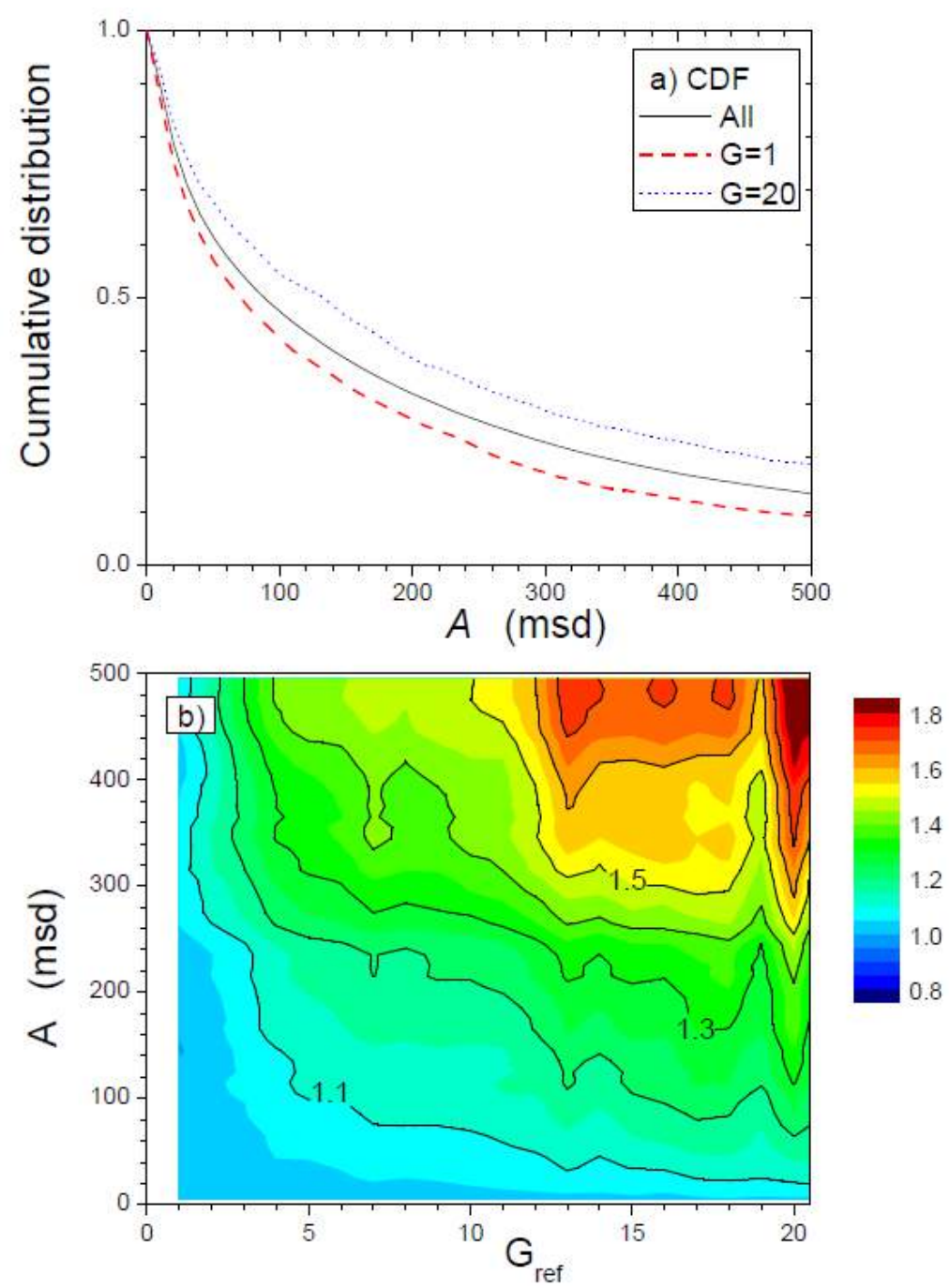

Figure 6.9: Size distribution of sunspot groups from the synthetic RGO data. Panel a): CDF of sunspot group size above a given threshold $A$. The solid line is for all sunspot groups, while the red (blue) lines show the CDF for low (high) activity days. Panel b): 2D map of the CDF as function of the activity level and the group size, normalized to the $\mathrm{CDF}$ at $G=1$. Taken from Usoskin et al. (2016b). 


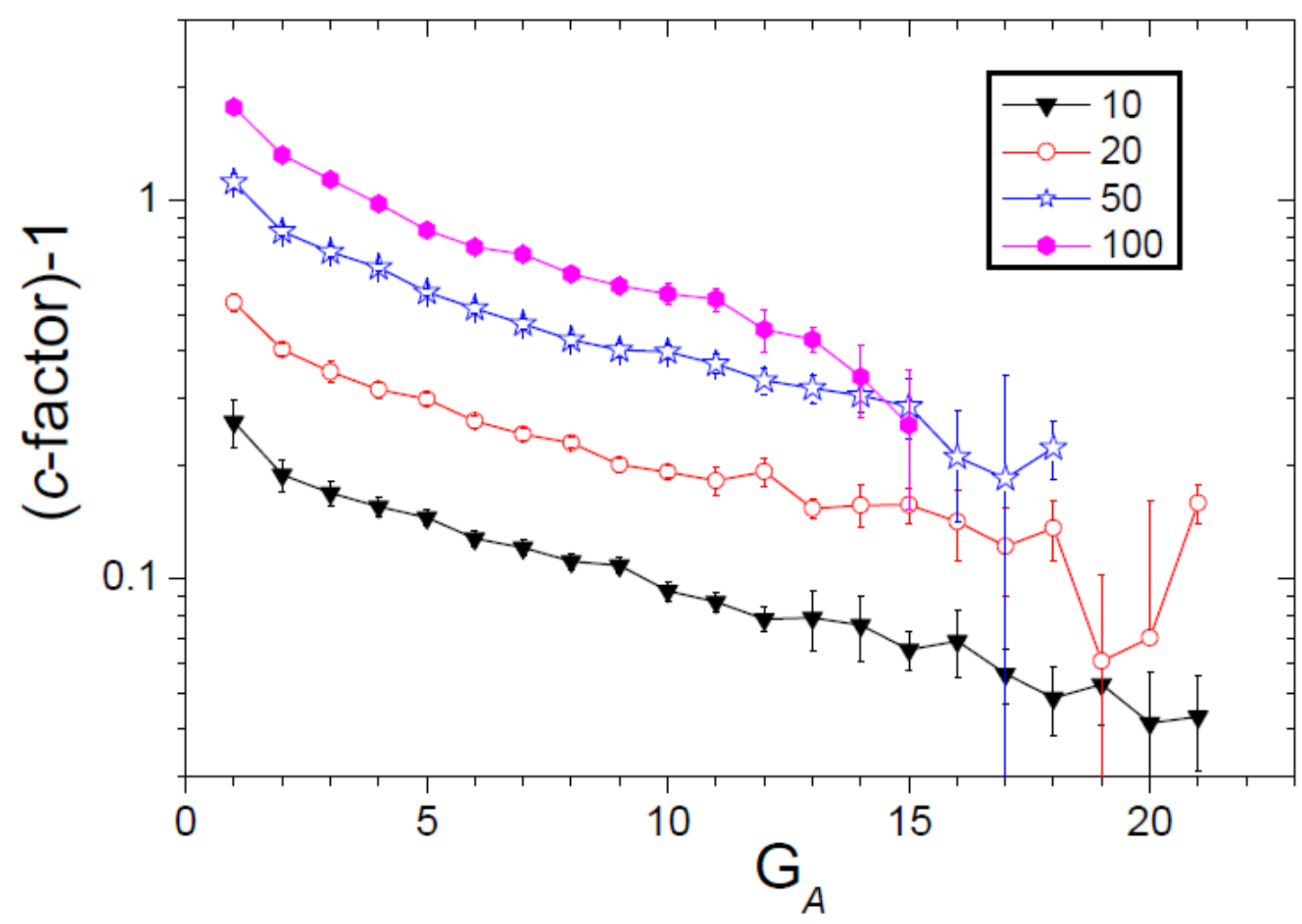

Figure 6.10: Ratio of the "small" (i.e. smaller than the given value of the threshold $[A]$ in units of msd as denoted in the inset) to "large" sunspot groups as a function of the number of "large" groups, in the total daily number of sunspot groups for the RGO dataset for the period 1916-1976. This corresponds to the correction $c$-factor minus 1 (see text). The error bars represent the standard statistical errors of the ratio. Taken from Usoskin et al. (2016b).

of the reference observer to occur for every possible count $G$ of the secondary observer (see Sect. 7.3 .3 for more details on how the matrices are created). Figure 6.11a) shows one example matrix for the synthetic data from RGO with acuity threshold $A=30$ msd and the original RGO data $(A=0 \mathrm{msd})$. To make the relation better visible we plot the matrix for the difference between the records of the observers (Fig. 6.11b)). The $c$ factor is also plotted in Fig. 6.11 c). Figures 6.12 and 6.13 show difference matrices derived for the synthetic data from RGO and Catania for acuity thresholds 10, 50, 100, and $200 \mathrm{msd}$. The observer with higher acuity threshold always reports fewer counts. We notice that the relation between the counts of two observers is non-linear and tends to saturate for higher $G$ values. The saturation level increases with acuity threshold. We find a similar behaviour with the synthetic data from the RGO, Rome, and Catania observatories, suggesting that this is the general effect of different acuities on sunspot observations. The mean of the difference values within each column asymptotically approaches a constant offset, which we approximate with the following function:

$$
\left\langle G^{*}\right\rangle-G=R_{0}+B e^{-a G},
$$

where $\left\langle G^{*}\right\rangle$ is the mean count of the reference observer (here, with $A=0 \mathrm{msd}$ ) for a given 
count of the secondary observer $G . R_{0}$ is the asymptotic value, while $B$ corresponds to the difference of $\left\langle G^{*}\right\rangle$ for $G=0$ to the asymptotic value, $a$ is the exponential rate. The best-fit parameters of $R_{0}, B$, and $a$ are derived with the weighted least mean squares method. $\left\langle G^{*}\right\rangle$ is fixed for a pair of observers, hence there are only two unknown parameters. Figures 6.12 and 6.13 also show the fits with green curves. The fit represents all $G$ counts accurately, but tend to lie higher than the last few points in each matrix. This implies that the observer with a higher acuity threshold is more accurate when more spots are present on the surface. However, these last bins contain a very limited number of days (for example in Fig. 6.12a) the bin for $G_{A}=24$ is based on 1 day). Therefore they are not statistically significant.

Figure 6.14 shows the fit parameters of Eq. 6.5 on synthetic data from RGO for various acuity thresholds. The parameters are found to increase almost smoothly with increasing acuity threshold.

\subsubsection{A test: Wolf vs. Wolfer}

Here we analyse the relation between the counts of two prominent observers in the 19th century: Rudolf Wolf and Alfred Wolfer, both from the Zürich observatory. They have been considered as primary observers in the WSN/ISN series and define a significant portion of the series in the 19th and early 20th centuries. The data of Wolf have been calibrated to those by Wolfer with a $k$-factor of 1.66 . This $k$-factor was initially suggested by Wolfer himself for the WSN/ISN, and has been used ever since for the ISN and group sunspot number series of SvSc16 (Clette et al. 2014, Svalgaard and Schatten 2016). We did the same analysis as in Sect. 6.2.3 including all days on which both observers reported their values. These are for 4385 days during the period 1876-1893. Figure 6.15 shows the relation matrix between Wolf and Wolfer, implying that Wolf reports fewer groups than Wolfer for the same day. This is consistent with Wolf having a higher acuity threshold. The matrix shows similar behaviour as that derived for the synthetic data (Fig. 6.11). In the plots we also show the $k$-factor of 1.66 with a yellow line. One can see that the $k$-factor of 1.66 lies consistently higher than the average values of the bins for $G>4$, while it is slightly lower for $G<4$. This means that the linear scaling with $k=1.66$ applied to the data of Wolf would on average tend to overestimate the activity during active periods and underestimate it during less active periods. We also note that when Wolf reported no groups, Wolfer reported $0.401_{-0.13}^{+0.06}$ groups on average. Figure 6.15 ; shows the corrections factor, $c$, between Wolf and Wolfer and illustrates that the ratio of the missed to seen groups is highly non-linear. In Fig. 6.16 we show the group counts of Wolfer and Wolf during August 1893. The shown counts of Wolf are corrected using Eq. 6.5 (black) and $k=1.66$ (blue). It becomes obvious that the scaling with the constant $k$-factor results in overestimating the higher counts, on average by 3-4 counts when $G^{*}>10$. The correction with the non-linear method presented here, results in a better agreement between the counts of the two observers. This approach is used in Chapter 7 to reconstruct a new group sunspot number series. 

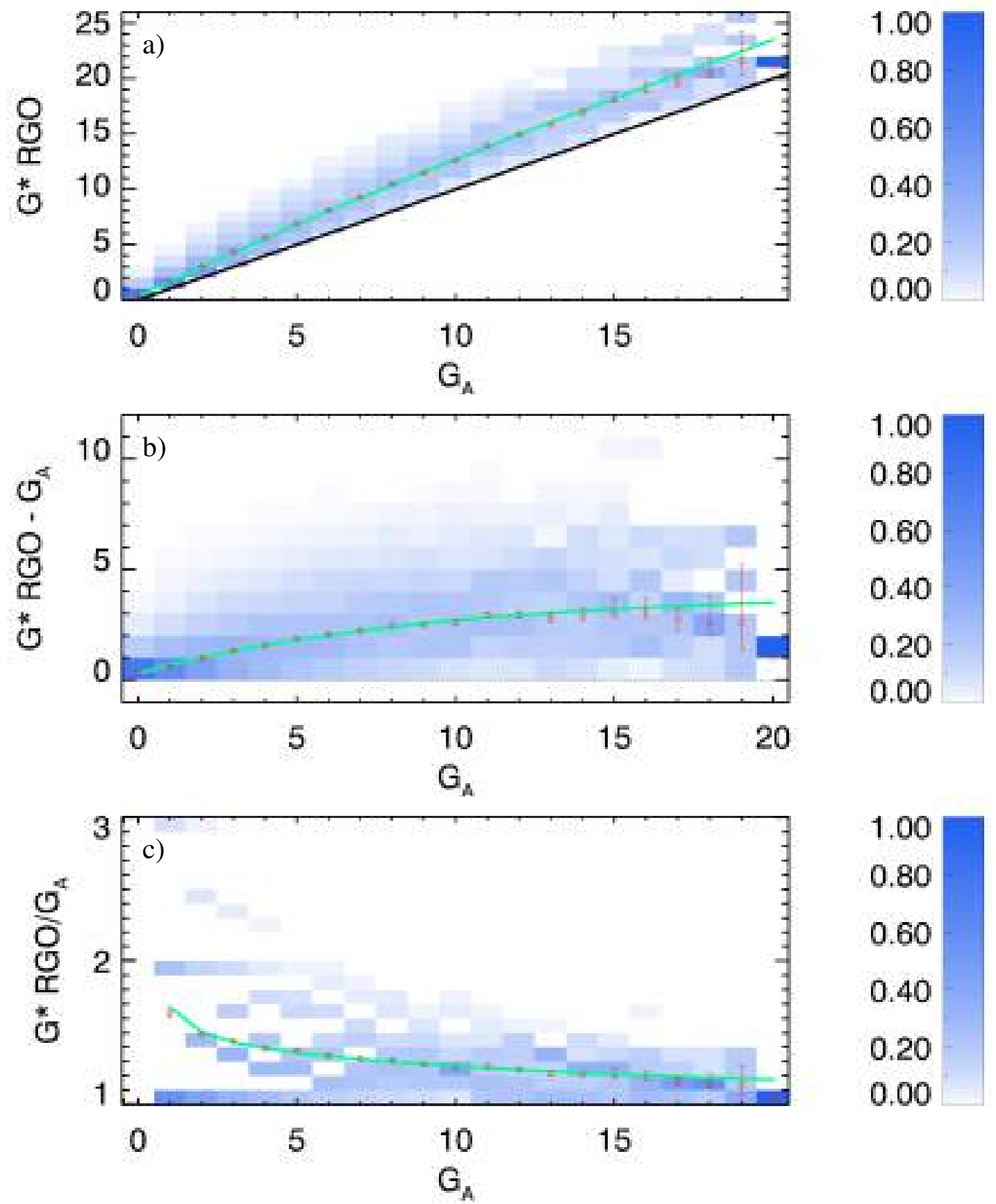

Figure 6.11: The relationship between daily sunspot-group numbers as counted by a synthetic observer created from RGO data with acuity threshold $A=30 \mathrm{msd}$ (secondary observer, $G_{A}$ ) and RGO (primary, $G^{*}$ ) from 1916-1976. Panel a) shows the PDF of the scatter plot of $G^{*}$ vs. $G_{A}$ : the black line has a slope of unity. Panel b) shows the difference, $G^{*}-G$ vs. $G_{A}$. Panel c) shows the correction factor, $c$ as a function of $G_{A}$. The red circles with error bars depict the mean $G^{*}$ values for each $G_{A}$ column and their $1 \sigma$ uncertainty. The green curves are the best fits from Eq. 6.5 . 

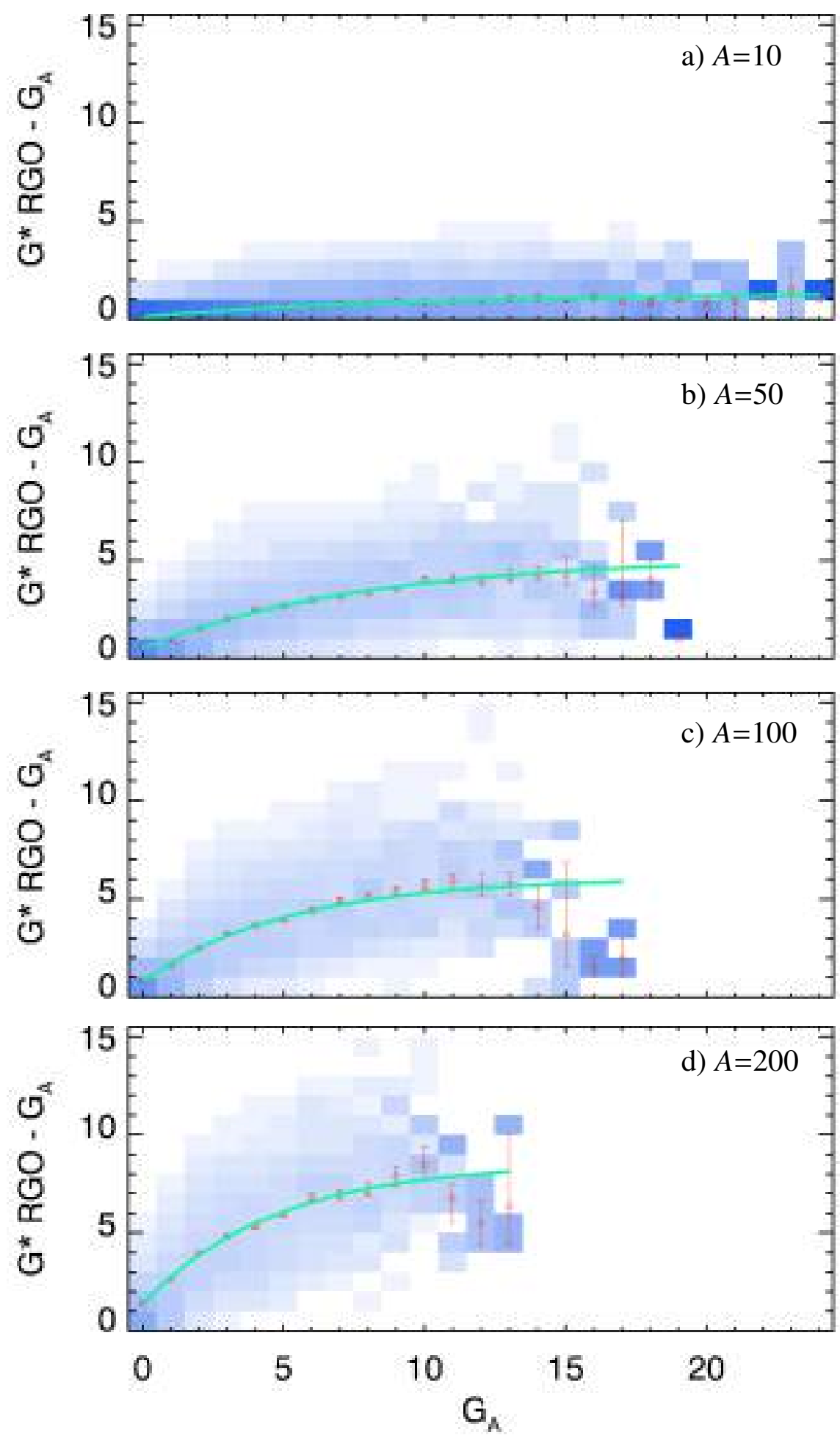

Figure 6.12: Difference matrices, $G^{*} \mathrm{RGO}-G_{A}$ vs. $G_{A}$, where $G^{*} \mathrm{RGO}$ are the counts from the original RGO data and $G_{A}$ the counts from the RGO synthetic data with acuity threshold $A$. Panels a)-d) are for acuity threshold 10, 50, 100, and $200 \mathrm{msd}$. The green curves are the best fits from Eq. 6.5 

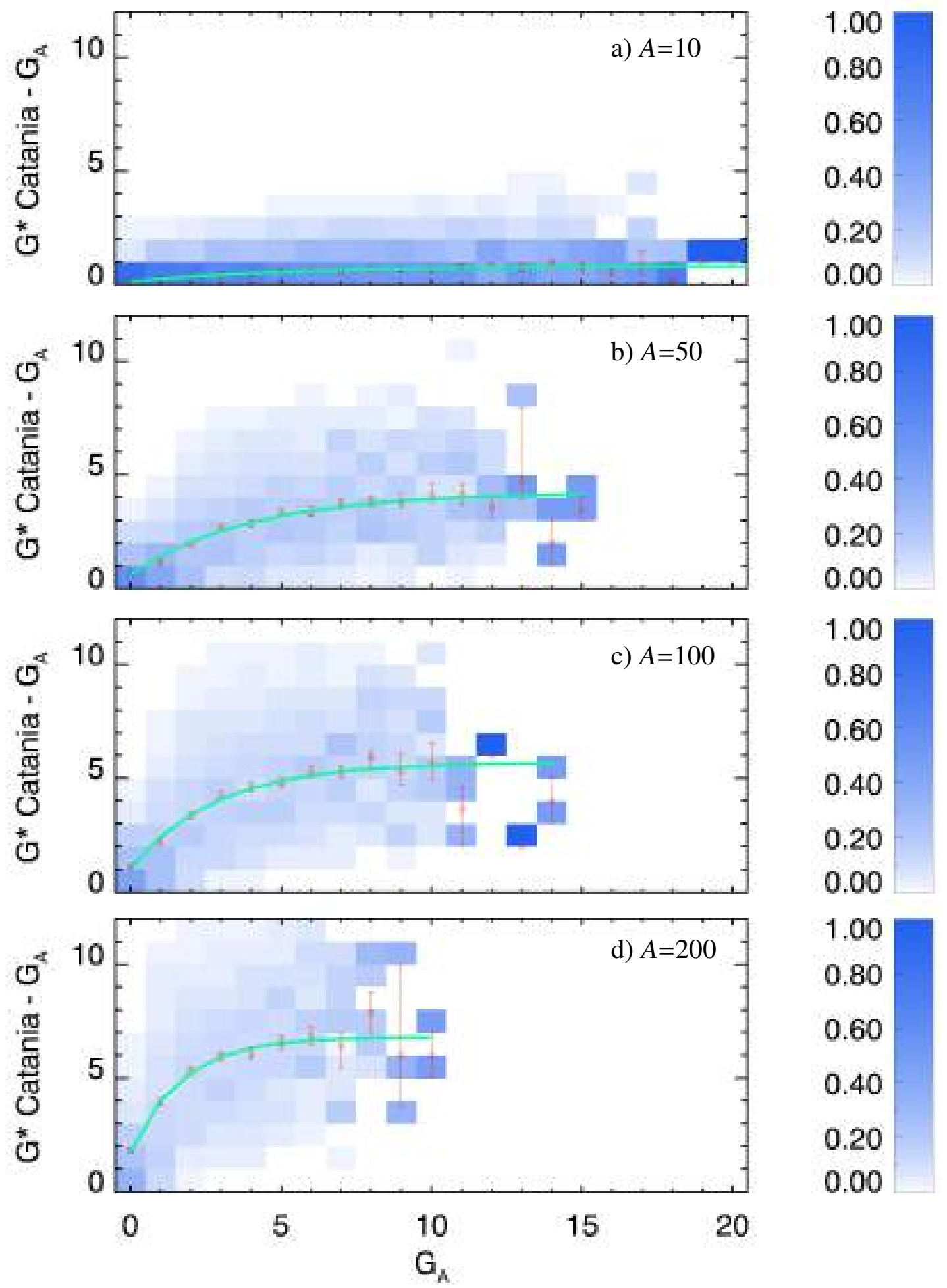

Figure 6.13: Same as Fig. 6.12 but for data from the Catania observatory. 


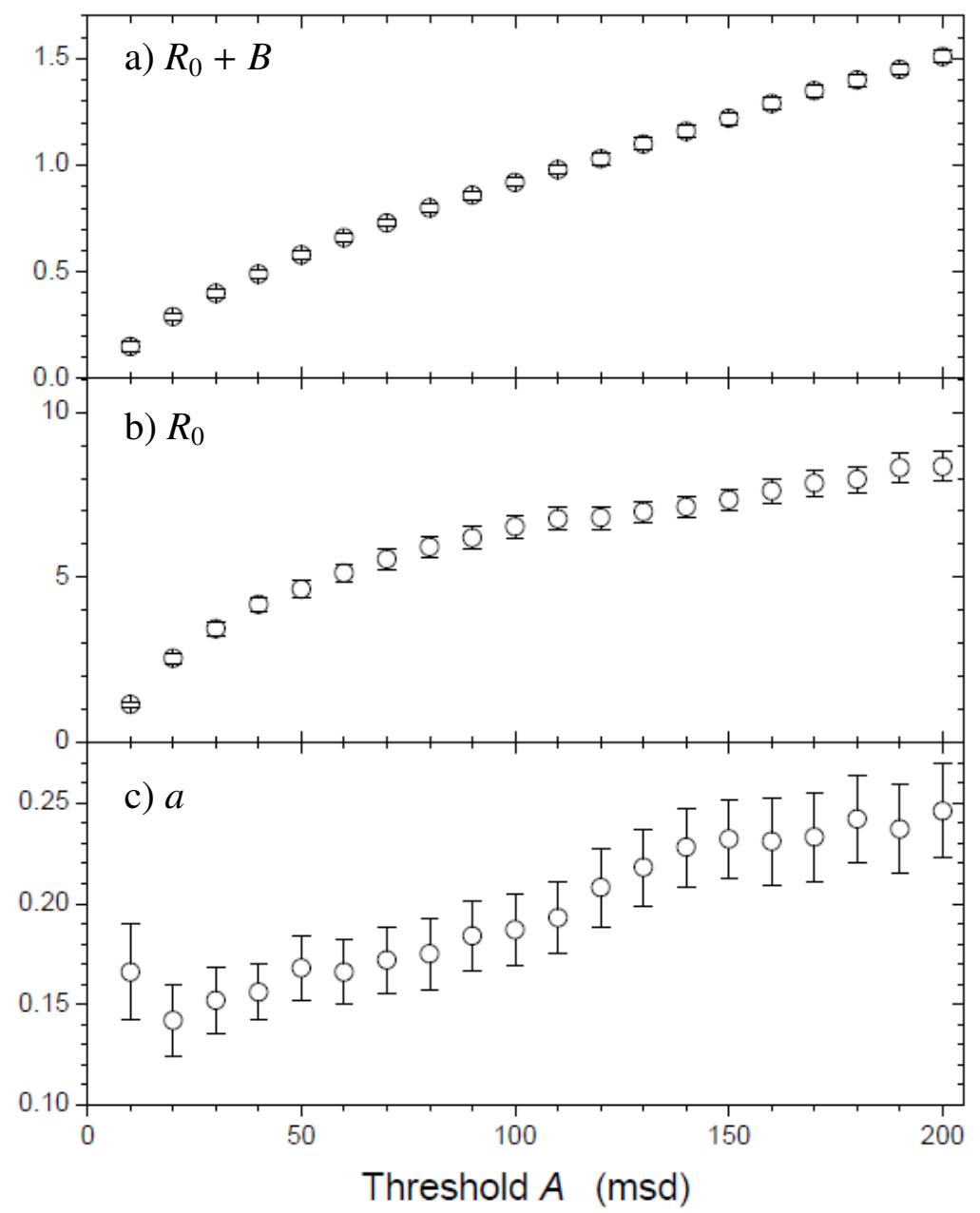

Figure 6.14: Dependence of the best-fit parameters of Eq. 6.5 on the acuity threshold $A$ using the reference dataset. The parameters are shown with the $68 \%$ confidence-interval uncertainties. Taken from Usoskin et al. (2016b). The legends of the plot have been modified to be consistent with the notation used in this work.

\subsection{Summary and conclusions}

The sunspot number series is a very important dataset, since it is the only available direct information about the activity of the Sun between 1610 and 1876, when RGO also started recording other spot properties (areas, position). The data pose a challenge as to how to calibrate them and produce one homogeneous time-series. Many attempts to perform this task applied a simple linear relation, which as we showed does not represent the data well. Such methods effectively assume that the fraction of small to big spots remains constant throughout the solar cycle. However, our analysis of the dependence of the size of sunspot groups on the activity level of the Sun shows that the fraction of small spots decreases with activity. We studied the relation between the records of observers with different capabilities using synthetic observers and found that this relation is strongly non-linear. We further modelled this relation using an exponential function with an asymptotic approach to the difference of the counts. We find that this approach allows 

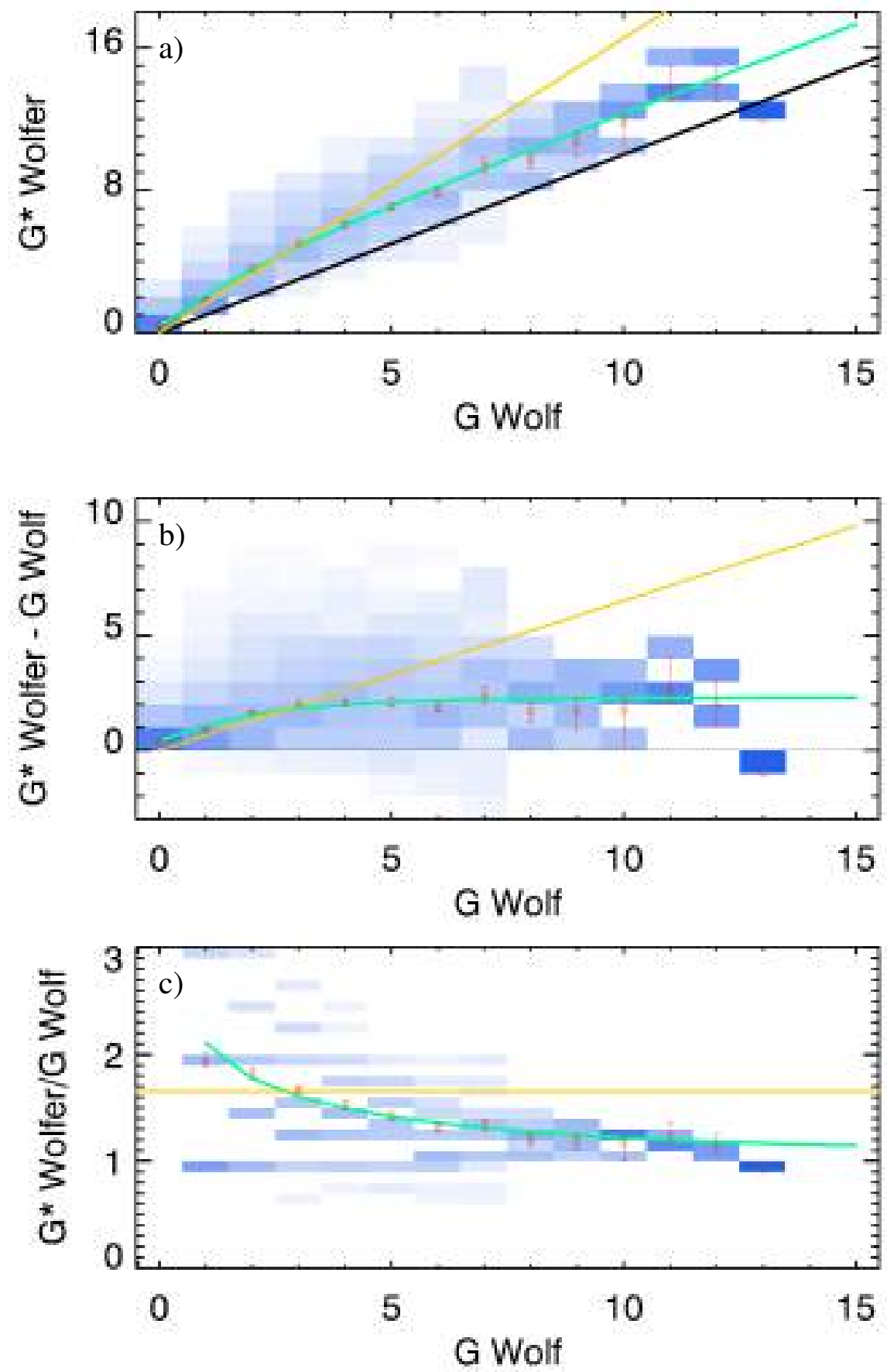

Figure 6.15: Same as Fig. 6.11 but using the records of Wolf (secondary observer, $G$ ) and Wolfer (primary, $G^{*}$ ) from 1876-1893. The yellow line shows the $k$-factor of 1.66 often used to calibrate the counts of Wolf to Wolfer. 


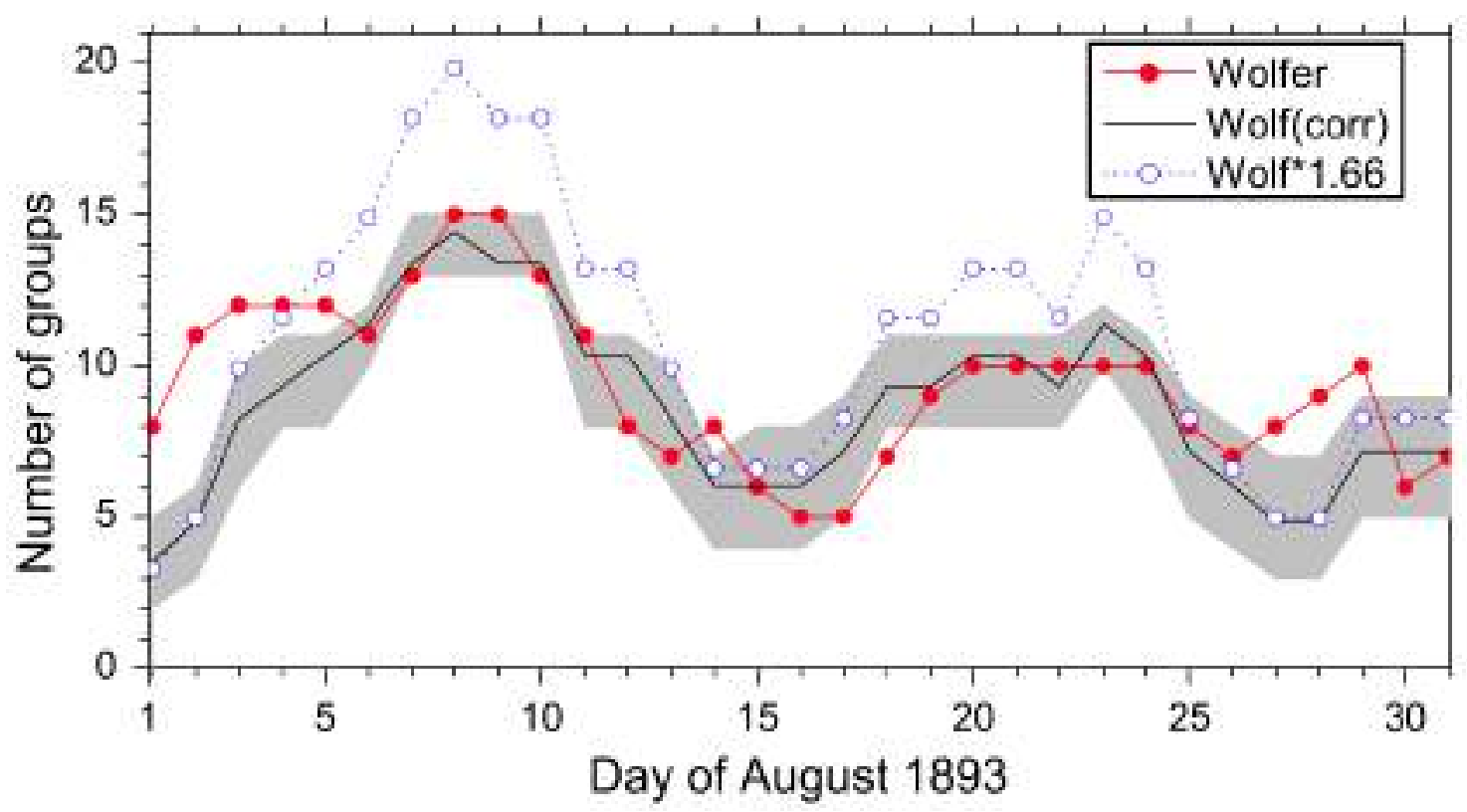

Figure 6.16: Daily number of sunspot groups for August 1893. The red curve shows the number of groups reported by Wolfer, the black curve with its $68 \%$ confidence interval denotes the data by Wolf normalized to Wolfer by the method described here, and the blue dotted curve shows the data by Wolf scaled with the $k$-factor of 1.66 . Taken from Usoskin et al. (2016b).

a better calibration of the records of two observers, while the commonly used method of linear $k$-factors results in potential overestimation of high activity periods. 



\title{
7 New reconstruction of the sunspot group numbers since 1739 using direct calibration and "backbone" methods
}

The contents of this chapter are identical to the printed version of Chatzistergos, T., Usoskin, I. G., Kovaltsov, G. A., Krivova, N. A., Solanki, S. K., A\&A, 602, A69, 2017, reproduced with permission (CESO.

\begin{abstract}
Context. The group sunspot number (GSN) series constitute the longest instrumental astronomical database providing information on solar activity. This database is a compilation of observations by many individual observers, and their inter-calibration has usually been performed using linear rescaling. There are multiple published series that show different long-term trends for solar activity.

Aims. We aim at producing a GSN series, with a non-linear non-parametric calibration. The only underlying assumptions are that the differences between the various series are due to different acuity thresholds of the observers, and that the threshold of each observer remains constant throughout the observing period.

Methods. We used a daisy chain process with backbone (BB) observers and calibrated all overlapping observers to them. We performed the calibration of each individual observer with a probability distribution function (PDF) matrix constructed considering all daily values for the overlapping period with the $\mathrm{BB}$. The calibration of the BBs was carried out in a similar manner. The final series was constructed by merging different BB series. We modelled the propagation of errors straightforwardly with Monte Carlo simulations. A potential bias due to the selection of BBs was investigated and the effect was shown to lie within the $1 \sigma$ interval of the produced series. The exact selection of the reference period was shown to have a rather small effect on our calibration as well.

Results. The final series extends back to 1739 and includes data from 314 observers. This series suggests moderate activity during the 18th and 19th century, which is significantly lower than the high level of solar activity predicted by other recent reconstructions applying linear regressions.
\end{abstract}

Conclusions. The new series provides a robust reconstruction, based on modern and nonparametric methods, of sunspot group numbers since 1739, and it confirms the existence 
7 New reconstruction of the sunspot group numbers since 1739 using direct calibration and "backbone" methods

of the modern grand maximum of solar activity in the second half of the 20th century.

\subsection{Introduction}

Observations of sunspots on the solar disc have been performed regularly since the advent of telescopes in the early 17th century. These measurements constitute the longest ongoing observational programme in astrophysics, providing important insights into solar activity and variability on centennial timescales.

However, these observations have been carried out by different people, with different instruments, at various locations. In some cases observations were taken for a different purpose but were also later used to define sunspot numbers. The definition of a sunspot group might have changed with time, gaps exist within the series of individual observers, and the various series do not necessarily all overlap with each other. Even for the same observer, the quality of the record may vary with time owing to, for example gaining experience, ageing of the observer (e.g. deteriorating eyesight), change of instrumentation, or varying conditions at the observing location. There have been several attempts to harmonize these measurements and to produce a homogeneous composite series. The first effort was made by Rudolf Wolf from Zürich who introduced the Wolf sunspot number (WSN) in 1848 (Wolf 1850, continued and updated as the international sunspot number, ISN), given by the formula

$$
R_{s}=k(10 G+S),
$$

where $k$ is a weighting factor to normalize the various observers with each other, $S$ the number of sunspots, and $G$ the number of sunspot groups. It is important that, for the sake of homogeneity, data from only one primary observer were used for each day. If the data from the primary observer were not available for a given day, data from the secondary, tertiary, etc., observer were used, but only one observation was used per day, ignoring all other available data. The original records and notebooks of Wolf are not readily available now, implying that WSN cannot be re-constructed from scratch. This series contains annual values back to 1700 , while monthly and daily values go back to 1749 and 1818, respectively. Since 1981 the WSN/ISN series has been synthesized by the Royal Observatory of Belgium (Clette et al. 2007), adapted to include all available observers for each day, rather than only the primary observer. The WSN/ISN series has been recently updated as version 2.0 by correcting for some proposed inhomogeneities (Clette et al.2014).

More than a century after the work by Wolf, Hoyt and Schatten (1998) introduced the group sunspot number (GSN) series (HoSc98, hereafter), which is based on the number of sunspot groups only, neglects individual spots and includes data from all observers on the same day. The daily GSN is defined as

$$
R_{g}=\frac{12.08}{N} \sum_{i} k_{i} G_{i}
$$

where $k_{i}$ is the individual correction factor of the $i$ th observer, $G_{i}$ is the GSN reported by the $i$ th observer, $N$ is the total number of observers on the given day, and the constant 12.08 was introduced to match the average level of $R_{g}$ to that of $R_{s}$ over the period 18741976. The GSN series was designed to be more robust than WSN/ISN since it only 
considers sunspot groups and reduces uncertainties in the counts of individual sunspots. In addition, the GSN series includes a much greater number of raw data than WSN and is extended further back in time to 1610. An important advantage is that for the GSN series, a complete database of the raw data (published as Hoyt and Schatten 1998, and revised recently by Vaquero et al. 2016) is available, which makes it possible to reconstruct the entire series from scratch.

The homogenization and cross-calibration of the data recorded by earlier observers was always performed through a daisy-chaining sequence of linear scaling normalization of the various observers, using the $k$-factors. This means that starting with a reference observer, the $k$-factors are derived for overlapping observers. The latter data are in turn used as the reference for the next overlapping observers, etc. As is apparent, this leads to error accumulation in time when moving further away from the reference observer.

It has become obvious that the old series need to be revised because of the newfound data and the outdated methodology based on constant $k$-factors. The issue with such methods is twofold. Firstly, such methods assume that counts by two observers are proportional to each other, which is generally not correct. Secondly, the $k$ - factors are assumed to be constant for the entire operational period of each observer, whereas in reality the acuity of the observers and sensitivity of the instruments may vary with time. A dedicated activity of the research community (Clette et al.2014) has led to several new sunspot series discussed below.

Cliver and Ling (2016, ClLi16, hereafter) have attempted to revise the GSN series using essentially the same methodology as Hoyt and Schatten (1998). They claim, however, that the earlier part of the Royal Greenwich Observatory (RGO hereafter) data (i.e. 41 yr before 1915) might suffer from uneven quality owing to the purported learning curve process. Therefore, they corrected the GSN values over this period by normalizing them to the data by Wolfer using a second degree polynomial fit. The inhomogeneity of the early RGO data is still a matter of debate, however. Other studies did not find any extensive problem with RGO data: Sarychev and Roshchina (2009), Clette et al. (2014), and Lockwood et al. (2016c) reported as potentially problematic periods before 1880, 1900, and 1877, respectively, while data from Aparicio et al. (2014) and Carrasco et al. (2013) do not exhibit any apparent trend with respect to RGO data after $\sim 1885$ and 1890, respectively. Thus, the period of 1874-1915 used by ClLi16 to "recalibrate" the RGO dataset is not well defined. The ClLi16 series covers the period 1841-1980 and yields the highest level of sunspot activity in the mid-19th century among all available reconstructions.

Svalgaard and Schatten (2016, SvSc16, hereafter) also used the method of daisychaining $k$-factors. But these authors introduced five key observers (called "backbones", BB hereafter) to calibrate each overlapping secondary observer to these BBs. Thus, they seemingly reduced the number of daisy-chain steps because some daisy-chain links are moved into the $\mathrm{BB}$ compilation rather than being eliminated. The problem with this method is that most of the BB observers did not overlap with each other. Thus their inter-calibration was performed via series extended using secondary observers with lower quality and poorer statistics. In the end, this introduces even more daisy-chain steps, since each $\mathrm{BB}$ observer is normalized to the neighbouring observer using a three-step procedure. The SvSc16 series also reduced the number of sunspot groups after 1940 by $7 \%$ to take into account the possible effect of the introduction of the Waldmeier classification of sunspot groups (Waldmeier 1939). However Lockwood et al. (2016ad) have 
7 New reconstruction of the sunspot group numbers since 1739 using direct calibration and "backbone" methods

questioned the necessity for such a correction for the GSN. The SvSc16 series covers the period 1610-2015 and suggests a rather high level of solar activity in the 18th and, especially, 17th centuries.

All of these sunspot number series used calibration methods based on the linear scaling regression to derive constant $k$-factors. However, this linear $k$-factor method has been demonstrated to be unsuitable for such studies (Lockwood et al. 2016e, Usoskin et al. $2016 \mathrm{~b}$ c), leading to errors in the reconstructions that employ them.

An alternative method was proposed by Usoskin et al.(2016c, UEA16, hereafter), who calibrated each observer directly to the reference dataset, avoiding the daisy chain and error accumulation. The method is based on comparison of the active day fraction statistics of an observer with that in the reference dataset (RGO data for the period 1900-1976). The quality of each observer is characterized by the acuity observational threshold so that the observer is assumed to miss all sunspot groups that are smaller than this threshold, and to report all sunspot groups that are larger than this threshold. The acuity threshold for each observer is found by matching their active day fraction statistic with that of an artificially created reference dataset. The UEA16 series covers the period 1749-1995 and yields a moderate level of sunspot activity in the 18th and 19th centuries, lying between the HoSc98 and SvSc16 series.

Another revision of the GSN series was carried out by Lockwood et al. (2014) who corrected it for some apparent inhomogeneities. However, since this study is close to the HoSc98 series, we do not consider it separately here.

Thus, presently there are a number of sunspot reconstructions using different methods of calibration and yielding results that are inconsistent with each other. The most critical implication of these series is that they yield different long-term trends for the activity of the Sun (Lockwood et al. 2016c, Kopp et al. 2016). Over the 19th and 20th centuries, ClLi16 and SvSc16 show no trend, while HoSc98 and UEA16 show an increase in solar activity.

In an attempt to bridge the methodologies underlying previous studies and present more accurate error estimates, we present here a recalibration of the GSN data using an amendment of the most direct non-parametric calibration method described in Usoskin et al. (2016b). Similarly to SvSc16, we incorporate BB observers. However, the calibration of overlapping observers is performed with a non-linear non-parametric probability distribution function (PDF) derived from sunspot group counts for days when two observers overlap. This allows us to account for the error propagation in a straightforward manner. Calibration of the different resulting BB series is achieved with daisy chaining.

The data we use are introduced in Sect. 7.2. The procedure, including information about all individual BB observers and their processing is described in Sect. 7.3. Our composite series is presented and compared with other existing series in Sect. 7.4, where we also discuss the stability of our method and potential problems of our series. We summarize our results in Sect.7.5. 


\subsection{Data}

We employ the database ${ }^{1}$ of the sunspot group numbers recorded by individual observers that was recently published by Vaquero et al. (2016) as an update of the HoSc98 database. Observers are uniquely identified by their identification number in the database. Here we use these identification numbers as well.

We apply the following filters to these data:

- Data by Wolfer (1880-1928, id 338) were merged with those by Billwiller and Wolfer (1876-1879, id 335). The two series were combined together to a single series, since they do not directly overlap. The two series differ in that the former includes observations solely by Wolfer, while the latter includes observations made by both Wolfer and Billwiller. By merging these two series together, we can increase the length of the Wolfer series and its overlap with observations by Schmidt.

- Data from Flaugergues, H., Aubenas (1794-1795, id 22) were also merged with those from Flaugergues, H., Viviers (1788-1830, id 227) using the same procedure. These two datasets were obtained by the same observer, Flaugergues, who performed the bulk of his observations in Viviers, Ardéche, but who relocated to Aubenas for a period of about two years.

The dataset from Aubenas contains merely 91 observations for these two years, a period of otherwise sparse observations (we have only nine records from all other observers used here). The overlap of the observations of Flaugergues from Aubenas to other observers is less than three days and does not provide adequate statistics to properly calibrate this series. Considering that the two locations are close to each other in the south of France, we make the assumption that the observing conditions were not significantly different. This enables us to merge the two Flaugergues series. Furthermore, because of the poor overlap with other series, inclusion of these data does not affect the rest of our series.

The HoSc98, ClLi16, SvSc16, and UEA16 series were downloaded from the SILSO 2 (Royal Observatory of Belgium) website.

\subsection{Calibration process}

\subsubsection{Algorithm and primary observers}

We have developed an automated algorithm to perform the calibration of sunspot records by individual observers which includes the following steps:

- First, we selected primary BB observers who provided long and high-quality observations.

- Next, we calibrated the data from all other observers, denoted as secondary observers hereafter, to the primary BB observers using periods of overlapping observations (sufficient overlap is required, see Sect. 7.3.3), and produced the $\mathrm{BB}$

\footnotetext{
${ }^{1}$ Available at http://haso. unex.es/?q=content/data

${ }^{2}$ http://www.sidc.be/silso/groupnumberv3
} 
7 New reconstruction of the sunspot group numbers since 1739 using direct calibration and "backbone" methods

series', which are composites of data from the BB observer and all other observers calibrated to him/her.

- Individual BB series were cross-calibrated to each other, using the daisy-chain procedure.

- Finally, the composite series of daily GSN was constructed by averaging the calibrated BB series.

The calibration was carried out using a direct non-parametric method to a single reference dataset with a straightforward propagation of errors. No regression was used and the acuity of the observers was assumed constant over their entire observing life. The method is described in detail in Sect. 7.3.2

The selected sequence of the primary BB observers is Kanzelhöhe, RGO, Wolfer, Schmidt, Schwabe, Flaugergues, and Horrebow (see Table 7.1). The BB observers were selected to be those with sufficiently long observational records of high quality. We also used Schubert, Zucconi, and Hagen as stand-alone BBs. Because of the lacking bridge in the data in the middle of the 18th century, we were unable to directly calibrate these three observers to a single observer acting as a BB. Thus we did this by the extended statistics of the calibrated BB series. These observers are important since they cover periods over the 18th century when no other data are available. Our reference observer is RGO (but restricted to the period between 1900-1976) and all other BB series were calibrated to the level of RGO.

All data from RGO prior to 1900 were ignored when considering the primary BB observer because of the disputed inhomogeneity, as discussed in the Introduction. We discuss the effect of this decision on our calibration in Sect. 7.4.2.3.

\subsubsection{Secondary observers}

Each BB series was also filled with all available secondary observers calibrated to the primary BB observers. As secondary observers we selected all the observers that have at least one nominal year of overlap with the primary BB observer. To avoid a distortion of statistics, each observer was included only in one BB. The assignment of observers to the BBs was made based on the length of the overlapping period and by trying to match observers with comparable quality BB observers. The only two successive BB observers whose observations do not overlap in time are Horrebow and Flaugergues. The bridging was made using Staudacher data. In this case, we chose Horrebow as the BB over Staudacher, because he observed more frequently and the data are of higher quality. Unfortunately, we were not able to go further back in time than Hagen (1739), because of the very sparse observations over this period with no observer making observations both before and after 1739 with adequate data to perform the calibration. Table 7.1 and Figure 7.1 provide key information about the BB observers and series.

All the observers we used for various BBs are listed in Tables 7.2 through 7.8. Figure 7.2 shows the number of days within each year covered by (a) the different BB series (i.e. including both primary and secondary observers) and by (b) our final composite series. One can see that the coverage is very good after ca 1800, but very poor in 1780-1795. This poorly covered period has led to large uncertainties in the daisy-chain method in the 18 th century. 


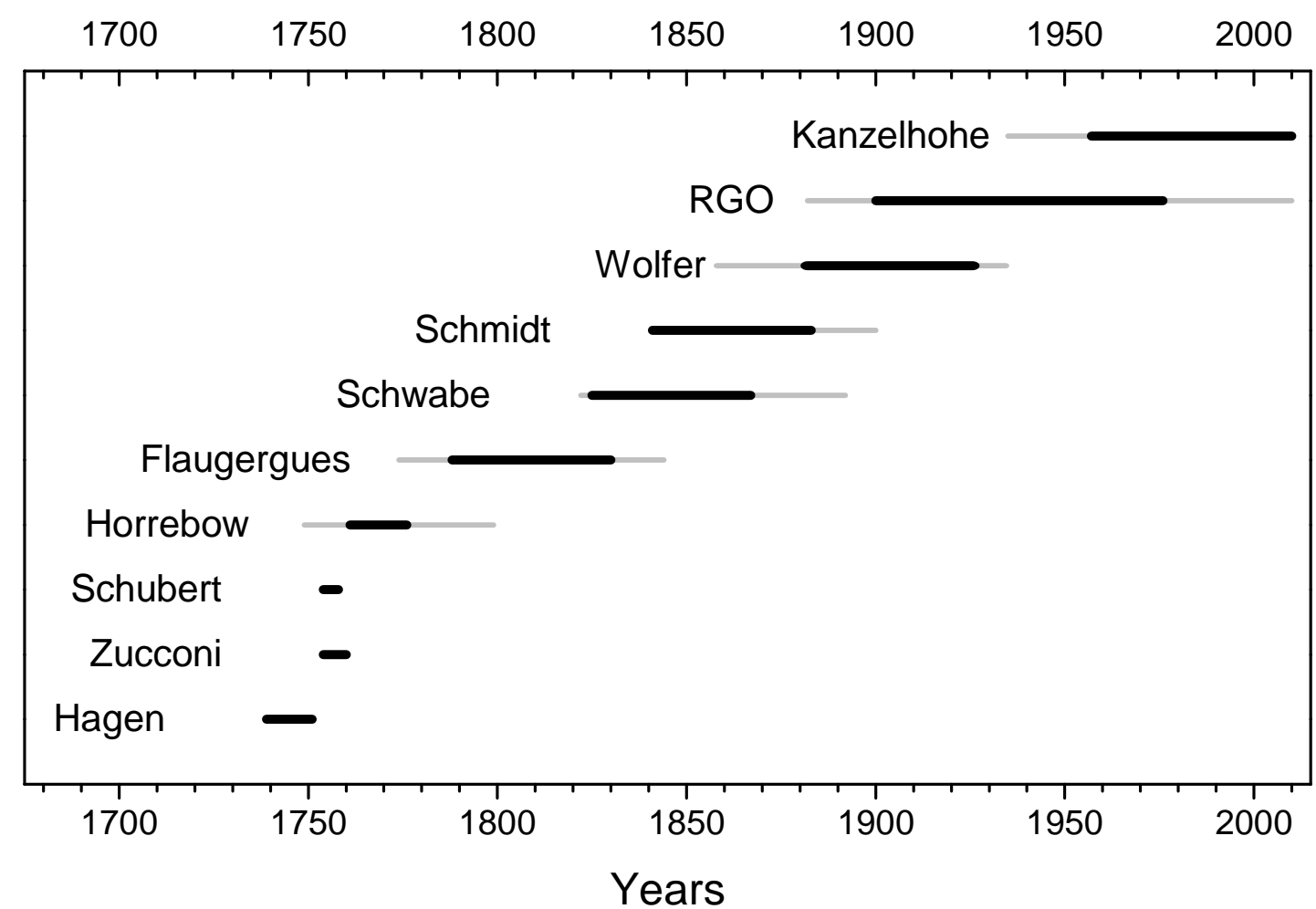

Figure 7.1: Temporal coverage by the BBs used here. Solid black lines represent the primary BB observers, while grey lines depict the extension of the BBs using calibrated secondary observers.

\subsubsection{Construction of the backbone series}

We started by building a direct calibration matrix (cf. Usoskin et al. 2016b) between the secondary observer to be calibrated and the primary BB observer for the days when both have observations. If, on a given day, $N_{1}$ and $N_{2}$ groups were recorded by the primary and secondary observers, respectively, then unity was added to the row $N_{1}$ and column $N_{2}$ of the matrix. In this way, the matrix was filled with all the overlapping days. Then the matrix was normalized such that each of its values were divided by the total sum over the corresponding column. Thus, we obtained a matrix of probability density functions (PDF) to find a value of $G^{*}$ reported by the primary observer for each day with the given value $G$ reported by the secondary observer. This allows a direct calibration of the secondary observer to the primary observer by replacing the $G$ value with the PDF of $G^{*}$. This is the most straightforward method for calibration applied directly to the data.

However, this matrix can potentially have some gaps due to poor statistics and limited range of overlap between the observers. In such cases, we fill the gaps by fitting the statistically significant part of the matrix with a function

$$
\left\langle G^{*}\right\rangle-G=R_{0}+B e^{-a G},
$$

where $\left\langle G^{*}\right\rangle$ are the mean counts of the primary observer (i.e., the mean of the PDF of each column of the matrix) for a given count of the secondary observer $G, R_{0}, B$, and $a$ are constants calculated for each pair of observers individually. We used the weighted 
7 New reconstruction of the sunspot group numbers since 1739 using direct calibration and "backbone" methods

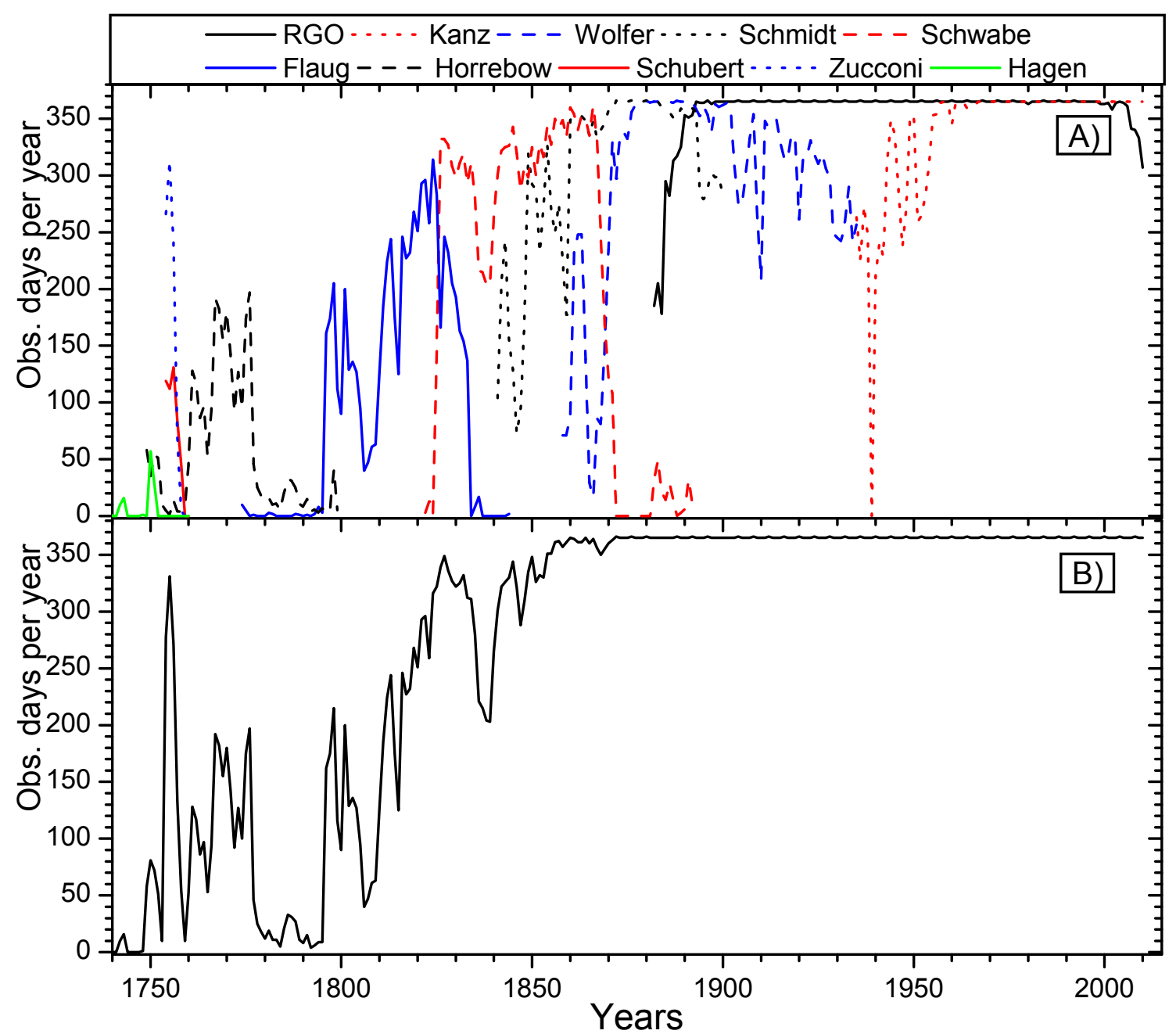

Figure 7.2: Annual coverage (number of observational days per year) by the different BB series (coloured curves in panel a)) and by our final composite series (panel b)).

least mean squares to find the best-fit parameters. This functional shape (asymptotic exponential approach to a constant offset in the difference) was proposed by Usoskin et al. (2016b) and found suitable for this kind of dependence, using synthetic data that were based on RGO sunspot group area data.

Only those columns of the matrix that contain more that 20 overlapping days were included into the fitting procedure. If the fit deviated by more than one group from the actual mean $\left\langle G^{*}\right\rangle$, such columns were excluded, and the fit was redone. In such cases we refilled the column matrices using a PDF derived with a bootstrap Monte Carlo (MC, hereafter) simulation. For this, we randomly selected half of the overlapping days from the two observers, reconstructed the matrix using this half-statistics and recalculated the fit for the matrix. This process was repeated 1000 times. The result of this simulation was used as a PDF for the corresponding column in the matrix.

An example of the matrix is shown in Fig. 7.3a) for Winkler (secondary observer, $G$ ) and RGO (primary reference observer, $\left.G^{*}\right)$ over the period of their overlap (1900 - 1910 with 2480 common days). It is apparent that RGO typically reported more groups than Winkler for the same day, since most of the matrix values lie above the line expected for 
a perfect match between the two (black line). The matrix of the difference, $G^{*}-G$ versus $G$, is shown in Fig. 7.3 b). The red circles with error bars represent the mean $\left\langle G^{*}\right\rangle$ value in each $G$ column and its (asymmetric) $1 \sigma$ intervals. The green curve shows the best fit of the functional form of Eq. (7.3). It is obvious that the relation between $G^{*}$ and $G$ is non-linear and cannot be represented by a simple linear scaling $k$-factor. One can see that, because of the limited overlap, the matrix is well constructed only for $G<9$. For higher values, the fit (Eq. (7.3) has to be used. The full matrix with the values filled with the MC method for $G>8$ is shown in Fig. $7.3 \mathrm{c}$ ).

Each secondary observer was calibrated to the BB observer by replacing, from the matrix, every daily count $G$ with the PDF of the calibrated counts $G^{*}$. In this way we directly convert the observations of the secondary observer to the BB condition without making any assumption about the type of relationship (e.g. linearity) and with a straightforward error estimate.

For each BB we constructed a composite series by averaging all the PDFs of all the available observations for every day, so that again, instead of one count for each day, we get a distribution based on all available observers. This composite of averaged PDFs includes possible errors in a straightforward way.

Only observers with a sufficiently long record of relatively good quality were included into the analysis. The selection of secondary observers was made using the following criteria:

1. The overlap with the primary BB observer should be not less than 20 common days of observations. This criterion was not applied for early years (see Sect.7.3.3.1).

2. Observers with an overall record longer than $10 \mathrm{yr}$ were considered only if their overlap with the primary BB observer was at least $4 \mathrm{yr}$. This is merely to make sure that long-running observers are not calibrated with a small fraction of their observations that might not be representative.

3. In cases in which we need to perform the fit to extrapolate to missing values in the matrix, we requested the conversion matrix for a selected observer to have sufficient data to cover at least three $G$-value bins. This is necessary since the function described by Eq. (7.3) has three parameters.

4. The matrix should cover, with sufficient statistics, at least one-quarter of the range of counts reported by the secondary observer.

5. Observers were excluded from the analysis if the difference matrix (see an example in Fig. 7.3b)) had an average offset of more than two groups for the $G$ values from 0 to 5 .

6. Observers, whose data could not be fitted accurately enough $\left(\chi^{2}\right.$ per degree of freedom $<6$ ), were also excluded.

After the calibration process of all observers, we compared each individual observer with the composite BB series they were part of. We excluded those that showed significant and systematic discrepancies. Four observers were removed as they showed such differences, namely Taipei observatory (Id 456), Lunping (Id 457), Mojica, Cochabamba, 
7 New reconstruction of the sunspot group numbers since 1739 using direct calibration and "backbone" methods

Bolivia (Id 628), and XE (Id 715). We also excluded the Locarno station (Id 614), because of the possible lack of stability after 1980 (Clette et al.2016).

There are also some special cases, which are described below in detail.

\subsubsection{Sparse data: Schwabe and earlier backbones}

Because of the lack of data for the first years of the Schwabe BB, we have not applied the criterion 1 from the list above to his data. Furthermore, while constructing the calibration matrix we considered observations not only during overlapping days but also within \pm 1 day; if there was no direct overlap, we first checked one day earlier and then one day later, making sure that no more than one pair entered the matrix. Possible errors due to short-lived groups are negligible compared to the gain of the increased statistical sample (Willis et al. 2016b, Usoskin et al. 2016c). These relieved constraints were also applied to the BBs covering earlier periods, when the statistics were poor.

\subsubsection{Correcting for low quality observations: Flaugergues, Schubert, Zucconi, and Hagen backbones}

For most BBs, we were able to match observers with a relatively similar quality. This was not the case for Flaugergues, though. Flaugergues' data are very important, because they are the only record covering a relatively extended period in the early 1800 s. However, the $G$ values he reported are significantly lower than those by other observers during that period, implying that his observations are of lower quality (higher acuity observational threshold). Therefore, a calibration of all other observers, with higher quality data, directly to Flaugergues would reduce their quality while increasing the uncertainties. In order to avoid that, we made use of a corrected Flaugergues series, calibrated to the mean level of the other observers of the period. In order to make the correction, we assumed that the acuity threshold for Flaugergues is $A=100 \mathrm{msd}$, which is greater than for any other observer (Usoskin et al. 2016c). In this case the acuity threshold for Flaugergues does not even have to be the correct one, but it only should allow us to calibrate the overlapping observers without downgrading their quality. Applying the $100 \mathrm{msd}$ threshold and the method described in Usoskin et al. (2016b), we obtained the following parameters for Eq. (7.3) for Flaugergues: $a=0.18, R=6.94$, and $B=6.03$. Then other observers were calibrated to this "corrected" Flaugergues series.

The same process with the same threshold was used for the Schubert, Zucconi, and Hagen BBs.

\subsubsection{Inter-calibration of backbone series}

Once the $\mathrm{BB}$ series were constructed and calibrated to the primary $\mathrm{BB}$ observer, different $\mathrm{BB}$ series had to be inter-calibrated to each other. We used the RGO BB as the reference one, and the others were calibrated to it using a daisy chain. The calibration of the BB series was performed using a procedure similar to that for the individual observers, by constructing the cross-calibration matrix between the whole BB series this time. However in this case, we have, for each day, not a single $G$ value but a PDF from each observer (now the entire composite BB series is considered an observer). In order to account for that, we constructed the calibration matrix using a MC simulation as described below. 

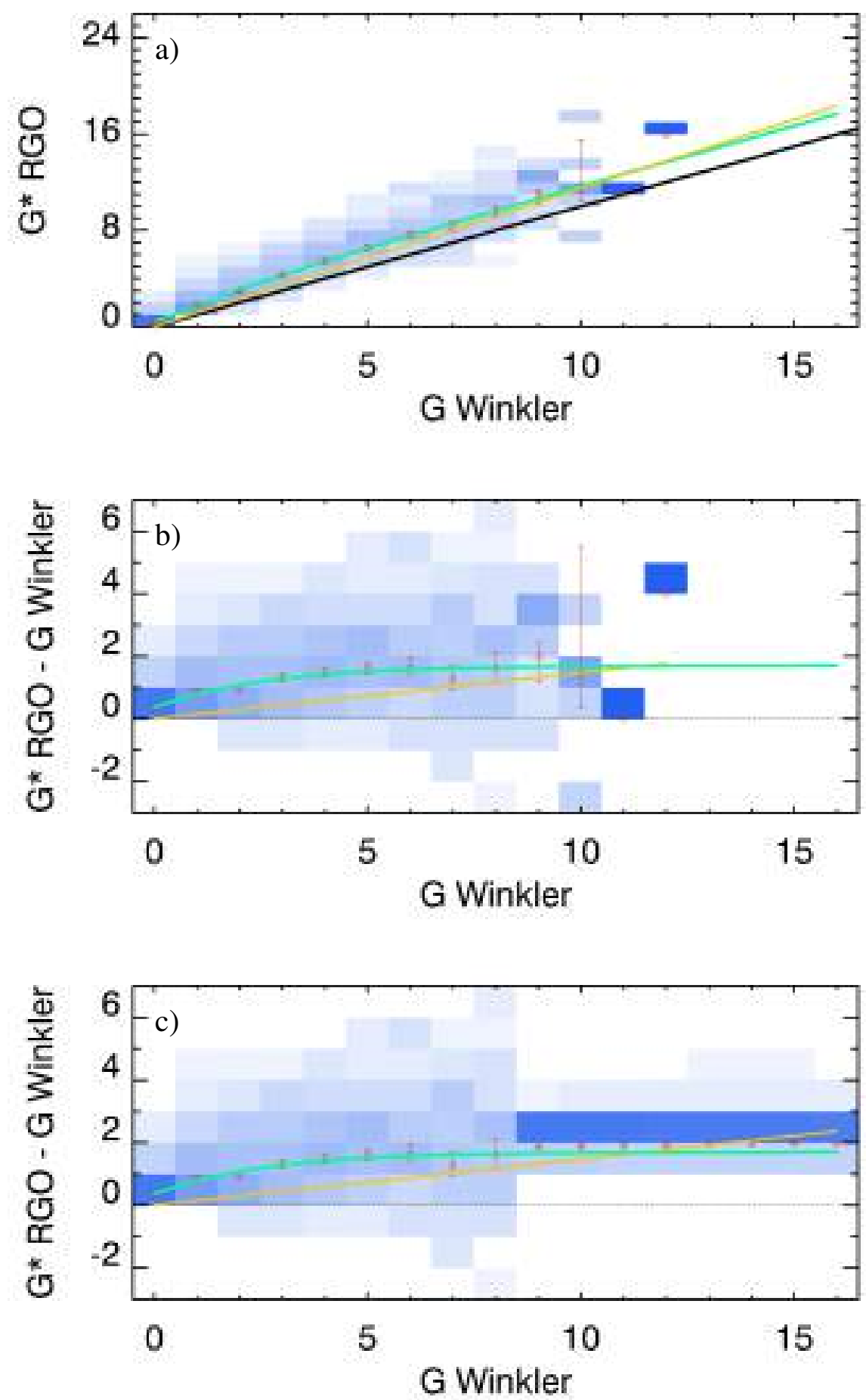

Figure 7.3: Example of the construction of the calibration matrix for Winkler (secondary observer, $G$ ) to RGO (primary, $G^{*}$ ) over 1900-1910. Panel a) shows the original distribution matrix $G^{*}$ vs. $G$ : the black line has a slope of unity. Panel b) shows the difference, $G^{*}-G$ vs. $G$. Panel c) is the same as b) but the empty columns for $G^{*}>8$ have been filled with the results of the MC simulation. The red circles with error bars depict the mean $G^{*}$ values for each $G$ column and their $1 \sigma$ uncertainty. The yellow line shows the $k$-factor used in Hoyt and Schatten (1998). 
7 New reconstruction of the sunspot group numbers since 1739 using direct calibration and "backbone" methods

For each day with simultaneous observations from both "observers" (the BB series), we randomly selected $G$ values corresponding to the PDFs and filled the matrix. This process was performed 1000 times for each day, and the final matrix was computed as the average among all the individual matrices.

Monte Carlo simulations were used to calibrate the secondary BB to the reference one accounting for the error propagation. We randomly picked a $G$ value from the PDF for each day of the secondary BB series and obtained, from the matrix, the PDF of the $G^{*}$ values for the reference BB. This was repeated 1000 times and the average PDF of the $G^{*}$ values was considered as the calibrated PDF of the secondary BB series for that day.

The procedure is illustrated in Fig. 7.4, which shows the result of the calibration of the secondary Wolfer BB series to the primary RGO BB series. It is evident from the panel a) that the RGO BB $G$ values are systematically higher than those of the Wolfer $\mathrm{BB}$ (the difference is positive), implying that RGO is a better observer than Wolfer. After the calibration (panel b)), the two series match each other so that the mean difference is consistent with zero in the entire range of $G$ values implying that the calibration was carried out correctly.

This procedure works well for all the BBs. However, the results for the Horrebow BB series are very uncertain. The overlap of this series with the Flaugergues BB series is short and occurs only during activity minima around 1775 and 1795 , which gives merely four points ( $G$ values) to perform the fit and to extrapolate to the rest of the range of values. Since the method gives a realistic estimate of the uncertainties, this is clearly expressed in large error bars for the 18th century.

\subsubsection{Construction of the final series}

After all the BBs were calibrated to the reference RGO series, the final composite series was produced. First, for each day, all the available BB series values (in the form of a PDF) were merged into a single PDF for that day. From the daily PDFs of the calibrated $G$ values we produced the monthly $G$ values using a MC simulation. For this, for each day with available data within a month, we randomly selected a $G$ value from the final daily PDF and then computed the monthly value as the arithmetic mean of these daily values. This procedure was repeated 1000 times, and the PDF of the monthly values was constructed for each month. This MC method considers all the uncertainties straightforwardly. Finally, we collected the mean and asymmetric $\pm 1 \sigma$ uncertainty level (a Table is available at the CDS).

Next, the annual numbers of sunspot groups with their asymmetric $\pm 1 \sigma$ uncertainties were calculated from the monthly values in the same manner as monthly values from the daily values. The final annual series is given in Table 7.9 and shown in Figure 7.5. The GSN in years without reliable values are denoted by -99 .

\subsection{Validation of the results}

\subsubsection{Comparison with other series}

Other published GSN series are also shown in Fig. 7.5, but without the uncertainties. While all the series are dominated by the 11-yr solar cycle, the centennial variability 

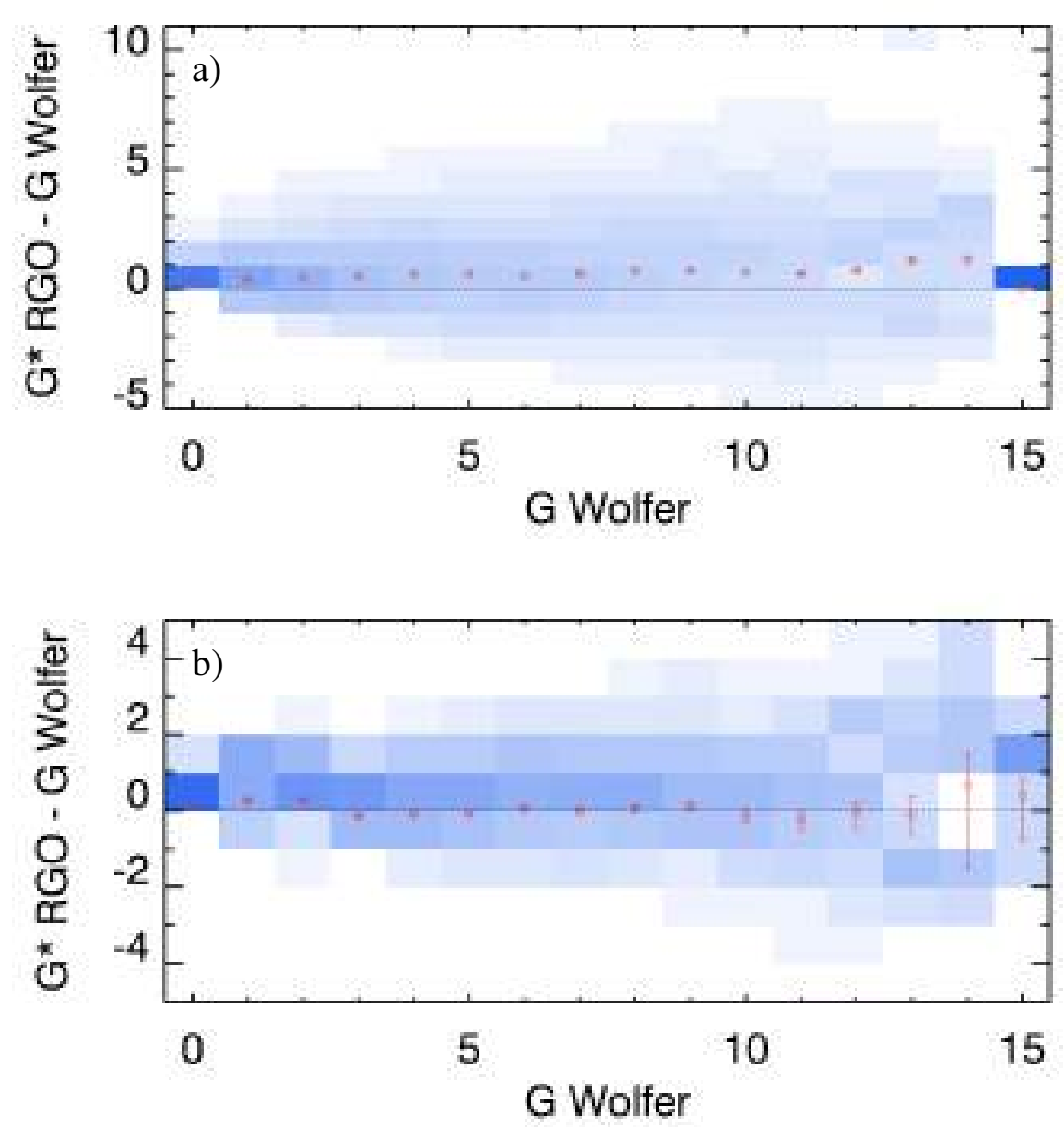

Figure 7.4: Difference between the Wolfer and RGO backbones. Panel a) shows an uncalibrated matrix after the full MC filling; panel b) shows the same matrix after the calibration. The red circles depict the average values in every column with their $1 \sigma$ uncertainty ranges.

differs among different reconstructions. The ClLi16 and SvSc16 series are systematically higher than our reconstruction in the 19th and 18th centuries, while the HoSc98 series is somewhat lower. The present result is close to UEA16 and lies between the 'high' and 'low' models.

Figures 7.6 and 7.7 show the difference between various other series and the result presented here.

One can see that all the series agree with each other in the 20th century, except the SvSc16 series which is systematically lower than all others, although still within the error bars.

The UEA16 series is very close to our series during cycle maxima, while there are noticeable differences around the minima. The two series diverge for cycles 2 (our series is lower than UEA16), 8-9 (ours is higher), and 21-22 (ours is lower). The differences in cycles $22-23$ can be explained by different observers used: while UEA16 used only RGO and Koyama over that period, we used here more than 150 observers, which allows us to estimate the activity more accurately. 
7 New reconstruction of the sunspot group numbers since 1739 using direct calibration and "backbone" methods

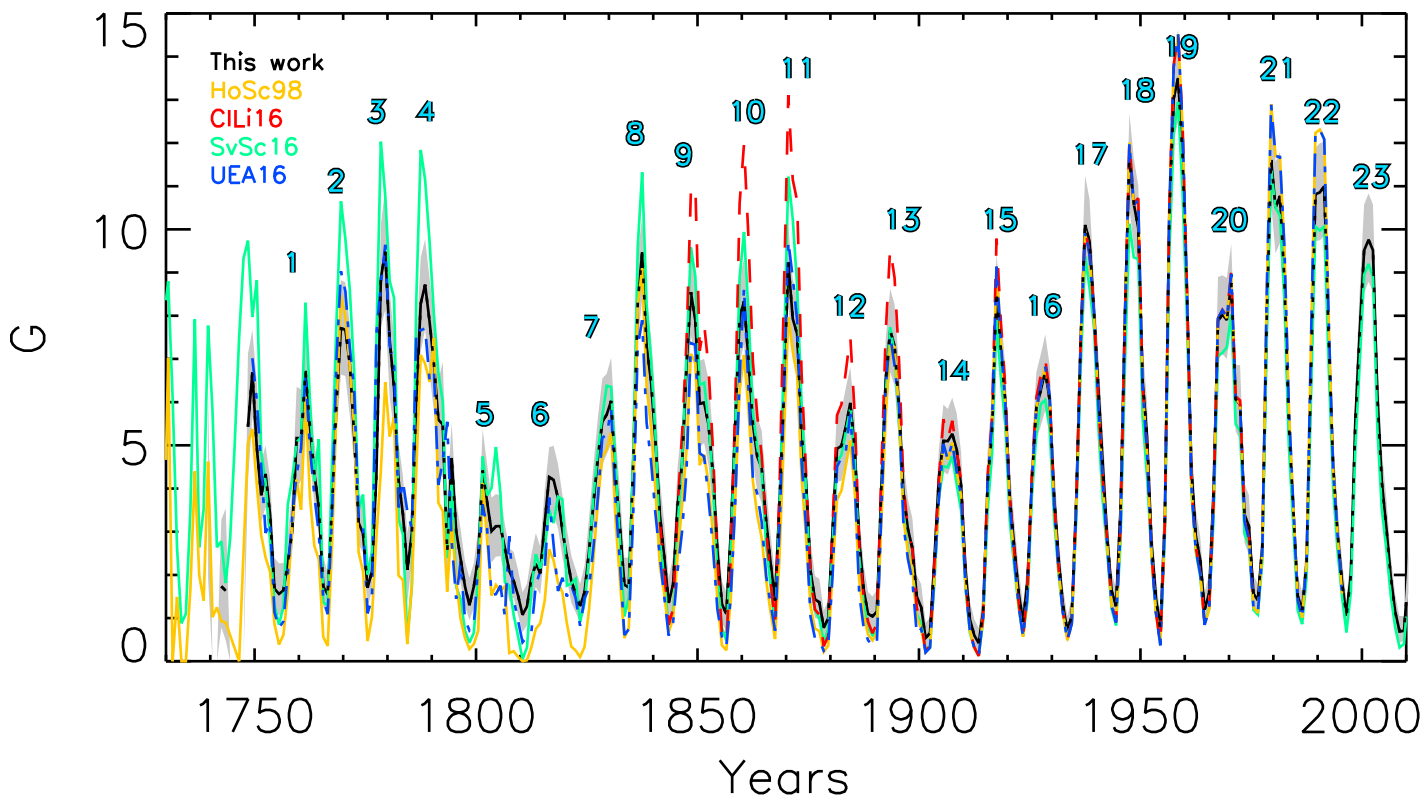

Figure 7.5: Annually averaged number of sunspot groups. This work is indicated in black with the $\pm 1 \sigma$ area shaded; HoSc98 is indicated in yellow; UEA16 is shown in blue; SvSc16 is shown in green; and ClLi16 is indicated in red. Numbers on top of the curves denote the conventional solar cycle numbering.

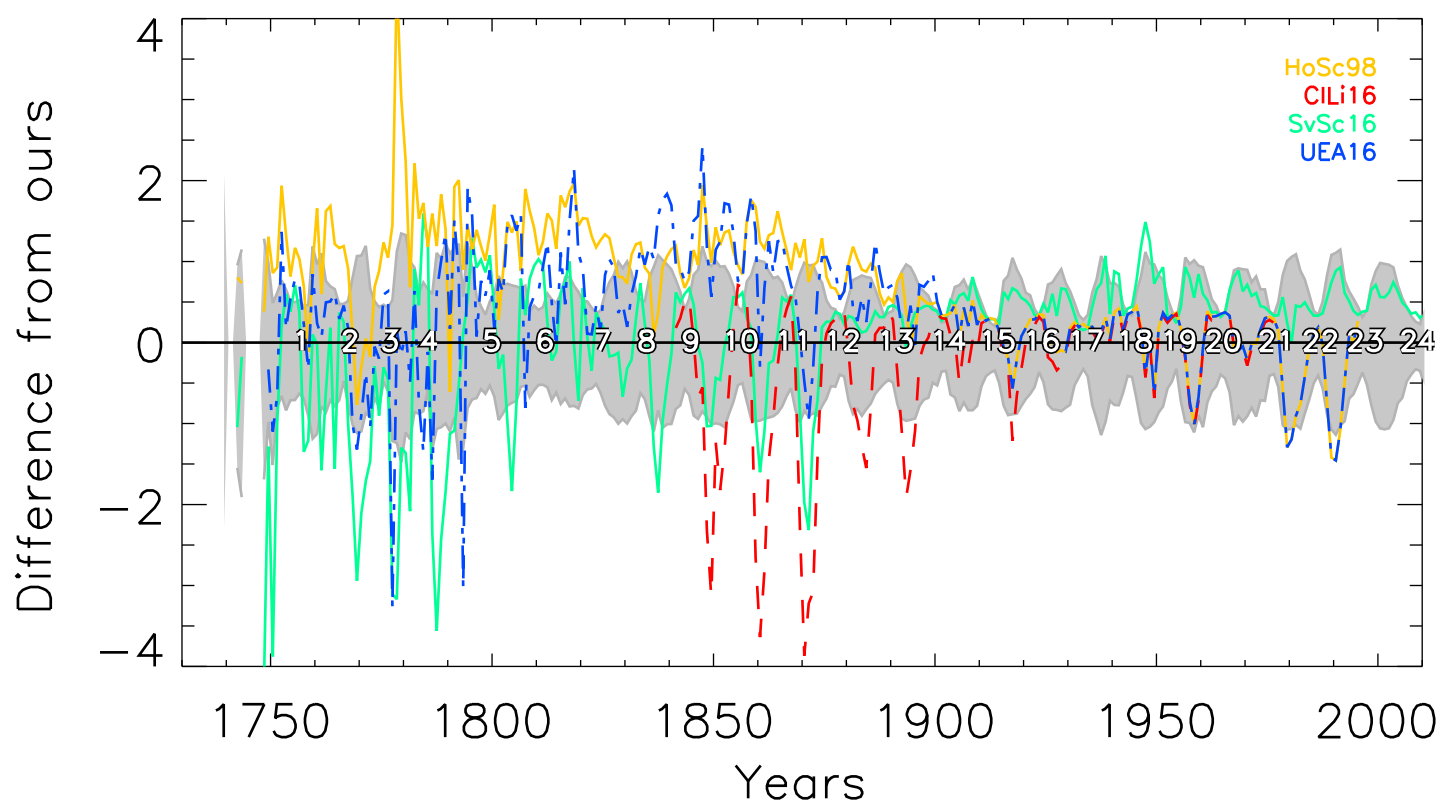

Figure 7.6: Differences of the annual GSN between our series and other series (as denoted in the legend). Positive values imply that our series is higher. The grey shading denotes the $\pm 1 \sigma$ range of our series. The numbers denote the conventional solar cycle numbering. 
During the solar cycle minima our series agrees with SvSc16, but there are distinct differences during the maxima. The SvSc16 series gives higher values over the cycles 1-5 and 8-11, while lower values are found for almost all cycles over the 20th century. These differences can be at least partly explained by the $-7 \%$ ad hoc adjustment applied by SvSc16 to the data after 1940 and by the choice of Koyama as the reference observer (see also a discussion about this in Sect.7.4.2).

Over the 20th century, the ClLi16 series is essentially the same as that of HoSc98, but they deviate over the 19th century so that maxima in the ClLi16 series are 3-4 groups higher than in HoSc98, and hence also than in ours. Keeping in mind that we ignored the RGO data before 1900 and used Wolfer as the reference for that period, the higher values by ClLi16 suggest a possible overcorrection of the RGO series by these authors. This is in agreement with the findings of Lockwood et al. (2016c).

In Figure 7.8 we show the secular trends of different series considered here, using the non-parametric SSA (singular spectrum analysis, Vautard et al.1992). The SSA method is based on decomposition of a time series into several components with distinct temporal behaviours. It is very convenient for the identification of long-term trends and quasiperiodic oscillations, especially in the conditions when the secular trend is subdominant with respect to the main periodicity. As the secular trend we consider the first SSA components of the SN series. We used the time window for the SSA in the range of 80-100 $\mathrm{yr}$, where the result is stable. All series show that the activity level was highest in the late 20th century, corresponding to the modern grand maximum, but the relative enhancement differs among series. The greatest increase over the last $200 \mathrm{yr}$ (defined as the ratio of the values in 2000 and in 1750$)$ is observed for the HoSc98 series $(\approx 2.6)$, followed by the UEA (1.9) and our final series (1.7). Finally, SvSc16 series yields 1.3. Thus, the modern grand maximum is observed in all series. According to this work, this grand maximum is weaker than that in the HoSc16 series but greater than in the SvSc16 series.

\subsubsection{Tests of stability}

\subsubsection{Choice of backbone observers}

As primary BB observers, we selected those with sufficiently long observational periods of the best quality for each epoch. This is illustrated in Fig. 7.9, which shows the difference matrices for Wolf and Schmidt for two cases: Schmidt is considered as the primary observer and Wolf as the secondary (panel a)) and vice versa (panel b)). It is apparent that Schmidt was a better quality observer and is more appropriate to be chosen as the primary BB observer. By choosing Wolf as the BB observer, we would need to degrade Schmidt and other observers.

To test whether our final series is robust against the choice of the primary BB observers, we repeated the same analysis for different BB combinations. We used all possible combinations of high-quality long-lasting observers over four different intervals: (1) RGO (1900-1976), Koyama (1947-1984), Mt Wilson (1923-1958), (2) Wolfer (18801928), Quimby (1889-1921), (3) Schmidt (1841-1883), Spoerer (1861-1893), Weber (1859-1883), Wolf (1848-1893), (4) Schwabe (1826-1867), and Stark (1813-1836). This led to 48 alternative reconstruction series. Additionally, we constructed two more series by replacing Kanzelhöhe (1957-2010) with Cragg (1947-2009) and Locarno (19582010 ) and keeping all the other BBs as in the main series. Thus the total number of var- 
7 New reconstruction of the sunspot group numbers since 1739 using direct calibration and "backbone" methods

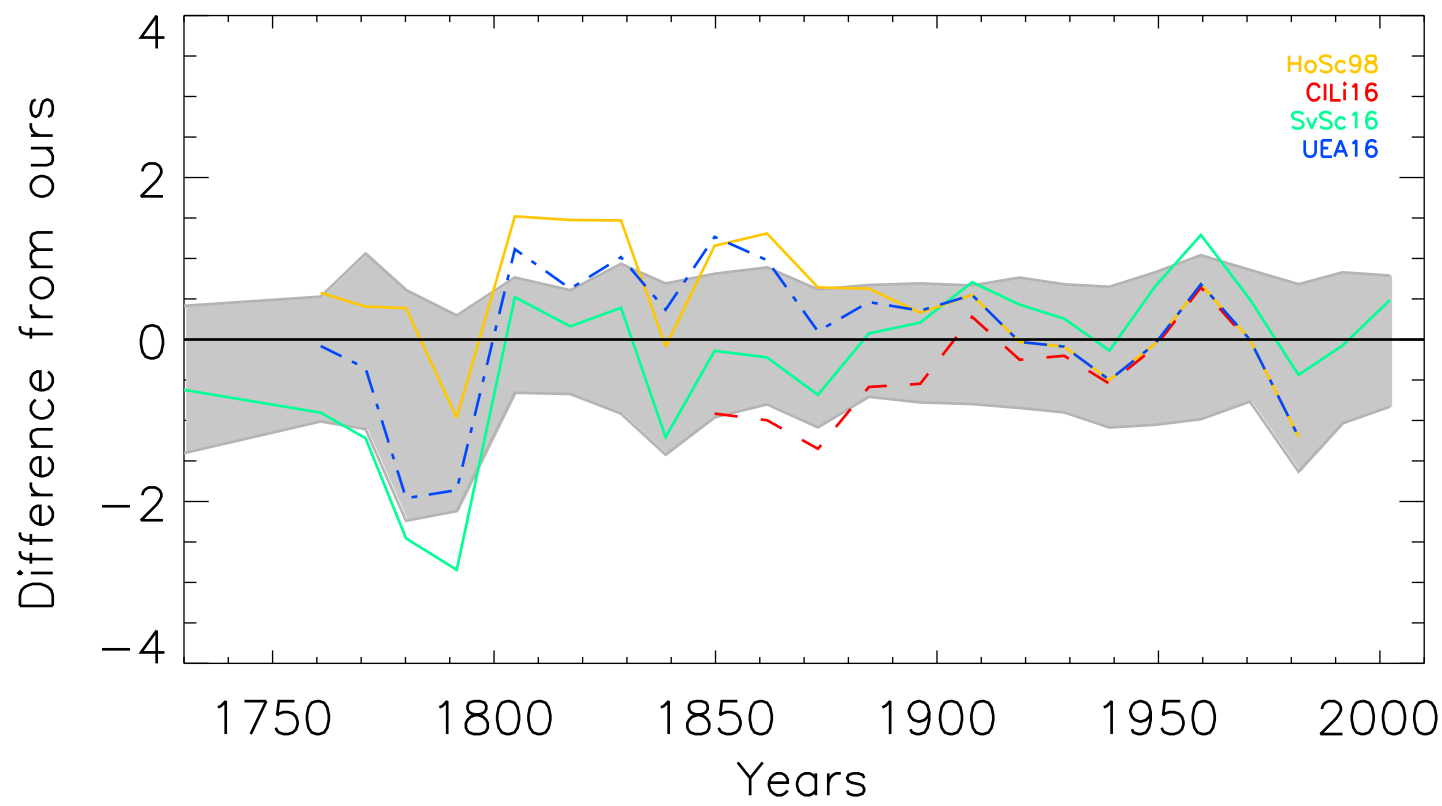

Figure 7.7: Differences of the solar cycle averaged GSN between our series and other series (as denoted in the legend). Positive values imply that our series is higher. The grey shading denotes the $\pm 1 \sigma$ range of our series.

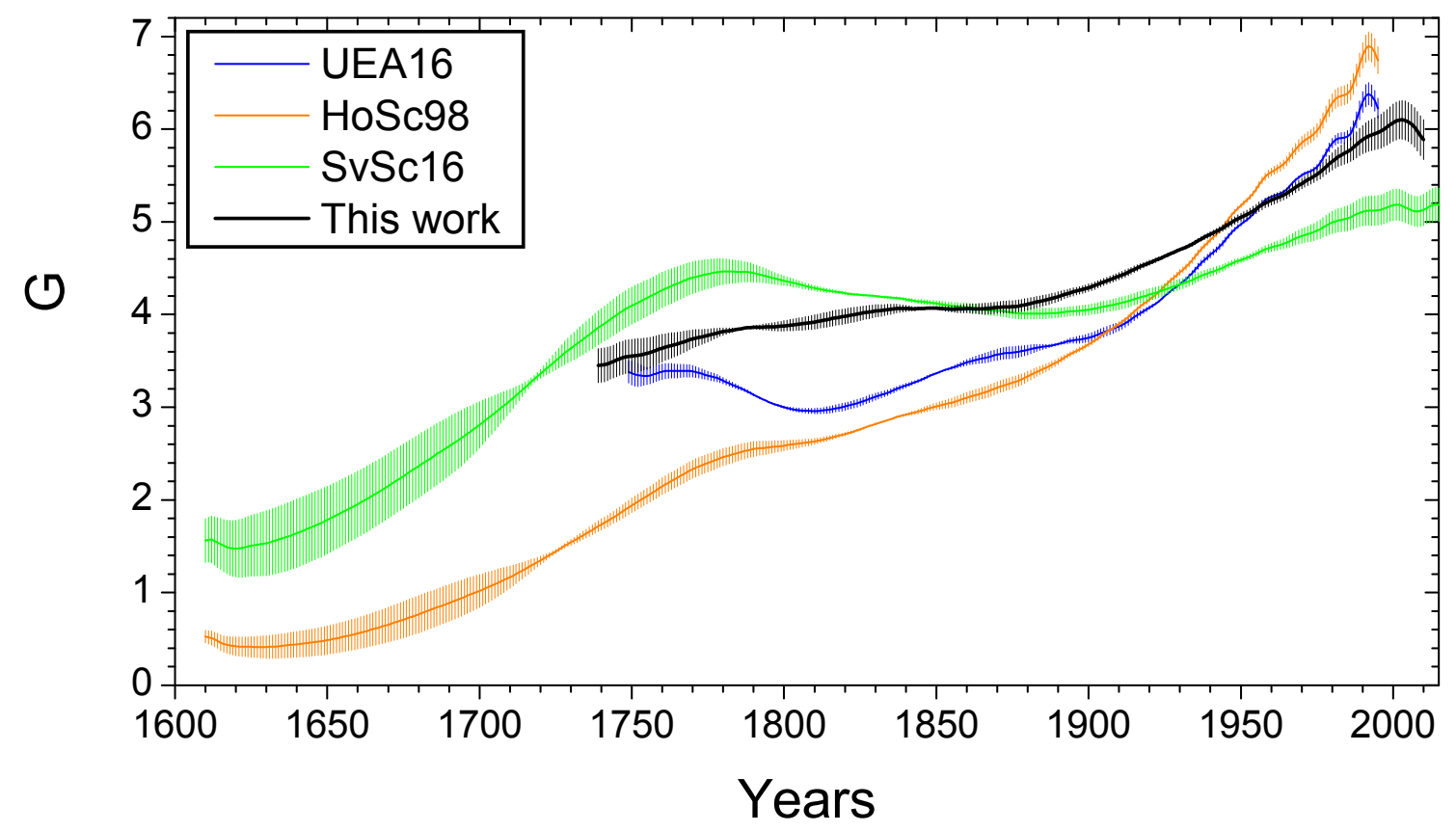

Figure 7.8: The long-term secular trend in different SN series, studied here, defined as the first SSA component. The shading represents only statistical uncertainties of the SSA method. 

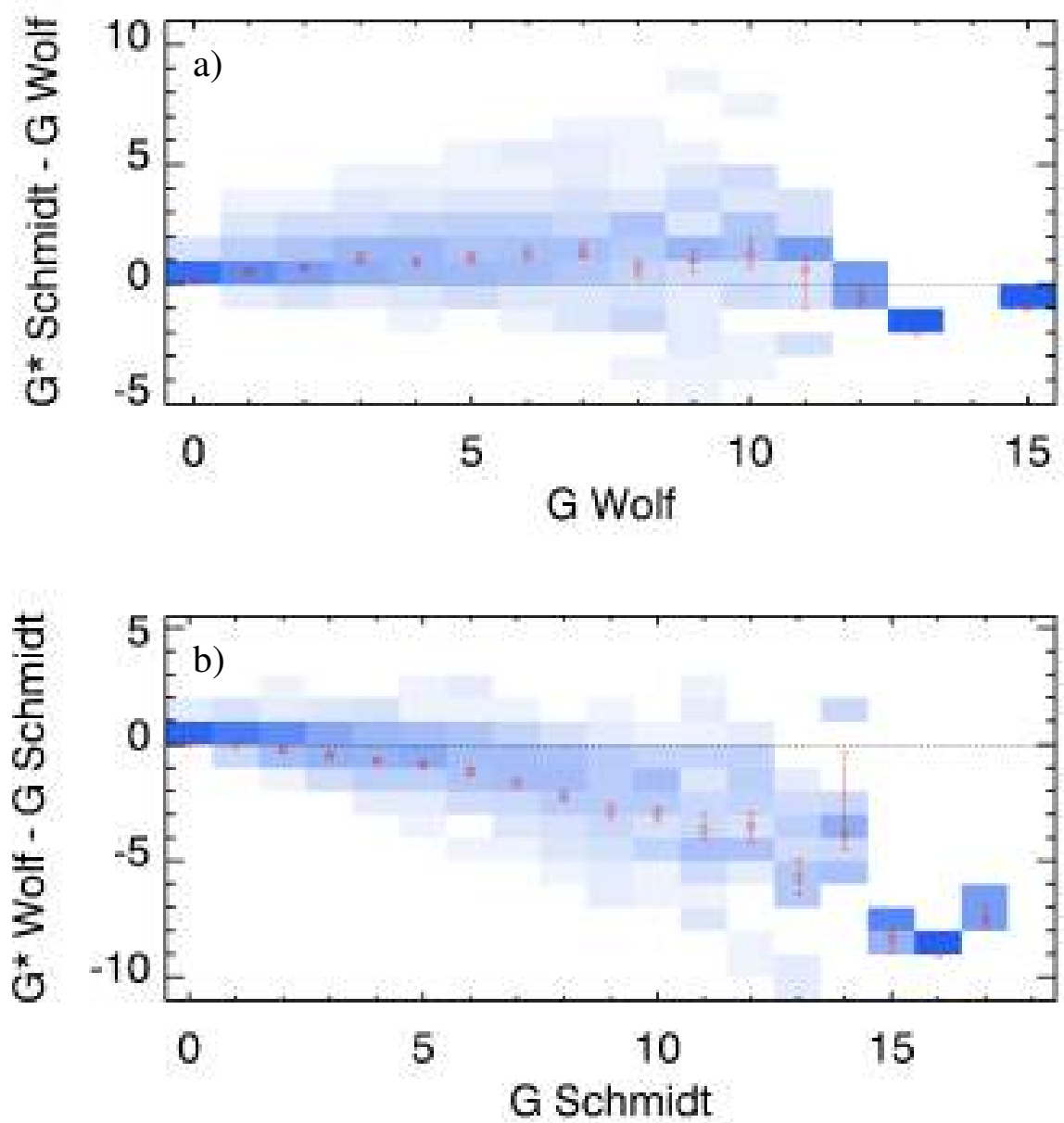

Figure 7.9: Matrices of the $G$ value difference between Wolf and Schmidt, where Schmidt (panel a)) and Wolf (panel b)) are selected as reference observers.

ious GSN reconstructions was 50. We also included Flaugergues and Horrebow BBs in all series, but excluded the stand-alone BBs. The reference observer was chosen between RGO, Koyama, and Mt Wilson. Locarno has been excluded from all composites and our main series, however, we include it here as a BB to evaluate its effects on the calibration. We note that Quimby, as an individual observer, has overlap only with RGO, Wolf and Spoerer, while Stark has no overlap with any other BB observer used here. Thus, many of these auxiliary series result from disconnected BBs and are sometimes based on poor statistics. They can be used to assess uncertainties related to the BB selection, but as individual series, they are much less reliable than our main composite series. In this process, we did not exclude any other observers except those automatically rejected by the code (Sect.7.3.3). The selection of observers within the BBs was performed automatically and may, of course, differ from those listed in Tables 7.2 7.8.

Figures 7.10 and 7.11 show the differences between our main series and the different auxiliary series, described above. The difference is mostly within the $\pm 1 \sigma$ interval. Moreover, if the three main BB observers, i.e. RGO, Wolfer, and Schwabe, are fixed, the differences among the reconstructed series are quite small (Fig.7.11) and, thus, the choice 
7 New reconstruction of the sunspot group numbers since 1739 using direct calibration and "backbone" methods

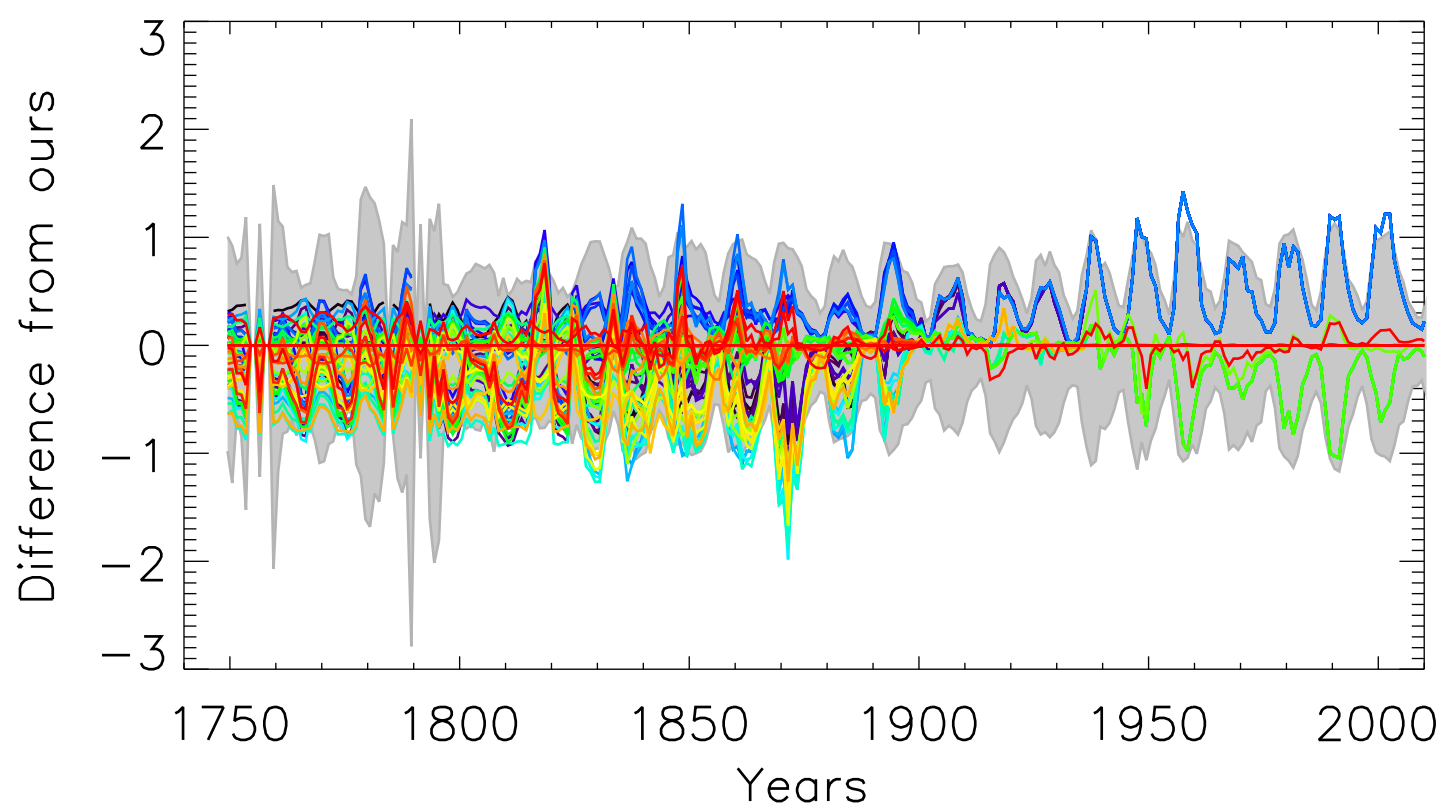

Figure 7.10: Difference between the main reconstructed series and all 50 auxiliary series produced with different backbone combinations. Annual values are shown. Grey shaded area indicates the $\pm 1 \sigma$ uncertainties of the main series.

of other BBs is not important. Using Koyama as the BB observer instead of RGO leads to systematically lower counts of sunspot groups (see blue curve in Fig. 7.10), but these counts are still within the $1 \sigma$ error bars.

Thus, we can conclude that the method is stable regarding the exact choice of the BB observers with the potential uncertainty lying within the formal error bars.

\subsubsection{Shape of the matrix}

The majority of the calibration matrices constructed for individual observers have a shape (see Fig. 7.3) similar to that expected from synthetic data with an artificial acuity threshold applied (Usoskin et al. 2016b). This implies that the quality of an observer can be adequately quantified by his/her acuity observational threshold. However, distorted behaviour was found for some observers during periods of high solar activity, so that an observer, who is "poor" (counting less groups than the reference observer) during periods of low and moderate activity, may appear to report more groups during solar activity maxima as if he/she were a better observer than the reference observer. This is caused by the low statistics and such columns in the matrix were replaced by the fit (Sect. 7.3.3). In the case in which this behaviour occurred over an extended region of the matrix, the observers were rejected by the code.

\subsubsection{Quality of the RGO dataset}

We also tested how crucial the choice of the exact reference period of the RGO dataset is. We repeated the same analysis, but considering the RGO dataset to start in 1874 and 
in 1916. Since a change of the reference period affects the statistics used for the calibration, allocation of some individual observers to specific BBs was automatically changed and was different than in Tables 7.2 through 7.8 . Figure 7.12 shows the differences between the main series proposed here and these two alternative series. The result within the Kanzelhöhe BB is not affected at all, and for the rest of the BBs the difference is significantly smaller than the error bars, which are on average 0.14 and 0.10 for the annual values using RGO data for the periods of 1874-1976 and 1916-1976, respectively. At the same time, the use of the reference period shortened to after 1916 significantly decreases statistics, ignoring $42 \mathrm{yr}$ of RGO data. Thus, we conclude that the present reconstruction is also robust against the choice of the reference period of the RGO dataset.

\subsubsection{Other issues}

Our method may suffer from an intrinsic problem related to a possible overestimate of $G$ for periods of low activity. If a secondary "poor" observer reports no spots, the method corrects it to a finite non-zero value of $G^{*}$ (see e.g. Fig. 7.3). This is different from the linear $k$-factor method (e.g. SvSc16), in which zero values of a low-quality observer are always translated to zero values of the high-quality reference observer.

We explicitly assume, similar to all other $\mathrm{SN}$ reconstructions, that the observational record of any observer is error free in the sense that they report exactly the number of sunspot groups that should be visible to them on the Sun on a given day (cf. Spearman 1904, Dudok de Wit et al.2017). If this assumption were violated (e.g. weather or health conditions may temporarily reduce the acuity of the observer), the method would tend to slightly underestimate the reconstructed values at high activity levels, while overestimating the values at activity minima. However, at present there is no way to assess these kinds of errors and we have to rely on this assumption. We note that this also affects all other methods, including the linear $k$-factor.

We also assume (as is done in all other reconstructions) that the observational quality of an observer is constant in time. On the other hand, if it changed over time, especially outside the calibration period, it may introduce some additional uncertainties in the final result. However, in this work we cannot account for that and have to make the assumption on the constancy of the quality of the observer, as done by all the other reconstructions as well.

\subsection{Summary and conclusions}

We present a new reconstruction of the number of sunspot groups since 1739, along with realistic uncertainties, with daily, monthly, and annual time resolutions. The reconstruction is based on the daisy-chain normalization of individual observers via so-called "backbones" built up on the records of the key observers of different epochs. In contrast to most of the previous works, based on a simple linear $k$-factor scaling (e.g. Hoyt and Schatten 1998, Clette et al. 2014, Svalgaard and Schatten 2016), our reconstruction employs a direct non-parametric calibration of observers by linking the values during days of simultaneous observations (Usoskin et al.2016b). This method is based on the assumption that the quality of the data of the various observers is maintained throughout their observing period, which may not be well validated (Lockwood et al. 2016c). This will be studied 
7 New reconstruction of the sunspot group numbers since 1739 using direct calibration and "backbone" methods

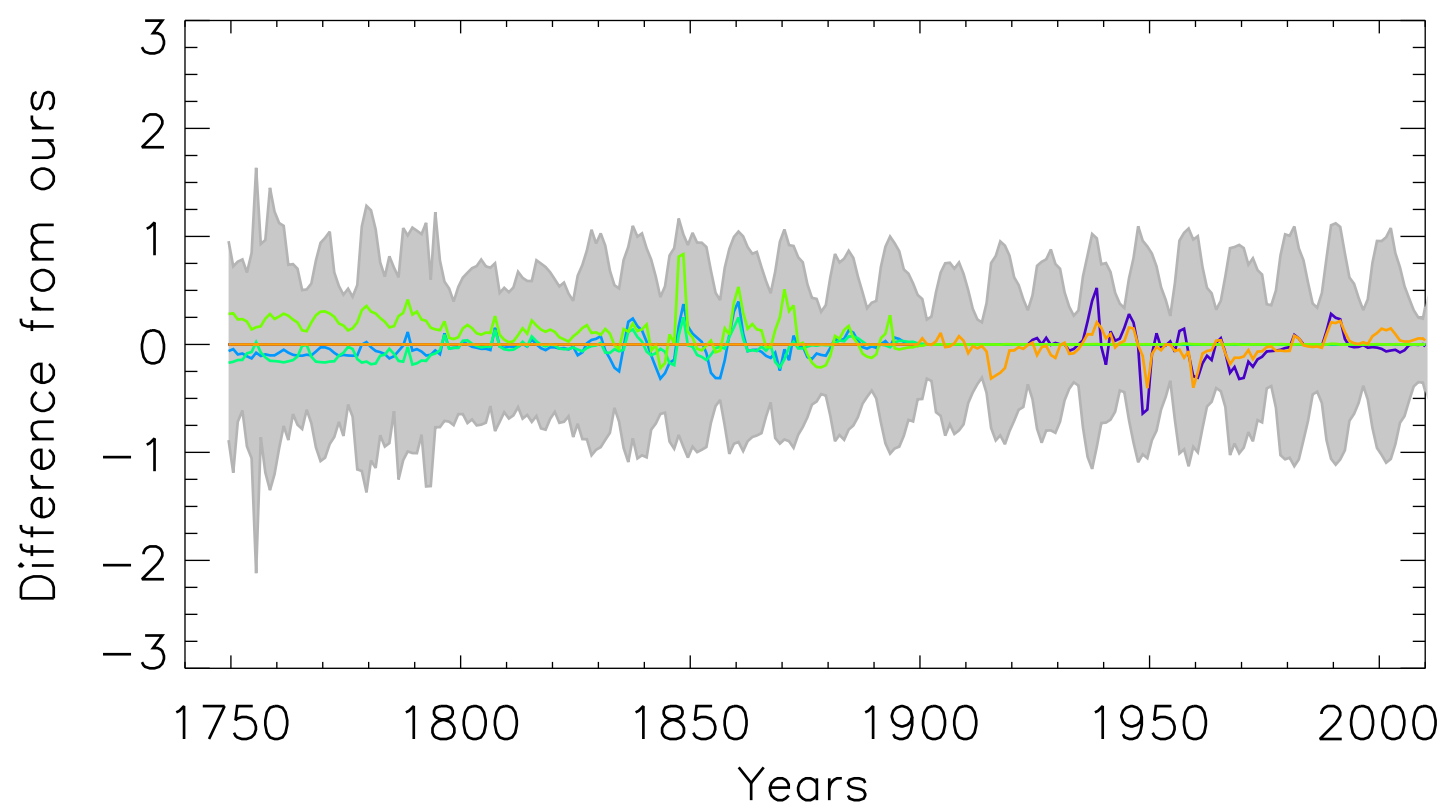

Figure 7.11: Difference between the main reconstructed series and the auxiliary series produced with different backbone combinations that include RGO, Wolfer, and Schwabe. Grey shaded area represents the $\pm 1 \sigma$ uncertainties of the main series.

elsewhere. We also assume, as all other methods do, that daily records of each observer are error free. A further assumption is that the main differences between the observers is due to their different observing capabilities. This assumption is used merely to extrapolate for the values that are missing from the overlapping period. Thus this method works with a minimum number of assumptions and allows for a direct comparison of two observers with different observational skills. Uncertainties of the reconstruction were assessed using a Monte Carlo method applied to the derived PDFs. This approach accounts naturally for the error propagation without making additional assumptions (e.g. about the normality and independence of errors). In other words, we present a highly advanced daisy-chain reconstruction of GSN based on the most direct calibration of observers.

We tested the sensitivity of the method to the choice of the BB observers and of the reference period. We found that the reconstruction was robust and the result remained within the provided uncertainties.

The new series has been compared with other published GSN reconstructions, i.e. HoSc98, ClLi16, SvSc16, and UEA16. The new series lies close to UEA16, but is slightly higher than that in the 18th century. In contrast, it is systematically lower than CILi16 in the 19th century and lower than SvSc16 in the 18th century. The latter two series are based on the $k$-factor scaling, which is shown to overestimate solar activity during solar cycle maxima (Lockwood et al. 2016e, Usoskin et al. 2016b c). The new series confirms the existence of the modern grand maximum of activity in the second half of the 20th century, when sunspot cycles were significantly higher than during the 19th and 18th centuries.

The new GSN series provides a robust reconstruction of solar activity (the number of sunspot groups) with a realistic estimate of uncertainties and forms a basis for further investigation of centennial variability of solar activity over the last $270 \mathrm{yr}$. 


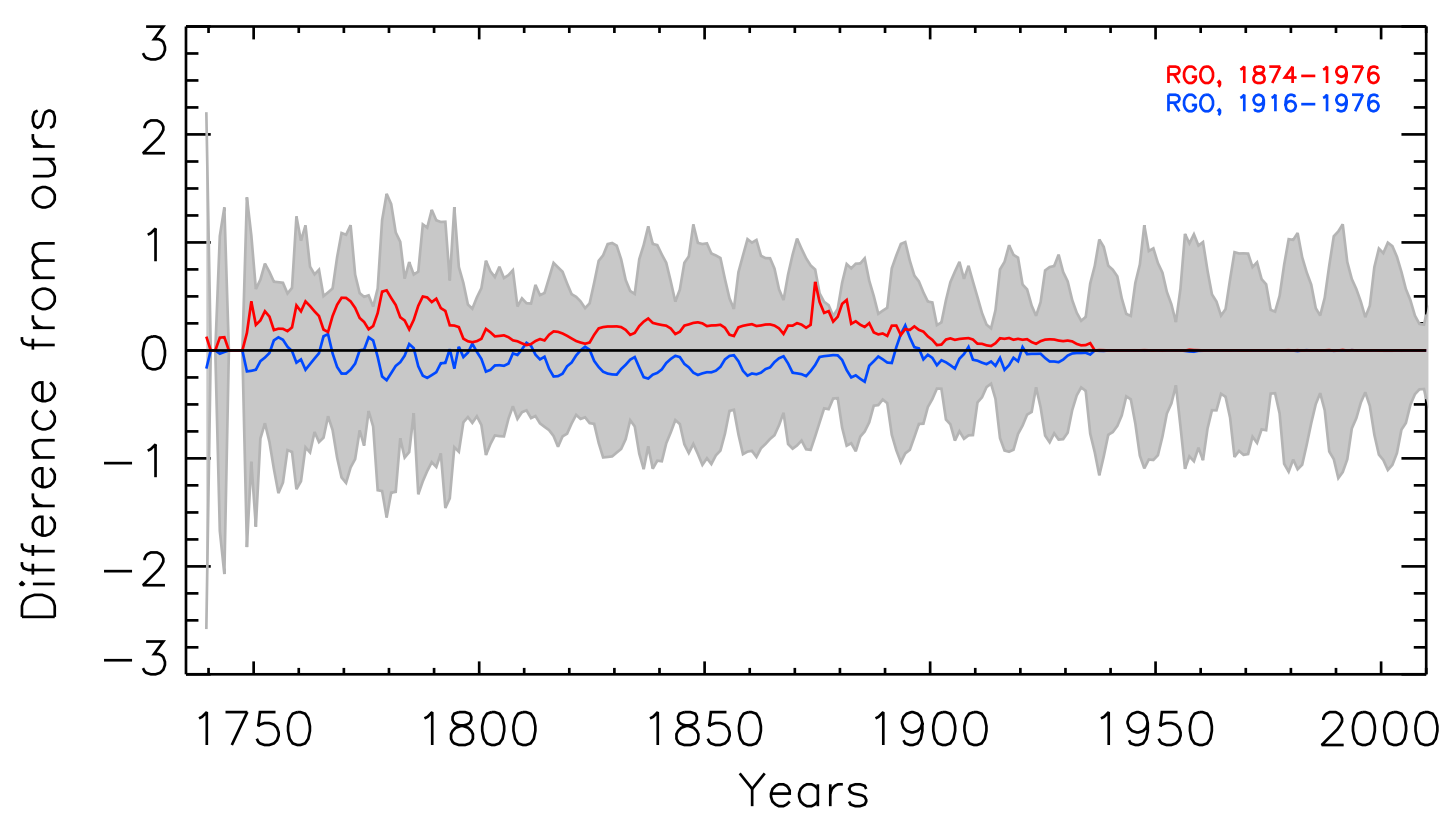

Figure 7.12: Differences between the main annual reconstructed $G$ series and those based on the reference RGO dataset for 1874-1976 and for 1916-1976 (blue and red, respectively). The grey shaded area depicts the $\pm 1 \sigma$ uncertainties of the main series.

\section{Appendix A: List of observers}

In Tables 7.2 7.8 we list all observers that were used in each BB series. The tables contain information on the Id of the observer in the Vaquero et al. (2016) database, the observer's name, the first year of observations employed here, the last year of observations employed here, the number of daily observations $N_{\mathrm{d}}$ used, and the number of overlap days of observations with the backbone observer $M_{\mathrm{d}}$ (for Schwabe, Flaugergues, and Horrebow BBs the values for \pm 1 days are also given). The backbone observer is listed first and the others are sorted based on their Id.

\section{Appendix B: Additional table}


7 New reconstruction of the sunspot group numbers since 1739 using direct calibration and "backbone" methods

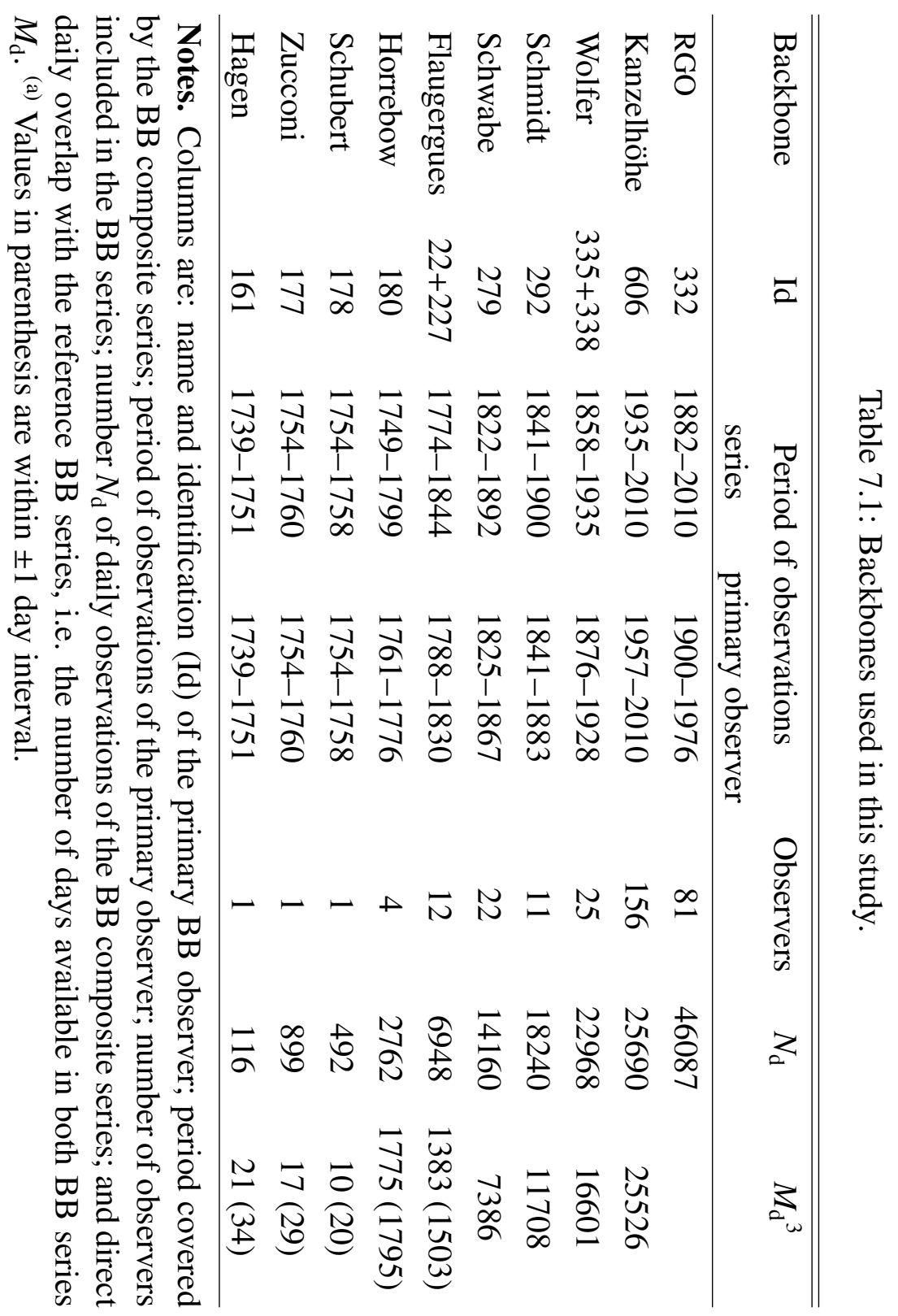


Table 7.2: List of observers used for the RGO backbone.

\begin{tabular}{|c|c|c|c|c|c|}
\hline Id & Observer & Start & End & $\mathbf{N}_{\mathrm{d}}$ & $\mathbf{M}_{\mathrm{d}}$ \\
\hline 332 & RGO & 1900 & 1976 & 28124 & \\
\hline 341 & Winkler, Jena & 1882 & 1910 & 6161 & 2480 \\
\hline 345 & Konkoly, Ogyalla & 1885 & 1905 & 3531 & 965 \\
\hline 347 & Stonyhurst College Obs. & 1886 & 1935 & 4534 & 4338 \\
\hline 352 & Quimby, Philadelphia & 1889 & 1921 & 10860 & 7428 \\
\hline 358 & Mount Holyoke College & 1890 & 1925 & 2799 & 2774 \\
\hline 361 & Schwab, Kremsmunster & 1892 & 1909 & 3619 & 2060 \\
\hline 362 & Catania & 1893 & 1918 & 7620 & 5417 \\
\hline 366 & Sykora, Charkow & 1894 & 1910 & 1883 & 1248 \\
\hline 368 & Lewitzky, Jurjew & 1895 & 1907 & 1279 & 647 \\
\hline 370 & Broger, Zurich & 1896 & 1935 & 9492 & 8600 \\
\hline 376 & Woinoff, Moscow & 1898 & 1919 & 2881 & 2758 \\
\hline 378 & Freyberg, St. Petersburg & 1898 & 1903 & 530 & 393 \\
\hline 380 & Kleiner, Zobten & 1899 & 1918 & 1965 & 1823 \\
\hline 381 & Kitschigin, Spitzbergen & 1900 & 1900 & 102 & 102 \\
\hline 382 & Subbotin, St. Petersburg & 1900 & 1908 & 1017 & 1017 \\
\hline 383 & Gorjatschy, Moscow & 1901 & 1908 & 603 & 603 \\
\hline 384 & Larionoff, Mohilew & 1901 & 1903 & 202 & 202 \\
\hline 385 & Struve, Charkow & 1901 & 1902 & 179 & 179 \\
\hline 386 & Guillaume, Lyon & 1902 & 1925 & 6340 & 6340 \\
\hline 387 & Schatkow, Kola & 1902 & 1910 & 1057 & 1057 \\
\hline 388 & Messerschmitt, Munchen & 1902 & 1910 & 1715 & 1715 \\
\hline 389 & Stempell, Hannover & 1903 & 1925 & 2760 & 2760 \\
\hline 390 & Amherst College Observatory & 1903 & 1906 & 672 & 672 \\
\hline 392 & Morosoff, Moscow & 1904 & 1909 & 58 & 58 \\
\hline 394 & Wasnetzoff, Moscow & 1905 & 1912 & 455 & 455 \\
\hline 395 & Belar, Laibach & 1906 & 1906 & 144 & 144 \\
\hline 396 & Hrase, Prague & 1906 & 1916 & 1748 & 1748 \\
\hline 397 & Brunner, Chur & 1906 & 1906 & 127 & 127 \\
\hline 398 & Bodocs, Ogyalla & 1906 & 1916 & 1674 & 1674 \\
\hline 399 & Ginori, Florence & 1907 & 1907 & 114 & 114 \\
\hline 402 & Sykora, Taschkent & 1907 & 1907 & 155 & 155 \\
\hline 403 & Biske, Zurich & 1908 & 1909 & 377 & 377 \\
\hline 405 & Lucchini, Florence & 1908 & 1914 & 1190 & 1190 \\
\hline 406 & Guerrieri, Capodimonte & 1908 & 1910 & 943 & 943 \\
\hline 407 & Braak, Batavia & 1909 & 1925 & 1586 & 1586 \\
\hline 408 & Stefko, Leysin & 1909 & 1913 & 260 & 260 \\
\hline 409 & Schwarz, Kremsmunster & 1910 & 1914 & 654 & 654 \\
\hline 411 & Kavan, Prague & 1911 & 1913 & 771 & 771 \\
\hline 412 & Moye, Montpellier & 1911 & 1925 & 4744 & 4744 \\
\hline 413 & Miloradowitsch, Pulkowo & 1913 & 1914 & 143 & 143 \\
\hline 414 & Buttlar, Simsdorf & 1914 & 1925 & 1898 & 1898 \\
\hline 417 & Bugoslawsky, Moscow & 1916 & 1918 & 411 & 411 \\
\hline 419 & Reed, Kennebunk, Maine & 1917 & 1917 & 33 & 33 \\
\hline
\end{tabular}


7 New reconstruction of the sunspot group numbers since 1739 using direct calibration and "backbone" methods

Table 7.2: continued.

\begin{tabular}{llcccc}
\hline Id & Observer & Start & End & $\mathbf{N}_{\mathrm{d}}$ & $\mathbf{M}_{\mathrm{d}}$ \\
\hline 427 & Mt. Wilson, Full Disk & 1923 & 1958 & 11666 & 11666 \\
428 & Brunner, Zurich & 1926 & 1944 & 4901 & 4901 \\
429 & Buser, Arosa & 1928 & 1937 & 2722 & 2722 \\
431 & Brunner, W., Zurich & 1929 & 1944 & 3262 & 3262 \\
432 & N.A.O., Japan, $k=0.75$ & 1930 & 1930 & 244 & 244 \\
433 & N.A.O., Japan, $k=0.65$ & 1931 & 1934 & 920 & 920 \\
434 & N.A.O., Japan, $k=0.70$ & 1935 & 1948 & 1293 & 1293 \\
436 & Waldmeier, Zurich & 1936 & 1947 & 1615 & 1615 \\
437 & N.A.O., Japan, $k=0.55$ & 1936 & 1936 & 207 & 207 \\
438 & Protitch, M., Belgrade & 1936 & 1954 & 3357 & 3357 \\
439 & N.A.O., Japan, $k=0.60$ & 1937 & 1944 & 2059 & 2059 \\
440 & Rapp, Locarno-monti & 1941 & 1944 & 1298 & 1298 \\
441 & Valencia Obs., Valencia & 1920 & 1956 & 5734 & 5734 \\
442 & Waldmeier, Arosa & 1942 & 1944 & 308 & 308 \\
443 & Djurkovic, P.M., Belgrade & 1946 & 1946 & 159 & 159 \\
444 & Oskanjan, V., Belgrade & 1947 & 1949 & 331 & 331 \\
445 & Koyama, H., Tokyo & 1947 & 1996 & 9848 & 5746 \\
446 & U.S. Naval Observatory & 1948 & 1956 & 3211 & 3211 \\
447 & National Astron. Obs., Japan & 1949 & 1993 & 12243 & 7689 \\
448 & Simic, M., Belgrade & 1949 & 1950 & 158 & 158 \\
449 & Dizer, M., Kandilli Obs. & 1949 & 1954 & 691 & 691 \\
451 & San Miguel Obs., Argentina & 1952 & 1965 & 1274 & 1274 \\
452 & Ozguc, A., Kandilli Obs. & 1955 & 1968 & 1931 & 1931 \\
454 & Rome Observatory & 1958 & 1989 & 7104 & 4758 \\
458 & Dogan, N., Ankara & 1974 & 1975 & 455 & 455 \\
464 & Luft, H. & 1924 & 1988 & 10628 & 7536 \\
486 & Athenes Eugenides, Greece & 1967 & 1982 & 2386 & 1877 \\
493 & Athenes III, Elias, Greece & 1949 & 1995 & 7611 & 4441 \\
610 & Luft 2, U.S.A. & 1958 & 1988 & 4992 & 2662 \\
612 & Looks, Chile & 1967 & 1987 & 3678 & 1906 \\
655 & Potsdam, Germany & 1950 & 1999 & 5436 & 2740 \\
658 & Quezon, Philippines & 1957 & 2010 & 10606 & 3709 \\
667 & Roma 3, Italy & 1950 & 2000 & 4213 & 654 \\
671 & Santiago, Chile & 1957 & 2005 & 3781 & 1356 \\
679 & Skalnate, Slovakia & 1950 & 2010 & 9200 & 4379 \\
681 & San Miguel, Argentina & 1967 & 2010 & 9400 & 2402 \\
701 & Uccle, Belgium & 1949 & 2010 & 13283 & 5033 \\
736 & Cragg, T., Los Angeles & 1947 & 2009 & 17726 & 8900 \\
\hline & & & & & \\
\hline
\end{tabular}


Table 7.3: Same as Table 7.2 but for the Kanzelhöhe backbone.

\begin{tabular}{|c|c|c|c|c|c|}
\hline Id & Observer & Start & End & $\mathbf{N}_{\mathrm{d}}$ & $\mathbf{M}_{\mathrm{d}}$ \\
\hline 606 & Kanzelhöhe Treffen, Austria & 1957 & 2010 & 12862 & \\
\hline 435 & Madrid Observatory, Madrid & 1935 & 1986 & 11931 & 3453 \\
\hline 453 & Lee Observatory, Bierut & 1956 & 1975 & 6532 & 3251 \\
\hline 459 & Space Environment Laboratory & 1977 & 1995 & 6922 & 4764 \\
\hline 460 & Debrechen Heliophysical Obs. & 1977 & 1977 & 365 & 268 \\
\hline 461 & Catania Observatory & 1978 & 1987 & 3288 & 2055 \\
\hline 462 & Air Force Network & 1981 & 1991 & 3572 & 2623 \\
\hline 463 & British Astron. Assoc. & 1992 & 1995 & 1002 & 806 \\
\hline 470 & N.O.A.A., U.S.A. & 1983 & 1994 & 2713 & 2071 \\
\hline 472 & Astr. Centre Ardenne, Belgium & 1992 & 2003 & 1220 & 955 \\
\hline 473 & Andries Son, Belgium & 2003 & 2010 & 1187 & 958 \\
\hline 474 & Antares, Italy & 1994 & 1995 & 170 & 145 \\
\hline 476 & Aguilar, Valencia, Spain & 1985 & 1988 & 967 & 729 \\
\hline 477 & Ahnert, Germany & 1981 & 1988 & 1244 & 975 \\
\hline 478 & Andrew Johnston, Australia & 2009 & 2010 & 221 & 168 \\
\hline 479 & Alcober Valencia Spain & 1985 & 1990 & 1177 & 896 \\
\hline 481 & Ankara, Turkey & 1977 & 1990 & 2898 & 2074 \\
\hline 483 & Philippe Wittelsheim, France & 1989 & 2010 & 3984 & 3255 \\
\hline 487 & Australian Obs. Coonabarabran, Australia & 1988 & 2007 & 5717 & 4325 \\
\hline 488 & G.O.A.S., Argentina & 1987 & 1993 & 563 & 421 \\
\hline 489 & Observ. Paul Ahnert, Cottbus, Germany & 1992 & 2010 & 4463 & 3431 \\
\hline 490 & Donostia, Spain & 1991 & 1993 & 225 & 188 \\
\hline 491 & Athenes Nat. Obser. (1) 127, Greece & 1981 & 1998 & 4247 & 3218 \\
\hline 492 & Athenes Nat. Obser. (2) 109, Greece & 1981 & 1999 & 4391 & 3303 \\
\hline 494 & A4 Sanvito 32404, Italy & 1986 & 2010 & 5971 & 4642 \\
\hline 495 & Balseiro, Uruguay & 1983 & 1985 & 333 & 250 \\
\hline 499 & Obs.Jordano Dimitrovgrad, Bulgaria & 1995 & 2005 & 1107 & 835 \\
\hline 500 & Bullon, Valencia, Spain & 1982 & 2010 & 5225 & 4083 \\
\hline 501 & Bortolotti Mauro, Italia & 1997 & 2009 & 3695 & 2989 \\
\hline 502 & Boscat Michael, Ca & 2008 & 2010 & 466 & 397 \\
\hline 504 & Basrah, Iraq & 1986 & 1986 & 228 & 168 \\
\hline 505 & Broxton Tony, U.K. & 2008 & 2010 & 625 & 508 \\
\hline 506 & Bucharest, Romania & 1981 & 1998 & 3828 & 2940 \\
\hline 507 & Bob Vanslooten, Netherlands & 2009 & 2010 & 294 & 227 \\
\hline 509 & Beyazit Obser., Turkey & 1981 & 1998 & 4532 & 3374 \\
\hline 512 & Courdurie Marcq En Baroeul, France & 1989 & 2010 & 3516 & 2670 \\
\hline 515 & Claeys Vedrin, Belgium & 1988 & 2010 & 5334 & 4169 \\
\hline 518 & Capricorno, Campinas, Brazil & 1981 & 2009 & 3064 & 2233 \\
\hline 521 & Hans Coeckelberghs, Belgium & 2006 & 2010 & 390 & 339 \\
\hline 522 & Fernandez Ruis, Santander, Spain & 1992 & 2010 & 4059 & 3215 \\
\hline 523 & Culgoora Narrabri, Australia & 1985 & 2010 & 4528 & 3484 \\
\hline 524 & De Backer Boom, Belgium & 1983 & 2010 & 5485 & 4325 \\
\hline 527 & Deman, Belgium & 1986 & 2010 & 568 & 471 \\
\hline 529 & Desrues, France & 1981 & 1985 & 1289 & 933 \\
\hline
\end{tabular}


7 New reconstruction of the sunspot group numbers since 1739 using direct calibration and "backbone" methods

Table 7.3: continued

\begin{tabular}{|c|c|c|c|c|c|}
\hline Id & Observer & Start & End & $\mathbf{N}_{\mathrm{d}}$ & $\mathbf{M}_{\mathrm{d}}$ \\
\hline 530 & Dubois Langemark, Belgium & 1985 & 2010 & 6545 & 5071 \\
\hline 533 & Vasquez Carlos, Argentina & 1991 & 2000 & 776 & 581 \\
\hline 534 & Ebro, Roquetes, Spain & 1949 & 2010 & 16266 & 10698 \\
\hline 536 & Eleizalde, Caracas, Venezuela & 1989 & 1999 & 3159 & 2411 \\
\hline 436 & Waldmeier, Zurich & 1936 & 1947 & 1615 & 1615 \\
\hline 548 & Observatory Frantiska, Czech Republic & 1997 & 2010 & 1657 & 1402 \\
\hline 549 & Stefaniks, Obs. Prague, Czech Republic & 1997 & 2010 & 1551 & 1308 \\
\hline 550 & Fujimori Nagano, Japan & 1968 & 2010 & 10558 & 7724 \\
\hline 552 & Gema Araujo, Spain & 2000 & 2010 & 3105 & 2494 \\
\hline 553 & Andre Gabriel, Belgium & 2006 & 2010 & 1497 & 1249 \\
\hline 554 & Grognard, Belgium & 1981 & 1991 & 572 & 396 \\
\hline 555 & Gerard Dinant, Belgium & 1981 & 2007 & 5031 & 3867 \\
\hline 557 & Gillissen, Belgium & 1981 & 1993 & 2543 & 1925 \\
\hline 558 & German Morales, Cochabamba, Bolivia & 1995 & 2010 & 4534 & 3530 \\
\hline 560 & Gollkowsky Rudolstadt, Germany & 1982 & 1997 & 874 & 711 \\
\hline 562 & Schott Lutz, Gerd, Germany & 2001 & 2010 & 2259 & 1839 \\
\hline 563 & Guillery Pulligny, France & 1985 & 2005 & 2914 & 2395 \\
\hline 565 & Huancayo, Peru & 1983 & 2006 & 1093 & 830 \\
\hline 566 & Hardie Jordanstown, N.Ireland & 1989 & 1999 & 2427 & 1825 \\
\hline 567 & Hancharia, Italy & 1995 & 1998 & 434 & 356 \\
\hline 568 & Helwan, Egypt & 1967 & 2010 & 9743 & 6914 \\
\hline 571 & Mahmoud S, Mosque Society, Egypt & 1995 & 2005 & 942 & 691 \\
\hline 572 & Holloman, U.S.a. & 1983 & 2010 & 7498 & 5697 \\
\hline 573 & Hvezdaren Presov, Slovakia & 1994 & 2010 & 3749 & 3013 \\
\hline 576 & Hazel Collett, United-kingdom & 2003 & 2007 & 779 & 624 \\
\hline 577 & Hurbanovo, Slovakia & 1969 & 2010 & 7859 & 6386 \\
\hline 578 & Hvezdaren Kysucke, Slovakia & 1993 & 2010 & 4290 & 3414 \\
\hline 581 & Iskum, Budapest, Hungary & 1989 & 1999 & 655 & 553 \\
\hline 582 & Iseo, Italy & 1994 & 2005 & 1628 & 1389 \\
\hline 583 & Jambol, Bulgaria & 1991 & 2003 & 698 & 532 \\
\hline 584 & Astro. De Reux Ciney, Belgium & 1992 & 2010 & 3363 & 2647 \\
\hline 585 & Jef Claes, Belgium & 2006 & 2010 & 799 & 654 \\
\hline 586 & Dragesco Jean, France & 2002 & 2005 & 774 & 599 \\
\hline 587 & Jahn Jost, West-Germany & 1987 & 1993 & 628 & 485 \\
\hline 588 & Observatory Haskovo, Bulgaria & 1998 & 2001 & 240 & 186 \\
\hline 589 & Jorge Luis Garcia, Spain & 1996 & 2010 & 1166 & 936 \\
\hline 591 & Johnston Gwynedd, England & 1991 & 2009 & 3267 & 2486 \\
\hline 592 & Havana Solar Station, Cuba & 2001 & 2010 & 2582 & 2057 \\
\hline 595 & Jeffrey Carels, Belgium & 2006 & 2010 & 1027 & 874 \\
\hline 596 & Kawaguchi, Japan & 1981 & 2010 & 8122 & 6151 \\
\hline 597 & Kandilli, Turkey & 1950 & 2010 & 11250 & 7889 \\
\hline 598 & Karjali, Bulgaria & 1992 & 1999 & 552 & 436 \\
\hline 599 & Kladno, Czech Republic & 1993 & 2008 & 3507 & 2855 \\
\hline
\end{tabular}


Table 7.3: continued

\begin{tabular}{|c|c|c|c|c|c|}
\hline Id & Observer & Start & End & $\mathbf{N}_{\mathrm{d}}$ & $\mathbf{M}_{\mathrm{d}}$ \\
\hline 600 & Koyama, Japan & 1981 & 1996 & 3250 & 2401 \\
\hline 601 & Observatory Rokycany, Czech Republic & 1997 & 2001 & 351 & 291 \\
\hline 602 & Kislovodsk, Russia & 1981 & 2010 & 9069 & 6880 \\
\hline 607 & Larguier, France & 1985 & 1994 & 2274 & 1754 \\
\hline 613 & Lieve Meeus, Belgium & 2005 & 2010 & 909 & 766 \\
\hline 615 & Learmouth, Australia & 1983 & 2010 & 7466 & 5614 \\
\hline 616 & Larissa Observatory, Greece & 1989 & 2010 & 4751 & 3837 \\
\hline 617 & Lunping, Republic Of China & 1981 & 1998 & 2965 & 2279 \\
\hline 618 & Manila, Philippines & 1971 & 1988 & 5103 & 3562 \\
\hline 620 & Mac Kenzie, Dover, United-kingdom & 1981 & 2010 & 8389 & 6421 \\
\hline 621 & Madrid, Spain & 1978 & 1986 & 1036 & 734 \\
\hline 622 & Meadows Peter, U.K. & 2008 & 2010 & 566 & 478 \\
\hline 625 & Michaux, Belgium & 1986 & 1990 & 319 & 251 \\
\hline 626 & Murmansk, Russia & 1994 & 2010 & 3041 & 2431 \\
\hline 627 & Milano, Italy & 1994 & 2010 & 1805 & 1505 \\
\hline 629 & Roberto De Manzano, Italy & 2003 & 2010 & 1984 & 1684 \\
\hline 630 & Mochizuki Urawa, Saitama, & 1978 & 2010 & 8007 & 5984 \\
\hline 631 & Mira Grimbergen, Belgium & 1987 & 2010 & 2193 & 1719 \\
\hline 632 & Smolyan, Bulgaria & 1990 & 2008 & 856 & 673 \\
\hline 634 & Juri Gagarin, Eilenburg & 1992 & 2010 & 1818 & 1428 \\
\hline 636 & Obs. Copernicus, Varna, Bulgaria & 1995 & 2002 & 494 & 352 \\
\hline 639 & Nijmegen, Netherlands & 1983 & 2010 & 5344 & 4168 \\
\hline 640 & Barnes, Auckland, New-zealand & 1985 & 2010 & 4037 & 3089 \\
\hline 642 & Obs. Solar Bernard Lyot, Brazil & 1995 & 1996 & 178 & 121 \\
\hline 645 & O.M.A. Americana, Brasil & 1987 & 1994 & 802 & 601 \\
\hline 646 & Ondrejov Observ., Czech-re & 1991 & 2010 & 4711 & 3890 \\
\hline 645 & O.M.A. Americana, Brasil & 1987 & 1994 & 802 & 601 \\
\hline 646 & Ondrejov Observ., Czech-republic & 1991 & 2010 & 4711 & 3890 \\
\hline 649 & Vlasim, Czech Republic & 1989 & 1992 & 436 & 360 \\
\hline 650 & Palehua, Hawai & 1983 & 1997 & 3512 & 2637 \\
\hline 651 & Perroni, Brazil & 1981 & 1986 & 1413 & 1021 \\
\hline 652 & Pasternak, Berlin, & 1984 & 2010 & 5429 & 4331 \\
\hline 654 & Lormont, France & 1991 & 1997 & 691 & 555 \\
\hline 656 & Observatory Prostejov, Czech Republic & 1998 & 2010 & 1529 & 1273 \\
\hline 657 & Pyong Yang, Korea & 1985 & 2003 & 4324 & 3306 \\
\hline 659 & Ramey, Puerto-rico & 1983 & 2003 & 5957 & 4505 \\
\hline 666 & Rokycany - Luzicka, Czech Republic & 1997 & 2001 & 424 & 348 \\
\hline 668 & Paulo Roberto Moser, Brazil & 2010 & 2010 & 172 & 144 \\
\hline 669 & Rasson Mons, Belgium & 1988 & 1997 & 2126 & 1626 \\
\hline 670 & Rodriguez, Venezuela & 1986 & 1989 & 950 & 720 \\
\hline 672 & Siracusa II, Lapichino, Italia & 1986 & 1995 & 365 & 294 \\
\hline 673 & Sjoerd Dufoer, Belgium & 2007 & 2010 & 366 & 323 \\
\hline 674 & Sergio Fabiani, Bolivia & 1995 & 1995 & 133 & 105 \\
\hline 675 & Sigma Octante, Cochabamba, Bolivia & 1981 & 2010 & 5258 & 4049 \\
\hline
\end{tabular}


7 New reconstruction of the sunspot group numbers since 1739 using direct calibration and "backbone" methods

Table 7.3: continued

\begin{tabular}{llcccc}
\hline Id & Observer & Start & End & $\mathbf{N}_{\mathrm{d}}$ & $\mathbf{M}_{\mathrm{d}}$ \\
\hline 677 & Smith Marlyn, U.K. & 2008 & 2010 & 379 & 313 \\
678 & San Jose, Buenos Aires, Argentina & 1986 & 1996 & 702 & 531 \\
683 & Sobota, Slovakia & 1992 & 2010 & 5258 & 4280 \\
685 & Saudi Arabia, Jeddah & 1981 & 2010 & 5477 & 4154 \\
688 & Suzuki, Japan & 1981 & 2010 & 7839 & 5954 \\
691 & Trento, Italy & 1994 & 1994 & 48 & 48 \\
692 & Thomas Teague, United Kingdom & 2005 & 2010 & 219 & 168 \\
693 & Central Weather Bureau, Republic Of China & 1981 & 2010 & 5898 & 4564 \\
694 & Tangjungsari, Indonesia & 1984 & 1989 & 1358 & 1031 \\
696 & Taipei 2, Republic Of China & 1981 & 2005 & 3692 & 2796 \\
697 & Trieste, Italy & 1967 & 1993 & 2704 & 2074 \\
698 & Spaninks Tilburg, Netherlands & 1991 & 2010 & 3079 & 2424 \\
700 & Tony Tanti Naxxar, Malta & 1986 & 1998 & 2271 & 1769 \\
702 & U.L.B., Belgium & 1983 & 1986 & 594 & 463 \\
705 & Sliven, Bulgaria & 1989 & 2003 & 1301 & 985 \\
706 & Ventura Mosta, Malta & 1986 & 2003 & 3732 & 2834 \\
709 & Ruben Verboven, Belgium & 2006 & 2010 & 154 & 135 \\
713 & Monte Mor, Brazil & 2006 & 2010 & 729 & 625 \\
717 & Y Alarcos, Valencia, Spain & 1986 & 1994 & 587 & 441 \\
719 & Yvergneaux Ronse-renaix, Belgium & 1981 & 1997 & 3754 & 2844 \\
720 & Zagora, Bulgaria & 1990 & 2010 & 2851 & 2307 \\
721 & Zamora, Spain & 1993 & 1999 & 1138 & 865 \\
\hline
\end{tabular}


Table 7.4: Same as Table 7.2 but for the Wolfer backbone.

\begin{tabular}{llcccc}
\hline Id & Observer & Start & End & $\mathbf{N}_{\mathrm{d}}$ & $\mathbf{M}_{\mathrm{d}}$ \\
\hline $335+338$ & Wolfer, Zurich & 1876 & 1928 & 13533 & \\
329 & Secchi, Rome & 1871 & 1877 & 1530 & 298 \\
333 & Moncalieri & 1874 & 1893 & 3598 & 2422 \\
336 & Aguilar, Madrid & 1876 & 1882 & 1940 & 1381 \\
337 & Monthly Weather Review & 1877 & 1886 & 2383 & 1786 \\
339 & Ricco, Palermo & 1880 & 1892 & 3709 & 2668 \\
343 & Merino, Madrid & 1883 & 1896 & 3221 & 2394 \\
346 & Vogel, Potsdam & 1886 & 1886 & 162 & 135 \\
347 & Stonyhurst College Obs. & 1886 & 1935 & 4534 & 1835 \\
349 & Schmoll, Paris & 1888 & 1892 & 1359 & 1041 \\
350 & Haverford College Obs., PA & 1888 & 1899 & 2063 & 1547 \\
353 & Carleton College Observatory & 1889 & 1892 & 523 & 383 \\
355 & Smith Observatory & 1890 & 1891 & 258 & 192 \\
356 & Hadden, D.E., Alta, Iowa & 1890 & 1890 & 2964 & 2256 \\
359 & Schreiber, Kalocsa & 1891 & 1895 & 1173 & 976 \\
360 & Zona, Palermo & 1891 & 1891 & 282 & 233 \\
369 & Maier, Schaufling & 1895 & 1901 & 632 & 529 \\
373 & Oliver, A.I., Boston U., MA & 1897 & 1901 & 254 & 190 \\
375 & Jastremsky, B., Charkow & 1898 & 1900 & 149 & 111 \\
377 & Mirkowitsch, Jaroslaw & 1898 & 1900 & 135 & 111 \\
379 & Kaulbars, St. Petersburg & 1898 & 1901 & 649 & 508 \\
391 & Boston University Obs. & 1903 & 1906 & 359 & 239 \\
401 & Bemmelen, Batavia & 1907 & 1919 & 2748 & 1910 \\
415 & Schmid, St. Gallen & 1915 & 1915 & 225 & 173 \\
421 & Voss, Altona & 1918 & 1918 & 198 & 145 \\
465 & Wolf, R., Zurich (small Telescope) & 1858 & 1893 & 8285 & 4385 \\
\hline & & & & &
\end{tabular}

Table 7.5: Same as Table 7.2 but for the Schmidt backbone.

\begin{tabular}{llcccc} 
Id & Observer & Start & End & $\mathbf{N}_{\mathrm{d}}$ & $\mathbf{M}_{\mathrm{d}}$ \\
\hline 292 & Schmidt, Athens & 1841 & 1883 & 6970 & \\
298 & Wolf, R., Zurich & 1848 & 1893 & 18311 & 4153 \\
307 & Carrington, London & 1853 & 1860 & 1215 & 204 \\
311 & Weber, Peckeloh & 1859 & 1883 & 6983 & 4035 \\
318 & Spoerer, G., Anclam & 1861 & 1893 & 6281 & 2449 \\
323 & Ferrari, Rome & 1866 & 1879 & 478 & 429 \\
324 & Leppig, Leipzig & 1867 & 1881 & 2611 & 1979 \\
325 & Dawson, W.M., Spiceland, Ind & 1867 & 1890 & 1623 & 824 \\
328 & Tacchini, Rome & 1871 & 1900 & 7584 & 2388 \\
330 & Billwiller, Zurich & 1872 & 1875 & 308 & 286 \\
331 & Sawyer, E.F., Cambridgeport & 1872 & 1874 & 282 & 273 \\
342 & Janesch, Laibach & 1882 & 1887 & 1164 & 439 \\
\hline
\end{tabular}


7 New reconstruction of the sunspot group numbers since 1739 using direct calibration and "backbone" methods

Table 7.6: Same as Table 7.2 but for the Schwabe backbone.

\begin{tabular}{llccccc}
\hline Id & Observer & Start & End & $\mathbf{N}_{\mathrm{d}}$ & $\mathbf{M}_{\mathrm{d}}$ & $\mathbf{M}_{\mathrm{d}} \pm 1$ day \\
\hline 279 & Schwabe, H. Dessau & 1825 & 1867 & 11945 & 11945 & \\
255 & Stark, J.M., Augsburg & 1826 & 1836 & 1075 & 924 & 1029 \\
274 & Herschel, J., London & 1822 & 1837 & 122 & 37 & 61 \\
278 & Von Both, G., Breslau & 1825 & 1826 & 183 & 59 & 72 \\
280 & Hussey, T.J., England & 1826 & 1837 & 1207 & 879 & 1073 \\
282 & Lawson, H., Hereford & 1831 & 1832 & 200 & 151 & 180 \\
283 & Ruprecht, H., Ziegenhain & 1832 & 1832 & 39 & 31 & 35 \\
284 & Boguslawski, P.H.L., Breslau & 1832 & 1832 & 17 & 14 & 17 \\
285 & Bohm, J.G., Wien & 1833 & 1836 & 101 & 84 & 96 \\
290 & Petersen, A.C., Altona & 1840 & 1841 & 13 & 10 & 13 \\
294 & Peters, C.H.F., Clinton, NY & 1844 & 1870 & 1308 & 953 & 1028 \\
299 & Greisbach, T.J., England & 1850 & 1865 & 168 & 161 & 168 \\
300 & Sestini, Georgetown & 1850 & 1850 & 42 & 35 & 39 \\
304 & Pogson, N., London & 1851 & 1851 & 13 & 11 & 13 \\
305 & Tomaschek, Wien & 1852 & 1854 & 15 & 8 & 15 \\
306 & Borck, Cassel & 1852 & 1855 & 19 & 19 & 19 \\
308 & Flagstaff Obs., Melbourne & 1857 & 1858 & 16 & 15 & 16 \\
312 & Howlett, F., England & 1859 & 1892 & 766 & 505 & 537 \\
313 & Baxendall, J., Manchester & 1859 & 1859 & 7 & 7 & 7 \\
314 & Coast Survey, Washington & 1860 & 1862 & 475 & 430 & 460 \\
316 & Jenzer, Bern & 1861 & 1865 & 585 & 542 & 566 \\
320 & Waldner, Zurich & 1863 & 1864 & 41 & 39 & 41 \\
321 & Meyer, Zurich & 1864 & 1871 & 912 & 387 & 397 \\
\hline
\end{tabular}

Table 7.7: Same as Table 7.2 but for the Flaugergues backbone.

\begin{tabular}{llccccc}
\hline Id & Observer & Start & End & $\mathbf{N}_{\mathrm{d}}$ & $\mathbf{M}_{\mathrm{d}}$ & $\mathbf{M}_{\mathrm{d}} \pm 1$ day \\
\hline $22+227$ & Flaugergues, H., Aubenas and Viviers & 1788 & 1830 & 2101 & 2101 & \\
202 & Bode, J.E., Berlin & 1774 & 1822 & 68 & 26 & 32 \\
218 & Heinrich, P., Munich & 1781 & 1820 & 396 & 119 & 216 \\
236 & Herschel, W., London & 1794 & 1818 & 384 & 29 & 67 \\
238 & Gemeiner, A.T., Regensburg & 1797 & 1797 & 3 & 1 & 3 \\
245 & Lindener, B.A., Glatz & 1800 & 1827 & 519 & 114 & 210 \\
246 & Derfflinger, T., Kremsmunster & 1802 & 1824 & 789 & 47 & 101 \\
250 & Prantner, S.M.J., Wilten & 1804 & 1844 & 115 & 35 & 67 \\
258 & Tevel, C., Middelburg & 1816 & 1836 & 858 & 89 & 156 \\
260 & Watts, Cape Diamond, Quebec & 1816 & 1818 & 83 & 3 & 10 \\
262 & Adams, C.H., Edmonton & 1819 & 1823 & 977 & 34 & 66 \\
263 & Pastorff, J.W., Drossen & 1819 & 1833 & 1477 & 53 & 109 \\
273 & Arago, F.D., Paris & 1822 & 1830 & 923 & 85 & 145 \\
\hline
\end{tabular}


Table 7.8: Same as Table 7.2 but for the Horrebow backbone.

\begin{tabular}{|c|c|c|c|c|c|c|}
\hline Id & Observer & Start & End & $\mathbf{N}_{\mathrm{d}}$ & $\mathbf{M}_{\mathrm{d}}$ & $\mathbf{M}_{\mathrm{d}} \pm 1$ day \\
\hline 180 & Horrebow, C., Copenhagen & 1761 & 1776 & 1532 & 1532 & \\
\hline 174 & Lalande, J., Paris & 1752 & 1798 & 105 & 15 & 26 \\
\hline 185 & Warschauer & 1764 & 1766 & 3 & 2 & 3 \\
\hline 203 & Lievog, E., Copenhagen & 1776 & 1777 & 196 & 97 & 101 \\
\hline 466 & Staudach, J.C., Nuremberg & 1749 & 1799 & 1172 & 128 & 234 \\
\hline
\end{tabular}


7 New reconstruction of the sunspot group numbers since 1739 using direct calibration and "backbone" methods

Table 7.9: Annual values of the proposed GSN series with the asymmetric $1 \sigma$ intervals.

\begin{tabular}{|c|c|c|c|c|c|c|c|c|c|c|c|}
\hline ear & $\bar{G}$ & $\sigma_{+}$ & $\overline{\sigma_{-}}$ & Year & $\mathrm{G}$ & $\sigma_{+}$ & $\sigma_{-}$ & ICal & G & $\sigma_{+}$ & $\sigma_{-}$ \\
\hline 1739 & 4.01 & מר & 208 & 1779 & .48 & 1.48 & 1.35 & 1819 & 3.04 & & 067 \\
\hline 1740 & 9.00 & & 00 & 780 & & & & & .42 & & \\
\hline 41 & & & & & & & & & & & 51 \\
\hline 742 & 1.73 & & 5 & & 4.20 & 5 & & ?ר & .56 & & \\
\hline 743 & 1.6 & & & 1783 & 1 & & 2 & 23 & 28 & & 3 \\
\hline 1744 & 9.00 & 0 & 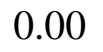 & 84 & 2.12 & 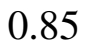 & 0.74 & 324 & 1.60 & & .3 \\
\hline 1745 & 00 & & .00 & & 97 & & 0.66 & 25 & .54 & & .60 \\
\hline 746 & .00 & & & & 0 & & 0 & & 3.67 & & 7 \\
\hline 1747 & 9.00 & 0. & & & .28 & & 1.11 & 827 & 4.71 & & .8 \\
\hline 8 & & & & & & & & & & & .00 \\
\hline 1749 & 6.68 & & & 39 & 37 & 0 & 1.22 & 29 & 5.71 & & 92 \\
\hline 50 & & & & & & & & & & & 00 \\
\hline 1751 & & & & 91 & 9 & 2 & & 31 & 4.34 & & 87 \\
\hline 52 & & & & & & & 1. & & 08 & & 6 \\
\hline 1753 & & & & & & & & & & & 52 \\
\hline 17 & & & & & 4.71 & & & & 1.70 & & 51 \\
\hline & & & & & & & & & & & 80 \\
\hline 1756 & & & & 6 & 2.38 & & & 36 & 8.32 & & 93 \\
\hline & & & & & & & & & 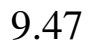 & & 08 \\
\hline 58 & & & & & & & & & 7.29 & & .02 \\
\hline & & & & & & & & & & & 96 \\
\hline 60 & & & & & 41 & & & & .12 & & 90 \\
\hline 51 & & & & & 2 & & & & 3.40 & & .77 \\
\hline 1762 & & & & & 9 & & 1 & 2 & 2.42 & & 63 \\
\hline 1763 & & & & & 1 & & & 3 & 1.35 & & 40 \\
\hline & & & & & & & & & 0 & & 54 \\
\hline 65 & & & & & 3 & & & & 61 & & 83 \\
\hline & & & & & & & & & & & 90 \\
\hline 7 & & & & & 9 & 3 & 0 & 47 & 82 & & 19 \\
\hline 1768 & & & & & & & & 8 & 8.55 & & .00 \\
\hline 1769 & 7 & & 1 & 9 & 2 & 7 & 0.46 & 9 & 39 & & 94 \\
\hline 1770 & & & & & & & & & 5.96 & & 01 \\
\hline 1771 & 6.89 & & & & 1.27 & & & 1 & 5.99 & & .94 \\
\hline & & & & & & & & & 5.48 & & .94 \\
\hline & & & & & 2.26 & & & & 4.33 & & .85 \\
\hline & & & & & 2.04 & & & & 2.55 & & .70 \\
\hline 1775 & & & & & 3.22 & 0.76 & 0.60 & 55 & 1.33 & & .44 \\
\hline & & & & & & & & & & & 0.39 \\
\hline 1777 & & & & & 4.20 & 0.79 & 0.81 & 1857 & 2.95 & 0.73 & 0.75 \\
\hline 778 & 8.86 & 12 & 1.12 & 18 & 3.78 & 0.78 & 0.70 & 1858 & 5.44 & 0.95 & 0.8 \\
\hline
\end{tabular}


Table 7.9: continued.

\begin{tabular}{|c|c|c|c|c|c|c|c|c|c|c|c|}
\hline Year & G & $\sigma_{+}$ & $\sigma_{-}$ & Year & $\mathrm{G}$ & $\sigma_{+}$ & $\sigma_{-}$ & Year & G & $\sigma_{+}$ & $\sigma_{-}$ \\
\hline 1859 & 7.94 & 0.97 & 1.02 & 1899 & 1.50 & 0.50 & 0.44 & 1939 & 8.10 & 0.72 & 0.73 \\
\hline 1860 & 8.34 & 0.96 & 1.01 & 1900 & 1.25 & 0.53 & 0.40 & 1940 & 6.31 & 0.77 & 0.74 \\
\hline 1861 & 7.01 & 0.94 & 0.99 & 1901 & 0.54 & 0.36 & 0.25 & 1941 & 4.55 & 0.78 & 0.69 \\
\hline 1862 & 5.50 & 0.93 & 0.88 & 1902 & 0.65 & 0.35 & 0.26 & 1942 & 2.86 & 0.62 & 0.46 \\
\hline 1863 & 4.74 & 0.91 & 0.79 & 1903 & 2.36 & 0.64 & 0.49 & 1943 & 1.63 & 0.41 & 0.36 \\
\hline 1864 & 4.47 & 0.87 & 0.81 & 1904 & 4.23 & 0.68 & 0.69 & 1944 & 1.27 & 0.43 & 0.33 \\
\hline 1865 & 3.23 & 0.79 & 0.74 & 1905 & 5.10 & 0.85 & 0.71 & 1945 & 3.55 & 0.70 & 0.63 \\
\hline 1866 & 2.27 & 0.66 & 0.57 & 1906 & 5.10 & 0.74 & 0.78 & 1946 & 8.07 & 0.99 & 0.82 \\
\hline 1867 & 1.41 & 0.56 & 0.46 & 1907 & 5.27 & 0.84 & 0.65 & 1947 & 11.62 & 1.07 & 1.12 \\
\hline 1868 & 3.62 & 0.84 & 0.68 & 1908 & 4.91 & 0.84 & 0.79 & 1948 & 10.59 & 1.04 & 0.98 \\
\hline 1869 & 6.21 & 0.98 & 0.85 & 1909 & 4.06 & 0.78 & 0.64 & 1949 & 10.04 & 0.98 & 0.96 \\
\hline 1870 & 9.24 & 0.84 & 0.99 & 1910 & 2.12 & 0.54 & 0.49 & 1950 & 6.47 & 0.86 & 0.83 \\
\hline 1871 & 7.93 & 0.91 & 0.95 & 1911 & 0.97 & 0.42 & 0.34 & 1951 & 5.19 & 0.79 & 0.77 \\
\hline 1872 & 7.58 & 0.87 & 0.96 & 1912 & 0.60 & 0.37 & 0.24 & 1952 & 2.74 & 0.57 & 0.55 \\
\hline 1873 & 5.27 & 0.90 & 0.82 & 1913 & 0.42 & 0.34 & 0.20 & 1953 & 1.46 & 0.48 & 0.39 \\
\hline 1874 & 4.18 & 0.79 & 0.74 & 1914 & 1.17 & 0.50 & 0.33 & 1954 & 0.74 & 0.32 & 0.27 \\
\hline 1875 & 2.09 & 0.69 & 0.52 & 1915 & 4.13 & 0.78 & 0.76 & 1955 & 3.33 & 0.73 & 0.57 \\
\hline 1876 & 1.44 & 0.54 & 0.43 & 1916 & 5.36 & 0.90 & 0.83 & 1956 & 10.29 & 0.98 & 1.05 \\
\hline 1877 & 1.37 & 0.54 & 0.41 & 1917 & 8.57 & 0.89 & 1.05 & 1957 & 13.03 & 0.99 & 0.97 \\
\hline 1878 & 0.77 & 0.40 & 0.29 & 1918 & 7.20 & 0.88 & 0.90 & 1958 & 13.50 & 1.11 & 1.02 \\
\hline 1879 & 0.99 & 0.42 & 0.37 & 1919 & 6.00 & 0.78 & 0.79 & 1959 & 11.71 & 0.90 & 0.95 \\
\hline 1880 & 3.03 & 0.72 & 0.57 & 1920 & 3.78 & 0.70 & 0.62 & 1960 & 8.53 & 1.05 & 0.93 \\
\hline 1881 & 4.93 & 0.88 & 0.77 & 1921 & 2.65 & 0.63 & 0.58 & 1961 & 4.45 & 0.74 & 0.74 \\
\hline 1882 & 4.98 & 0.82 & 0.80 & 1922 & 1.59 & 0.55 & 0.41 & 1962 & 2.91 & 0.60 & 0.53 \\
\hline 1883 & 5.43 & 0.90 & 0.83 & 1923 & 0.92 & 0.37 & 0.33 & 1963 & 2.35 & 0.54 & 0.46 \\
\hline 1884 & 5.98 & 0.82 & 0.81 & 1924 & 1.85 & 0.49 & 0.44 & 1964 & 1.20 & 0.39 & 0.32 \\
\hline 1885 & 4.88 & 0.75 & 0.80 & 1925 & 4.22 & 0.76 & 0.72 & 1965 & 1.58 & 0.44 & 0.39 \\
\hline 1886 & 2.79 & 0.63 & 0.66 & 1926 & 5.87 & 0.82 & 0.74 & 1966 & 3.97 & 0.61 & 0.64 \\
\hline 1887 & 1.66 & 0.57 & 0.51 & 1927 & 6.28 & 0.83 & 0.83 & 1967 & 7.88 & 1.02 & 0.89 \\
\hline 1888 & 1.10 & 0.51 & 0.32 & 1928 & 6.72 & 0.85 & 0.91 & 1968 & 8.03 & 0.91 & 0.92 \\
\hline 1889 & 1.04 & 0.46 & 0.37 & 1929 & 6.05 & 0.81 & 0.74 & 1969 & 7.90 & 0.98 & 0.90 \\
\hline 1890 & 1.15 & 0.49 & 0.39 & 1930 & 3.83 & 0.77 & 0.63 & 1970 & 8.75 & 0.91 & 0.90 \\
\hline 1891 & 3.98 & 0.76 & 0.75 & 1931 & 2.39 & 0.53 & 0.49 & 1971 & 6.07 & 0.80 & 0.74 \\
\hline 1892 & 6.51 & 0.94 & 0.88 & 1932 & 1.31 & 0.43 & 0.34 & 1972 & 5.94 & 0.91 & 0.82 \\
\hline 1893 & 7.66 & 0.97 & 0.96 & 1933 & 0.72 & 0.33 & 0.28 & 1973 & 3.40 & 0.71 & 0.66 \\
\hline 1894 & 7.31 & 0.95 & 0.93 & 1934 & 1.05 & 0.42 & 0.34 & 1974 & 3.12 & 0.72 & 0.66 \\
\hline 1895 & 5.86 & 0.94 & 0.82 & 1935 & 3.75 & 0.77 & 0.62 & 1975 & 1.57 & 0.41 & 0.39 \\
\hline 1896 & 3.85 & 0.78 & 0.68 & 1936 & 7.45 & 0.95 & 0.79 & 1976 & 1.41 & 0.39 & 0.34 \\
\hline 1897 & 3.05 & 0.70 & 0.63 & 1937 & 10.10 & 1.14 & 1.07 & 1977 & 2.65 & 0.55 & 0.50 \\
\hline 1898 & 2.63 & 0.68 & 0.58 & 1938 & 9.72 & 0.98 & 0.95 & 1978 & 7.92 & 1.02 & 0.83 \\
\hline
\end{tabular}


7 New reconstruction of the sunspot group numbers since 1739 using direct calibration and "backbone" methods

Table 7.9: continued.

\begin{tabular}{|cccc|cccc|cccc|}
\hline Year & $\mathrm{G}$ & $\sigma_{+}$ & $\sigma_{-}$ & Year & $\mathrm{G}$ & $\sigma_{+}$ & $\sigma_{-}$ & Year & $\mathrm{G}$ & $\sigma_{+}$ & $\sigma_{-}$ \\
\hline 1979 & 11.61 & 1.08 & 1.01 & 1990 & 10.86 & 1.14 & 1.11 & 2001 & 9.76 & 1.07 & 0.97 \\
1980 & 10.50 & 1.07 & 1.06 & 1991 & 11.00 & 1.03 & 1.15 & 2002 & 9.52 & 1.06 & 0.96 \\
1981 & 10.78 & 1.02 & 1.09 & 1992 & 7.46 & 0.97 & 0.77 & 2003 & 6.13 & 0.96 & 0.93 \\
1982 & 8.85 & 1.06 & 0.89 & 1993 & 4.53 & 0.69 & 0.63 & 2004 & 4.15 & 0.75 & 0.71 \\
1983 & 5.52 & 0.88 & 0.78 & 1994 & 2.96 & 0.64 & 0.54 & 2005 & 3.09 & 0.68 & 0.55 \\
1984 & 3.56 & 0.70 & 0.59 & 1995 & 1.83 & 0.51 & 0.44 & 2006 & 1.95 & 0.48 & 0.44 \\
1985 & 1.51 & 0.50 & 0.39 & 1996 & 1.05 & 0.37 & 0.31 & 2007 & 1.15 & 0.40 & 0.33 \\
1986 & 1.19 & 0.41 & 0.34 & 1997 & 2.00 & 0.52 & 0.40 & 2008 & 0.69 & 0.33 & 0.26 \\
1987 & 2.28 & 0.56 & 0.50 & 1998 & 5.53 & 0.77 & 0.71 & 2009 & 0.70 & 0.35 & 0.23 \\
1988 & 6.65 & 0.88 & 0.83 & 1999 & 7.61 & 0.98 & 0.88 & 2010 & 2.03 & 0.53 & 0.43 \\
1989 & 10.81 & 1.07 & 1.08 & 2000 & 9.51 & 1.06 & 0.98 & & & & \\
\hline
\end{tabular}




\section{Summary and outlook}

Understanding of the mechanisms of solar influence on climate requires long and reliable reconstructions of past solar irradiance. A critical input to such reconstructions are direct or indirect indices of solar magnetic activity. They need to be as reliable as possible, homogeneous, self-consistent and with realistic uncertainty estimates, which is usually not the case. The main goal of this thesis is to construct new or to critically revise existing datasets of historical solar observations. Two types of data, which are of prime importance for irradiance models are addressed in this thesis: facular and sunspot observations from historical Ca II K spectroheliograms and sunspot counting, respectively.

In the introduction (Chapter 1) we give a brief overview of the basic underlying solar physics, solar irradiance and its connection to Earth's climate. In Chapter 2 we describe the historical and modern $\mathrm{Ca}$ II $\mathrm{K}$ data and provide information on the observational and photographic process. An overview of the previously published processing techniques and plage time series is also given. Chapters $3-7$ present our work on the historical and modern data.

In Chapter 3 we present a new technique to process and photometrically calibrate historical Ca II K spectroheliograms. We have developed a method to compute the CLV profile and to account for all possible artefacts and large-scale inhomogeneities on the historical observations. The accuracy of the method was evaluated on synthetic observations. These synthetic data were created by using modern CCD-based Ca II K observations as their basis, but degraded such that a variety of problems having known characteristics were introduced. We show that our method to account for the limb darkening and artefacts is superior to other methods presented in the literature. Application of our method to a synthetic dataset with a random set of problems, thus mimicking historical data of poor quality, showed that pixel-by-pixel errors were on average less than $6.5 \%$. We have processed subsets of images from 7 available historical datasets and showed that the technique returns consistent results for diverse data without the need for adaptations or modifications. We test segmentation schemes on modern data in order to evaluate their stability in time and aptness for application on the historical data (Chapter 4).

In Chapter 5 we re-evaluate the relation between the $\mathrm{Ca}$ II K contrast and the magnetic field strength. We employ full disc Rome/PSPT Ca II K filtergrams along with SDO/HMI magnetograms and continuum images. Comparing pixel values from near co-temporal observations we confirm a monotonic relationship which we approximate with a power law function. We derive the best fit parameters of the power law function from all available observations. Testing these parameters for observations taken at different levels of activity showed no significant variance. We also find the exponents to be independent of the disc position. We use these parameters to reconstruct magnetograms from the $\mathrm{Ca}$ II $\mathrm{K}$ observations and show that we replicate the magnetic field (without the polarity informa- 
tion) over the disc with unsigned differences that are on average $\sim 30 \mathrm{G}$.

In Chapter 6 we first review the existing sunspot data and their cross-calibration methods. We then study the dependence of sunspot areas on the activity level and show that during high activity periods large spots dominate the distribution. This poses a problem to existing cross-calibration methods that assume that the relation between the records of observers is linear and constant in time. Taking into account that different observers differ in their observing capabilities, which is expressed in the smallest size of a sunspot they can detect, the relation between the records of observers could be linear if the fraction of small spots (which a "poor" observer would miss) was constant in time. We studied the relation of the sunspot group measurements between observers that have different acuities with the aid of synthetic data, created from the RGO, Rome, and Catania sunspot area records. We show that the relation is strongly non-linear for observers with different observing capabilities and that methods applying simple linear regressions potentially overestimate the activity levels.

We have also developed a technique to cross-calibrate the records by individual observers using a non-linear and non-parametric method with minimum assumptions based on the daily statistics of the common days of observation (Chapter 7 ). We used a method similar to the backbone methodology to calibrate the measurements of observers earlier in time, with the improvement that the records of the backbone observers directly overlap with each other. The backbone observers were chosen to be those of the best quality and long period of records. An important aspect of this work is that we are able to accurately account with Monte Carlo simulations for the propagation of errors, which has never been done for previous series. Our reconstruction of the group sunspot number series shows that the activity level over the 18th and 19th centuries was moderate, as suggested by earlier reconstructions, and supports the high level of activity over the 20th century, known as the grand modern maximum.

The work carried out here contributes to our understanding of solar variability on time-scales of decades to centuries and sets the grounds for more accurate analyses of historical observations of the Sun. This has significant implications for reconstructions of past solar irradiance changes and thus for our understanding of the role of the Sun in global climate change.

More work can and has to be done with the historical solar data. For example, the tests with the synthetic data can be expanded based on other modern CCD-based Ca II $\mathrm{K}$ observations taken with narrower bandwidths than the one used in the Rome/PSPT observations used in this study. The different segmentation schemes need to be further tested. We can make use of intensity synthetic data and test the segmentation methods against a variety of inhomogeneities. It is also important to check how strongly the results depend on smearing, due to e.g. seeing, which can also be tested with synthetic data. Analysis of other modern CCD-based archives of Ca II K data, such as those from Chrotel, Kanzelhöhe, or MLSO/PSPT can help assess the segmentation methods. Further careful analysis is needed to obtain final plage and network area series from the historical Ca II K archives. Any residual problems from the processing of the data have to be identified and the corresponding data have to either be excluded or reprocessed. The differences between the series resulting from individual archives and their sources need to be understood, e.g. the characteristics of the observatories such as different pass-bands. A composite series can be constructed, thus providing a better coverage with fewer data gaps. The rest of the 
digitised historical Ca II $\mathrm{K}$ data can be processed and their results used to complement the ones we have already processed. Inclusion of more datasets can help to reduce any gaps in the time coverage and help determine uncertainties in the case that there is more than one observation per day. There are numerous archives of Ca II K observations that have not been digitised yet. It is important to carefully digitise all available observations. The time-series of plage areas can be extended to the present with results from modern observations.

Based on our work on the relation between the magnetic field strength and Ca II K brightness, we can use a larger sample of CCD-based observations, covering more than a solar cycle, to reconstruct magnetograms and compare our results for the total magnetic flux with those from other measurements. We can test the performance of the reconstruction of magnetograms with narrower band Ca II K observations, which resemble more the historical observations. Then we can use the photometrically calibrated historical Ca II K data to produce pseudo-magnetograms covering the whole 20th century. These can in turn be used as input to perform improved irradiance reconstructions. The reconstructed magnetograms can also provide information on how strongly has the network flux changed over the last century and if there is any trend in its variation.

In our work on the sunspot number series we made the assumption that the quality of the observers remained constant throughout their observing periods. The next step would be to assess the stability of the long-running observers over shorter time-intervals and to evaluate if there are systematic changes in their observations. Another option would be to check how the acuities of the observers that were derived with this method vary with time. One way this could be done is by direct comparisons between the PDF matrices of observers, thus getting a relative acuity difference between them. As was stated, there is a wall in 1739 due to missing overlap between sunspot observers and the calibration cannot be applied prior to 1739. Acquiring these relative acuities of the observers after 1739 employed in our series, can potentially provide a way to make the leap and calibrate the records of observers prior to 1739. Archives of sunspot data that include both the group sunspot number and the sunspot number can be used to evaluate the consistency of individual observers and study effects of grouping with time. We can use the method we developed to process the historical Ca II K spectroheliograms to process the historical photographic archives of white-light observations. These data can be used then to determine sunspot areas, along with information on sunspot location and contrast. 



\section{Bibliography}

Abbot, C. G.: 1919, Proceedings of the National Academy of Sciences of the United States of America 5(9), 383

Abbot, C. G.: 1933, in Smithsonian miscellaneous collections, Vol. 85, Washington Smithsonian Institution

Abbot, C. G.: 1952, in Smithsonian miscellaneous collections, Vol. 117, Washington Smithsonian Institution

Abbot, C. G.: 1963, in Smithsonian miscellaneous collections, Vol. 146, Washington Smithsonian Institution

Abney, W. d.: 1889, Journal of the Camera Club 3

Adelberger, E. G., García, A., Robertson, R. G. H., Snover, K. A., Balantekin, A. B., Heeger, K., Ramsey-Musolf, M. J., Bemmerer, D., Junghans, A., Bertulani, C. A., Chen, J.-W., Costantini, H., Prati, P., Couder, M., Uberseder, E., Wiescher, M., Cyburt, R., Davids, B., Freedman, S. J., Gai, M., Gazit, D., Gialanella, L., Imbriani, G., Greife, U., Hass, M., Haxton, W. C., Itahashi, T., Kubodera, K., Langanke, K., Leitner, D., Leitner, M., Vetter, P., Winslow, L., Marcucci, L. E., Motobayashi, T., Mukhamedzhanov, A., Tribble, R. E., Nollett, K. M., Nunes, F. M., Park, T.-S., Parker, P. D., Schiavilla, R., Simpson, E. C., Spitaleri, C., Strieder, F., Trautvetter, H.-P., Suemmerer, K., and Typel, S.: 2011, Reviews of Modern Physics 83, 195

Adhémar, J.: 1842, Révolutions de la mer, Carilian-Goeury et V. Dalmont, Paris

Čalogović, J., Albert, C., Arnold, F., Beer, J., Desorgher, L., and Flueckiger, E. O.: 2010, Geophysical Research Letters 37, L03802

Anders, E. and Grevesse, N.: 1989, Geochimica et Cosmochimica Acta 53, 197

Antia, H. M.: 1998, Astronomy and Astrophysics 330, 336

Antonucci, E., Azzarelli, L., Casalini, P., and Cerri, S.: 1977, Solar Physics 53, 519

Aparicio, A. J. P., Vaquero, J. M., Carrasco, V. M. S., and Gallego, M. C.: 2014, Solar Physics 289, 4335

Arlt, R.: 2008, Solar Physics 247, 399 
Arlt, R., Leussu, R., Giese, N., Mursula, K., and Usoskin, I. G.: 2013, Monthly Notices of the Royal Astronomical Society 433, 3165

Asvestari, E.: 2016, Ph.D. thesis, University of Oulu, Oulu

Asvestari, E., Usoskin, I. G., Cameron, R. H., and Krivova, N. A.: 2016, in I. Dorotovic, C. E. Fischer, and M. Temmer (eds.), Coimbra Solar Physics Meeting: Ground-based Solar Observations in the Space Instrumentation Era, Vol. 504 of Astronomical Society of the Pacific Conference Series, p. 269, San Francisco

Asvestari, E., Usoskin, I. G., Kovaltsov, G. A., Owens, M. J., Krivova, N. A., Rubinetti, S., and Taricco, C.: 2017, Monthly Notices of the Royal Astronomical Society 467(2), 1608

Athay, R. G.: 1976, The solar chromosphere and corona: Quiet sun, Vol. 53 of Astrophysics and Space Science Library, Springer Netherlands

Auvergne, M., Bodin, P., Boisnard, L., Buey, J.-T., Chaintreuil, S., Epstein, G., Jouret, M., Lam-Trong, T., Levacher, P., Magnan, A., Perez, R., Plasson, P., Plesseria, J., Peter, G., Steller, M., Tiphène, D., Baglin, A., Agogué, P., Appourchaux, T., Barbet, D., Beaufort, T., Bellenger, R., Berlin, R., Bernardi, P., Blouin, D., Boumier, P., Bonneau, F., Briet, R., Butler, B., Cautain, R., Chiavassa, F., Costes, V., Cuvilho, J., CunhaParro, V., de Oliveira Fialho, F., Decaudin, M., Defise, J.-M., Djalal, S., Docclo, A., Drummond, R., Dupuis, O., Exil, G., Fauré, C., Gaboriaud, A., Gamet, P., Gavalda, P., Grolleau, E., Gueguen, L., Guivarc'h, V., Guterman, P., Hasiba, J., Huntzinger, G., Hustaix, H., Imbert, C., Jeanville, G., Johlander, B., Jorda, L., Journoud, P., Karioty, F., Kerjean, L., Lafond, L., Lapeyrere, V., Landiech, P., Larqué, T., Laudet, P., Le Merrer, J., Leporati, L., Leruyet, B., Levieuge, B., Llebaria, A., Martin, L., Mazy, E., Mesnager, J.-M., Michel, J.-P., Moalic, J.-P., Monjoin, W., Naudet, D., Neukirchner, S., NguyenKim, K., Ollivier, M., Orcesi, J.-L., Ottacher, H., Oulali, A., Parisot, J., Perruchot, S., Piacentino, A., Pinheiro da Silva, L., Platzer, J., Pontet, B., Pradines, A., Quentin, C., Rohbeck, U., Rolland, G., Rollenhagen, F., Romagnan, R., Russ, N., Samadi, R., Schmidt, R., Schwartz, N., Sebbag, I., Smit, H., Sunter, W., Tello, M., Toulouse, P., Ulmer, B., Vandermarcq, O., Vergnault, E., Wallner, R., Waultier, G., and Zanatta, P.: 2009, Astronomy and Astrophysics 506, 411

Babcock, H. D.: 1959, The Astrophysical Journal 130, 364

Babcock, H. W. and Babcock, H. D.: 1955, The Astrophysical Journal 121, 349

Babcock, T. A.: 1976, AAS Photo Bulletin 13, 3

Baliunas, S. L., Donahue, R. A., Soon, W. H., Horne, J. H., Frazer, J., Woodard-Eklund, L., Bradford, M., Rao, L. M., Wilson, O. C., Zhang, Q., Bennett, W., Briggs, J., Carroll, S. M., Duncan, D. K., Figueroa, D., Lanning, H. H., Misch, T., Mueller, J., Noyes, R. W., Poppe, D., Porter, A. C., Robinson, C. R., Russell, J., Shelton, J. C., Soyumer, T., Vaughan, A. H., and Whitney, J. H.: 1995, The Astrophysical Journal 438, 269 
Baliunas, S. L., Horne, J. H., Porter, A., Duncan, D. K., Frazer, J., Lanning, H., Misch, A., Mueller, J., Noyes, R. W., Soyumer, D., Vaughan, A. H., and Woodard, L.: 1985, The Astrophysical Journal 294, 310

Ball, W. T., Unruh, Y. C., Krivova, N. A., Solanki, S., Wenzler, T., Mortlock, D. J., and Jaffe, A. H.: 2012, Astronomy and Astrophysics 541, A27

Balmaceda, L. A., Solanki, S. K., Krivova, N. A., and Foster, S.: 2009, Journal of Geophysical Research: Space Physics 114(A7), n/a

Bard, E.: 1998, Geochimica et Cosmochimica Acta 62, 2025

Barthol, P., Gandorfer, A., Solanki, S. K., Schüssler, M., Chares, B., Curdt, W., Deutsch, W., Feller, A., Germerott, D., Grauf, B., Heerlein, K., Hirzberger, J., Kolleck, M., Meller, R., Müller, R., Riethmüller, T. L., Tomasch, G., Knölker, M., Lites, B. W., Card, G., Elmore, D., Fox, J., Lecinski, A., Nelson, P., Summers, R., Watt, A., Martínez Pillet, V., Bonet, J. A., Schmidt, W., Berkefeld, T., Title, A. M., Domingo, V., Gasent Blesa, J. L., Del Toro Iniesta, J. C., López Jiménez, A., Álvarez Herrero, A., Sabau-Graziati, L., Widani, C., Haberler, P., Härtel, K., Kampf, D., Levin, T., Pérez Grande, I., SanzAndrés, A., and Schmidt, E.: 2011, Solar Physics 268, 1

Basu, S. and Antia, H. M.: 1997, Monthly Notices of the Royal Astronomical Society 287, 189

Basu, S. and Antia, H. M.: 2008, Physics Reports 457, 217

Baumann, I. and Solanki, S. K.: 2005, Astronomy and Astrophysics 443, 1061

Beck, J. G. and Chapman, G. A.: 1993, Solar Physics 146, 49

Beck, J. W., Richards, D. A., Lawrence Edwards, R., Silverman, B. W., Smart, P. L., Donahue, D. J., Hererra-Osterheld, S., Burr, G. S., Calsoyas, L., Jull, A. J. T., and Biddulph, D.: 2001, Science 292, 2453

Beer, J., Blinov, A., Bonani, G., Finkel, R. C., Hofmann, H. J., Lehmann, B., Oeschger, H., Sigg, A., Schwander, J., Staffelbach, T., Stauffer, B., Suter, M., and Wötfli, W.: 1990, Nature 347(6289), 164

Beer, J., McCracken, K., and von Steiger, R.: 2012, Cosmogenic Radionuclides - Theory and Applications in the Terrestrial and Space Environments, Physics of Earth and Space Environments, Springer-Verlag Berlin Heidelberg, 1 edition

Beer, J., Vonmoos, M., and Muscheler, R.: 2006, Space Science Reviews 125, 67

Benestad, R. E.: 2006, Solar activity and earth's climate 2nd ed., Springer-Verlag Berlin Heidelberg, 2 edition

Berggren, A.-M., Beer, J., Possnert, G., Aldahan, A., Kubik, P., Christl, M., Johnsen, S. J., Abreu, J., and Vinther, B. M.: 2009, Geophysical Research Letters 36, L11801

Bertello, L., Ulrich, R. K., and Boyden, J. E.: 2010, Solar Physics 264, 31 
Bethge, C., Peter, H., Kentischer, T. J., Halbgewachs, C., Elmore, D. F., and Beck, C.: 2011, Astronomy and Astrophysics 534, A105

Bühler, D., Lagg, A., and Solanki, S. K.: 2013, Astronomy and Astrophysics 555, A33

Bolduc, C., Charbonneau, P., Barnabé, R., and Bourqui, M. S.: 2014, Solar Physics 289, 2891

Bonanno, A., Schlattl, H., and Paternò, L.: 2002, Astronomy E Astrophysics 390(3), 1115

Bonino, G., Cini Castagnoli, G., Bhandari, N., and Taricco, C.: 1995, Meteoritics 30

Bovelet, B. and Wiehr, E.: 2001, Solar Physics 201, 13

Bovelet, B. and Wiehr, E.: 2007, Solar Physics 243, 121

Brandt, P. N., Schmidt, W., and Steinegger, M.: 1990, Solar Physics 129, 191

Brandt, P. N. and Steinegger, M.: 1998, Solar Physics 177(1-2), 287

Brants, J. J. and Zwaan, C.: 1982, Solar Physics 80, 251

Bray, R. J. and Loughhead, R. E.: 1964, Sunspots, Chapman and Hall

Brown, T. M. and Christensen-Dalsgaard, J.: 1998, The Astrophysical Journal Letters 500, L195

Bruls, J. H. M. J. and Solanki, S. K.: 1995, Astronomy and Astrophysics 293, 240

Brumfiel, G.: 2012, Nature News

Caccin, B., Ermolli, I., Fofi, M., and Sambuco, A. M.: 1998, Solar Physics 177, 295

Caligari, P., Moreno-Insertis, F., and Schussler, M.: 1995, The Astrophysical Journal 441, 886

Carlsson, M. and Stein, R. F.: 1992, The Astrophysical Journal Letters 397, L59

Carlsson, M. and Stein, R. F.: 1997, The Astrophysical Journal 481, 500

Carrasco, V. M. S. and Vaquero, J. M.: 2016, Solar Physics pp 1-11

Carrasco, V. M. S., Vaquero, J. M., Gallego, M. C., and Trigo, R. M.: 2013, New Astronomy 25, 95

Carrington, R. C.: 1863, Observations of the spots on the sun from November 9, 1853, to March 24, 1861, made at Redhill, London [etc.] Williams and Norgate

Carroll, B. W. and Ostlie, D. A.: 2006, An Introduction to Modern Astrophysics, Pearson, San Francisco, 2 edition edition

Caughlan, G. R. and Fowler, W. A.: 1988, Atomic Data and Nuclear Data Tables 40, 283 
Chapman, G. A., Cookson, A. M., and Dobias, J. J.: 1996, Journal of Geophysical Research 101, 13541

Chapman, G. A., Cookson, A. M., and Dobias, J. J.: 1997, The Astrophysical Journal 482, 541

Chapman, G. A., Cookson, A. M., and Preminger, D. G.: 2012, Solar Physics 276, 35

Chapman, G. A., Cookson, A. M., and Preminger, D. G.: 2013, Solar Physics 283, 295

Chapman, G. A., Dobias, J. J., and Arias, T.: 2011, The Astrophysical Journal 728(2), 150

Charbonneau, P.: 2010, Living Reviews in Solar Physics 7(1), 3

Chatterjee, S., Banerjee, D., and Ravindra, B.: 2016, The Astrophysical Journal 827(1), 87

Chatzistergos, T., Ermolli, I., Solanki, S. K., and Krivova, N. A.: 2016, in I. Dorotovic, C. E. Fischer, and M. Temmer (eds.), Coimbra Solar Physics Meeting: Ground-based Solar Observations in the Space Instrumentation Era, Vol. 504 of Astronomical Society of the Pacific Conference Series, pp 227-231, San Francisco

Chatzistergos, T., Ermolli, I., Solanki, S. K., and Krivova, N. A.: 2017a, in preparation

Chatzistergos, T., Ermolli, I., Solanki, S. K., and Krivova, N. A.: 2017b, in preparation

Chatzistergos, T., Ermolli, I., Solanki, S. K., Krivova, N. A., Giorgi, F., and Yeo, K. L.: 2017c, in preparation

Chatzistergos, T., Usoskin, I. G., Kovaltsov, G. A., Krivova, N. A., and Solanki, S. K.: 2017d, Astronomy $\mathcal{E}$ Astrophysics 602, A69

Christensen-Dalsgaard, J., Gough, D. O., and Thompson, M. J.: 1991, The Astrophysical Journal 378, 413

Cimino, M.: 1967, Solar Physics 2, 375

Clette, F., Berghmans, D., Vanlommel, P., Van der Linden, R. A. M., Koeckelenbergh, A., and Wauters, L.: 2007, Advances in Space Research 40, 919

Clette, F. and Lefèvre, L.: 2016, Solar Physics 291(9-10), 2629

Clette, F., Lefèvre, L., Cagnotti, M., Cortesi, S., and Bulling, A.: 2016, Solar Physics 291(9-10), 2733

Clette, F., Svalgaard, L., Vaquero, J. M., and Cliver, E. W.: 2014, Space Science Reviews 186(1-4), 35

Cliver, E. W.: 2016, Solar Physics 291(9-10), 2891

Cliver, E. W., Clette, F., Svalgaard, L., and Vaquero, J. M.: 2015, Central European Astrophysical Bulletin 39, 1 
Cliver, E. W. and Ling, A. G.: 2016, Solar Physics 291(9-10), 2763

Cnossen, I., Liu, H., and Lu, H.: 2016, Journal of Geophysical Research (Atmospheres) 121, 7781

Coddington, O., Lean, J. L., Pilewskie, P., Snow, M., and Lindholm, D.: 2015, Bulletin of the American Meteorological Society 97(7), 1265

Connelly, J. N., Bizzarro, M., Krot, A. N., Nordlund, k., Wielandt, D., and Ivanova, M. A.: 2012, Science 338(6107), 651

Cortesi, S., Cagnotti, M., Bianda, M., Ramelli, R., and Manna, A.: 2016, Solar Physics 291(9-10), 3075

Coulter, R. L. and Kuhn, J. R.: 1994, in Solar Active Region Evolution: Comparing Models with Observations, Vol. 68 of Astronomical Society of the Pacific Conference Series, p. 37, Astronomical Society of the Pacific, San Francisco

Courtillot, V. and Le Mouel, J. L.: 1988, Annual Review of Earth and Planetary Sciences 16, 389

Covington, A. E.: 1969, Journal of the Royal Astronomical Society of Canada 63, 125

Cox, A. N.: 2000, Allen's astrophysical quantities, Springer-Verlag New York, 4 edition

Criscuoli, S. and Ermolli, I.: 2008, Astronomy and Astrophysics 484, 591

Croll, J.: 1875, Climate and Time in Their Geological Relations: A Theory of Secular Changes of the Earth's Climate, D. Appleton

Crouch, A. D., Charbonneau, P., Beaubien, G., and Paquin-Ricard, D.: 2008, The Astrophysical Journal 677, 723

Crucifix, M., Loutre, M. F., and Berger, A.: 2006, Space Science Reviews 125, 213

Cubasch, U., Voss, R., Hegerl, G. C., Waszkewitz, J., and Crowley, T. J.: 1997, Climate Dynamics 13(11), 757

Cubasch, U., Zorita, E., Kaspar, F., Gonzalez-Rouco, J. F., von Storch, H., and Prömmel, K.: 2006, Advances in Space Research 37, 1629

Curto, J. J., Solé, J. G., Genescà, M., Blanca, M. J., and Vaquero, J. M.: 2016, Solar Physics

Dacie, S., Démoulin, P., van Driel-Gesztelyi, L., Long, D. M., Baker, D., Janvier, M., Yardley, S. L., and Pérez-Suárez, D.: 2016, Astronomy and Astrophysics 596, A69

Dainty, J. C. and Shaw, R.: 1974, Image science. Principles, analysis and evaluation of photographic-type imaging processes, Academic Press, London

Danilovic, S., Schüssler, M., and Solanki, S. K.: 2010, Astronomy and Astrophysics 513, A1 
Dasi-Espuig, M., Jiang, J., Krivova, N. A., and Solanki, S. K.: 2014, Astronomy and Astrophysics 570, A23

Dasi-Espuig, M., Jiang, J., Krivova, N. A., Solanki, S. K., Unruh, Y. C., and Yeo, K. L.: 2016, Astronomy and Astrophysics 590, A63

Deland, M. T. and Cebula, R. P.: 2012, Journal of Atmospheric and Solar-Terrestrial Physics 77, 225

Delaygue, G. and Bard, E.: 2011, Climate Dynamics 36, 2201

Denker, C., Johannesson, A., Marquette, W., Goode, P. R., Wang, H., and Zirin, H.: 1999, Solar Physics 184, 87

Dewitte, S., Crommelynck, D., and Joukoff, A.: 2004, Journal of Geophysical Research (Space Physics) 109, A02102

Dickinson, R. E.: 1975, Bulletin of the American Meteorological Society 56, 1240

Dikpati, M. and Gilman, P. A.: 2009, Space Science Reviews 144, 67

Dima, M. and Voiculescu, M.: 2016, Climate Dynamics 47, 667

Dizer, M.: 1968, Solar Physics 3, 491

Donadini, F., Korte, M., and Constable, C.: 2010, Space Science Reviews 155, 219

Donadini, F., Korte, M., and Constable, C. G.: 2009, Geochemistry, Geophysics, Geosystems 10(6)

Donnelly, R. F., White, O. R., and Livingston, W. C.: 1994, Solar Physics 152, 69

Dorman, L. I.: 2012, Annales Geophysicae 30(1), 9

Druffel, E. R. M., Robinson, L. F., Griffin, S., Halley, R. B., Southon, J. R., and Adkins, J. F.: 2008, Paleoceanography 23(2), PA2209

Dudok de Wit, T., Kopp, G., Fröhlich, C., and Schöll, M.: 2017, Geophysical Research Letters p. 2016GL071866

Duncan, D. K., Vaughan, A. H., Wilson, O. C., Preston, G. W., Frazer, J., Lanning, H., Misch, A., Mueller, J., Soyumer, D., Woodard, L., Baliunas, S. L., Noyes, R. W., Hartmann, L. W., Porter, A., Zwaan, C., Middelkoop, F., Rutten, R. G. M., and Mihalas, D.: 1991, The Astrophysical Journal Supplement Series 76, 383

Dunne, E. M., Gordon, H., Kürten, A., Almeida, J., Duplissy, J., Williamson, C., Ortega, I. K., Pringle, K. J., Adamov, A., Baltensperger, U., Barmet, P., Benduhn, F., Bianchi, F., Breitenlechner, M., Clarke, A., Curtius, J., Dommen, J., Donahue, N. M., Ehrhart, S., Flagan, R. C., Franchin, A., Guida, R., Hakala, J., Hansel, A., Heinritzi, M., Jokinen, T., Kangasluoma, J., Kirkby, J., Kulmala, M., Kupc, A., Lawler, M. J., Lehtipalo, K., Makhmutov, V., Mann, G., Mathot, S., Merikanto, J., Miettinen, P., Nenes, A., Onnela, 
A., Rap, A., Reddington, C. L. S., Riccobono, F., Richards, N. A. D., Rissanen, M. P., Rondo, L., Sarnela, N., Schobesberger, S., Sengupta, K., Simon, M., Sipilä, M., Smith, J. N., Stozkhov, Y., Tomé, A., Tröstl, J., Wagner, P. E., Wimmer, D., Winkler, P. M., Worsnop, D. R., and Carslaw, K. S.: 2016, Science p. aaf2649

Eddy, J. A.: 1976, Science 192, 1189

Eggleton, P.: 2011, Evolutionary Processes in Binary and Multiple Stars, Cambridge University Press

Eldrett, J. S., Ma, C., Bergman, S. C., Lutz, B., Gregory, F. J., Dodsworth, P., Phipps, M., Hardas, P., Minisini, D., Ozkan, A., Ramezani, J., Bowring, S. A., Kamo, S. L., Ferguson, K., Macaulay, C., and Kelly, A. E.: 2015, Cretaceous Research 56, 316

Ermolli, I.: 2001, Memorie della Societa Astronomica Italiana 72, 545

Ermolli, I., Berrilli, F., and Florio, A.: 2003a, Astronomy and Astrophysics 412, 857

Ermolli, I., Caccin, B., Centrone, M., and Penza, V.: 2003b, Memorie della Societa Astronomica Italiana 74, 603

Ermolli, I., Criscuoli, S., Centrone, M., Giorgi, F., and Penza, V.: 2007a, Astronomy and Astrophysics 465, 305

Ermolli, I., Criscuoli, S., and Giorgi, F.: 2011, Contributions of the Astronomical Observatory Skalnate Pleso 41, 73

Ermolli, I., Criscuoli, S., Uitenbroek, H., Giorgi, F., Rast, M. P., and Solanki, S. K.: 2010a, Astronomy and Astrophysics 523, 55

Ermolli, I., Cristaldi, A., Giorgi, F., Giannattasio, F., Stangalini, M., Romano, P., Tritschler, A., and Zuccarello, F.: 2017, ArXiv e-prints 1701, arXiv:1701.06440

Ermolli, I., Fofi, M., Bernacchia, C., Berrilli, F., Caccin, B., Egidi, A., and Florio, A.: 1998, Solar Physics 177, 1

Ermolli, I., Krivova, N., and Solanki, S. K.: 2010b, in 38th COSPAR Scientific Assembly, Vol. 38 of COSPAR Meeting, p. 133

Ermolli, I., Marchei, E., Centrone, M., Criscuoli, S., Giorgi, F., and Perna, C.: 2009a, Astronomy and Astrophysics 499, 627

Ermolli, I., Matthes, K., Dudok de Wit, T., Krivova, N. A., Tourpali, K., Weber, M., Unruh, Y. C., Gray, L., Langematz, U., Pilewskie, P., Rozanov, E., Schmutz, W., Shapiro, A., Solanki, S. K., and Woods, T. N.: 2013, Atmospheric Chemistry E Physics 13, 3945

Ermolli, I., Solanki, S. K., Tlatov, A. G., Krivova, N. A., Ulrich, R. K., and Singh, J.: 2009b, The Astrophysical Journal 698, 1000

Ermolli, I., Tlatov, A., Solanki, S. K., Krivova, N. A., and Singh, J.: 2007b, in The Physics of Chromospheric Plasmas, Vol. 368, p. 533 
Feynman, J. and Ruzmaikin, A.: 2014, Journal of Geophysical Research (Space Physics) 119, 6027

Fligge, M. and Solanki, S. K.: 2000, Geophysical Research Letters 27, 2157

Fligge, M., Solanki, S. K., and Beer, J.: 1999, Astronomy and Astrophysics 346, 313

Fligge, M., Solanki, S. K., and Unruh, Y. C.: 2000a, Astronomy and Astrophysics 353, 380

Fligge, M., Solanki, S. K., and Unruh, Y. C.: 2000b, Space Science Reviews 94, 139

Fligge, M., Solanki, S. K., Unruh, Y. C., Froehlich, C., and Wehrli, C.: 1998, Astronomy and Astrophysics 335, 709

Floyd, L. E., Cook, J. W., Herring, L. C., and Crane, P. C.: 2003, Advances in Space Research 31, 2111

Fontenla, J., White, O. R., Fox, P. A., Avrett, E. H., and Kurucz, R. L.: 1999, The Astrophysical Journal 518, 480

Fontenla, J. M., Avrett, E., Thuillier, G., and Harder, J.: 2006, The Astrophysical Journal 639, 441

Fontenla, J. M., Harder, J., Livingston, W., Snow, M., and Woods, T.: 2011, Journal of Geophysical Research (Atmospheres) 116, 20108

Forbush, S. E.: 1954, Journal of Geophysical Research 59, 525

Forbush, S. E.: 1958, Journal of Geophysical Research 63, 651

Foster, G. L., Royer, D. L., and Lunt, D. J.: 2017, Nature Communications 8, 14845

Foukal, P.: 1993, Solar Physics 148, 219

Foukal, P.: 1996, Geophysical Research Letters 23, 2169

Foukal, P.: 1998, Geophysical Research Letters 25, 2909

Foukal, P.: 2002, Geophysical Research Letters 29, 2089

Foukal, P.: 2015, The Astrophysical Journal 815, 9

Foukal, P., Bertello, L., Livingston, W. C., Pevtsov, A. A., Singh, J., Tlatov, A. G., and Ulrich, R. K.: 2009, Solar Physics 255, 229

Foukal, P. and Duvall, Jr., T.: 1985, The Astrophysical Journal 296, 739

Foukal, P. and Lean, J.: 1986, The Astrophysical Journal 302, 826

Foukal, P. and Lean, J.: 1988, The Astrophysical Journal 328, 347

Foukal, P. and Lean, J.: 1990, Science 247, 556 
Foukal, P. and Milano, L.: 2001, Geophysical Research Letters 28, 883

Frame, T. H. A. and Gray, L. J.: 2009, Journal of Climate 23(8), 2213

Frazier, E. N.: 1971, Solar Physics 21, 42

Fredga, K.: 1971, Solar Physics 21, 60

Fröhlich, C.: 2006, Space Science Reviews 125, 53

Fröhlich, C.: 2013, Space Science Reviews 176, 237

Fröhlich, C. and Lean, J.: 1997, in Correlated Phenomena at the Sun, in the Heliosphere and in Geospace, Vol. 415, p. 227

Fröhlich, C. and Lean, J.: 2004, Astronomy and Astrophysics Review 12, 273

Fuller, N., Aboudarham, J., and Bentley, R. D.: 2005, Solar Physics 227, 61

García, R. A., Turck-Chièze, S., Jiménez-Reyes, S. J., Ballot, J., Pallé, P. L., Eff-Darwich, A., Mathur, S., and Provost, J.: 2007, Science 316, 1591

Garcia, A. and Mouradian, Z.: 1998, Solar Physics 180, 495

Garcia, A., Sobotka, M., Klvana, M., and Bumba, V.: 2011, Contributions of the Astronomical Observatory Skalnate Pleso 41, 69

Gardner, I. C.: 1947, Validity of the cosine-fourth-power law of illumination, National Bureau of Standards

Gary, G. A.: 2001, Solar Physics 203, 71

Gemma Frisius, Phalesius, P. L., and De Bonte, G. A.: 1545, Gemmae Frisii medici et mathematici De radio astronomico $\mathcal{E}$ geometrico liber, in quo multa quae ad geographiam, opticam, geometriam $\mathcal{E}$ astromiam utiliss. sunt, demonstrantur., apud Greg. Bontium, \& Lovanii : apud Petrum Phalesium, Antverpiae, OCLC: 68848378

Georgieva, K., Kilcik, A., Nagovitsyn, Y., and Kirov, B.: 2016, in Solar and solarterrestrial Physics, Vol. 1701, pp 61-66

Georgieva, K., Nagovitsyn, Y., and Kirov, B.: 2015, Geomagnetism and Aeronomy 55, 1026

Giorgi, F., Ermolli, I., Centrone, M., and Marchei, E.: 2005, Memorie della Societa Astronomica Italiana 76, 977

Giovanelli, R. G. and Jones, H. P.: 1982, Solar Physics 79, 267

Gleissberg, W.: 1939, The Observatory 62, 158

Gloeckler, G. and Fisk, L. A.: 2015, The Astrophysical Journal Letters 806, L27

Gnevyshev, M. N.: 1938, Mitteilungen der Nikolai-Hauptsternwarte zu Pulkowo 16, B36 
Gokhale, M. H. and Zwaan, C.: 1972, Solar Physics 26, 52

Goldman, D. and Chen, J.-H.: 2005, in Tenth IEEE International Conference on Computer Vision, 2005. ICCV 2005, Vol. 1, pp 899-906 Vol. 1

Golovko, A. A., Golubeva, E. M., Grechnev, V. V., Myachin, D. Y., Trifonov, V. D., and Khlystova, A. I.: 2002, in Solar Variability: From Core to Outer Frontiers, Vol. 506, pp 929-932, ESA Publications Division

Gondoin, P., Gandolfi, D., Fridlund, M., Frasca, A., Guenther, E. W., Hatzes, A., Deeg, H. J., Parviainen, H., Eigmüller, P., and Deleuil, M.: 2012, Astronomy and Astrophysics 548, A15

Goslar, T., Arnold, M., Tisnerat-Laborde, N., Czernik, J., and Wi $\backslash$ c $\{$ e $\}$ ckowski, K.: 2000, Nature 403, 877

Gough, D. O.: 1981, Solar Physics 74, 21

Gray, L. J., Beer, J., Geller, M., Haigh, J. D., Lockwood, M., Matthes, K., Cubasch, U., Fleitmann, D., Harrison, G., Hood, L., Luterbacher, J., Meehl, G. A., Shindell, D., van Geel, B., and White, W.: 2010, Reviews of Geophysics 48, 4001

Grevesse, N. and Sauval, A. J.: 2002, Advances in Space Research 30, 3

Grossmann-Doerth, U., Knölker, M., Schüssler, M., and Solanki, S. K.: 1994, Astronomy and Astrophysics 285, 648

Haberreiter, M., Delouille, V., Mampaey, B., Verbeeck, C., Del Zanna, G., and Wieman, S.: 2014, Journal of Space Weather and Space Climate 4, A30

Haberreiter, M., Schmutz, W., and Hubeny, I.: 2008, Astronomy and Astrophysics 492, 833

Hagenaar, H. J., DeRosa, M. L., and Schrijver, C. J.: 2008, The Astrophysical Journal 678, 541

Hagenaar, H. J., Schrijver, C. J., and Title, A. M.: 1997, The Astrophysical Journal 481, 988

Haigh, J. D.: 1994, Nature 370, 544

Haigh, J. D.: 1996, Science 272, 981

Haigh, J. D.: 1999, Journal of Atmospheric and Solar-Terrestrial Physics 61, 63

Haigh, J. D.: 2003, Philosophical Transactions of the Royal Society of London Series A 361, 95

Haigh, J. D.: 2007, Living Reviews in Solar Physics 4, 2

Hale, G. E.: 1893, Memorie della Societa Degli Spettroscopisti Italiani 21, 68 
Hale, G. E. and Ellerman, F.: 1903, Publications of the Yerkes Observatory 3, I.1

Hale, G. E., Ellerman, F., Nicholson, S. B., and Joy, A. H.: 1919, The Astrophysical Journal 49, 153

Hall, J. C.: 2008, Living Reviews in Solar Physics 5

Hall, J. C., Lockwood, G. W., and Skiff, B. A.: 2007, The Astronomical Journal 133, 862

Hanaoka, Y.: 2013, Journal of Physics Conference Series 440, 2041

Hardy, R.: 1991, Journal of the British Astronomical Association 101, 261

Hartley, D. E., Villarin, J. T., Black, R. X., and Davis, C. A.: 1998, Nature 391, 471

Harvey, K. and Harvey, J.: 1973, Solar Physics 28, 61

Harvey, K. L. and Martin, S. F.: 1973, Solar Physics 32, 389

Harvey, K. L. and Recely, F.: 1984, Solar Physics 91, 127

Harvey, K. L. and White, O. R.: 1999, The Astrophysical Journal 515, 812

Hathaway, D. H.: 2015, Living Reviews in Solar Physics 12

Hathaway, D. H. and Choudhary, D. P.: 2008, Solar Physics 250, 269

Hathaway, D. H. and Harvey, K. L.: 1990, in Bulletin of the American Astronomical Society, Vol. 22, p. 873

Hathaway, D. H., Upton, L., and Colegrove, O.: 2013, Science 342, 1217

Hathaway, D. H., Wilson, R. M., and Reichmann, E. J.: 1994, Solar Physics 151, 177

Heath, D. F. and Schlesinger, B. M.: 1986, Journal of Geophysical Research 91, 8672

Hennessey, J. J.: 1969, Solar Physics 9, 496

Herschel, W.: 1801, Philosophical Transactions of the Royal Society of London 91, 265

Hickey, J. R., Stowe, L. L., Jacobowitz, H., Pellegrino, P., Maschhoff, R. H., House, F., and Vonder Haar, T. H.: 1980, Science 208, 281

Hirtenfellner-Polanec, W., Temmer, M., Pötzi, W., Freislich, H., Veronig, A. M., and Hanslmeier, A.: 2011, Central European Astrophysical Bulletin 35, 205

Hoffmann, D. L., Beck, J. W., Richards, D. A., Smart, P. L., Singarayer, J. S., Ketchmark, T., and Hawkesworth, C. J.: 2010, Earth and Planetary Science Letters 289(1-2), 1

Howard, R.: 1959, The Astrophysical Journal 130, 193

Howard, R., Boyden, J. E., Bruning, D. H., Clark, M. K., Crist, H. W., and Labonte, B. J.: 1983, Solar Physics 87, 195 
Howard, R. F.: 1992, Solar Physics 137, 51

Howe, R.: 2009, Living Reviews in Solar Physics 6, 1

Hoyt, D. V. and Schatten, K. H.: 1998, Solar Physics 179, 189

Hua, Q., Barbetti, M., Fink, D., Kaiser, K. F., Friedrich, M., Kromer, B., Levchenko, V. A., Zoppi, U., Smith, A. M., and Bertuch, F.: 2009, Quaternary Science Reviews 28, 2982

Hubrecht, J. B.: 1912, Monthly Notices of the Royal Astronomical Society 73, 5

Hudson, H. S., Silva, S., Woodard, M., and Willson, R. C.: 1982, Solar Physics 76, 211

Hurter, F. and Driffield, V. C.: 1890, Journal of the Society of Chemical Industry 9(5), 455

Jackman, C. H., Marsh, D. R., Vitt, F. M., Garcia, R. R., Fleming, E. L., Labow, G. J., Randall, C. E., López-Puertas, M., Funke, B., von Clarmann, T., and Stiller, G. P.: 2008, Atmos. Chem. Phys. 8(3), 765

James, T. H. and Higgins, G. C.: 1960, Fundamentals of Photographic Theory, Morgan \& Morgan

Jiang, J., Cameron, R. H., Schmitt, D., and Schüssler, M.: 2011, Astronomy and Astrophysics 528, A82

Johannesson, A., Marquette, W. H., and Zirin, H.: 1998, Solar Physics 177, 265

Jung, J., Oh, S., Yi, Y., Evenson, P., Pyle, R., Jee, G., Kim, J.-H., Lee, C., and Sohn, J.: 2016, Journal of Astronomy and Space Sciences 33, 345

Kahil, F., Riethmüller, T. L., and Solanki, S. K.: 2017, The Astrophysical Journal Supplement Series 229(1), 12

Kariyappa, R. and Pap, J. M.: 1996, Solar Physics 167, 115

Kariyappa, R. and Sivaraman, K. R.: 1994, Solar Physics 152, 139

Karoff, C., Inceoglu, F., Knudsen, M. F., Olsen, J., and Fogtmann-Schulz, A.: 2015, Astronomy and Astrophysics 575, A77

Keil, S. L., Balasubramaniam, K. S., Smaldone, L. A., and Reger, B.: 1999, The Astrophysical Journal 510, 422

Keil, S. L., Henry, T. W., and Fleck, B.: 1998, in K. S. Balasubramaniam, J. Harvey, and D. Rabin (eds.), Synoptic Solar Physics, Vol. 140 of Astronomical Society of the Pacific Conference Series, p. 301

Keil, S. L. and Worden, S. P.: 1984, The Astrophysical Journal 276, 766

Kiepenheuer, K. O.: 1969, Mitteilungen der Astronomischen Gesellschaft Hamburg 26, 42 
Kiepenheuer, K. O.: 1974, Mitteilungen der Astronomischen Gesellschaft Hamburg 35, 60

Kilcik, A., Yurchyshyn, V. B., Abramenko, V., Goode, P. R., Ozguc, A., Rozelot, J. P., and Cao, W.: 2011, The Astrophysical Journal 731, 30

Kirkby, J., Curtius, J., Almeida, J., Dunne, E., Duplissy, J., Ehrhart, S., Franchin, A., Gagné, S., Ickes, L., Kürten, A., Kupc, A., Metzger, A., Riccobono, F., Rondo, L., Schobesberger, S., Tsagkogeorgas, G., Wimmer, D., Amorim, A., Bianchi, F., Breitenlechner, M., David, A., Dommen, J., Downard, A., Ehn, M., Flagan, R. C., Haider, S., Hansel, A., Hauser, D., Jud, W., Junninen, H., Kreissl, F., Kvashin, A., Laaksonen, A., Lehtipalo, K., Lima, J., Lovejoy, E. R., Makhmutov, V., Mathot, S., Mikkilä, J., Minginette, P., Mogo, S., Nieminen, T., Onnela, A., Pereira, P., Petäjä, T., Schnitzhofer, R., Seinfeld, J. H., Sipilä, M., Stozhkov, Y., Stratmann, F., Tomé, A., Vanhanen, J., Viisanen, Y., Vrtala, A., Wagner, P. E., Walther, H., Weingartner, E., Wex, H., Winkler, P. M., Carslaw, K. S., Worsnop, D. R., Baltensperger, U., and Kulmala, M.: 2011, Nature 476, 429

Kitagawa, H. and Plicht, J. v. d.: 2006, Radiocarbon 42(3), 369

Kitai, R., Ueno, S., Maehara, H., Shirakawa, S., Katoda, M., Hada, Y., Tomita, Y., Hayashi, H., Asai, A., Isobe, H., Goto, H., and Yamashita, S.: 2013, Data Science Journal 12(0), WDS213

Knölker, M. and Schüssler, M.: 1988, Astronomy and Astrophysics 202, 275

Kodera, K. and Kuroda, Y.: 2002, Journal of Geophysical Research (Atmospheres) 107, 4749

Koechlin, L.: 2015, in SF2A-2015: Proceedings of the Annual meeting of the French Society of Astronomy and Astrophysics, pp 21-24

Kontogiannis, I., Belehaki, A., Tsiropoula, G., Tsagouri, I., Anastasiadis, A., and Papaioannou, A.: 2016, Advances in Space Research 57, 418

Kopp, G.: 2014, Journal of Space Weather and Space Climate 4, A14

Kopp, G.: 2016, Journal of Space Weather and Space Climate 6, A30

Kopp, G., Krivova, N., Wu, C. J., and Lean, J.: 2016, Solar Physics 291(9-10), 2951

Kopp, G., Lawrence, G., and Rottman, G.: 2005, in G. Rottman, T. Woods, and V. George (eds.), The Solar Radiation and Climate Experiment (SORCE), pp 129-139, Springer New York

Kopp, G. and Lean, J. L.: 2011, Geophysical Research Letters 38, L01706

Kopp, G. and Rabin, D.: 1992, Solar Physics 141, 253

Krimigis, S. M., Decker, R. B., Roelof, E. C., Hill, M. E., Armstrong, T. P., Gloeckler, G., Hamilton, D. C., and Lanzerotti, L. J.: 2013, Science 341, 144 
Krivova, N. A., Balmaceda, L., and Solanki, S. K.: 2007, Astronomy and Astrophysics $\mathbf{4 6 7}, 335$

Krivova, N. A. and Solanki, S. K.: 2002, Astronomy and Astrophysics 394, 701

Krivova, N. A. and Solanki, S. K.: 2004a, Astronomy and Astrophysics 417, 1125

Krivova, N. A. and Solanki, S. K.: 2004b, Advances in Space Research 34, 361

Krivova, N. A., Solanki, S. K., and Beer, J.: 2002, Astronomy and Astrophysics 396, 235

Krivova, N. A., Solanki, S. K., Fligge, M., and Unruh, Y. C.: 2003, Astronomy and Astrophysics 399, L1

Krivova, N. A., Solanki, S. K., and Floyd, L.: 2006, Astronomy and Astrophysics 452, 631

Krivova, N. A., Solanki, S. K., and Schmutz, W.: 2011, Astronomy and Astrophysics 529, 81

Krivova, N. A., Solanki, S. K., Wenzler, T., and Podlipnik, B.: 2009, Journal of Geophysical Research (Atmospheres) 114

Krivova, N. A., Vieira, L. E. A., and Solanki, S. K.: 2010, Journal of Geophysical Research (Space Physics) 115, 12112

Kulmala, M., Riipinen, I., Nieminen, T., Hulkkonen, M., Sogacheva, L., Manninen, H. E., Paasonen, P., Petäjä, T., Dal Maso, M., Aalto, P. P., Viljanen, A., Usoskin, I., Vainio, R., Mirme, S., Mirme, A., Minikin, A., Petzold, A., Hõrrak, U., Plaß-Dülmer, C., Birmili, W., and Kerminen, V.-M.: 2010, Atmos. Chem. Phys. 10(4), 1885

Laken, B., Wolfendale, A., and Kniveton, D.: 2009, Geophysical Research Letters 36(23), L23803

Laken, B. A. and Čalogović, J.: 2013, Journal of Space Weather and Space Climate 3, A29

Laken, B. A., Pallé, E., Čalogović, J., and Dunne, E. M.: 2012, Journal of Space Weather and Space Climate 2, A18

Lal, D. and Peters, B.: 1967, Handbuch der Physik 46, 551

Langley, S. and Abbot, C.: 1900, Annals of the Astrophysical Observatory of the Smithsonian Institution 1, 7

Larkin, A., Haigh, J. D., and Djavidnia, S.: 2000, Space Science Reviews 94, 199

Le Mouël, J.-L., Lopes, F., and Courtillot, V.: 2017, Solar Physics 292, \#43

Lean, J.: 2000, Geophysical Research Letters 27, 2425

Lean, J., Beer, J., and Bradley, R.: 1995, Geophysical Research Letters 22, 3195 
Lean, J. and Foukal, P.: 1988, Science 240, 906

Lean, J. L. and Brueckner, G. E.: 1989, The Astrophysical Journal 337, 568

Lee, C. O., Luhmann, J. G., Hoeksema, J. T., Sun, X., Arge, C. N., and Pater, I. d.: 2011, Solar Physics 269(2), 367

Lefebvre, S., Ulrich, R. K., Webster, L. S., Varadi, F., Javaraiah, J., Bertello, L., Werden, L., Boyden, J. E., and Gilman, P.: 2005, Memorie della Societa Astronomica Italiana 76, 862

Legrand, J.-P.: 1987, L’Astronomie 101, 5

Leighton, R. B.: 1959, The Astrophysical Journal 130, 366

Lemaire, J. F. and Stegen, K.: 2016, Solar Physics 291, 3659

Leussu, R., Usoskin, I. G., Arlt, R., and Mursula, K.: 2013, Astronomy and Astrophysics 559, A28

Leussu, R., Usoskin, I. G., Pavai, V. S., Diercke, A., Arlt, R., Denker, C., and Mursula, K.: 2017, Astronomy $\mathcal{E}$ Astrophysics 599, A131

Lites, B. W., Centeno, R., and McIntosh, S. W.: 2014, Publications of the Astronomical Society of Japan 66, S4

Litvinenko, Y. E. and Wheatland, M. S.: 2015, The Astrophysical Journal 800, 130

Liu, S.-Y.: 1974, The Astrophysical Journal 189, 359

Livingston, W. and Sheeley, Jr., N. R.: 2008, The Astrophysical Journal 672, 1228

Livingston, W. and Wallace, L.: 2003, Solar Physics 212, 227

Livingston, W., Wallace, L., White, O. R., and Giampapa, M. S.: 2007, The Astrophysical Journal 657, 1137

Livingston, W. C., Harvey, J., Pierce, A. K., Schrage, D., Gillespie, B., Simmons, J., and Slaughter, C.: 1976, Applied Optics 15, 33

Lockwood, G. W., Henry, G. W., Hall, J. C., and Radick, R. R.: 2013, in Chavez (ed.), New Quests in Stellar Astrophysics III: A Panchromatic View of Solar-Like Stars, With and Without Planets, Vol. 472 of Astronomical Society of the Pacific Conference Series, p. 203

Lockwood, G. W., Skiff, B. A., Henry, G. W., Henry, S., Radick, R. R., Baliunas, S. L., Donahue, R. A., and Soon, W.: 2007, The Astrophysical Journal Supplement Series 171, 260

Lockwood, M.: 2001, Journal of Geophysical Research 106, 16021

Lockwood, M., Owens, M. J., and Barnard, L.: 2014, Journal of Geophysical Research (Space Physics) 119, 5172 
Lockwood, M., Owens, M. J., and Barnard, L.: 2016a, Solar Physics 291(9-10), 2843

Lockwood, M., Owens, M. J., Barnard, L., Scott, C. J., Usoskin, I. G., and Nevanlinna, H.: 2016b, Solar Physics 291(9-10), 2811

Lockwood, M., Owens, M. J., Barnard, L., and Usoskin, I. G.: 2016c, The Astrophysical Journal 824, 54

Lockwood, M., Owens, M. J., Barnard, L., and Usoskin, I. G.: 2016d, Solar Physics 291(9-10), 2829

Lockwood, M., Scott, C. J., Owens, M. J., Barnard, L., and Willis, D. M.: 2016e, Solar Physics 291(9-10), 2785

Lockyer, W. J. S.: 1909, Monthly Notices of the Royal Astronomical Society 70, 14

Lodders, K.: 2003, The Astrophysical Journal 591, 1220

Loughhead, R. E. and Bray, R. J.: 1958, Australian Journal of Physics 11, 177

Loukitcheva, M., Solanki, S. K., and White, S. M.: 2009, Astronomy and Astrophysics 497, 273

Luthardt, L. and Rößler, R.: 2017, Geology p. G38669.1

Maher, B. A.: 2016, Quaternary Science Reviews 154

Makarov, V. I., Tlatov, A. G., and Callebaut, D. K.: 2004, Proceedings of the International Astronomical Union 2004(IAUS223), 49

Marsh, N. D. and Svensmark, H.: 2000, Physical Review Letters 85, 5004

Martinez Pillet, V.: 1997, in B. Schmieder, J. C. Del Toro Iniesta, and M. Vazquez (eds.), 1st Advances in Solar Physics Euroconference. Advances in Physics of Sunspots, Vol. 118 of Astronomical Society of the Pacific Conference Series, p. 212

Martinez Pillet, V., Moreno-Insertis, F., and Vazquez, M.: 1993, Astronomy and Astrophysics 274, 521

Martínez Pillet, V., Lites, B. W., and Skumanich, A.: 1997, The Astrophysical Journal 474, 810

Mathew, S. K., Solanki, S. K., Lagg, A., Collados, M., Borrero, J. M., and Berdyugina, S.: 2004, Astronomy and Astrophysics 422, 693

Matthes, K., Kuroda, Y., Kodera, K., and Langematz, U.: 2006, Journal of Geophysical Research (Atmospheres) 111, D06108

Mattig, W. and Schroeter, E. H.: 1977, Mitteilungen der Astronomischen Gesellschaft Hamburg 41, 81

Mavromichalaki, H., Gerontidou, M., Paschalis, P., and Paouris, E.: 2016, ArXiv e-prints 1612, arXiv:1612.08343 
Mayaud, P. N.: 1973, A Hundred Year Series of Geomagnetic Data, 1868-1967: Indices Aa, Storm Sudden Commencements, IUGG Publications Office

McIntosh, P. S.: 1981, in L. E. Cram and J. H. Thomas (eds.), The Physics of Sunspots, pp 7-54

McIntosh, P. S.: 1990, Solar Physics 125, 251

McIntosh, S. W., Leamon, R. J., Hock, R. A., Rast, M. P., and Ulrich, R. K.: 2011, The Astrophysical Journal Letters 730, L3

Meehl, G. A. and Arblaster, J. M.: 2009, Journal of Climate 22(13), 3647

Meehl, G. A., Arblaster, J. M., Branstator, G., and Loon, H. v.: 2008, Journal of Climate 21(12), 2883

Meehl, G. A., Washington, W. M., Wigley, T. M. L., Arblaster, J. M., and Dai, A.: 2003, Journal of Climate 16(3), 426

Mees, C. E. K.: 1942, The Theory of the Photographic Process, The Macmillan company, New York, NY, 1st edition

Mein, P. and Ribes, E.: 1990, Astronomy and Astrophysics 227, 577

Meunier, N., Roudier, T., and Rieutord, M.: 2008, Astronomy and Astrophysics 488, 1109

Michel, E., Baglin, A., Weiss, W. W., Auvergne, M., Catala, C., Aerts, C., Appourchaux, T., Barban, C., Baudin, F., Briquet, M.-L., Carrier, F., Debosher, J., De Ridder, J., Garcia, R. A., Garrido, R., Gutierrez, J., Kallinger, T., Lefevre, L., Neiner, C., Poretti, E., Samadi, R., Sarro, L., and COROT Team: 2008, Communications in Asteroseismology 157, 69

Mickaelian, A. M., Nesci, R., Rossi, C., Weedman, D., Cirimele, G., Sargsyan, L. A., Erastova, L. K., Gigoyan, K. S., Mikayelyan, G. A., Massaro, E., Gaudenzi, S., Houck, J., Barry, D., D’Amante, L., and Germano, P.: 2007, Astronomy and Astrophysics 464, 1177

Miesch, M. S., Brun, A. S., DeRosa, M. L., and Toomre, J.: 2008, The Astrophysical Journal 673, 557

Mironova, I. A., Aplin, K. L., Arnold, F., Bazilevskaya, G. A., Harrison, R. G., Krivolutsky, A. A., Nicoll, K. A., Rozanov, E. V., Turunen, E., and Usoskin, I. G.: 2015, Space Science Reviews 194, 1

Mitalas, R. and Sills, K. R.: 1992, The Astrophysical Journal 401, 759

Münzer, H., Hanslmeier, A., Schröter, E. H., and Wöhl, H.: 1989, in R. J. Rutten and G. Severino (eds.), Solar and Stellar Granulation, No. 263 in NATO ASI Series, pp 217-218, Springer Netherlands, DOI: 10.1007/978-94-009-0911-3_25

Mohler, O. C. and Dodson, H. W.: 1968, Solar Physics 5, 417 
Moraal, H. and Stoker, P. H.: 2010, Journal of Geophysical Research (Space Physics) 115, A12109

Moran, T., Foukal, P., and Rabin, D.: 1992, Solar Physics 142, 35

Moreno-Insertis, F. and Vazquez, M.: 1988, Astronomy and Astrophysics 205, 289

Morita, S., Shibata, K., Ueno, S., Ichimoto, K., Kitai, R., and Otsuji, K.-I.: 2010, Publications of the Astronomical Society of Japan 62, 901

Moss, W.: 1942, Monthly Notices of the Royal Astronomical Society 102, 86

Muñoz-Jaramillo, A., Sheeley, N. R., Zhang, J., and DeLuca, E. E.: 2012, The Astrophysical Journal 753, 146

Myhre, G., Shindell, D., Bréon, F.-M., Collins, W., Fuglestvedt, J., Huang, J., Koch, D., Lamarque, J.-F., Lee, D., Mendoza, B., Nakajima, T., Robock, A., Stephens, G., Takemura, T., and Zhang, H.: 2013, in T. Stocker, D. Qin, G.-K. Plattner, M. Tignor, S. Allen, J. Boschung, A. Nauels, Y. Xia, V. Bex, and P. Midgley (eds.), Climate Change 2013: The Physical Science Basis. Contribution of Working Group I to the Fifth Assessment Report of the Intergovernmental Panel on Climate Change, pp 659740, Cambridge University Press, Cambridge, United Kingdom and New York, NY, USA

Nagovitsyn, Y. A.: 1997, Astronomy Letters 23, 742

Nagovitsyn, Y. A., Pevtsov, A. A., and Livingston, W. C.: 2012, The Astrophysical Journal Letters 758, L20

Nagovitsyn, Y. A., Tlatov, A. G., and Nagovitsyna, E. Y.: 2016, Astronomy Reports 60(9), 831

Naqvi, M. F., Marquette, W. H., Tritschler, A., and Denker, C.: 2010, Astronomische Nachrichten 331, 696

Nesme-Ribes, E., Meunier, N., and Collin, B.: 1996, Astronomy and Astrophysics 308, 213

Neuhäuser, R., Arlt, R., Pfitzner, E., and Richter, S.: 2015, Astronomische Nachrichten 336, 623

Neuhäuser, R. and Neuhäuser, D. L.: 2016, Astronomische Nachrichten 337, 581

Ney, E. P.: 1959, Nature 183, 451

Nindos, A. and Zirin, H.: 1998, Solar Physics 179, 253

Notes from Mt Wilson observatory: 1947, Publications of the Astronomical Society of the Pacific 59(346), 36

Obridko, V. N. and Badalyan, O. G.: 2014, Astronomy Reports 58, 936 
Ogurtsov, M. G., Nagovitsyn, Y. A., Kocharov, G. E., and Jungner, H.: 2002, Solar Physics 211, 371

Oliver, R., Ballester, J. L., and Baudin, F.: 1998, Nature 394, 552

Ortiz, A. and Rast, M.: 2005, Memorie della Societa Astronomica Italiana 76, 1018

Oster, L., Schatten, K. H., and Sofia, S.: 1982, The Astrophysical Journal 256, 768

Owens, M. J. and Forsyth, R. J.: 2013, Living Reviews in Solar Physics 10, 5

Owens, M. J., McCracken, K. G., Lockwood, M., and Barnard, L.: 2015, Journal of Space Weather and Space Climate 5, A30

Paillard, D.: 2001, Reviews of Geophysics 39, 325

Parenti, S., Bromage, B. J. I., Poletto, G., Noci, G., Raymond, J. C., and Bromage, G. E.: 2000, Astronomy and Astrophysics 363, 800

Parker, E. N.: 1955, The Astrophysical Journal 122, 293

Parker, E. N.: 1958, The Astrophysical Journal 128, 664

Patterson, C.: 1956, Geochimica et Cosmochimica Acta 10, 230

Peck, C. L. and Rast, M. P.: 2015, The Astrophysical Journal 808, 192

Penza, V., Caccin, B., Ermolli, I., Centrone, M., and Gomez, M. T.: 2003, in Solar Variability as an Input to the Earth's Environment, Vol. 535 of ESA Special Publication, pp 299-302

Pesnell, W. D., Thompson, B. J., and Chamberlin, P. C.: 2012, Solar Physics 275, 3

Petrovay, K. and van Driel-Gesztelyi, L.: 1997, Solar Physics 176, 249

Pevtsov, A. A., Virtanen, I., Mursula, K., Tlatov, A., and Bertello, L.: 2016, Astronomy and Astrophysics 585, A40

Pierce, A. K. and Slaughter, C. D.: 1977, Solar Physics 51, 25

Pierce, A. K., Slaughter, C. D., and Weinberger, D.: 1977, Solar Physics 52, 179

Pierce, J. R. and Adams, P. J.: 2009, Geophysical Research Letters 36, L09820

Pilcher, J. R., Baillie, M. G. L., Schmidt, B., and Becker, B.: 1984, Nature 312(5990), 150

Potgieter, M. S.: 2013, Living Reviews in Solar Physics 10, 3

Pouillet, C.-S.-M. .-. A. d. t.: 1838, Mémoire sur la chaleur solaire: sur les pouvoirs rayonnants et absorbants de l'air atmosphérique et sur la température de l'espace, Bachelier, Paris 
Preminger, D. G., Walton, S. R., and Chapman, G. A.: 2001, Solar Physics 202, 53

Preminger, D. G., Walton, S. R., and Chapman, G. A.: 2002, Journal of Geophysical Research (Space Physics) 107, 1354

Priest, E. R.: 2003, Solar magnetohydrodynamics, Vol. 21 of Geophysics and Astrophysics monographs, Springer Netherlands

Priyal, M., Banerjee, D., Karak, B. B., Muñoz-Jaramillo, A., Ravindra, B., Choudhuri, A. R., and Singh, J.: 2014, The Astrophysical Journal Letters 793, L4

Priyal, M., Singh, J., Ravindra, B., Priya, T. G., and Amareswari, K.: 2013, Solar Physics

Pötzi, W.: 2008, Central European Astrophysical Bulletin 32, 9

Pulkkinen, T. I., Nevanlinna, H., Pulkkinen, P. J., and Lockwood, M.: 2001, Space Science Reviews 95, 625

Rabin, D.: 1992, The Astrophysical Journal 391, 832

Radick, R. R., Lockwood, G. W., and Baliunas, S. L.: 1990, Science 247, 39

Rast, M. P.: 2003a, The Astrophysical Journal 597, 1200

Rast, M. P.: 2003b, in GONG+ 2002. Local and Global Helioseismology: the Present and Future, Vol. 517 of ESA Special Publication, pp 163-172

Rast, M. P., Fox, P. A., Lin, H., Lites, B. W., Meisner, R. W., and White, O. R.: 1999, Nature 401, 678

Rast, M. P., Ortiz, A., and Meisner, R. W.: 2008, The Astrophysical Journal 673, 1209

Ravindra, B., Priya, T. G., Amareswari, K., Priyal, M., Nazia, A. A., and Banerjee, D.: 2013, Astronomy and Astrophysics 550, 19

Reames, D. V.: 2013, Space Science Reviews 175, 53

Rüedi, I., Solanki, S. K., Livingston, W., and Stenflo, J. O.: 1992, Astronomy and Astrophysics 263, 323

Reimer, P. J., Bard, E., Bayliss, A., Beck, J. W., Blackwell, P. G., Ramsey, C. B., Buck, C. E., Cheng, H., Edwards, R. L., Friedrich, M., Grootes, P. M., Guilderson, T. P., Haflidason, H., Hajdas, I., Hatté, C., Heaton, T. J., Hoffmann, D. L., Hogg, A. G., Hughen, K. A., Kaiser, K. F., Kromer, B., Manning, S. W., Niu, M., Reimer, R. W., Richards, D. A., Scott, E. M., Southon, J. R., Staff, R. A., Turney, C. S. M., and Plicht, J. v. d.: 2013, Radiocarbon 55(4), 1869

Reiners, A.: 2012, Living Reviews in Solar Physics 9

Rempel, M., Schüssler, M., and Tóth, G.: 2000, Astronomy and Astrophysics 363, 789

Rezaei, R., Schlichenmaier, R., Beck, C. A. R., Bruls, J. H. M. J., and Schmidt, W.: 2007, Astronomy and Astrophysics 466, 1131 
Rigozo, N. R., Echer, E., Vieira, L. E. A., and Nordemann, D. J. R.: 2001, Solar Physics 203, 179

Roudier, T. and Muller, R.: 1987, Solar Physics 107, 11

Roy, I. and Haigh, J. D.: 2012, Journal of the Atmospheric Sciences 69(4), 1446

Saarni, S., Muschitiello, F., Weege, S., Brauer, A., and Saarinen, T.: 2016, Quaternary Science Reviews 154

Sarychev, A. P. and Roshchina, E. M.: 2009, Solar System Research 43, 151

Scherrer, P. H., Bogart, R. S., Bush, R. I., Hoeksema, J. T., Kosovichev, A. G., Schou, J., Rosenberg, W., Springer, L., Tarbell, T. D., Title, A., Wolfson, C. J., Zayer, I., and MDI Engineering Team: 1995, Solar Physics 162, 129

Scherrer, P. H., Schou, J., Bush, R. I., Kosovichev, A. G., Bogart, R. S., Hoeksema, J. T., Liu, Y., Duvall, T. L., Zhao, J., Title, A. M., Schrijver, C. J., Tarbell, T. D., and Tomczyk, S.: 2012, Solar Physics 275, 207

Schmutz, W., Fehlmann, A., Finsterle, W., Kopp, G., and Thuillier, G.: 2013, in American Institute of Physics Conference Series, Vol. 1531, pp 624-627

Schou, J., Antia, H. M., Basu, S., Bogart, R. S., Bush, R. I., Chitre, S. M., ChristensenDalsgaard, J., Di Mauro, M. P., Dziembowski, W. A., Eff-Darwich, A., Gough, D. O., Haber, D. A., Hoeksema, J. T., Howe, R., Korzennik, S. G., Kosovichev, A. G., Larsen, R. M., Pijpers, F. P., Scherrer, P. H., Sekii, T., Tarbell, T. D., Title, A. M., Thompson, M. J., and Toomre, J.: 1998, The Astrophysical Journal 505, 390

Schou, J., Borrero, J. M., Norton, A. A., Tomczyk, S., Elmore, D., and Card, G. L.: 2012a, Solar Physics 275, 327

Schou, J., Kosovichev, A. G., Goode, P. R., and Dziembowski, W. A.: 1997, The Astrophysical Journal Letters 489, L197

Schou, J., Scherrer, P. H., Bush, R. I., Wachter, R., Couvidat, S., Rabello-Soares, M. C., Bogart, R. S., Hoeksema, J. T., Liu, Y., Duvall, T. L., Akin, D. J., Allard, B. A., Miles, J. W., Rairden, R., Shine, R. A., Tarbell, T. D., Title, A. M., Wolfson, C. J., Elmore, D. F., Norton, A. A., and Tomczyk, S.: 2012b, Solar Physics 275, 229

Schove, D. J.: 1955, Journal of Geophysical Research 60, 127

Schove, D. J.: 1979, Solar Physics 63, 423

Schröder, W.: 1992, Journal of geomagnetism and geoelectricity 44(2), 119

Schrijver, C. J., Cote, J., Zwaan, C., and Saar, S. H.: 1989, The Astrophysical Journal 337, 964

Schrijver, C. J., Hagenaar, H. J., and Title, A. M.: 1997, The Astrophysical Journal 475, 328 
Schrijver, C. J., Livingston, W. C., Woods, T. N., and Mewaldt, R. A.: 2011, Geophysical Research Letters 38, 6701

Schwabe, M.: 1844, Astronomische Nachrichten 21, 233

Seleznyov, A. D., Solanki, S. K., and Krivova, N. A.: 2011, Astronomy and Astrophysics 532, A 108

Shapiro, A. I., Schmutz, W., Rozanov, E., Schoell, M., Haberreiter, M., Shapiro, A. V., and Nyeki, S.: 2011, Astronomy and Astrophysics 529, 67

Shapiro, A. I., Solanki, S. K., Krivova, N. A., Schmutz, W. K., Ball, W. T., Knaack, R., Rozanov, E. V., and Unruh, Y. C.: 2014, Astronomy and Astrophysics 569, A38

Shapley, A. H.: 1946, Popular Astronomy 54, 351

Shaviv, N. J.: 2002, Physical Review Letters 89, 051102

Sheeley, N. R.: 1967, The Astrophysical Journal 147, 1106

Sheeley, Jr., N. R.: 2008, The Astrophysical Journal 680, 1553

Sheeley, Jr., N. R., Cooper, T. J., and Anderson, J. R. L.: 2011, The Astrophysical Journal 730, 51

Sheeley, Jr., N. R., Stauffer, J. R., Thomassie, J. C., and Warren, H. P.: 2017, The Astrophysical Journal 836, 144

Shindell, D., Faluvegi, G., Lacis, A., Hansen, J., Ruedy, R., and Aguilar, E.: 2006, Journal of Geophysical Research (Atmospheres) 111, D08302

Shindell, D., Rind, D., Balachandran, N., Lean, J., and Lonergan, P.: 1999, Science 284, 305

Simon, G. W. and Weiss, N. O.: 1968, The Astronomical Journal Supplement 73, 76

Singh, J., Belur, R., Raju, S., Pichaimani, K., Priyal, M., Gopalan Priya, T., and Kotikalapudi, A.: 2012, Research in Astronomy and Astrophysics 12, 472

Singh, J. and Ravindra, B.: 2012, Bulletin of the Astronomical Society of India 40

Siscoe, G. L.: 1980, Reviews of Geophysics and Space Physics 18, 647

Skumanich, A., Lean, J. L., Livingston, W. C., and White, O. R.: 1984, The Astrophysical Journal 282, 776

Skumanich, A., Smythe, C., and Frazier, E. N.: 1975, The Astrophysical Journal 200, 747

Smith, W. J.: 2007, Modern Optical Engineering, McGraw-Hill Education, 4 edition edition 
Snow, M., Weber, M., Machol, J., Viereck, R., and Richard, E.: 2014, Journal of Space Weather and Space Climate 4, A04

Sobotka, M.: 2003, Astronomische Nachrichten 324, 369

Sobotka, M., Vázquez, M., Sánchez Cuberes, M., Bonet, J. A., and Hanslmeier, A.: 2000, The Astrophysical Journal 544, 1155

Sokoloff, D.: 2004, Solar Physics 224, 145

Solanki, S. and Krivova, N.: 2009, Landolt Börnstein 4B, 4123

Solanki, S. K.: 2003, Astronomy and Astrophysics Review 11, 153

Solanki, S. K., Barthol, P., Danilovic, S., Feller, A., Gandorfer, A., Hirzberger, J., Riethmüller, T. L., Schüssler, M., Bonet, J. A., Martínez Pillet, V., del Toro Iniesta, J. C., Domingo, V., Palacios, J., Knölker, M., Bello González, N., Berkefeld, T., Franz, M., Schmidt, W., and Title, A. M.: 2010, The Astrophysical Journal Letters 723, L127

Solanki, S. K., Finsterle, W., Rüedi, I., and Livingston, W.: 1999, Astronomy and Astrophysics 347, L27

Solanki, S. K. and Fligge, M.: 1998, Geophysical Research Letters 25

Solanki, S. K. and Fligge, M.: 1999, Geophysical Research Letters 26

Solanki, S. K. and Fligge, M.: 2002, Journal of Atmospheric and Solar-Terrestrial Physics 64, 677

Solanki, S. K., Krivova, N. A., and Haigh, J. D.: 2013, Annual Review of Astronomy and Astrophysics 51(1), 311

Solanki, S. K., Krivova, N. A., Schüssler, M., and Fligge, M.: 2002, Astronomy and Astrophysics 396, 1029

Solanki, S. K. and Marsch, E.: 2006, in SOHO-17. 10 Years of SOHO and Beyond, Vol. 617 of ESA Special Publication

Solanki, S. K., Montavon, C. A. P., and Livingston, W.: 1994, Astronomy and Astrophysics 283, 221

Solanki, S. K., Riethmüller, T. L., Barthol, P., Danilovic, S., Deutsch, W., Doerr, H.-P., Feller, A., Gandorfer, A., Germerott, D., Gizon, L., Grauf, B., Heerlein, K., Hirzberger, J., Kolleck, M., Lagg, A., Meller, R., Tomasch, G., van Noort, M., Blanco Rodríguez, J., Gasent Blesa, J. L., Balaguer Jiménez, M., Del Toro Iniesta, J. C., López Jiménez, A. C., Orozco Suarez, D., Berkefeld, T., Halbgewachs, C., Schmidt, W., Álvarez Herrero, A., Sabau-Graziati, L., Pérez Grande, I., Martínez Pillet, V., Card, G., Centeno, R., Knölker, M., and Lecinski, A.: 2017, The Astrophysical Journal Supplement Series 229, 2

Solanki, S. K. and Steiner, O.: 1990, Astronomy and Astrophysics 234, 519 
Solanki, S. K., Usoskin, I. G., Kromer, B., Schüssler, M., and Beer, J.: 2004, Nature 431, 1084

Solanki, S. K., Walther, U., and Livingston, W.: 1993, Astronomy and Astrophysics 277, 639

Solomon, S., Crutzen, P. J., and Roble, R. G.: 1982, Journal of Geophysical Research: Oceans 87(C9), 7206

Spearman, C.: 1904, American Journal of Psychology 15, 88

Spurk, M., Friedrich, M., Hofmann, J., Remmele, S., Frenzel, B., Leuschner, H. H., and Kromer, B.: 1998, Radiocarbon 40(3), 1107

Steinegger, M., Brandt, P. N., and Haupt, H. F.: 1996, Astronomy and Astrophysics 310, 635

Steinegger, M., Brandt, P. N., and Schmidt, W.: 1990, in Astronomische Gesellschaft Abstract Series, Vol. 5, p. 42

Steinhilber, F., Abreu, J. A., Beer, J., Brunner, I., Christl, M., Fischer, H., Heikkila, U., Kubik, P. W., Mann, M., McCracken, K. G., Miller, H., Miyahara, H., Oerter, H., and Wilhelms, F.: 2012, Proceedings of the National Academy of Science 109, 5967

Steinhilber, F., Beer, J., and Fröhlich, C.: 2009, Geophysical Research Letters 36, L19704

Stenflo, J. O. and Harvey, J. W.: 1985, Solar Physics 95, 99

Stephenson, F. R.: 1990, Philosophical Transactions of the Royal Society of London Series A 330, 499

Stephenson, F. R., Willis, D. M., and Hallinan, T. J.: 2004, Astronomy and Geophysics 45, 6.15

Stix, M.: 2004, The sun : an introduction, Springer-Verlag Berlin Heidelberg

Stone, E. C., Cummings, A. C., McDonald, F. B., Heikkila, B. C., Lal, N., and Webber, W. R.: 2013, Science 341, 150

Sütterlin, P.: 1998, Astronomy and Astrophysics 333, 305

Sütterlin, P., Wiehr, E., and Stellmacher, G.: 1999, Solar Physics 189, 57

Stuiver, M. and Polach, H. A.: 1977, Radiocarbon 19(3), 355

Suess, H. E.: 1955, Science 122(3166), 415

Suess, H. E.: 1980, Radiocarbon 22(2), 200

Sukhodolov, T., Usoskin, I., Rozanov, E., Asvestari, E., Ball, W. T., Curran, M. A. J., Fischer, H., Kovaltsov, G., Miyake, F., Peter, T., Plummer, C., Schmutz, W., Severi, M., and Traversi, R.: 2017, Scientific Reports 7, 45257 
Suzuki, Y.: 1967, Publications of the Astronomical Society of Japan 19, 220

Svalgaard, L., Cagnotti, M., and Cortesi, S.: 2017, Solar Physics 292(2), 34

Svalgaard, L. and Schatten, K. H.: 2016, Solar Physics 291(9-10), 2653

Svensmark, H. and Friis-Christensen, E.: 1997, Journal of Atmospheric and SolarTerrestrial Physics 59, 1225

Svensmark, J., Enghoff, M. B., Shaviv, N. J., and Svensmark, H.: 2016, Journal of Geophysical Research: Space Physics 121(9), 2016JA022689

Tanaka, H., Castelli, J. P., Covington, A. E., Krüger, A., Landecker, T. L., and Tlamicha, A.: 1973, Solar Physics 29, 243

Tapping, K. F.: 1987, Journal of Geophysical Research 92, 829

Tapping, K. F., Boteler, D., Charbonneau, P., Crouch, A., Manson, A., and Paquette, H.: 2007, Solar Physics 246, 309

Tapping, K. F. and Morton, D. C.: 2013, in Journal of Physics Conference Series, Vol. 440

Taylor, P. O.: 1985, Journal of the American Association of Variable Star Observers (JAAVSO) 14, 28

Tebabal, A., Damtie, B., Nigussie, M., Bires, A., and Yizengaw, E.: 2015, Journal of Atmospheric and Solar-Terrestrial Physics 135, 64

Title, A. M., Tarbell, T. D., Topka, K. P., Ferguson, S. H., Shine, R. A., and SOUP Team: 1989, The Astrophysical Journal 336, 475

Tlatov, A. G.: 2012, Astrophysics and Space Science Proceedings 30, 33

Tlatov, A. G., Dormidontov, D. V., Kirpichev, R. V., Pashchenko, M. P., and Shramko, A. D.: 2015, Geomagnetism and Aeronomy 55(7), 961

Tlatov, A. G., Pevtsov, A. A., and Singh, J.: 2009, Solar Physics 255, 239

Todd, M. C. and Kniveton, D. R.: 2001, Journal of Geophysical Research: Atmospheres 106(D23), 32031

Tourpali, K., Schuurmans, C. J. E., van Dorland, R., Steil, B., and Brühl, C.: 2003, Geophysical Research Letters 30, 1231

Travaglini, G.: 2017, Solar Physics 292(1), 23

Turney, C. S. M., Fifield, L. K., Hogg, A. G., Palmer, J. G., Hughen, K., Baillie, M. G. L., Galbraith, R., Ogden, J., Lorrey, A., Tims, S. G., and Jones, R. T.: 2010, Quaternary Science Reviews 29, 3677

Usoskin, I. G.: 2017, Living Reviews in Solar Physics 14(1), 3 
Usoskin, I. G., Arlt, R., Asvestari, E., Hawkins, E., Käpylä, M., Kovaltsov, G. A., Krivova, N., Lockwood, M., Mursula, K., O’Reilly, J., Owens, M., Scott, C. J., Sokoloff, D. D., Solanki, S. K., Soon, W., and Vaquero, J. M.: 2015, Astronomy and Astrophysics 581, A95

Usoskin, I. G., Gallet, Y., Lopes, F., Kovaltsov, G. A., and Hulot, G.: 2016a, Astronomy and Astrophysics 587, A150

Usoskin, I. G., Hulot, G., Gallet, Y., Roth, R., Licht, A., Joos, F., Kovaltsov, G. A., Thébault, E., and Khokhlov, A.: 2014, Astronomy and Astrophysics 562, L10

Usoskin, I. G., Kovaltsov, G. A., and Chatzistergos, T.: 2016b, Solar Physics 291(12), 3793

Usoskin, I. G., Kovaltsov, G. A., Lockwood, M., Mursula, K., Owens, M., and Solanki, S. K.: 2016c, Solar Physics 291(9-10), 2685

Usoskin, I. G., Kovaltsov, G. A., Mishina, L. N., Sokoloff, D. D., and Vaquero, J.: 2017, Solar Physics 292, \#15

Usoskin, I. G., Mursula, K., Arlt, R., and Kovaltsov, G. A.: 2009, The Astrophysical Journal Letters 700, L154

Usoskin, I. G., Mursula, K., and Kovaltsov, G. A.: 2001, Astronomy and Astrophysics 370, L31

Usoskin, I. G., Mursula, K., and Kovaltsov, G. A.: 2002, Geophysical Research Letters 29, 2183

Usoskin, I. G., Mursula, K., and Kovaltsov, G. A.: 2003a, in Solar Variability as an Input to the Earth's Environment, Vol. 535, pp 161-164

Usoskin, I. G., Mursula, K., and Kovaltsov, G. A.: 2003b, Astronomy and Astrophysics 403, 743

Usoskin, I. G., Mursula, K., and Kovaltsov, G. A.: 2003c, Solar Physics 218, 295

Usoskin, I. G., Mursula, K., Solanki, S., Schüssler, M., and Alanko, K.: 2004, Astronomy and Astrophysics 413, 745

Usoskin, I. G., Solanki, S. K., and Kovaltsov, G. A.: 2007, Astronomy and Astrophysics 471, 301

Usoskin, I. G., Solanki, S. K., Taricco, C., Bhandari, N., and Kovaltsov, G. A.: 2006, Astronomy and Astrophysics 457, L25

Vaquero, J. M.: 2007, Advances in Space Research 40, 929

Vaquero, J. M., Gallego, M. C., Acero, F. J., and García, J. A.: 2007, in P. Heinzel, I. Dorotovic, and R. J. Rutten (eds.), The Physics of Chromospheric Plasmas, Vol. 368 of Astronomical Society of the Pacific Conference Series 
Vaquero, J. M., Gallego, M. C., Usoskin, I. G., and Kovaltsov, G. A.: 2011, The Astrophysical Journal Letters 731, L24

Vaquero, J. M., Svalgaard, L., Carrasco, V. M. S., Clette, F., Lefèvre, L., Gallego, M. C., Arlt, R., Aparicio, A. J. P., Richard, J.-G., and Howe, R.: 2016, Solar Physics 291(910), 3061

Vaquero, J. M. and Vázquez, M.: 2009, The Sun Recorded Through History: Scientific Data Extracted from Historical Documents, Vol. 361 of Astrophysics and Space Science Library, Springer New York, New York, NY

Vautard, R., Yiou, P., and Ghil, M.: 1992, Physica D Nonlinear Phenomena 58, 95

Vernazza, J. E., Avrett, E. H., and Loeser, R.: 1981, The Astrophysical Journal Supplement Series 45, 635

Vögler, A. and Schüssler, M.: 2007, Astronomy and Astrophysics 465, L43

Vidal-Madjar, A.: 1975, Solar Physics 40, 69

Vieira, L. E. A., Solanki, S. K., Krivova, N. A., and Usoskin, I.: 2011, Astronomy and Astrophysics 531, 6

Viereck, R. A., Floyd, L. E., Crane, P. C., Woods, T. N., Knapp, B. G., Rottman, G., Weber, M., Puga, L. C., and DeLand, M. T.: 2004, Space Weather 2, S10005

Viereck, R. A. and Puga, L. C.: 1999, Journal of Geophysical Research 104, 9995

Vogler, F. L., Brandt, P. N., Otruba, W., and Hanslmeier, A.: 2005, Hvar Observatory Bulletin 29, 79

Vonmoos, M., Beer, J., and Muscheler, R.: 2006, Journal of Geophysical Research (Space Physics) 111, A10105

Vázquez, M. and Vaquero, J. M.: 2010, Solar Physics 267, 431

Vázquez, M., Vaquero, J. M., Gallego, M. C., Roca Cortés, T., and Pallé, P. L.: 2016, Solar Physics 291, 613

Wagner, G., Beer, J., Masarik, J., Muscheler, R., Kubik, P. W., Mende, W., Laj, C., Raisbeck, G. M., and Yiou, F.: 2001, Geophysical Research Letters 28, 303

Waldmeier, M.: 1939, Astronomische Mitteilungen der Eidgenössischen Sternwarte Zurich 14, 439

Waldmeier, M.: 1955, Ergebnisse und Probleme der Sonnenforschung., Vol. 22 of Probleme der kosmischen Physik, Akademische Verlagsgesellschaft

Walton, S. R., Chapman, G. A., Cookson, A. M., Dobias, J. J., and Preminger, D. G.: 1998, Solar Physics 179, 31

Walton, S. R. and Preminger, D. G.: 1999, The Astrophysical Journal 514, 959 
Wang, J., Zhou, G., Jin, C., and Li, H.: 2012, Solar Physics 278, 299

Wang, Y.-M., Lean, J. L., and Sheeley, Jr., N. R.: 2005, The Astrophysical Journal 625, 522

Wang, Y.-M. and Sheeley, Jr., N. R.: 1989, Solar Physics 124, 81

Warren, H. P., Byers, J. M., and Crump, N. A.: 2017, The Astrophysical Journal 836, 215

Webber, W. R. and McDonald, F. B.: 2013, Geophysical Research Letters 40, 1665

Wenzler, T., Solanki, S. K., Krivova, N. A., and Fröhlich, C.: 2006, Astronomy and Astrophysics 460, 583

White, O. R. and Livingston, W.: 1978, The Astrophysical Journal 226, 679

White, O. R. and Livingston, W. C.: 1981, The Astrophysical Journal 249, 798

White, W. B., Lean, J., Cayan, D. R., and Dettinger, M. D.: 1997, Journal of Geophysical Research 102, 3255

Wöhl, H.: 2005, Hvar Observatory Bulletin 29, 319

Willamo, T., Usoskin, I. G., and Kovaltsov, G. A.: 2017, Astronomy and Astrophysics in press

Williams, P. E. and Pesnell, W. D.: 2011, Solar Physics 270, 125

Willis, D. M., Henwood, R., Wild, M. N., Coffey, H. E., Denig, W. F., Erwin, E. H., and Hoyt, D. V.: 2013, Solar Physics 288, 141

Willis, D. M., Wild, M. N., Appleby, G. M., and Macdonald, L. T.: 2016a, Solar Physics 291(9-10), 2553

Willis, D. M., Wild, M. N., and Warburton, J. S.: 2016b, Solar Physics 291(9-10), 2519

Willson, R. C.: 1997, Science 277, 1963

Willson, R. C., Gulkis, S., Janssen, M., Hudson, H. S., and Chapman, G. A.: 1981, Science 211(4483), 700

Willson, R. C. and Hudson, H. S.: 1988, Nature 332, 810

Willson, R. C. and Mordvinov, A. V.: 2003, AGU Fall Meeting Abstracts 31

Wilsey, R. B. and Pritchard, H. A.: 1926, Journal of the Optical Society of America 12, 661

Wilson, O. C.: 1978, The Astrophysical Journal 226, 379

Wittmann, A. D. and Xu, Z. T.: 1987, Astronomy and Astrophysics Supplement Series 70, 83 
Wolf, R.: 1850, Astronomische Mitteilungen der Eidgenössischen Sternwarte Zurich 1, 27

Woods, T. N., Tobiska, W. K., Rottman, G. J., and Worden, J. R.: 2000, Journal of Geophysical Research 105, 27195

Worden, J. R., White, O. R., and Woods, T. N.: 1998a, The Astrophysical Journal 496, 998

Worden, J. R., White, O. R., and Woods, T. N.: 1998b, Solar Physics 177(1-2), 255

Worden, J. R., Woods, T. N., and Bowman, K. W.: 2001, The Astrophysical Journal 560, 1020

Xiang, B., Wang, B., Lauer, A., Lee, J.-Y., and Ding, Q.: 2014, Climate Dynamics 43, 259

Yang, S. and Zhang, J.: 2014, The Astrophysical Journal 781, 7

Yau, K. K. C. and Stephenson, F. R.: 1988, Quarterly Journal of the Royal Astronomical Society 29, 175

Yeo, K. L., Ball, W. T., Krivova, N. A., Solanki, S. K., Unruh, Y. C., and Morrill, J.: 2015a, Journal of Geophysical Research (Space Physics) 120, 6055

Yeo, K. L., Feller, A., Solanki, S. K., Couvidat, S., Danilovic, S., and Krivova, N. A.: 2014a, Astronomy and Astrophysics 561, 22

Yeo, K. L., Krivova, N. A., and Solanki, S. K.: 2015b, in The Solar Activity Cycle, Vol. 53 of Space Sciences Series of ISSI, p. 137, Springer New York

Yeo, K. L., Krivova, N. A., and Solanki, S. K.: 2017, Journal of Geophysical Research: Space Physics p. 2016JA023733

Yeo, K. L., Krivova, N. A., Solanki, S. K., and Glassmeier, K. H.: 2014b, Astronomy and Astrophysics 570, A85

Yeo, K. L., Solanki, S. K., and Krivova, N. A.: 2013, Astronomy and Astrophysics 550, 95

Zacharias, P.: 2014, Surveys in Geophysics 35(4), 897

Zank, G. P. and Müller, H.-R.: 2003, Journal of Geophysical Research (Space Physics) 108, 1240

Zhao, J. and Li, H.: 2012, Research in Astronomy and Astrophysics 12, 1681

Zharkova, V. V., Ipson, S. S., Zharkov, S. I., Benkhalil, A., Aboudarham, J., and Bentley, R. D.: 2003, Solar Physics 214, 89

Zirin, H.: 1974, Solar Physics 38(1), 91 
Zirin, H. and Popp, B.: 1989, The Astrophysical Journal 340, 571

Zolotova, N. V. and Ponyavin, D. I.: 2011, The Astrophysical Journal 736, 115

Zolotova, N. V. and Ponyavin, D. I.: 2015, The Astrophysical Journal 800, 42

Zolotova, N. V. and Ponyavin, D. I.: 2016, Solar Physics 291(9-10), 2869

Zuccarello, F., Contarino, L., and Romano, P.: 2011, Contributions of the Astronomical Observatory Skalnate Pleso 41, 85

Zwaan, C.: 1978, Solar Physics 60, 213

Zwaan, C.: 1985, Solar Physics 100, 397 



\section{Scientific contributions}

\section{Refereed publications}

- Usoskin I. G., Kovaltsov G. A., and Chatzistergos T. 2016, "Dependence of the Sunspot-Group Size on the Level of Solar Activity and its Influence on the Calibration of Solar Observers", Solar Physics 291(12), 3793

- Chatzistergos T., Usoskin I. G., Kovaltsov G. A., Krivova N. A., Solanki S. K. 2017, "New reconstruction of the sunspot group number since 1739 using the direct calibration and 'backbone' methods", in A\&A , 602, A69

\section{Conference proceedings}

- Chatzistergos T., Ermolli I., Solanki S. K., and Krivova N. A. 2016, "Exploiting Four Historical Ca II K Spectroheliogram Archives", in publications of the Astronomical Society of the Pacific, Vol. 504, Coimbra, 227-231

\section{Conference contributions}

- Chatzistergos T., Ermolli I., Solanki S. K., and Krivova N. A., "Exploiting Four Historical Ca II K Spectroheliogram Archives: Preliminary results from four archives", in 2nd Coimbra solar physics meeting, Coimbra, Portugal, 5-9/10/2015 (talk)

- Chatzistergos T., Ermolli I., Solanki S. K., and Krivova N. A., "Examination of spectroheliogram archives", in Space climate symposium 6, Levi, Finland, 47/04/2016 (talk)

- Chatzistergos T., Ermolli I., Solanki S. K., and Krivova N. A., "The potential of historical spectroheliograms for Sun-climate studies", in 9th IAGA - ICAMA/IAMAS - ROSMIC/VarSITI/SCOSTEP workshop on Long-Term Changes in the Atmosphere, Kühlungsborn, Germany, 19-23/09/2016 (talk)

- Chatzistergos T., Ermolli I., Solanki S. K., and Krivova N. A., "Exploring Historical Ca II K Spectroheliogram Archives", in XIVth Hvar astrophysical colloquium, Hvar, Croatia, 26-30/09/2016 (talk) 
- Chatzistergos T., Ermolli I., Solanki S. K., and Krivova N. A., "Handling Historical Ca II K Spectroheliogram Archives", in AGU fall meeting, San Francisco, U.S.A., $12-16 / 12 / 2016$ (poster)

- Chatzistergos T., Usoskin I. G., Kovaltsov G. A., Krivova N. A., and Solanki S. K., "Non-linear re-calibration of group sunspot number series back to 1739", in AGU fall meeting, San Francisco, U.S.A., 12-16/12/2016 (talk and poster)

- Chatzistergos T., Usoskin I. G., Kovaltsov G. A., Krivova N. A., and Solanki S. K., "New calibration of the group sunspot number series using a non-linear nonparametric method", in DPG meeting, Bremen, Germany, 13-17/03/2017 (talk) 


\section{Acknowledgements}

Special thanks to my supervisors Sami Solanki and Natalie Krivova for their guidance, full support and for giving me the opportunity to carry out this work. My gratitude to Natalie Krivova for being there whenever I had a problem, her patience with me knowing that it would never be short.

Mille grazie al gruppo solare presso l'osservatorio di Roma, Ilaria, Alice, Carla, Fabio, Fabrizio, Marco, Marco, Ronaldo, per la loro gentilezza e ospitalità. Un ringraziamento speciale a Ilaria Ermolli per il suo sostegno e tutto l'aiuto di cui ha fornito durante i miei studi.

Я хочу поблагодарить группу космической физики в университете Оулу за гостеприимство во время моих визитов. Особая благодарность Ilya Usoskin и Gennady Kovaltsov.

I want to thank the Max Planck Institute for Solar System Research (MPS) and the International Max Planck Research School for Solar System Science (IMPRS) for the funding they provided. Many thanks to Sonja Schuh for all her help and support throughout my studies at MPS.

I am indebted to Eleanna Asvestari, Ilaria Ermolli, Greg Kopp, David Marshall, and Jörn Warnecke for proof-reading parts of this thesis.

I want to thank my office mates, David Martin Belda, Franzisca Zeuner, Iulia Chifu, Kok Leng Yeo, Maria Andriopoulou for making the office hours more fun.

The IMPRS band members, Ankit Barik, Earl Bellinger, Alessandro Cilla, Hans Huybrighs, Abbey Ingram, Fatima Kahil, Felix Mackebrandt, David Marshall, Helge Mißbach, Emanuele Papini, Bastian Proxauf, Kenny Roffo, and Holly Waller for helping me have a break with our rehearsals on Mondays, and giving me enough reasons to complain and be bossy.

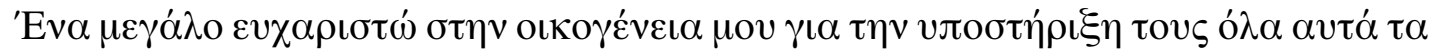

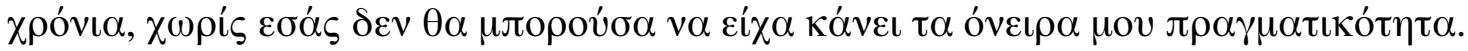





\section{Curriculum vitae}

\section{Personal data}

Name: Theodosios Chatzistergos

Day of birth: 14/09/1986

Place of birth: Kos, Greece

\section{Education}

- 10/2013-06/2017 PhD

Max Planck Institute for Solar System Research, Göttingen, Germany

Georg-August-Universität, Göttingen, Germany.

International Max Planck Research School for Solar System Science. Stipend holder.

- 09/2012-08/2013 MSc (Astrophysics)

Queen Mary University, London, England. Thesis: "Satellite perturbations on planetary rings". Supervisor: Prof. Carl D. Murray.

- 02/2005-12/2011 BSc (Physics).

Faculty of Physics at National and Kapodistrian University of Athens, Greece. Thesis: "The atmosphere of Saturn's satellite, Titan, using data from the space mission Cassini-Huygens". Supervisor: Prof. Xenophon Moussas.

\section{Publications}

- Usoskin I. G., Kovaltsov G. A., and Chatzistergos T. 2016, "Dependence of the Sunspot-Group Size on the Level of Solar Activity and its Influence on the Calibration of Solar Observers", Solar Physics 291(12), 3793

- Chatzistergos T., Usoskin I. G., Kovaltsov G. A., Krivova N. A., Solanki S. K. 2017, "New reconstruction of the sunspot group number since 1739 using the direct calibration and 'backbone' methods", in A\&A, 602, A69, 2017 
Theodosios Chatzistergos: Analysis of historical solar observations and long-term changes in solar irradiance

The Sun has the potential to affect Earth's climate. However, the short time span covered by direct measurements of the solar irradiance makes any meaningful conclusions about solar influence on climate difficult. Models have been developed that reconstruct the past solar irradiance from the available long-term proxies of solar magnetic activity. The most widely used dataset is the sunspot number series. However, it only gives information on the dark regions on the solar disc. Another type of data that might provide information critical for past irradiance reconstructions are the $\mathrm{Ca}$ II $\mathrm{K}$ spectroheliograms. Until now they have not been used due to the various artefacts plaguing them. In this thesis we present a new method to automatically process and photometrically calibrate the historical Ca II K spectroheliograms. We also reassess the relation between the $\mathrm{Ca}$ II $\mathrm{K}$ brightness and the magnetic field strength. This opens prospects for these data to be used to derive information about the evolution of the solar magnetic field and the solar irradiance over the whole 20th century. We also recalibrate the historical record of the group sunspot number. In particular, we show that the assumption of the linear relation between records by different observers is not valid in general and produce a new compilation with a direct non-parametric and non-linear cross-calibration of the individual records. 